New Home Cemetery (41FB334): Archaeological Search Exhumation, and Reinterment of Multiple Historic Graves along FM 1464, Sugar Land, Fort Bend County, Texas

Mary Cassandra Hill

Jeremy W. Pye

Follow this and additional works at: https://scholarworks.sfasu.edu/ita

Part of the American Material Culture Commons, Archaeological Anthropology Commons, Environmental Studies Commons, Other American Studies Commons, Other Arts and Humanities Commons, Other History of Art, Architecture, and Archaeology Commons, and the United States History Commons

Tell us how this article helped you.

This Article is brought to you for free and open access by the Center for Regional Heritage Research at SFA ScholarWorks. It has been accepted for inclusion in Index of Texas Archaeology: Open Access Gray Literature from the Lone Star State by an authorized editor of SFA ScholarWorks. For more information, please contact cdsscholarworks@sfasu.edu. 


\section{New Home Cemetery (41FB334): Archaeological Search Exhumation, and Reinterment of Multiple Historic Graves along FM 1464, Sugar Land, Fort Bend County, Texas}

\section{Licensing Statement}

This is a work for hire produced for the Texas Department of Transportation (TxDOT), which owns all rights, title, and interest in and to all data and other information developed for this project under its contract with the report producer. The report may be cited and brief passages from this publication may be reproduced without permission provided that credit is given to TxDOT and the firm that produced it. Permission to reprint an entire chapter, section, figures or tables must be obtained in advance from the Supervisor of the Archeological Studies Branch, Environmental Affairs Division, Texas Department of Transportation, 125 East 11th Street, Austin, Texas, 78701 
NEW HOME CEMETERY (41FB334):

ARCHAEOLOGICAL SEARCH, EXHUMATION,

AND REINTERMENT OF MULTIPLE HISTORIC

GRAVES ALONG FM 1464, SUGAR LAND,

4. FORT BEND COUNTY, TEXAS

\section{(CSJ 1415-02-032)}

by

Dr. Mary Cassandra Hill

Jeremy W. Pye

Principal Investigator

Duane E. Peter

for

Texas Department of Transportation

Environmental Affairs Division

ARCHEOLOGICAL STUDIES PROGRAM REPORT NUMBER 145

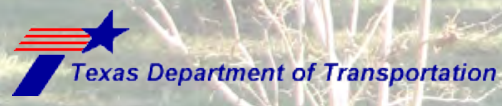

and

TeXas Antiquities Permit No. 5804

MISCELLANEOUS REPORTS OF INVESTIGATIONS

NUMBER 545

\section{GMI}

December 2012 



\title{
NEW HOME CEMETERY (41FB334): ARCHAEOLOGICAL SEARCH, EXHUMATION, AND REINTERMENT OF MULTIPLE HISTORIC GRAVES ALONG FM 1464, SUGAR LAND, FORT BEND COUNTY, TEXAS \\ (CSJ 1415-02-032)
}

\author{
by \\ Mary Cassandra Hill \\ Jeremy W. Pye \\ with contributions by \\ Melissa M. Green \\ Lindsey Skelton \\ Adam Leroy \\ Teresa Nugent
}

Principal Investigator

Duane E. Peter

for

Texas Department of Transportation

Environmental Affairs Division

118 East Riverside Drive

Austin, Texas 78704

Archeological Studies Program Report Number 145

TEXAs ANTIQUities PERMit Number 5804

MISCELLANEOUS REPORTS OF INVESTIGATIONS

NUMBER 545

Geo-Marine, Inc.

$2201 \mathrm{~K}$ Avenue, Suite A2

Plano, Texas 75074

December 2012 
Copyright (C) 2012

\title{
Texas Department of Transportation (TxDOT)
}

This is a work for hire produced for the Texas Department of Transportation (TxDOT), which owns all rights, title, and interest in and to all data and other information developed for this project under Contract No. 579 xx SA005, Work Authorization 08. Brief passages from this publication may be reproduced without permission, provided that credit is given to TxDOT and Geo-Marine, Inc. Permission to reprint an entire chapter, section, figures, or tables must be obtained in advance from the Supervisor of the Archeological Studies Program, Environmental Affairs Division, Texas Department of Transportation, 125 East 11th St., Austin, Texas 787012483. Copies of this publication have been deposited with the Texas State Library in compliance with the State Depository requirements.

\author{
Printed by Sir Speedy \\ Richardson, Texas
}

published by:

Texas Department of Transportation

Environmental Affairs Division

Archeological Studies Program

Scott Pletka, Ph.D., Supervisor

Archeological Studies Program, Report No. 145

and

Geo-Marine, Inc.

Plano, Texas

Miscellaneous Reports of Investigations Number 545

GMI No. 22005.00.08

ISBN: 978-1-935545-12-5 


\section{MANAGEMENT SUMMARY}

The Texas Department of Transportation (TxDOT) plans improvements to Farm-to-Market road (FM) 1464 between Stratford Creek Drive and Oyster Creek, Sugar Land, Texas, in eastern Fort Bend County, southwest of downtown Houston. Those proposed improvements will expand the FM 1464 right-of-way about 20 feet eastward into an approximately 328-foot length adjacent to New Home Cemetery, which is at the intersection of FM 1464 and Orchard Lake Estates Drive. This report recounts the recovery, analysis, and reinterment of the human remains, funerary objects and furnishings, and all associated artifacts associated with burials found in the right-ofway adjacent to New Home Cemetery (41FB334) as proscribed by contractual agreements between Geo-Marine, Inc., and TxDOT.

Previous investigations within the right-of-way during the spring of 2010 had encountered human remains and associated funerary objects, prompting further excavation of the right-of-way bordering the cemetery. Geo-Marine was subsequently contracted by TxDOT to search, exhume, analyze, and rebury any human remains found within the right-of-way and under the existing roadbed of FM 1464. From November 15, 2010, to December 17, 2010, Geo-Marine conducted the first phase of fieldwork with the archaeological search, exhumation, and analysis of multiple historic graves. A total of 24 unmarked burials-11 complete skeletons and 13 partial-was recovered. The comprehensive osteological analyses included the identification of sex, age, ethnicity, pathology, nutrition, and other health history, plus possible/probable cause of death. The detailed analyses revealed that these individuals were engaged in an active lifestyle mostly associated with physical labor. This lifestyle unfortunately led to severe physical pain and suffering, and at times early death. During that investigative phase, funerary materials, including casket handles, nails, and thumbscrews, were sent to the funerary hardware specialist to determine typology, material, and timeframe. Based on the funerary analyses, the timeframe for the burials ranged from 1895 through the late 1960s. In addition, a small area indicating a prehistoric component was identified near the north end of the scraped area, but contained no evidence of the historic cemetery.

The second phase of the project began on May 2, 2011, with the systematic scraping of the area located under the old roadbed. No burials or human remains were encountered during that phase. Only a small amount of possible funerary material was recovered from underneath the roadway; the majority of items encountered were associated with modern or industrial trash. Reinterment 
occurred on May 9, 2011, during the second phase of search and recovery. The 24 individuals removed and recorded during Phase I were reinterred, along with all associated funerary objects and furnishings as well as with all associated artifacts, within the existing New Home Cemetery. 


\begin{abstract}
The Texas Department of Transportation (TxDOT) plans improvements to Farm-to-Market road (FM) 1464 between Stratford Creek Drive and Oyster Creek, Sugar Land, Texas, in eastern Fort Bend County, southwest of downtown Houston. Those proposed improvements will expand the FM 1464 right-of-way about 20 feet eastward into an approximately 328-foot length adjacent to New Home Cemetery (41FB334), which is at the intersection of FM 1464 and Orchard Lake Estates Drive.

Geo-Marine was contracted by TxDOT to search, exhume, analyze, and rebury any human remains found within the right-of-way and under the existing roadbed of FM 1464. From midNovember to mid-December 2010, Geo-Marine conducted Phase I fieldwork - the archaeological search, exhumation, and analysis of multiple historic graves. A total of 24 unmarked burials-11 complete skeletons and 13 partial-was recovered. The comprehensive osteological analyses, which included the identification of sex, age, ethnicity, pathology, nutrition, and other health history, plus possible/probable cause of death, revealed that these individuals were engaged in an active lifestyle, mostly associated with physical labor that led to severe physical pain and suffering, and at times early death. During Phase I, analyses of funerary materials, including markers, burial containers, casket handles, plaques, nails, and thumbscrews, was undertaken to determine typology, material, and timeframe. Based on the funerary analyses, the timeframe for the burials ranged from 1895 through the late 1960s. Additionally, a small area containing prehistoric materials was also encountered, but the historic cemetery did not appear to overlap.

On May 2, 2011, Phase II of the project began with the systematic scraping of the area located under the old roadbed. No burials or human remains were encountered during that phase. On May 9, 2011, the 24 individuals removed and recorded during Phase I were reinterred, along with all associated funerary objects, furnishings, and all associated artifacts, within the existing New Home Cemetery.
\end{abstract}





\section{TABLE OF CONTENTS}

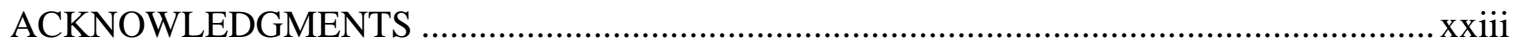

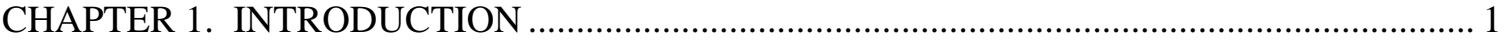

CHAPTER 2. ENVIRONMENTAL SETTING AND HISTORIC BACKGROUND ................... 7

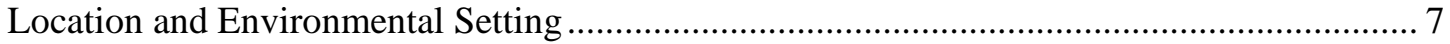

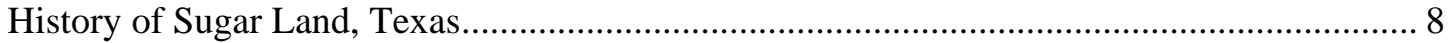

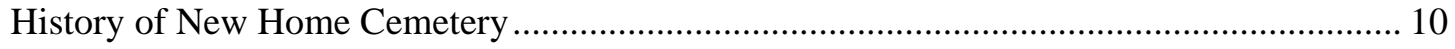

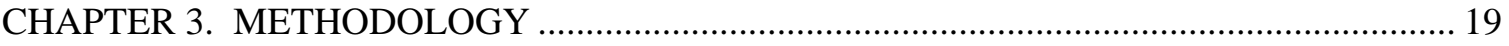

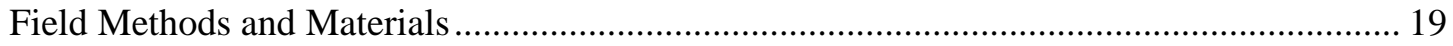

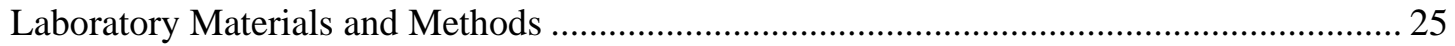

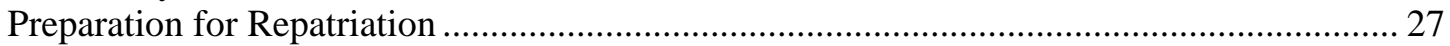

CHAPTER 4. BIOARCHAEOLOGICAL DESCRIPTION AND REPATRIATION

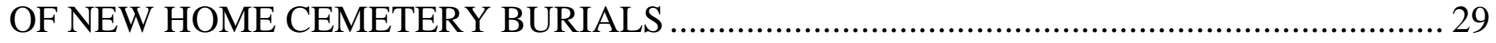

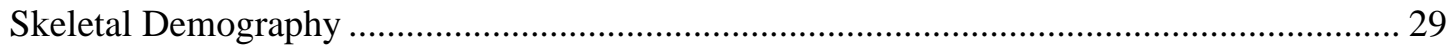

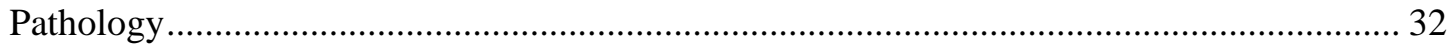

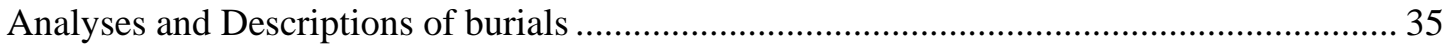

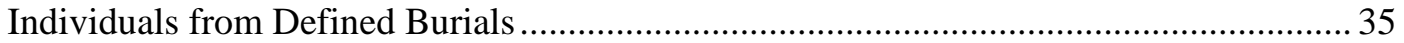

Burial 1: Undetermined Age; Undetermined Sex …................................................ 35

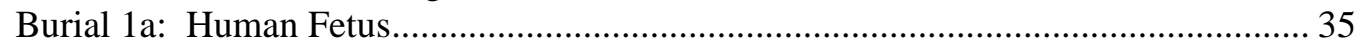

Burial 2: Adolescent, Approximately $15 \pm 2$ Years; Undetermined Sex ....................... 35

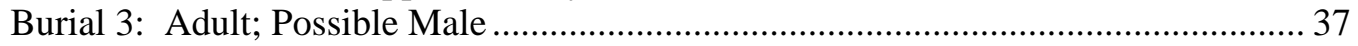

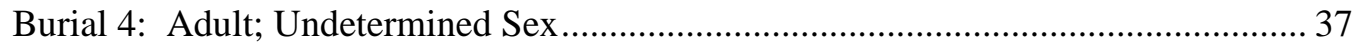

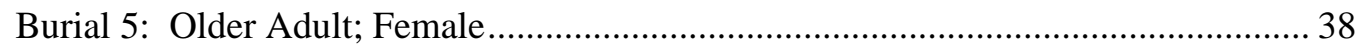

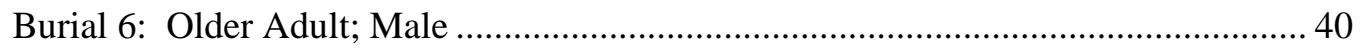

Burial 7: Young Adult (Early 20s); Possible Male (Ambiguous) ................................ 41

Burial 8: Possible Young Adult; Possible Male ............................................................. 43

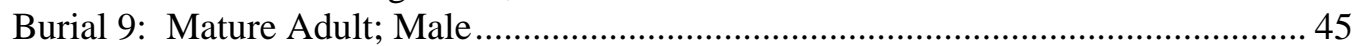

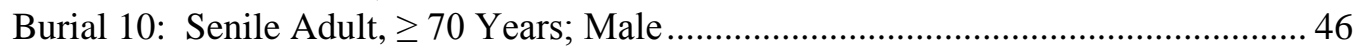

Burial 11: Adult; Probable Female (Ambiguous) .......................................................... 48

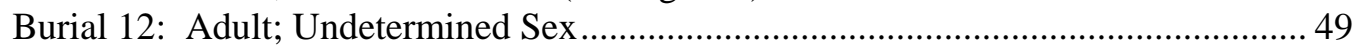

Burial 13: Younger Adult; Female ......................................................................... 49 
Table of Contents

(cont'd)

Burial 14: Senile Adult, Late 70s to Early 80s; Female ................................................. 50

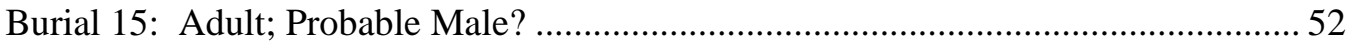

Burial 16: Young Adult, 24 to 27 Years; Male .......................................................... 52

Burial 17: Adult; Undetermined Sex (Possibly Male??) ............................................. 55

Burial 18: Mature Adult, 58 to 71 Years; Male............................................................ 55

Burial 19: Senile Adult 70.4 to 82.3 Years; Female..................................................... 57

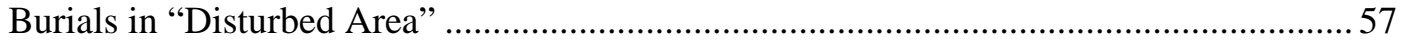

Coffin 1: Mature Adult (46 to 58 Years); Undetermined Sex..................................... 58

Coffin 2: Young Adult (Late Teens/Early 20s); Undetermined Sex ............................. 59

Coffin 3: Undetermined Age; Undetermined Sex ........................................................... 59

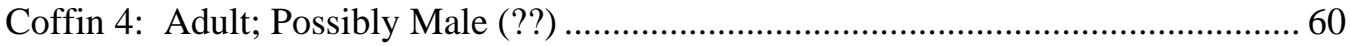

Coffin 5: Senile Adult (Early 70s to Early 80s); Probable Female ........................................ 60

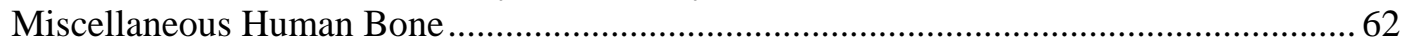

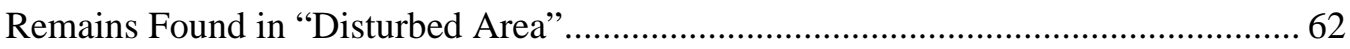

Bone Recovered from the Backdirt Pile or Surface Collection...................................... 63

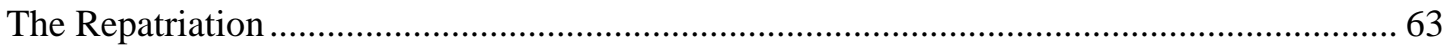

CHAPTER 5. TYPOLOGY AND ANALYSIS OF MORTUARY AND PERSONAL

ARTIFACTS RECOVERED FROM EXCAVATIONS AT NEW HOME CEMETERY .......... 73

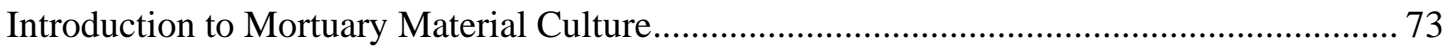

Typology Methodology and Comparative Materials......................................................... 74

United States Patent Records: Utility Patents $(1839-1965)(n=2,160)$.............................. 74

United States Patent Records: Design Patents $(1843-1965)(\mathrm{n}=455)$................................ 74

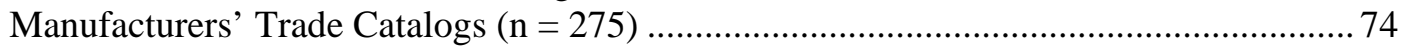

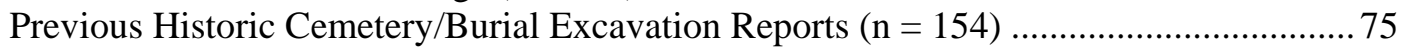

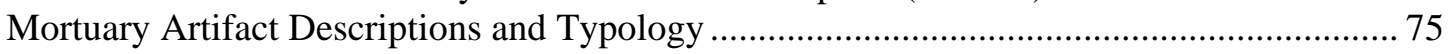

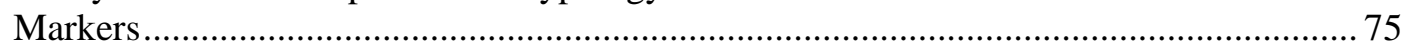

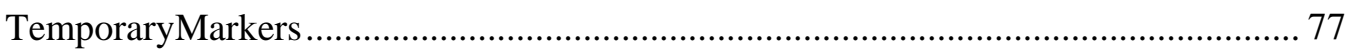

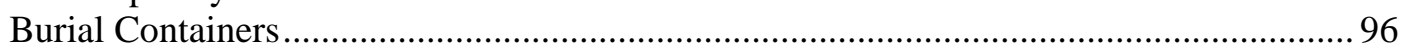

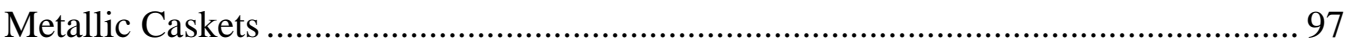

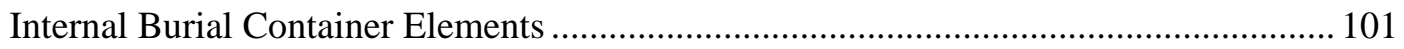

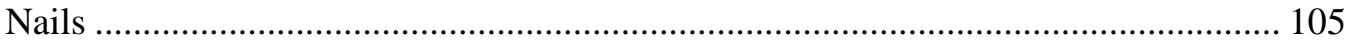

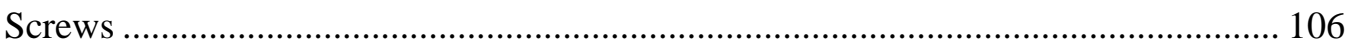

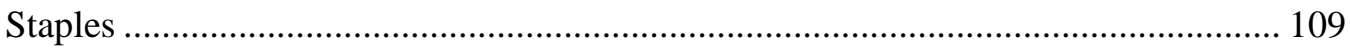

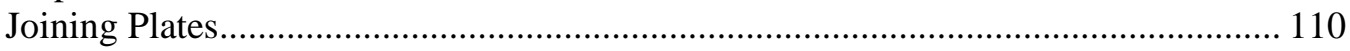

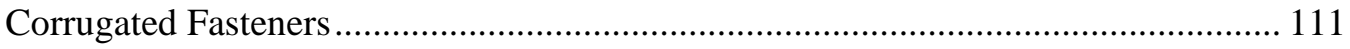

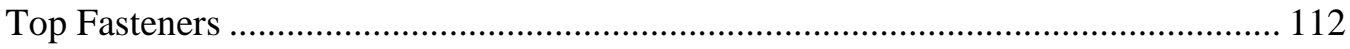

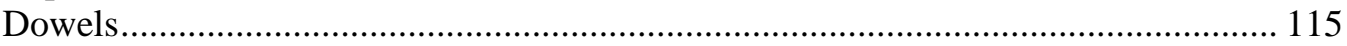

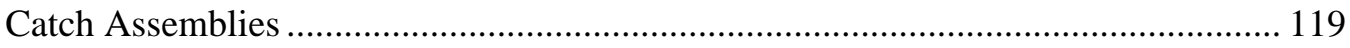

Separable Stop Hinge Assemblies.......................................................................... 130

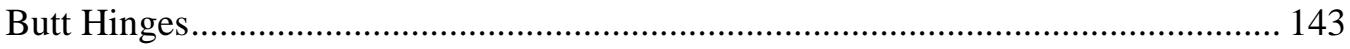

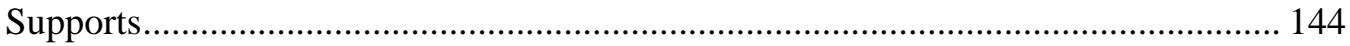

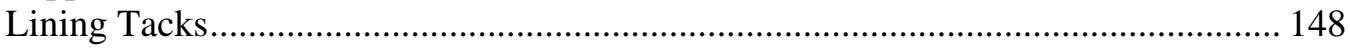


Table of Contents

(cont'd)

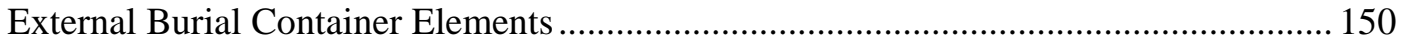

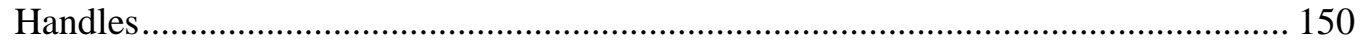

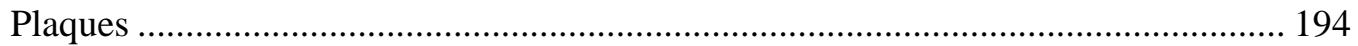

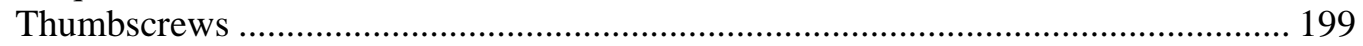

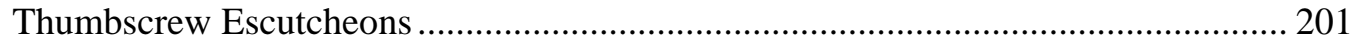

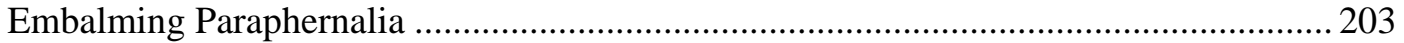

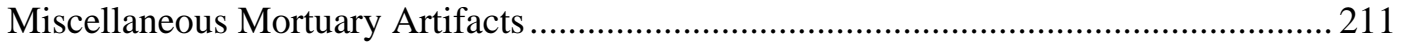

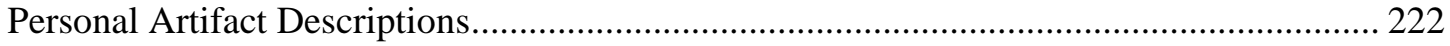

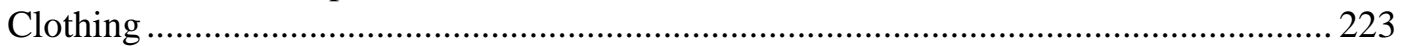

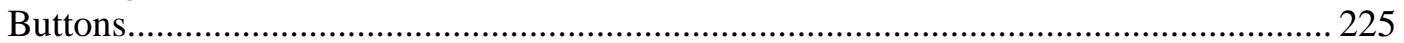

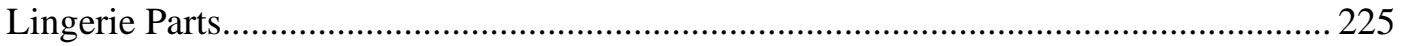

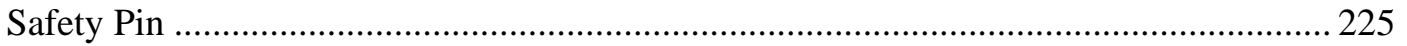

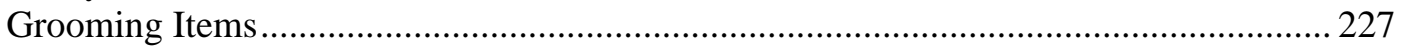

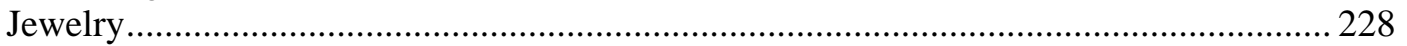

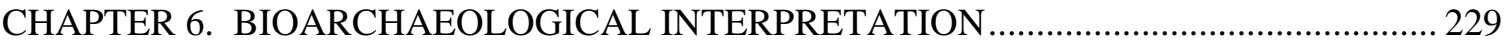

African and African-American Burial Practices and the Southern Folk Tradition ................ 229

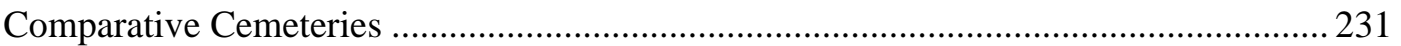

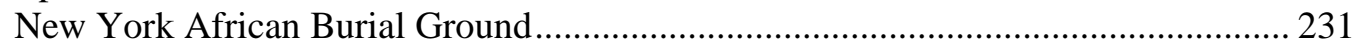

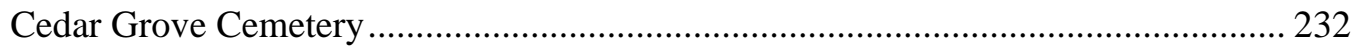

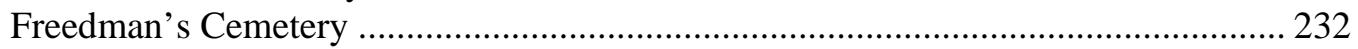

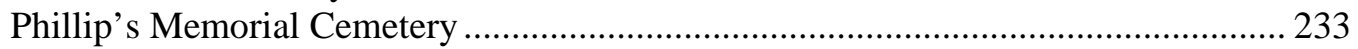

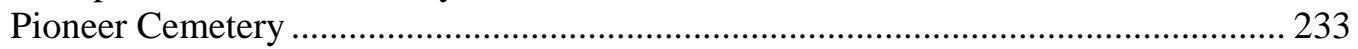

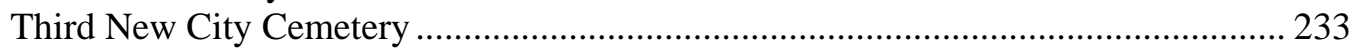

New Home Cemetery Burials in Context ..................................................................... 234

Cemeteries Located in Groves of Trees .................................................................. 234

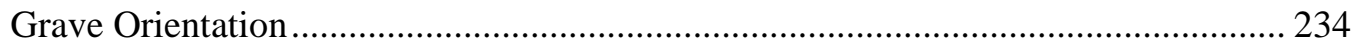

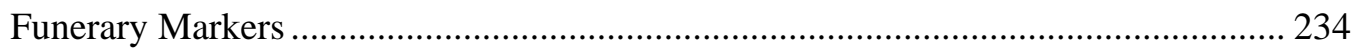

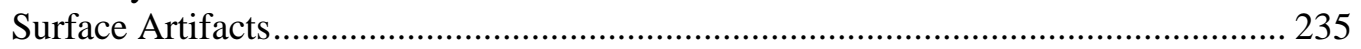

Burial Containers and Hardware ............................................................................... 237

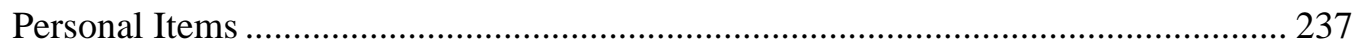

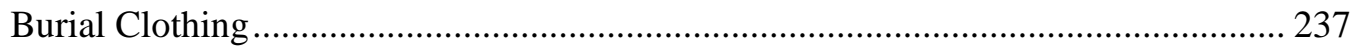

Chronology of New Home Cemetery Burials ................................................................ 238

Quality of Life: Health and Socioeconomics at New Home Cemetery ........................... 240

Demography and Skeletal Analysis ........................................................................... 240

Society and Access to Health Care.......................................................................... 246

Burial Socioeconomics and Social Support ........................................................... 248

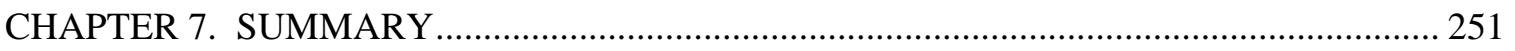

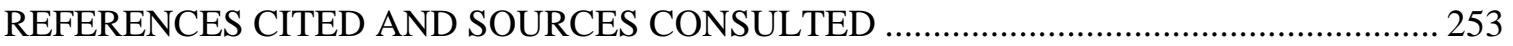


Table of Contents

(cont'd)

APPENDICES:

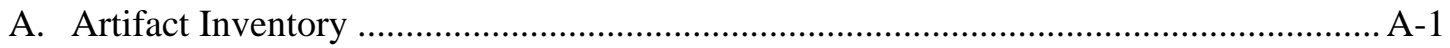

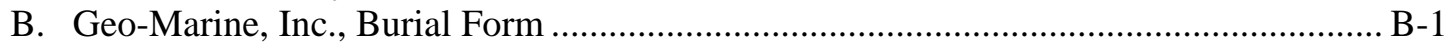

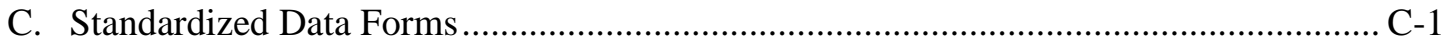

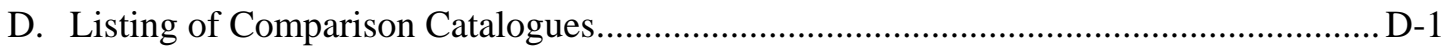

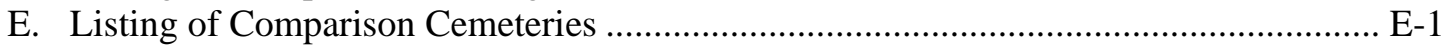

F. Evidence of Prehistoric Occupation and Lithic Artifact Raw Data ...................................-1 


\section{LIST OF FIGURES}

1. Topographic map showing the general location of the project area in Fort Bend

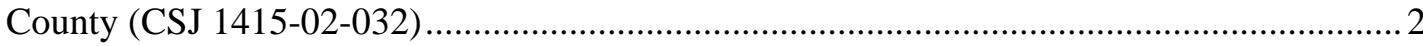

2. Plan map of Phase I investigations along FM 1464 at New Home Cemetery

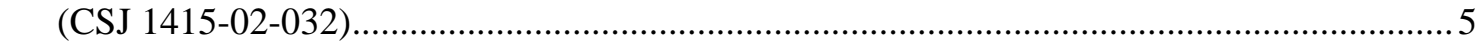

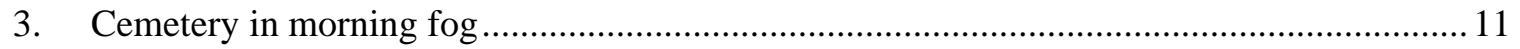

4. NE area of cemetery, looking east ................................................................................... 13

5. Small broken crosses in open area of cemetery, view west .................................................. 14

6. Open area of cemetery looking west from eastern margin of cemetery ................................ 14

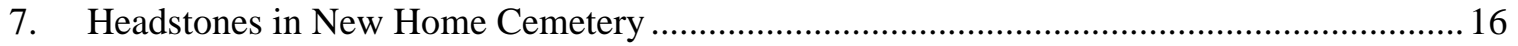

8. Row of stones on southern margin of cemetery, looking north ........................................... 17

9. New Home Cemetery sign, view northeast ................................................................... 17

10. Aerial imagery showing the Phase I and Phase II project areas adjacent to

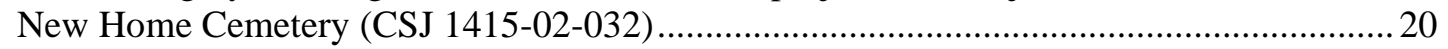

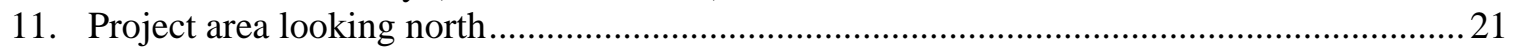

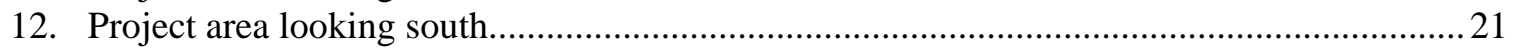

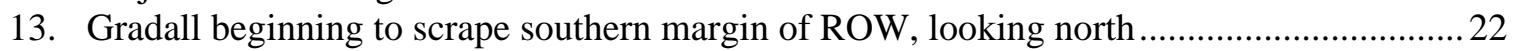

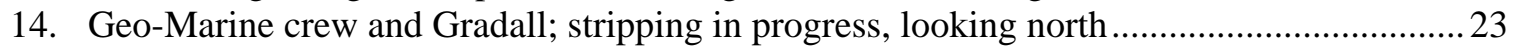

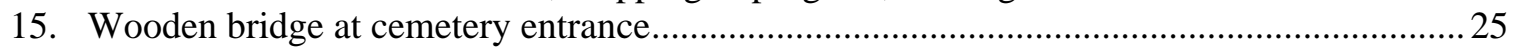

16. Plan map of the southern portion of Phase I and Phase II project areas adjacent

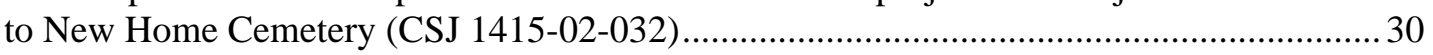

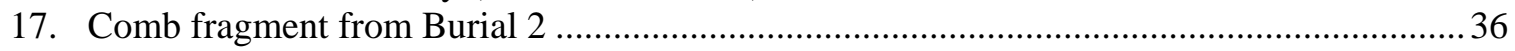

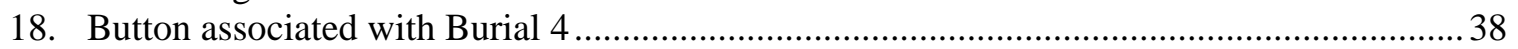

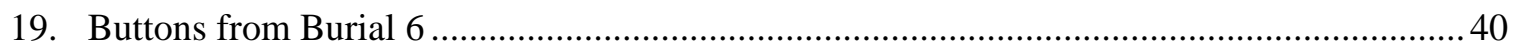

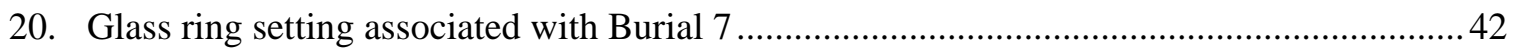

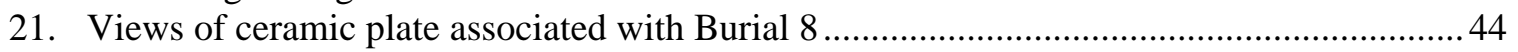

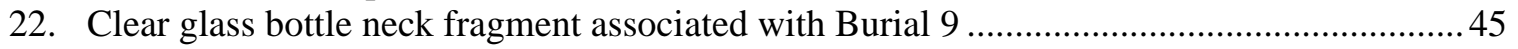

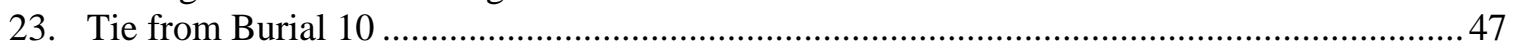

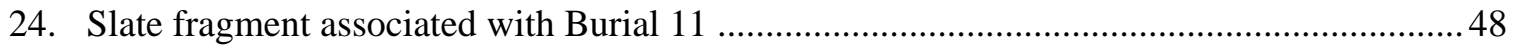

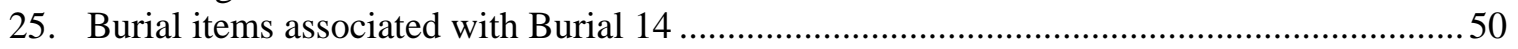

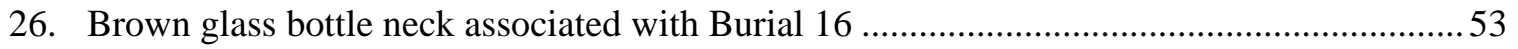

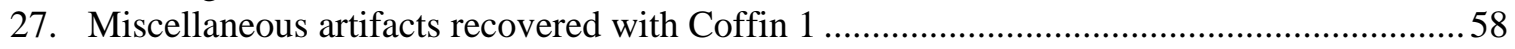

28. Matching pair of caskets (Coffins 3 [left] and 4 [right]), looking west ................................ 59 
List of Figures

(cont'd)

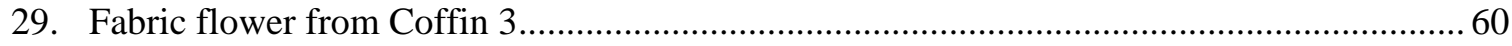

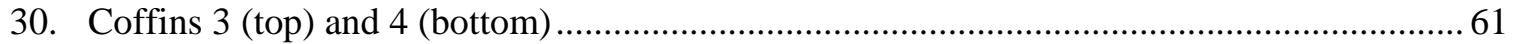

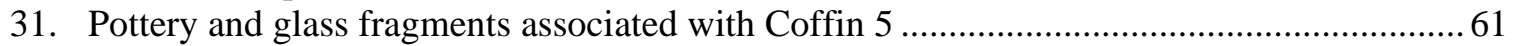

32. Mr. and Mrs. Jones and M. C. Hill monitor excavation of the trench, facing east ................ 64

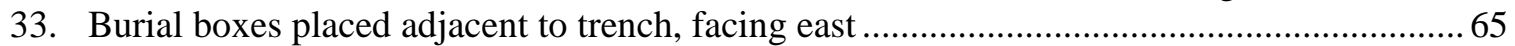

34. Geo-Marine crew measuring and placing burial boxes in trench ........................................66 66

35. Geo-Marine crew placing burial boxes in trench, facing east.............................................67 67

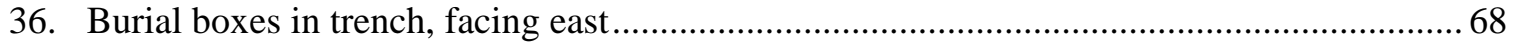

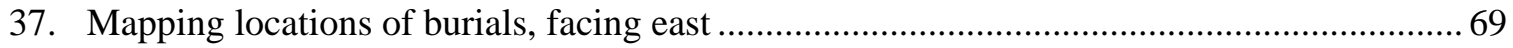

38. Plan map of the reinterment locations within New Home Cemetery (CSJ 1415-02-032)..... 70

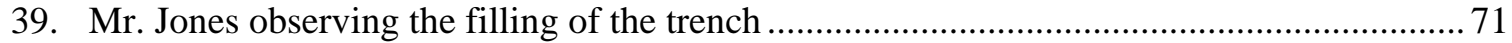

40. Placement of markers adjacent to boxes in trench, facing east............................................ 71

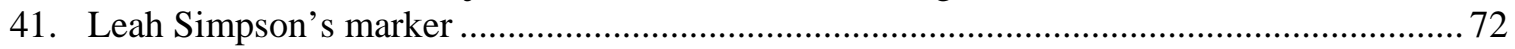

42. Geo-Marine crew measuring and placing marker stones on surface of trench,

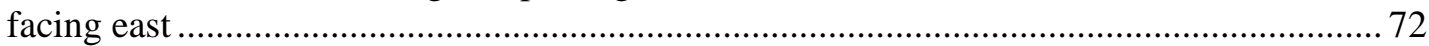

43. U.S. Utility Patent No. 473296 assigned in 1892 to John A. Coffey for a grave marker ....... 76

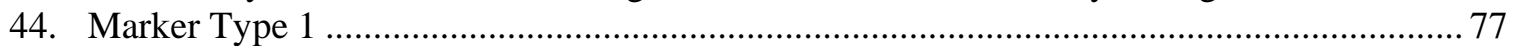

45. Comparison of Marker Type 1 and and low-resolution laser-scanned image

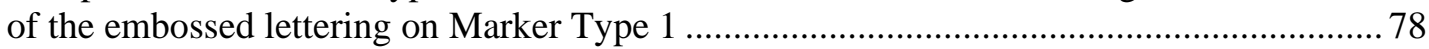

46. U.S. Utility Patent No. 1276798 (a) and No. 1573268 (b) assigned, respectively, in 1918 and 1926, to William F. Norman for grave markers............................................... 79

47. Advertisement for Norman's Grave Marker in the April 11, 1919, issue of the

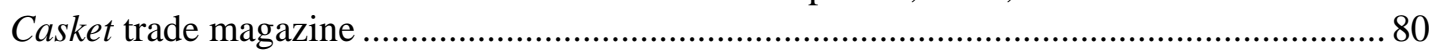

48. Advertisement for Norman's Grave Marker in the May 15, 1929, issue of the

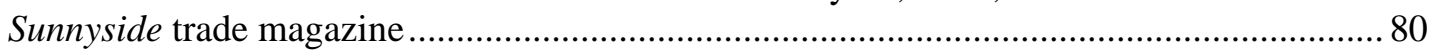

49. Regular Norman Grave Marker, which is similar to Marker Type 1, depicted on page 38 of the 1934 J. Oliver Johnson, Inc., catalog ....................................................... 81

50. Norman Series Temporary Grave Markers on page C172 of the 2002a Kelco Supply

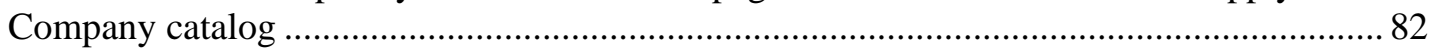

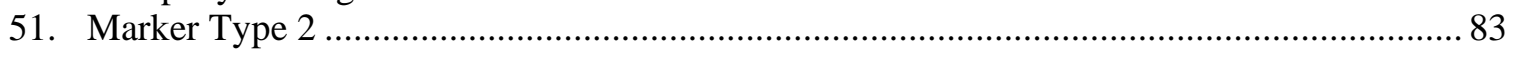

52. U.S. Utility Patent No. 1,651,780 asigned in 1927 to Marion G. Slawson for a grave marker .

53. Shield-shaped "Perfection Grave Marker," which matches Marker Type 2, depicted on page 39 of the 1934 J. Oliver Johnson, Inc., catalog.

54. Shield-shaped temporary grave marker, which matches Marker Type 2, depicted on page 163 of a 1930s Undertakers' Supply Company catalog.............................. 86

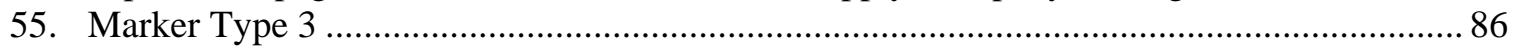

56. U.S. Utility Patent No. 2,083,873 assigned in 1937 to Edward C. Sisson et al. for an inscription tablen and temporary marker.

57. Aluminum inscription plate for "Leah Simpson" (Burial 5) associated with Marker Type 3

58. Sisto Aluminum Marker No. 5783, which matches Marker Type 3, depicted on page 91 of the ca. 1930s Undertakers' Supply Company catalog 


\section{List of Figures}

(cont'd)

59. Sisto Grave Marker No. 8317, which matches Marker Type 3, depicted on page 168 of the ca. 1950 Royal Bond, Inc., catalog

60. Sisto Marker No. 47869-5, which matches Marker Type 3, depicted on page

C173 of the 2002a Kelco Supply Company catalog...

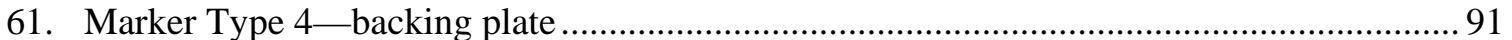

62. U.S. Utility Patent No. 1,589,199 assigned in 1926 to J. M. McNeill for a grave marker .... 92

63. “McNeill Grave Marker” No. 8394, which matches Marker Type 4, depicted on page 169 of the circa 1950 Royal Bond, Inc., catalog.

64. Slotted temporary grave marker No. 900, which matches Marker Type 4, depicted on page C171 of the 2002a Kelco Supply Company catalog ................................... 94

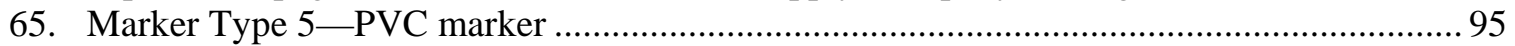

66. Field photo showing PVC grave marker in situ in an undisturbed area of New Home

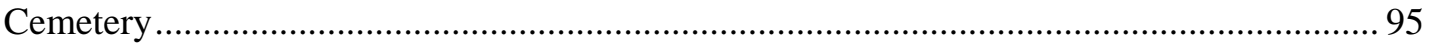

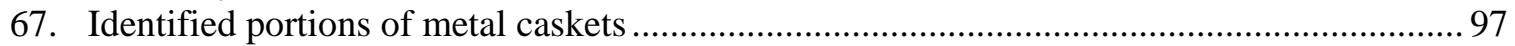

68. Examples of casket shell types from Thacker Casket, Inc., Training Module 4....................98

69. Gasketed vs. nongasketed caskets from Thacker Casket, Inc., Training Module 4...............99

70. Examples of casket hardware types from Thacker Casket, Inc., Training Module 4 ............99

71. Examples of finish types from Thacker Casket, Inc., Training Module 4........................... 100

72. Field photo showing Coffins 3 and 4 (Metal Casket Type 1) after uncovering

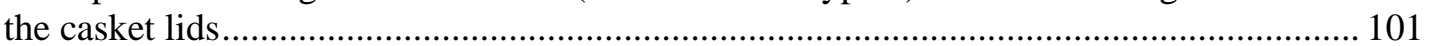

73. Field photo showing Coffin 4 (Metal Casket Type 1) after removal from grave feature .... 102

74. Blue-brushed, stainless steel accoseal casket depicted on a 1999 Aurora Casket Company advertising card that is similar to Metal Casket Type 1 ...................................... 102

75. New Pointe Triton Grey 20-gauge steel casket depicted on a 1998 Batesville Casket Company advertising card that shows handles similar to Handle Type 23 found on Metal Casket Type 1

76. 1883 advertisement for Stein Manufacturing Company’s “Patent Fastener” for casket tops

77. Selection of cut nails offered for sale on page 251 of the 1865 Russell \& Erwin Manufacturing Company general catalog....

78. U.S. Utility Patent No. 4,704 assigned in 1846 to T. J. Sloan for wood screws

79. Selection of American gimlet screws offered for sale on page 126 of the 1865

Russell \& Erwin Manufacturing Company general catalog

80. U.S. Utility Patent No. 2,046,343 assigned in 1936 to Henry F. Phillips for a screw ......... 108

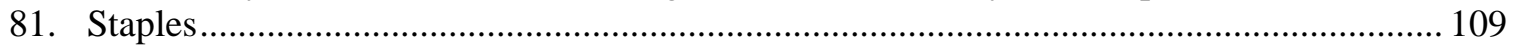

82. Blind staples offered for sale on page 149 of the 1865 Russell \& Erwin Manufacturing Company general catalog.

83. Joining plates

84. Corrugated fasteners

85. U.S. Utility Patent No. 300536 assigned in 1884 to A. H. Walker for a corrugated fastener.

86. U.S. Utility Patent No. 406,545 assigned in 1889 to Ferdinand W. Starr for a corrugated fastener. 
List of Figures

(cont'd)

87. U.S. Utility Patent No. 377325 assigned in 1888 to William J. Noble for a coffin fastener

88. U.S. Utility Patent No. 401663 assigned in 1889 to William A. Sparks for a coffin fastener

89. Top Fastener Type 1 -foot hook and plate .

90. Top Fastener Type 1-spring and head plate

91. Top Fastener No. 45, which is similar to Top Fastener Type 1, as illustrated on page 77 of the 1920s-1930s Langenau Manufacturing Company catalog

92. Dowel Type 1 pictured with Stop Hinge Escutcheon Type

93. Dowel No. 9, which is a similar match to Dowel Type 1, as illustrated on page 30 of the 1920s-1930s Langenau Manufacturing Company catalog

94. Dowel Type 2

95. Dowel No. 174, which is a match to Dowel Type 2, as illustrated on page 33 of the 1920s-1930s Langenau Manufacturing Company catalog

96. U.S. Utility Patent No. 2634997 assigned to William R. Gallowitz for a spring latch mechanism.

97. U.S. Utility Patent No. 275,503 assigned in 1883 to W. C. Langenau for a

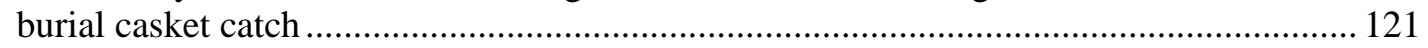

98. Catch Type 1 and Catch Escutcheon Type 1.............................................................. 122

99. Catch No. 114, which is similar to Catch Type 1, as illustrated on page 8 of the 1920s-1930s Langenau Manufacturing Company catalog .............................................. 123

100. U.S. Utility Patent No. 416002 assigned in 1889 to W. C. Langenau for a coffin

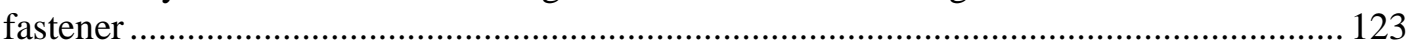

101. Catch Type 2 and Catch Escutcheon Type 1................................................................ 124

102. Catch No. 169, which is similar to Catch Type 2, as illustrated on page 20 of the 1920s-1930s Langenau Manufacturing Company catalog ........................................ 125

103. Catch Type 3 and Catch Escutcheon Type 2 2................................................................... 125

104. Catch No. B2, which is similar to Catch Type 3, as illustrated on page 10 of the

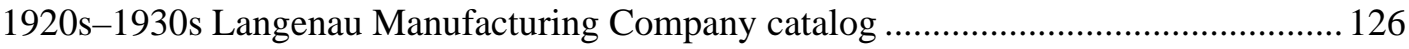

105. Catch Type 4 and Catch Escutcheon Type 1.................................................................. 126

106. U.S. Utility Patent No. 2031302 assigned in 1936 to W. L. Clark for a spring catch.........127

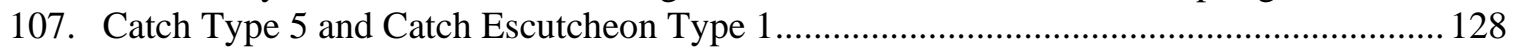

108. U.S. Utility Patent No. 2,504,716 assigned in 1950 to D. A. Morand for a spring catch

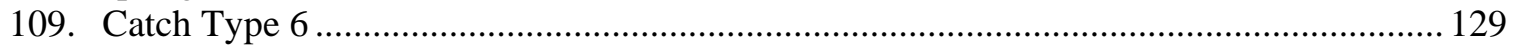

110. Page 22 of the 1920s-1930s Langenau Manufacturing Company catalog showing rectangular escutcheons similar to Catch Escutcheon Type 1

111. Escutcheon No. 30, which is similar to Catch Escutcheon Type 2, as illustrated on page 22 of the 1920s-1930s Langenau Manufacturing Company catalog.

112. Illustration of a separable stop hinge assembly on page 65 of the 1920s-1930s Langenau Manufacturing Company catalog

113. U.S. Utility Patent No. 382160 assigned in 1888 to Edward and Britain Holmes for a burial casket fastener 


\section{List of Figures}

(cont'd)

114. Stop Hinge Type 1 and Stop Hinge Escutcheon Type 1

115. Separable Stop Hinge No. 34 1/2, which is similar to Stop Hinge Type 1, as illustrated on page 59 of the 1920s-1930s Langenau Manufacturing Company catalog.....

116. Stop Hinge Type 2 and Stop Hinge Escutcheon Type 1

117. Stop Hinge Type 2 and Stop Hinge Escutcheon Type 2 ....

118. Separable Stop Hinge No. 34, which is similar to Stop Hinge Type 2, as illustrated on page 59 of the 1920s-1930s Langenau Manufacturing Company catalog..................... 135

119. Stop Hinge Type 3 and Stop Hinge Escutcheon Type 3 .

120. Separable Stop Hinge No. 214, which is a match to Stop Hinge Type 3, as illustrated on page 61 of the 1920s-1930s Langenau Manufacturing Company catalog.....

121. Stop Hinge Type 4 and Stop Hinge Escutcheon Type 4 .

122. Separable Stop Hinge No. 148, which is a match to Stop Hinge Type 4, as illustrated on page 61 of the 1920s-1930s Langenau Manufacturing Company catalog.....

123. Stop Hinge Type 5 and Stop Hinge Escutcheon Type 5.

124. Stop Hinge Type 5 and Stop Hinge Escutcheon Type 6 ....

125. Separable Stop Hinge No. 33, which is a match to Stop Hinge Type 5, as illustrated on page 63 of the 1920s-1930s Langenau Manufacturing Company catalog..................... 138

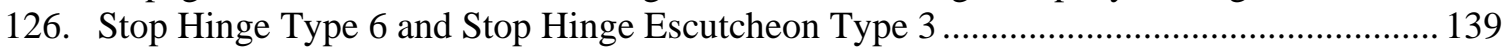

127. Separable Stop Hinge No. 28, which is a match to Stop Hinge Type 6, as illustrated on page 61 of the 1920s-1930s Langenau Manufacturing Company catalog.....

128. Escutcheon No. 27, which is similar to Stop Hinge Escutcheon Type 1, as illustrated on page 60 of the 1920s-1930s Langenau Manufacturing Company catalog....

129. Escutcheon No. 24, which is similar to Stop Hinge Escutcheon Type 2, as illustrated on page 60 of the 1920s-1930s Langenau Manufacturing Company catalog.....

130. Escutcheon Nos. 2 and 3, which are similar to Stop Hinge Escutcheon Type 3, as illustrated on page 62 of the 1920s-1930s Langenau Manufacturing Company catalog.

131. Escutcheon Nos. 4 and 5, which are similar to Stop Hinge Escutcheon Type 4, as illustrated on page 62 of the 1920s-1930s Langenau Manufacturing Company catalog.....

132. Escutcheon No. 26, which is similar to Stop Hinge Escutcheon Type 5, as illustrated on page 64 of the 1920s-1930s Langenau Manufacturing Company catalog.....

133. Escutcheon No. 29, which is similar to Stop Hinge Escutcheon Type 6, as illustrated on page 64 of the 1920s-1930s Langenau Manufacturing Company catalog. 
List of Figures

(cont'd)

134. Escutcheon No. 25, which is similar to Stop Hinge Escutcheon Type 7, as illustrated on page 64 of the 1920s-1930s Langenau Manufacturing Company catalog

135. Selection of hinges offered for sale on page 115 of the 1865 Russell \& Erwin

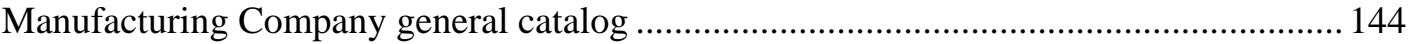

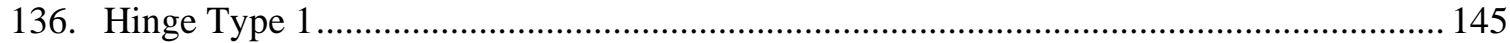

137. Hinge Type 2

138. Casket exhibiting supports on page 91 of a 1910-1920 John Marsellus

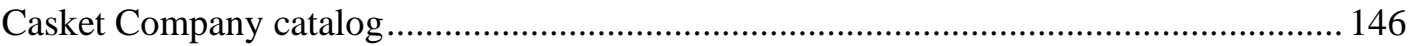

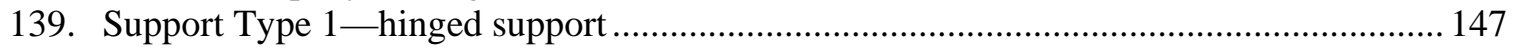

140. Support No. 280 illustrated on page 69 of the 1920s-1930s Langenau

Manufacturing Company catalog that is a match to Support Type 1 ............................... 147

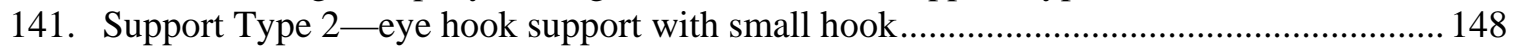

142. Support Type 2-eye hook support with large hook ................................................... 148

143. Selection of lining tacks illustrated on page 105 of the 1901 Gate City Coffin

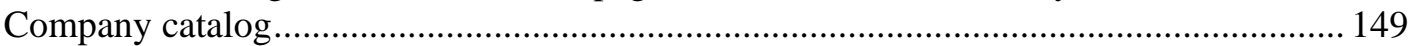

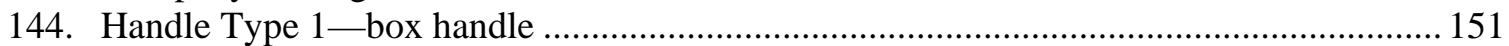

145. Atlas steel box handle illustrated on page 264 of the 1912 Cincinnati Coffin

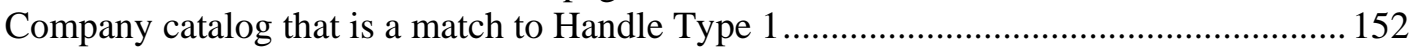

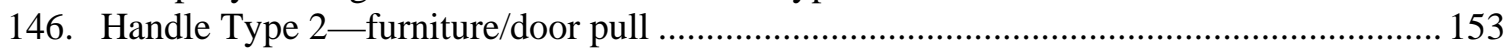

147. Selection of door pulls advertised on page 143 of the 1865 Russell \& Erwin

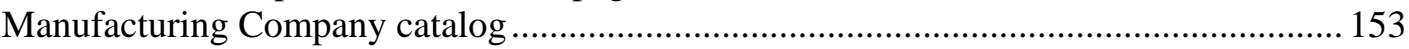

148. U.S. Design Patent No. 30,535 assigned in 1899 to Edward R. Sargent

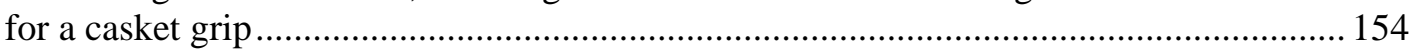

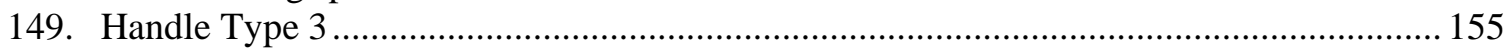

150. Handle Hold Cover No. 436 as advertised on page 71 in the 1920s-1930s

Langenau Manufacturing Company catalog, which is similar to Handle Type 3 .............. 155

151. Handle Type 4-double-lug swing-bail ......................................................................... 156

152. Commonwealth. Illustration of Paramount Casket No. 1 exhibiting handles similar to Handle Type 4 on page 100 of a ca. 1890s Cincinnati Coffin Company

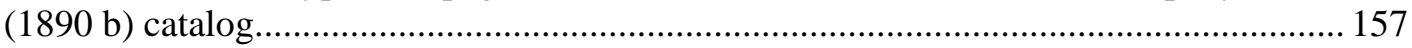

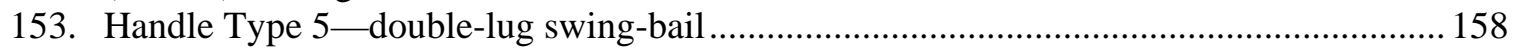

154. Swing-bail handle No. 2105 illustrated on page 106 of the 1921 Sargent \&

Company catalog that is similar to Handle Type 5 …....................................................... 158

155. Drawing page 1 of U.S. Utility Patent No. 1,106,731 assigned in 1914 to

Edward R. Sargent for a casket handle

156. Drawing page 2 of U.S. Utility Patent No. 1,106,731 assigned in 1914 to

Edward R. Sargent for a casket handle 160

157. U.S. Utility Patent No. 97827 assigned in 1869 to C. Strong for a coffin handle with early components of a short-bar handle

158. Handle Type 6-double-lug, short-bar handle with an octagonal swell

159. U.S. Utility Patent No. 444973 assigned in 1891 to Max Bremer for a coffin handle exhibiting a swell-bar form. 163 
List of Figures

(cont'd)

160. U.S. Utility Patent No.964562 assigned in 1910 to Edward R. Sargent for a casket handle

161. Casket exhibiting a short-bar handle, similar to Handle Type 6, on page 30 of the ca. 1910 Dallas Coffin Company catalog.

162. Parsons Casket Hardware Company 1947 Bar Size Code page 1 ......................................165

163. Parsons Casket Hardware Company 1947 Bar Size Code page 2...................................... 165

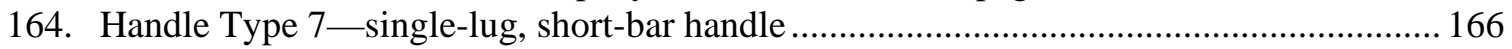

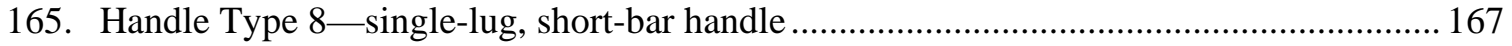

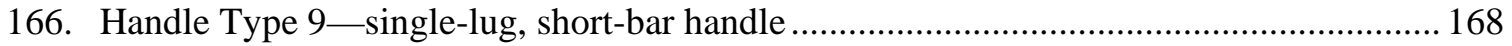

167. Handle Type 10 — single-lug, short-bar handle ................................................................. 169

168. Examples of types of extension handles offered for sale in the 1906 catalog of the

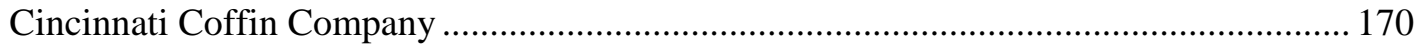

169. Early example of an extension bar handle illustrated on page 12 of the 1867 Crane, Breed \& Company catalog ....

170. Casket with swing-bar extension handle advertised on page 13 of the 1885 Stein Manufacturing Company catalog...

171. Early example of double-arm lug swing-bar extension handles on casket advertised on page 19 of the July-August 1893 National Casket Company Bulletin......... 172

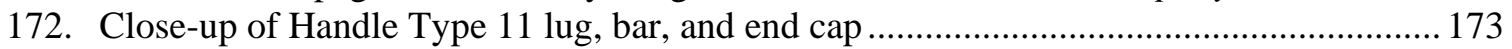

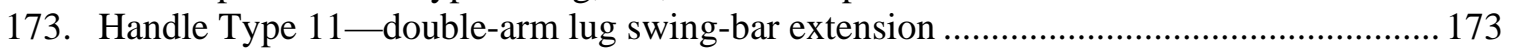

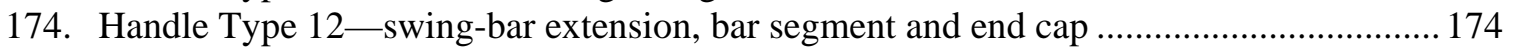

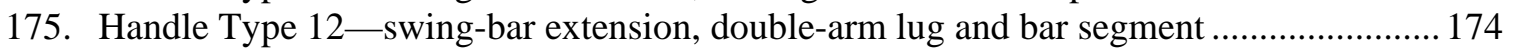

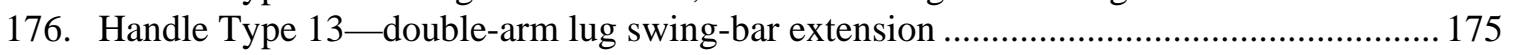

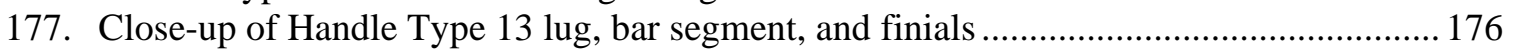

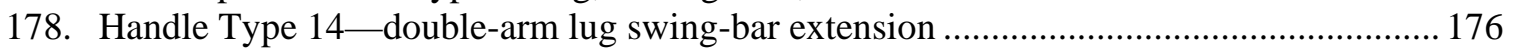

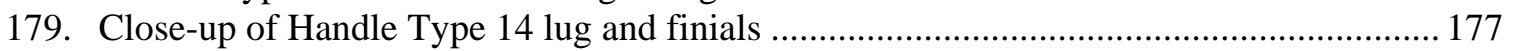

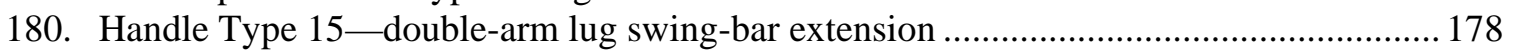

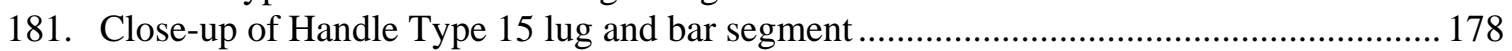

182. Handle Type 16 - double-arm swing-bar extension ......................................................... 179

183. Cloth-covered casket from a 1999 advertising card produced by Aurora Casket Company displaying a swing-bar extension handle similar to Handle Type 16 ................. 180

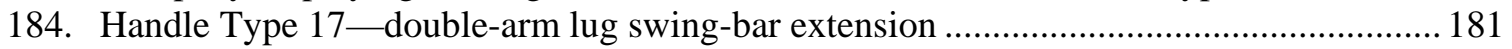

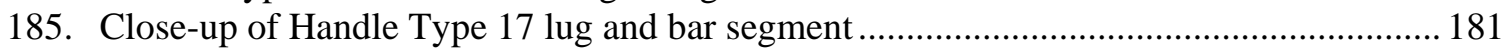

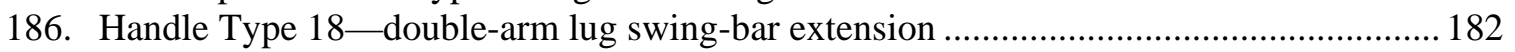

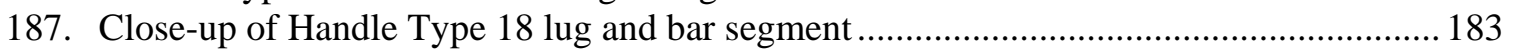

188. Carved hardwood stationary extension handles mounted on caskets offered for sale on page 40 of the 1903 National Casket Company illustrated price list ................ 183

189. Modern stationary extension bar shown on page 133 in the 1936 Boyertown Burial Casket Company Catalog "L".

190. Handle Type 19 — stationary extension side brace and bar segment.................................. 185

191. Handle Type 19—stationary extension corner brace ........................................................... 185

192. Cloth-covered casket from a 1999 advertising card produced by Aurora Casket Company displaying a stationary extension handle similar to Handle Type 19 .... 186 


\section{List of Figures}

(cont'd)

193. Handle Type 20 — stationary extension side brace and bar segment................................. 187

194. Handle Type 20 — stationary extension corner brace ...................................................... 187

195. Handle Type 21—stationary extension handle side braces and bar fragments ................. 188

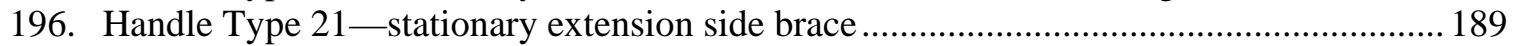

197. Handle Type 22—stationary extension corner brace ................................................... 189

198. Handle Type 23-stationary extension handle from Coffin 4 shown in field photo ......... 190

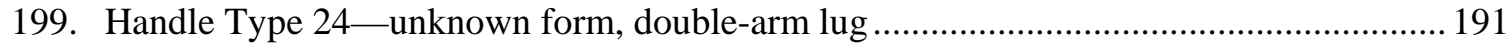

200. Handle Type 25-unknown form, finials, and lug fragments.......................................... 192

201. Short-bar handle No. 369 with finials matching those of Handle Type 25

illustrated in the ca. 1904 Gate City Coffin Company ......................................................... 192

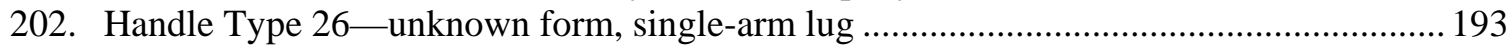

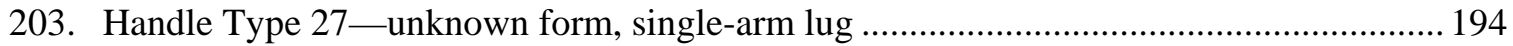

204. Handle Type 28 — unknown form, finial and segment of bar......................................... 195

205. Examples of early “coffin plates” from ca. 1797 anonymous English hardware

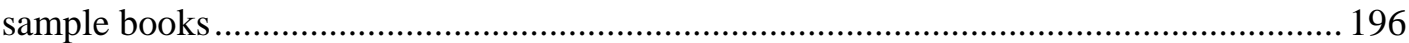

206. Plaque Type 1—“At Rest” plaque with wood recovered from Burial 18 .........................197

207. Possible reconstruction of the Plaque Type 1 original appearance based on examples of plaques advertised for sale in early twentieth-century catalogs..................... 198

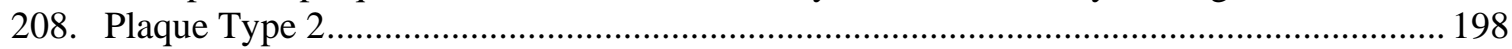

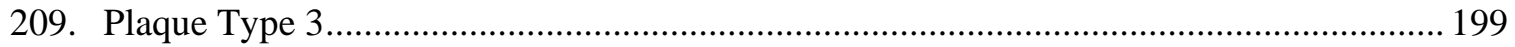

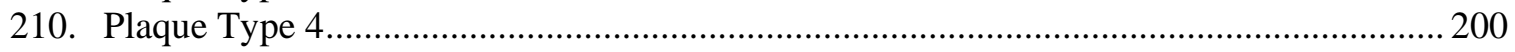

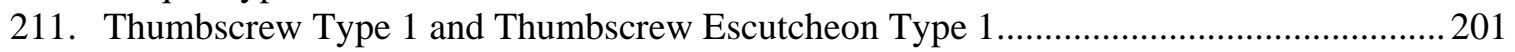

212. Thumbscrew No. 59 illustrated on page 100 of the 1901 Gate City Coffin

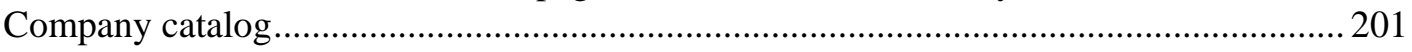

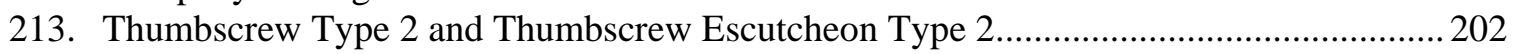

214. Embalming Paraphernalia Type 1 -trocar button ............................................................ 203

215. U.S. Utility Patent No. 2437381 assigned in 1948 to Arthur V. Cullen for an

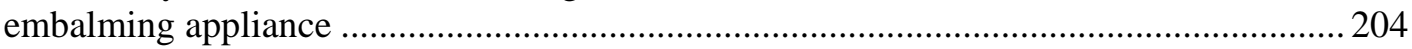

216. Trocar Button No. 2119 illustrated on page 49 of the ca. 1950s Royal Bond, Inc.,

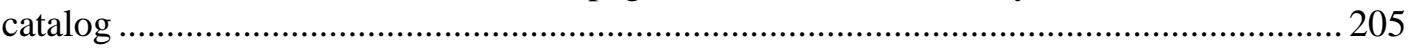

217. Embalming Paraphernalia Types 2-7-plastic embalming fluid bottles from Coffin 4; bottle A shows Type 2 C-45 Cavity Chemical ....................................................2206

218. Embalming Paraphernalia Type 8 (eye caps) and Type 9 (mouth former)........................ 209

219. Morgan's Eye Cap as illustrated on page 216 of the 1940 Dominion Manufacturers, Ltd., catalog.

220. U.S. Utility Patent No. 3195215 assigned in 1965 to Charles W. Rector for the "Natural Expression Former".

221. Natural Expression Vinyl Mouth Former illustrated on page 185 of the 1966 Superior Funeral Supply Company catalog

222. Miscellaneous Artifact Type 1-foam from caskets......................................................... 213

223. U.S. Utility Patent No. 430964 assigned in 1890 to Louis G. Kregel for burial casket padding.

224. Miscellaneous Artifact Type 2-netting... 


\section{List of Figures \\ (cont'd)}

225. Field image of Coffin 4 showing netting (Miscellaneous Artifact Type 2) in situ ............ 216

226. Miscellaneous Artifact Type 3-unidentified housing plate ..........................................217

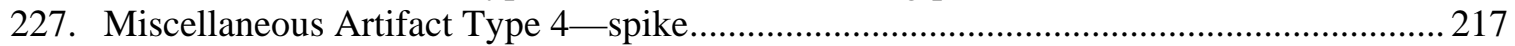

228. Miscellaneous Artifact Type 5-unidentified casket hardware …................................... 218

229. Miscellaneous Artifact Type 6 - unidentified hardware .................................................. 218

230. Glass Stop No. 8 illustrated on page 49 of the 1920s-1930s Langenau Manufacturing Company catalog

231. Miscellaneous Artifact Type 7-unknown ferrous artifact............................................. 220

232. Miscellenaous Artifact Type 8-dome-headed bolt with square nut................................ 220

233. Assortment of bolts offered for sale on page 257 of the 1865 Russell \& Erwin

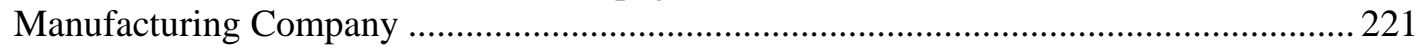

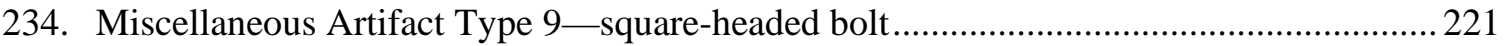

235. Miscellaneous Artifact Type 10 —barbed wire and plain wire .......................................... 222

236. Coffin 4: man's blue suit jacket and suit sleeve with white shirt; close-up of

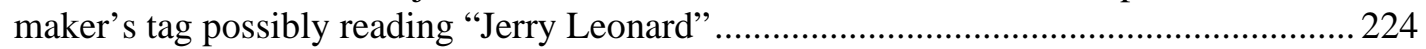

237. Personal items recovered from Burials 2, 5, 7, and 9 at New Home Cemetery ................. 226

238. Personal items recovered from Burials 14, 17, and 18 at New Home Cemetery ................ 227

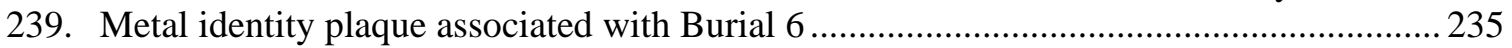

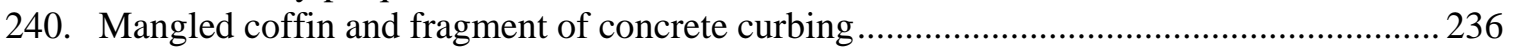

241. Plan map of New Home Cemetery project area showing chronology of burials ................ 243 



\section{LIST OF TABLES}

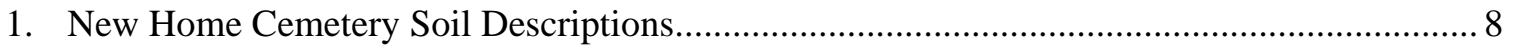

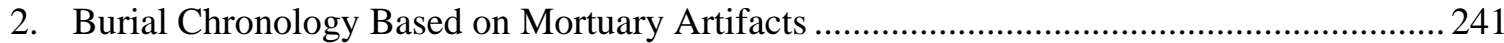

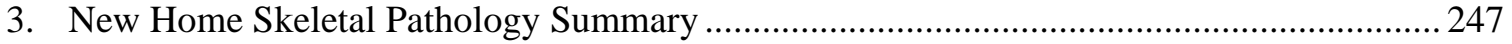





\section{ACKNOWLEDGMENTS}

For a project like this, there are always many people who should be recognized, because without their participation this project would not have happened. First and foremost are Allen C. Bettis Jr. of TxDOT ENV, Archeology Studies; and Mr. Woodrow W. Jones, owner of New Home Cemetery. Thank you, Allen, for giving us this opportunity and your support throughout the project. Mr. Jones also is thanked for his support and all additional oral information he was able to supply about the cemetery. Special thanks go to Fort Bend District Engineer James V. Hunt, Houston District Environmental Coordinator Lance Olenius, and TxDOT FM 1464 Project Manager Jim Ross and their teams, in particular Chris Patterson and Royce Macha, for the support in traffic control and day-to-day scheduling of heavy machinery and operators. Our appreciation is extended to heavy machinery operators Mike Warnasch, Rick Wrobliski, Roberto "Sharkey" Espinosa, Servando "Rod" Rodriguez, and Bennie Krpec, all of whom had a gentle touch and helped in identifying grave shafts and material.

Without the hard work of the osteological field crew, this project could never have been completed. In addition to excavation duties, two osteological/archaeological field technicians, Lindsey Skelton and Teresa Nugent, helped with the cleaning and photography of all materials recovered from the investigations. Lindsey also did an extraordinary job of keeping all corporate and project records straight throughout the project. Adam Leroy, archaeologist/historian, conducted the detailed archival and deed title research, giving us a better understanding of the history of New Home Cemetery. Arlo McKee ably served as archaeologist and geoarchaeologist during the Phase I investigation and kept the gradall operators on track. Archaeological field technicians Karl Huebchen and David Treichel assisted in the reinterment during Phase II.

Laser scanning of corroded temporary marker Type 1 was undertaken by Christopher Goodmaster in the Geo-Marine laboratory. Erin King produced the graphics in the report. Sharlene Allday did a wonderful job of editing the report and pulling all the sections together, and Denise Pemberton's expertise in making the report visually pleasing and easy to read was invaluable. 



\title{
CHAPTER 1 INTRODUCTION
}

\author{
by Mary Cassandra Hill
}

The Texas Department of Transportation (TxDOT) plans improvements to Farm-to-Market road (FM) 1464 between Stratford Creek Drive and Oyster Creek, Sugar Land, Texas, in eastern Fort Bend County, southwest of downtown Houston. Those proposed improvements will expand the FM 1464 right-of-way (ROW) about 20 feet (ft; 6 meters [m]) eastward into an approximately $328-\mathrm{ft}(100-\mathrm{m})$ length adjacent to New Home Cemetery (41FB344), which is at the intersection of FM 1464 and Orchard Lake Estates Drive (Figure 1). This report recounts the recovery, analysis, and reinterment of the human remains, funerary objects and furnishings, and all artifacts associated with burials found in the ROW adjacent to New Home Cemetery, as proscribed by contractual mitigation agreements between Geo-Marine, Inc. (Geo-Marine), and TxDOT.

The mitigation was mandated following the recommendations from a PBS\&J survey of the proposed area of expansion of FM 1464 ROW that would impact approximately 2.494 acres of land, adjacent to and including a portion of the existing New Home Cemetery, between Stratford Creek Drive and Oyster Creek (Cordova and Anderson 2010). The previous investigations by PBS\&J within the ROW during the spring of 2010 had encountered human remains and associated funerary objects, prompting further excavation of the ROW bordering the cemetery. Three of the seven trenches that were excavated exposed human remains, personal effects, and burial container hardware. These materials then were covered with plastic, tarps, and plywood, and the trenches backfilled.

Geo-Marine was subsequently contracted by TxDOT to search, exhume, analyze, and rebury any human remains found within the ROW and under the existing roadbed of FM 1464 (Geo-Marine project number 22005.00.07.03/22005.00.08). A principal goal of the work by Geo-Marine was the determination of the extent to which those remains and any others were intact, given that the project area had been subjected to previous roadway construction episodes, intrusion by buried utilities, modification by drainage ditches, and previous burial relocation activities by a commercial funeral company. Additionally, a biological profile description would be assembled for each of the individuals exhumed from the project area. Lastly, Geo-Marine would conduct the reinterment within the existing cemetery of all human remains, funerary accoutrements, and associated artifacts in a respectful and ethical manner, per the expressed concerns of existing cemetery landowner Mr. Woodrow W. Jones, the descendent community, and all contractual agencies. 


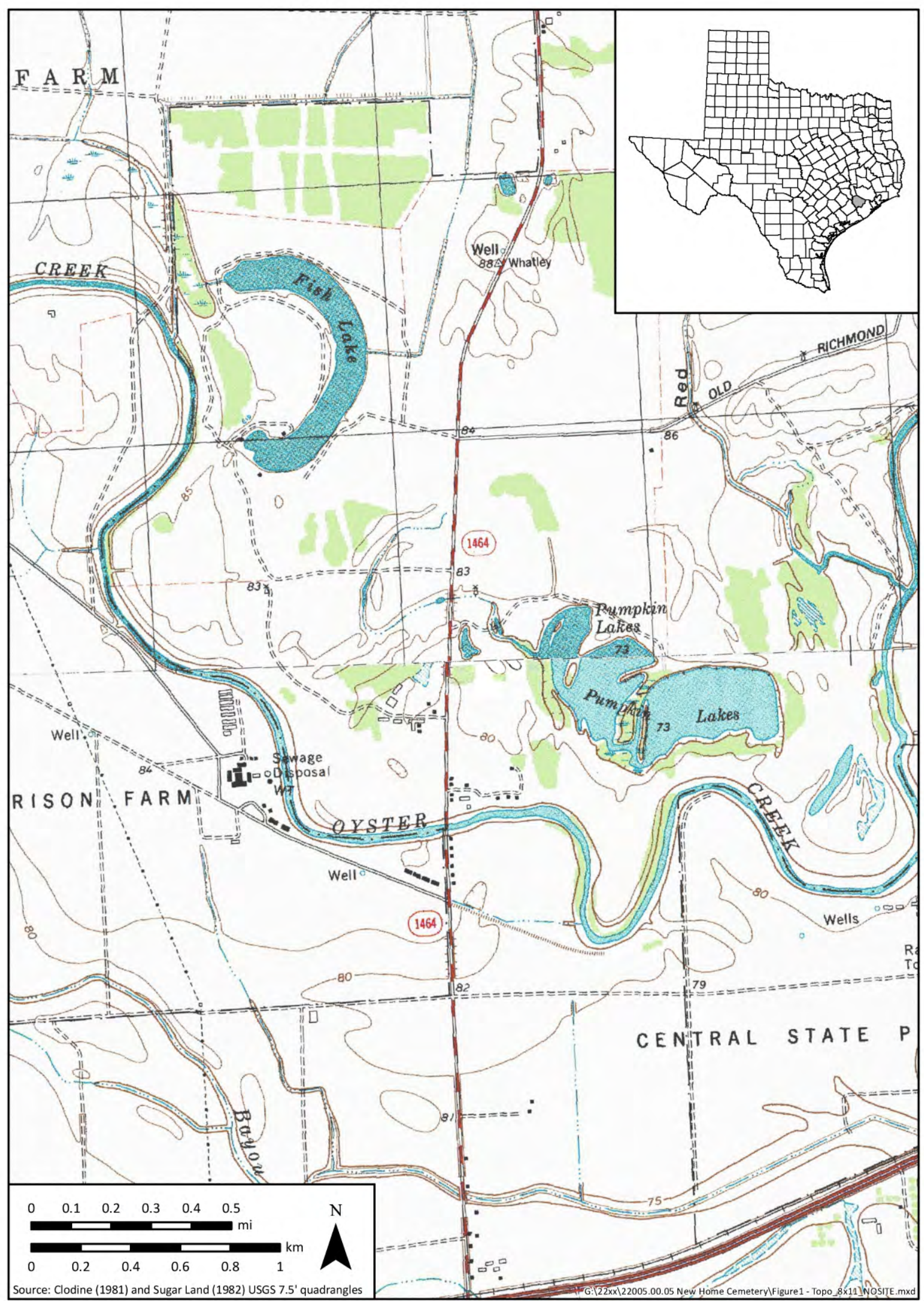

Figure 1. Topographic map showing the general locatin of the project area in Ford Bend County (CSJ 1415-02032). 
In 1895, New Home Missionary Baptist Church purchased land from B. A. Everts with the intent of establishing a place of worship and a place of rest for the African Americans of the Sugar Land community (Fort Bend County Deed Records, Book 4:pgs 454-455). As the town of Sugar Land began to grow and develop, the state of Texas purchased a small tract of the New Home property in 1950 to construct FM 1464 to accommodate the demands of the growing community. Unfortunately, the locations of many of the burials within the New Home grounds were lost over the years as the area continued to develop. Upon additional construction and widening of the road in 2006, the state of Texas faced the issue of relocating several graves adjacent to the road. In total, 26 interments from New Home Cemetery were encountered, exhumed, and relocated within the cemetery grounds (Adams 2006). By 2010, the state of Texas once again confronted the same predicament, with needed additional improvement and expansion of FM 1464. Thus, in late 2010, Geo-Marine began the investigations at the cemetery. Work for this project was conducted intermittently from November 15, 2010, until May 10, 2011.

From November 15, 2010, to December 17, 2010, Geo-Marine conducted the first phase of fieldwork with the archaeological search, exhumation, and analysis of multiple historic graves. Twenty-four unmarked graves, containing 11 complete and 13 partial human burials, were recovered. Of the 24 graves encountered, only one extended beyond the ROW into the existing cemetery property. In addition to the human remains, numerous funerary materials, including three complete metal caskets and a plethora of personal and mortuary artifacts, were recovered from the ROW. All of the 24 graves were recorded using a total data station (TDS), geographic positioning system (GPS), and field maps to accurately record the original burial site of each individual. For the 11 complete skeletons, TDS and GPS points were collected, if possible, at the top of the cranium. For the partial remains, TDS and GPS points were collected for all disarticulated human remains. In addition, TDS and GPS points were recorded for noteworthy casket hardware and other artifacts. Field maps of the burials were drawn with measurements of the grave shaft, individual human remains, mortuary hardware, and other artifacts before removal. All human remains, funerary materials, and other goods were stored in climate-controlled storage units in Sugar Land and Plano, Texas, to ensure preservation and security before reinterment.

The demographic and osteological analyses of the 24 burials continued from January 4, 2011, to January 14, 2011. All observations of the skeletal remains were recorded according to the Standards for Data Collection for Human Skeletal Remains (the Standards; Buikstra and Ubelaker 1994), with additional references pertinent to analyses. The comprehensive analyses included the identification of sex, age, ethnicity, pathology, nutrition, and other health history, plus possible/probable cause of death. The remains were then photographed and placed into permanent burial boxes with the burial number engraved into brass identification plates on the outside of the box. The detailed osteological analyses, which will be discussed in subsequent chapters, revealed that these individuals were engaged in an active lifestyle mostly associated with physical labor. This lifestyle unfortunately led to severe physical pain and suffering, and at times early death.

Also in early 2011, funerary materials, including casket handles, nails, and thumbscrews, were sent to the funerary hardware specialist to determine typology, material, and timeframe. Based on the mortuary artifact analyses, the timeframe for the burials ranged from 1895 through the late 1960s. Only one individual was identified by name, and the remaining 23 were unfortunately left as "Unknown." The analyses and results from the mortuary artifacts will be discussed in the following chapters. 
A small area with prehistoric materials was encountered in the northern portion of the project area (Figure 2). New Home Cemetery did not encroach into this small feature area, as indicated by encountering no historic funerary materials or human remains. This feature is discussed only in Appendix $\mathrm{F}$ at the end of this report.

The second phase of the project began on May 2, 2011, with the systematic scraping of the area located under the old roadbed. No burials or human remains were encountered during this phase; however, several modern utility lines were uncovered running north to south underneath a series of three old roadbeds. The utility lines consisted of a PVC (polyvinyl chloride) water/wastewater line, a concrete storm sewer line, a PVC gas line, a fiber optic cable line, and numerous PVC lines tied into a sprinkler system. Only a small amount of possible funerary material was recovered from underneath the roadway. The majority of items encountered were associated with modern or industrial trash. The extensive amount of utilities under the road resulted in the area being highly disturbed.

Reinterment occurred on May 9, 2011, during the second phase of search and recovery. The 24 individuals removed and recorded during Phase I were reinterred within the existing New Home Cemetery. An area of approximately 55 square meters in the southwest quadrant of the cemetery was chosen, based on the recommendations of cemetery owner Mr. Woodrow Jones. The chosen area was reported to have been frequently flooded in the past and was built up and leveled by $\mathrm{Mr}$. Jones. Based on the flooding history of the cemetery, Mr. Jones believed that a low area in the cemetery that was subject to frequent inundation would not contain any previously interred individuals. The scraping/trenching of the prospective burial area yielded no human remains, and the 24 individuals could be laid to rest. Headstones made of natural stone were prepared and placed over the new graves of the 24 individuals. Twenty-three of the headstones bore the word "Unknown," but the twenty-fourth headstone, Burial 5, was engraved with "Leah Simpson / October 16, 1883 to May 17, 1954.” After the soil was replaced and the headstones were set into position, the center of each headstone was recorded using TDS and GPS to ensure accurate and detailed account of each individual's new place of rest. The archaeological search, exhumation, analysis, and relocation of unmarked historic burials within the ROW were concluded on May 10, 2011.

During the entirety of this project, Duane Peter served as Principle Investigator, and Melissa Green was the Project Manager. Dr. Mary Cassandra Hill served as senior osteologist and field director for excavations. Arlo McKee fulfilled the duties of both archaeologist and geoarchaeologist, while Jeremy W. Pye carried out the duties of funerary hardware specialist. Staff osteologists included Lindsey Skelton and Teresa Nugent, and Adam Leroy was a field technician and historian. Archaeological Field Technicians Karl Huebchen and David Treichel assisted in the reinterment of remains. 


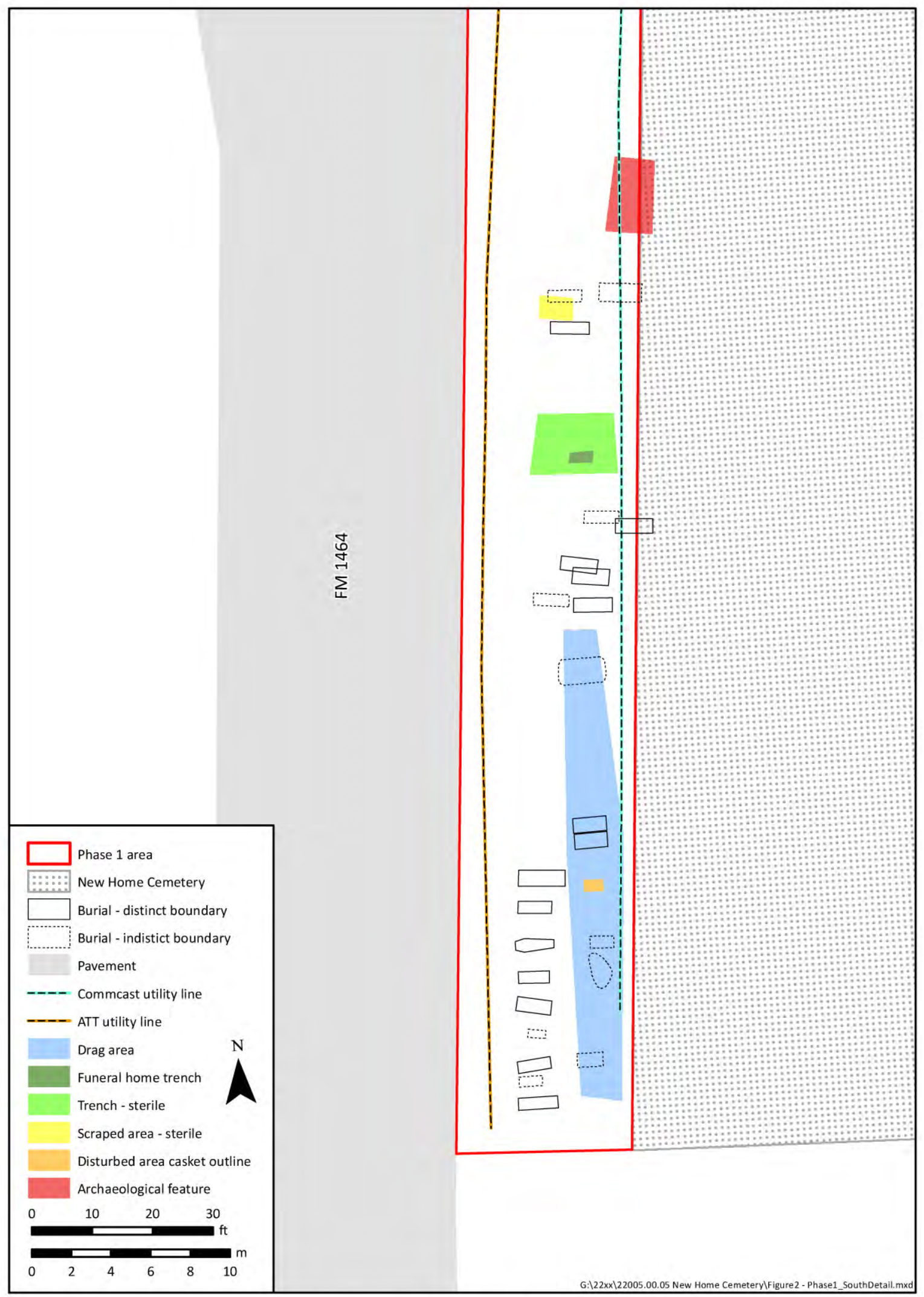

Figure 2. Plan map of Phase I investigations along FM 1464 at New Home Cemetery (CSJ 1415-02-032). 



\section{CHAPTER 2 \\ ENVIRONMENTAL SETTING AND HISTORIC BACKGROUND}

by Mary Cassandra Hill, with contributions by Adam Leroy and Lindsey Skelton

\section{LOCATION AND ENVIRONMENTAL SETTING}

New Home Cemetery is located in northeast Fort Bend County, Texas, on the Sugar Land U.S. Geological Survey (USGS) quad (2995-312). Current references state that Sugar Land is one of the fastest-growing and most affluent cities in Texas (Arcadia Publishing 2010; Sugar Land, Texas 2011). Located approximately 25 miles southwest of Houston, it has the greatest number of master-planned communities in Fort Bend County, which is the location of the largest number of master-planned communities in the United States. New Home Cemetery is located in the middle of several of these large planned communities. Large stone walls built by the housing developer border the cemetery to the north, south, and east. Newly built large homes are directly adjacent to the cemetery toward the east and south. Orchard Lake Estates Drive forms the northern border, and FM 1464 serves as the western border.

New Home Cemetery is situated approximately $570 \mathrm{~m}(1,870 \mathrm{ft})$ north of Oyster Creek, which was a paleochannel from the Brazos River. Oyster Creek occupies the eastern side of the Brazos valley for most of its length and drains the Brazos/Colorado delta (Abbott 2001). In Sugar Land, groundwater discharge is extremely high and sufficient to sustain a relatively permanent flow to the creek (Abbott 2001). Evidence of the high-water table was observable at approximately 1.7$1.8 \mathrm{~m}(5.5-6 \mathrm{ft})$ below surface during excavation.

Soils of the project area consist of a Belk Clay with fine, mixed, thermic Entic Hapluderts (vertisol), and a small portion of the eastern edge of the cemetery is a Sumpf Clay with very-fine, mixed, thermic Aeric Endoaquerts (vertisol) (Soil Survey Staff 2010). The soil series originates from a clay alluvium of the Quaternary age with the underlying geological unit being Qal Alluvium (Cordova and Anderson 2010).

A soil description profile by the geoarchaeologist revealed that the top approximately 40 centimeters (cm; 15.8 inches [in]) were disturbed silty clay loam fill; no A horizon soil existed below the disturbed fill, possibly due to erosion. Mr. Jones reported that upon purchase of the cemetery in 1995, soil was brought in to help preserve the cemetery by allowing the burials to be more deeply buried. Table 1 provides the soil description profile: 
Table 1

New Home Cemetery Soil Descriptions

\begin{tabular}{|c|c|c|}
\hline Soil Horizon & Depth (in cm) & Soil Description \\
\hline Disturbed 1 & $0-20$ & Mottled 10YR 3/2 and 7.5YR 4/6 silty clay loam \\
\hline Disturbed 2 & $20-35$ & $\begin{array}{l}\text { Mottled 10YR } 4 / 6 \text { with root channels filled with } 10 \mathrm{YR} 3 / 2 \text { silty clay } \\
\text { loam }\end{array}$ \\
\hline Be1 & $35-60$ & $\begin{array}{l}\text { 7.5YR } 3 / 2 \text { root channels filled with } 7.5 Y R 4 / 4 \text { clay with many fine to } \\
\text { medium slick inside faces }\end{array}$ \\
\hline Be2 & $60-110$ & $\begin{array}{l}\text { 5YR } 3 / 4 \text { mottled with } 10 \text { YR } 3 / 2 \text { clay loam with common continuous } \\
\text { dry coats on ped faces }\end{array}$ \\
\hline $\mathrm{Ab}$ & $110-140$ & $\begin{array}{l}\text { 7.5YR } 3 / 2 \text { clay loam with snail shell fragments and many continuous } \\
\text { common coated fine charcoal threads; boundary is clear and smooth }\end{array}$ \\
\hline $\mathrm{BCb}$ & $140-190$ & 7.5YR 4/3 silt loam; boundary is diffuse \\
\hline
\end{tabular}

\section{HISTORY OF SUGAR LAND, TEXAS}

Current references state that Sugar Land is one of the fastest-growing and most affluent cities in Texas (Arcadia Publishing 2010; Sugar Land, Texas, 2011). The headquarters for Imperial Sugar is in Sugar Land, and the company's distribution center and main refinery were once located there. It also is the location of many international energy, software, engineering, and product industries, as well as the headquarters for Western Airways and a major manufacturing facility for Nalco Chemical Company. This profile of active economic growth reflects the history of the area from its inception in the early 1800s. The majority of the following information has been derived from The City of Sugar Land (Arcadia Publishing 2010).

The area originally was part of an extensive land grant between the Mexican government and Stephen Fuller Austin when he and the first 300 settlers moved into the territory. Per the agreement, Austin sold the land in parcels, measured in leagues, to individuals who already had become successful in the large-scale production of crops such as sugar, rice, tobacco, and cotton on their families' plantations across the southeastern states and territories. The settlers brought sugarcane stalks with them because they had been told that the environmental conditions would be perfect for its cultivation. Modern-day Sugar Land is located on the acreage that Austin originally selected for himself. However, Austin never occupied his land; he divided it into five leagues that were quickly purchased by five different planters. New Home Cemetery falls within the Jesse H. Cartwright land grant patented in 1827 (Dase 2004).

One of these planters was Samuel M. Williams, whose land consisted of the area that would later become Sugar Land. Samuel Williams sold his league to his brother, Nathaniel. Although Nathaniel owned the plantation, it was managed by another brother, Matthew, whose workers grew corn, cotton, and sugarcane on the land of Oakland Plantation, which quickly became the hub of economic and social life between the Brazos River and Oyster Creek. The first sugar mill for Austin's colony had been established by 1828, and just 22 years later, 45 large plantations were producing millions of pounds of sugar a year (Texas Beyond History 2002). 
All of the plantations in the area, including Oakland, went through a series of rapid transformations in the years between 1850 and the early 1900s. When Matthew Williams died, his brothers sold Oakland Plantation to Benjamin Franklin Terry (famed for Terry's Texas Rangers) and William Jefferson Kyle. They combined it with their own landholdings and renamed the plantation Sugar Land. It was one of the largest plantations in Texas. Throughout the fission-fusion of the lands' changing ownership, the crops and labor force remained essentially the same. The production and processing of sugarcane were central to the economy, with other crops playing large but secondary roles.

Prior to the Civil War, workers on the plantations were enslaved Africans or Afro-Caribbeans, and sometimes Native Americans. The planters who settled the area had brought their slaves with them. All aspects of the workers' lives were under the direct control of the landowners. After the Civil War, with nowhere to go and very restricted prospects for employment or independent ownership of land, the majority of the emancipated workers and their families remained on the plantations. Emancipation presented a new series of problems, primarily because the workers were responsible for their own subsistence and they had to participate in the larger economic system. Agricultural production was dependent on a system of tenant farming and sharecropping, with the landowners remaining in control. This was true for all plantations throughout the U.S. southern states and territories. Both tenant farming and sharecropping relied on contractual agreements between the landowner(s) and the laborer(s) or farmer(s), and included strict rules and regulations concerning work and behavior. The landowner provided housing, equipment, and tools, and plants or seed for both tenant farming and sharecropping. However, in tenant farming, the laborers were given wages out of which they provided their own clothing and food. In sharecropping, the landowners provided an advance of food and clothing in exchange for a share of the crops being produced (Roark 1977).

The explanation for the cover photograph for The City of Sugar Land (Arcadia Publishing 2010) reveals a great deal about the working class of Sugar Land after emancipation:

Before Sugar Land became a model company town attracting families, a refinery superintendent wrote that squalid conditions supported a population of "many ex-convicts and about every nationality anyone could mention. Sugar boilers, centrifugal operators, and other skilled [workers] were mostly from Louisiana. We never lacked for labor, and Sugar Land seemed to be the dump for deserters from ships in Galveston, hoboes needing a couple of bucks, and hikers.”

This is hardly a complimentary description of life along the Brazos River and Oyster Creek. In fact, it was referred to as "the Hell Hole of the Brazos" (Arcadia Publishing 2010:23, 26). Without slave labor, the conglomerate of plantations struggled for economic survival. To that end, the transformation to tenant farming and sharecropping was augmented with labor provided by the state's convict lease system. While other states' sugar plantations, most notably Louisiana, were worked by wage labor after emancipation, Texas relied almost entirely on the convict labor force:

In 1882, 12 of the 18 Texas sugar plantations utilized 800 , or more than one-third, of the state's prison inmates. The cost per worker actually exceeded the prevailing wage scale, but the labor force, as under slavery, was constant and guaranteed [Wilke 2011].

Squalid housing facilities consisted of canvas tents set upon wooden foundations. It was essentially a primitive camp for seasonal, transient workers. Oyster Creek and the Brazos River were subject to constant flooding, and the resultant swampy land attracted vermin and pestilence. 
Living and work conditions became so deplorable that a law was passed in 1910 that prohibited the leasing of convicts (Texas Beyond History 2002). By this time, Sugar Land Plantation had grown to almost 20 square miles and was held by the partnership of Isaac Kempner and William Eldridge. It included a raw sugar mill and sugarcane refinery, known as the Imperial Sugar Company. With an eye on the future, and mindful of the needs of their workers, Kempner and Eldridge were determined to transform their businesses and landholdings into a model company town. They practiced a business model known as welfare capitalism. They designed neighborhoods for workers and their families that provided a positive environment and amenities, including churches, schools, medical facilities, and commercial businesses. This was not required by law, nor was it beneficial to their industry (Arcadia Publishing 2010:8). Kempner and Eldridge hoped to attract family-oriented workers, enticing people from as far away as Germany and other foreign countries.

However, as with the rest of the small towns in Texas and the United States in general prior to the 1950s, Sugar Land was racially segregated. In the horseshoe bend of Oyster Creek north of the refinery, houses were built for Hispanics and African Americans. The homes did not have the same amenities as those for Euro-American neighborhoods; they only had limited electricity and water. In the 1950s, Imperial Sugar replaced those houses with brick homes that had all modern conveniences. The company maintained all residences and businesses with crews of painters, carpenters, electricians, plumbers, and laborers (Arcadia Publishing 2010:30-31). The company town remained a vibrant model for other similar ventures across the United States for at least five decades.

Without birth or death records, or other documents for the church and cemetery, the actual connection to Imperial Sugar is circumstantial for New Home Cemetery. Given the proximity to Imperial Sugar, Oyster Creek, and the state prison, it is likely that the church and cemetery were part of the network of the company town (Figure 3).

\section{HISTORY OF NEW HOME CEMETERY}

New Home Cemetery and associated New Home Missionary Baptist Church have been attributed to an African American ethno-cultural affiliation (Adams 2006; W. W. Jones, personal communication, November 2010), or possibly to a European and Mexican Hispanic affiliation (Texas Historical Commission Historic Sites files and the Texas Archeological Research Laboratory; Cordova and Anderson 2010). A document search for Fort Bend County conducted by Geo-Marine did not locate any early historic land plats that identified the location of New Home Cemetery. The fact that the cemetery was not demarcated on early maps may be an indication of the disenfranchised status of the individuals associated with the church and cemetery. Lack of documentation of cemeteries and/or lack of burial records for individuals interred in cemeteries usually suggests that those individuals belonged to a minority segment of the general population (Jordan 1982; Yalom 2008). Dates that appear on the few gravestones that are present indicate that the cemetery has been in use from at least as early as the late 1800s through the present. Conversations with Mr. Jones and also with individuals who visited the cemetery during the project revealed that the area encompassed by the cemetery and church grounds was at one time much larger than the present acreage. According to these informants, the cemetery extended, "all the way to Oyster Creek." However, it should be noted that the meander of Oyster Creek has varied considerably over time. They also stated that the church had burned, and that there had been a separate area for the interment of children that is no longer part of the cemetery. Over the past 100 years, New Home Cemetery has been completely enveloped by upscale homes and landscaped areas. 


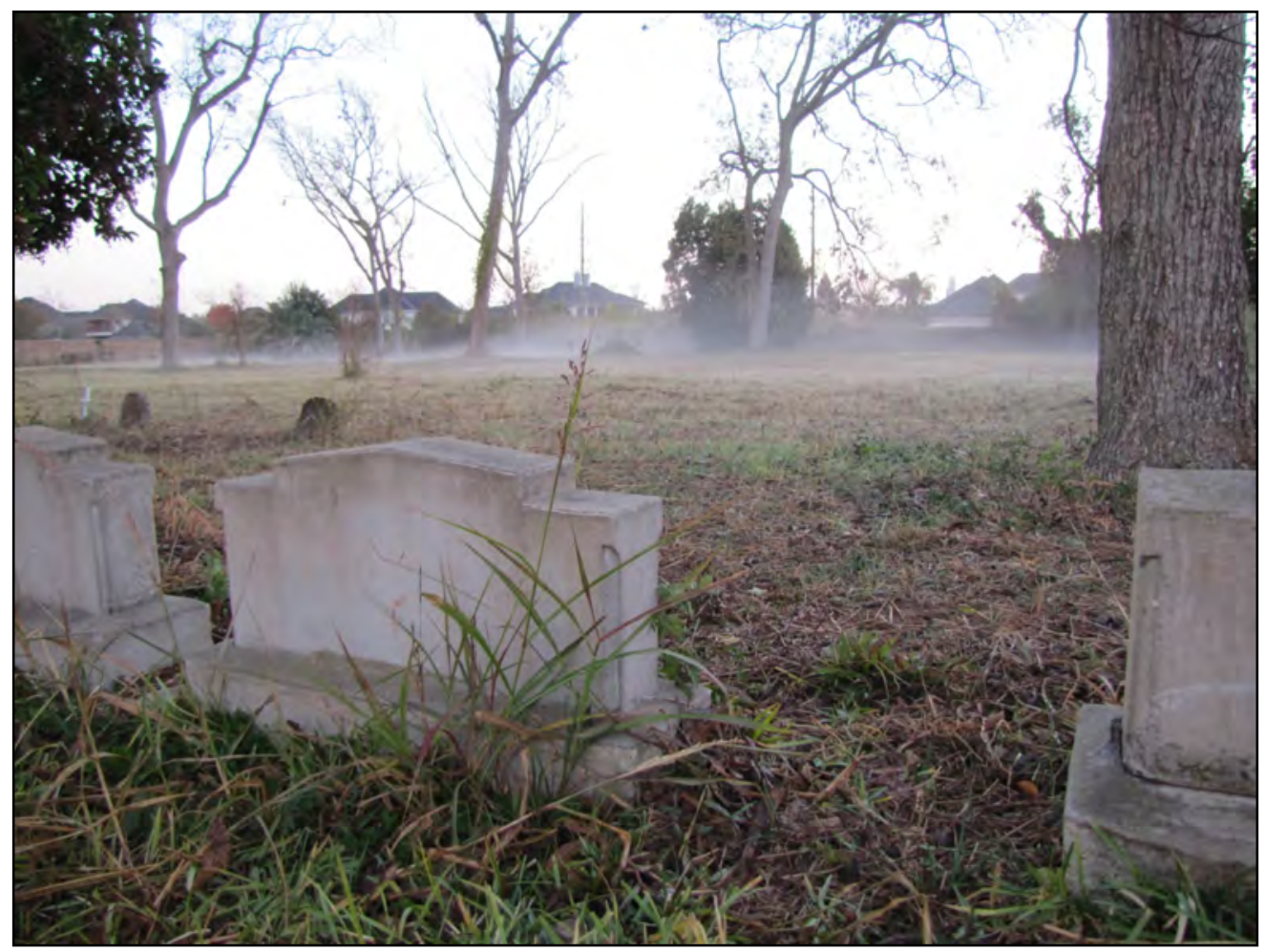

Figure 3. Cemetery in morning fog.

Historical research regarding the New Home Cemetery, located adjacent to FM 1464 in Sugar Land, Texas, was conducted at the Fort Bend County Clerk's Office at 301 Jackson St. in Richmond, Texas, and at the George Memorial Library in Richmond, Texas, resulting in the recovery of several pertinent documents (three property deeds and one newspaper article). Two online articles written about the proprietors of a local funeral home responsible for at least one of the burials at the New Home Cemetery disinterred in 2006 were also found. Information about the history of the cemetery was also provided by the property's current owner, Mr. Woodrow W. Jones, along with several sets of digital photographs depicting previous activities at the cemetery. No historic photographs of the cemetery or the former church building were recovered.

Records show that B. A. Everts purchased land from the Jesse Cartwright property on February 17, 1882 (Fort Bend County Deed Records, Book No. 3, pp. 395-396). This property consisted of a 50 -acre tract and was purchased for a total of $\$ 870$. As outlined in the subsequent deed between B. A. Everts and the New Home Mission Baptist Church (Fort Bend County Deed Records, Book No. 4, pp. 454-455), the church purchased 2.19 acres of the Everts property on December 7, 1895, for the sum of $\$ 50$. According to a July 17, 1950, deed between the New Home Mission Baptist Church and the state of Texas (Fort Bend County Deed Records, Book No. 275, pp. 268-269), a piece of the church's 1895 land purchase was sold to the state for the purpose of constructing FM 1464. This piece of land measured 40 feet wide by 315 feet long (0.036 acres) and was purchased for $\$ 1$. This land is the current ROW on the east side of FM 1464. 
According to Mr. Jones, a church building was built sometime shortly after the 1895 purchase, and subsequent to that, burials began to take place on the property. Jones stated that some of the original church building foundation can still be seen at the cemetery. He also claimed that the New Home Cemetery was almost exclusively a burial ground for the area's local African American community members into the 1980s.

Since no efforts to protect the burials had been made prior to the 1950 construction of FM 1464, Mr. Jones stated that in 2006, pending the TxDOT eventual widening of FM 1464, he personally hired the McWilliams Funeral Home of Hempstead, Texas, to undertake the task of exhuming bodies from the road ROW . This was done so that grave contents could be removed from the area to be affected and reburied within the current cemetery boundary. During this process, Denise Adams, a reporter for the Fort Bend Herald, authored an article that appeared in the paper on July 16, 2006, that discussed some points of the project. According to Adams, the funeral home was responsible for the removal of a total of 26 "sets of remains" (Adams 2006) over a period of nearly two years. Adams stated in the article that a funeral home tag that contained the name of the Fort Bend Fraternal Undertaking Company, formerly located in Richmond, Texas, 5 miles to the west of Sugar Land, was recovered from one of the excavated graves. A current attempt to track down burial records from this funeral home was unsuccessful. Additional research, however, found that the Fort Bend Fraternal Undertaking Company was first founded in 1927 by several individuals. Most notably among them were Mrs. Arizona Fleming, an African American civil rights activist whose work was instrumental in reestablishing suffrage for African Americans in Fort Bend County, and her partner Andrew Webster Jackson, also an African American civil rights activist, teacher, Master Mason, and writer. Fleming served as the secretary and manager of the company, eventually becoming its sole proprietor. Jackson was the company's first president. The Fort Bend Fraternal Undertaking Company closed sometime in the 1950s.

Map research was conducted primarily at the George Memorial Library and online at the Portal to Texas History. Some additional research was carried out at the Fort Bend Historical Commission office located inside the Fort Bend County Courthouse building. The most current maps of the area were created from Google Earth software. This research revealed several maps of the Fort Bend County area ranging in dates from 1882 to 2008.

As discussed above, the New Home Mission Baptist Church purchased the plot of land that would become the New Home Cemetery from Everts on May 25, 1895. However, neither the 1882 nor the 1892 or 1893 map of Fort Bend County displayed the Everts property boundaries. One 1936 Texas Highway Department map was examined, but showed only county roadways. No other maps of the area between 1893 and the 1950s were found.

Because of the limited number of cartographic materials, it was not possible to determine the precise location of the original 2.19 acres of the cemetery grounds pre-1950. The next map to be examined was a 1950s county map. Though this map did show the location of several cemeteries and churches, it did not include the location of the New Home Cemetery. In 1962, the Zingery Map Company, Inc., of Houston, Texas, released a detailed map book displaying detailed illustrations of all sections of Fort Bend County. This book, however, contained no indication of the location of the cemetery. Using a measurement tool on Google Earth, it was determined that the cemetery is situated roughly 1,700 feet north of Oyster Creek along FM 1464 (Clodine Reddick Road). Using that measurement and the scale on the Zingery map ( 1 inch = 1,000 feet), the cemetery appeared to be within the Paul E. Schumann property. The only map found that identified the cemetery location was the 1999 USGS Sugar Land quadrangle. Google Earth software, updated in 2008, contained no marker identifying the New Home Cemetery. 
In its current configuration, New Home Cemetery is a pecan grove with scattered plantings of ligustrum trees, amaryllis lilies, snow drops, canna lilies, nandina, wax myrtles (also known as southern bayberry or candleberry), crepe myrtles, and several varieties of palms. The pecan trees in the grove appear to be all of the same variety, producing a small, thin-shelled nut variety that grows wild throughout the southeastern United States. These are very large, mature trees that probably were there when the church and cemetery were in active use. (Presented in more detail later in the report, natural groves of trees are integral locations for African American folk cemeteries; and Geo-Marine did indeed recover amaryllis lily bulbs still in situ with one of the graves). The open areas are covered by thick, wild grasses, plus some sparse areas of tall, overgrown St. Augustine and centipede grass. Wild privet bushes and wild vines, including smilex, honeysuckle, and wild grapes, have overtaken certain areas of the open space, and also form an impenetrable barrier along the southern margin of the property. Landowner Woodrow $\mathrm{W}$. Jones has undertaken general maintenance of the property, but individuals who have relatives interred in the cemetery occasionally visit and clean those areas specific to their family members (Figures 4-6).

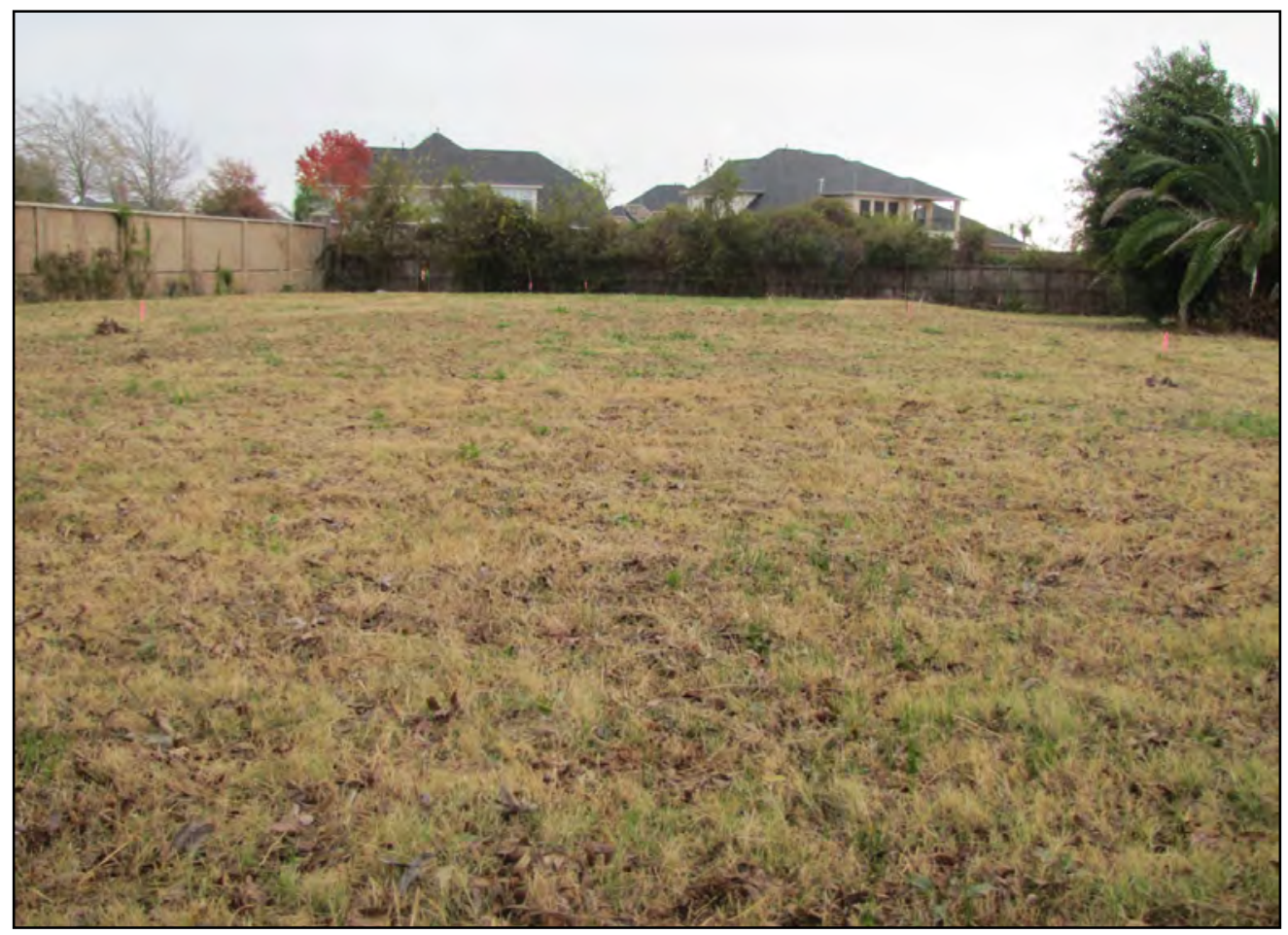

Figure 4. NE area of cemetery, looking east.

The ground surface is lumpy and uneven. Conversations between landowner Jones and field director M. C. Hill revealed that the original surface was traversed by a small, natural creek. It had zones that sloped significantly and tended to hold water. In order to alleviate the drainage problems, large loads of soil were brought in to fill the majority of the open area in the mid-tolate 1990s. This increased the soil overburden by as much as 18 inches to 2 feet in some areas of the cemetery. The information about the overfilled areas was very useful later in the project when the location for repatriation of the remains was selected (see The Repatriation section in this report). 


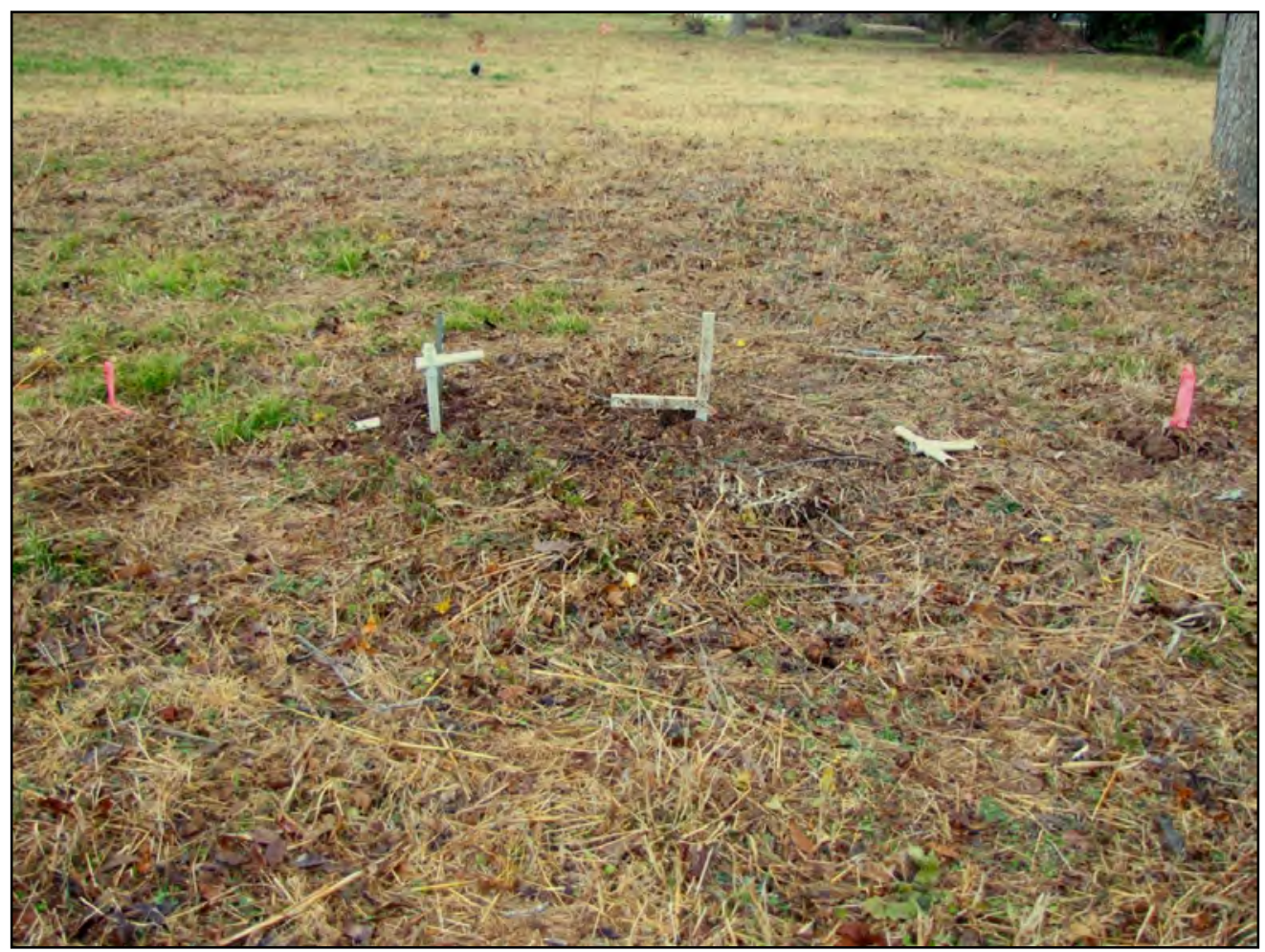

Figure 5. Small broken crosses in open area of cemetery, view west.

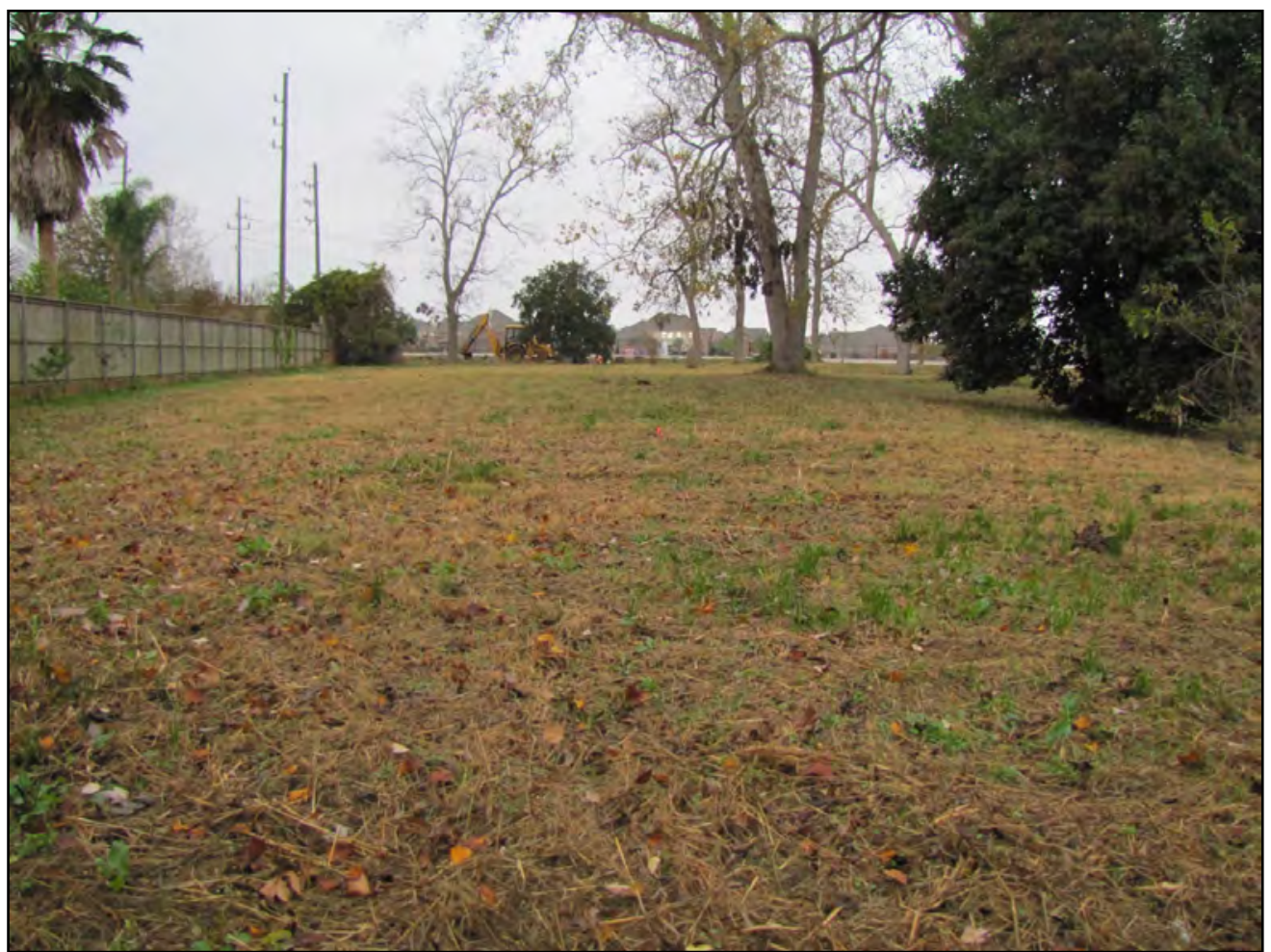

Figure 6. Open area of cemetery looking west from eastern margin of cemetery. 
There are few formal markers or gravestones. All are modest in appearance, ranging from simple crosses fashioned from small pieces of white PVC pipe, to handmade concrete markers with hand lettering, to commercial-grade gray granite headstones with commercial engraving (Figure 7). The markers/graves in the southwestern aspect of the cemetery are in rows oriented east/west with the heads west (Figure 8), but those in the northwestern aspect are randomly arranged in small clusters with differing orientations per cluster. There are no markers in the majority of open space within the cemetery.

The cemetery itself is identified by a modest iron sign that can be easily overlooked unless one is looking closely (Figure 9). Two single-syllable words, "New Home," convey an ironically immense message about the beliefs or sentiments of the people who are interred there, as well as those of the individuals who participated in their respective interments.

Mr. Jones negotiated with a local commercial funeral home to disinter graves that were in the portion of the cemetery to be sold to TxDOT for the expanded ROW. In addition to the relocation of those graves, Mr. Jones paid for new grave markers. As reported by PBS\&J and confirmed by Geo-Marine, the removal and relocation of those graves was neither complete for the ones that were disinterred, nor comprehensive for the area as a whole. In other words, skeletal elements and funerary containers were left in situ (but greatly disturbed), and there were other graves that were still intact and in situ despite the assurance to Mr. Jones that relocation was complete. 


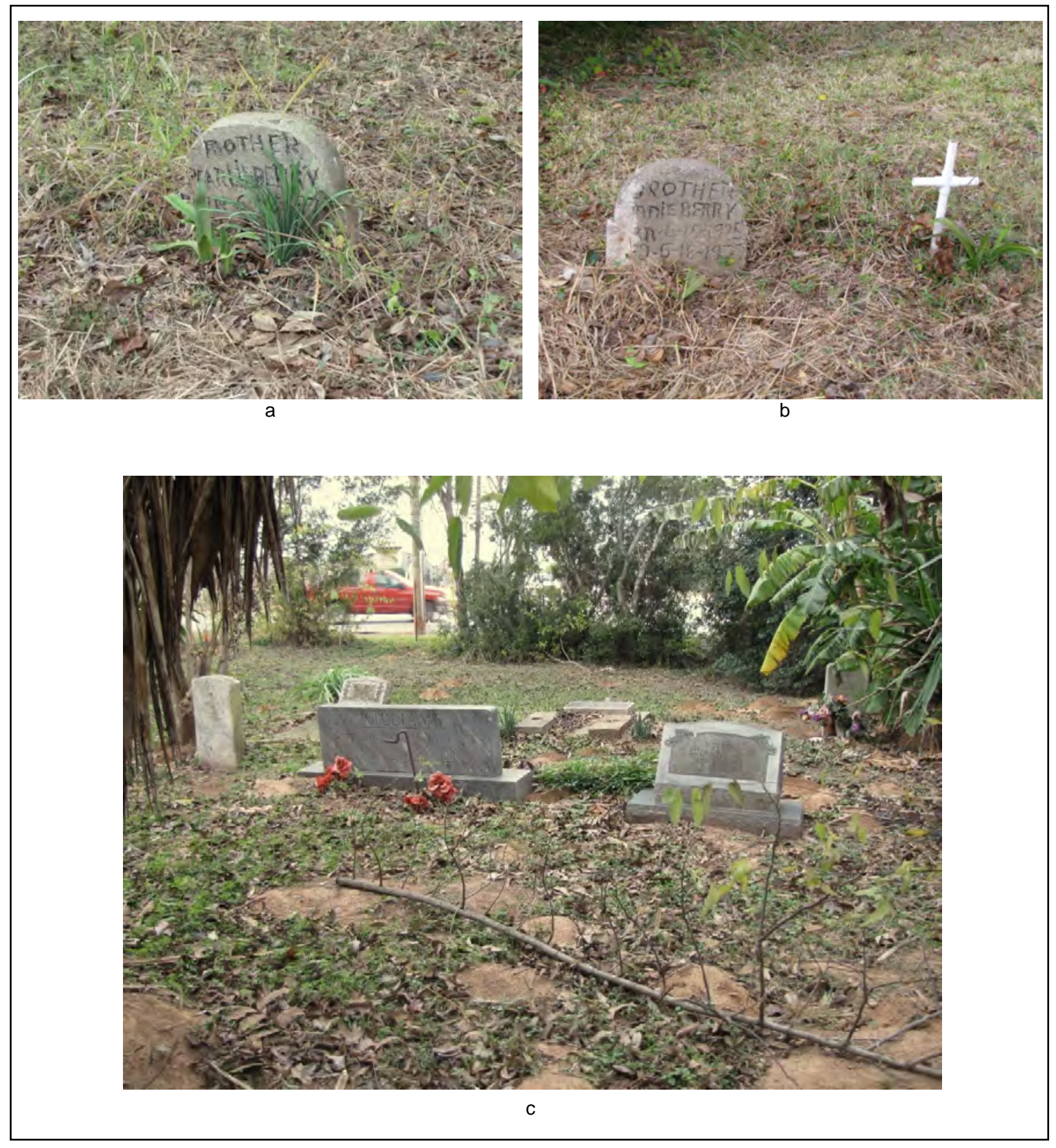

Figure 7. Headstones in New Home Cemetery: (a) hand engraved; (b) hand engraved and PVC pipe; and (c) family plot in northern end of cemetery. 


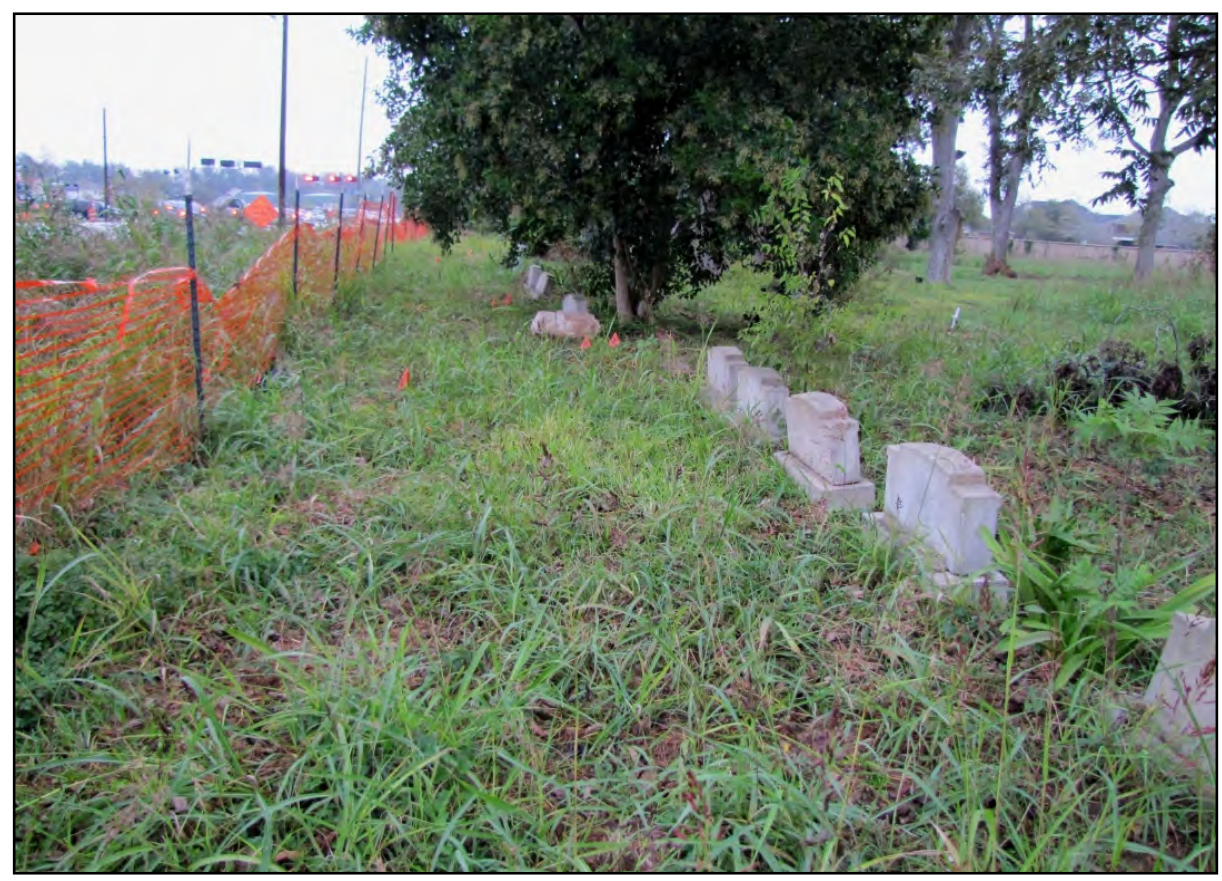

Figure 8. Row of stones on southern margin of cemetery, looking north.

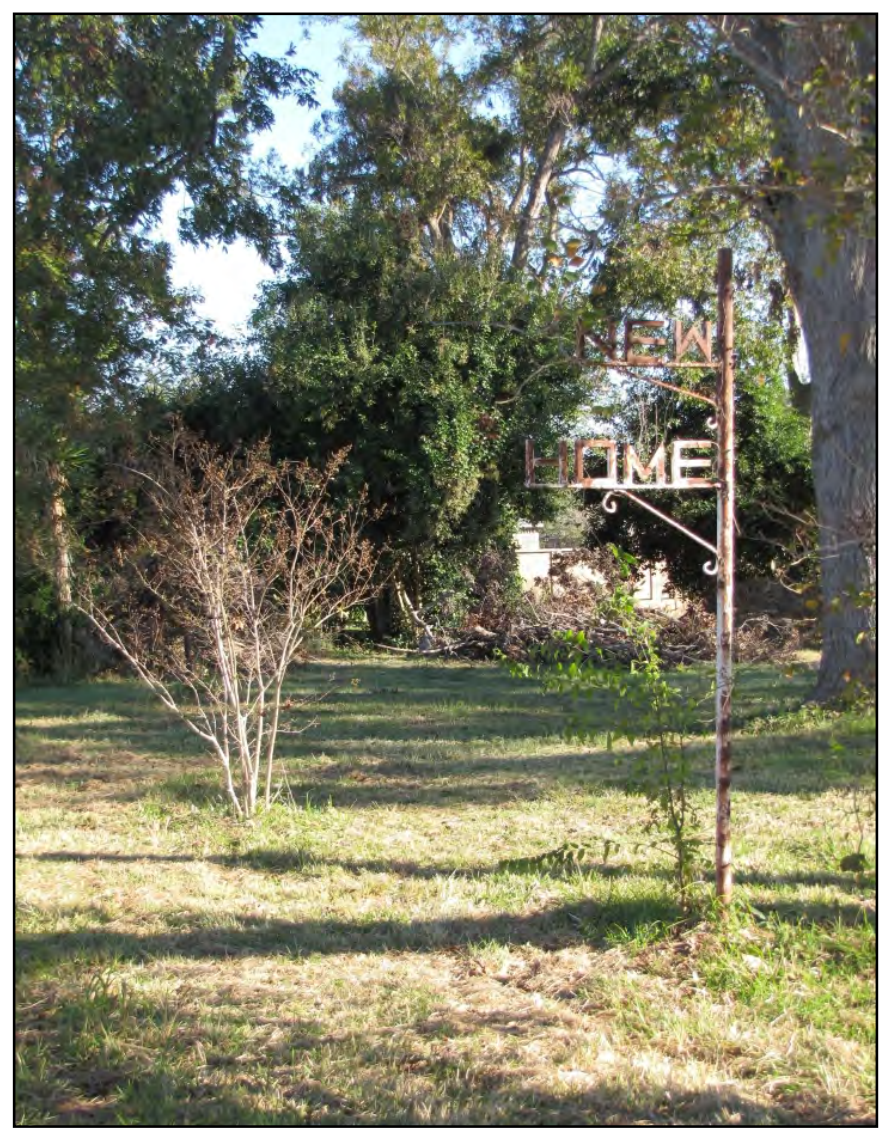

Figure 9. New Home Cemetery sign, view northeast. 



\title{
CHAPTER 3 METHODOLOGY \\ by Mary Cassandra Hill
}

\begin{abstract}
All aspects of the field and laboratory proceedings were subject to the highest level of professional standards as dictated by the Register of Professional Archaeologists, the Society of American Archaeology, the American Association of Physical Anthropologists, and the Paleopathology Association. The Geo-Marine crew was composed of field director/senior osteologist M. Cassandra Hill, archaeology/osteology technical assistants Lindsey Skelton and Teresa Nugent, and archaeology/historian technical assistant Adam Leroy for the first series of excavations and lab sessions. Field director/senior osteologist Hill and archaeology/osteology technical assistants Skelton and Nugent were also present for the second series of excavations. Additional personnel included archaeologist Arlo McKee, who is a soils specialist, during the first week of the first session, and archaeology assistants Karl Huebchen and David Treichel, who participated in the repatriation at the end of the second session. Time and budgetary constraints determined the field and laboratory strategies. Power stripping with heavy machinery was the essential method for accomplishment of rapid removal of soil overburden.
\end{abstract}

\section{FIELD METHODS AND MATERIALS}

Fieldwork was undertaken in two phases. Phase I consisted of stripping and subsequent excavation of any burials found in the expanded ROW between the cemetery and FM 1464 (approximately 0.21 acre), and Phase II work consisted of stripping and subsequent excavation of any burials found beneath the current FM 1464 roadbed after removal of the pavement (approximately 0.22 acre) (Figure 10).

The strategy for excavation was to conduct controlled removal of the overburden soils of the project area in an expeditious manner, beginning with the southern margin and systematically moving north. In the event that human remains were encountered, the crew would focus on hand excavation of those remains, and the field director and power equipment would then move north of the discovery and continue stripping southward to meet it. The stripping proceeded in this south-north/south-north pattern throughout the first phase of the project (Figures 11 and 12). 


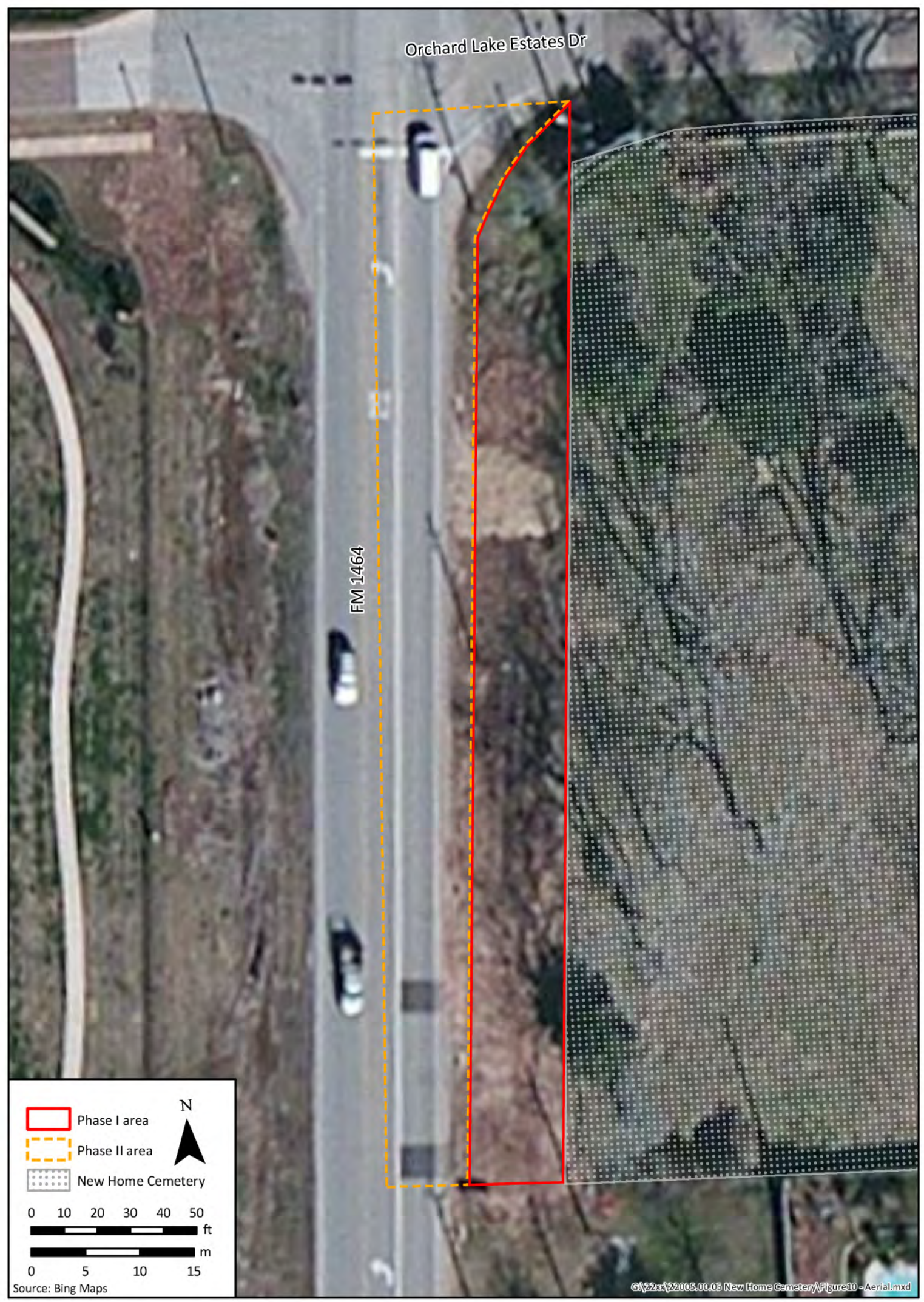

Figure 10. Aerial imagery showing the Phase I and Phase II project areas adjacent to New Home Cemetery (CSJ 1415-02-032). 


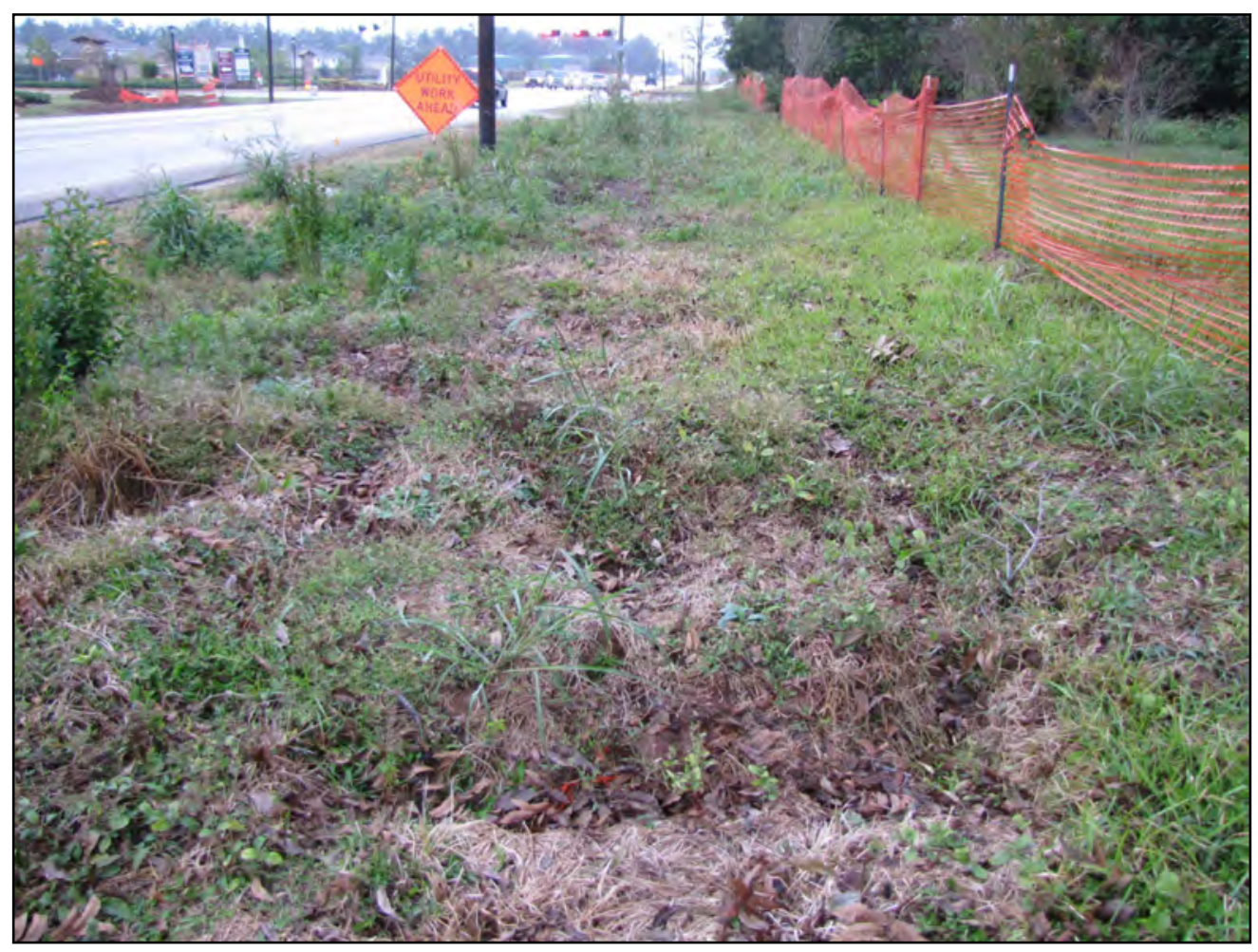

Figure 11. Project area, looking north.

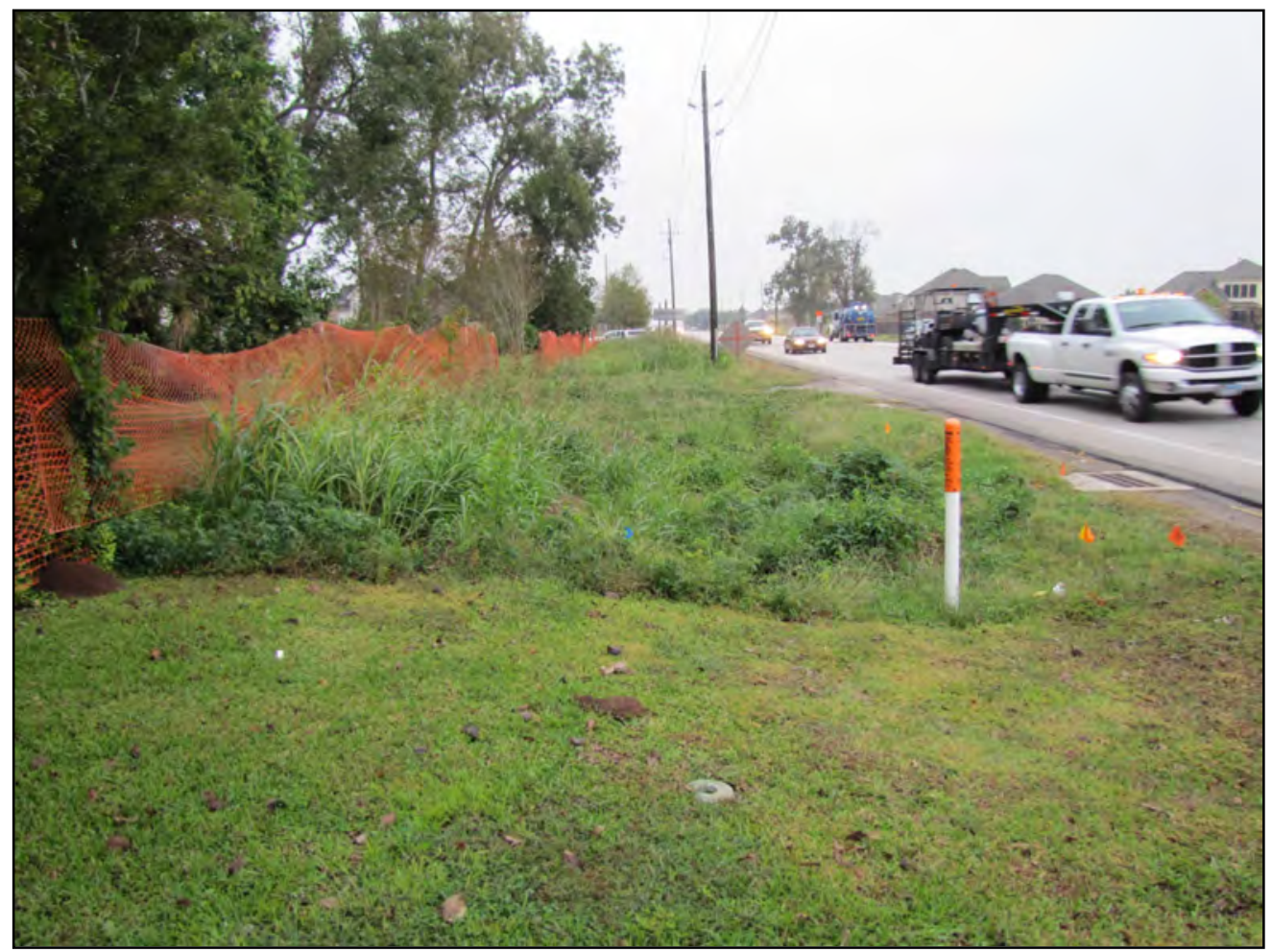

Figure 12. Project area, looking south. 
After a grid and benchmarks were established with a standard optical transit total station for the entire site, stripping began in the southwestern corner. This location was the area where PBS\&J had located what appeared to be intact graves (Cordova and Anderson 2010). All stripping was under direct supervision and instruction of the field director, with field assistants standing adjacent to the margin of the area being stripped. Areas equivalent to two bucket-widths were stripped to a maximum depth of $7 \mathrm{ft}(2.1 \mathrm{~m})$. However, it became increasingly clear that the soils were wetter and held more moisture at a depth of approximately $5.5-6 \mathrm{ft}(1.7-1.8 \mathrm{~m})$, and by $7 \mathrm{ft}$, the soils were soupy and unsafe. In addition to power equipment personnel (Mike Warnasch, Rick Wrobliski, and Sharkey Espinosa), TxDOT provided a safety monitor (Jim Ross) throughout the project (Figures 13 and 14).

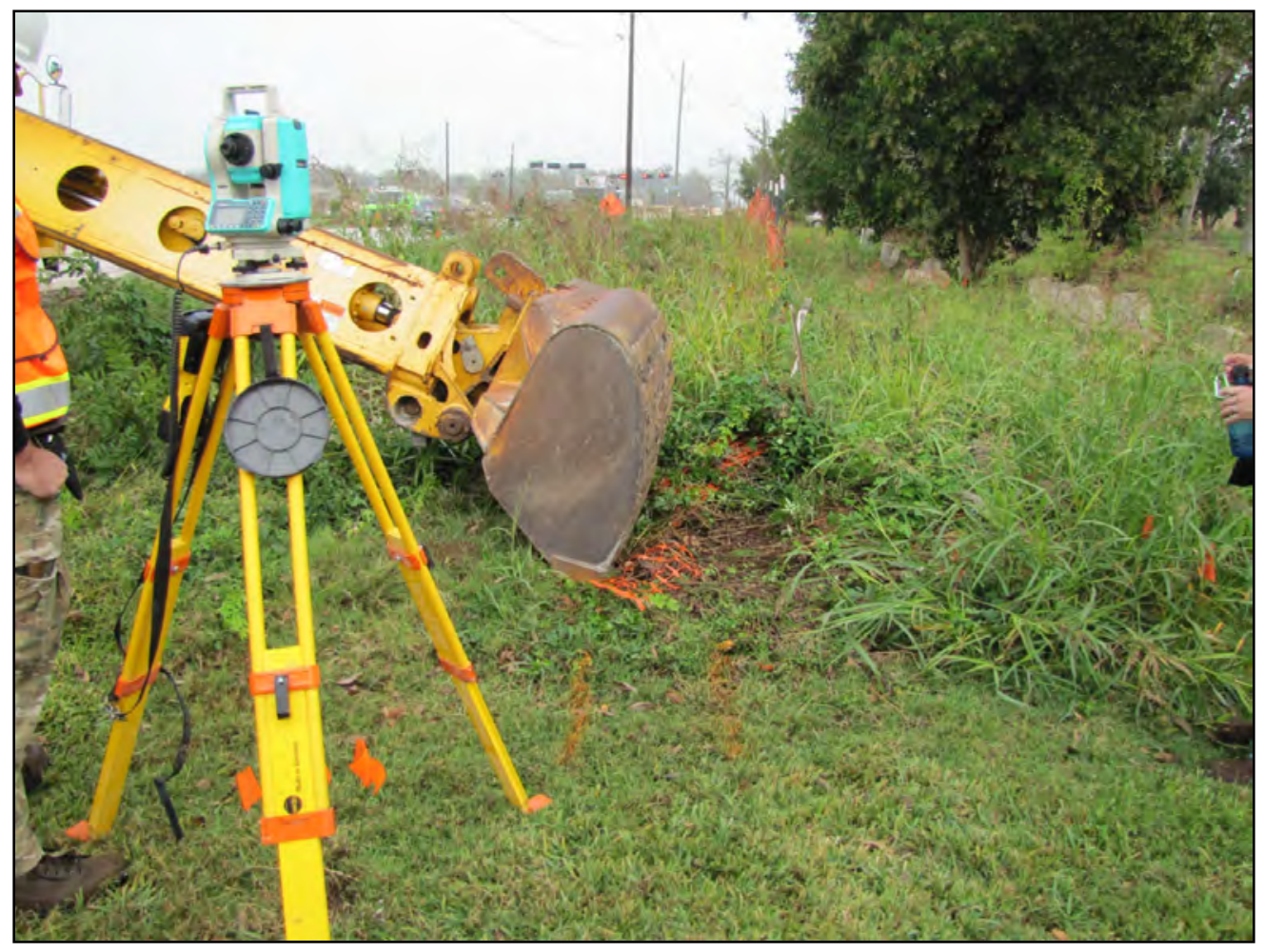

Figure 13. Gradall beginning to scrape southern margin of ROW, looking north.

The field director and field assistants monitored all soils as they were deposited on the outside margin of the open unit. The field assistants subsequently scanned the deposits and removed any materials. If a significant artifact was encountered, its location was plotted with the total station, and excavation continued. Power stripping was careful and methodical, with soil being stripped in thin layers of approximately 3-6 cm (1.2-1.8 in) per scrape (see Figure 14).

Originally, power stripping was accomplished with a standard Gradall trackhoe outfitted with a smooth-edge bucket. The Gradall was positioned perpendicular to the existing roadway at the beginning of the first week, but due to the size of the Gradall and the proximity to moving traffic, the position was changed to parallel to the roadway by the end of the week. The Gradall proved to be too large and unwieldy for the south-north-south movement back and forth in such a 


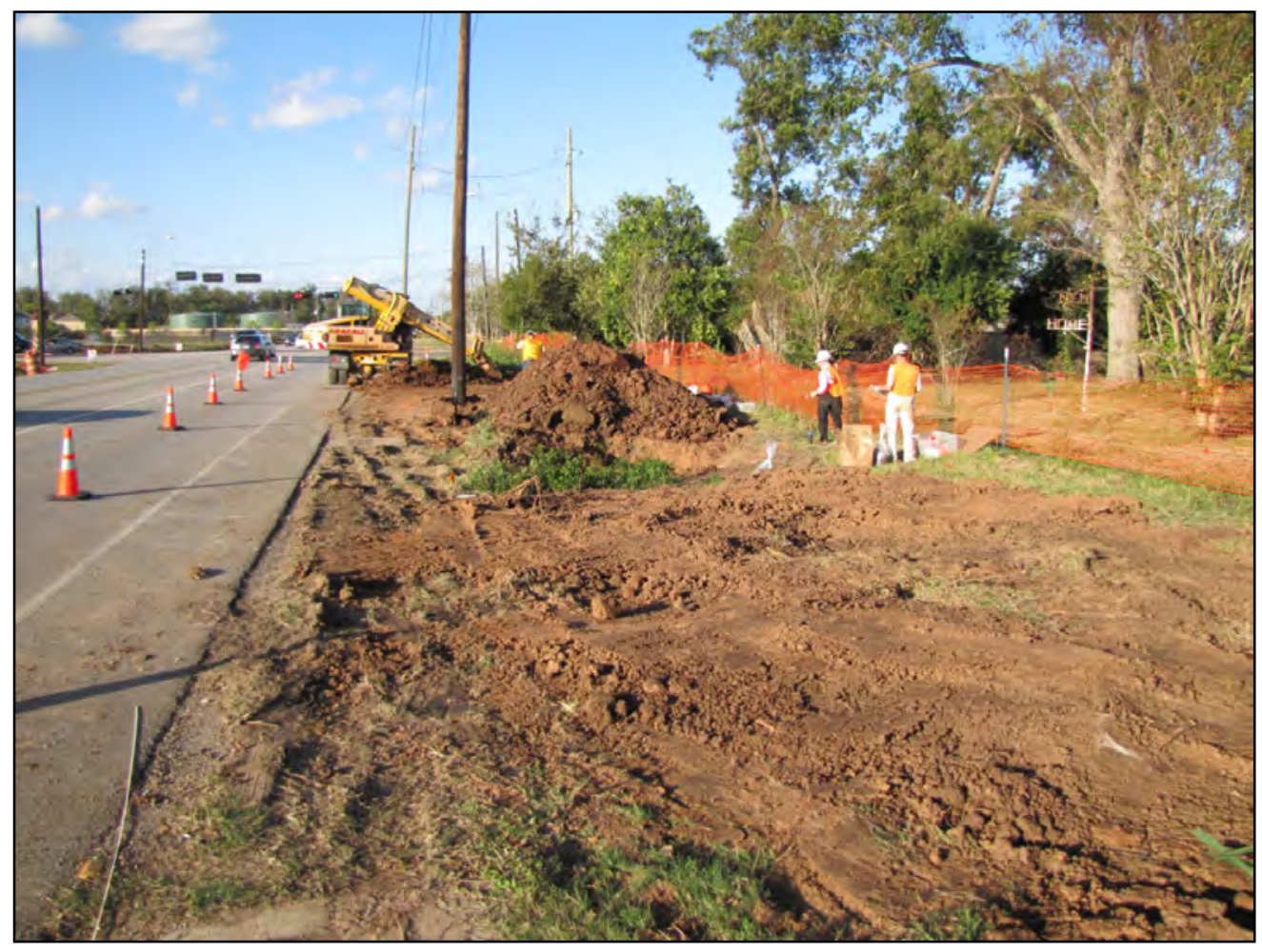

Figure 14. Geo-Marine crew and Gradall; stripping in progress, looking north.

narrowly confined space adjacent to moving traffic, particularly when it became clear that there were more burials than had been initially anticipated, and they were located throughout the project zone. Subsequently, the power stripping was accomplished by a standard backhoe outfitted with a smooth bucket, and it was positioned parallel to the roadway for the remainder of the first phase of excavation.

The soils had been subjected to varied disturbances, including the installation of numerous public utilities, the construction of a drainage ditch, and the construction of a concrete and brick fence for the border of the cemetery that also included plantings of crepe myrtles. Over the years, these trees had formed massive root balls and a web of roots. The trees had been cut prior to the beginning of the project, and the portion of the fence that was aboveground also had been removed. However, very large concrete anchor pillars were still in place just below the surface. As a consequence of all of the aforementioned alterations to the landscape, the soils were exceedingly difficult to assess. No grave shafts were discerned; all interments were encountered by discovery.

A metal detector was used as a supplementary device for locating burial containers when excavation reached a level of approximately 3.5-4 ft (1.1-1.6 m) below surface. Once the outline of a possible burial containers was encountered, mechanical stripping ceased. The remainder of the exposure and recovery of remains was accomplished by manual excavation, using standard trowels, a variety of brushes, and an array of bamboo tools provided by the field director. No metal tools were used on the skeletal elements themselves. 
After each skeleton was exposed, a scaled drawing and photographs were completed with the remains in situ. Notes were made, and bags were labeled for all artifacts and skeletal segments. The skeleton was removed in sections: cranium and mandible, torso, pelvis, right arm and hand, left arm and hand, right leg and foot, and left leg and foot. During the exposure of the remains, soil that was surrounding the remains was kept in a large plastic bag and labeled. This "sentimental soil” was kept for each individual. Additionally, fragments of wood from burial containers were placed in bags and retained for possible identification of the wood, and for eventual reinterment with the remains

Prior to removal, the field director reviewed the burial and took notes on various observations. The remains of each individual were placed in a plain cardboard box that was labeled appropriately; it was taken immediately to the truck. All remains were handled in a respectful manner. Artifacts were packaged separately. After all remains and artifacts had been removed, the area underneath the grave(s) was power stripped to a depth of approximately $7 \mathrm{ft}(2.1 \mathrm{~m})$, per contractual agreement, in order to ascertain that there were no burials at a lower depth. In one instance, Burial 15 was discovered partially underneath Burial 12.

After the entire project area was fully excavated, the decision was made that the Geo-Marine crew would return to the project area when the asphalt was removed from the existing roadway in preparation for the new road. Nine intact burials had been located immediately adjacent to the existing roadway during the first phase excavations. In order to certify that no human remains were underneath the road, Geo-Marine conducted the same power stripping protocol for the full length of road that passed in front of the cemetery. Stripping proceeded from the southern margin to the northernmost margin, using the backhoe at first, but then changing to the Gradall because of the density of underground utility lines. Some of the utilities, in fact, proved impenetrable for the backhoe. No additional graves were encountered. However, a wooden bridge foundation at the entrance of the cemetery was identified, as well as the remainder of the brick and concrete foundation for the aforementioned fence (Figure 15).

At least three road-resurfacing episodes were identified. These, plus the numerous buried utilities, probably obliterated any graves that may have been located there. The descriptions of items recovered by PBS\&J (Cordova and Anderson 2010) for the historic sites located north and south of the cemetery match items recovered from the cemetery itself. However, the extent to which these historic areas may have been related to the church and cemetery is unclear.

Mindful of public health concerns pertaining to the excavation of historic cemeteries (Bybee 2003a; Sledzik 2000; Ubelaker 1995), a "cleaning station” was provided by the field director. This consisted of antibacterial soap, antibacterial foaming hand sanitizer, and individual antibacterial sanitizing hand wipes. These materials were placed next to the water canister each day and were available to all personnel throughout the field and laboratory sessions. Historic cemeteries are known to be problematic for individuals who are involved in the disinterment of remains; this is particularly true for cemeteries that date to the postbellum period (after the Civil War) and into the early twentieth century (Bybee 2003a; Sledzik 2000; Ubelaker 1995). In fact, Geo-Marine did discover embalming fluid containers and tubing inside two of the caskets that were disinterred. 


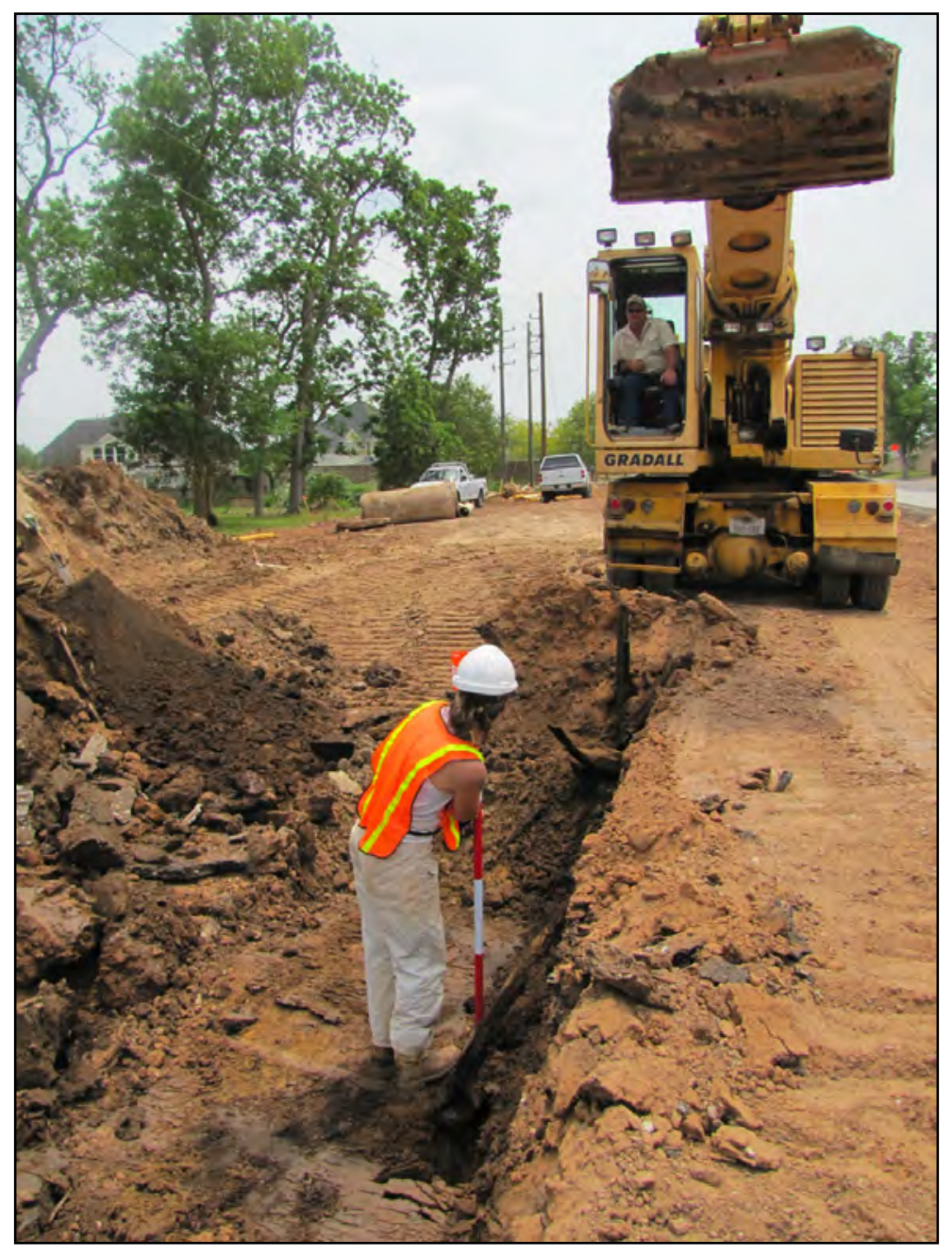

Figure 15. Wooden bridge at cemetery entrance.

\section{LABORATORY MATERIALS AND METHODS}

The same "cleaning station" materials were used throughout the laboratory sessions, with the addition of nitrile disposable gloves. Because there was no source of water available onsite, the Geo-Marine crew was provided access to water spigots within the TxDOT staging area that was a secure space not far from the cemetery. The area was surrounded by a tall chain-link fence and was secured with a series of gates and locks. Because the public thoroughfare passed by the staging area, a tent was set up for privacy. Tarps were secured to the sides of the tent and the section of fence that bordered a small private residence, and the Geo-Marine truck was positioned in such a manner that the Geo-Marine crew could not be viewed from the roadway. In that manner, privacy was maintained throughout the cleaning activities.

The boxes containing the remains were transported from the climate-controlled storage facility to the staging area. The remains were placed in small plastic screening tubs that had 0.25 -inch hardware mesh forming the bottom of the tub. This was placed on top of window screen mesh, and water was gently washed over the remains to remove the majority of soil adhering to the 
remains. This first wash was accomplished by the field director in order to make note of vital information. The remains were then transferred to window screens placed on sawhorses for the second, fine-tuned cleaning by the osteology assistants. Using an array of hair-bristled brushes and small bamboo and wooden picks, each burial was cleaned and placed on screens to dry. Once thoroughly dry, the remains were wrapped in clean, dry toweling and returned to their respective containers. Photographs were taken of specific observations throughout the cleaning procedures. All of the burials were completely cleaned before the second phase of the laboratory work was initiated.

After cleaning, human remains were taken to a secure, climate-controlled room in Sugar Land. The senior osteologist and the two osteology technical assistants completed all assessments following the guidelines in Standards for Data Analysis (Buikstra and Ubelaker 1994). Standard texts for osteology identification and analyses were referenced throughout the assessment phase; these included Bass (1987), Hillson (1996), Reichs (1998), Steele and Bramblett (1988), White and Folkens (1991), and numerous others. All individual skeletons were assessed for age, sex, pathology and anomaly, cause of death (if possible), and possible ethnic affinity. Additionally, the senior osteologist completed inventories and any possible assessments on all isolated bones that were not assigned to a particular burial; these elements presumably belong to the individuals whose graves were relocated prior to the current mitigations. Stature was not assessed because it was obvious from macroscopic examination that there was probable population admixture. Assessments for stature (and actually, other types of assessment) are highly dependent on population-specific-derived standards (for example, formulae derived by discriminant function analyses). Many of the formulae and/or computer programs have been derived from modern skeletal collections that include forensic cases or certain segments of the community at large that may or may not represent the general living population. For instance, the Montague Cobb Collection at Howard University (the largest comparative study collection of African American skeletons) is primarily composed of the unclaimed poor or indigent from the Washington, D.C., morgue. These may not be appropriate for comparison to historic cemetery samples.

In order to minimize interobserver error, specific aspects of the analysis were assigned to each osteologist. The senior osteologist was responsible for the compilation of all folders, plus all visual assessments of pathology and anomaly, plus the inventory homunculus drawings (blank drawings of the skeleton that are color-coded). The folders contained all paperwork, including field drawings, notes, burial forms, and all analysis forms for each individual as well as each of the coffins, and all of the miscellaneous and isolated bones. Teresa Nugent completed the skeletal anthropometric forms, inventory scoring, and age and sex scoring. Lindsey Skelton completed the dental assessments, odontometrics, and dental inventory and homunculus forms. Standardized metrical instruments were utilized: spreading calipers, sliding calipers, steel and fiberglass metric tapes, and osteometric board. The Arizona State University (Turner et al. 1991) system of dental casts was utilized to assess nonmetric variation in tooth form. The inventories for all dental and skeletal remains were recorded as text and as numerical codes. The remains were extensively photographed with a digital camera. Only macroscopic visual assessments were recorded for pathology and anomaly; there was not enough time for numerical coding. Time and budgetary constraints did not allow radiographic or microscopic assessment.

Artifacts that were discovered in the soil matrix during the cleaning of the remains were placed in appropriately labeled bags and placed with the other artifacts that were recovered in the field. All artifacts recovered from the overburden plus artifacts from the burials were transported to GeoMarine. Lindsey Skelton and Melissa Green cleaned, photographed, and conducted analysis of 
personal artifacts recovered from burial excavations. Most mortuary hardware was temporarily transferred to mortuary hardware specialist Jeremy W. Pye at the University of Florida for analysis and photographing.

When the analyses were completed, all human remains and mortuary hardware, along with the remainder of the burial artifacts were transported to Geo-Marine and stored in a secure space to await the notice to proceed for the second series of excavations. When notice to proceed was given, the remains were returned to Sugar Land for repatriation.

A copy of the Geo-Marine burial form is presented in Appendix B. Copies of the standardized data forms that were utilized for the analysis of the human remains comprise Appendix C. Although no photographs of the remains appear in the report, all photographs and analysis forms are archived and available for review through Geo-Marine and the Texas Historical Commission.

\section{PREPARATION FOR REPATRIATION}

Wooden boxes were constructed at Geo-Marine. Each box was labeled with a brass tag that had been engraved with the burial number. The sentimental soil and wood fragments from the burial containers that had been retained from each respective burial were placed inside the box; the remains were placed on top of the soil, being mindful of the placement of the cranium at the "head" of the burial box. All personal artifacts and funerary hardware were placed with the remains and the box was sealed. Grave markers were made for each of the burials. Each stone was labeled with a single word: "Unknown," except for Burial 5, whose actual name, "Leah Simpson," was inscribed. All burials and grave markers were transported to Sugar Land where they were placed in a secure, climate-controlled storage facility until time for repatriation. 



\section{CHAPTER 4 BIOARCHAEOLOGICAL DESCRIPTION AND REPATRIATION OF NEW HOME CEMETERY BURIALS}

by Mary Cassandra Hill

A minimum of 24 separate funerary events was represented by the remains and artifacts that were disinterred during this project (Figure 16). However, they were not of equal state of preservation and completeness due to the extensive, intrusive activities that had altered the landscape prior to the final recovery efforts. Additionally, scattered human bone elements and fragments were recovered from the "disturbed area" that were not assignable to any of the discrete interments. These skeletal elements were identified and analyzed individually, and then were included as a separate category within the biological descriptions. Eleven (45.8 percent) of the 24 burials contained articulated skeletons. Twelve (50 percent) were only partially represented, and one coffin contained no human remains. There were 16 individuals for whom sex determination was possible: 10 males and 6 females. Even though the individuals do not meet the criteria for a representative population sample, they reflect certain aspects of what life was like for the working class of Sugar Land.

\section{SKELETAL DEMOGRAPHY}

After years of contentious exchanges between and among archaeologists, biological anthropologists, descendent communities, activists, and various private and public funding agencies, the Paleopathology Association took on the monumental task of assembling a committee that would compile a list of biological information. This information would be considered absolutely essential to any study of human existence, past or present. The committee polled the association members at large. Technical methodologies were reviewed and then assembled in a cogent format for dissemination to the professional anthropology community at large. The reasons for the resultant volume, Standards for Data Collection from Human Skeletal Remains (Buikstra and Ubelaker 1994), were that decades of disparate methodologies and theoretical tenets had resulted in volumes of texts and reports for which their data were irreproducible by other researchers. Comparisons could not be made across data sets in many instances. The passage of the Native American Graves Protection and Repatriation Act (NAGPRA) legislation forced biological anthropologists into a dilemma of reburying skeletal collections without meaningful scientific data documentation. The Standards represents the general consensus agreements for the methods that would be used for all sets of human skeletal 


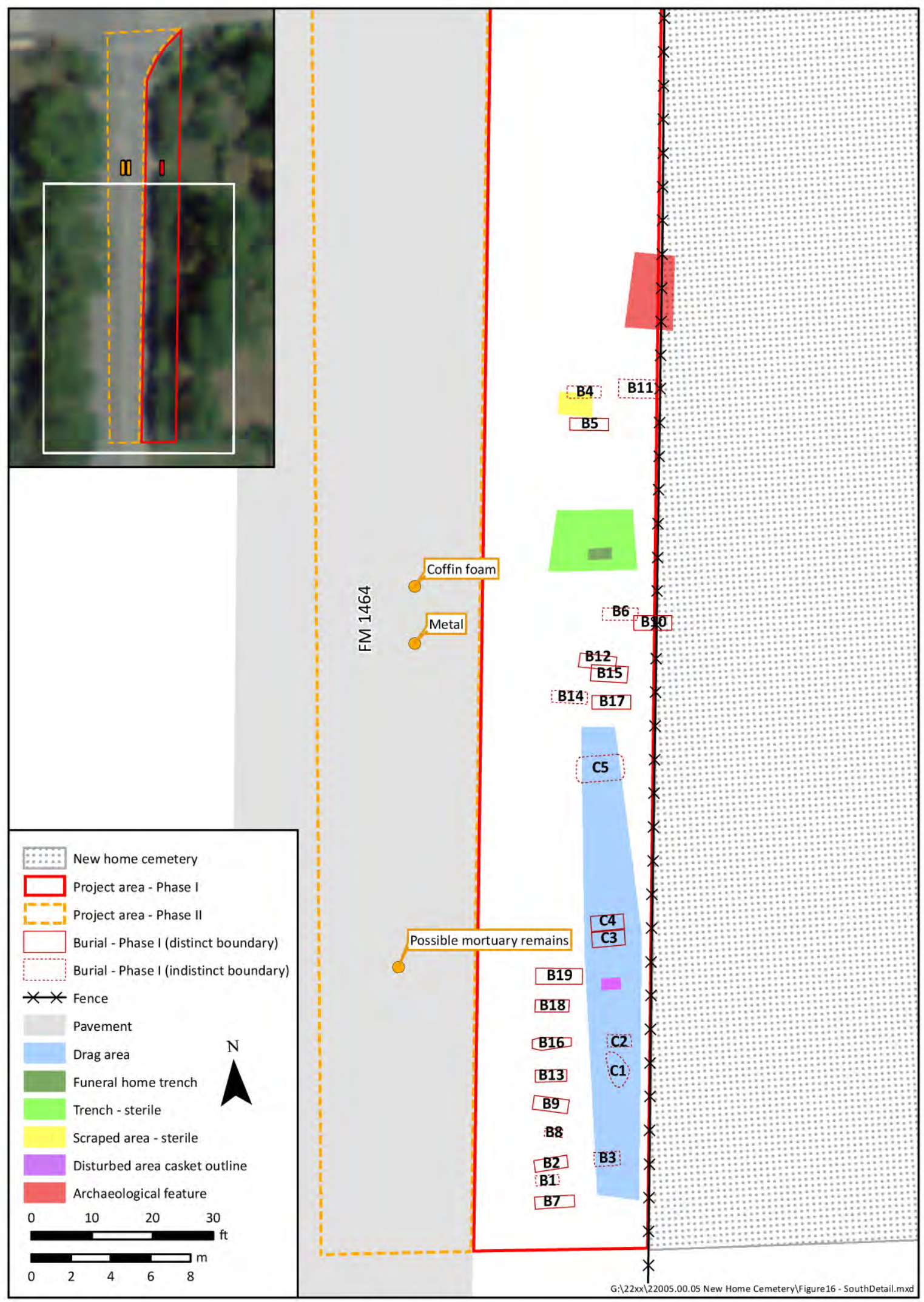

Figure 16. Plan map of the southern portion of Phase I and II project areas adjacent to New Home Cemetery (CSJ 1415-02-032). 
assemblages. The most important point to take into consideration is that for most skeletal studies (particularly for cultural resources management reports), there is only one chance to document the remains. Knowledge of the methodologies summarized in the Standards is absolutely essential. In addition to the Standards, Bass (1987) and the authors in Işcan and Kennedy (1989) offer excellent reviews of skeletal assessment methodologies.

Age is a continual process from conception to death. Thus, any measures of age would be the sum of all processes of development and degeneration. The hard tissues include both dental and skeletal elements. For younger individuals, age is assessed by examining the development of dental crowns and roots; the formation and eventual coalescence of skeletal ossification centers for the cranial base, mandible, and all postcranial elements (enchondral bone); and the formation/shape and eventual coalescence of the upper elements of the cranial vault (intramembranous bone). In other words, the size and shape of bones and teeth are evaluated on a continuum. The tooth crowns push through the hard tissues of the upper and lower jaws at an expected rate. This is the eruption sequence. Growth (maturation) is evaluated by measuring the lengths and widths of postcranial bones, and by measuring the overall size and shape of the cranium and mandible. For individuals who have reached the maximum potential for growth (adults), the processes of degeneration are evaluated by examining the effects of wear and tear over time. These include the wearing away of the surfaces and eventual loss of the teeth, the changes in the surfaces of articulation of certain bones (the pubic symphyses, the sacroiliac articular platforms, the major joint surfaces), the loss of vertical height of the spinal elements, and the formation of bone spurs from the articular cartilage of the central tips of the ribs. Appropriate references, charts, drawings, and photographs for each of these indicators are presented in the Standards. Because the actual chronological ages may not ever be known, individual comparisons of population samples are evaluated on a more or less equal basis, if the Standards are applied equally across the board.

The determination of $\boldsymbol{S e x}$ is only attempted on individuals who have reached or passed adolescence, because that is when hormones that affect growth and maturation work in tandem with the hard tissues to create the differences between males and females. The skeletal and dental traits that are utilized most frequently include the size and shape of certain teeth; overall bone size in general; sizes of the heads of the femur, humerus, and radius; size and shape of the innominate and sacrum (pelvic bones), certain aspects of the innominate (sciatic notch, auricular platform, and preauricular sulcus); and size and shape characteristics of the cranium and mandible. Although overall size may be used to discern sexual dimorphism, one must keep in mind that notable size differences exist among various populations around the world. There may even be notable size differences among members of a single family. This is of particular consideration when assessing African Diasporic samples because there is pronounced variation in size among different groups (for example, the Bushmen and the Maasai).

Ethnic Affinity/Ancestry is most frequently determined by examining certain aspects of the cranium, mandible, and teeth, as well as anterior bowing and metaphyseal flattening of the femora. (The metaphyses are the flared, spongy parts at the ends of the tubular bones). The three primary ethnic populations into which individuals are sorted are Mongoloid/Asian, Caucasoid/European, and Negroid/African. However, most researchers will agree that it is exceedingly difficult to make assignations to a particular affinity (unless DNA samples are taken) because of the amount of genetic admixture that has taken place even within the last 100 years. In addition to those listed above, numerous other skeletal traits also may be examined; these are reviewed in Bass (1987), Işcan and Kennedy (1989), and the Standards (Buikstra and Ubelaker 
1994). The traits include the size and shape of the orbits (eye sockets), the shape of the nasal opening, the shape and texture of the cranial vault, alveolar prognathism (the forward-projection of the upper and lower jaws and teeth), and nonmetric variation in certain dental characteristics (for example, Uto-Aztecan premolar identified most frequently in Native American groups, shovel-shaped incisors identified most frequently in Native American groups, and Carabelli's cusp identified most frequently in Euro-American groups). Additionally, postcranial observations, such as bowing of the diaphyses (more frequent in European and Asian) and flattening of the distal femoral metaphyses (more frequent in African), may be cited for ethnic affinity. However, both of these parameters are affected by the development of muscle mass from repetitive activities.

\section{PATHOLOGY}

The purpose of this section is to provide a brief, general discussion of the types of pathology that can be identified on skeletal tissues and also to provide an explanation for some of the pathology that was identified and evaluated for each of the individuals in the sample. More-specific reviews of the pathology appear in the individual descriptions. For purposes of comparison, only general categories are utilized in the majority of paleopathology reports. This is to avoid problems that arise because of the absence of the full spectrum of diagnostic criteria that are utilized for living patients. Pathologists utilize four descriptive categories to diagnose an abnormality: rubror, calor, dolor, and tumor (color, temperature, pain, and swelling). Soft tissues have the capability to express any of these. Skeletal tissues are limited in their response capabilities to pathological perturbation. In fact, only two processes, bone formation and bone resorption (and sometimes a combination of the two) work to produce lesions on bone. The following is a list of categories of skeletal pathology. Excellent sources for skeletal pathology references include Jaffe (1972), Işcan and Kennedy (1989), and Ortner and Putschar (1981).

Markers of Occupational Stress (MOS) are any areas of tendon or ligament attachments that appear outside of the range of "normal" variation. Tendons attach muscles to bone surfaces, and the areas where they attach are called entheses. Ligaments attach bones to other bones, and their locations for attachments are called syndesmoses. Abnormalities of these attachment locations are referred to as enthesopathy or syndesmopathy, respectively. However, many researchers only use the term, "enthesopathy" to describe all pathology of connective tissue insertions. Sometimes these areas form bone spurs, or ossifications, at the attachment sites. These are called enthesophytes and syndesmophytes, respectively. Bone spurs also may form on any bone surface, usually at the margins of articulation, and these are called osteophytes.

MOS also may include certain types of fractures that are pathognomonic (characteristic or diagnostic) for specific activities or mechanisms of injury. "Axial loading” encompasses a suite of traumatic injuries associated with exceeding the maximum capacities or physiological limits of endurance. It refers to excessive force(s) applied in a vertical direction from the top to the bottom of one or more skeletal elements and is associated with weight-bearing stress. Excess weight applied to the vertebrae may result in the extrusion of the cartilaginous pads that are between consecutive elements; this will leave a "punched-in" or gouged mark on the top and/or bottom of the centrum, called a Schmorl's Node. Axial loading may also be associated with blunt force trauma, such as a blow to the top of the head. Axial loading injuries include cranial base fracture, fracture of the first cervical vertebra (Jefferson fracture), osteophytosis (bone spurs) of the cervical vertebrae, fractures of the pedicles of the vertebrae (the points where the neural arches 
connect to the centra), and linear/vertical splitting of the bones of the legs. Kennedy (1989) provides an excellent, comprehensive discussion of MOS, including a summary chart that also provides secondary sources for specific markers on individual bones.

Degenerative Pathology occurs as a normal process of aging, but it also may be influenced by certain excessive and repetitive activities that eventually lead to the erosion of the articular surfaces, and in advanced cases, the breakdown of the underlying bone. Cartilage covers the articular surfaces and provides a protective buffer. When this protective layer breaks down, the resultant friction of bone on bone destroys the surfaces. There may even be enough friction to produce a thickened, highly polished secondary surface (eburnation). Excessive hard-work activities may result in premature aging of the skeletal tissues.

Trauma is an injury or insult to the body that may be caused by chemical, thermal, or other extrinsic agent, or by violence. Trauma may be an instantaneous event, or it may be cumulative (such as tiny microfractures that eventually lead to a larger, pathological fracture). Trauma may be accidental or nonaccidental. Accidental trauma would include broken toes, or fractures to the lower portion of the forearm resulting from falls (Colles' fracture). Fractures of the sternal ends of ribs may result from an accidental fall, a crushing injury, or from a beating (nonaccidental trauma). Small, rounded indentations on the ectocranial (outside) surface are called Pond fractures. Pond fractures may be accidental (such as a kick in the head by a horse), or they may be nonaccidental (such as striking an individual with a hammer or baton). Pond fractures may be delivered with enough force to produce a small fragment/spall of bone on the endocranial surface; these are usually fatal. Fractures of the central aspect of the forearm are called parry fractures, and they are nonaccidental, defense wounds (when a person uses his/her arm to shield the face and head). Multiple fractures of two or more adjacent ribs, particularly if the fractures are bilateral, are caused by crushing injury; these can be accidental (crushing by machinery) or nonaccidental (a severe beating). The result is Flail Chest, in which the ribs move freely from side to side during inspiration, rather than expanding in and out with normal respiration. If not treated immediately, the result is fatal. Boxer's fractures are identified on the metacarpals (the tubular bones that form the body—palm and back—of the hands. These are only a few of the many types of trauma to bones. Kozin and Berlet (1992) provide an excellent summary of fractures, their classifications, and mechanisms of injury.

Infection/Inflammation on bone is most often identified by the presence of fibrous, woven bone that has been applied to the surface (cortex) by osteoblastic (bone forming) activity associated with some form of stimulation of the periosteum (the fibrous outer covering of bone). This woven bone may be applied in layers. It is referred to as "periostitis" in the overwhelming majority of references. Over time, the periostitis may be remodeled through osteoclastic (bone cells that remove bone) activity combined with osteoblastic activity (the bone resorption and bone formation mentioned above). Osteoblasts are bone cells that make new bone. Osteoclasts are bone cells that remove bone. These two processes may be active or healed, or a combination of both at the time of death. Periostitis is not always caused by infection, however. Other conditions will stimulate the periosteum to form new woven bone. An example would be venous stasis (poor circulation) that would cause swelling in the soft tissues, putting pressure on the periosteum to the point that it becomes irritated. Thus, infection is not the cause of the periostitis. It is very important not to rely too heavily on reductionist explanations for complex physiology. 
There are and were thousands of infectious agents in the environment. A few of these are indeed pathognomonic for skeletal tissues and can be identified by the presence of certain configurations of lesions. Skeletal tuberculosis and treponematosis (venereal and nonvenereal syphilis and yaws) are two. Prior to the development and distribution of broad-spectrum antibiotics in the 1950s, infections of any sort could eventually lead to mortality. Abscessed teeth were and continue to be causative agents in systemic infections that may lead to heart failure and death. In addition to bacterial infection, parasitic infections also affect health and mortality.

Metabolic Distress includes vitamin and mineral deficiencies. Porotic hyperostosis and cribra orbitalia are attributed to anemia in the paleopathology literature. They are lesions on the cranial vault and/or eye orbits that have a characteristic appearance of pitting and porosity that sometimes looks like volcanic pumice in the most extreme expressions. Most reports attribute these lesions to iron deficiency anemia or sickle cell anemia. These interpretations are antiquated (Hill 2001; Hill and Armelagos 1990). They do not take into account that there are multiple genetic anemias, not just sickle cell disease, or that anemia may occur for many vitamin and mineral deficiencies, not just iron deficiency. The porotic lesions on the cranial vault and in the orbits are pathognomonic for any of the anemias. Anemia is the physiological failure in transport and exchange of oxygen to all tissues. Deficiency in any of the primary nutrients, such as vitamins A, B, C, may result in a fragile red blood cell membrane that breaks apart before the oxygen is delivered to its target tissue (Hill 2001). The hemoglobin molecule may be complete, in terms of adequate iron for attachment of oxygen, but because of other nutritional deficiency, the transportation of oxygen fails. Porosity on the sides of the cranial vault and on the mandible has been attributed to scurvy (vitamin C deficiency). Vitamin D deficiency may result in rickets (poorly mineralized bone that bends due to normal weight bearing). Nutritional deficiency is always associated with multiple elements that are insufficient.

Skeletal Asymmetry/Growth Disruption may be an indicator of environmental stressors. They are classified as congenital abnormalities. Cranial asymmetry may occur when one or more cranial sutures close prematurely, resulting in a misshapen skull (Hill 2001). Skeletal asymmetry may also be observed in the long bones or any of the skeletal elements throughout the body. Cleft palate and spina bifida are two of the more familiar congenital abnormalities.

Dental Enamel Defects and Pathology include caries (cavities), hypoplasia (growth disruption of the dental enamel), hypocalcification (poorly mineralized enamel), hypercementosis (lumpy, overgrowth of the hard tissues of the tooth roots), calculus (plaque formation), periodontal disease (inflammation of the gums and bone that surrounds the tooth roots), and abscessing. Caries, periodontal disease, calculus, and abscessing are associated with poor diet and poor dental care, and infectious agents that may be introduced secondarily, due to the breakdown of the hard tissues. Hypoplasia and hypocalcification are indicative of stressors that are sufficient enough or of prolonged duration to affect the growth of the dental hard tissues. Hypoplasia can be in the form of lines or pits on the tooth crown surface, and indicate periods of growth failure. These can be measured and then compared to chronological standards to determine when the insult/perturbation occurred. They are indicators of childhood stress. Similarly, hypocalcification is the generalized, poorly mineralized appearance of the tooth crown. It is indicative of generalized stress throughout the formation of the crown. Hypercementosis is excessive formation of matrix on the tooth roots and is associated with generalized, poor dental health, as in the loss of the alveolar bone that surrounds and supports the teeth. 


\section{ANALYSES AND DESCRIPTIONS OF BURIALS}

The information on the individuals whose remains were recovered is presented in narrative format, similar to Nawroki et al. (1998). The narrative format is used in order to focus on the aspect of humanity that frequently is absent from cultural resources management summaries.

\section{Individuals from Defined Burials}

\section{Burial 1: Undetermined Age; Undetermined Sex}

On the first day of the recovery, fragments of long bones, lying parallel to each other and also in alignment (femora and tibiae?), plus wood fragments from a burial container, were exposed between Burial 7 and Burial 2. These remains were on the western margin of the proposed ROW, immediately adjacent to the existing roadway. Burials 7, 1, 2, 8, and 9 were buried parallel, very close to one another. All were oriented east/west with crania west. They had been impacted by Gradall activity during previous Phase II evaluations and subsequently were covered with black plastic, a tarp, and large pieces of painted plywood. The Geo-Marine crew was not able to fully expose and recover the remains of Burial 1 due to flooding of the unit by a ruptured sprinkler pipe. The long bones had relatively small and gracile appearance, suggestive of an adolescent and/or a female.

\section{Burial 1a: Human Fetus}

The tiny fragments of bone that were recovered from the Gradall bucket when removing the pieces of plywood were assessed as cranium of a human fetus, but because of the circumstances of their recovery, it was unclear whether the fetal fragments belonged to Burial 1, or if the fetus was in the immediate area adjacent to Burial 1. The fragments of fetus cranium were designated Burial 1a.

The cranial fragments exhibited striations and slight areas of "crusty" subperiosteal apposition, plus thickening of the diploë (the internal, spongy bone of the cranial components of the vault) of the postorbital area (the area just behind the eye sockets) of the frontal bone. All are indicative of metabolic distress (Hill 2001). A fetus that exhibits metabolic distress actually provides possible information about the mother. That is, the mother probably was in metabolic distress as well.

\section{Burial 2: Adolescent, Approximately $15 \pm 2$ Years; Undetermined Sex}

The grave was impacted by previous Phase II survey activity, and covered with black plastic and large pieces of painted plywood. Fragments of wood, metal handles, and a metal identity plaque were recovered, along with the human remains. Preservation was only fair, due to penetration of the grave by large roots. Tiny mother-of-pearl buttons, a large, fabric-covered, metal button, fragments of white cotton fabric, and a small fragment of a comb (Figure 17) were recovered during the exhumation and subsequent laboratory cleaning procedures. 


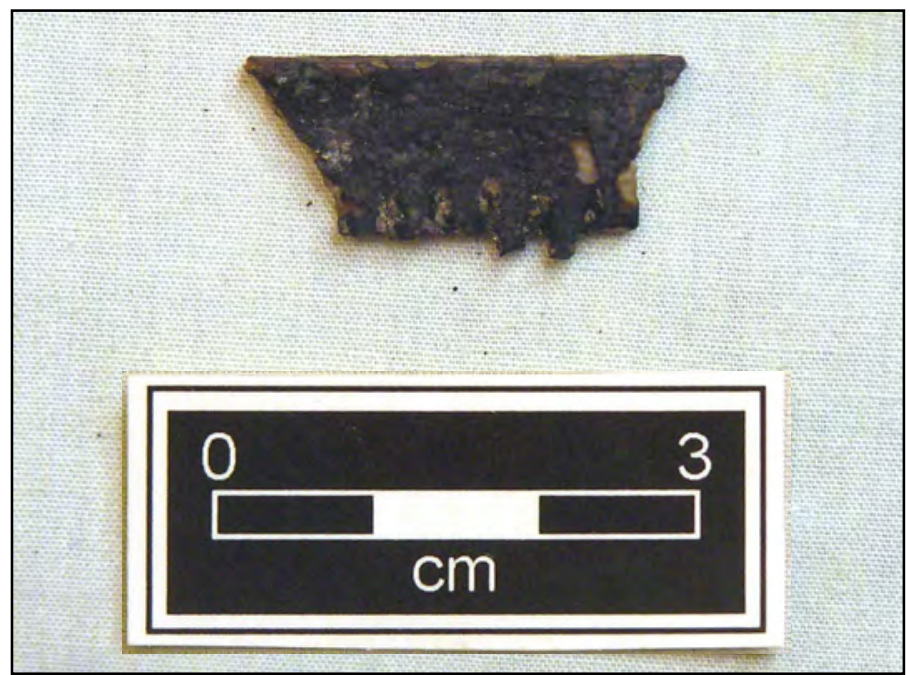

Figure 17. Comb fragment from Burial 2.

Observations of the skeleton included the following. The skeleton was of mature size, but the majority of long bone epiphyses (end plates) were not fused, indicating an age of adolescence, approximately 15 years. The skeletal morphology was gracile, attributable in large part to the age at the time of death, but perhaps also attributable to sex. The assessment scores for greater sciatic notch and preauricular sulcus of the pelvic bones were in the male range, but the nuchal crest (back of cranium) and mental eminence (chin) were in the female range (perhaps related to age, as well as sex). The humeri (upper bones of the arms) had septal apertures (holes at the elbows that allow hyperextension; large on left and small on right), which are observed more frequently in females (Bass 1987).

The teeth exhibited hypocalcification and hypoplasia. There also was a dental trait (Uto-Aztecan premolar) that is suggestive of Native American admixture. The vertebral centra exhibited large, hypervascular, resorptive pitting (indicative of increased demand for oxygen transport by increased red blood cell production). The cranium exhibited "hammered silver" indentations on the endocranial surfaces (inside the vault), particularly those bones forming the cranial base and lower portion of the vault (parietals, temporals, occipital). The area around the foramen magnum (occipital base) was rugose, and there was an "old" (i.e., perimortem; not fractured during excavation) fracture of the cranial base (darkly stained; angled/beveled margins of the fracture). The endocranial surface had a large fragment of bone spalled from the fracture site. The right temporal bone also exhibited a fracture that was part of the trauma to the cranial base. It produced an angled, beveled fracture that resulted in the separation of the petrous portion (ear canal) from the squamous portion on the endocranial surface. Multiple ossicles were in the lambdoid suture. Higher frequencies of lambdoid ossicles have been noted for African American and Native American populations, as well as with the cultural practice of head-binding, and with cranial suture development associated with hydrocephalous (a condition more commonly known as "water-head"; increased cerebrospinal fluid inside the cranium).

The hypocalcification, hypoplasia, and hypervascular pitting are diagnostic for metabolic stress. The "hammered silver" endocranial surfaces in combination with the multiple ossicles in the lambdoid suture are indicative of increased endocranial pressure. The rugose cranial base (accentuated entheses and syndesmoses for muscles and ligaments of the cranial base) and the 
cranial base fracture are diagnostic of axial loading (i.e., carrying heavy loads on the head and shoulders). Differential diagnosis would have to include the possibility of blunt force trauma that produced the ring fracture of the cranial base, even though there was no evidence of trauma to the superior aspect (top) of the cranial vault.

This individual had a short life that was characterized by multiple stressors, probably nutritional and socio-cultural. He/she habitually carried heavy loads on the head and shoulders, and died as a consequence of perimortem fracture of the cranial base. Death may not have been immediate; it may have occurred after several days, with leakage of cerebrospinal fluid, possible hemorrhage, and possible sepsis.

\section{Burial 3: Adult; Possible Male}

This rectangular, wooden casket with metal handles had been previously impacted by funeral company activity and by previous Phase II evaluations. The grave fill was a very distinctive, dark black, oily, dense clay, with a pungent odor. The skeletal fragments that were recovered appeared to be from a single individual. They included fragments of right maxilla with teeth, right zygomatic, left and right ribs, thoracic vertebra (approximately T8), left scapula, right innominate, right radius, left and right hands, left fibula, and right foot.

The overall morphology was relatively large and robust, and the teeth were relatively large compared to other individuals in this skeletal sample. The vertical diameter of the humeral head was 48.13 millimeters $(\mathrm{mm})$. A slight nasal sill was on the fragment of maxilla (possible European admixture).

The first cervical vertebra, C1, exhibited notable arthritic changes (lipping and apposition) of the articular facet for the dens of C2 (the little knob of bone that allows the rotation of the skull), possibly associated with an incomplete burst fracture (Jefferson fracture) of the ring on the left aspect. The thoracic vertebra exhibited arthritic accentuation of the articular margins of the apophyseal and zygapophyseal facets (top and bottom facets that stack on top of each other). Notable calculus was on the cingula (the interface of the crown and the root) of the teeth, and the teeth also exhibited hypercementosis (lumpy roots). The right maxillary first premolar was turned slightly distal in the socket.

\section{Burial 4: Adult; Undetermined Sex}

This was a decomposed steel casket (pressed metal over wood) discovered while scraping with the Gradall. At first, it was not recognized as an actual casket because of its disintegrated appearance. It consisted of fragments of wood, metal, decomposed foam rubber and shreds of polyester fibers, mashed flat and oriented east/west in the profile of the scraped area in which Geo-Marine was monitoring. Mangled casket extension handle and three skeletal elements were recovered from the backdirt pile, and a plastic button was recovered from the bits remaining in the profile. The casket apparently had been previously opened by a funeral company and the majority of skeletal remains were removed. Subsequently, the casket was closed and reburied by heavy machinery. 
The skeletal elements that were recovered were two phalanges of the right hand (complete) and a fragment of the third cervical vertebra. The digits exhibited enthesopathy/syndesmopathy of the flexors: the proximal phalanx had moderate-to-pronounced marginal lipping of the proximal articular rim plus notable accentuation of the flexor tendons and syndesmopathy of the articular ligaments; the distal phalanx had moderate lipping of the proximal articular surface and accentuation of the flexor tendon entheses. There also was a tiny sesamoid bone. All of these are indicative of hyperactivity of the hands. The cervical vertebra exhibited slight accentuation of the articular margins. The skeletal elements were small, and the button looked more like one from a woman's clothing (Figure 18). However, an assessment of possible female is purely conjecture.

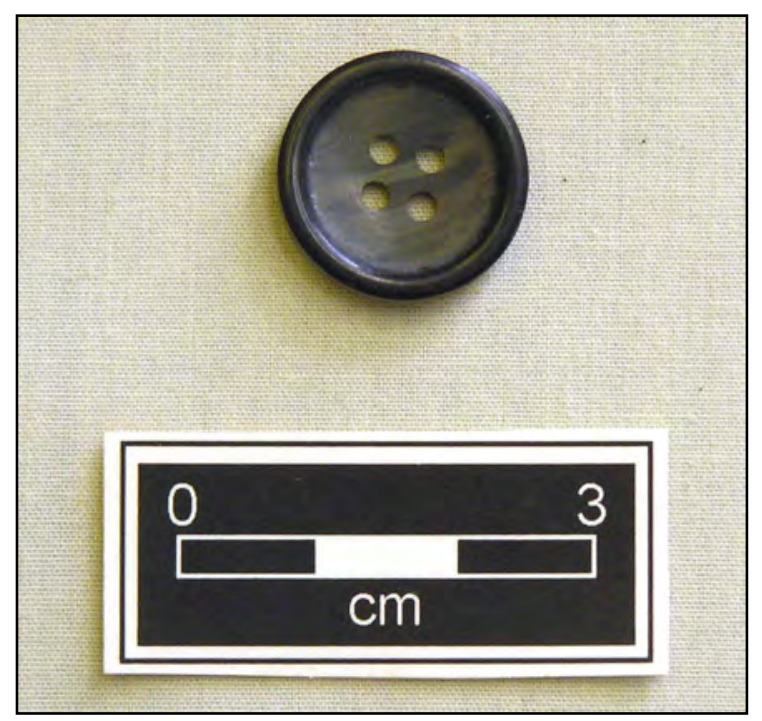

Figure 18. Button associated with Burial 4.

\section{Burial 5: Older Adult; Female}

This individual was interred in a rectangular wooden casket with metal handles. The casket outline was black, dense, sticky clay that had an oily, iridescent appearance and unpleasant odor. The skeletal elements were darkly stained due to the dark coffin fill. The person was supine and oriented east/west with cranium west. The pelvis, legs, and feet were inside pantyhose, and a film of nitre was underneath the pelvis when Geo-Marine removed the lower portion of the skeleton. During cleaning, a lingerie buckle was discovered underneath the cranium, and brass stains were on the right temporal and left parietal that were probably indicative of earrings, although no jewelry was recovered. Skeletal preservation was fair. A temporary grave marker (Marker Type 3, see Marker Type 3 in Chapter 5) that was recovered from the backdirt that had been scraped from immediately above the skeleton was cleaned in the laboratory and revealed the following on the inscription tablet: "Leah Simpson, Born October 10, 1883, Died May 17, 1954."

The skeletal assessment included the following. The overall morphology was small and gracile, and assessment scores and anthropometric measures that could be taken were all in the female range. The orbital borders of the frontal bone were very thin, and the frontal sinuses were small. The mandible exhibited morphological appearance indicative of senile age changes. All of the cranial sutures were fused and obliterated (advanced age). Inferior collars (a ruff of bone) were present on both auditory meati (openings for the ear canals). 
The skeletal elements were very fragile and exhibited advanced osteoporosis and osteopenia. The cortices were exceedingly thin and brittle. There was pronounced thickening of the cranial diploë; hyperostosis frontalis interna (lumpy bone deposited on the inside of the frontal bone that is a condition related to advanced age and/or hormonal changes); numerous small pacchionian pits (small, benign pits) on the endocranial surfaces; and deeply etched meningeal vessels on the endocranial surfaces. Despite the pronounced thickening of the diploë, there was no evidence of porotic hyperostosis or cribra orbitalia, but there was notable resorptive pitting of the greater wings of the sphenoid (vitamin and mineral deficiency associated with metabolic stress and scurvy). The maxillae and mandible were almost completely edentulous except for a root stump for the right maxillary first premolar and shallow sockets for the left mandibular second molar (no tooth was present, indicating antemortem evulsion just prior to death). The maxillae and mandible exhibited extreme resorption of the alveolar tissues. The mandibular gonial angles and rami (angles of the jaw) were as thin as eggshell and exhibited notable inversion. There was a small osteoma (benign bone tumor) on the inferior margin of the left aspect of the mandibular mental eminence (chin). Examination of the gonial angles of the mandibular rami determined that there probably had been some sort of large, soft tissue mass on the right side just inferior to the corpus, resulting in notable thinning of the corpus and gonial angle (possibly a goiter, given the presence of other markers of nutritional deficiency).

All long bones exhibited accentuation of entheses and syndesmoses. The distal humeri articular margins exhibited moderate-to-pronounced lipping. There was no evidence of advanced arthritis of the femoral heads, but there was platymeria (anterior/posterior flattening) of the proximal femoral metaphyses, indicative of development of the musculature of the upper legs. The anterior aspects of both femoral necks exhibited Poirier's facets, with the left side being more notable. These facets are indicative of hyperflexion of the hip, such as habitual squatting or sitting with the legs crossed (Kennedy 1989). There was a perimortem subtrochanteric fracture of the lateral aspect of the left femoral proximal metaphysis (spontaneous hip fracture). Additionally, the right femoral diaphysis and both fibulae and tibiae exhibited perimortem, linear fractures indicative of axial loading: the cortices were too thin and shattered into numerous linear strips of bone in response to the initial fracture of the hip.

There also was evidence on the metatarsal metaphyses and metaphysis of the right ulna of small, smooth-walled lesions within the trabeculae of the metaphyses (possibly indicative of a pathological condition that contributed to the osteopenia of the skeletal elements, making them more vulnerable to spontaneous fracture). The differential diagnosis for these lesions would include possible gout, systemic infection, or carcinoma.

There was active and sclerotic (smooth; healed) bone formation on the ectocranial surface/margin of the foramen magnum, and a small cloaca (drainage opening/sinus) on the right gonial angle of the mandible.

Death occurred proximate to the spontaneous pathological fracture of the hip and associated fractures of the tibiae and fibulae. The actual cause of death may have been a pulmonary embolus (a fatty marrow clot that breaks free and then travels from the fracture site to the lung or heart, where it occludes normal pulmonary function). 


\section{Burial 6: Older Adult; Male}

This individual was interred in a rectangular wooden casket, oriented east/west with cranium west. Large mother-of-pearl buttons (on the chest; Figure 19) and fragments of white cotton fabric were recovered, suggesting that he was wearing a white cotton nightshirt, pajamas, or shroud garment. All remains and coffin materials were recovered ex situ due to impact by the Gradall.

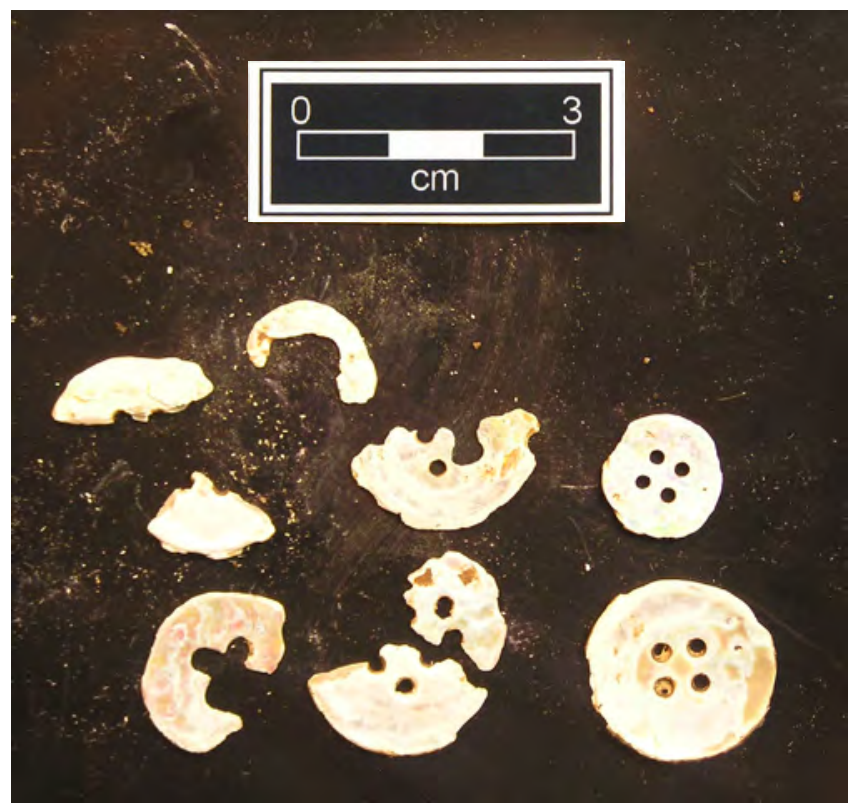

Figure 19. Buttons from Burial 6.

The skeletal elements that were present were large and robust in overall morphology. In general, there was notable arthropathy of the joints. The cortices of the ribs were notably thin and brittle. Observations for the cranium included the following. There was normal thickness of the diploë. The principal cranial sutures were fused. The right maxilla was edentulous (cannot asses the left side). The mastoid processes were short and rugose. The temporal bones exhibited temporal foramen, mastoid foramen, and "notched" temporal squamous. The mandible was very fragmented, but exhibited the following: senile morphology; squared mental eminence (chin); edentulous; and small condyle relative to the large size of the other skeletal elements. The occipital squamous had an extremely pronounced enthesophyte (hyperostosis) located along the nuchal crest, indicative of massive attachment for trapezius muscle attachment on the back of the head and neck.

The first cervical vertebra exhibited healed nonunion burst fractures of the anterior and posterior aspects of the ring (Jefferson fracture); and although it is possible that the fractures occurred from a singular injury, they may have resulted from repeated injury. Jefferson fracture of C1 is diagnostic of axial loading. The lower thoracic and lumbar vertebrae exhibited pronounced-tosevere arthropathy, including erosive pitting, lipping, and osteophyte formation (extreme on the right side, between L4 and L5). 
The right scapula (shoulder blade) exhibited pronounced rugosity of the lateral border, resorptive pitting of the metaphysis of the glenoid (shoulder socket), pronounced lipping of the rim of the glenoid, plus active and sclerotic inflammatory apposition on the inferior aspect of the acromion process (part of the shoulder). The left clavicle (collar bone) had thin, brittle cortices and was notably flattened (superior/inferior); it also exhibited enthesopathy of deltoideus on the inferior/lateral surface. The arms had the following pathology: enthesopathy of deltoid, latissimus, and teres major insertions on the proximal humeri; pronounced rotator cuff arthropathy; severe arthropathy of the joints of the elbows and wrists; and bony plaque apposition on the surface of the right humerus head. The elements of the hands exhibited moderate lipping of the articular margins.

Assessment of the elements of the legs included the following. The femora exhibited platymeria (flattening) of the proximal metaphyses, plus large bony plaque formation on the surface of the right femoral head (cannot assess the left femoral head). There was a large bony plaque formation for Poirier's facet (hyperflexion of the hip) on the left anterior proximal femur (cannot assess the right femur). The tibiae exhibited platycnemia (medial/lateral flattening associated with development of the calf muscles). There were moderate arthritic changes of the distal femoral articular surfaces, but pronounced arthritic changes of the proximal tibiae. The linea aspera (attachment areas on the back of the leg) on the posterior aspects of the femoral diaphyses were not particularly well defined, nor were the soleus insertions on the posterior aspects of the tibiae (back of the calf).

The innominates exhibited narrow sciatic notches, severe arthritic changes of the auricular and postauricular surfaces (sacroiliac joint surface), very slight preauricular sulci, and thinning of the ilia (blades). The innominates also exhibited pronounced rugosity of the ischia, "Weaver's bottom"; this is associated with sitting for prolonged periods of time in one position (Kennedy 1989). The acetabula (hip sockets) of the innominates exhibited lipping and apposition of tiny, bony plaques on the inferior surfaces. The sacrum was fused to the innominate on the left side by a large, sclerotic bridge of bone across the sacroiliac joint. The sacrum also exhibited pronounced lipping on the left side of the superior articular platform and severe arthropathy of the left articular facet.

This individual had a lifetime of heavy manual labor, lifting and carrying heavy loads on his head, shoulders, and back. This may have involved the use of a tump line (thus the formation of the large enthesophyte on the back of the skull).

\section{Burial 7: Young Adult (Early 20s); Possible Male (Ambiguous)}

This grave was discovered during the previous Phase II survey. It was covered with black plastic and a large piece of painted plywood. The casket and lower legs had been impacted. The body was oriented east/west, with cranium west. Skeletal and dental preservation was excellent. Tiny mother-of-pearl shell buttons were recovered from the center of the torso and the wrists, and brass stains plus an elongated pinkish-red glass setting were recorded for the ring finger of the left hand (Figure 20). 


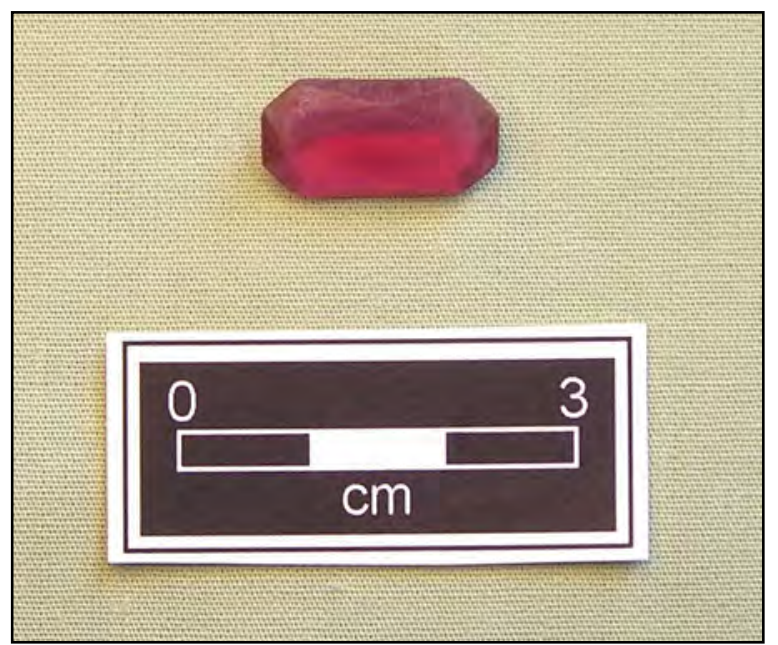

Figure 20. Glass ring setting associated with Burial 7.

Skeletal traits were ambiguous for age and sex determination. Some of the skeletal traits include the following: smooth nasal aperture, inferior collars on both auditory meati, temporal foramen (one on each temporal), remnant mastoid sutures, incomplete closure/fusion of the petrous portion to temporal squamous on the right side but not the left side, and bilateral parietal foramen (one on each parietal). The basilar suture was fragmented but at least partially closed, yet all of the other cranial sutures were open (early 20s). The cranial vault was smooth and relatively small, with sharp orbital borders and relatively shallow orbits. The frontal sinuses were small. There was moderate vermiculate appearance (tiny pits on the bone surface of the brow ridge) of the supraorbital region (usually associated more frequently with males). The mastoid processes were short and blunt. The bones of the arms were large, but the entheses were not particularly well developed. The diameter of the head of the radius was $24 \mathrm{~mm}$. The vertical diameter of the head of the humerus was $44 \mathrm{~mm}$ (indeterminate sex; Bass 1987:151). The curvature of the sacrum was more male than female; and the diameter of the head of the femur was $45 \mathrm{~mm}$ (the sectioning point for male; Bass 1987). The sciatic notches of the innominates were medium width. There were no septal apertures on the humeri. All long bone epiphyses were fused. The manubrium and corpus sterni (elements of the breast bone) were separate. The teeth were relatively large. The mandibular morphology was more male than female (substantial corpus, but the condyles were relatively short). Dental development was complete, and dental attrition was minimal. The sternal ends of the few rib fragments that could be assessed for age appeared relatively young (Phase 0b to $1 \mathrm{~b}=$ late teens to early 20s; Bass 1987; Işcan et al. 1985). The medial epiphyses of the clavicles were incompletely fused (approximately 21 years). The fifth sacral vertebra was not fused to the rest of the sacral elements, and there was retention of a nonunion line between S1 and S2.

One of the most prominent observations for this individual was that the right maxillary central incisor had a gold crown; there was notable alveolar resorption, leaving very little hard tissue for apical support of the tooth. There was evidence of light-to-moderate calculus on the buccal and lingual surfaces of the cingula of the mandibular anterior dentition. The left maxillary lateral incisor exhibited a possible apical abscess. In general, the individual had moderate-topronounced periodontal disease, with resorption of the alveolus most notable for the maxillary and mandibular molars. There was subsequent development of hypercementosis. 
There was moderate thickening of the parietal diploë, plus tiny, pinpoint cribra parietalis parallel to the sagittal suture (completely healed). The occipital squamous also exhibited moderate thickening of the diploë, but with no obvious cribra on the ectocranial surface. These are indicative of childhood anemia. There was notable asymmetry of the occipital condyles, with active and sclerotic bone formation around the foramen magnum on the ectocranial surface (no inflammation on the endocranial surface). There was no evidence of cribra orbitalia, but there was a very thin "crust" of active bone formation in the lateral aspect of the left orbit (possibly an infection of the eye).

There was a small, healed injury/trauma to a medial phalanx of the left hand. Slight resorptive pitting was on the proximal metaphyses of both humeri, reflective of hypervascular activity. The left scapula had active and sclerotic bone formation on the superior and inferior surfaces of the acromion process, and slight erosive activity on the inferior aspect of the glenoid cavity (neither was present on the right scapula). There were contact facets on the acromion processes of the scapulae indicative of hyperflexion of the shoulders, possibly associated with carrying activity (contact with the lateral ends of the clavicles). Enthesopathic pits were evident on the medial/inferior aspects of the clavicles.

The sternum had normal appearance. Developmental asymmetry of the articular facets of the first cervical vertebra, C1, was assessed, along with failure of complete closure (possible spina bifida) of the anterior inferior aspect of the "ring" of C1. There were small Schmorl's nodes on the superior and inferior surfaces of the centra of T8, T9, and T12, plus a small Schmorl's node on the inferior surface of T10. Additionally, there was notable accentuation of the syndesmoses of the internal common ligaments (inside the vertebral foramen) of the lower thoracic vertebrae. There was asymmetry of the pedicles, appophyseal facets, and spinous process of L4, slight Schmorl's node on the superior surface of the centrum of L3, and asymmetry of the alae of the sacrum. All of these are indicative of axial loading. There was a possible perimortem crushing injury to the chest (Flail Chest), evidenced by numerous sharply angled fractures with darkly stained margins. There also was possible perimortem crushing of the right innominate, evidenced by an incomplete fracture through the acetabulum. There was erosive pitting in the superior margins of both acetabula, and active woven bone formation on the inferior/interior aspect of the right sciatic notch (unobservable on the left side).

Poirier's facets were on both proximal metaphyses of the femoral necks, more pronounced on the right femur. The left tibia exhibited notable platycnemia (media/lateral flattening).

Analysis of the skeletal remains suggest that this person worked hard, lifting and carrying heavy loads, and died from a crushing injury to the torso. Although it is promising to be able to infer cause of death, no individual identification could be made based on this information.

\section{Burial 8: Possible Young Adult; Possible Male}

This grave had been impacted originally by previous Phase II activities and subsequently covered with plastic, a tarp, and large pieces of plywood. It was then impacted when the coverings were being removed. Geo-Marine recovered everything that was discerned including this small twentieth century German saucer (Figure 21). 


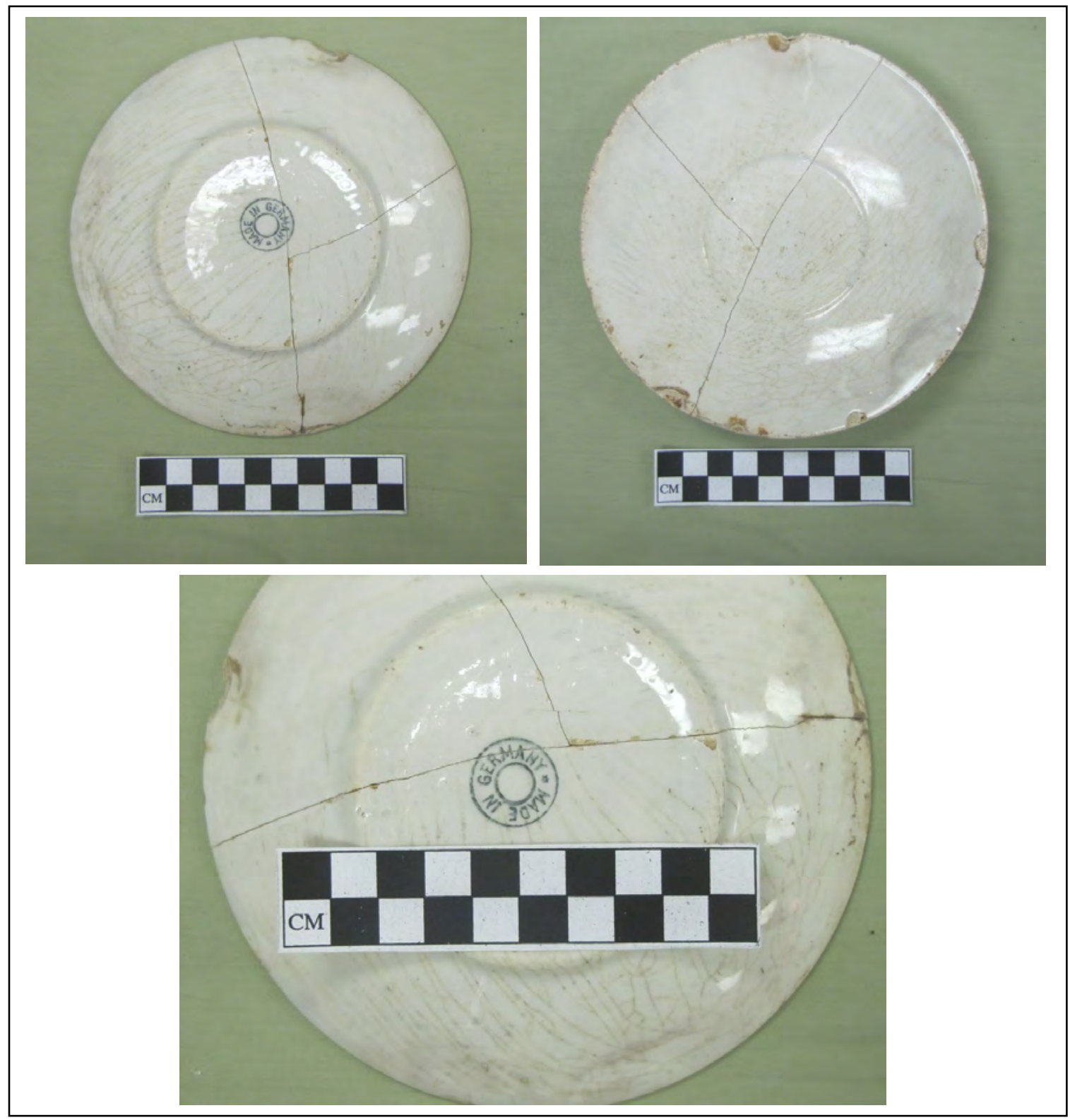

Figure 21. Views of ceramic plate associated with Burial 8.

The individual was represented by elements of both legs, plus lower left arm. The femoral head diameter was in the male range $(46 \mathrm{~mm})$, but the long bone diaphyses were relatively slender, with no pronounced development of entheses. The femoral and tibial metaphyses exhibited hypervascular resorptive pitting. This pitting has been associated with increased demand for oxygen transport (Hill 2001). The femoral and tibial diaphyses were "normal" (i.e., no platycnemia or platymeria), but the distal femoral metaphyses exhibited slight anterior/posterior flattening. Without radiographs to confirm the differential diagnosis, the pitting and anterior/posterior flattening suggest increased marrow activity associated with metabolic distress. There was no evidence of arthropathy (joint pathology) for any of the elements that were present. 


\section{Burial 9: Mature Adult; Male}

This grave was one of several located on the western margin of the ROW. The rectangular casket was oriented east/west along the long axis, with the cranium west. Fragments of barbed wire and amaryllis bulbs were found around the perimeter of the grave. The casket was adorned with an identity plaque over the pelvic region, and personal items, including a safety pin, comb, scissors, straight razor, as well as a clear glass bottle fragment, were recovered from the burial (Figure 22). A mother-of-pearl shell button was also recovered from the pelvic area.

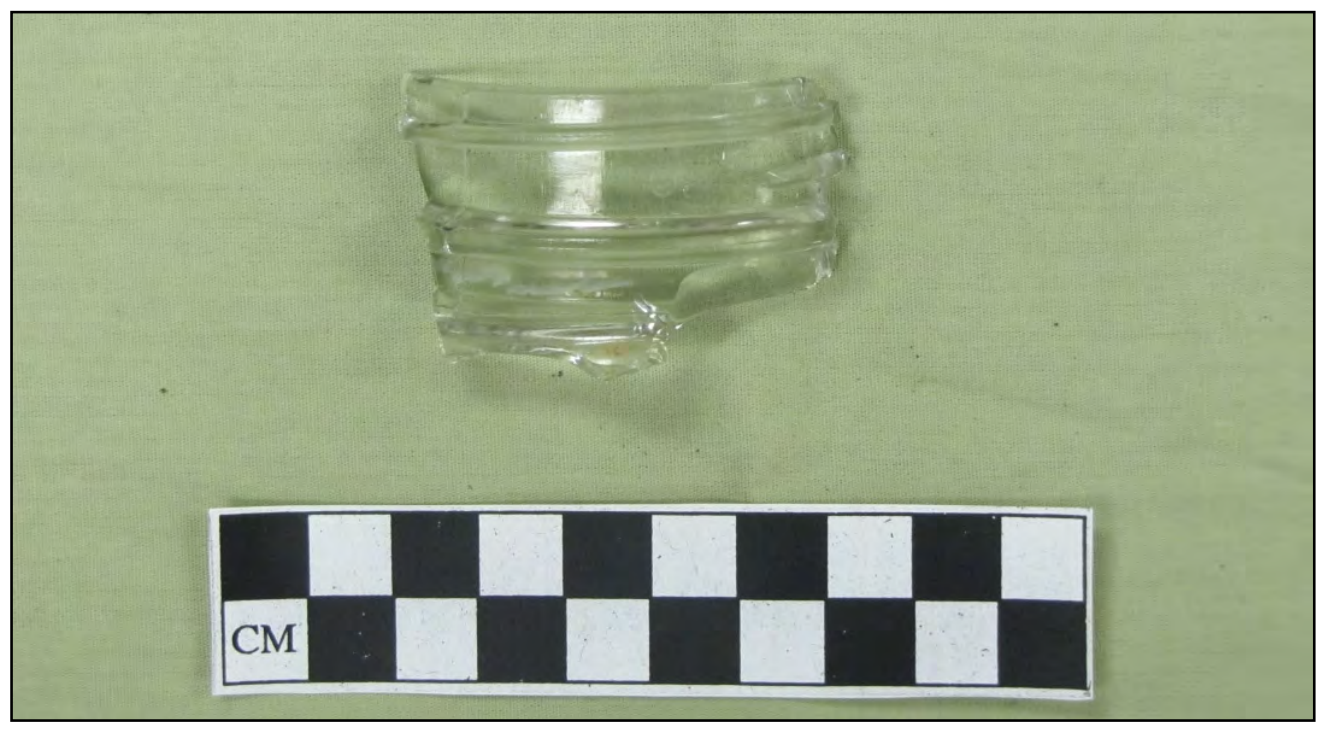

Figure 22. Clear glass bottle neck fragment associated with Burial 9.

The skeletal elements of this individual were notably large and robust, and had very heavy bone density. All joint surfaces appeared to have at least moderate arthropathy, but some were extreme, particularly the elements of the torso. Similarly, the skeletal elements were all textbook examples of enthesopathy and syndesmopathy.

The shoulders exhibited pronounced rotator cuff inflammation/arthropathy, with large, porous contact facets on the lateral aspects of the clavicles and the acromion processes of the scapulae (shoulder sockets). There was notable erosive pitting and eburnation on the lateral/anterior aspects of the surfaces of the acromion processes. The medial clavicles and the sternum exhibited extreme syndesmopathy of the sterno-clavicular articulations (large syndesmophytes). The joints of the elbows, wrists, knees, and ankles were all affected by arthritic changes, including syndesmopathy, lipping, erosive pitting, and isolated areas of eburnation caused by bone-on-bone friction. The diaphyses of the femora were notably thickened medial/lateral, and flattened anterior/posterior; the platymeria affected the entire upper halves of the diaphyses. The right femur exhibited a large Poirier's facet (hyperflexion of the hip) (cannot assess the left side). The femora also exhibited lateral torsion of the proximal halves. Similarly, the tibiae exhibited platycnemia (medial/lateral flattening), plus pronounced lateral torsion of the entire diaphyses. The soleus insertions on the posterior aspects of the tibial diaphyses were enthesopathic. The linea aspera of the femoral diaphyses were enthesopathic, but did not exhibit pillastering (a large, thick ridge of bone on the back of the upper leg bone). There was a large enthesophyte on the right proximal aspect of the right fifth metatarsal. 
The most extreme pathology involved the entire torso: fusion of thoracic and lumbar vertebrae with lateral scoliosis; sclerotic, "melted wax" apposition on the thoracic vertebrae (a thick coating of sclerotic bone that encased the vertebral column); and extreme collapse and osteophyte bridging of the lumbar vertebral centra. The heads and tubercles of the ribs exhibited pronounced-to-extreme arthritic lipping and some were completely fused to the costal facets of the thoracic vertebrae (on the right side). The left first rib was fractured antemortem and exhibited healing, but the shattered fragments were still very clearly apparent. A shattered first rib is usually fatal because of the possibility of puncture of the carotid or subclavian arteries, but in this case, it was not. The sacrum was fused to the innominates.

The maxillae and mandible exhibited pronounced dental disease including pronounced attrition, abscessing, antemortem evulsion, and pronounced calculus. The mandible exhibited senile age changes in morphology, and a perimortem fracture of the corpus. The maxillae exhibited a very unusual pathology that suggested some sort of injury to the left side that resulted in notable hypoplasia and hypocalcification of the left maxillary first permanent molar, malocclusion, and bony overgrowth of the alveolus of the first, second, and third permanent molars. It may have been the same trauma that shattered the left first rib that is described above. The cranium was large and heavy, and exhibited the following: thickened diploë with enlarged diploic veins, sclerotic porotic hyperostosis on the ectocranial surface, and perimortem fracture of the cranial base. Cranial traits included tiny, pointed mastoid on the right side, and small blunt mastoid on the left side; thin orbital borders; vermiculate appearance of the supraorbital region. In addition to the porotic hyperostosis, the ectocranial surface exhibited small isolated deposits of sclerotic bone (small "blobs," irregular appearance). The etiology is undetermined, but possibly may have been related to scalp infection. There were large inferior collars on the auditory meati, and the meati were narrow and oval-shaped.

This person had experienced extreme load stress throughout his life, and died proximate to a fracture of the cranial base. At some point in older childhood, he had received a massive blow to the left side of the neck and face.

\section{Burial 10: Senile Adult, $\geq 70$ Years; Male}

This rectangular, wooden casket with metal handles was discovered along the eastern margin of the ROW. It was a more recent interment. The individual was completely skeletonized, but he was clothed in a suit and tie (Figure 23). The waistband of his underwear was present, but the remainder of the garment was not (it was cotton, a natural fiber, therefore decomposed). Since the suit, tie, and socks were still present, they were made from man-made fibers. Shirt buttons also were present, but the shirt fabric had decomposed (cotton, silk, or linen). The burial was oriented east/west, with cranium west and facing east. Also, celluloid eye caps, a mouth former, and two trocar buttons, used in preparation of the corpse by a funeral company, were recovered from the remains (see Chapter 5 for discussions of these items).

The individual was represented by a complete skeleton, in excellent condition, with pathologies suggesting that this person had a lifetime of heavy manual lifting and carrying. Additionally, the skeleton had an unusual combination of male and female skeletal traits. There was a large, ossified cricoid cartilage (“Adam's Apple”), which is a male trait. The innominates exhibited the following: medium-width sciatic notches, no preauricular sulci, and male morphology of the pubic rami, plus senile age changes of the auricular surfaces and pubic symphyses. The cranium was gracile. 


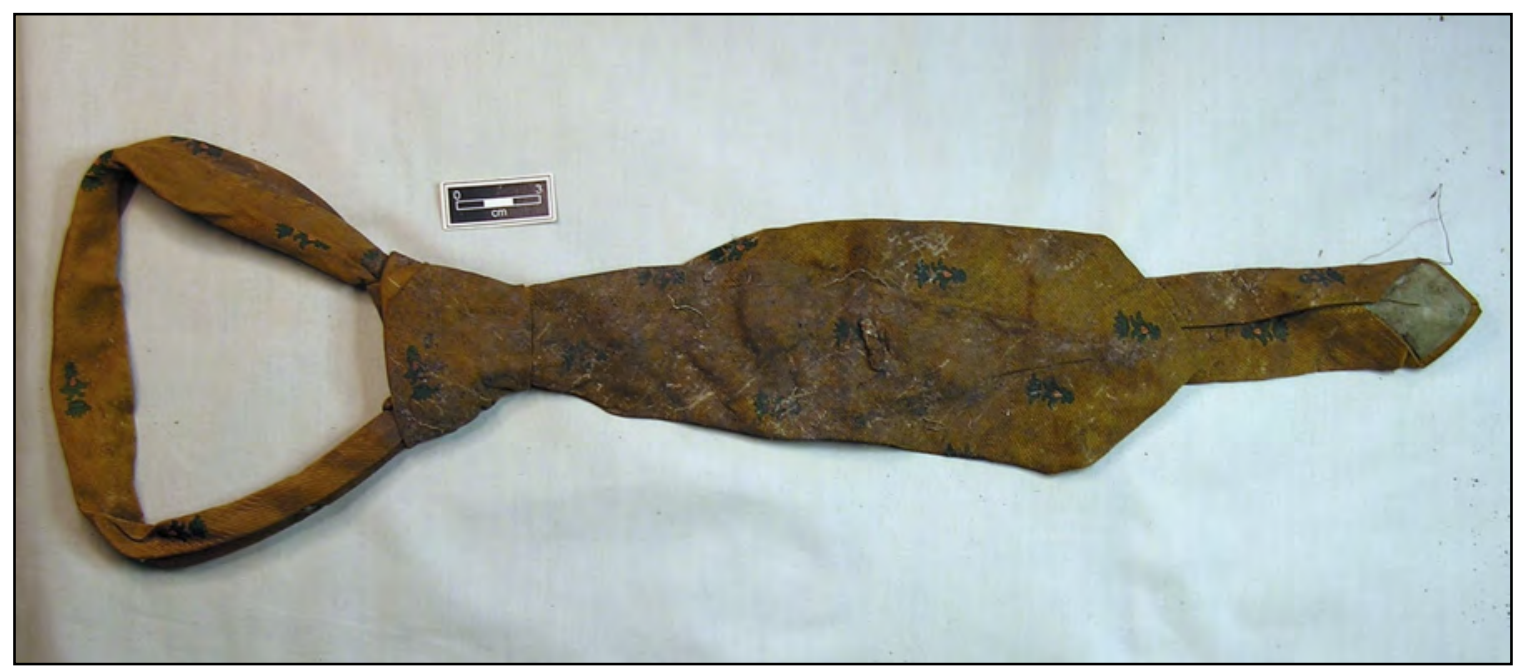

Figure 23. Tie from Burial 10.

The skeletal elements were large and robust, and had notably heavy bone density. All of the joints throughout the entire skeleton exhibited at least moderate arthropathy, but some exhibited pronounced pathology. These included the acetabula (hip sockets), the sacroiliac articular surfaces, the proximal and distal aspects of the radii and ulnae (lower arm bones), cervical vertebrae C3-C7, lumbar vertebrae L1-L5, the manubrium and corpus of the sternum, and the medial and lateral aspects of the clavicles. The joints of the hands exhibited pronounced arthropathy (enthesopathy and syndesmopathy).

There was extreme enthesopathy of the following: posterior femora (pillastering of the linea aspera), posterior tibiae (soleus attachments), proximal femora, iliac crests, proximal halves of the humeri (all entheses), and anterior patellae (extreme enthesopathy of the patellar tendons on the anterior aspects). The pillastering of the linea aspera on the femora has been associated with power-lift behavior and also with equestrian activities (horseback riding) (Kennedy 1989).

In addition to the pathology of $\mathrm{C} 3-\mathrm{C} 7$, the second cervical vertebra had wear facets on the dens (odontoid process; related to friction from the internal transverse ligament of C1), plus a thin line of eburnation on the lateral margin of the superior left articular facet of C2. There was notable spinal canal stenosis (narrowing of the spinal canal), particularly of the lumbar vertebrae. Lumbar vertebrae L4 and L5 exhibited extreme pathology associated with an apparent spondylolisthesis of L5 (separation fracture of the spinous process from the centrum, with forward dislocation). The fractures were through the pedicles and subsequently healed, essentially obliterating the pedicles.

The shoulders exhibited pronounced rotator cuff inflammation. The lateral aspects of the clavicles had pronounced inflammatory response to joint pathology, including contact facets for the acromion processes of the scapulae. The manubrium of the sternum and the medial aspects of the clavicles had extreme arthropathy, forming a bridge of bone where ligaments had been. There was extreme ossification of the sternal ends of the ribs, Phase 8 or greater, indicating an individual of advanced age greater than 70 years (Bass 1987; Işcan et al.1985). There were numerous isolated fragments of ossifications in the chest area, and also notable arthritic changes of the heads and tubercles of the ribs. 
The maxillae and mandible were edentulous, and the mandible had multiple abscesses (sclerotic, indicating long-standing infection). The mandible exhibited senile morphology: very gracile, with a very slightly squared mental eminence (chin). The cranium was smooth and gracile, with very small, pointed mastoid processes. The cranial base was not rugose. The foramen magnum was relatively narrow and oval-shaped. The auditory meati were very narrow (possible diminished hearing), and exhibited small inferior collars.

\section{Burial 11: Adult; Probable Female (Ambiguous)}

This burial was discovered along the eastern margin of the ROW. It was deeper than other burials encountered by the crew. It had been opened previously by a funeral company, and the majority of remains had been removed. The soil surrounding the casket was dense clay, with obvious bioturbation and inclusions such as carbon flecks and fire-cracked rock from the archaeological zone that was part of the cemetery. The casket fill was oily, black clay, with a distinctive, unpleasant odor. Geo-Marine recovered fragments of a possible slate marker or writing tablet (Figure 24) and a burial container handle on the surface, plus fragments of white cotton fabric approximately $2 \mathrm{ft}$ below surface, before subsequently encountering the casket.

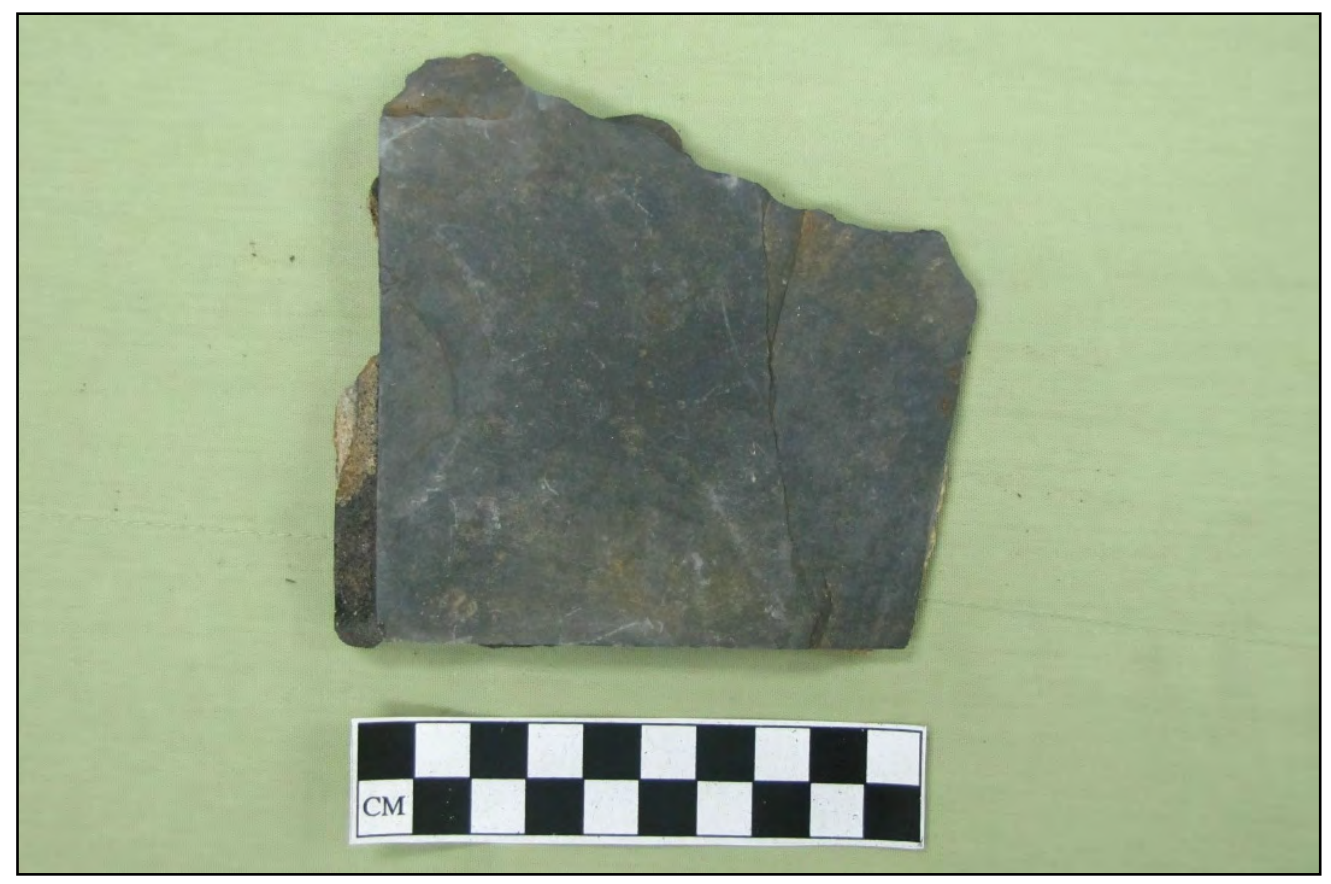

Figure 24. Slate fragment associated with Burial 11.

The skeletal fragments that were recovered were darkly stained from the black fill inside the casket. The individual was an adult, and the overall morphology was petite and notably gracile. The remains included fragments of left and right hands, left and right ribs, left scapula, right clavicle, one cervical vertebra, one thoracic vertebra, and one lumbar vertebra. The articular margins of the hands exhibited slight accentuation. All cortices were thin and brittle (osteopenia). 


\section{Burial 12: Adult; Undetermined Sex}

This burial was discovered along the eastern margin of the ROW sitting partially atop Burial 15. It had been opened previously, and the majority of the contents from the wooden casket had been removed by a funeral company. The Geo-Marine crew found elements of left and right hands, plus fragments of two ribs, long bone cortex fragment, and a fragment of orbital plate of the frontal bone. The carpals, metacarpals, and phalanges exhibited accentuation of the articular margins; and the flexor tendon entheses were accentuated. The articular lipping and pitting of the interarticular syndesmoses of the carpals are indicative of strenuous activity of the wrists (Kennedy 1989).

\section{Burial 13: Younger Adult; Female}

The casket within this burial was wood, with metal handles, and it was placed inside a wooden outer box. There was a metal identity plaque over the pelvis. The body was oriented east/west, with cranium west and facing east. The left arm was flexed at the elbow, with the left hand resting on the pelvis. The right arm was extended by the right side and the hand was pronated.

The individual was represented by an almost complete skeleton, in good-to-excellent preservation. The skeletal elements were relatively small, and exhibited smooth, gracile overall morphology. The cranial vault was smooth and gracile, except for a well-defined nuchal ridge on the occipital bone. The mastoid processes were small and rounded. The primary cranial sutures were open and had sharp, well-defined convolutions. The teeth did not have notable attrition. The innominates exhibited preauricular and postauricular sulci, indicative of parturition (giving birth).

The mandibular third permanent molars were impacted. The roots of the maxillary teeth had eroded into/pierced the maxillary sinuses; this would have caused more serious health problems, if she had lived to an older age. There was slight calculus and slight periodontal disease for all dentition, and two maxillary permanent incisors had gold-capped crowns. The temporal bones of the cranium had unusual morphology: long (anterior/posterior) and low (superior/inferior), possibly indicative of low cranial base height.

The clavicles were normal, and the inferior margins of the acromion processes of the scapulae exhibited only slight, sclerotic apposition. The articular surfaces for the ribs were normal. The knee joints exhibited enthesopathic pitting and sclerotic apposition of the medial posterior aspects of the femoral metaphyses, plus hypervascular pitting of both femoral and tibial metaphyses. The proximal metaphyses of the femora exhibited platymeria and enthesopathy. The articular margins for the long bones of the arms, legs, and hands, exhibited slight accentuation (not true lipping). The inferior surfaces of the lumbar vertebral centra exhibited slight, centrally located depressions.

The right pedicle of thoracic vertebra T11 exhibited a healed fracture, with no subluxation. Also, there was notable sclerotic apposition bridging sacral vertebrae S1 and S2, but not for the other sacral vertebrae. Both of these spinal pathologies indicate early episodes of axial loading.

The cranial base was rugose, particularly around the foramen magnum. There was a perimortem

ring fracture of the cranial base (occipital foramen magnum), plus a perimortem fracture of the squamous portion of the right temporal. The first cervical vertebra, C1, exhibited a burst fracture 
(Jefferson burst fracture; Harris classification; Kozin and Berlet 1992): angled, beveled fragments; darkly stained (i.e., perimortem). Additionally, the first thoracic vertebra, T1, exhibited perimortem fractures of the superior aspects of both pedicles, but not fractured on the inferior aspects.

\section{Burial 14: Senile Adult, Late 70s to Early 80s; Female}

This burial was located along the western margin of the ROW. It contained a wooden casket with metal handles and was oriented east/west, with cranium west. White buttons with metal rings and pins were recovered during the exhumation of the remains. The buttons are those that are used on uniforms, usually white broadcloth. These types of buttons are found most frequently on uniforms worn by nurses and nurses' aides, domestics, and cosmetologists, but may be worn for any occupation that requires a uniform. The buttons are removable, because the garments require rigorous washing, starching, and pressing. This individual had these buttons, indicating that she was dressed in a uniform when she was interred. It suggests that her uniform was an important part of her life; it was important to her and to those who attended her burial. Also found in association with Burial 14 were a fabric flower and a complete Mason jar (Figure 25).

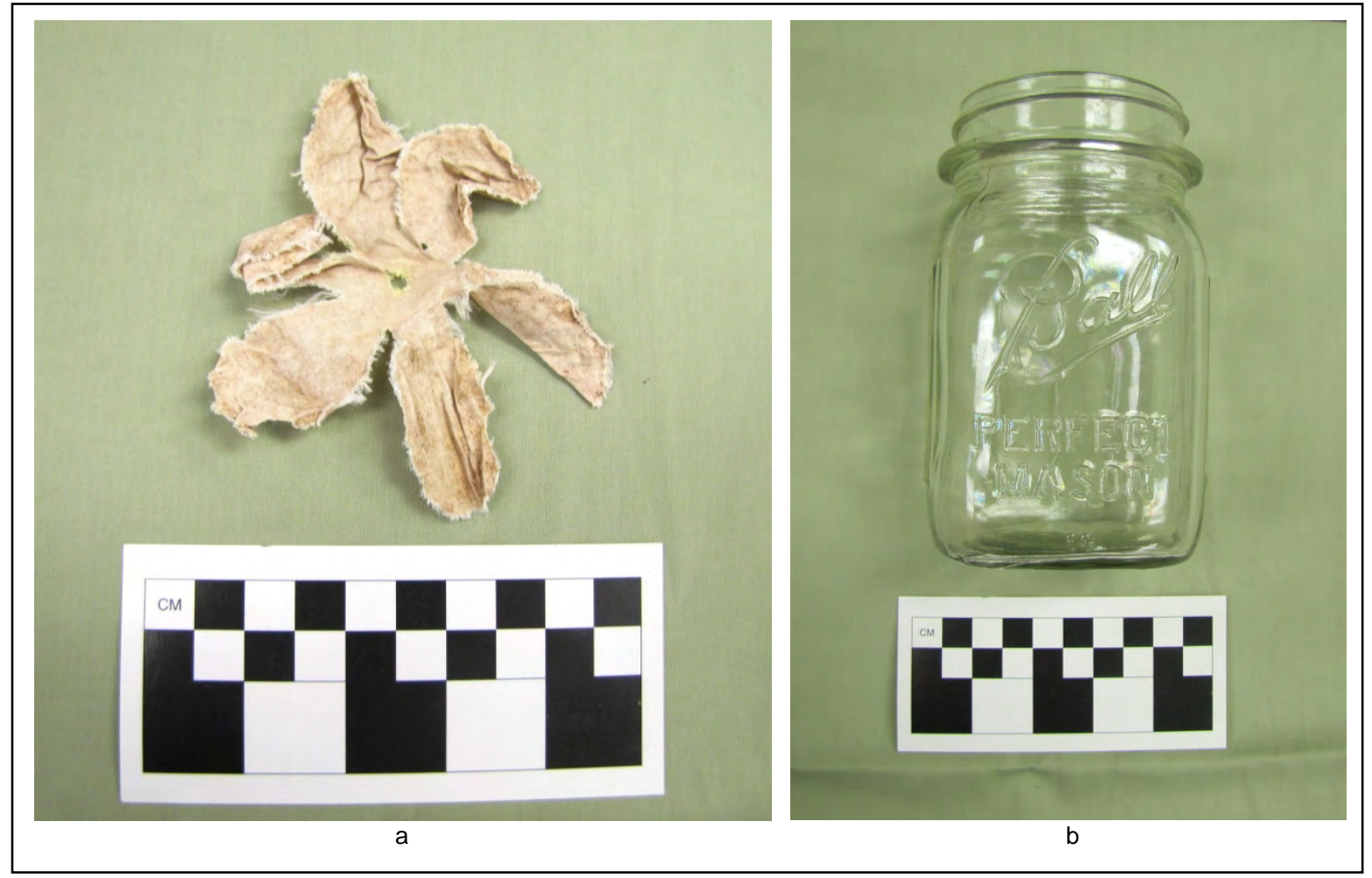

Figure 25. Burial items associated with Burial 14: (a) fabric flower; and (b) Mason jar.

The overall morphology of the skeletal elements was gracile and notably petite. The cranium and mandible were small, smooth, and gracile. The mastoid processes were small and pointed. The supraorbital borders were sharp, thin, and gracile. The mandible had pronounced senile age 
changes in the overall morphology. The temporal squama had unusual morphology: long (anterior/posterior) and narrow (superior/inferior), with pronounced "notch" at petrous/squamous interface, but not quite enough to form ossicles. The sciatic notches were wide; the femoral heads, radius head, and head of humerus were all notably small; and the clavicles were very small and gracile. The elements of the hands also were very small and gracile. The ribs were slender and very small for an adult, and had thin, brittle cortices. The age indicated by sternal end ossification was Phase 8b to 8c (late 70s to early 80s) (Bass 1987; Işcan et al. 1985).

The cortices of all elements were thin and brittle, and osteoporotic. The auricular surfaces of the innominates exhibited pronounced arthropathy (erosion of the surfaces and the postauricular area), and the pubic symphyses were eroded to the point of being almost completely destroyed, probably related to multiple parturition (multiple pregnancies).

The cranium had slightly thickened diploë, and heavy density (weight) compared to the overall size. The endocranial surface of the frontal bone exhibited "hammered silver" appearance that is diagnostic for increased endocranial pressure (refer to Hill 2001). The endocranial surface also exhibited tiny pinpoint, sclerotic resorptive pitting on the frontal and parietals, plus several small and medium-sized pacchionian pits. The "hammered silver" endocranial surface was more notable on the left aspect of the frontal bone, and the cranium was asymmetric (i.e., deviated from the sagittal plane) to the left side. This is attributable to premature closure of one of the smaller sutures of the cranial base, usually during fetal and neonatal development (Hill 2001). The orbital plates exhibited tiny pinpoint, sclerotic cribra and vascular channels (healed). There were tiny pinpoint, sclerotic cribra on the occipital squamous (healed), but there were no obvious cribra on the other bones of the cranial vault. There was notable sclerotic apposition on the ectocranial surface of the foramen magnum (cranial base), but not on the endocranial surface.

The maxilla fragment was edentulous and exhibited pronounced-to-extreme alveolar resorption. Similarly, the mandible was edentulous except for one molar that was probably only clinging to the gingival tissue and had an extreme carious lesion with an associated, large alveolar abscess. The left and right mandibular molars were abscessed, and the left mandibular gonial angle had been dramatically modified by the antemortem presence of a soft tissue mass (possibly a goiter, or a soft tissue abscess). There was no evidence of active inflammatory bone formation associated with this gonial angle, indicating that it had been a benign soft tissue mass.

The entheses of the skeletal elements of the legs were gracile. There were very slight, sclerotic periosteal striations on the cortical surfaces of the tibiae. The articular surfaces of distal femora and proximal tibiae exhibited pronounced erosive arthropathy (lipping, pitting, and eburnation), as did the articular surfaces of the patellae. The proximal femoral metaphyses/diaphyses were platymeric and the tibial diaphyses were platycnemic. There was severe lipping, plus erosive pitting and slight-to-moderate eburnation of the lateral condyles of the distal femora. The difference between the arthropathy of the knees (pronounced) compared to the ankles (moderate) suggests a lot of work-related kneeling activity. The arthropathy of the hip joints also was pronounced. There was active periostitis on the plantar surfaces of the calcanei (soles of the feet; associated with hyperflexion of the feet).

The clavicle and scapula had notable arthropathy of the shoulder, as did the sternal end of the clavicle. The proximal right humerus (could not assess the left humerus) had pronounced rotator cuff inflammation, as indicated by the entheses and syndesmoses. Also, there was enthesopathy of the medial epicondyle of the distal right humerus (ulnar collateral ligament, flexors of the 
forearm, and pronator teres). The skeletal elements of the hands also exhibited notable arthropathy, including slight eburnation of some of the articular surfaces. A small osteoma was on the lateral aspect of the proximal phalanx of the right hand.

The posterior ring of the first cervical vertebra, C1, exhibited nonunion, possibly as spina bifida (a rare location for this disorder), or as a healed burst fracture (Jefferson's fracture). The centra and appophyseal facets for the remainder of the entire spinal column (C2 through L5) exhibited notable arthropathy: erosive pitting and lipping. There was pronounced spinal canal stenosis of the thoracic and lumbar vertebrae, pronounced osteoporosis of the centra, pronounced arthropathy, plus narrowing of all spinous processes of the lower thoracic and lumbar vertebrae. Pathology of the cranial base and cervical vertebrae reflected axial loading, with additional evidence provided by the reminder of the spinal column.

\section{Burial 15: Adult; Probable Male?}

This burial was discovered partially beneath Burial 12. The remains were housed within a rectangular wooden casket with metal handles. The remaining skeletal elements included the left arm and hand, essentially intact and in articulated position within the casket, plus numerous disarticulated fragments scattered in the western two-thirds of the casket. Despite being fragmented, preservation was fair-to-good. The elements were relatively large and robust. The measurements were indicative of a male (left humerus transverse diameter of head=46 $\mathrm{mm}$; left radius diameter of head $=27 \mathrm{~mm}$ ). There was severe arthropathy of the hand, particularly the bones of the wrist that exhibited severe arthritic lipping and extreme eburnation of the joint surfaces. The joint margins of the humerus, radius, and ulna also exhibited lipping. The acetabulum (hip socket) of the right innominate exhibited moderate-to-pronounced lipping, and the ischium exhibited pronounced rugosity ("Weaver's bottom," indicative of sitting for long periods of time in one position; Kennedy 1989). The humerus exhibited accentuation of the rotator cuff entheses and syndesmoses, and the deltoid enthesis was well developed. The medial epiphysis of the left clavicle exhibited syndesmopathy (pitting). The articular facet of the right rib exhibited pronounced arthritic lipping. The left maxillary canine exhibited calculus on the cemento-enamel juncture and moderate attrition of the cusp, exposing a pinpoint area of dentin.

\section{Burial 16: Young Adult, 24 to 27 Years; Male}

This burial was on the western margin of the ROW. The grave was an articulated skeleton oriented east/west with cranium west, facing east. It was contained within a "pinched toe" hexagonal wooden coffin that had four metal handles: two located in the area of the shoulders and two in the area of the ankles. The coffin was inside a wooden outer box. Soil inside the inner box was dark gray. There was a small pile of thumbscrews and plates on the left side of the coffin, and they appeared not to have been used. The plates were tightly stacked together as if they were never separated. An identity plaque was over the pelvis. A brown glass fragment was also found in association with the burial (Figure 26). 


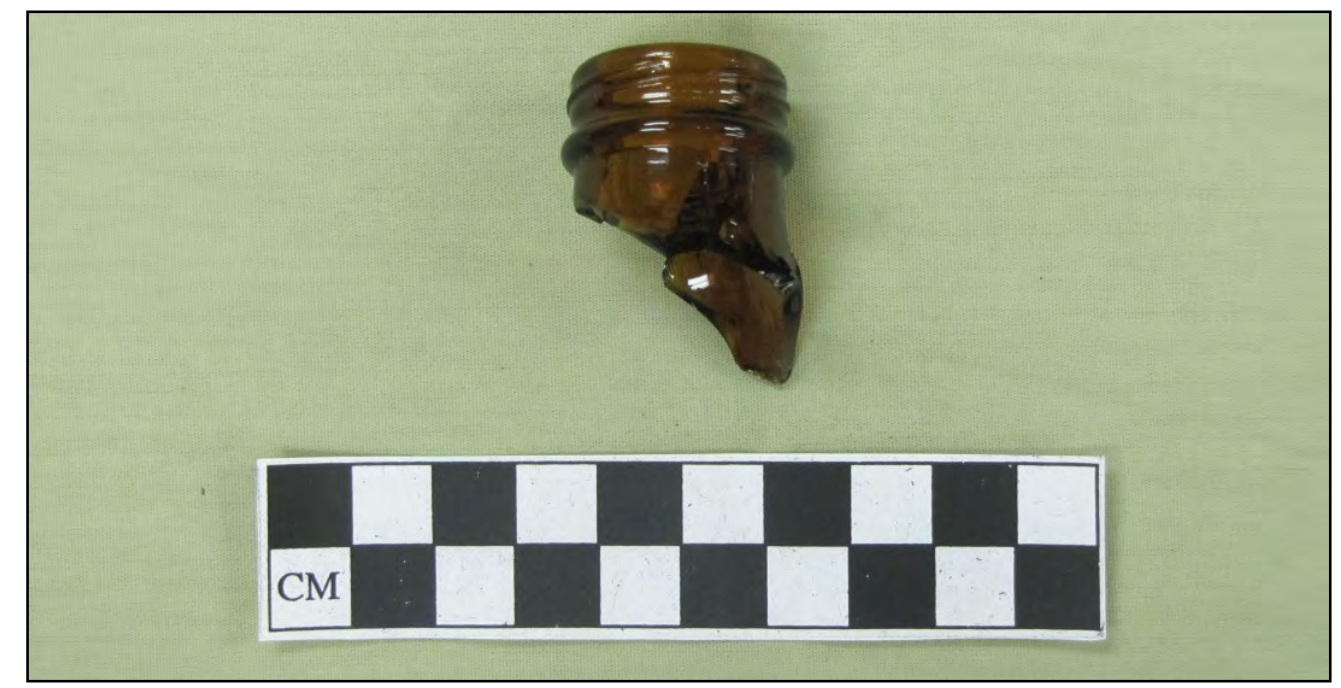

Figure 26. Brown glass bottle neck associated with Burial 16.

The remains were in excellent condition of preservation. The body was supine, oriented east/west. The skeletal elements were very large and robust, with heavy bone density. The teeth also were well preserved and large, with large roots. Measurements of skeletal elements (head of humerus, head of radius, and head of femur) were indicative of a male. The cranial vault sutures were open and had well-defined convolutions. The auditory meati were large and oval-shaped and had small inferior collars, and there were remnant mastoid sutures. The mastoid processes were long and pointed. The cranial base was notably rugose. There was a healed blunt force trauma (Pond fracture) to the right aspect of the frontal bone. Other observations for the cranium, maxillae, and mandible included the following: U-shaped dental arcade; small enthesophyte at inion on the occipital; tiny, healed, pinpoint cribra on the occipital squamous and on the parietals on each side of the sagittal suture (approximately $11 \mathrm{~mm}$ to $15 \mathrm{~mm}$ along the sagittal suture on each side; porotic hyperostosis); minute sclerotic bony plaques inside orbits (cribra orbitalia, healed); pronounced temporal lines on the frontal bone, but no accessory frontal sulci (blood vessel lines on the ectocranial surface); cerebral convolution impressions on the endocranial surface of the occipital squamous and immediately posterior to the orbital plates on the frontal bone (evidence of endocranial pressure). For a [male] African American, the mandible morphology is more female; the teeth were relatively small and crowded; the cranial vault was rounded and exhibited parietal bossing; and the mastoid processes were relatively small. The cranial base was very rugose (axial loading).

There was crowding of the anterior dentition (central and lateral incisors) of the mandible. There was minimal attrition of the occlusal surfaces of the dentition. The permanent third molars were slightly impacted and were crenulated. There was evidence of periodontal disease. The right maxillary central incisor exhibited pitted hypoplasia and hycalcification, and the right maxillary lateral incisor exhibited hypoplasia. There was hypoplasia of the upper fourth of the crowns of the maxillary and mandibular first permanent molars. The maxillary left M3 was destroyed by a carious lesion, possibly related to being impacted. The right maxillary central incisor exhibited pitted hypoplasia and hypocalcification. The maxillary right lateral incisor also had hypocalcification. The third molars (maxillary and mandibular) were crenulated. 
The cervical vertebrae had notable pathology that included the following. C1 was fragmented, but exhibited notable thickening of the anterior aspect of the ring. The second cervical vertebra exhibited notable thickening of the spinous process and centrum, plus notable asymmetry of the inferior apophyseal facets. It appeared that the left pedicle was fractured antemortem and subsequently healed. The spinous processes of C3 and C4 were fractured antemortem and subsequently healed. The superior and inferior apophyseal facets of C3 also exhibited notable asymmetry. C7 spinous process was notably thickened and there was a very large, smooth platform (avulsion fracture/clay shoveler's fracture) on the tip of the process for trapezius muscle attachment. The inferior apophyseal facets for C7 also exhibited asymmetric morphology.

The zygapophyseal facets for T11, T12, and L1 exhibited notable arthritic changes, and the costal facets on T11 and T12 showed notable arthritic changes (particularly on the left side). The second lumbar vertebra, L2, exhibited notable asymmetric thickening of the inferior left apophyseal facet. L1 and L2 exhibited notable thickening of the pedicles and spinous processes.

Sternal end ossification of the ribs was Phase 3 (24 to 27 years; Isçan et al. 1985). Age-related changes to the pubic symphyses and auricular surfaces of the innominates indicated an age of mid-30s to 50s, but these surfaces can be altered by work-related activities (and/or parturition in females), making an individual appear to be older than his or her actual chronological age.

The manubrium and corpus sterni (elements of the breast bone) were separate and exhibited tiny hypervascular pitting on the dorsal and ventral aspects (evidence of increased demand for red blood cell/oxygen transport). The lateral aspects of the clavicles had large contact facets for the acromion processes of the scapulae. All appendicular (arms and legs) joint surfaces exhibited slight-to-moderate accentuation of the articular margins. The proximal aspects of the humeri exhibited rotator cuff enthesopathy and syndesmopathy.

A large erosive lesion was on the medial articular facet of the right patella (osteochondritis dissecans), but not on the left patella. The distal metaphyses of the femora and proximal metaphyses of the tibiae exhibited large hypervascular foramena, which are indicative of increased demand for oxygen transport (Hill 2001).

The markers of occupational stress were extensive on the arms and legs (analysis is available for review). Essentially, the elements of the arms, hands, legs, and feet exhibited pronounced pathology associated with deep squatting, lifting, and carrying heavy loads, as did the spinal column and cranial base (axial loading).

The pelvis exhibited male morphology: elongated innominates; long, narrow sacrum; narrow, Cshaped sciatic notches; no preauricular sulci; male morphology of the pubic rami; and large acetabula (hip sockets). It also exhibited notable spinal canal stenosis of the sacrum (narrowing of the canal for the spinal cord); enthesopathy of the "necks" of the acetabula; notable erosive activity at the pubic symphyses; and large enthesopathic pits retro to the articular platforms of the sacral alae (wide "wings" of the sacrum). 


\section{Burial 17: Adult; Undetermined Sex (Possibly Male??)}

This rectangular wooden casket with metal handles was oriented east/west on the eastern margin of the ROW. The casket was in situ, but apparently had been opened by previous funeral company disturbances, and the majority of the skeletal remains had been removed. The casket was still relatively intact; all skeletal elements were found within the casket; and because the vertical height of the casket was still relatively intact, the skeletal elements were recorded on essentially three levels as the excavators proceeded more deeply into the casket.

The skeletal remains that were recovered included cervical and thoracic vertebrae, sacrum, fragments of two left ribs, right patella, right radius and ulna, and right and left hands. Two buttons from clothing also were recovered. The elements that were present were large and robust, with heavy bone density. The radius head diameter was large $(26 \mathrm{~mm})$. The radial tuberosity had moderate arthritic lipping of the medial/posterior margin. All articular margins on all of the skeletal elements exhibited slight-to-moderate bony accentuation. The articular margins of the hands exhibited moderate accentuation, plus accentuated pitting formation of the interarticular syndesmoses of the carpals. The flexor tendon entheses of the hands also were accentuated by enthesophyte formation. The diaphyses of the radius and ulna were smooth, with essentially no entheses development. The manubrium of the sternum was unusually long and slender, and the sterno-clavicular facets exhibited moderate-to-pronounced arthritic changes. The sacrum exhibited "male" morphology, plus asymmetry of S5, spinal canal stenosis, spina bifida occulta (the nondebilitating form of spina bifida), pronounced arthritic lipping of the left appophyseal facet, large pits (accessory sacroiliac facets) on both postsacroiliac articular surfaces (associated with carrying heavy loads on the lower back), and a large syndesmophyte on the right margin of the centrum of S1. The right patella exhibited a healed avulsion fracture of the lateral aspect (the vastus lateralis enthesis; associated with stabilizing loads placed on the knee or standing up with a heavy load from a deep squat position). The left superior apophyseal facet of T9 exhibited arthritic changes, as did the inferior (left) apophyseal facet of T10. Cervical vertebra C7 exhibited arthritic degenerative changes of the left superior aspect of the centrum and left inferior appophyseal facet, plus slight lateral collapse of the left aspect of the centrum.

\section{Burial 18: Mature Adult, 58 to 71 Years; Male}

This articulated skeleton of a mature individual had a lifetime of hard manual labor combined with severe antemortem trauma to the left side that left him partially debilitated. He was interred within a wooden casket that had metal handles. The grave was oriented east/west along the long axis, with cranium west, facing east. Preservation was good to excellent. A metal identity plaque was found over the pelvis.

Some of the skeletal elements exhibited heavy bone density, but the cortices were brittle, indicating osteopenia. All joint surfaces exhibited at least mild arthropathy; several joints had pronounced pathology; and there was evidence of antemortem trauma.

The proximal humeri, lateral clavicles, and lateral scapulae exhibited advanced rotator cuff enthesopathy; this was further compounded by a severe trauma to the left proximal humerus and lateral clavicle that destroyed the proximal half of the humerus. It was completely healed, but disuse atrophy of the lower left arm was apparent by the dramatic difference in appearance of the bones of the two forearms. The injury resembled a healed gunshot wound, but this could not be 
confirmed due to lack of access to radiography. Because the flexors are so much stronger than the extensors, the disuse atrophy would have resulted in positional behavior of holding the arm in a "claw" position, close to the chest (Aprille Andress, R.N., personal communication). This disability would have affected his capacity for work and also may have had social stigma.

The proximal femoral metaphyses exhibited platymeria and slight anterior torsion. The linea aspera (line of muscle attachments on the back of the femora) were enthesopathic. The tibial diaphyses (shafts) were platycnemic (flattened) and exhibited lateral torsion, particularly the right tibia. The left proximal tibia metaphysis and diaphysis exhibited a healed subepicondylar fracture (proximal one-fourth transverse or oblique fracture; see Kozin and Berlet 1992). This fracture may have occurred at the same time as the trauma of the humerus. The tibial fracture was well reduced and healed. The right patella exhibited arthropathy of the articular surface, plus rugosity of the vastus enthesis. The distal metaphyses of the femora and proximal metaphyses of the tibiae exhibited notable hypervascular pitting, and the joint surfaces of the knees exhibited advanced arthropathy. Due to postmortem fragmentation, the internal structures of the long bone metaphyses could be assessed; they had the "moth-eaten" appearance of advanced age.

Observations of the torso included the following. All vertebrae (cervical, thoracic, lumbar) exhibited arthropathy associated with axial loading. The sacrum was long and narrow. The second cervical, C2, exhibited a "pencil-shaped" odontoid process, attributable to probable friction from the internal transverse ligament of $\mathrm{C} 1$. The spinous processes of the cervical vertebrae had been fractured repeatedly (tiny microfractures), resulting in thin points, rather than their normal bifurcated appearance. Thoracic vertebrae T7-T12 all exhibited Schmorl's nodes on the centra and pronounced osteophyte formation. Similarly, the lumbar vertebral centra (L1-L5) exhibited collapse of the superior and inferior central aspects, and L4 and L5 exhibited severe osteophyte formation on the appophyseal facets and the margins of the centra. The sternum was very porous and brittle. The sternal ends of the ribs were assessed as Phase 6c to 7c (58 to 71 years; Isçan et al. 1985).

The cortices of the diaphyses of the elements of the hands and feet were extremely thin and brittle (osteopenia). The navicular and cuneiform of the left foot exhibited extreme arthropathy, erosive pitting, eburnation, and osteophyte formation, probably related to the trauma of the left tibia, but also might have been a separate injury.

Cranial sutures were fused. There was deviation of the vault to the left side of the sagittal plane (the midline). There was vermiculate appearance of the supraorbital region (related to "hypermale" morphology). There was a small indentation (Pond fracture) to the right frontal, just superior to the orbit, indicative of antemortem blunt-force trauma. Other observations of the cranium included long, slender, pointed mastoid processes; small, oval-shaped auditory meati with large inferior collars; postorbital constriction; antemortem trauma to the left temporal, healed (best visualized on the endocranial surface-the petrous is flattened); remnant mastoid sutures on both mastoids; and a deep inferior sulcus on the endocranial surface of the right temporal petrous.

Observations for the maxillae and mandible included extremely long, slender roots on the few remaining teeth; severe dental pathology, including periodontal disease, abscess, antemortem evulsion; irregular attrition pattern; severe, active abscesses of the maxillary posterior teeth; and senile age changes of the morphology of the mandible. The left maxilla exhibited pronounced atrophy of the corpus: the sinus appeared to have collapsed, but in fact, there was a sulcus (a 
sunken area) that had formed inferior to the maxilla-zygomatic suture (which had completely fused and was obliterated). The sulcus may have been related to the severe abscessing of the left maxillary posterior dentition.

\section{Burial 19: Senile Adult 70.4 to 82.3 Years; Female}

This articulated skeleton of an elderly individual was found within a wooden casket with metal handles. The grave was on the western margin of the ROW and was oriented east/west with cranium west, facing east. A more recent intrusion had crushed the northwest corner of the casket.

This person was represented by an almost complete skeleton. Preservation was fair-to-good. The skeletal elements were notably small and gracile, with thin, brittle cortices (pronounced osteopenia). Perhaps the most notable observation was the presence of a large, round ossified mass that was found on/in the right side of the pelvis. It was a discrete mass, with no evidence of metastasis.

The maxillae and mandible were almost edentulous, and the mandible exhibited pronounced senile age-change morphology. The cranial vault was very smooth and gracile, with very thin orbital borders and tiny, pointed mastoid processes. The auditory meati were oval-shaped and had very slight inferior collars. The few teeth that were present exhibited unusually long and slender roots, hypercementosis, caries, and abscessing. A large occipital bun was on the posterior cranium. The orbits exhibited tiny pinpoint, sclerotic cribra orbitalia (healed). A small, healed blunt-force trauma indentation, Pond fracture, was on the ectocranial surface of the left parietal.

There were small areas of osteophyte formation on the cervical and thoracic vertebrae, and pronounced degenerative changes of lumbar vertebrae L4 and L5. The second thoracic vertebra exhibited a healed fracture of the spinous process; this is possibly a "clay shoveler's fracture," related to heavy manual labor (shoveling). The ribs were very thin and brittle, and there were numerous sternal end ossifications, plus ossifications found within the area of the chest cavity (isolated islands of bone; Phase 8: 70.4 to 82.3 years; Işcan et al. 1985). Deep preauricular and postauricular sulci were present on the innominates, indicative of parturition. The blades of the innominates were extremely thin, and the ischia exhibited pronounced rugosity ("Weaver's bottom”) (Kennedy 1989).

Long bone traits included the following: small, thin, gracile diaphyses (shafts); anterior/posterior flattening of the distal femoral metaphyses; platycnemia and lateral torsion of the tibial diaphyses; left tibia exhibiting much more lateral torque than the right tibia; accentuation of the rotator cuff entheses and syndesmoses on the proximal humeri.

\section{Burials in "Disturbed Area"}

Five burials were identified within the disturbed area of the eastern margin of the ROW. Because there was such a large area of dragged disturbance, field personnel decided to assign separate numbers to these burials (Coffins 1-5) in order to distinguish them from the other burials previously discussed (Burials 1-19). Their descriptions and analyses of associated remains were as follows. 


\section{Coffin 1: Mature Adult (46 to 58 Years); Undetermined Sex}

The casket in this feature had been severely mangled by funeral company activity first, and then by previous evaluation activity. The previous activity included being impacted by the Gradall, and subsequently covered with plastic, a tarp, and painted plywood. It was fully exposed, evaluated, and disinterred by the Geo-Marine crew. The casket was metal, with a white exterior finish and polyester and foam rubber interior lining; several miscellaneous glass and plastic artifacts and shell were associated with the casket (Figure 27).

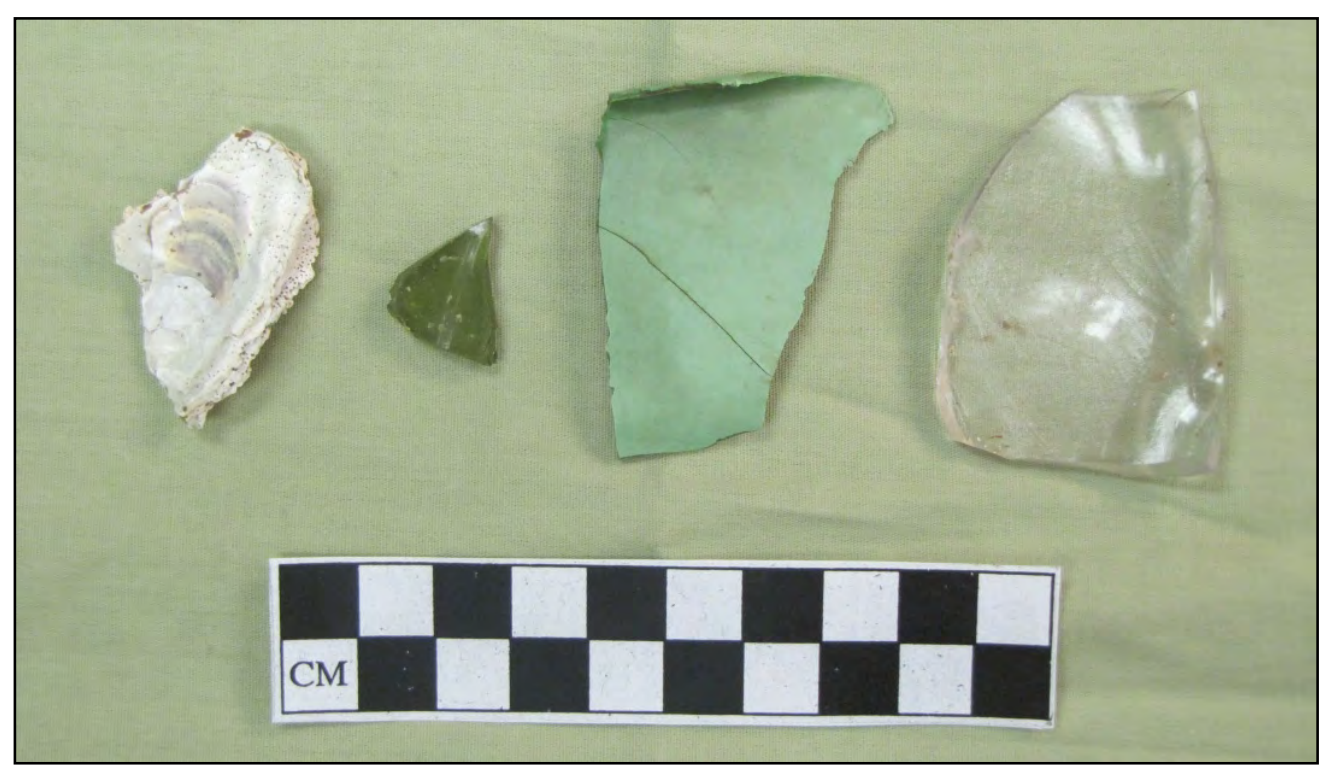

Figure 27. Miscellaneous artifacts recovered with Coffin 1.

The skeletal elements included cervical vertebrae (C3 and C5; complete), a fragment of left rib (approximately \#4), a fragment of long bone cortex, a fragment of scapula, and a fragment of spinous process from a thoracic vertebra (approximately T3 or T4). The fragments of spinous process, scapula, and long bone cortex had normal appearances. The rib fragment had slight, active periostitis on the cortical surface of the sternal end and a sternal end age assessment of $5 \mathrm{c}$ to $6 \mathrm{c}$ (46 years to 58 years; Işcan et al. 1985). The vertebrae exhibited slight-to-moderate arthritic lipping on the appophyseal facets, more notable on the right side; tiny areas of eburnation on the articular margins of the right inferior facet of C5; and small lumps of increased sclerotic apposition on the inferior aspect of the spinous process of C5. Both C3 and C5 exhibited small Schmorl's nodes on the superior and inferior aspects of the centra, adjacent to the neural arch of each. The centrum of C3 also exhibited a small osteophyte on the inferior/anterior/ right aspect. There was a linear bony apposition on the interior surface of the centrums of both C3 and C5 inside the neural canals, possibly associated with the internal common ligament. 


\section{Coffin 2: Young Adult (Late Teens/Early 20s); Undetermined Sex}

The remnants of this casket probably were impacted originally by the funeral company, since it was located along the eastern margin of the ROW. It subsequently was impacted by the Gradall during the previous Phase II evaluation. It was immediately north of Coffin 1 and was covered by black plastic, a tarp, and pieces of painted plywood. The excavation by Geo-Marine revealed a wood and metal casket with polyester and foam-rubber lining.

Only two fragments of adult ribs were recovered. The sternal end of one rib had an age assessment of $1 \mathrm{~b}$ to $1 \mathrm{c}$ (young adult; late teens to early 20s; Işcan et al. 1985).

\section{Coffin 3: Undetermined Age; Undetermined Sex}

This casket was buried side-by-side with Coffin 4 (Figure 28). The caskets were exactly alike and were so close together that they must have been interred in a single burial service. A singular border constructed of 2-x-4 lumber encompassed both caskets. The caskets were metal, with pale blue exterior finish and polyester and foam rubber lining (see discussion of metal caskets in Chapter 5).

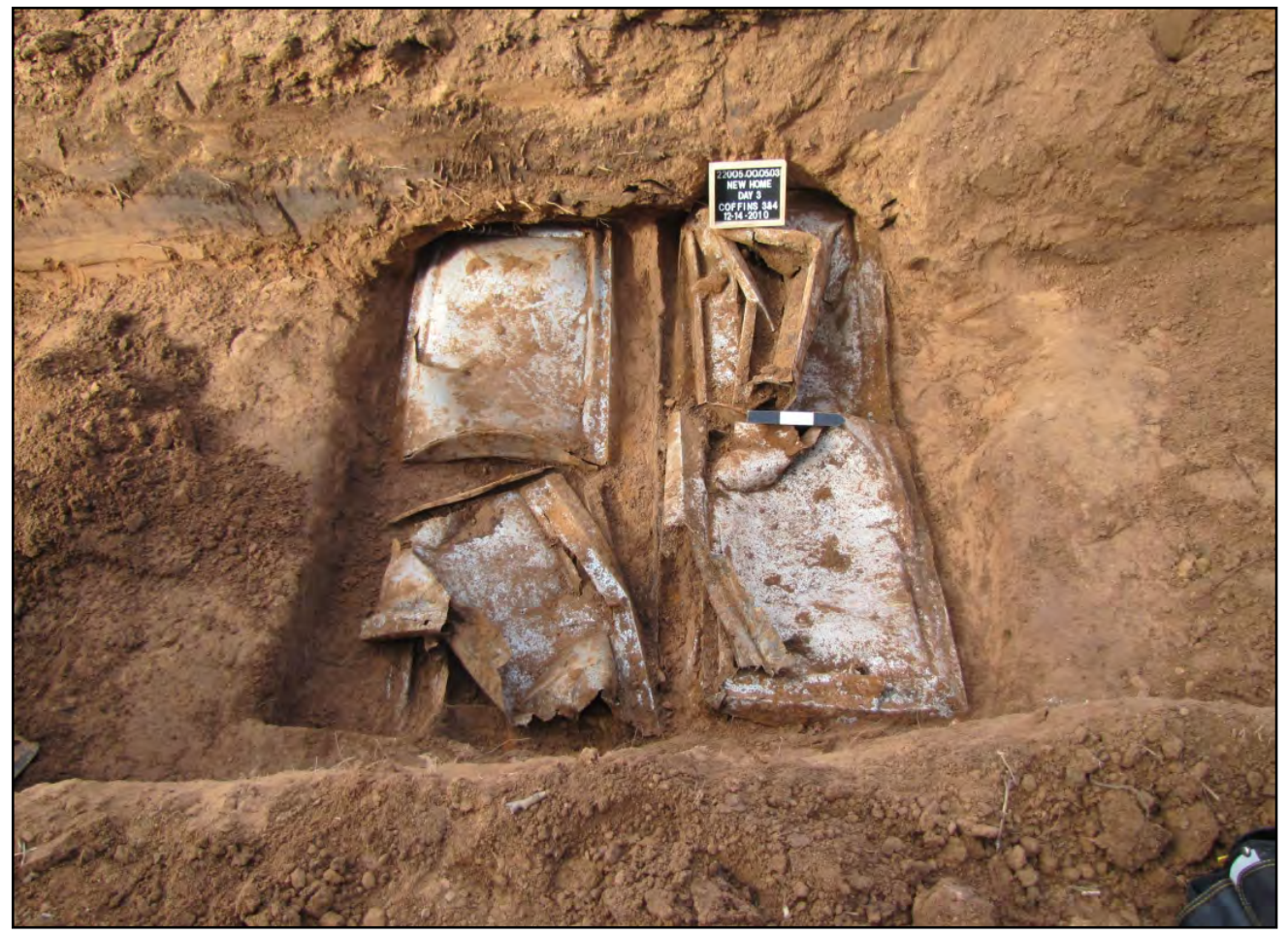

Figure 28. Matching pair of caskets (Coffins 3 [left] and 4 [right]), looking west.

Geo-Marine crew did not find any skeletal elements associated with this casket. However, a fabric flower boutonnière was inside the casket (Figure 29). Several small, white, plastic buttons were found after both caskets were disinterred; it was unclear to which casket the buttons belonged. 


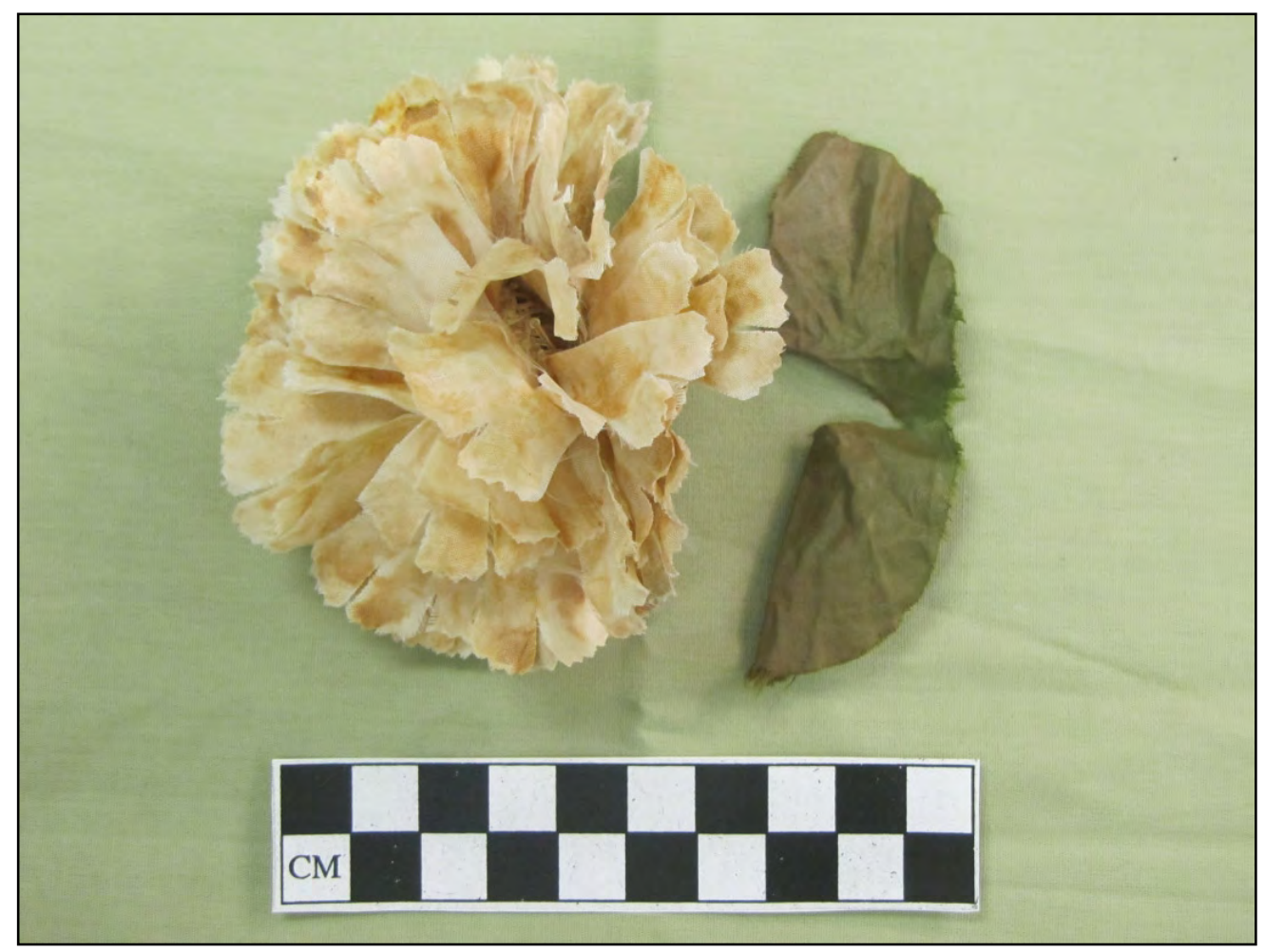

Figure 29. Fabric flower from Coffin 3.

\section{Coffin 4: Adult; Possibly Male (??)}

This burial was the second of a pair of matching caskets (the first of which was Coffin 3) that were buried side-by-side in what may have been a single burial event (Figure 30). Fragments of clothing and four skeletal elements were recovered. A plastic bag containing embalming fluid bottles and another large plastic bag also were inside Coffin 4 (see discussion of embalming fluid bottles in Chapter 5).

The skeletal elements were relatively large compared to others recovered from the series of burials. The skeletal elements were the left fifth metacarpal and proximal phalanx, right eleventh rib, and a fragment of the dorsal aspect of the neural arch of the sacrum. The head of the rib exhibited arthritic pitting and lipping of the articular margin. The metacarpal had a small, smooth-walled lesion on the dorsal surface of the head. The metacarpal and phalanx exhibited slight accentuation of the articular margins.

\section{Coffin 5: Senile Adult (Early 70s to Early 80s); Probable Female}

This rectangular wooden casket was oriented east/west and was adjacent to the eastern margin of the ROW. A large puddle of rust had accumulated underneath the black plastic, tarp, and painted plywood that covered it due to the presence of metal hardware. Several pottery and glass fragments were associated with Coffin 5 (Figure 31). 


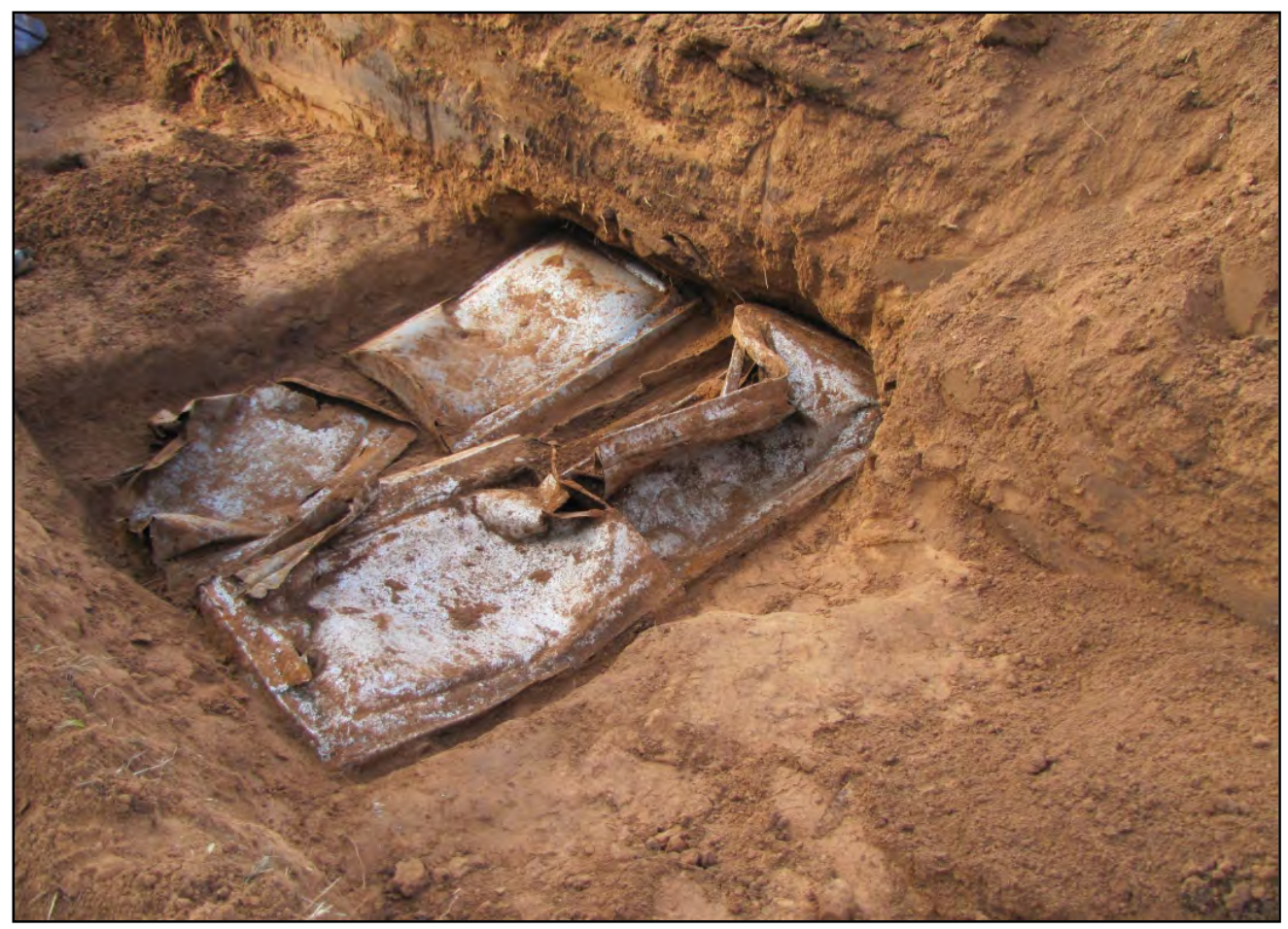

Figure 30. Coffins 3 (top) and 4 (bottom).

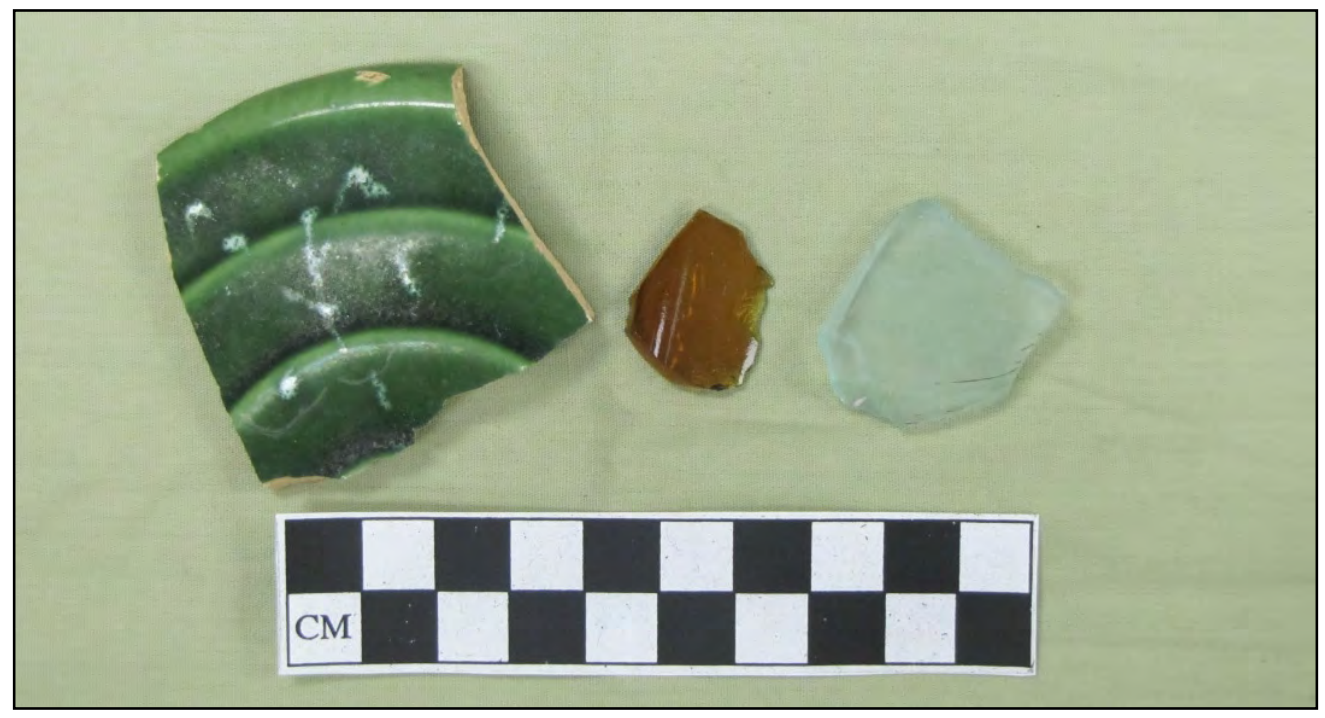

Figure 31. Pottery and glass fragments associated with Coffin 5.

The skeletal elements included fragments of cervical vertebrae (C3 and C5), thoracic vertebrae (T1 and T4), left and right ribs, right arm (humerus, radius, hand), right patella, left and right pubic symphyses, and right foot (one metatarsal and two proximal phalanges). There was pronounced erosion of the pubic symphyses, comparable to that associated with parturition. The sternal end of one of the rib fragments had an age assessment of 7c to 8c (early 70s to early 80s; 
Işcan et al. 1985). The bone cortices were notably thin and brittle (osteoporosis and osteopenia). The radius fragment was relatively small and gracile. The patella exhibited pronounced enthesopathy of the patellar tendon insertion on the superior margin, but no arthritis of the patellar articular facets. Cervical vertebra C3 exhibited arthritic pitting and lipping on the right superior and inferior aspects of the centrum.

\section{Miscellaneous Human Bone}

For the purposes of this study, miscellaneous human bone was defined as that which could not be assigned to any of the defined burials in the project area. This category was divided into two subcategories: human bone from the "disturbed area" and human bone discovered in surface and backdirt collection.

\section{Remains Found in "Disturbed Area”}

Isolated skeletal elements and fragments were observed throughout the disturbed area that was along the eastern margin of the ROW. The bone was left behind by the funeral company during disinterment activities and/or was dragged by the Gradall during the Phase II evaluation of the property. When possible, these elements were included in the inventories for each of the respective coffins that were identified within the same disturbed area. However, if it was not possible to conclusively associate the bones to any of the coffins, the remains were collected individually. The following skeletal elements were assigned to this group.

- Nine fragments of adult ribs with no obvious pathology or anomaly

- One fragment of adult innominate (acetabulum; unsided)

- Two tiny, amorphous cortical fragments

- One adult left humerus: proximal epiphysis missing; large and robust with notably heavy bone density; enthesopathy of the deltoid, latissimus, and teres major entheses (points of attachment on the bone surface); erosive arthritic pitting of the trochlea; patches of active periostitis on the proximal half of the diaphysis; diameters at (approximate) midshaft $=24$ $\mathrm{mm}$ anterior/posterior, $21 \mathrm{~mm}$ medial/lateral

- One fragment of adult thoracic vertebra spinous process (approximately T8), with no obvious pathology or anomaly

- One fragment of adult thoracic vertebra centrum, notably osteoporotic and exhibiting arthritic changes of the margins

- Three fragments of adult metatarsals; large and robust in overall morphology, with no obvious pathology or anomaly

- One adult metacarpal with no obvious pathology or anomaly

- One adult proximal phalanx (hand) with no obvious pathology or anomaly

- One adult medial phalanx (hand) with no obvious pathology or anomaly

- Adult cervical vertebrae, C3 and C4, fused together; pronounced arthritic and degenerative changes; possible Klippel-Feil syndrome or fusion due to axial loading stress. Klippel-Feil is the congenital fusion of two middle-to-lower cervical vertebrae. It gives the person an appearance of having a very short neck, as if the head is sitting on top of the shoulders. It also limits rotation of the head. 


\section{Bone Recovered from the Backdirt Pile or Surface Collection}

Human bone that was randomly discovered while walking around the site or collected from the backdirt pile included the following:

- One fragment of an adult left twelfth rib that exhibited a healed fracture of the sternal tip

- One almost complete, adult, proximal phalanx of the right second finger (no discernible pathology or anomaly)

- Two small, amorphous cortical fragments (no discernible pathology or anomaly)

- One fragment of diaphysis of clavicle (undetermined side; undetermined age; no discernible pathology or anomaly).

\section{THE REPATRIATION}

The morning of May 9, 2011, began with a telephone conversation between landowner Woodrow Jones and field director Cassandra Hill. The Geo-Marine crew (Hill, Skelton, and Nugent) then drove to the cemetery, where they were joined by TxDOT personnel (Mike Warnasch and Sharkey Espinosa, the backhoe and bulldozer drivers). Hill reviewed the excavation strategy with TxDOT and Geo-Marine personnel. After Mr. and Mrs. Jones arrived, everyone moved to the area in the cemetery that had been selected for the repatriation. Geo-Marine crewmembers David Treichel and Karl Huebchen arrived to assist in the transportation and placement of the recovered metal caskets, new burial containers, and marker stones.

According to Mr. Jones, the original surface had been more steeply sloped in this location, and a larger amount of backfill was deposited in order to make it equally level to the rest of the cemetery. The field director's expectation was that the likelihood of exposing in situ graves would be minimal if the trench was excavated to a maximum depth of approximately 4 feet. In situ graves had been discovered at an average depth of approximately $4-5$ feet below surface. An additional 18 inches to 2 feet of soil had been added to the soil's surface in this location. Thus, digging 2 feet into the built-up surface plus 2 feet into the original surface would still be approximately 2 feet above any interments that might be encountered. If graves were encountered, the trench would be moved to another part of the cemetery, and excavation would begin anew. This would be repeated until a suitable area could be found.

As excavation of the trench proceeded, the trench itself was monitored by the field director and the backdirt pile was monitored by the other Geo-Marine crewmembers (Figure 32). When the trench had been excavated to the width and approximate two-thirds of the length estimated to accommodate all materials and remains, the four Geo-Marine crew went to the secure storage facility to begin transport of the metal caskets. The trench was completed successfully, with only a few minutes passing before the crew returned with the first of the caskets. The casket was offloaded into the bucket of the backhoe, which then was lowered into the end of the trench. The bucket gently emptied the casket fragments onto the floor of the trench. Each casket required a separate trip to the storage facility. After all of the metal casket remnants were deposited, the crew went to the storage facility to retrieve all of the wooden burial boxes that contained the remains, artifacts, and sentimental soil for each respective grave. Each box was identified by an engraved brass plate that had the burial number in the center of the plate. All miscellaneous or random bones were included in a separate burial container. 


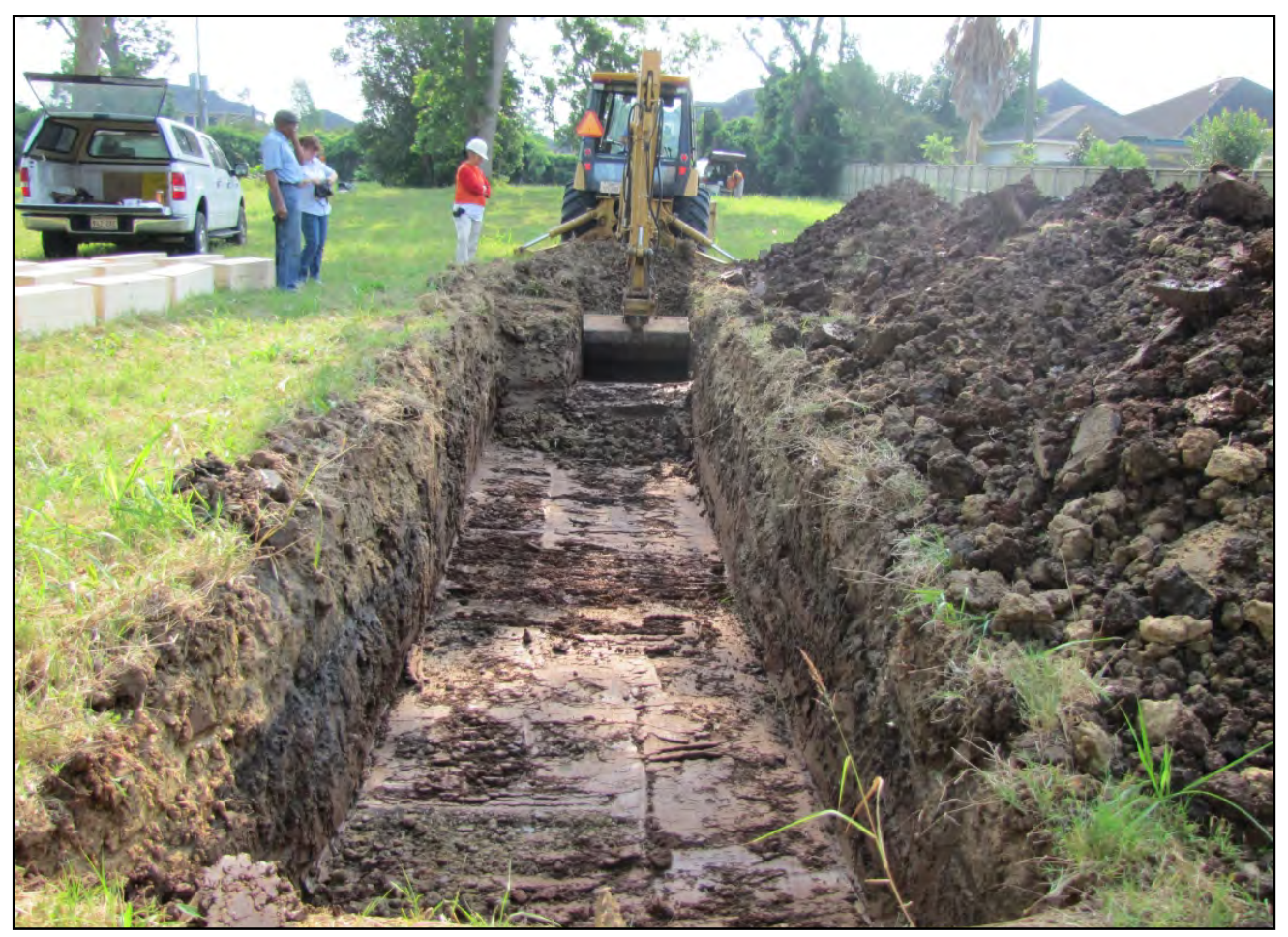

Figure 32. Mr. and Mrs. Jones (left, foreground) and M. C. Hill (right, background) monitor excavation of the trench, facing east.

All burial boxes were unloaded from the truck and placed along the long axis of the trench (Figure 33). Measurements were taken in order to determine how many could be accommodated within each row. It was determined that three boxes would be placed in each row, with approximately 18 inches between the coffins, and approximately 6 inches between the edge of the boxes and the walls of the trench (Figure 34). All boxes were carefully placed in this manner, until the entire length of the trench was filled (Figures 35 and 36). The arrangement was supervised by the field director and by Mr. Jones. Mr. Jones also participated in the placement of the boxes themselves. In order to prevent the boxes from rolling over or being displaced during the filling of the trench, the field director instructed the crew to place clods of soil between and around all boxes, essentially anchoring them in place. After the total station was used to mark the four corners of each box (Figure 37), a map of the location of each reburial was subsequently produced (Figure 38). When this was completed, and Mr. Jones was satisfied with the configuration, the trench was backfilled, beginning with the eastern end of the trench and slowly moving toward the western end. Soil was carefully deposited by the backhoe driver(s) (Figure 39).

As the trench was being filled, the four Geo-Marine crewmembers returned to the storage facility and retrieved the marker stones. The stones were placed along the margin of the trench, stacked in groupings of three, adjacent to their respective boxes (Figure 40). When the closure of the trench was complete, measurements were taken and the stones were placed on the surface above each of the boxes. Particular care was taken that the stone for Leah Simpson (the only stone with a name on it) was located directly above the box containing her remains, Box \#5 (Figure 41). All other stones were engraved with the word, "UNKNOWN" (Figure 42). 


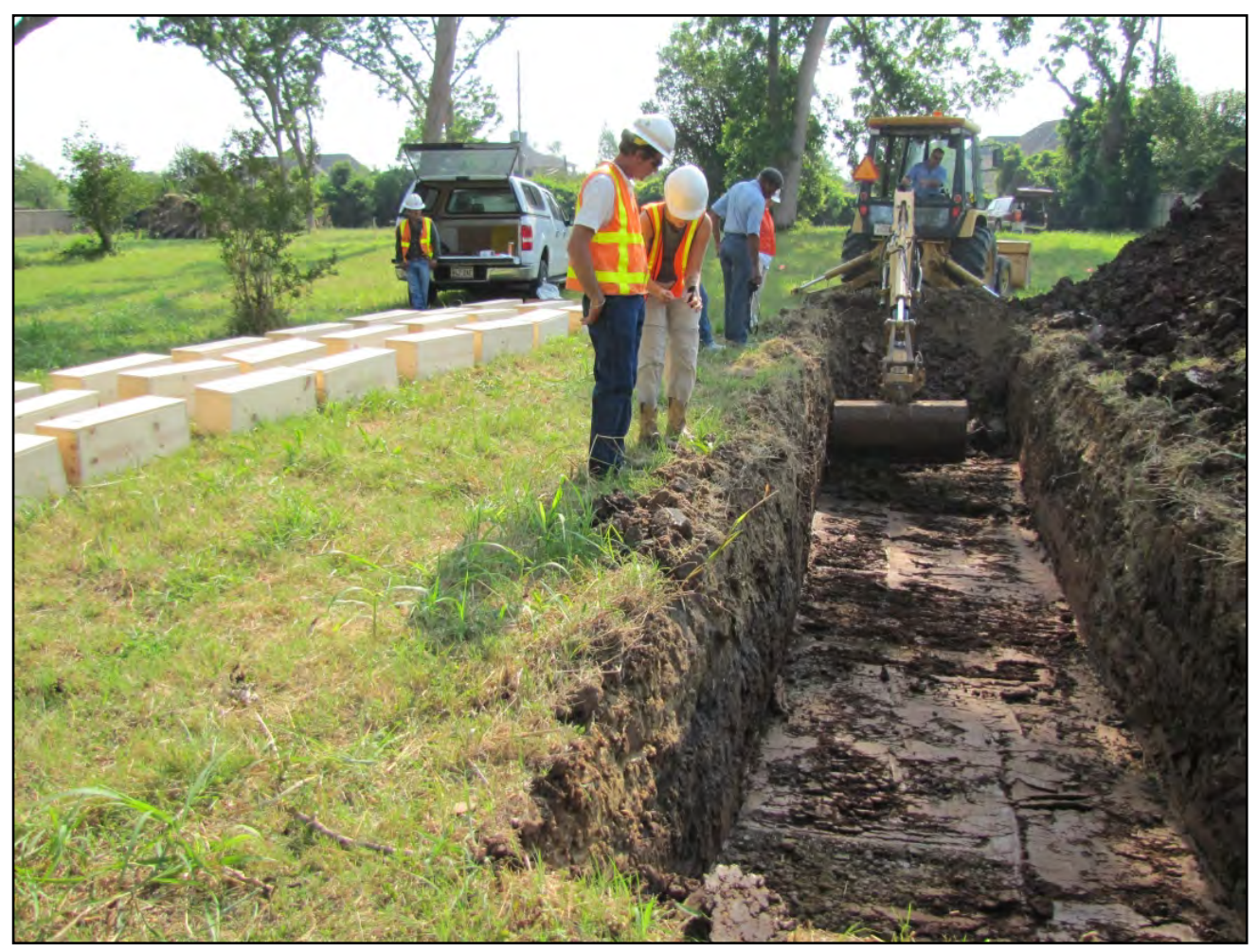

Figure 33. Burial boxes placed adjacent to trench, facing east.

The repatriation was complete by the end of the day. Although Mr. Jones had sent notices to local church groups asking them to get in touch with him if they had relatives in New Home Cemetery, Mr. and Mrs. Jones were the only individuals from the local community who were present at the reinterment. Mr. Jones stated that he would landscape the area where the repatriated remains were located. After parting conversations with Mr. and Mrs. Jones, the group adjourned.

The area of mitigation was mapped throughout the disinterment and repatriation. Maps produced by Geo-Marine located the graves in their original positions and also in their new locations. These maps are archived with all other forms of documentation pertaining to the graves. 


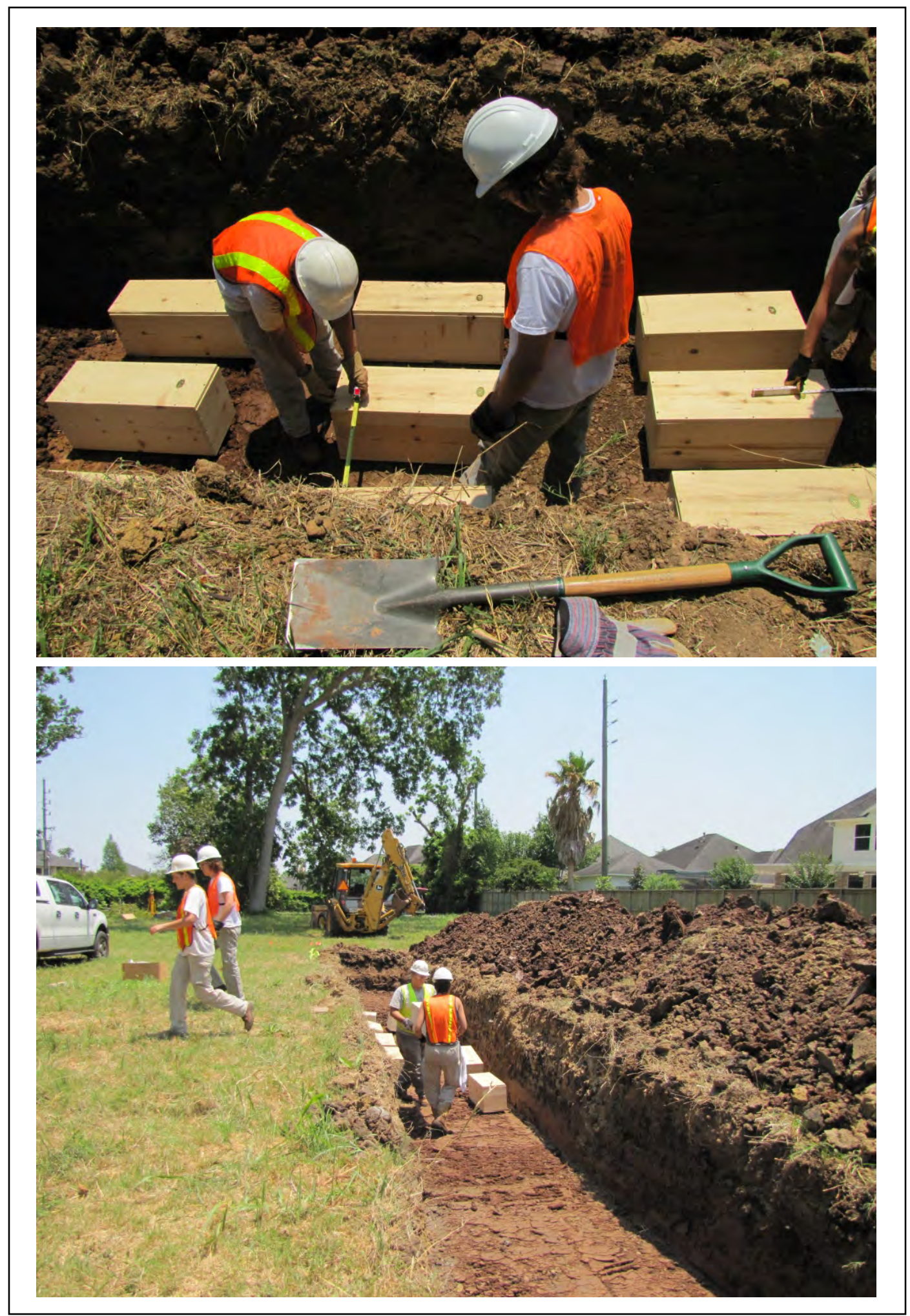

Figure 34. Geo-Marine crew measuring and placing burial boxes in trench. 


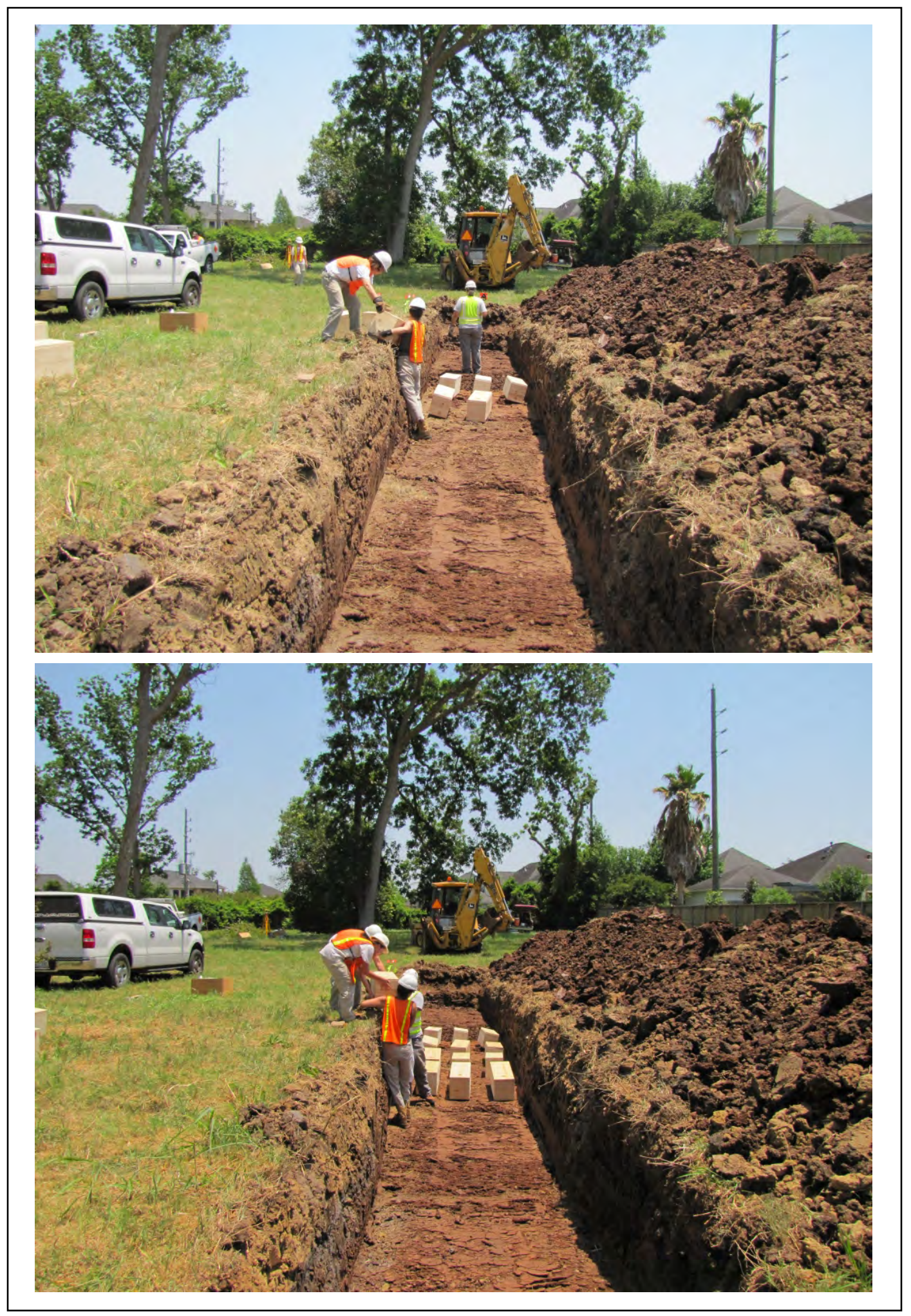

Figure 35. Geo-Marine crew placing burial boxes in trench, facing east. 


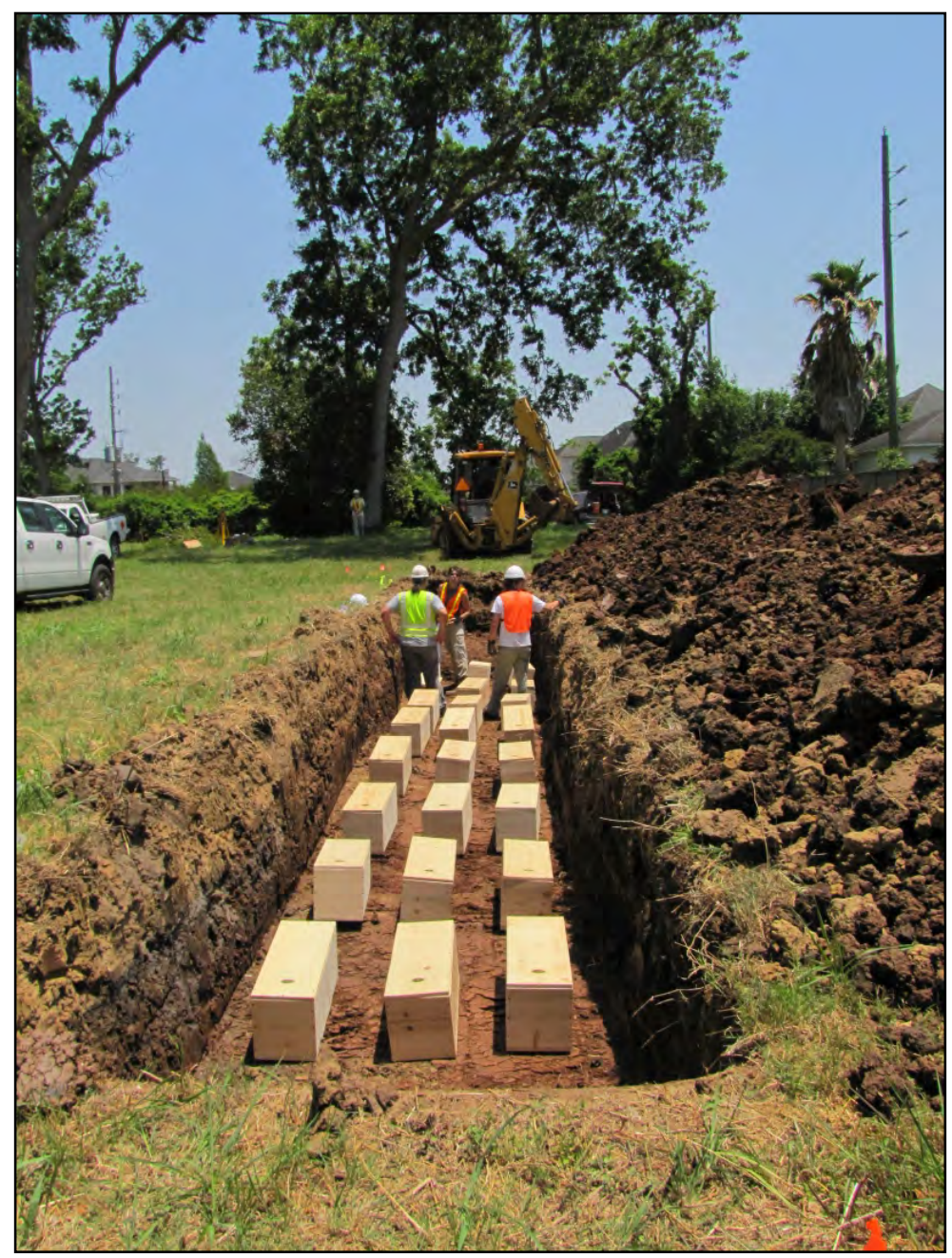

Figure 36. Burial boxes in trench, facing east. 


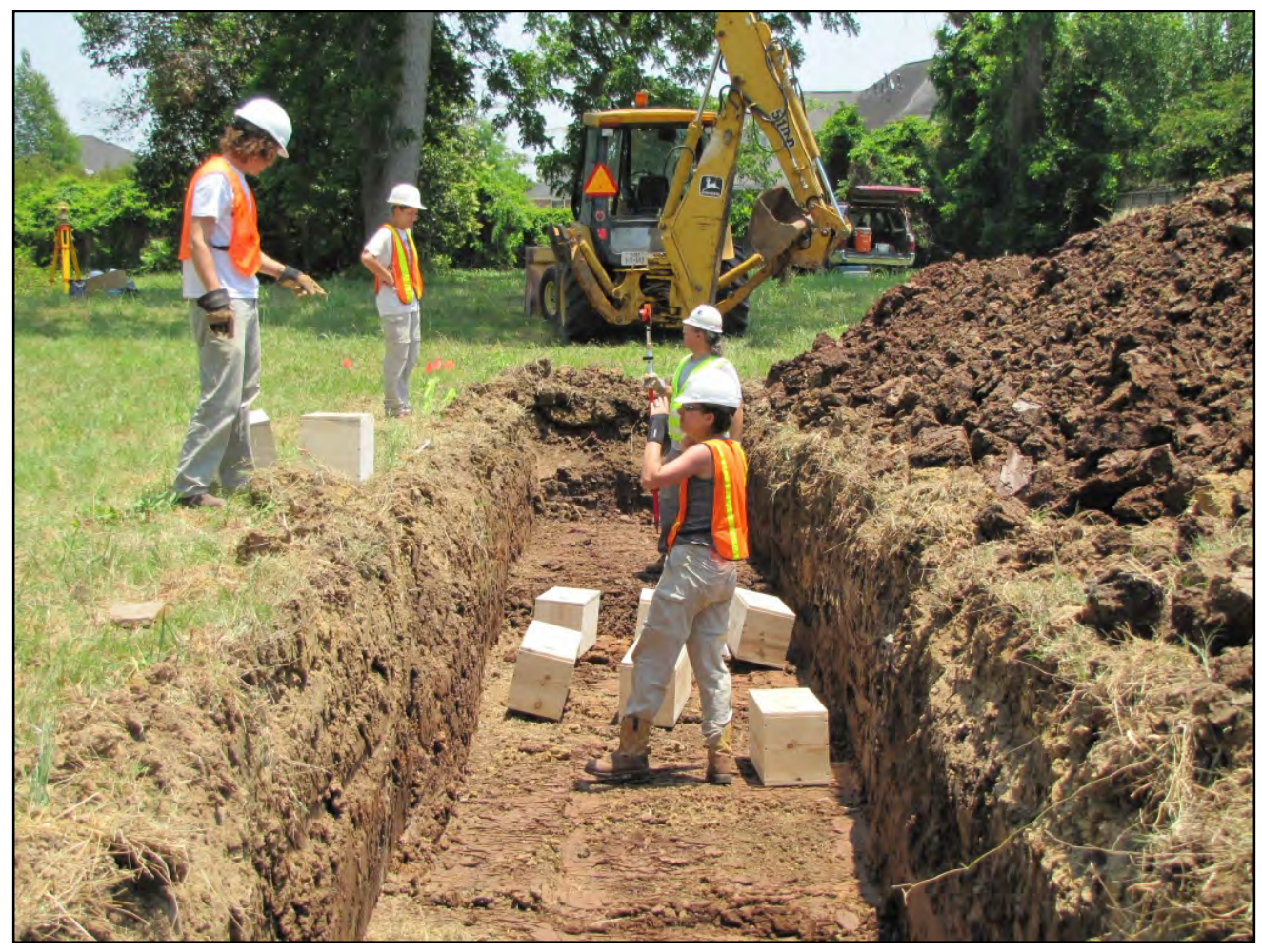

Figure 37. Mapping locations of burials, facing east. 


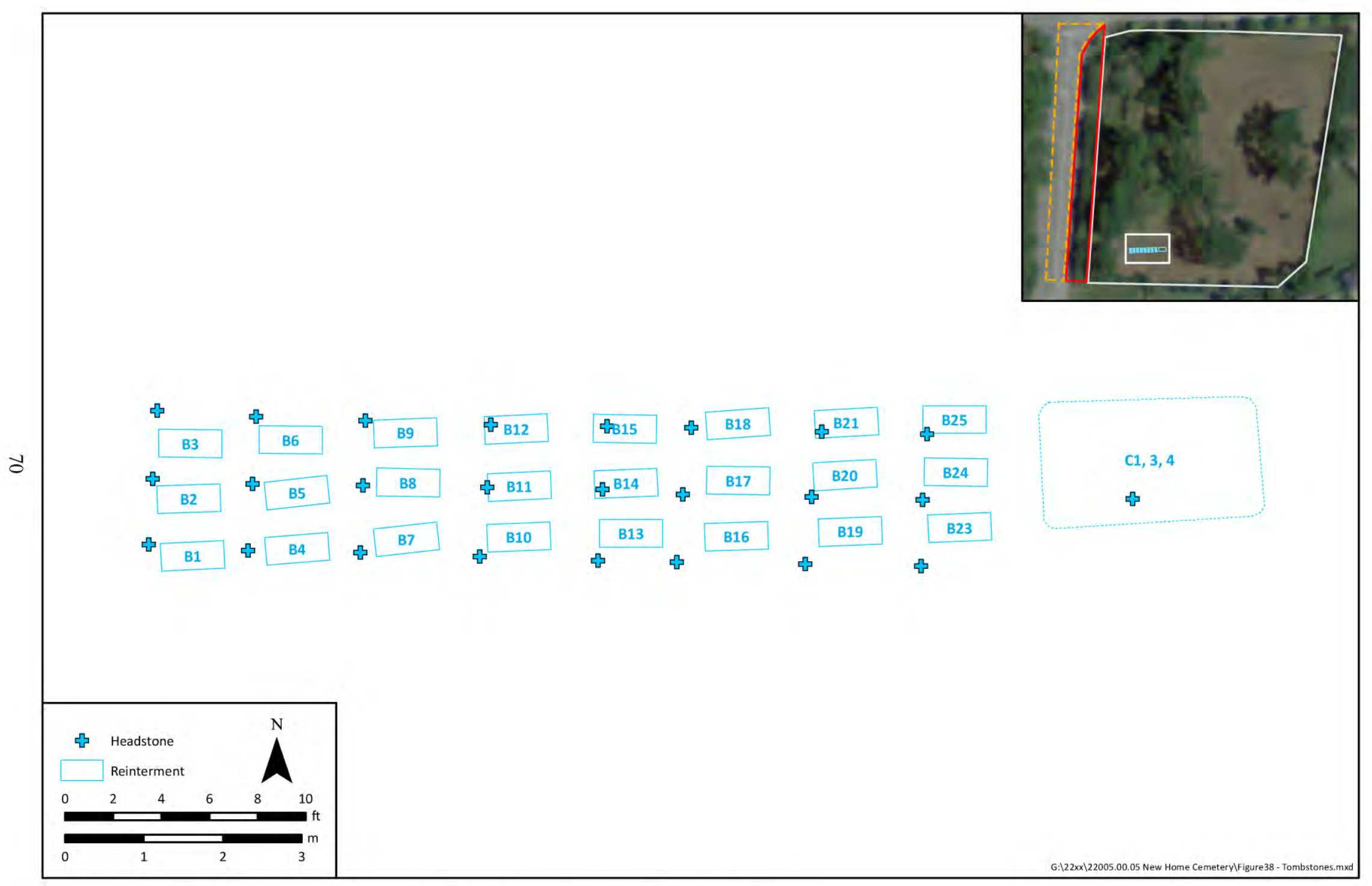

Figure 38. Plan map of the reinterment locations within New Home Cemetery (CSJ 1415-02-032). 


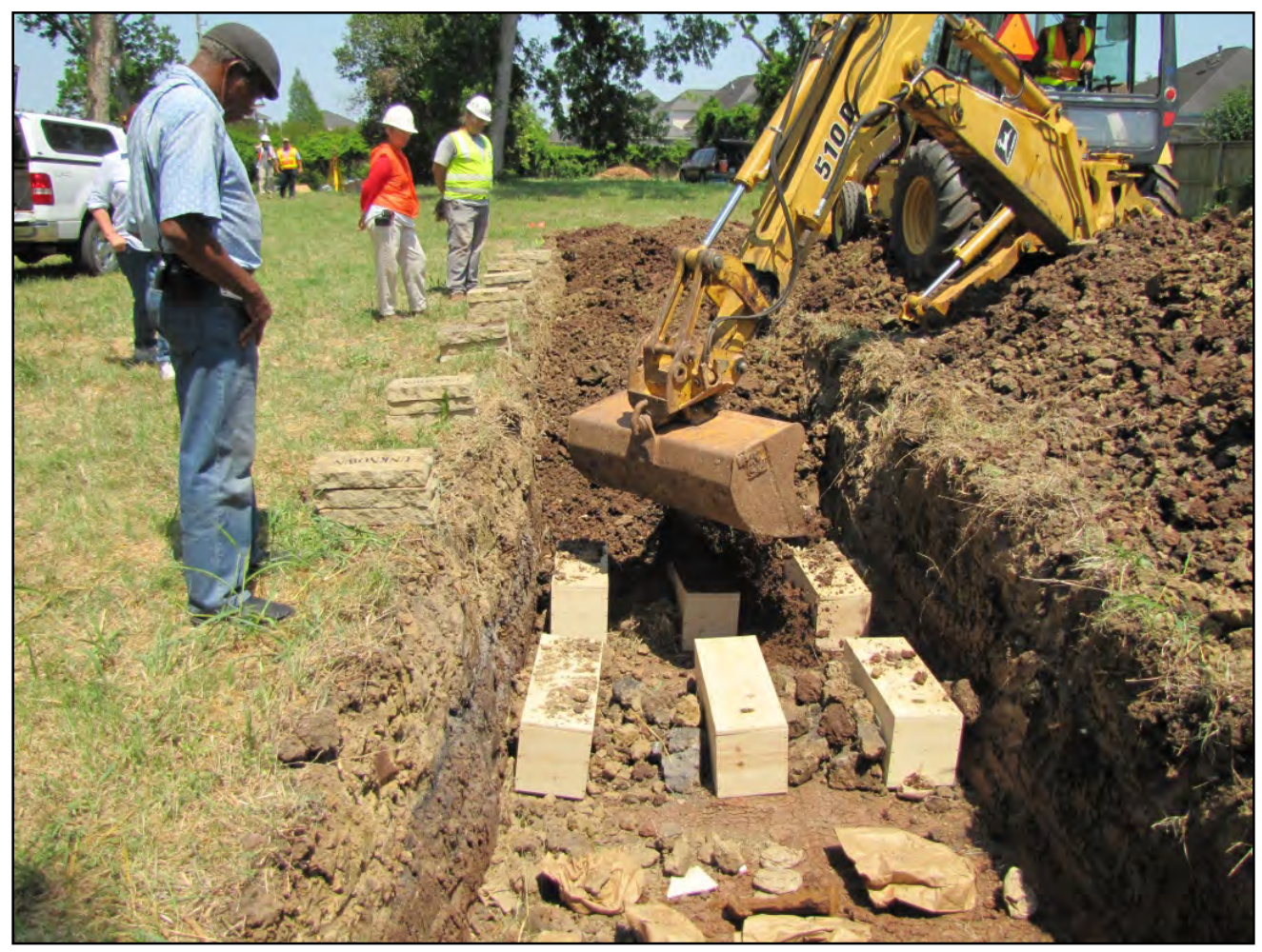

Figure 39. Mr. Jones observing the filling of the trench.

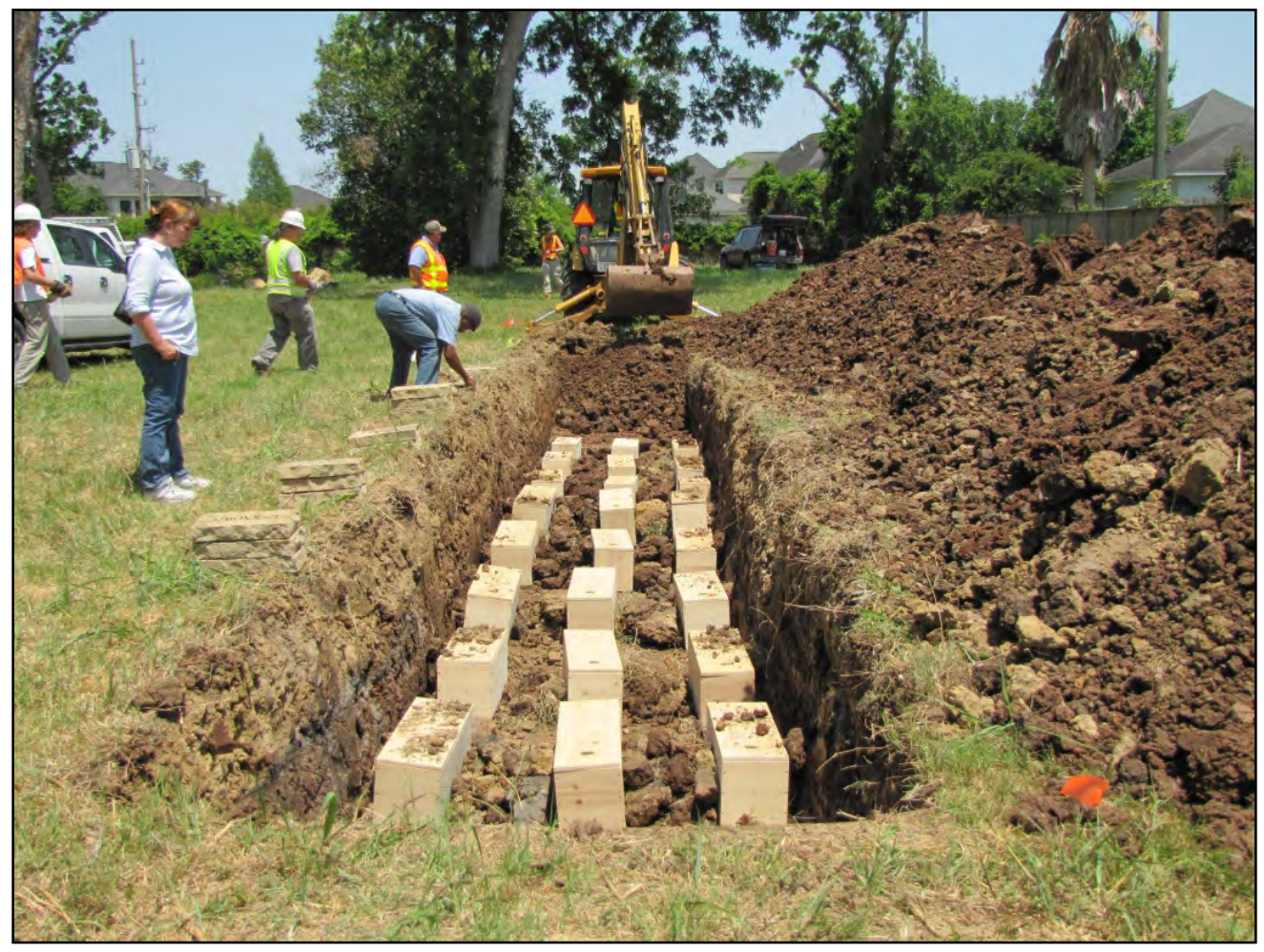

Figure 40. Placement of markers adjacent to boxes in trench, facing east. 


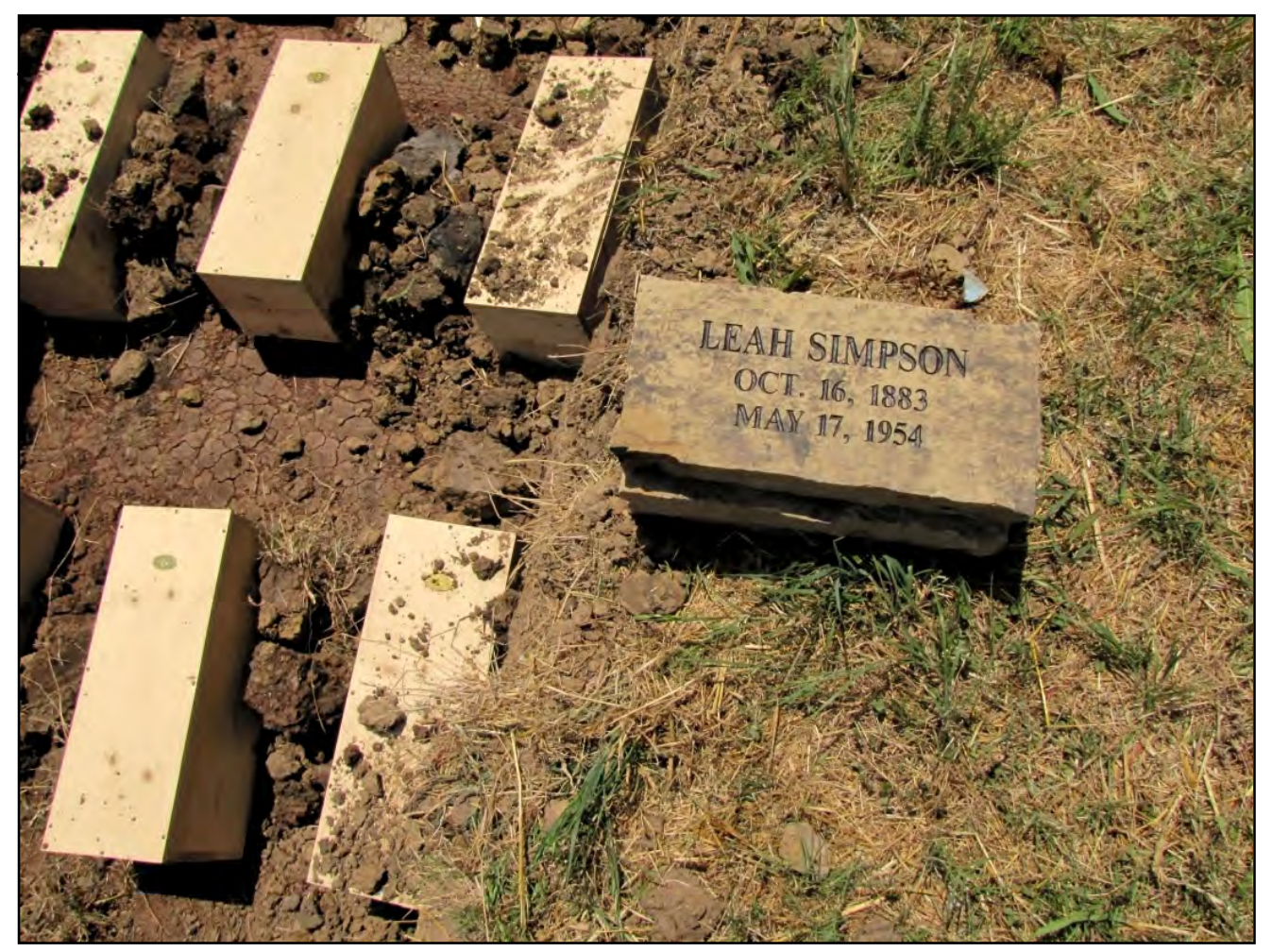

Figure 41. Leah Simpson’s marker.

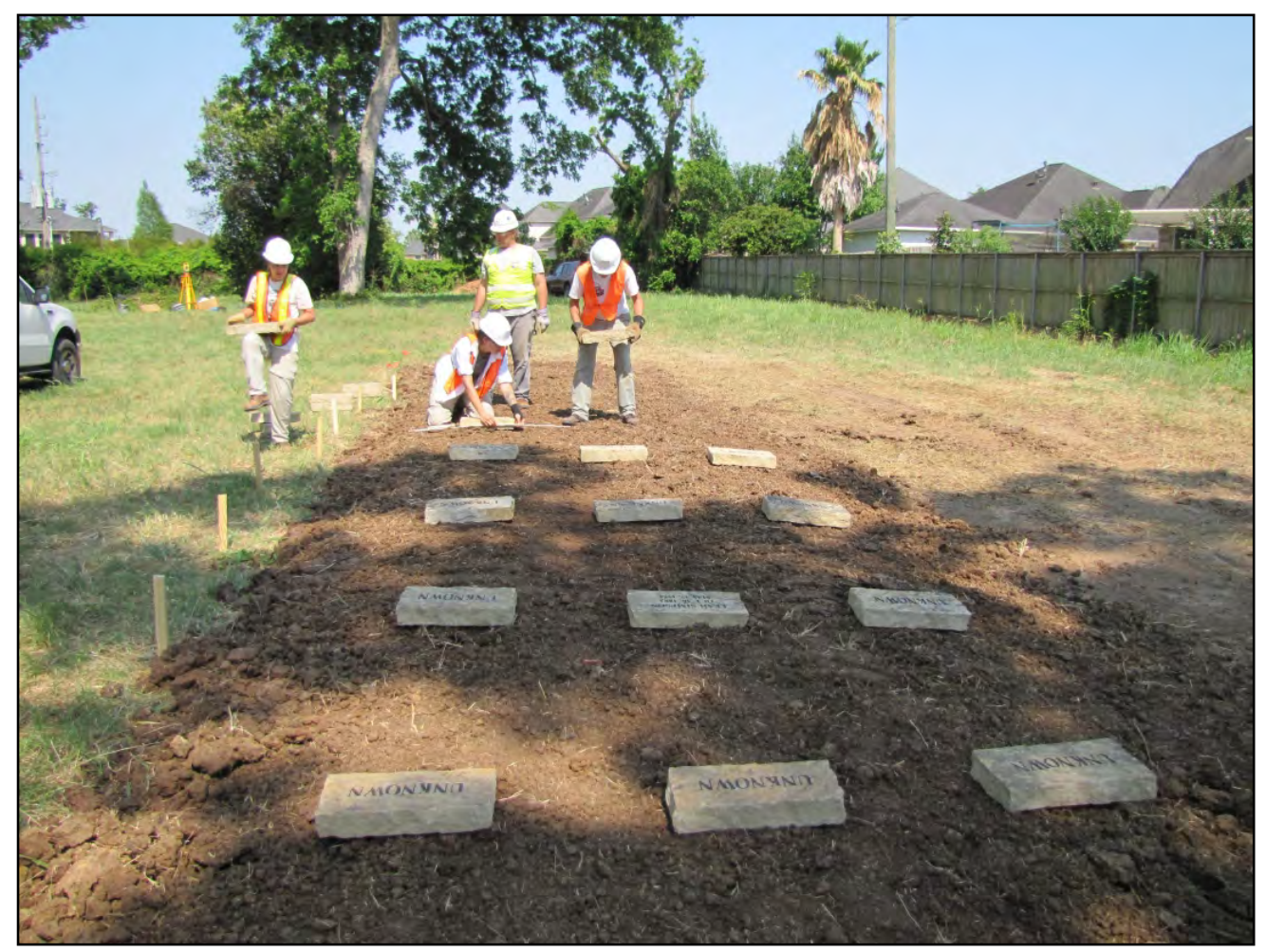

Figure 42. Geo-Marine crew measuring and placing marker stones on surface of trench, facing east. 


\section{CHAPTER 5 \\ TYPOLOGY AND ANALYSIS OF MORTUARY AND PERSONAL ARTIFACTS RECOVERED FROM EXCAVATIONS AT NEW HOME CEMETERY}

by Jeremy W. Pye, with contributions by Melissa M. Green and Lindsey Skelton

\section{INTRODUCTION TO MORTUARY MATERIAL CULTURE}

The analysis and historical study of burial container hardware and other mortuary artifacts is crucial in establishing a useful discourse between multiple lines of evidence recorded and recovered in historical cemetery investigations. Decorative burial container hardware and grave adornments can infer multiple social and aesthetic meanings. For some affluent members of society, ornate and expensive burial container hardware or monuments were used as a marker of social status (Burgess et al. 2007). Others used these objects as a means of masking social realities and presenting the illusion of wealth (Little et al. 1992). Hardware and grave markers in and of themselves held religious and ideological symbolic value in the development of the outward expression of the Victorian Beautification of Death movement in the late nineteenth century (Bell 1987, 1990). The nineteenth- and early twentieth-century perspective viewed the ornamentation of the funeral and the burial container as an extremely important part of the expression of sentiment and community restructuring.

Mortuary artifacts hold great value for archaeologists just as they did for mourners. Exact identification of types and styles of burial container hardware and other mortuary artifacts is vital in defining the chronology of burial, particularly when dated grave markers are absent or when markers have been displaced. Variations in hardware styles and forms, as well as materials of manufacture, indirectly reflect aspects of socioeconomic class, status, and/or community involvement in the funeral process (Bell 1987, 1990; Davidson 1999, 2004; Little et al. 1992; Pye 2007). Additionally, the specialized burial container hardware introduced into the archaeological record in early twentieth-century contexts reveals the deepening control of the professional funeral industry in the production and distribution of funeral merchandise. 


\section{TYPOLOGY METHODOLOGY AND COMPARATIVE MATERIALS}

The methods established by Davidson (1999) for the classification of hardware from the Freedman's Cemetery project, Dallas, Texas, have been applied to the New Home Cemetery materials. Essentially, a new type is designated when a different artifact form/style (or combination of elements) is encountered. Mainfort and Davidson (2006:120-121) give the example, "the first thumbscrew . . . was given the type designation Thumbscrew Type 1. . . if the next burial excavated uncovered a thumbscrew with an even slightly different design motif, [then] it was assigned a new type number (e.g., Thumbscrew Type 2).” Size variants (i.e., adultsized handles versus child-sized handles) were designated by numerical suffixes (e.g., Handle Type 12.1).

Following Davidson (1999) and Mainfort and Davidson (2006:121), it is acknowledged that these artifacts should be dated and contextualized through three lines of evidence: patent dates, dates derived from period hardware catalogs, and known dates of cemetery use. A fourth line of evidence-the estimated interment ranges of burials from previously excavated cemeteries - can be included, but must be critically analyzed based on the previous lines of evidence.

\section{United States Patent Records: Utility Patents $(1839-1965)(n=2,160)$}

Two thousand one hundred sixty utility patents related to burial containers, burial container hardware, and other types of mortuary merchandise dated between 1839 and 1965 were viewed through the United States Patent Office (2012) online database during this analysis. Searches were restricted between 1800 and 1965 and used the primary search terms, such as "casket," "coffin," "embalming," "corpse," etc. It is extremely likely that pertinent patents have escaped the search due to simplicity of the search terms, flaws in the PDF OCR process, and human error in database entry.

\section{United States Patent Records: Design Patents $(1843-1965)(n=455)$}

Four hundred fifty-five design patents related to burial containers, burial container hardware, and other types of mortuary merchandise that dated between 1843 and 1965 were viewed through the United States Patent Office (2012) online database during this analysis. Searches were restricted between 1800 and 1965 and used the primary search terms, such as "casket," "coffin," "embalming," "corpse," etc. It is extremely likely that pertinent patents have escaped the search due to simplicity of the search terms, flaws in the PDF OCR process, and human error in database entry.

\section{Manufacturers' Trade Catalogs $(n=275)$}

For this study, 275 period mortuary hardware trade catalogs, spanning the period between 1797 and the present, were examined for comparative purposes. These catalogs (see Appendix D) were either viewed at a number of different libraries, museums, or companies throughout the country, or are contained within the collections of the author or James Davidson. Most were viewed in their entirety, but some were only partial copies. 


\section{Previous Historic Cemetery/Burial Excavation Reports (n = 154)}

A total of 154 reports of excavated historical period cemeteries was also consulted (see Appendix E). When possible, identical matches to each artifact type are listed in the following analysis. In some instances, similar matches may be mentioned, and it should be noted that even though they are not listed, most artifacts have contemporary similar forms. Care should be taken in future studies to identify exact stylistic matches and not matches of simple form similarity.

\section{MORTUARY ARTIFACT DESCRIPTIONS AND TYPOLOGY}

An attempt will not be made in this report to describe the history and various iterations of all mortuary artifact forms. For more descriptive information about many of the nineteenth- and early twentieth-century general hardware types see Davidson (1999, 2004) and Mainfort and Davidson (2006). However, for general purposes, a contextual discussion of each mortuary artifact form encountered during the cemetery excavations will be presented, followed by descriptive discussions of each type as constructed within the current typology.

\section{Markers}

The way people choose to mark the graves of the deceased has varied widely through time and space. Throughout the history of the United States, bereaved families customarily memorialized a deceased loved one through the erection of a stone monument when economically feasible. It could sometimes take weeks, months, or even years for families to design, contract for, and set a stone. Temporary grave markers have been used by cemetery caretakers and funeral directors to help families locate and identify burial sites until installation of a permanent marker. Additionally, in the twentieth century, funeral directors used temporary grave markers as a means of advertising for their services. The industry promoted the use of temporary markers so that new gravesites would not look "neglected."

Temporary grave markers can take many forms, including fieldstones, wooden stakes or boards, formal aluminum or steel stakes with name plates or paper slips, or any number of objects that could be inserted to mark the location of a grave. The first known patent for a temporary grave marker was issued in 1892 (U.S. Utility Patent No. 473,296) to John A. Coffey (Figure 43). This invention consisted merely of a bent rod inserted into the ground from which an information plate could be suspended. The reverse face of the information plate was designed with a slot into which photographs or other mementos could be inserted. It is noteworthy that Coffey claimed his invention to be a temporary or permanent grave marker because temporary markers gain "permanent” status if they are placed as such or if they are never replaced. 


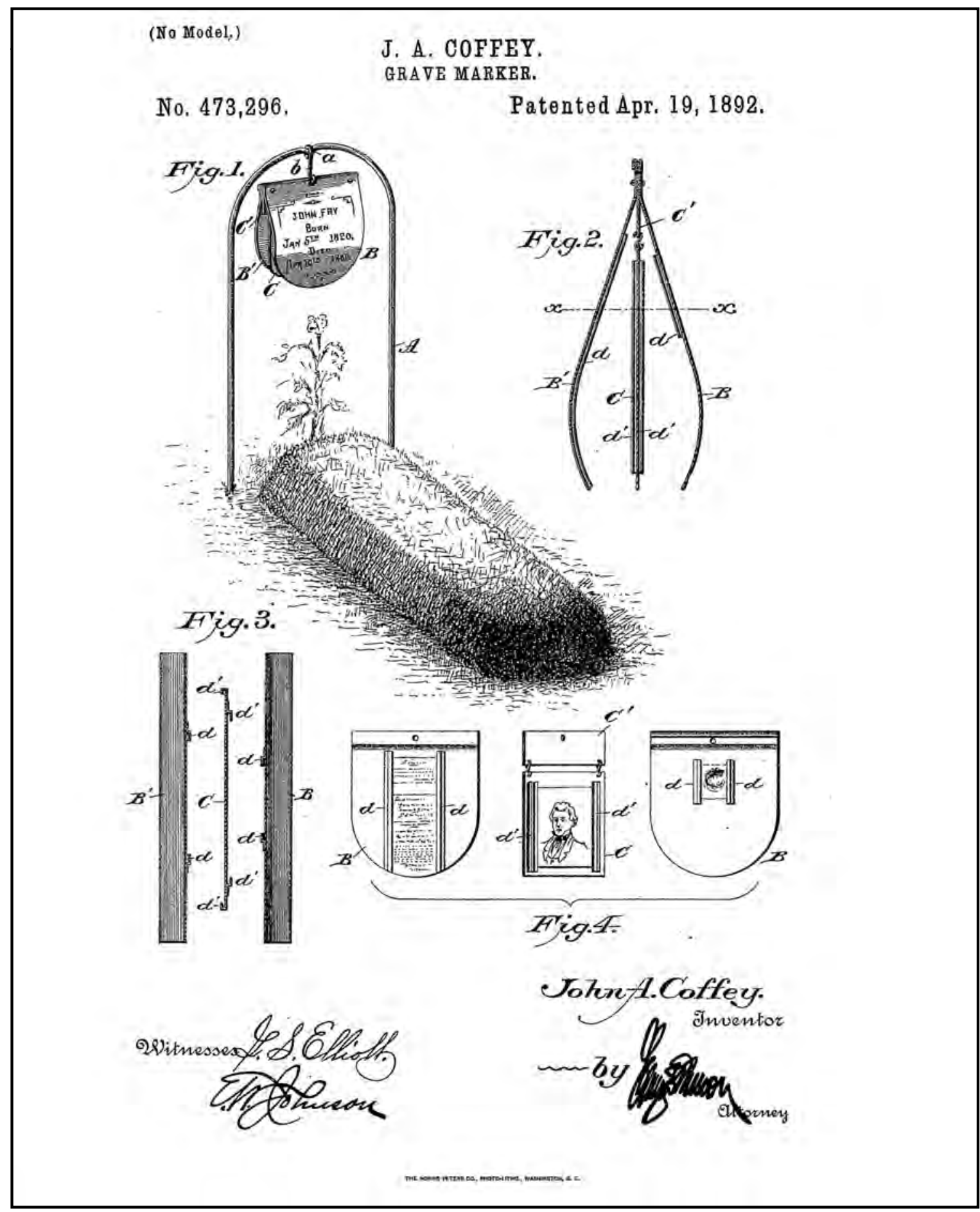

Figure 43. U.S. Utility Patent No. 473296 assigned in 1892 to John A. Coffey for a grave marker. 


\section{TemporaryMarkers}

\section{Marker Type 1}

Marker Type 1 (Figure 44) is a temporary grave marker present in the fill or at the surface of two burials (Burials 6 and 11) at New Home Cemetery, as well as one recovered from a disturbed area (see Appendix A). The marker consists of a galvanized steel frame and stake and a glass pane that would have covered a paper slip with the deceased's personal information.

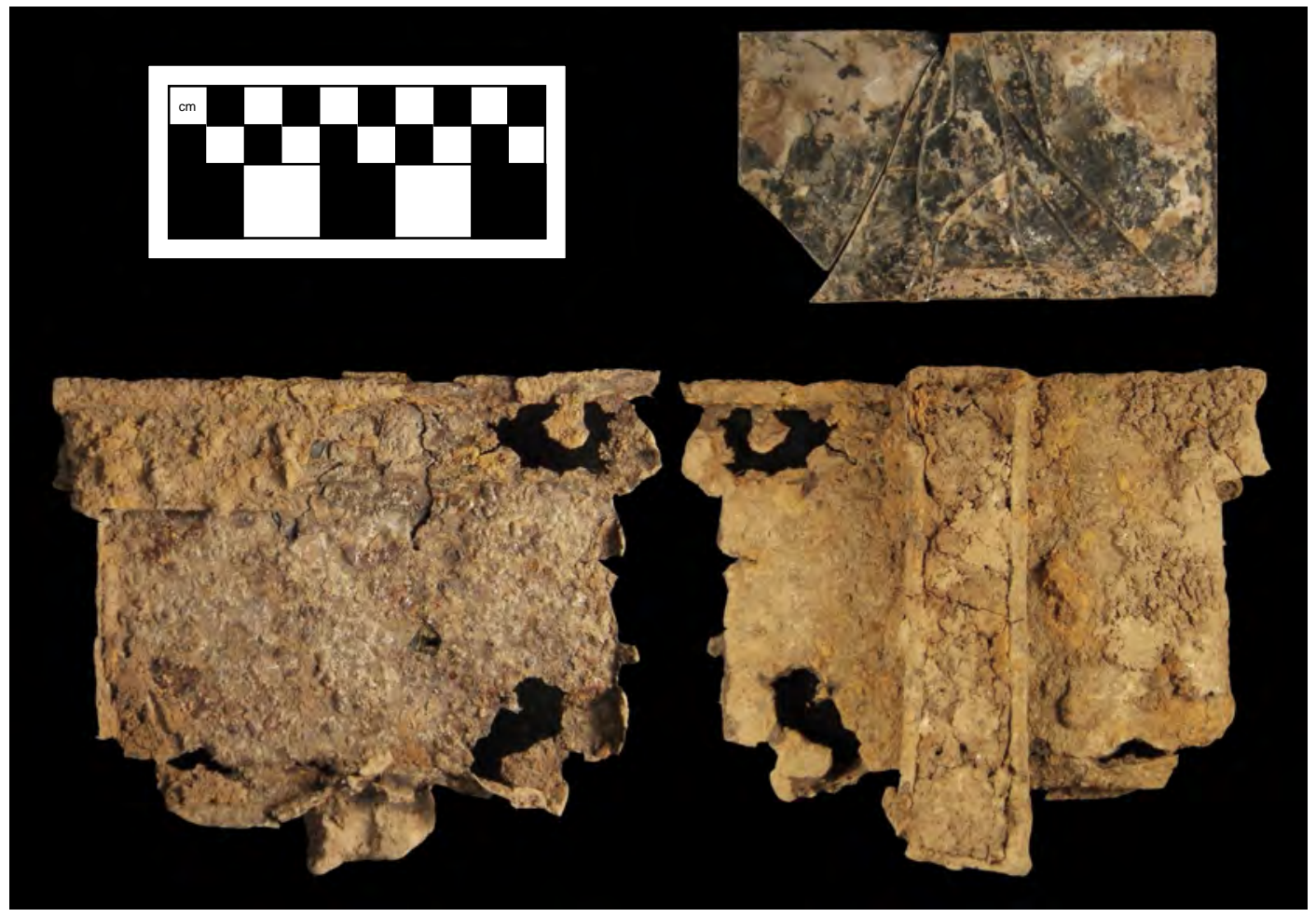

Figure 44. Marker Type 1.

Two of the frames have deteriorated because of heavy iron corrosion, but one appears to have been finished with a thin gray enamel layer. Embossed lettering was evident on the surface of this item, though corrosion and the lifting of the enamel layer made it somewhat difficult to discern the message. Therefore, Christopher Goodmaster of Geo-Marine subjected the artifact to a 3D laser scan. Figure 45 shows the upper artifact with the low-resolution version of the laser scanning result. Results of the surface enhancement algorithm were good; the marker discloses the following patent information:

NO PATENTED

1276798 AUG 261918

1573268 FEB 161926 


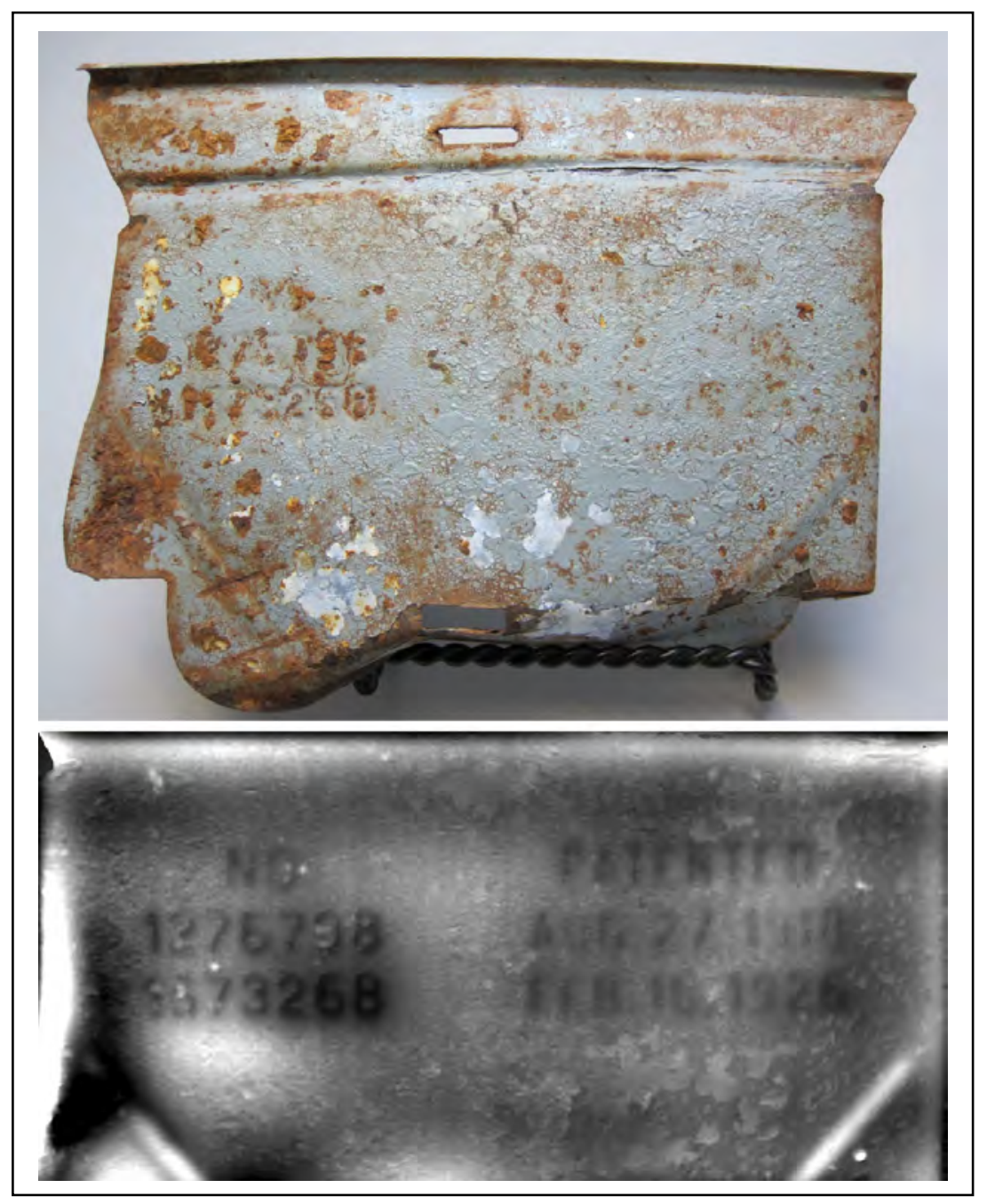

Figure 45. Comparison of Marker Type 1 (top) and and low-resolution laser-scanned image (bottom) of the embossed lettering on Marker Type 1.

Both of these patents (Figure 46) were issued to William F. Norman of Nevada, Missouri, and relate to improvements in grave markers that would yield a simple and cheap way to mark graves with the information of the deceased without the need to set more complicated monuments.

These temporary markers quickly increased in popularity among funeral directors as is evident in an advertisement for "Norman Grave Markers," which appeared in the April 1, 1919, issue of the industry trade journal Casket (Figure 47). At that time, the product was advertised in the journal as available for sale from the W. F. Norman Sheet Metal Mfg. Company for $\$ 42$ per gross. A second similar advertisement appears in the May 15, 1929, issue of the Sunnyside (Figure 48) depicting the form presented in Norman's second patent (U.S. Patents No. 1573268), which is also the same form present at New Home Cemetery (see Appendix D). 


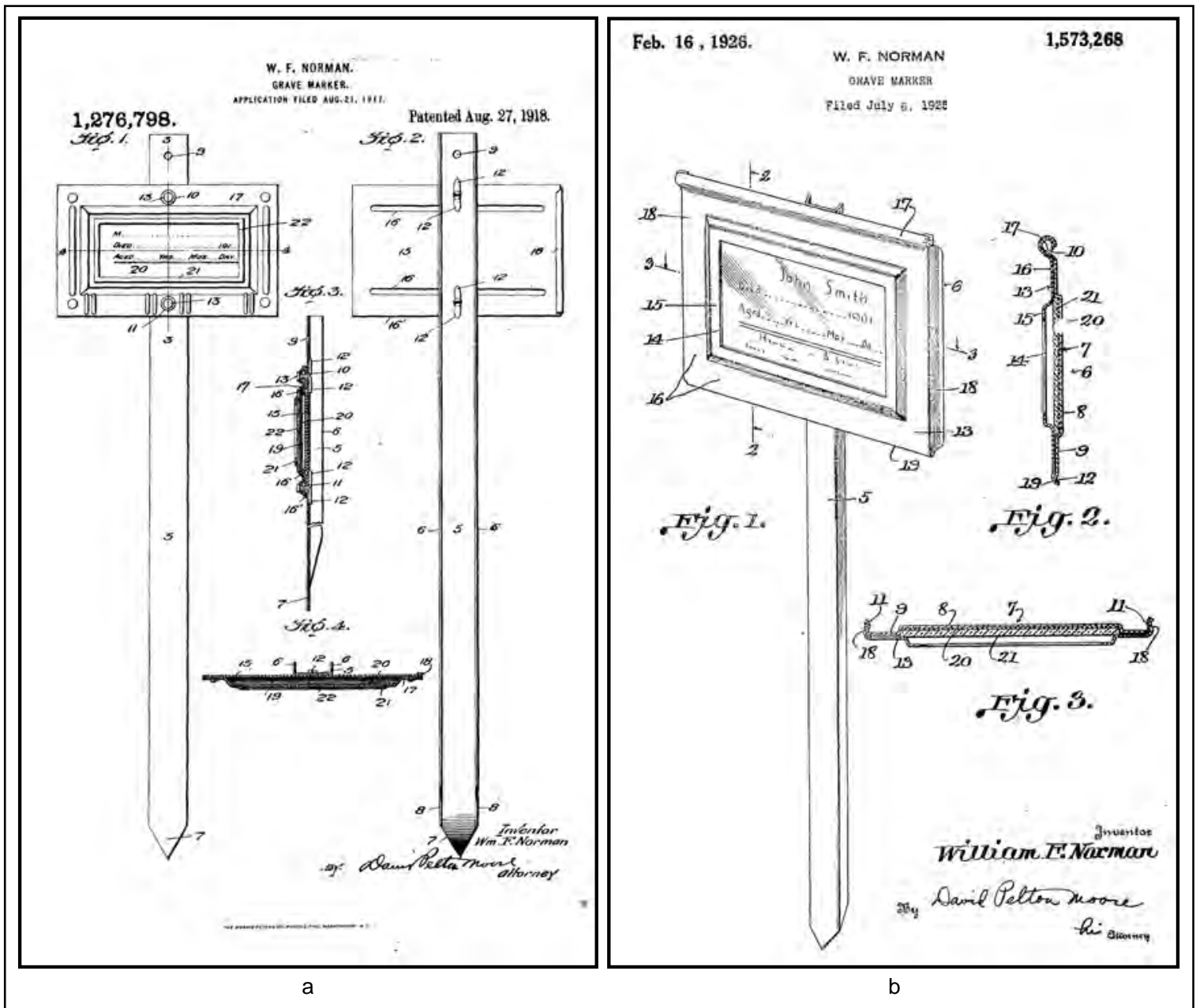

Figure 46. U.S. Utility Patent No. 1276798 (a) and No. 1573268 (b) assigned, respectively, in 1918 and 1926, to William F. Norman for grave markers.

In addition to being located in the above two trade journals, Marker Type 1 has been identified in six mortuary merchandise catalogs ranging in date from 1934 to 2010 (see Appendix D), and they are still manufactured today. There was a small and steady increase in price of $\$ 3$ between the 1929 Sunnyside advertisement and the 1934 J. Oliver Johnson, Inc., catalog. At that time one could also buy the Norman markers with a cross attachment for the top of the piece (Figure 49). Johnson appears to have been a wholesaler selling these items at base value because the Undertakers' Supply Company (ca. 1930s b) catalog advertised Norman markers at \$69 per gross, and $\$ 70$ in the ca. 1950 Royal Bond, Inc., catalog. There is a great increase in price between the 1950s and the late 1960s/early 1970s when these same markers were sold for $\$ 112$ per 100 by Tri-State Industries, Ltd (n.d.). Figure 50 shows that in 2002 the Kelco Supply Company (2002a) offered Norman markers that remained relatively unchanged since the publication of the 1929 Sunnyside advertisement. In 2002, they sold for $\$ 3.20$ each, which would be approximately $\$ 460$ per gross (Kelco Supply Company 2002b). 


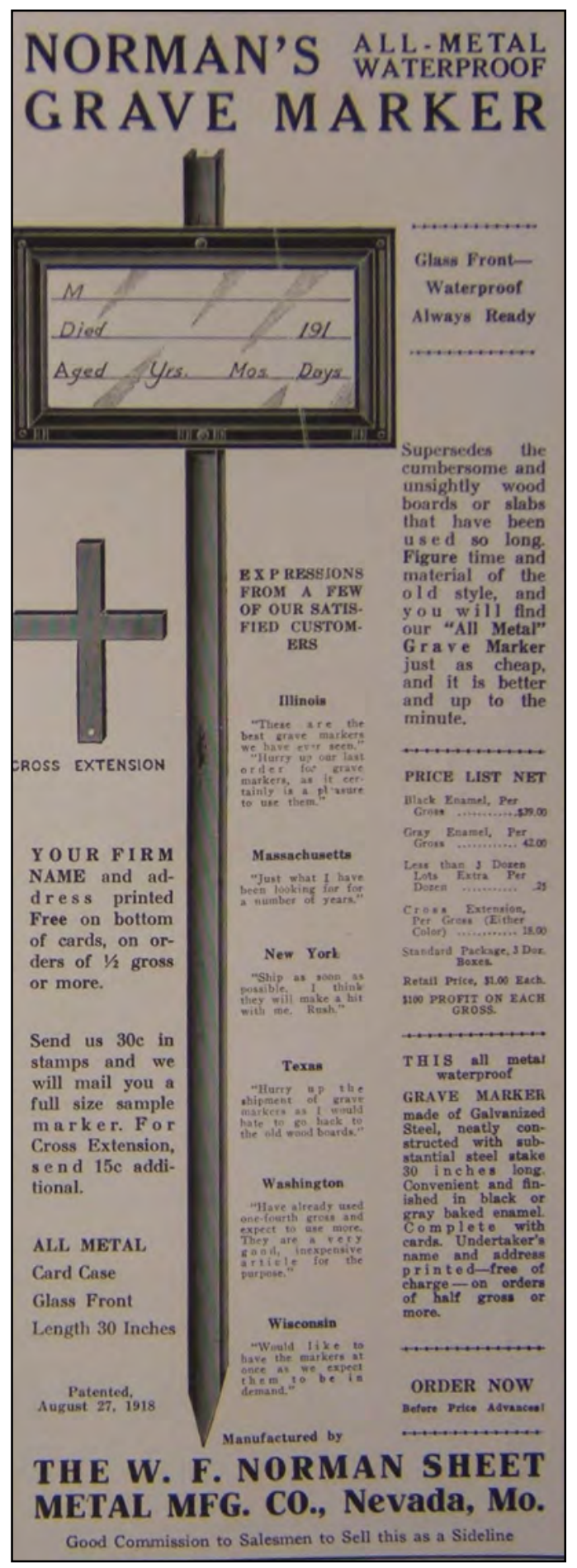

Figure 47. Advertisement for Norman's Grave Marker in the April 11, 1919, issue of the Casket trade magazine

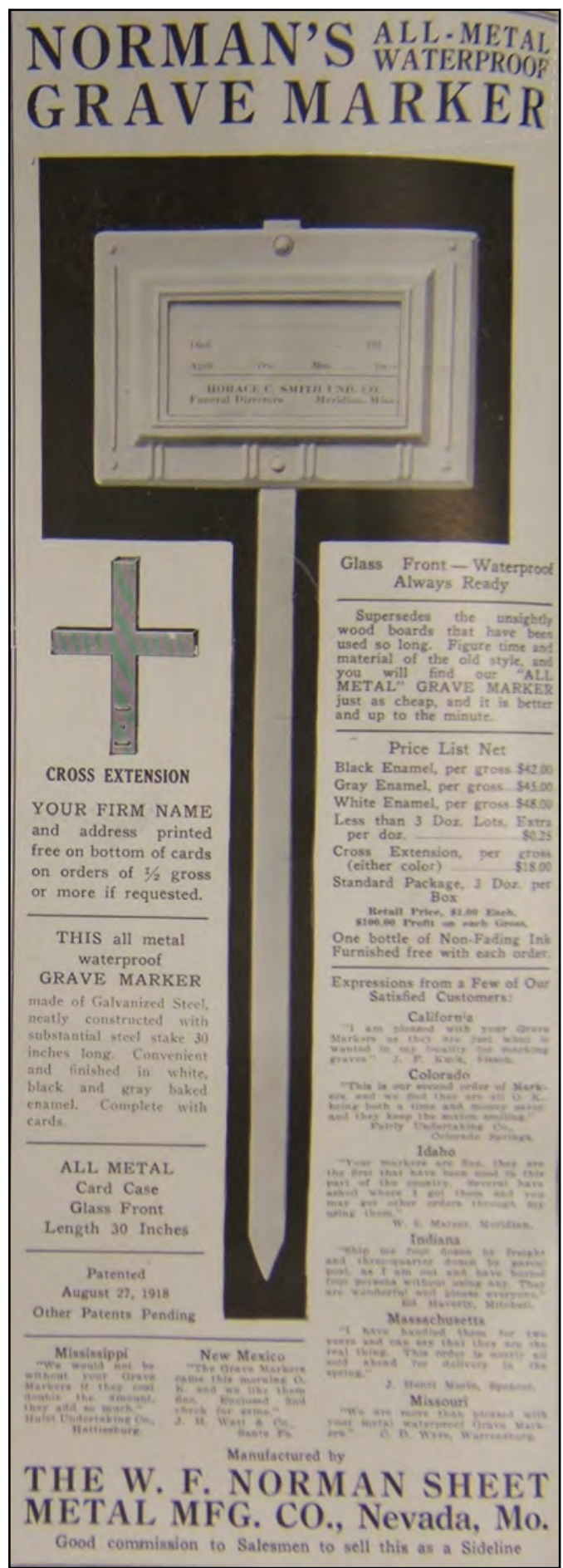

Figure 48. Advertisement for Norman's Grave Marker in the May 15, 1929, issue of the Sunnyside trade magazine. 


\title{
NORMAN GRAVE MARKERS AND CROSSES
}

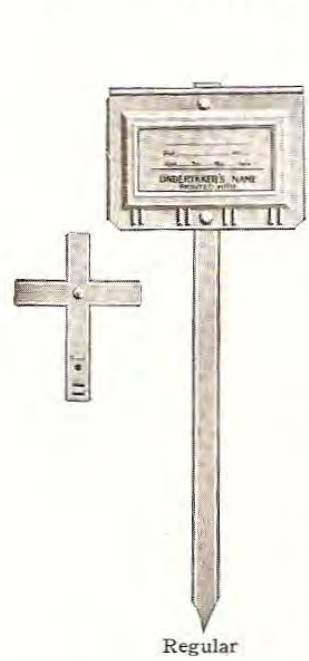

\author{
"Mark Every Grave"
}

\begin{abstract}
A popular line of Galvanized Metal Markers used for temporarily marking graves and lots. They are made with hinged receptacles for holding inscription cards which are protected by glass. Inscriptions are written with indelible ink. The cards are not affected by rain.

The Crown design can now be furnished with tilted head when desired, finished in any of the five standard colors.

Please be sure to specify style of design and color desired when sending your order. Either the Regular or the Crown design may be used for marking lot and grave numbers, and lots under Special or Annual Care.
\end{abstract}

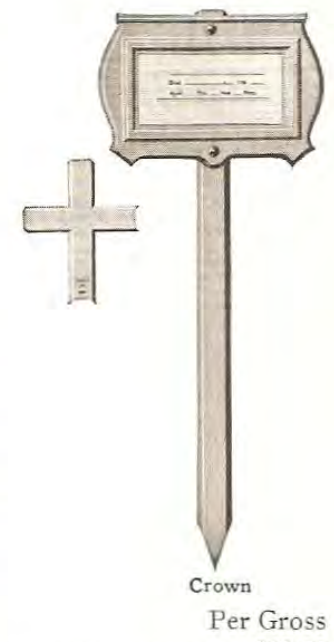

CD3801-Black Enamel Markers, Regular or Crown.................. \$48.00 CD3802-Gray, White, Purple and Orchid Markers................... 48.00 CD3803-Crown Tilted Markers, any of the above colors.............. 48.00

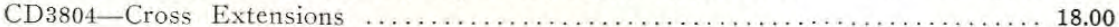
CD3805-White Crosses for Crown Tilted Markers placed on the front of the stake. 18.00

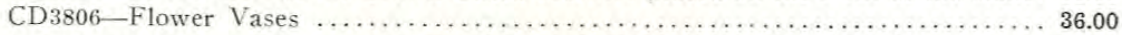
LESS THAN 3 DOZEN LOTS, ADD 25c PER DOZEN. CARDS AND INK ARE INCLUDED IN THESE PRICES.

Figure 49. Regular Norman Grave Marker, which is similar to Marker Type 1, depicted on page 38 of the $1934 \mathrm{~J}$. Oliver Johnson, Inc., catalog.

\section{Marker Type 2}

Marker Type 2 (Figure 51) is a temporary grave marker present at only one burial (Burial 12) in New Home Cemetery (see Appendix A). The marker consists of a galvanized steel stake and attached base plate upon which sits a glass pane that would have covered a paper slip with the deceased's personal information, though no paper remains. Mounted over the glass plate is a shield-shaped steel cover plate that is hinged to the rear frame for lifting. The rear base and shield exhibit heavy iron corrosion, but the stake reveals that the item had been finished with a thin gray enamel layer.

This shield-shaped temporary marker was patented by Marion G. Slawson, of Gibard, Kansas, in 1927 (Figure 52). The Slawson shield marker has only been identified in two mortuary merchandise catalogs from the early 1930s (Figures 53 and 54; see Appendix D). In the advertisements in both the 1934 J. Oliver Johnson, Inc., catalog and the early 1930s Undertakers' Supply Company (1930s a) catalog, these shield markers appear alongside other W. F. Normaninspired temporary markers, with the gray-finished variety selling for $\$ 42.00$ per gross. This variety of temporary marker does not appear in any later merchandise catalog available for comparisons during this analysis until it reappears in the Hydrol Chemical Company catalog in 


\section{TEMPORARY GRAVE MARKERS}

NORMAN SERIES includes six individual markers; each is constructed from heavy-duty galvanized steel with baked enamel finishes available in gray, green, black, brown and orchid to ensure long-lasting and rust-resistant use. Five of the markers are designed for upright use and are available in Crown, Royal and Regent frame styles. Crown and Royal styles have flat-surfaced faces and come mounted on either angled or straight stakes. Regent style has a convex-surfaced face and comes mounted on a straight stake only. The Ground Level marker is designed exclusively for ground level use and is available in only one frame style. All markers, except the Regent, include an unbreakable clear acrylic pane, the pane on the Ground Level marker is slightly larger. All markers include $51^{\prime \prime} \times 26^{\prime \prime}$ heavy paper stock decedent information cards with space for name, year of birth, year of death and age. Optional aluminum stock decedent information cards may be substituted for an additional charge. Writing on both types of decedent information cards should be done with an indelible marker. All markers include waterproof poly-envelopes with fold-over flaps that provide additional weather protection for the information cards; poly-envelopes for the Regient are extra thick since there is no acrylic pane. The frames and backplates are separate pieces that snap together to house the contents of the markers.

ORDERING: all markers and parts are packaged 25 per style per single color; orders must be in multiples of $\mathbf{2 5}$ markers per style per single color. Specify color choice for markers and standard heavy paper stock or optional aluminum stock for decedent information cards.

\section{ACCESSORIES \&}

REPLACEMENTS:

A. GROUND LEVEL STYLE. Frame is flat-surfaced and $6 \%$ $\times 43^{*}$; opening is $41^{\prime \prime}$ $\times 2 \%$; three spikes are 4"L; overall

height when installed is 1 . 8409-3

Pack of 100 Bristol Information Cards 3176-3

Pack of 100 Aluminum Stock Information Cards 3174-7

Pack of 100 Clear Acrylic Panes for Crown and Royal Styles

3177-1

Pack of 100 Clear Acrylic Panes for Ground Level Style 3178-6

Pack of 100 Protective

Poly-Envelopes for Crown, Royal \& Ground Level Styles 3179-8

Pack of 100

Protective

Poly-Envelopes for Regent Style 3180-1
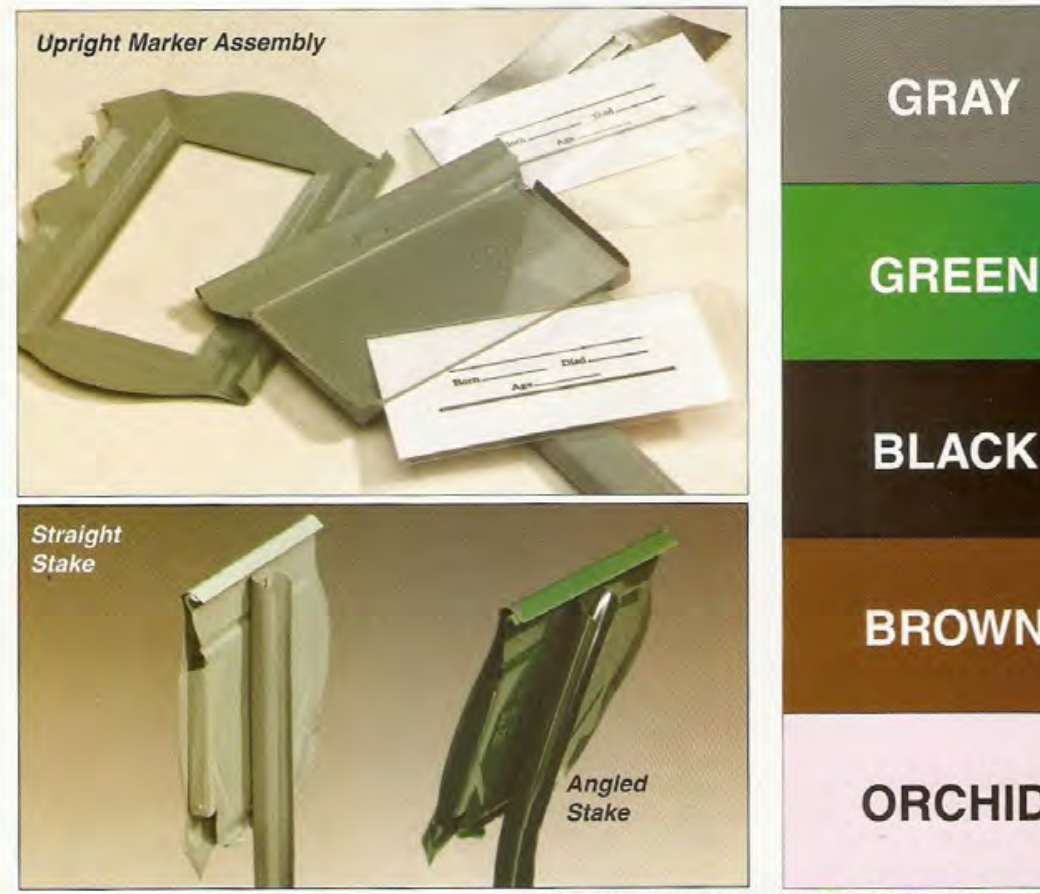

BLACK

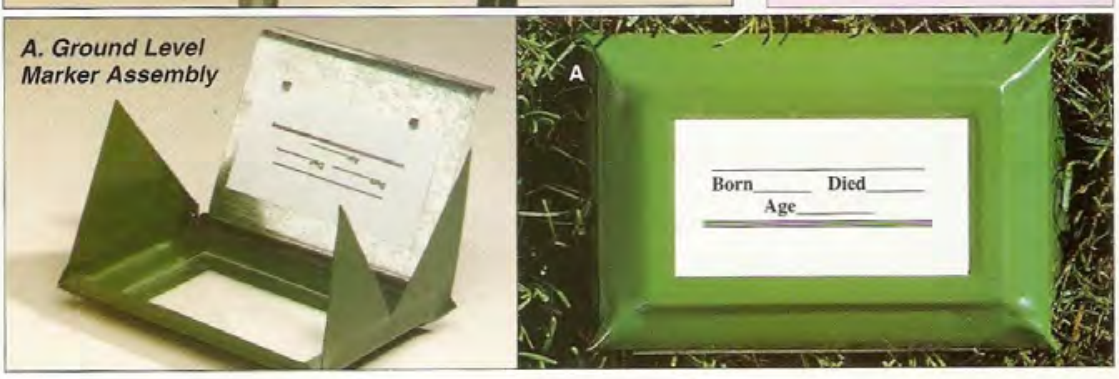

(K

DIRECTORY C172

Figure 50. Norman Series Temporary Grave Markers on page C172 of the 2002a Kelco Supply Company catalog. 


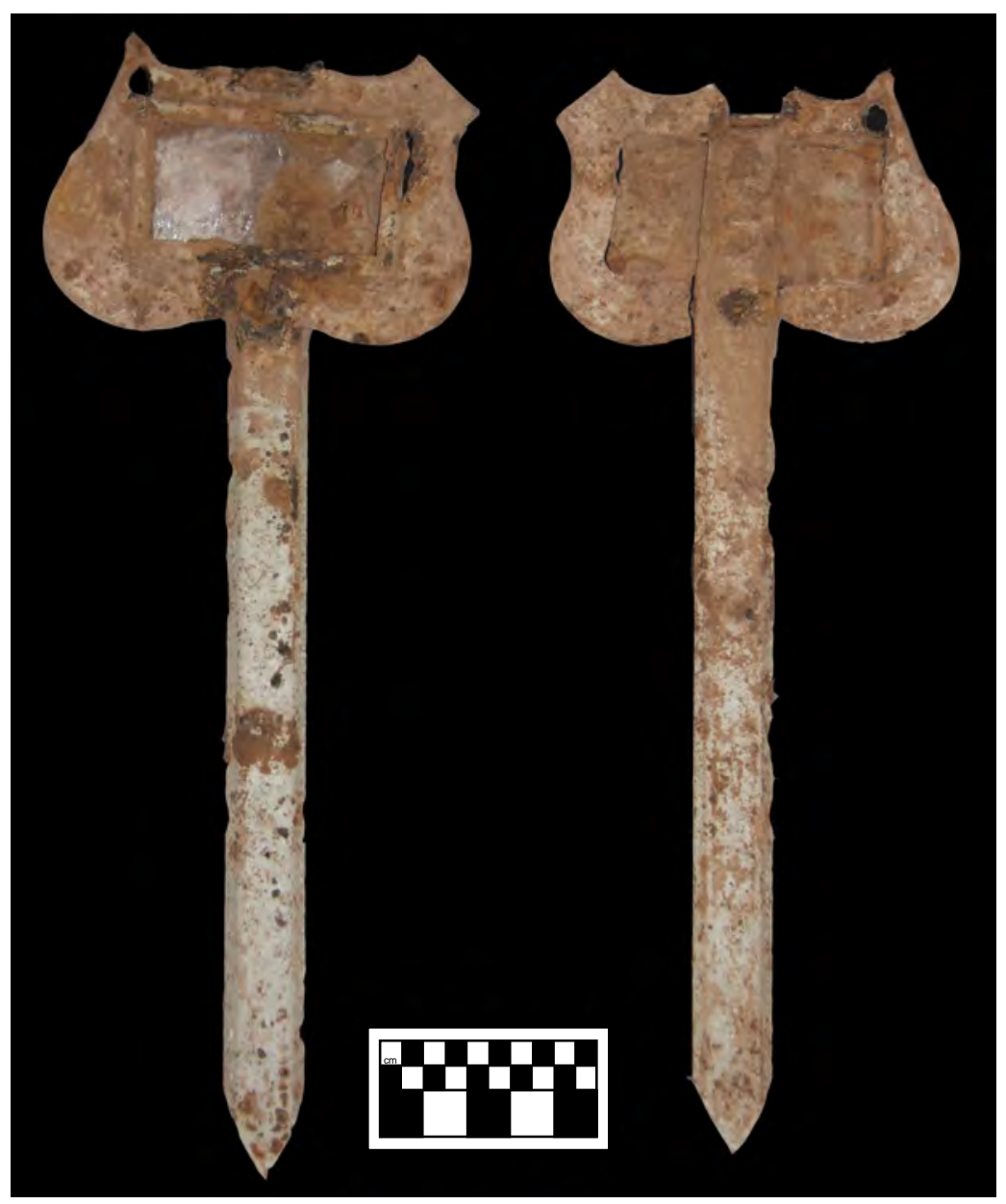

Figure 51. Marker Type 2.

2010 (see Appendix D). It is unclear why this marker seemingly disappeared from the market for this period, but it may have persisted on the fringes of the market, or might have been resurrected. Although the shield design is very similar to W. F. Norman's “Crown Style” (see Figure 49), it is possible that slight differences in design made the Norman markers more reliable and therefore more sustainable.

\section{Marker Type 3}

Marker Type 3 (Figure 55) is a temporary grave marker represented by at least two artifacts recovered from New Home Cemetery, one in the backfill of Burial 5, and the other collected from an unknown location on the surface (see Appendix A). The marker is made of stamped aluminum and consists of a pointed stake to which is affixed by welding or riveting a rectangular backing plate with clipped corners. Secured by these same attachments on the front of the backing plate is a holding plate, the edges of which are rolled inward in order to accept an impressionable aluminum ID card upon which personal information about the deceased could be inscribed. 


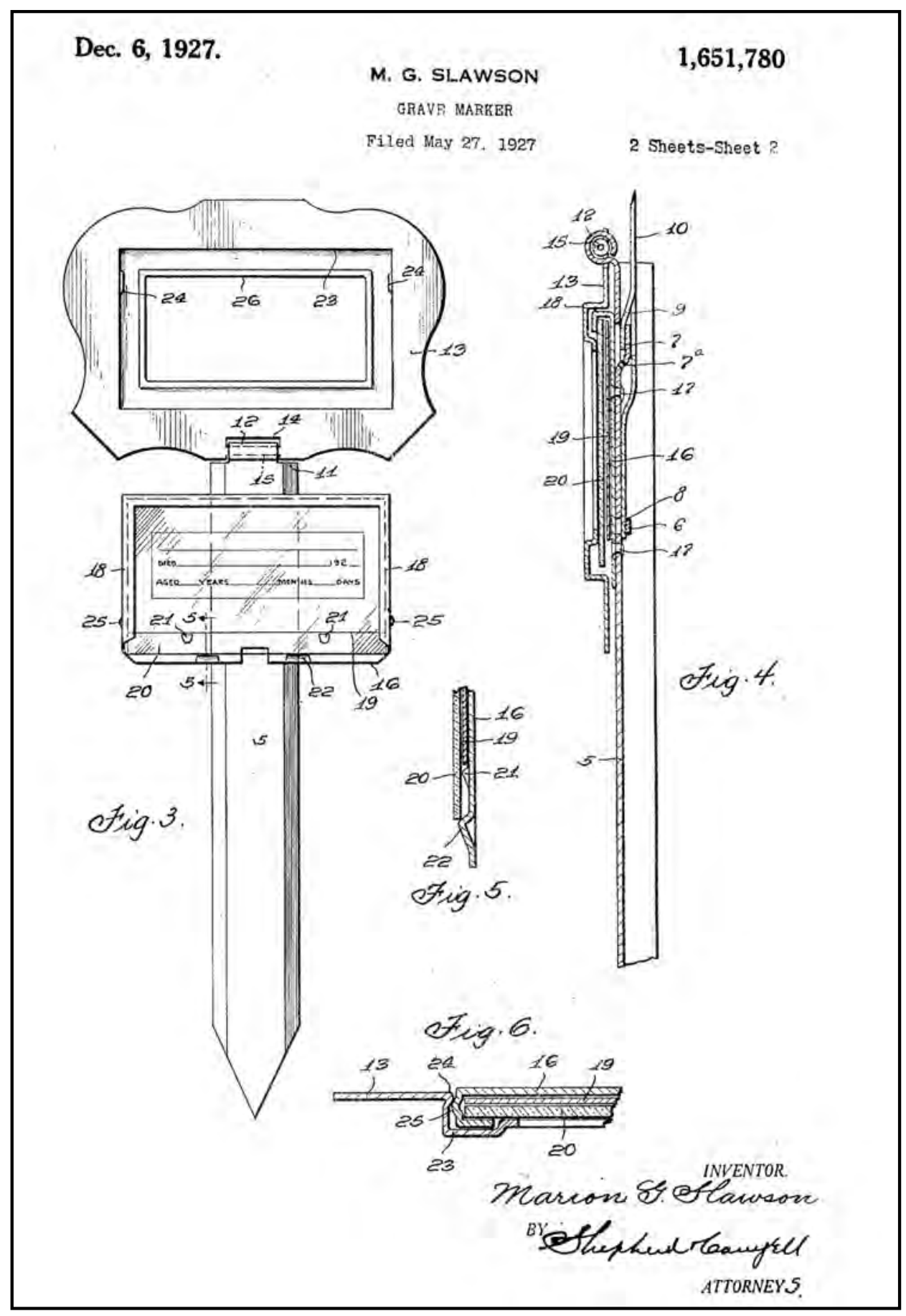

Figure 52. U.S. Utility Patent No. 1,651,780 asigned in 1927 to Marion G. Slawson for a grave marker. 


\section{PERFECTION GRAVE MARKERS}
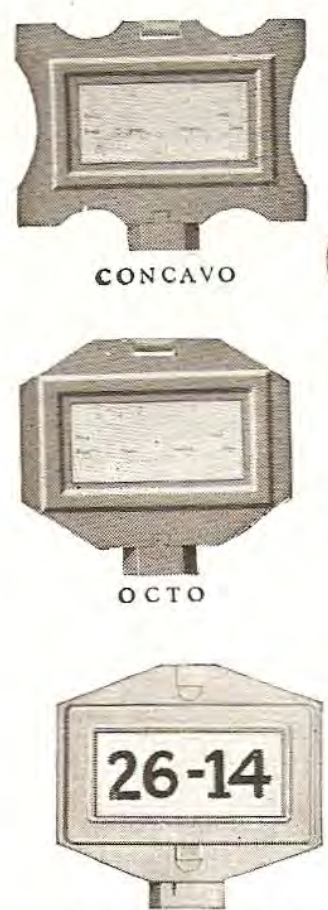

Lot Marker

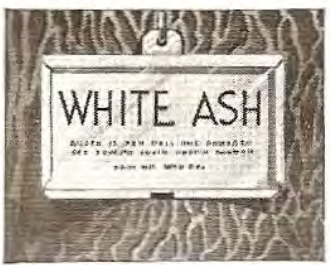

Tree Marker

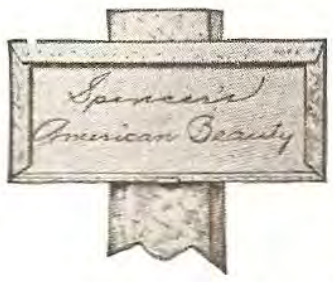

Nursery Row or Bed Marker

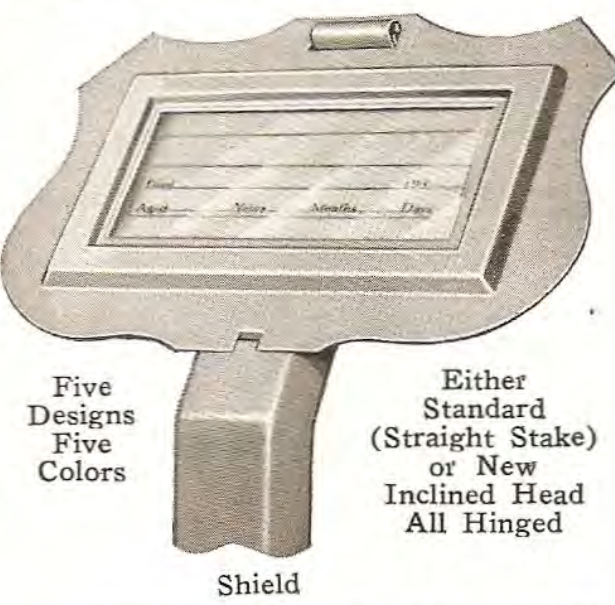

PERFECTION GRAVE AND LOT MARKERS are made of heavy gauge galvanized metal. Lacquer finished in durable colors. Printed Cards with space for name, date of death, age, etc., also bottle of non-fadeable ink with each order. No water can touch card. Packed three dozen in case.

Choice of various shapes and colors. CD3901-Black Enamel Markers

...Per Gross $\$ 40.00$ CD 3902 - Gray, White, Purple

and Orchid ......Per Gross 42.00 CD3903-Cross Extension, any

color ............... Ger Gross 18.00 CD3904-Inclined Heads......

Per Gross 45.00

Lot Markers may also be used for marking lots under Special Care, Annual Care or any other marking.

Less than 3 doz. lots add $25 \mathrm{c}$ per dozen.

Perfection Plant and Nursery Stakes Mark various plants in your Greenhouses and Nursery, clearly and neatly for benefit of prospective buyers.

These Stakes are made from heavy Tight-Coat galvanized sheet steel. Will last for years and may be used over and over again.

Cards protected by glass face.

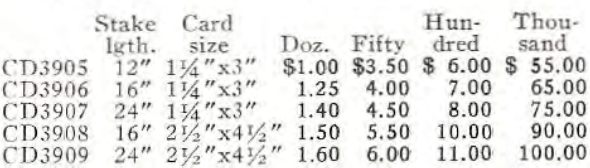

F. O. B. Chicago or Kansas Factory
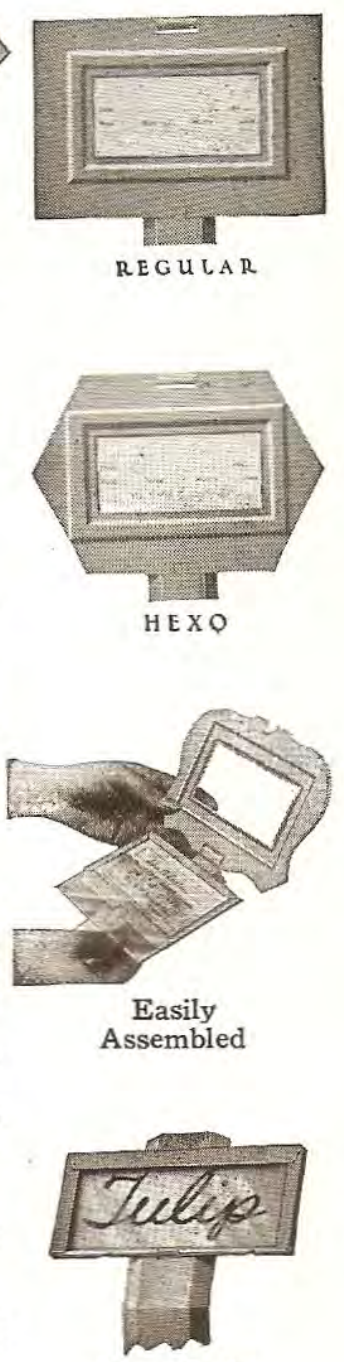

Plant Stake

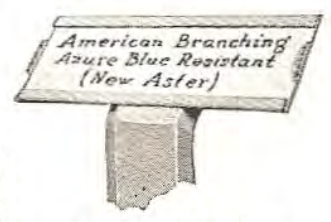

Garden Marker

\section{IF WE HAVE NOT LISTED YOUR NEEDS, WRITE US PLEASE}

Figure 53. Shield-shaped “Perfection Grave Marker,” which matches Marker Type 2, depicted on page 39 of the 1934 J. Oliver Johnson, Inc., catalog. 


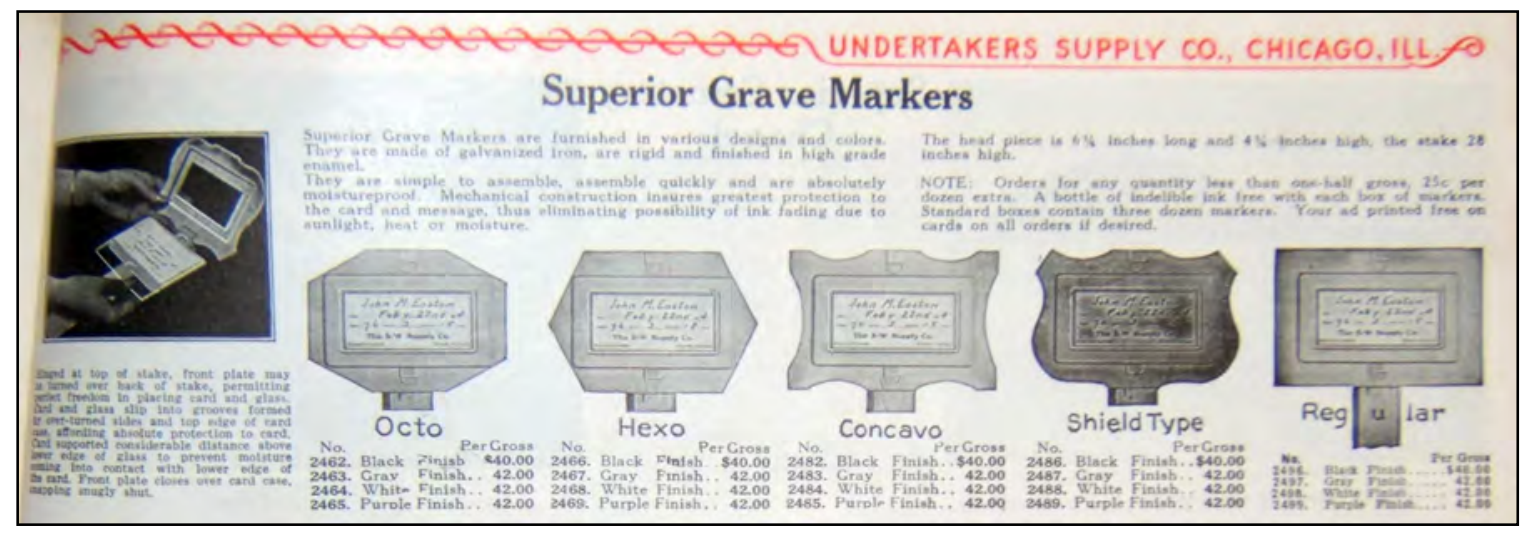

Figure 54. Shield-shaped temporary grave marker, which matches Marker Type 2, depicted on page 163 of a $1930 \mathrm{~s}$ Undertakers' Supply Company catalog.

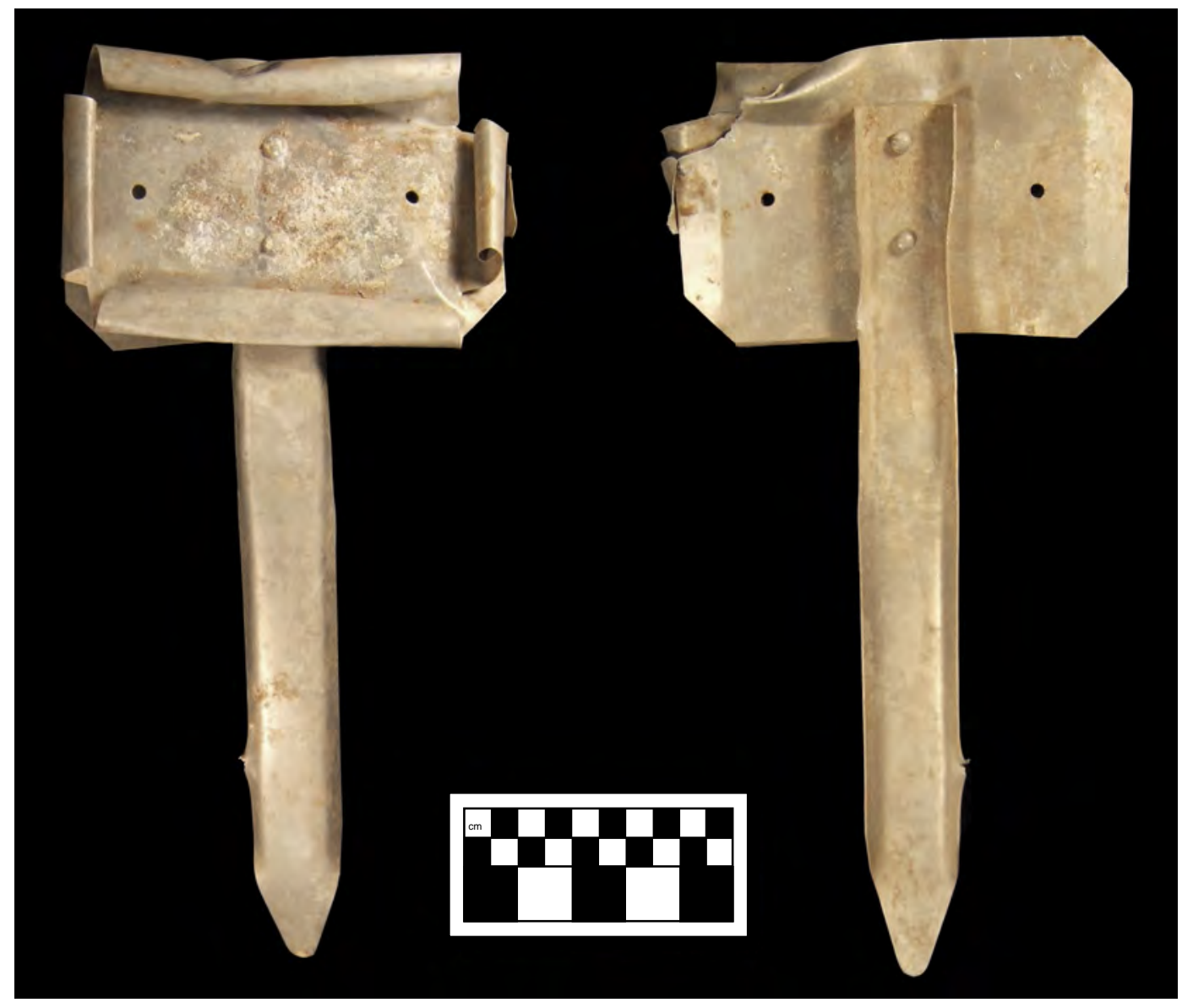

Figure 55. Marker Type 3. 
In 1935, Edward Sisson and Jeffrey Stokes, of Dallas, Texas, were issued a patent (U.S. Utility Patent No. 1997087) for an improvement in grave markers whose objective was first to create an inscription card that would not be "subject to fading, rusting, deterioration, washing off of inks and prints or obliterating by exposure to sun and storm," and second to create a frame that could easily be manipulated to accept the identification cards with no tools and little effort.

Marker Type 3 at New Home Cemetery represents the modern form and is an improvement on the original patent. This second patent (U.S. Utility Patent No. 2083873) (Figure 56) was awarded to Sisson and Stokes in 1937 (though the application was filed in 1934), and the marker became known commonly as a "Sisto Marker." As the patent illustrates and discusses, the identification card is produced by layering a sheet of cardboard and blotting paper, around which is wrapped a thin layer of tin or aluminum. The blotting paper is supposed to provide a cushion that will permit impressions to be made in the metal foil.

There was one example of this impressionable identification card in the New Home Cemetery collection associated with the Sisto marker recovered from Burial 5 (Figure 57). In addition to providing the above patent information, the identification card also discloses that Burial 5 likely was that of Leah Simpson who was born on October 10, 1883, and died on May 17, 1954. This marker was placed by the Fort Bend Fraternal Undertaking Company and would have been originally purchased by Fort Bend Fraternal Undertaking Company from the Mortuary Supply Company of Dayton, Ohio.

The Sisto markers quickly hit the market either immediately after the granting of the patent, or possibly even before through the parent company Sisto Mfg. Company of Dallas, Texas. Sisto markers appeared in four known merchandise catalogs ranging in date from the 1930s to 2010, and they are still produced today (see Appendix D). They are included in one 1930s catalog from the Undertakers' Supply Company (1930s b) (Figure 58) with the advertising propaganda, "No glass to break, no ink to smear." Compared with Markers 1 and 2 in the same time period, the Sisto marker would have been very expensive. Undertakers' Supply Company (1930s b) was selling a gross of Sisto markers for $\$ 85.50$ in the 1930s catalog, and the price increased to $\$ 89.90$ by the time of their appearance in the 1950s Royal Bond catalog (Figure 59). Figure 60 shows that in 2002 the Kelco Supply Company (2002b) was offering Sisto markers for \$47.35 per dozen.

\section{Marker Type 4}

Marker Type 4 (Figure 61) is a temporary grave marker represented by one artifact recovered from Burial 11 at New Home Cemetery (see Appendix A). The marker is made of stamped aluminum and the only portion present is the backing plate. The plate has three vertically oriented slots along each side made to accept three tab-in-slot character strips allowing for the decedent's personal information to be written out in interchangeable individual letters. There are also two round holes along the midline where it would have been affixed to an aluminum stake for insertion into the ground. 


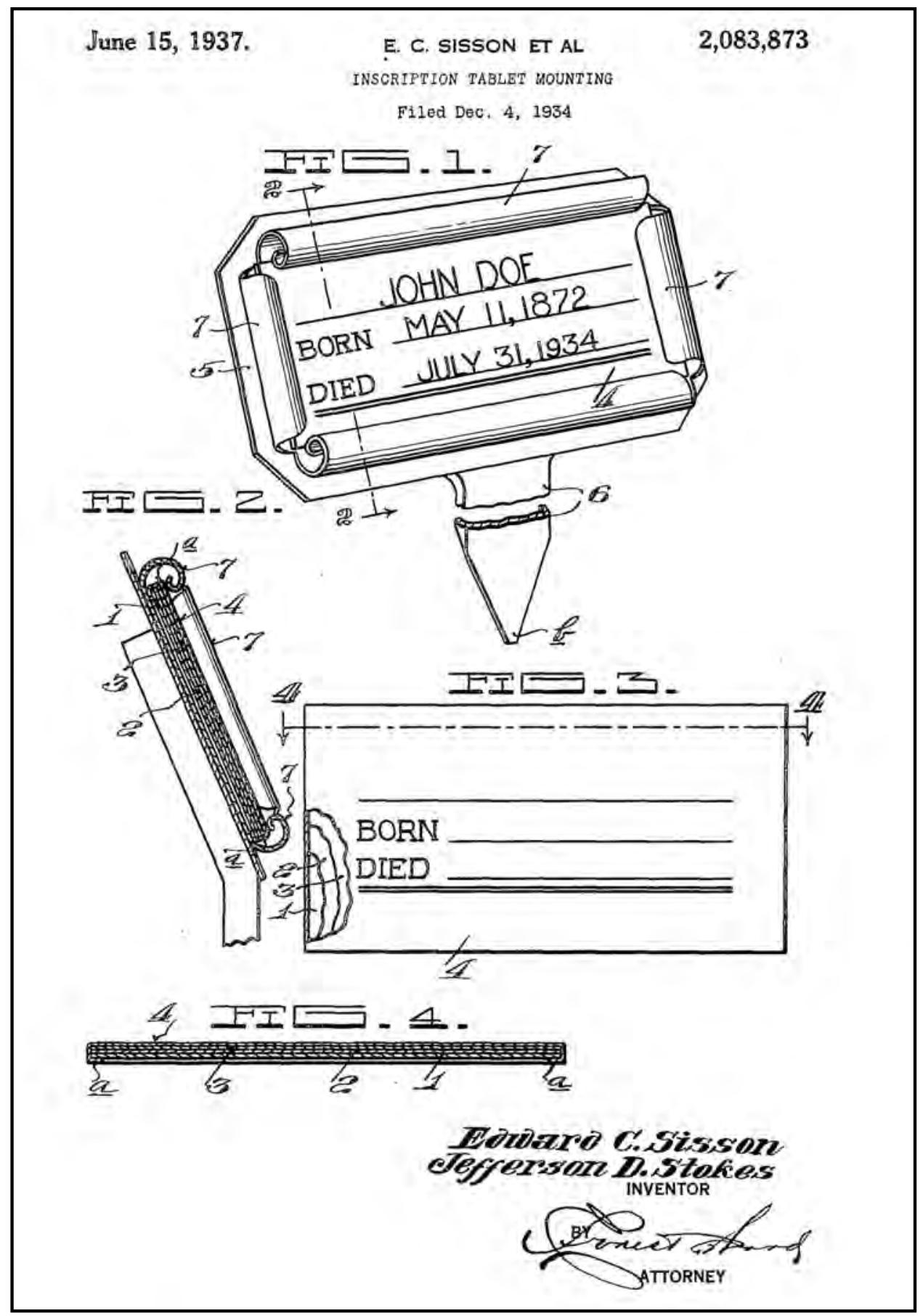

Figure 56. U.S. Utility Patent No. 2,083,873 assigned in 1937 to Edward C. Sisson et al. for an inscription tablet and temporary marker. 


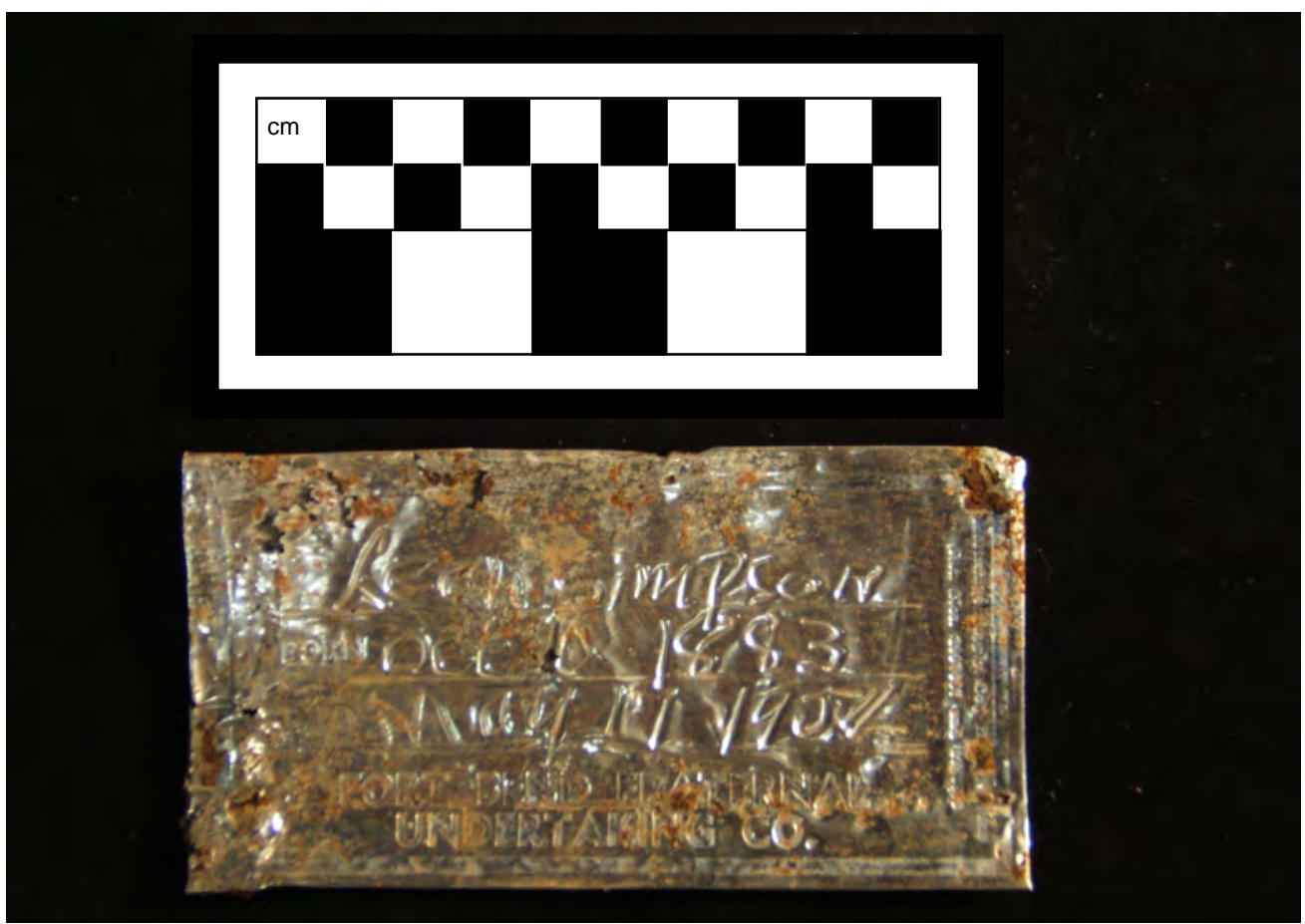

Figure 57. Aluminum inscription plate for “Leah Simpson”(Burial 5) associated with Marker Type 3.

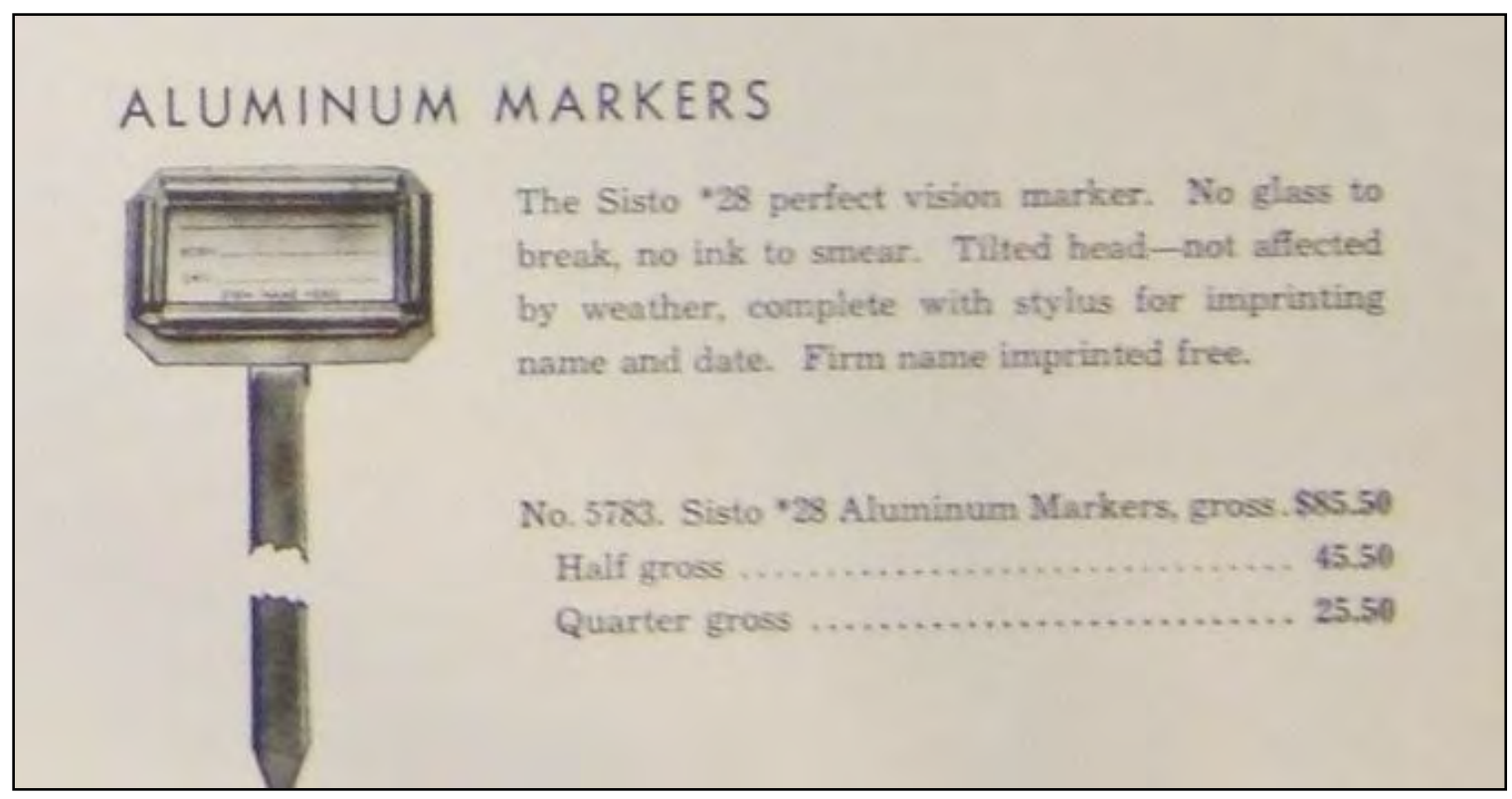

Figure 58. Sisto Aluminum Marker No. 5783, which matches Marker Type 3, depicted on page 91 of the ca. 1930s Undertakers' Supply Company catalog. 


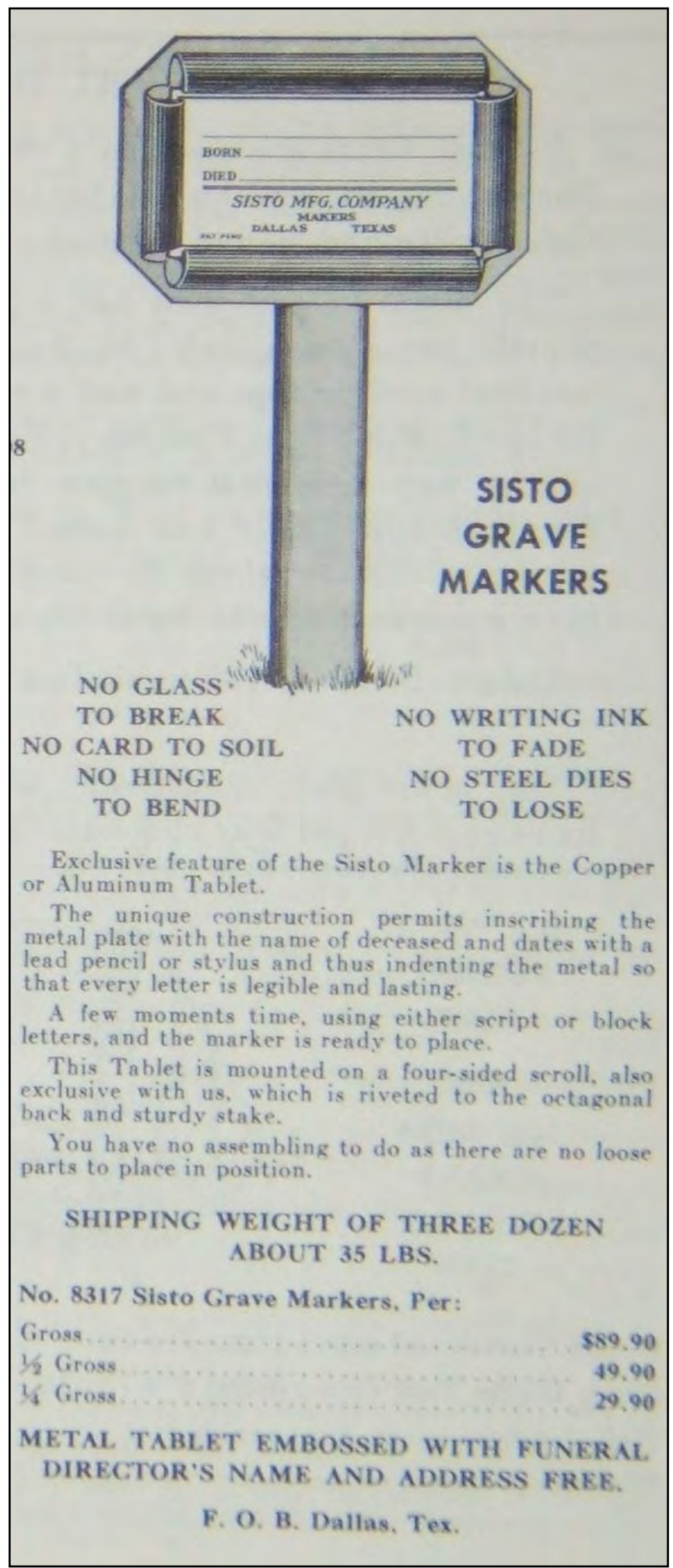

Figure 59. Sisto Grave Marker No. 8317, which matches Marker Type 3, depicted on page 168 of the ca. 1950 Royal Bond, Inc., catalog. 


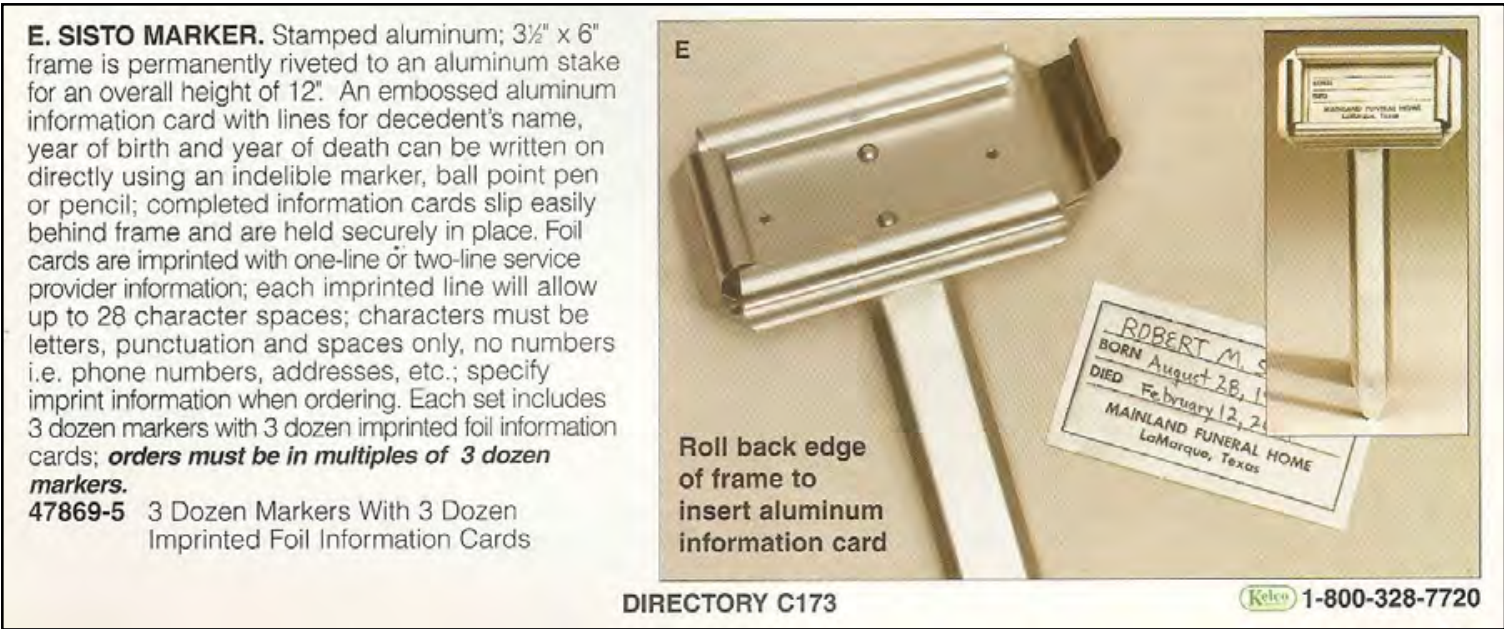

Figure 60. Sisto Marker No. 47869-5, which matches Marker Type 3, depicted on page C173 of the 2002a Kelco Supply Company catalog.

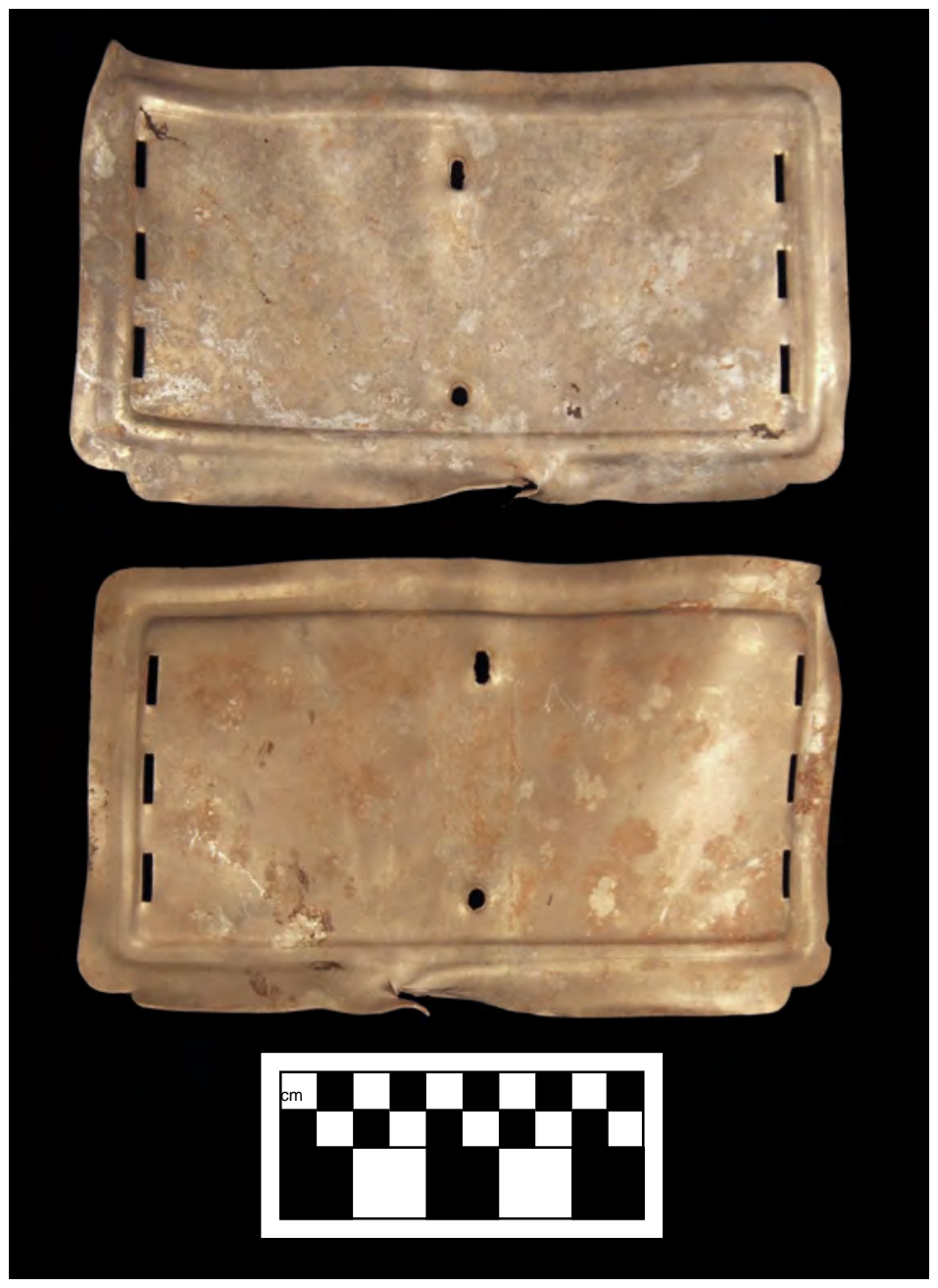

Figure 61. Marker Type 4-backing plate. 
No exact patent matches were located for this specific marker, but the first temporary marker with interchangeable lettering (U.S Utility Patent No. 1504565) was patented in 1924 to John M. McNeill of St. Joseph, Missouri. This first invention was nothing more than a triangular stake with a rounded top. The face of the stake contained a rectangular area with ridges upon which could be mounted the individual letters. An elaboration on McNeill's original design was patented in 1926 (U.S. Utility Patent No. 1589199) (Figure 62). Although not an exact match to New Home Cemetery Marker Type 4, the 1926 McNeill marker is clearly a close precursor.

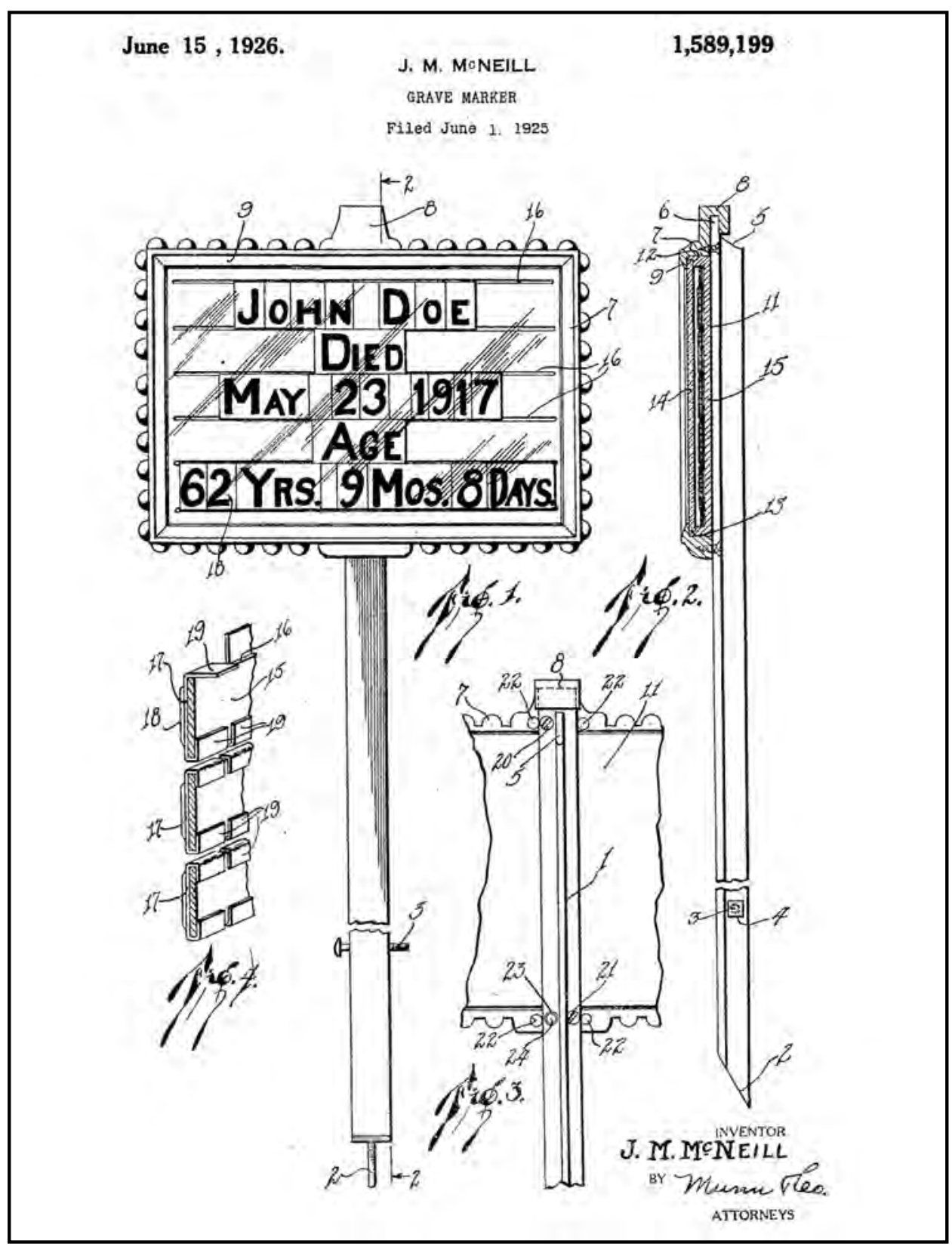

Figure 62. U.S. Utility Patent No. 1,589,199 assigned in 1926 to J. M. McNeill for a grave marker. 
It is not known for sure when Marker Type 4 entered the market, but it was definitely after the 1926 patent and possibly as late as the 1940s. No catalogs from the 1930s advertise this type of marker (see Appendix D). It does appear, however, as the "McNeill Grave Marker" in the 1950s Royal Bond, Inc., catalog at $\$ 39.60$ for three dozen markers and a box of 4,000 letters and numbers (Figure 63). It was sold simply as “Aluminum Grave Marker” for \$58.20 for three dozen in the 1970s Tri-State Industries catalog, and for $\$ 58.40$ per dozen in the 2002 Kelco Supply Company (2002b) catalog (Figure 64). It is still produced today; its most recent appearance is in the 2010 Hydrol Chemical Company catalog (see Appendix D).

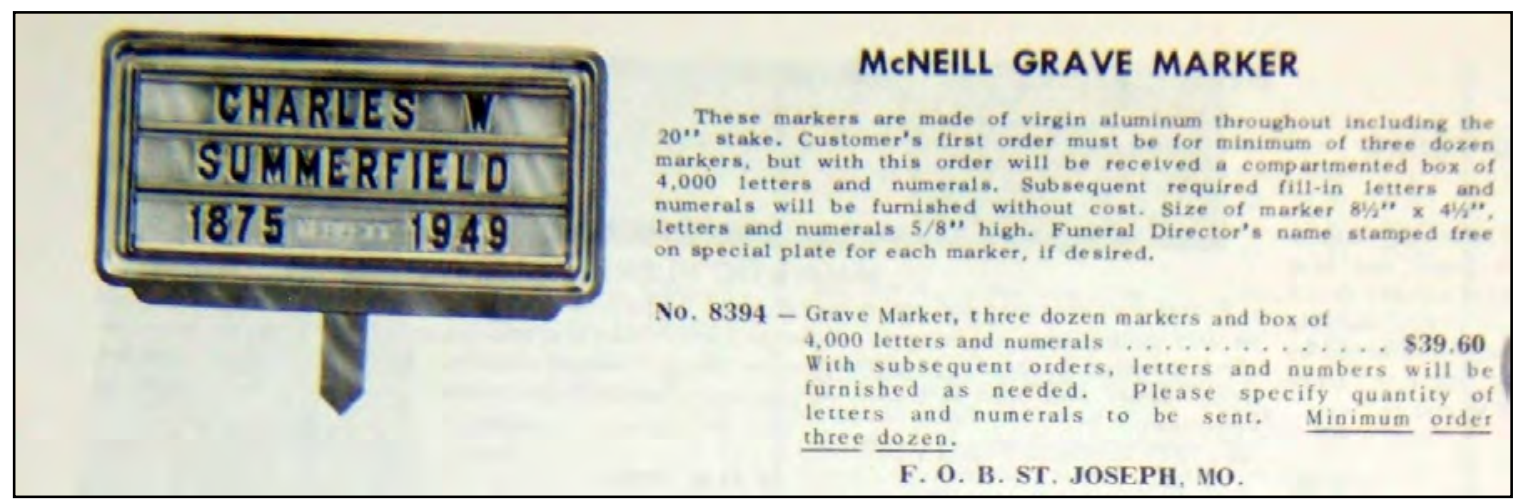

Figure 63. “McNeill Grave Marker” No. 8394, which matches Marker Type 4, depicted on page 169 of the ca. 1950 Royal Bond, Inc., catalog.

\section{Marker Type 5}

Marker Type 5 (Figure 65) represents one temporary or permanent grave marker that was recovered during surface scraping at New Home Cemetery. It is a fragment of a PVC pipe cross consisting of a 4-way pipe fitting and two short lengths of piping. Other PVC pipe crosses were noted in the undisturbed section of the cemetery (Figure 66).

PVC (polyvinyl chloride) was discovered as early as 1835, but it was not until Waldo Semon, a chemist working with the BF Goodrich Corporation, reinvented a plasticized PVC as an alternative to natural rubber (Walker 1990). Semon's 1934 U.S. Utility Patent No. 1983949 describes a method by which various additives could be combined with PVC to create a more rigid product that could be extruded to develop various products such as piping. Though there were uses of this early PVC pipe in Germany in the 1930s and 1940s, it was not until subsequent advances in technology in 1950 and the granting of 1953 U.S. Utility Patent No. 2646417 to Garland Jennings that modern PVC pipes were introduced into the market.

The 4-way fitting used in the construction of Marker Type 5 was produced by NIBCO, an extant plumbing supply company that has been in business for more than 105 years. Based on the historical information presented here, the PVC cross marker must have been placed at New Home Cemetery sometime after 1950, but more likely sometime after 1960. 


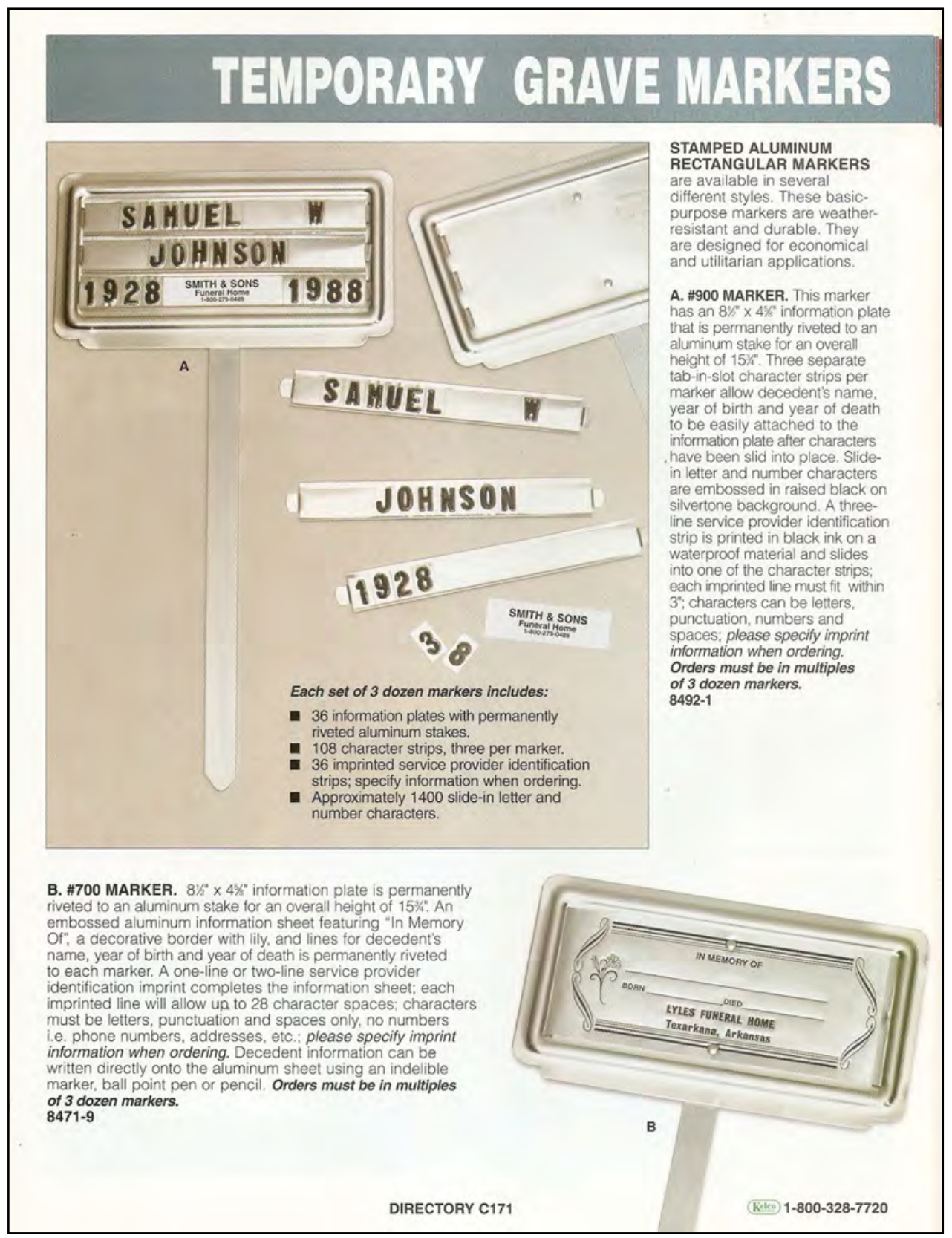

Figure 64. Slotted temporary grave marker No. 900, which matches Marker Type 4, depicted on page C171 of the 2002a Kelco Supply Company catalog. 


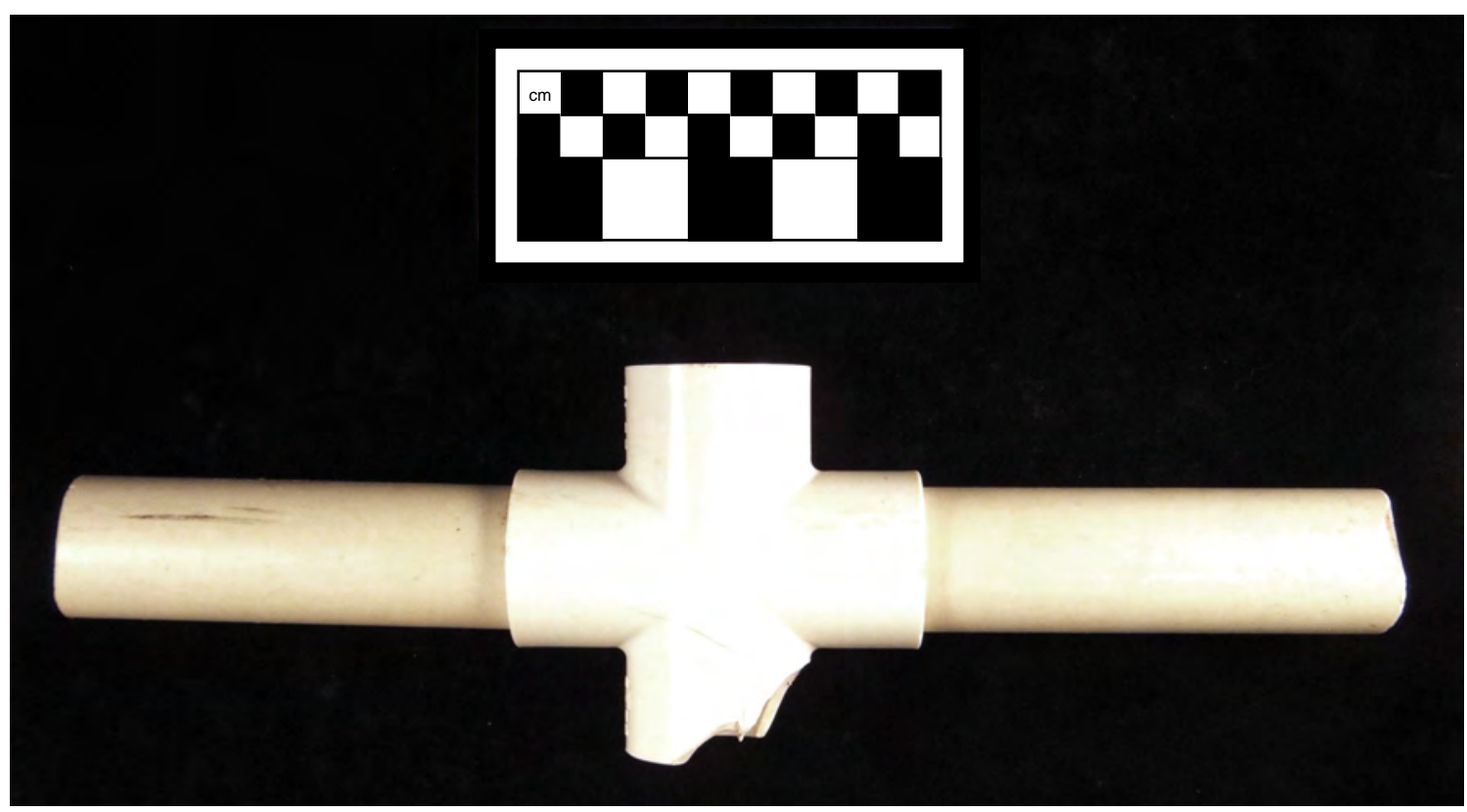

Figure 65. Marker Type 5-PVC marker.

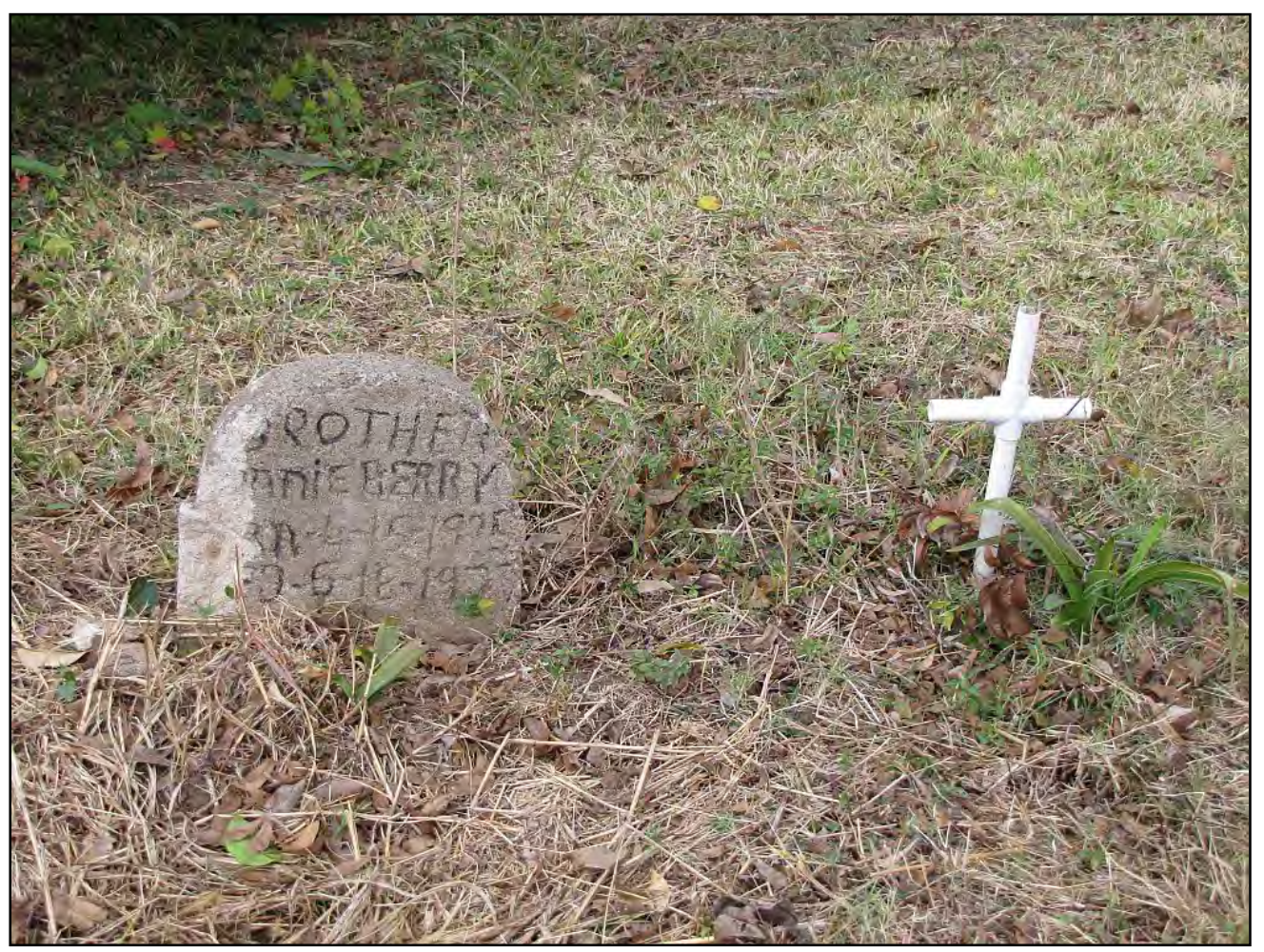

Figure 66. Field photo showing PVC grave marker in situ in an undisturbed area of New Home Cemetery. 


\section{Burial Containers}

In the archaeological literature discussing historic cemeteries, the terms "coffin" and "casket" have mistakenly been used interchangeably. The Columbia Guide to Standard American English states that the term "coffin" is generic, with "casket" being used as a euphemism for "coffin" for longer than a century in the United States (Oster et al. 2005:161). Nonetheless, these terms actually refer to different styles of burial containers that also reflect different cultural and historical meanings. Popular literature and even period mortuary catalogs sometimes propagate this confusion about the terms through their ambiguous use (Pye 2010a).

Although a variety of forms did exist between the sixteenth and nineteenth centuries, coffins were generally hexagonal in silhouette with the widest point at the shoulders, tapering to both the head and the foot ends. Within the archaeological literature, hexagonal coffins have been referred to as logical text such as "toe-pincher" (Burnston and Thomas 1981:II-5; Parrington et al. 1989:144; Trinkley and Hacker-Norton 1984:4), "pigeon-toed coffin” (Blakely and Beck 1982:188), "truncated diamond coffin" (McReynolds 1981:15), as well as the "shouldered coffin" (LeeDecker et al. 1995:50). Mainfort and Davidson (2006:104) observe that the hexagonal shape imitates the idealized human form. A close relationship between the coffin and the shape of the body is not surprising because the explicit purpose of the coffin was to act as a protective encasement for the deceased (Lang 1984:30).

The casket is a rectangular or parallel-sided container, which developed in the late nineteenth century and represents an important change in terminology and construction. Rectangular burial containers were already in use prior to the nineteenth century (Koch 1983) for all demographics, and primarily for the interment of infants and young children in the early nineteenth century (Bybee 2002; Davidson 1999). The shift to the term "casket," however, reflects a change in ideology as it pertains to death and burial in the United States. The term "casket," which is of French derivation, refers to a box used to hold and showcase valuables (Farrell 1980:10; Habenstein and Lamers 1985:168; Lang 1984:31). Tharp (1996:199) interpreted its use as a term for burial receptacle to mean that the container "held not an ugly corpse, but the valuable remains of a loved one and the mourners experienced a 'beautiful' death." Thus, the burial container evolved from a coffin meant to enclose the body to a casket whose intent was presentation (Lang 1984:31).

An attempt has been made by some scholars to use burial container shape as a temporal diagnostic. This is a problematic approach because the rectangular casket was introduced at different points in time in different regions throughout the country (Mainfort and Davidson 2006). This was not just because of production and distribution concerns, but also due to cultural aversion to changes in the burial container. The presence of hexagonal coffins in trade catalogs indicates that a persistent niche market existed for these containers even into the 1920s (Mainfort and Davidson (2006:109-110).

Most of the graves encountered during excavations at New Home Cemetery contained rectangular, wooden caskets. Burial 16 was the only example of a hexagonal coffin present on site. Even though burial container shape alone is not a good temporal indicator because of the reasons mentioned above, the presence of a hexagonal coffin suggests that this burial, at least, dates to the 1920s at the latest. Notably, five burials (Coffins 1, 2, 3, 4, and Burial 4) at New Home Cemetery contained metallic caskets. Because no concerted effort has been made in the available archaeological literature to describe metallic caskets, some effort is expended here in that task. 


\section{Metallic Caskets}

Metallic caskets are specifically mentioned in period mortuary catalogs as early as the 1858 Crane, Breed \& Company catalog. It was not until the 1880s, however, that their inclusion became more commonplace, and by 1950, metallic caskets had taken over the funeral industry, nearly supplanting all other types. Because they are an extremely important icon of the modern funeral industry, it is curious why they have received no attention in the archaeological literature, even though they have occasionally been discovered during relocations. A brief introduction to the description of important features of metallic caskets is therefore warranted.

Figure 67 shows an image of a casket offered for sale by Thacker Caskets, Inc., of Clinton, Maryland. The image shows many of the most important components of metallic caskets, including lining terms, hardware terms, as well as terms for aspects of the casket itself. There are typically seven features of a metallic casket that are the major determining factors of casket type, which are also important factors in cost: (1) type of metal; (2) type of shell; (3) type of lid; (4) gasketed vs. nongasketed; (5) type of hardware; (6) finish; and (7) type of interior.

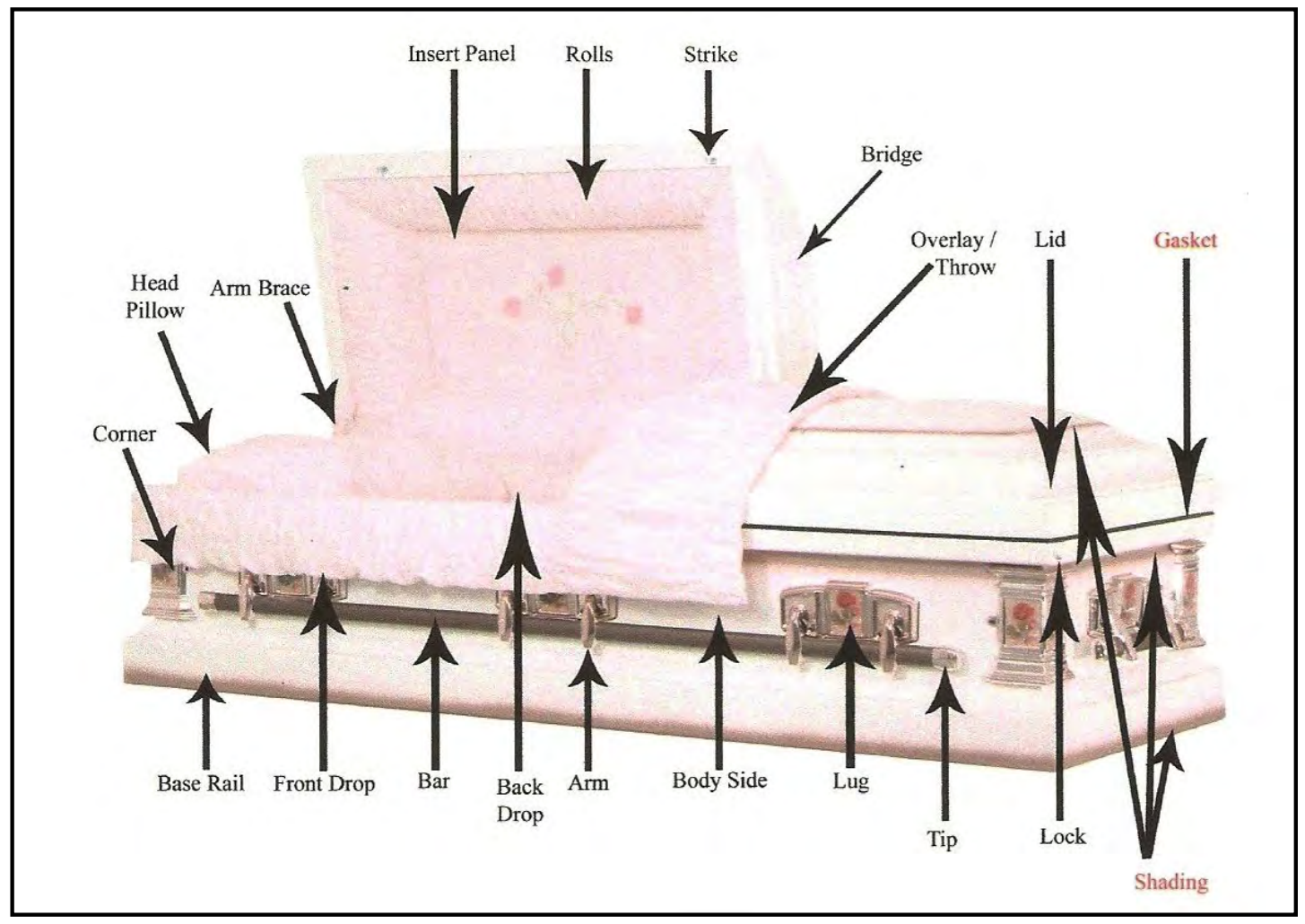

Figure 67. Identified portions of metal caskets (courtesy of Michael Beardsley, Thacker Casket, Inc.).

The metals most commonly used in casket construction are bronze, copper, stainless steel, as well as 16-gauge, 18-gauge, and 20-gauge steel. Presently, cold-rolled steel is the leading material used in the construction of caskets. Differences in steel caskets usually lie in the thickness of the metal used. Thickness is measured in gauge, where 20-gauge steel means that it would take 20 
steel sheets to create a stack 1 inch thick (Thacker Caskets, Inc., nd:1-2). The various types of metals used in construction of caskets have different strengths and can be formed into a variety of shell forms. Twenty-gauge steel, for instance, is the thinnest and can only be stamped into rectangular forms, while 16-gauge steel can be formed into urn-shaped ends such as that seen in Figure 68. Twenty-gauge steel caskets are typically cheaper than other varieties because less metal is used in their production (Thacker Caskets, Inc., n.d.:1-2).

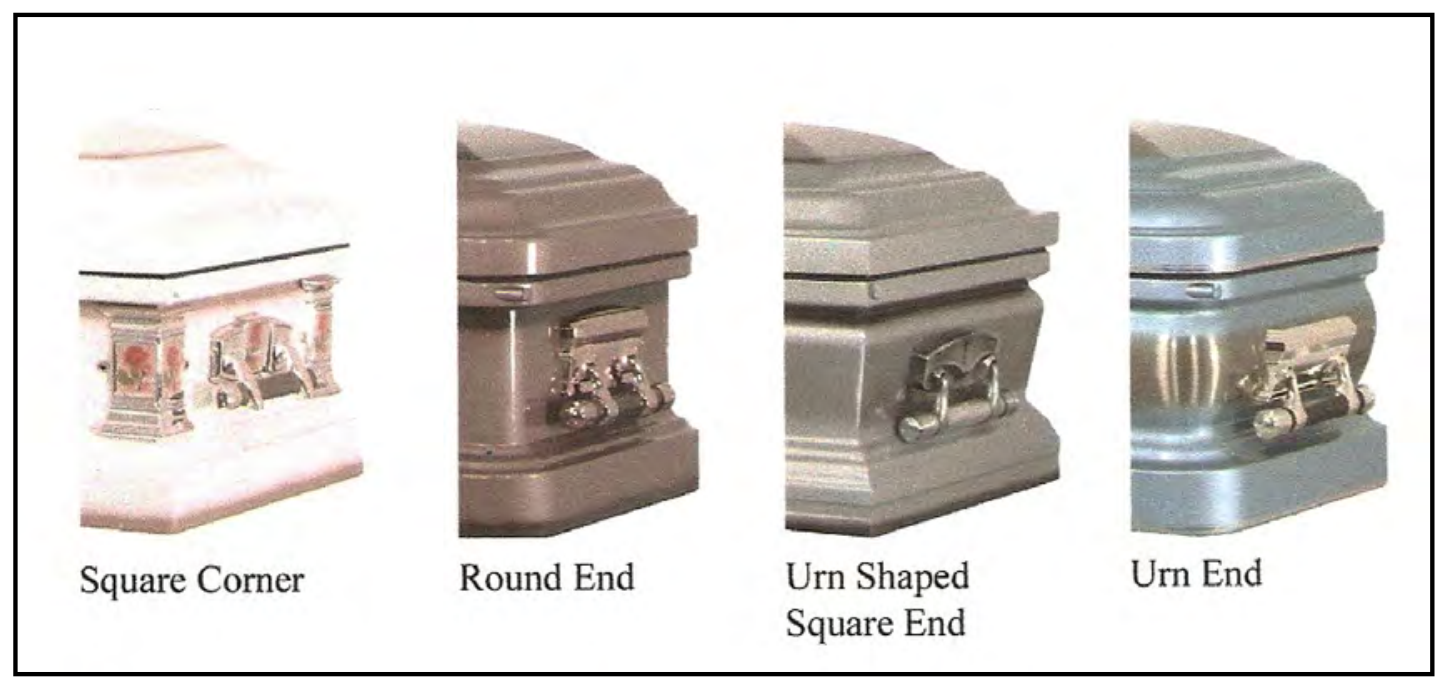

Figure 68. Examples of casket shell types from Thacker Casket, Inc., Training Module 4 (courtesty of Michael Beardsley, Thacker Casket, Inc.).

Other important features to consider while classifying caskets are the type of lid used and whether the caskets contain rubber gaskets and sealing/locking mechanisms or not (Figure 69). The two primary types of casket lid varieties are when the lid is one piece (in which case the casket would be called a "full couch") or when the lid over the lower half of the casket is separate from the lid over the upper half of the casket (in which case the casket would be called a "half couch"). Gaskets can be used with either lid type. Gasketed caskets are advertised as being "air-tight" and "water-proof" and claim to be the best option for protecting and preserving a deceased loved one for eternity. The truth of the matter is that gasketed caskets placed directly into a grave rarely hold up to the strain of being buried and the seals inevitably fail. It is possible for seals to hold if the casket is interred within a sturdy metal, concrete, or brick vault because the vault supports the weight of the soil, but unless the seal on the vault remains intact, water will still contact and possible corrode and enter the casket. Nongasketed caskets do not claim to deter the seepage of water into the caskets and are often more economical as a result.

External hardware also plays an important role in calculating cost of a metal casket. The most common hardware elements used on modern metal caskets are swing bar extension handles, corners, and stationary extension bars (Figure 70). There is great variation in materials used in the production of these hardware types, as well as in the forms and styles advertised in catalogs. External casket hardware will be discussed in greater detail later in this text. 


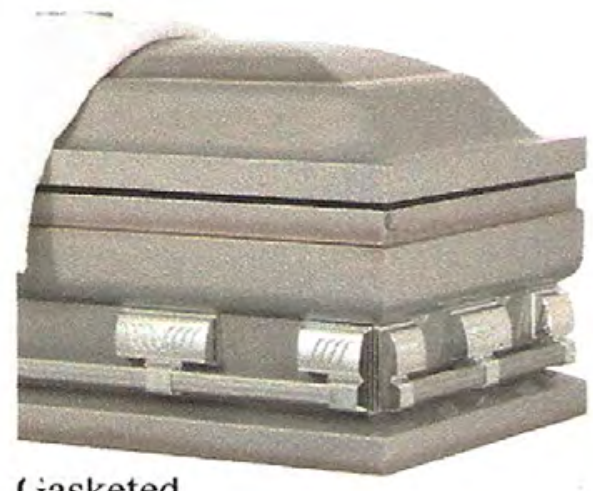

Gasketed

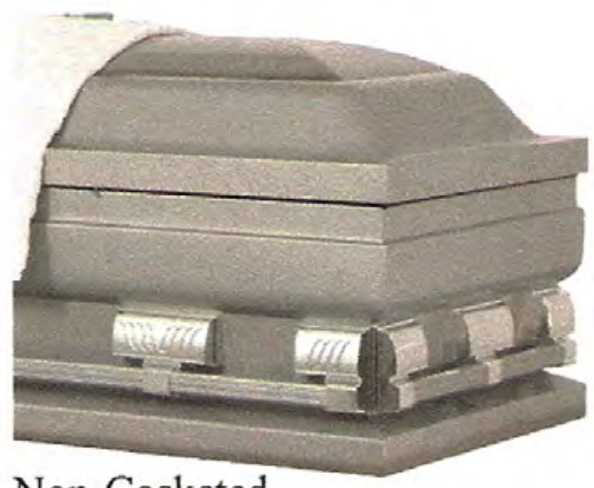

Non-Gasketed

Figure 69. Gasketed vs. nongasketed caskets from Thacker Casket, Inc., Training Module 4 (courtesty of Michael Beardsley, Thacker Casket, Inc.).

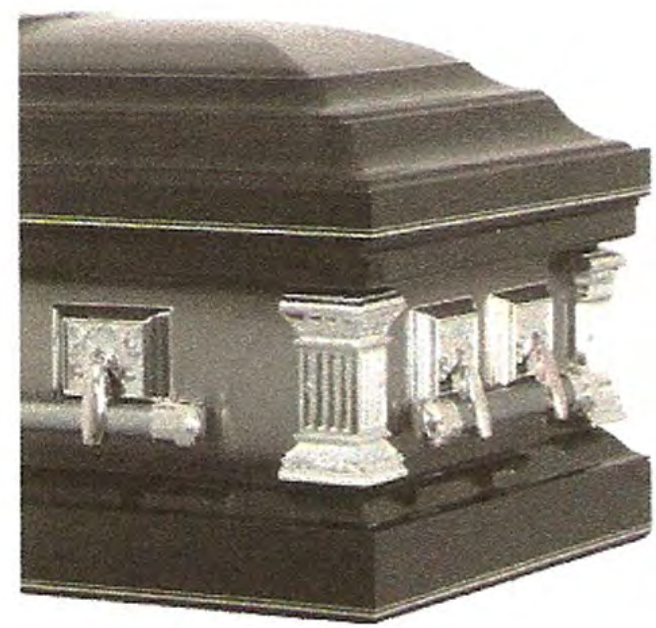

Swing Bar Hardware

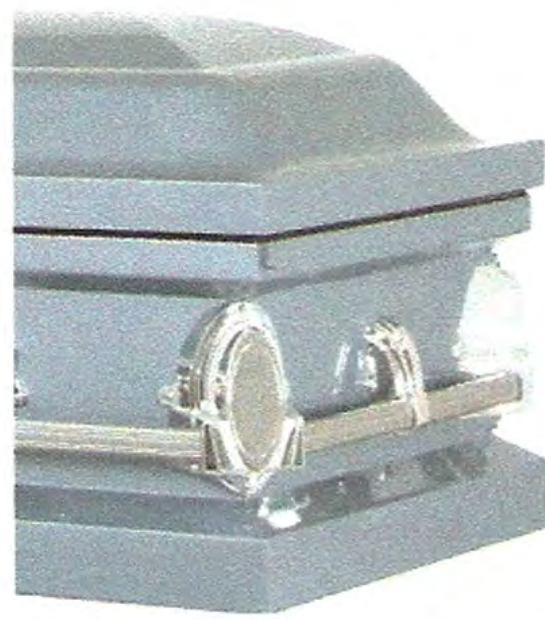

Stationary Bar Hardware

Figure 70. Examples of casket hardware types from Thacker Casket, Inc., Training Module 4 (courtesty of Michael Beardsley, Thacker Casket, Inc.).

Finish does not affect cost as much as some of the other features of a casket, but finish along with external hardware greatly affect presentation. Figure 71 shows three types of finishes offered currently by Thacker Caskets, Inc.: (1) brushed; (2) two-tone; and (3) shading. This is not an exhaustive list of finish types, but does give important examples. Caskets finished with a single, flat color tend to be less expensive than two-toned caskets. 


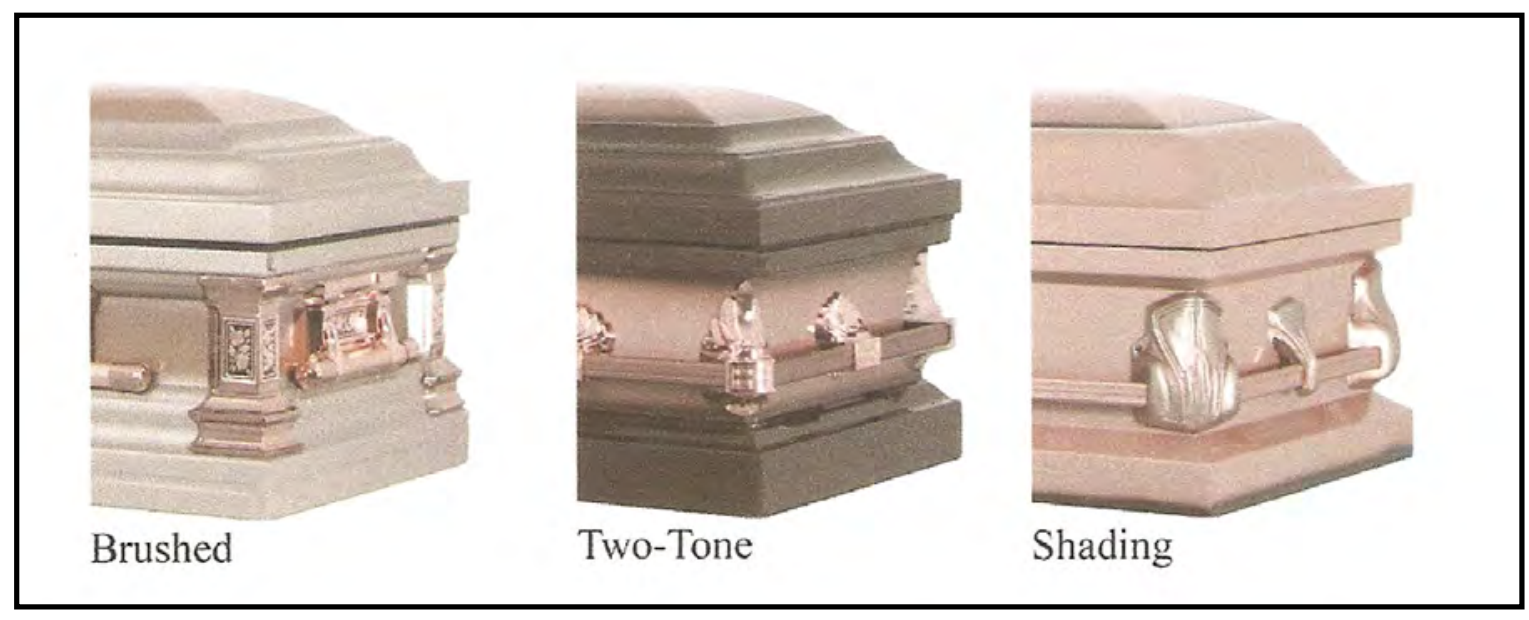

Figure 71. Examples of finish types from Thacker Casket, Inc., Training Module 4 (courtesty of Michael Beardsley, Thacker Casket, Inc.).

Finally, the interior treatment of the casket can shed light on quality of production and cost (see Pye 2010a, b for a more in-depth discussion of interior burial container treatments). The development of the funeral industry in the late nineteenth century capitalized on the ideological notion of death as a natural "sleep" by turning the burial container into a bed or couch-like vessel. Mattresses developed out of the layer of sawdust or other absorbent material upon which the head linings were placed (Janaway 1998:22). Linings could be produced from a variety of fabrics, which ran from very economical cotton weaves to more expensive silks (Pye 2010a, b). Various sorts of frills, laces, gimps, and other adornments were also used to decorate the interior of burial containers (Pye 2010a, b). Pillows were also often included in the burial container. Sets of linings could often be purchased from period catalogs that sometimes included mattresses, linings, and pillows of the same decorative style (Janaway 1998:22).

\section{Metal Casket Type 1}

As previously mentioned, Coffins $1,23,4$, and Burial 4 were features that contained metallic caskets of some type. Coffin 1 was damaged to the point that it was described as a crumpled mass of metal, and therefore its type could not be discerned. Field personnel noted that Burial 4 and Coffin 2 contained caskets with a stamped metal surface over wood, but no other details were provided to determine a type. Coffins 3 and 4, however, were mostly intact, were photographed in the field, and therefore it is these two identical caskets that make up Metal Casket Type 1 (see Appendix A). These caskets were not present in the laboratory for formal analysis so this description is based solely on field photographs and very brief notes made by the archaeological technicians.

The caskets were made of thin sheet steel, possibly 20-gauge, and were approximately 7 feet in length. The lids of the caskets were crushed inward, as were the side walls to a certain extent. The caskets have square corners, gently curved top moldings, and slightly domed platform lids with separate upper and lower halves with hinged support arms on each end (Figure 72). The lids of the casket exhibit a shiny pale blue finish. Evidence of rubber was noted along the casket body margin of Coffin 4 suggesting that a rubber gasket had been present. Lengths of foam (Miscellaneous Artifact Type 1) were found attached to the interior side walls of the casket, and 


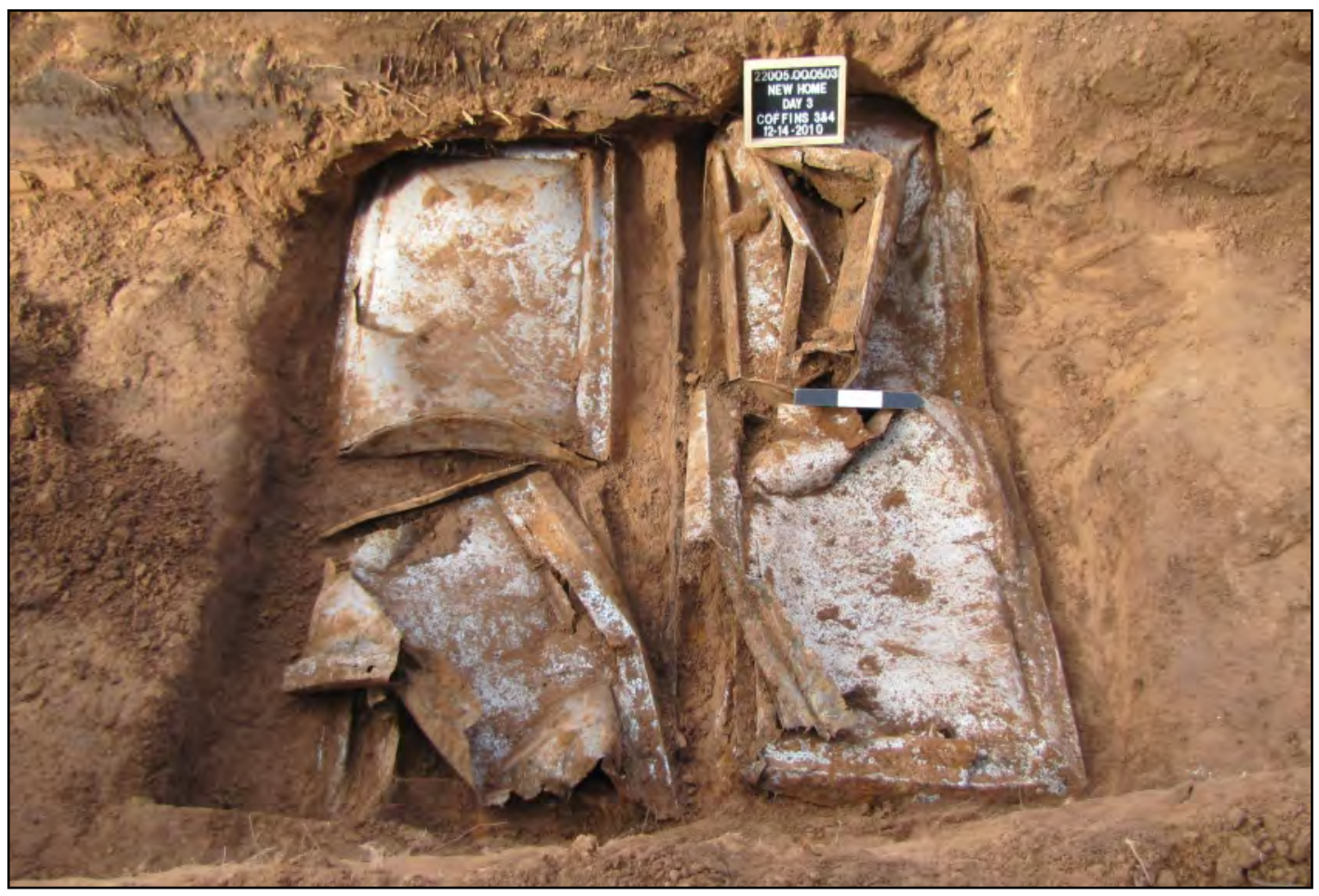

Figure 72. Field photo showing Coffins 3 and 4 (Metal Casket Type 1) after uncovering the casket lids.

netting (Miscellaneous Artifact Type 2) was attached to the upper margins and hung down along the side walls. Archaeologists stated that a polyester lining was present on the interior of the caskets as well. A field photograph (Figure 73) taken after removal of the mangled remains of Coffin 4 shows an archaeologist examining the casket remains, with a stationary handle (Handle Type 23) present on the casket fragment in the center background of the frame.

No definitive patent record, mortuary merchandise catalog, or archaeological artifact matches could be made based on available data (see Appendix D). Figure 74 depicts a type of bluebrushed stainless steel accoseal half-couch casket produced by the Aurora Casket Company with a light blue crepe interior. This casket appears to be very similar to Casket Type 1 in regard to finish, shell type, lid type, and the presence of gasket. It is also possible that the New Home Cemetery example shared the same type of lining. Crepe has been a very common lining fabric historically and can be produced using polyester threads. The Aurora casket differs in that Casket Type 1 did not appear to be stainless steel. Also, though the Aurora casket does exhibit stationary extension handles, the handles evidently used on Casket Type 1 more closely resemble those used on the Batesville Casket Company’s New Pointe Triton Series models (Figure 75).

\section{Internal Burial Container Elements}

Internal burial container hardware elements are critically important when conducting an analysis of historic cemetery artifact collections. Structurally, these elements are what held the burial container together and therefore elucidate key aspects of change and variation in construction technique. These changes in construction took place over time due to technological innovation, which means that internal hardware elements are often valuable temporal indicators. 


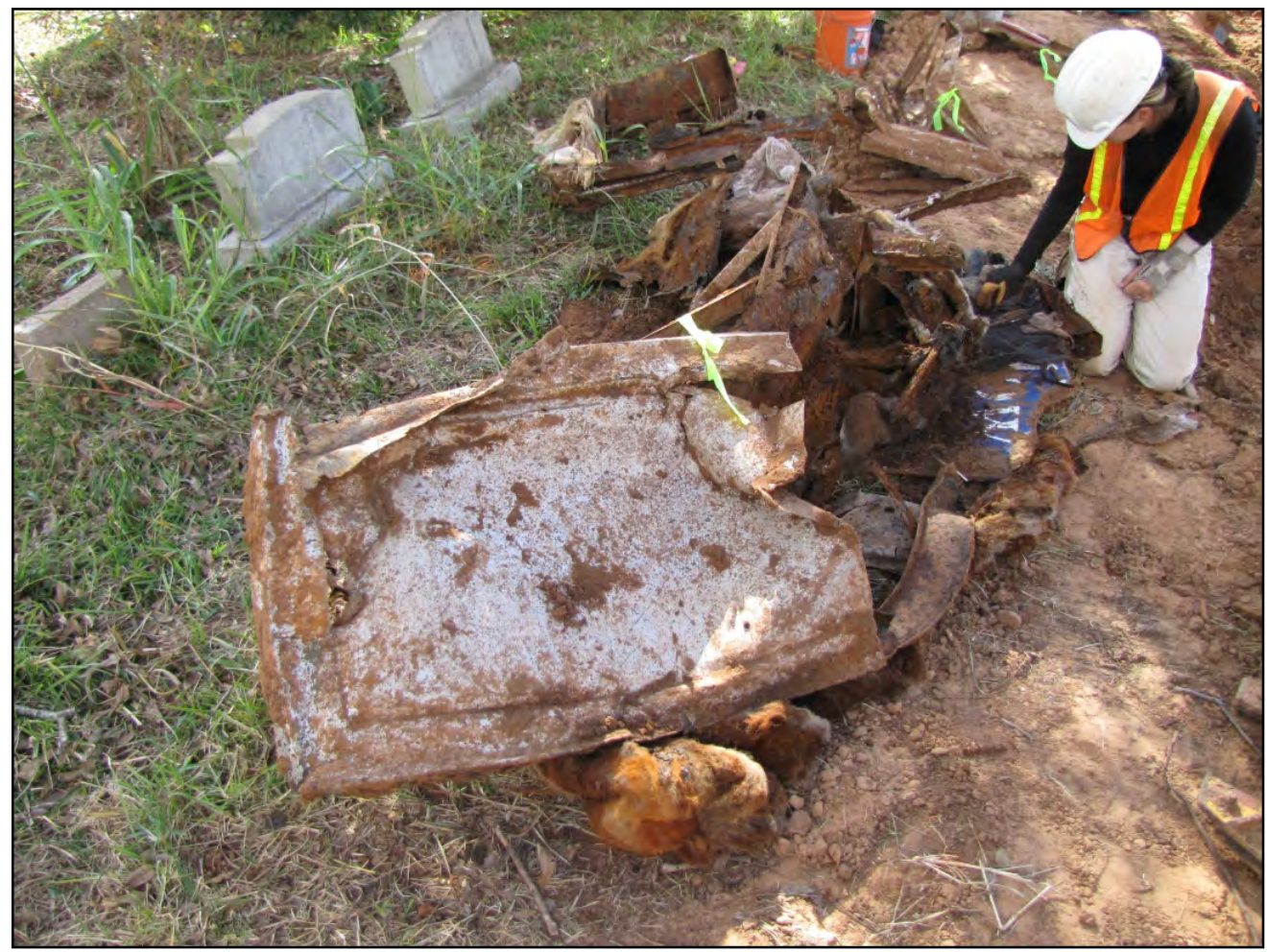

Figure 73. Field photo showing Coffin 4 (Metal Casket Type 1) after removal from grave feature.

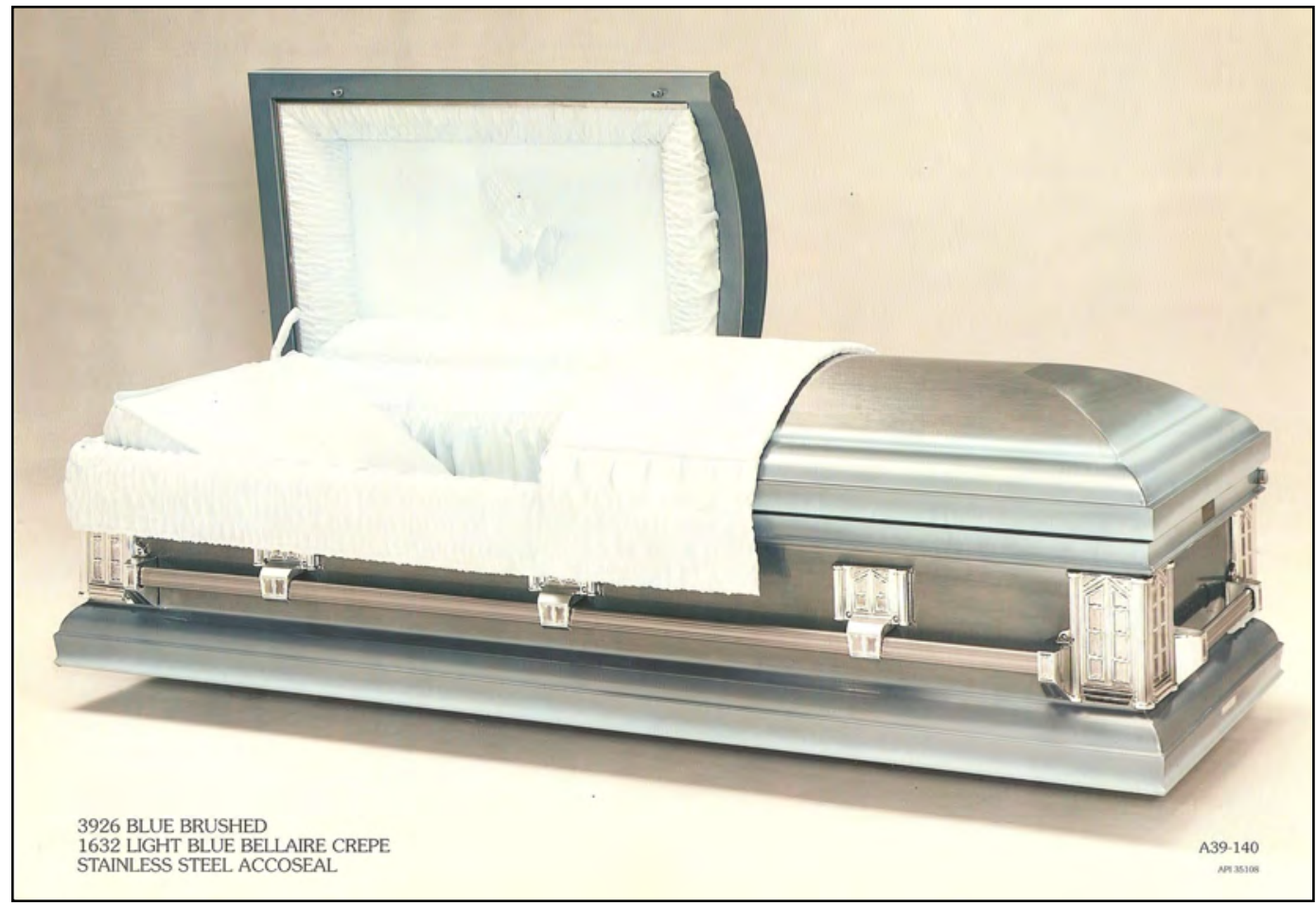

Figure 74. Blue-brushed, stainless steel accoseal casket depicted on a 1999 Aurora Casket Company advertising card that is similar to Metal Casket Type 1. 


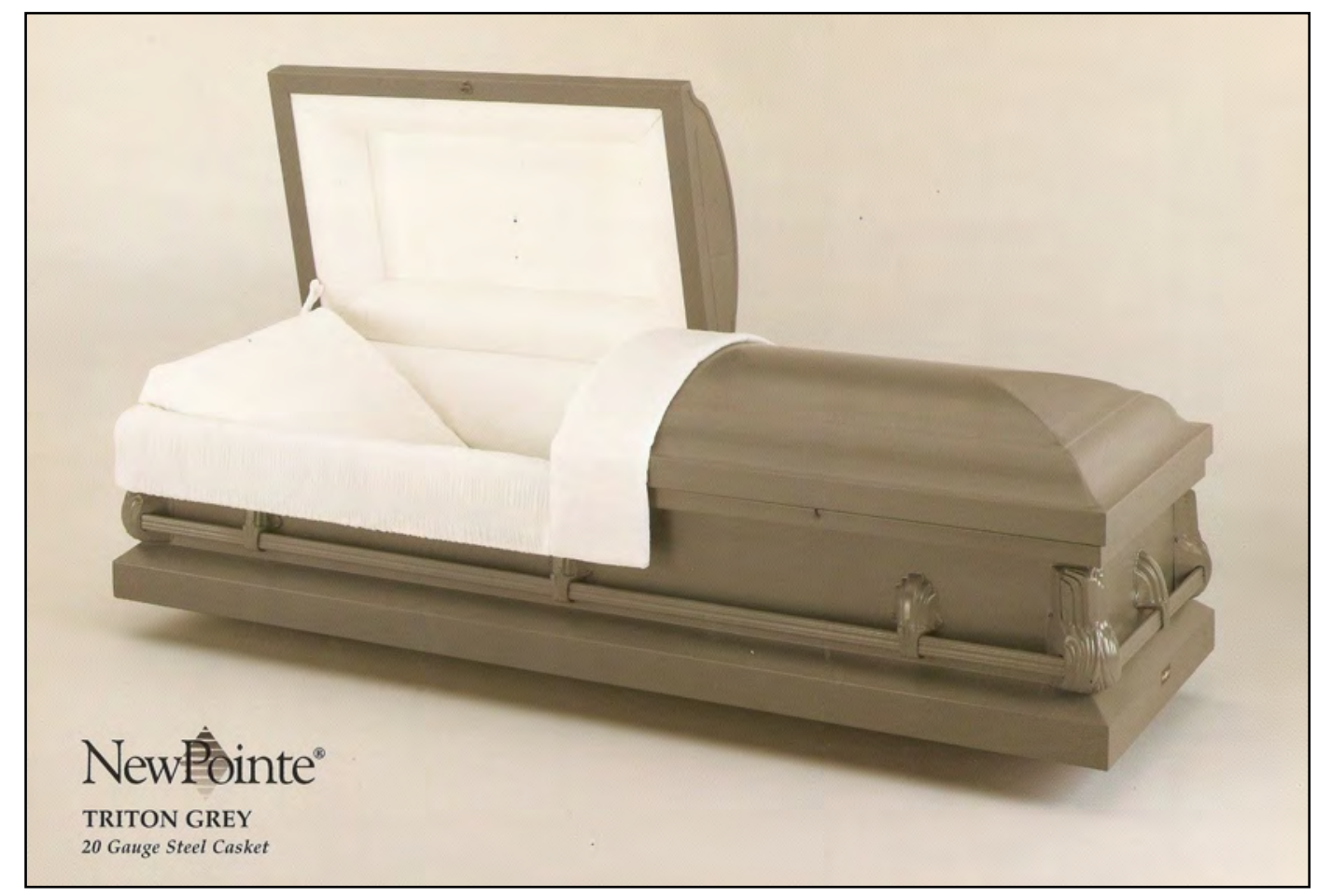

Figure 75. New Pointe Triton Grey 20-gauge steel casket depicted on a 1998 Batesville Casket Company advertising card that shows handles similar to Handle Type 23 found on Metal Casket Type 1.

Within historical archaeology and material culture studies, a vast literature exists on the production and history of nails (Adams 2002; Baackes 1896; Benson 1983; Edgerton 1897; Edwards and Wells 1993; Epstein 1981; Fontana 1965; Fontana and Greenleaf 1962; Jurney 1987; Loveday 1983; Michael 1974; Nelson 1963, 1968; Phillips 1989; Priess 1970, 1973; Wells 1993, 1998; Young 1991). Rarely, however, are nails given appropriate respect in historic cemetery archaeology reports.

Even less attention is given to the various esoteric complex fastening devices and other internal hardware used by professional burial container manufactures in the late nineteenth and twentieth centuries. These devices were used to secure the lid of the coffin or casket, secure the viewing window cover, allow the viewing window to slide and lock closed, secure drop casket sides closed, and support opening and hinging of burial container lids. An 1883 advertisement (Figure 76) from the Stein Manufacturing Company of Rochester, New York, presents, "The Most Serviceable Invention of the Age . . . Our 'Patent Fastener,' For Casket Tops.” Although it is not specifically evident from the illustration or patent research to what fastener they are referring, the ad attests to the fact that this "perfect boon to the funeral director" was a major turning point in the industry. Therefore, the fastener should be addressed in greater detail.

The few authors of historic cemetery excavation reports who attempt to responsibly address internal hardware elements are hindered by the general lack of preservation of ferrous materials or by a lack of knowledge of hardware function and/or hardware terminology. These internal hardware elements are often classified collectively as latches (Dockall, Powell, and Steele 1996), iron closures (Davidson 1999), or even in some cases as miscellaneous hardware (Mainfort and 


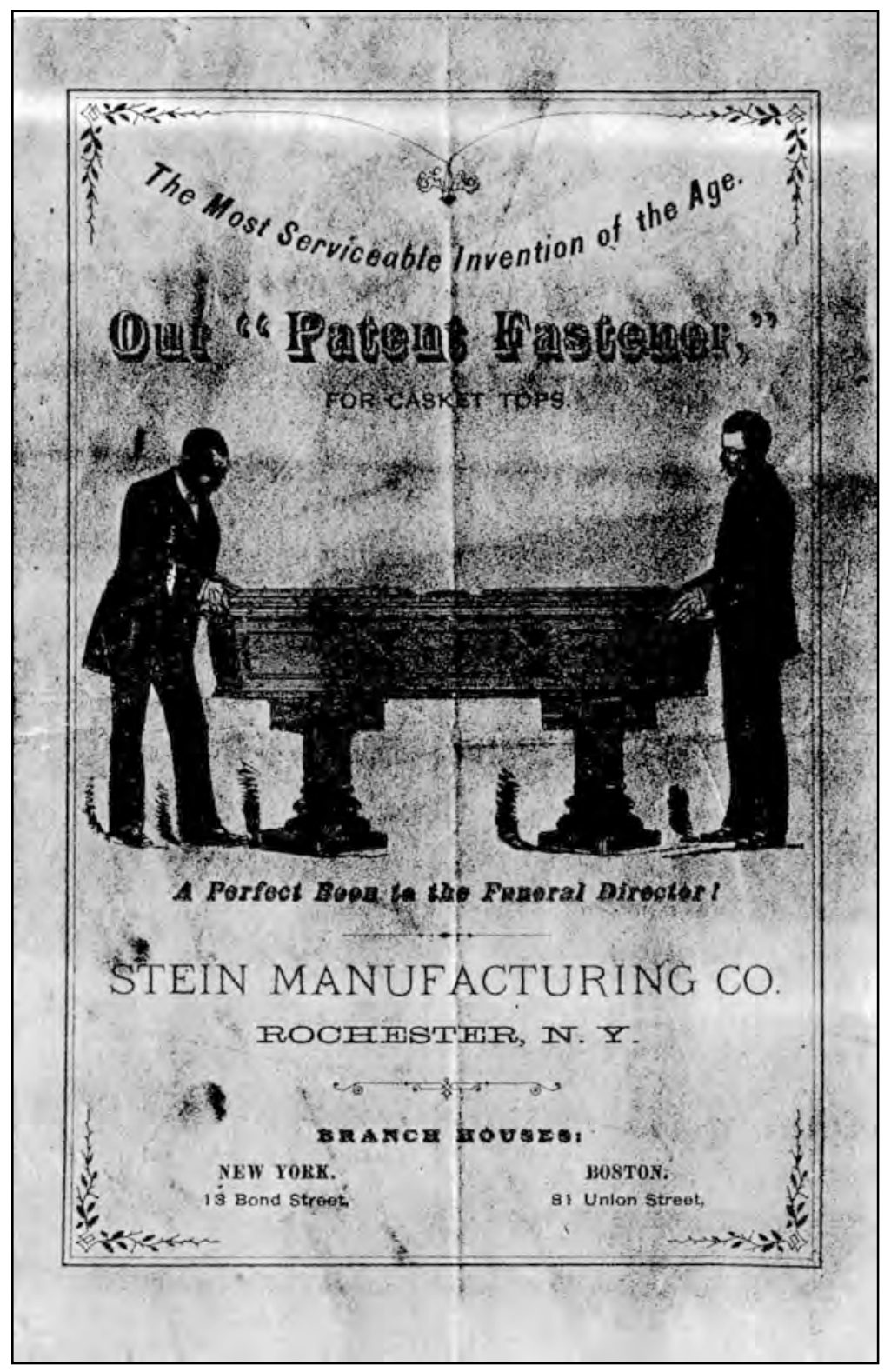

Figure 76. 1883 advertisement for Stein Manufacturing Company’s “Patent Fastener” for casket tops.

Davidson 2006). Mainfort and Davidson (2006) made the most concerted effort thus far to present adequate discussions of the few complex internal hardware elements recovered from burial in the Becky Wright and Eddy cemeteries near Fort Smith, Arkansas. Hopefully, the following descriptions of the New Home Cemetery internal hardware elements will provide a starting point for a more detailed discussion of these important historical artifacts. 


\section{Nails}

Nails were an essential and ubiquitous form of construction hardware used in the production of burial containers in the nineteenth and early twentieth centuries. There were three general types of nails in use in various regions and times in the nineteenth century: hand wrought, square cut, and wire. Hand wrought nails were commonly used during the seventeenth and eighteenth centuries until the introduction of the cut nail around 1800 (Mainfort and Davidson 2006:115116) (Figure 77). Cut nails declined in use toward the end of the nineteenth century as wire nails became available on the market and became more widely used in the casket industry.

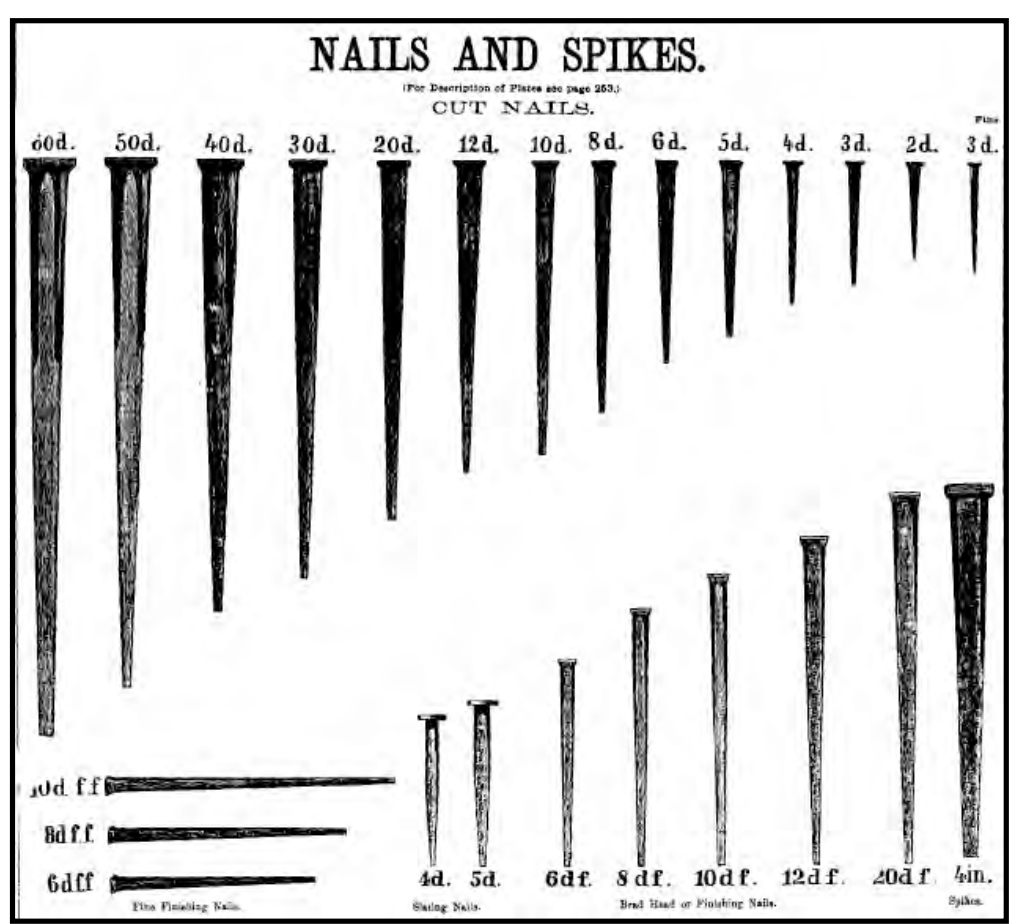

Figure 77. Selection of cut nails offered for sale on page 251 of the 1865 Russell \& Erwin Manufacturing Company general catalog.

Based on Edgerton (1897), Fontana (1965), Fontana and Greenleaf (1962), and other reliable sources, Mainfort and Davidson (2006) have placed the introduction of wire nails to common usage in the funeral industry between 1890 and 1900. In estimates of burial chronology, this date has been conventionalized to ca. 1895, and prior to that characterized as the exclusive use of cut nails, or the absence of wire nails. This dating has held true for comparable burials accurately dated by other artifact associations or historical record from Arkansas (Cande 1995:161-168, 249-251), Freedman's Cemetery in Dallas, Texas (Peter et al. 2000), and Meadowlark Cemetery, Kansas (Pye 2007).

The archaeological literature (see Appendix E) suggests that the most common sizes of nails used in the construction of coffins and caskets were smaller nails (i.e., 4d, 6d, and 8d), whereas larger nails (i.e., 10d and above) were more likely to be used in shipping containers for the transport of mass-produced burial containers (Davidson 1999; Mainfort and Davidson 2006). It is reasonable 
to expect that there would be a certain degree of uniformity in the sizes of nails used for massproduced coffins and caskets, and in fact, the most common nails listed in the archaeological literature have been $6 \mathrm{~d}$ and $8 \mathrm{~d}$ cut nails with a bias toward the use of $6 \mathrm{~d}$ nails in both the cut and wire varieties through time (Mainfort and Davidson 2006:101). This pattern plays out in the New Home Cemetery collection as well; however, nails ranging from $4 \mathrm{~d}$ to $10 \mathrm{~d}$ were also recovered (see Appendix A).

No in-depth analyses of nails from the New Home Cemetery excavations were conducted, mainly due to the poor preservation of the excavated ferrous materials. All burials contained at least some nails, and most of the nails recovered from excavations were wire nails placing the collection squarely in the twentieth century. It is noteworthy, however, that three cut nails were identified (one each from Burials 16 and 19, and one from disturbed context). This suggests that that these two burials took place sometime in the early use of this cemetery after the 1895 common adoption of wire nails in the funeral industry.

The term "clinched" nail refers to nails that have been intentionally bent at roughly a 90-degree angle toward the distal end. Nails are often intentionally bent in this manner when used to secure bracing or runners connecting multiple boards or when securing external hardware. The nail is driven through the required boards and then the protruding end is bent over. Mainfort and Davidson (2006:101) suggest that clinched nails would not have been used in the construction of primary burial containers. Instead, clinched nails would have been more commonly used in the construction of an outer shipping crate, or sometimes in a vaulted lid (Mainfort and Davidson 2006:101). Although the roughness of outer shipping crates would typically require this type of construction, the presence of clinched nails alone should never be used as a proxy for an outer box or commercial manufacture. Their presence can only be indicative of the functional aspect of their use in the construction of a container. In the New Home Cemetery collection, clinched nails were noted in at least 11 burials, as well as from disturbed contexts. Only five of these burials (Coffins 1 and 2, as well as Burials 2, 13, and 16) also contained other evidence of outer boxes, or an outer box was noted by field personnel (see Appendix A and burial descriptions in Chapter 4).

\section{Screws}

Mainfort and Davidson (2006:144-145) report that wood screws in some form have been around since the time of the ancient Greeks; however, prior to the nineteenth century, most screws had blunt points and could not self-start. The introduction of the gimlet wood screw, a screw that has a tapered body and a pointed tip, has been attributed to Thomas J. Sloan, who was issued a U.S. Utility Patent (No. 4,704) in 1846 (Figure 78). The mass production of these gimlet screws was initiated the same year by Sloan's introduction of the machine capable of producing said screws (U.S. Utility Patent No. 4,864). Even though, technically, a gimlet form had been introduced 10 years earlier by Thomas W. Harvey of Poughkeepsie Screw Company, as well as a machine capable of producing wood screws in 1834, most early gimlet and blunt-tip forms had to be handturned and therefore were more costly to consumers than later machine-made screws (Mainfort and Davidson 2006:145).

Plain gimlet screws were commonly employed as burial container lid closures in the nineteenth century, though the earliest mention of some type of screw being used in mortuary contexts dates to 1748 (Mainfort and Davidson 2006:145; Tharp 1996:226). In the known sample of general hardware and mortuary catalogues available for comparison, flat, round, oval, and fillister-headed 

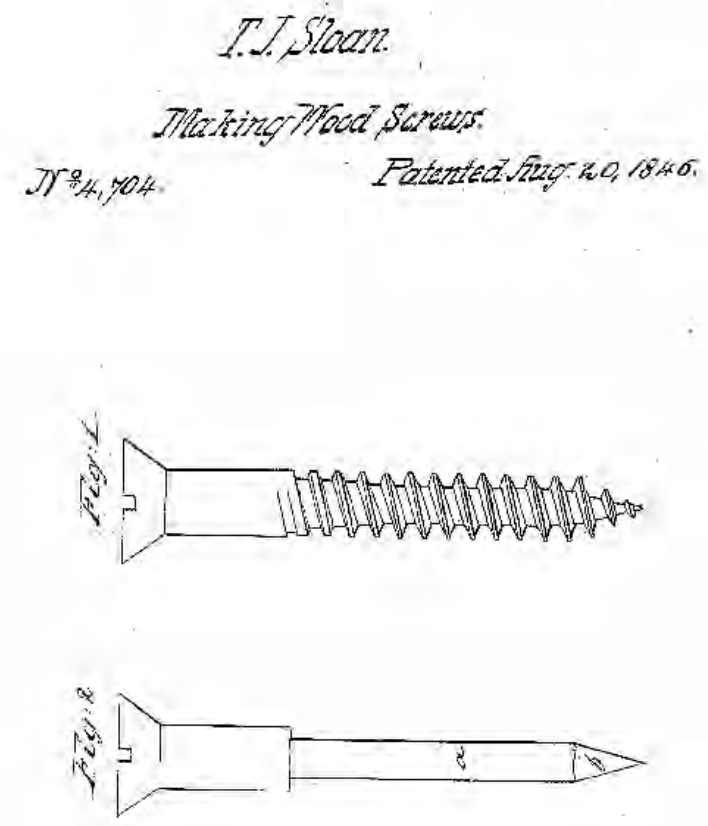

Figure 78. U.S. Utility Patent No. 4,704 assigned in 1846 to T. J. Sloan for wood screws.

gimlet screws were prominently advertised for sale (Figure 79). Mainfort and Davidson (2006:145) conclude after critical examination of archaeological literature of pre-1850 cemeteries that the presence of screws, particularly gimlet screws, was relatively rare. Additionally, in those burial containers where utilitarian gimlet screws were used as primary means of lid closure, there was an absence of formal coffin hardware such as coffin screws or thumbscrews. Forms of ornamental tacks, however, were often utilized to mask the use of ordinary screws (Mainfort and Davidson 2006:146).

\section{AMERICAN GIMLET-POINT SCREWS,}

FLAT, ROUND, OVAL AND FILLISTER HEAD.

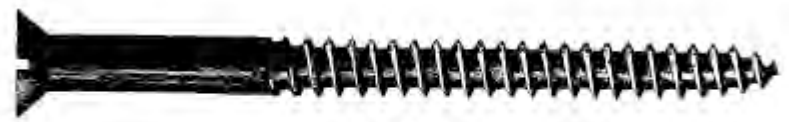

Flat Heac.

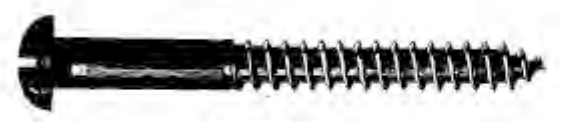

Forind Head,

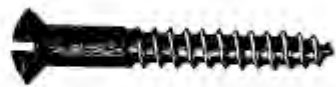

Oval Head.

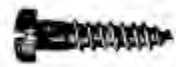

Fullister Head.

Figure 79. Selection of American gimlet screws offered for sale on page 126 of the 1865 Russell \& Erwin Manufacturing Company general catalog. 
All burials at New Home Cemetery contained utilitarian screws, most being used to secure hardware such as handles to burial containers. The heads of most of these screws, although masked by ferrous corrosion, did appear to be flat-headed gimlet forms. Of the few that were not corroded, both the traditional slotted and Phillips head screws were noted (Phillips head screws were identified in Burials 11, and 12, as well as with Coffin 1 and 3) (see Appendix A). Phillips screws are a fantastic temporal indicator because Henry F. Phillips did not file for this patent until 1934, and the patent was not granted until 1936 (U.S. Utility Patent No. 2046343) (Figure 80). Phillips was granted two other patents (U.S. Patents 2046840 and 2046837) on the same day for a screwdriver and the method for uniting a screw with a screwdriver. Based on this historical information, the four burials containing Phillips head screws must date to after 1936.

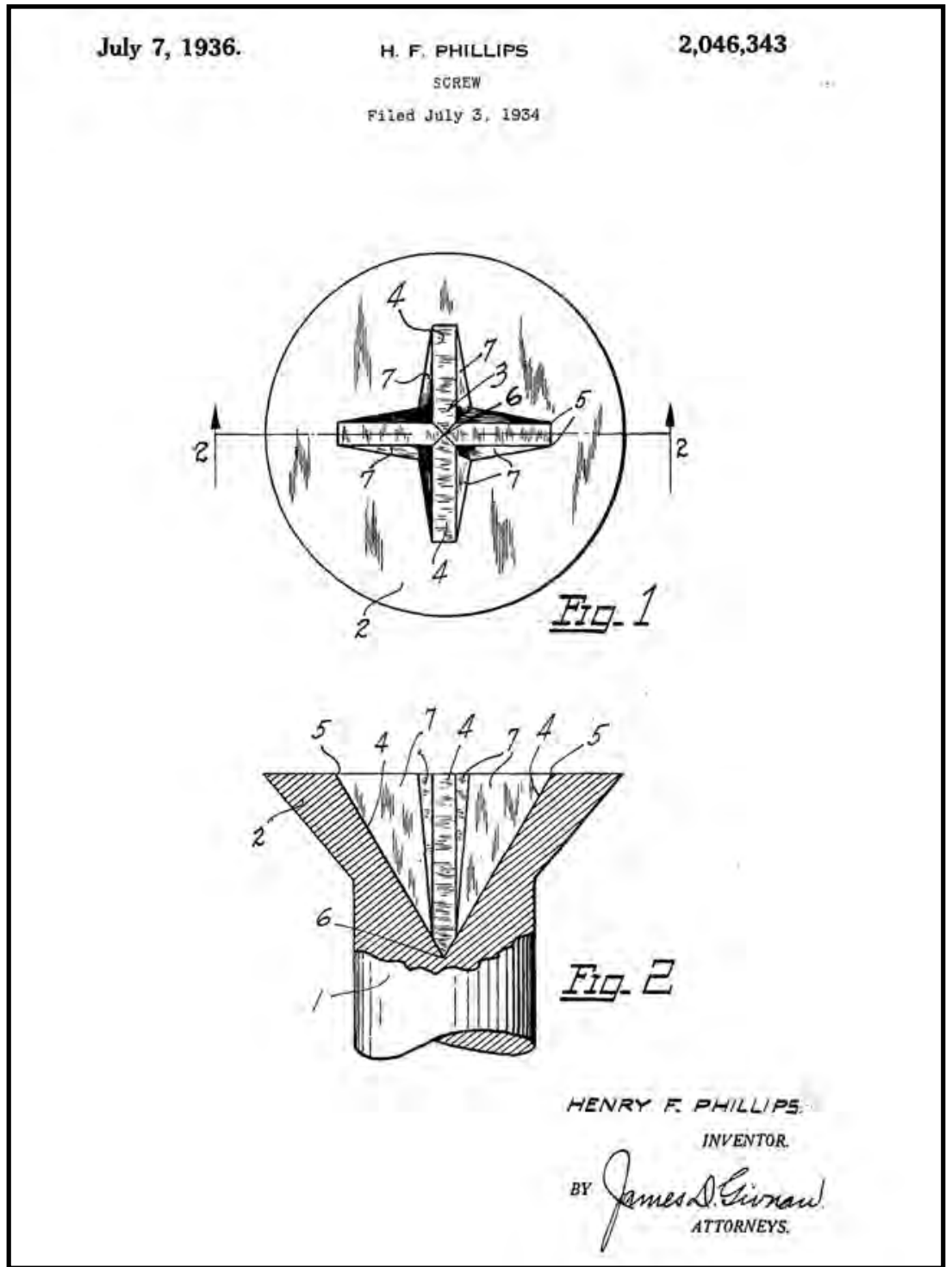

Figure 80. U.S. Utility Patent No. 2,046,343 assigned in 1936 to Henry F. Phillips for a screw. 


\section{Staples}

Staples are thin, bent-wire, ferrous metal fasteners used to join two boards together, affix wire or cloth to wooden surfaces, secure multiple sheets of paper together, as well as many other industrial uses. A description of the full history of the staple is beyond the scope of the present discussion, but U.S. patent documents reveal the use of the word "staple" in the context of a curved piece of securing hardware as early as the 1790s. Although staples likely existed in Europe prior to this point, it is not known exactly when the first U.S. patent for the modern type staple was issued. The first known patent illustrating a modern-looking staple was not issued until 1883 (U.S. Patent 274481) for an improvement in staples. The first known patent for a stapling device for construction purposes was issued to J. S. Bokenkotter in 1884 (U.S. Patent 302092). This device would have increased the efficiency of the technology and promoted the use of staples in construction and carpentry fields.

\section{Staple Type 1}

Staple Type 1 (Figure 81, see middle and right) is represented by six artifacts recovered from two burials (Burials 6 and 10) and the disturbed area in New Home Cemetery (see Appendix A). This type is noted as a common blind staple in the 1980 [1865] Russell \& Erwin Manufacturing Company catalog (Figure 82; see Appendix D). It has a gently squared top with rounded corners and blunted arms of equal length. Within the New Home Cemetery collection, there are three size variants of the common staple: 2-inch $(n=4), 1 \frac{1}{2}$-inch $(n=1)$, and 1-inch $(n=1)$.

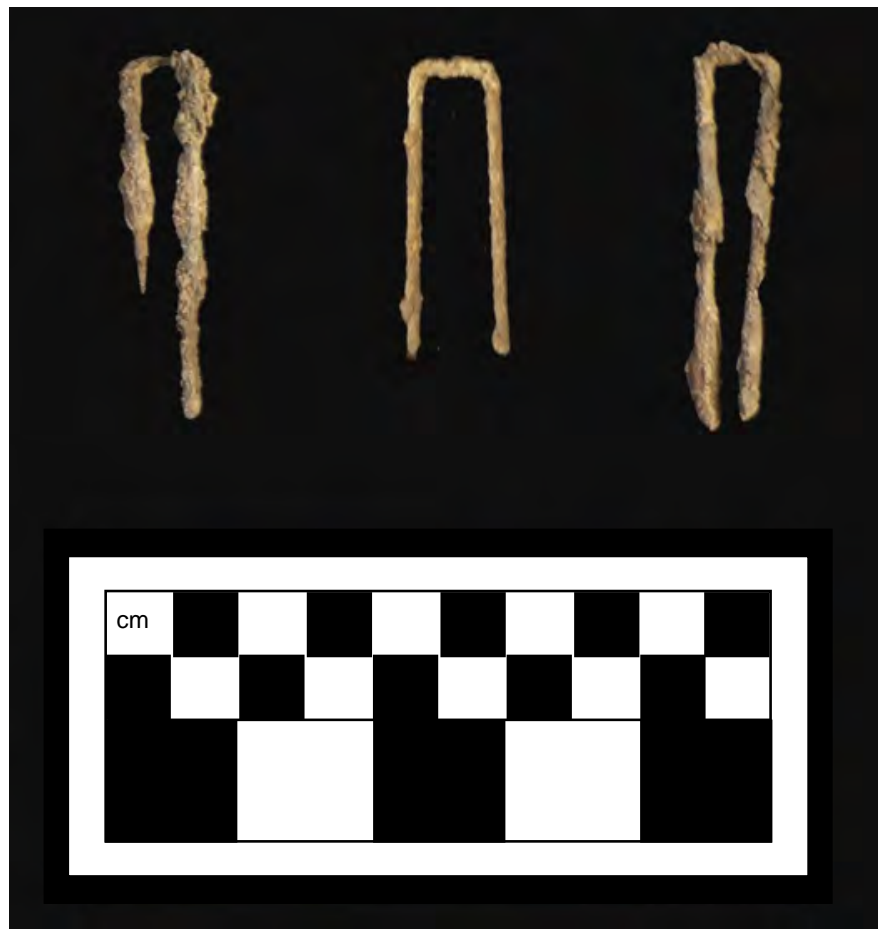

Figure 81. Staples. 


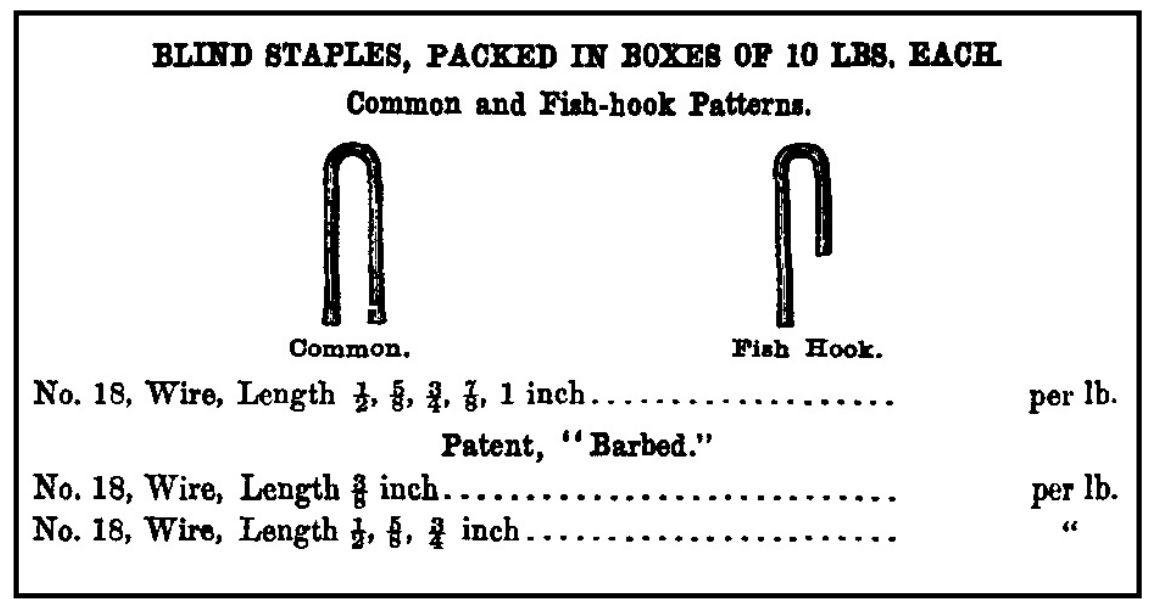

Figure 82. Blind staples offered for sale on page 149 of the 1865 Russell \& Erwin Manufacturing Company general catalog.

\section{Staple Type 2}

Staple Type 2 (see Figure 81, left) is represented by five artifacts recovered from two burials (Burials 6 and 10) in New Home Cemetery (see Appendix A). This type is noted as a fishhook blind staple in the 1980 [1865] catalog of the Russell \& Erwin Manufacturing Company (see Figure 82; see Appendix D). It has a gently squared top with rounded corners and blunted arms, with one arm substantially shorter. Within the New Home Cemetery collection, there are two size variants of the fishhook staple: 2-inch $(n=2)$ and 1-inch $(n=3)$.

\section{Joining Plates}

The arbitrary term "joining plate" refers to thin, rectangular, ferrous metal plates used to join two boards together in the construction of the burial container (Figure 83). Three size variants (3-cm, 4-cm, and 5-cm long) were recovered in nine graves in the New Home Cemetery, as well as in the disturbed areas (see Appendix A). The 3-cm variety was recovered from Burials 6 and 18 and the disturbed areas. On both faces of this variety are wood grains that meet at a centerline at a roughly 90-degree angle. This suggests that they were possibly used as biscuits at a mitered corner joint. The 4-cm variety was recovered from six burials (Coffin 5, and Burials 2, 3, 13, 14, and 19) and exhibits angled wood grains on only one face, suggesting they were also used at mitered corners, but possibly secured at the top or bottom of the corner and not within the joint. No nails or screws are associated with these plates, so they likely were secured with some type of adhesive. The 5-cm variety was recovered in Coffin 1 and Burial 13 and typically exhibits in-line wood grains on one face, suggesting they were used to join board ends on end. Identification of joining plates speaks to the construction technique and skill of the carpenter, availability of other resources for construction, and possibly even the cost of the burial container. 


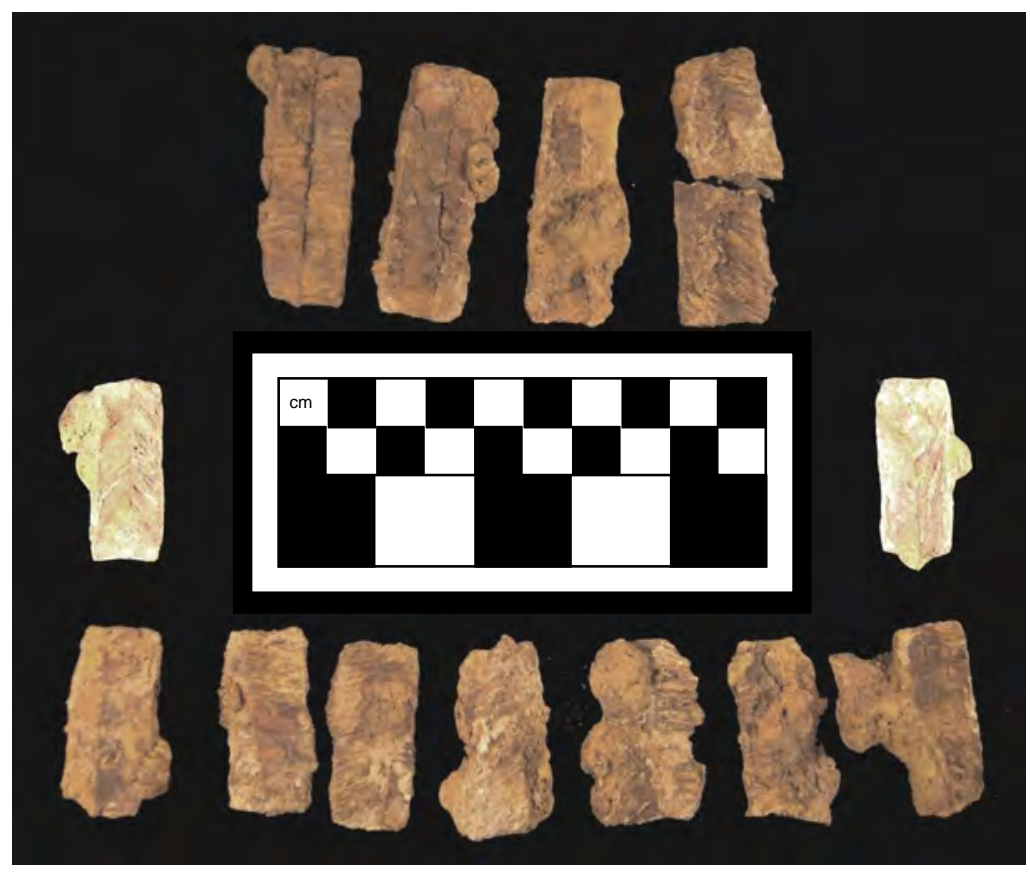

Figure 83. Joining plates.

\section{Corrugated Fasteners}

Corrugated fasteners (Figure 84) are small, ribbed pieces of sheet metal with sharp edges intended to be driven into wood to secure two pieces together. They are presently commonly used in the construction of picture frames and some types of furniture. These types of fasteners were first patented in 1884 by Albert H. Walker of Brooklyn, New York (U.S. Utility Patent No. 300536). The patent document was titled "Means of Uniting Wood," but as can be seen in Figure 85, the item was somewhat crude in design. Mainfort and Davidson (2006:168) cast doubt on whether Walker's fastener was ever actually produced and marketed. No known hardware catalogs depict similar items. Walker's patent was never reissued, and no patents were ever filed for machines to manufacture said fasteners.

A more streamlined version of the corrugated fastener appears in the 1887 patent granted to Ferdinand W. Starr of Springfield, Ohio (U.S. Utility Patent No. 366269). Starr was continually improving this piece of hardware and is responsible for nine of the 14 patents identified with some association with corrugated fasteners. He observed that the corrugated fasteners would be easier to insert if one edge was toothed, so he created the double pointed variety, which he patented in 1889 (U.S. Utility Patent No. 406545) (Figure 86). It is this version that catapulted this invention into mass production. In 1890, Adolph Samson patented the first machine to manufacture Starr's fastener (U.S. Utility Patent No. 419973). Starr himself produced a similar, though lower-scale machine in 1890 (U.S. Utility Patent No. 427632), as well as a driving device designed to make insertion easier and more efficient (U.S. Utility Patent No. 428701). Based on historical data, the presence of corrugated fasteners indicates that some New Home Cemetery burials took place after 1890 . 


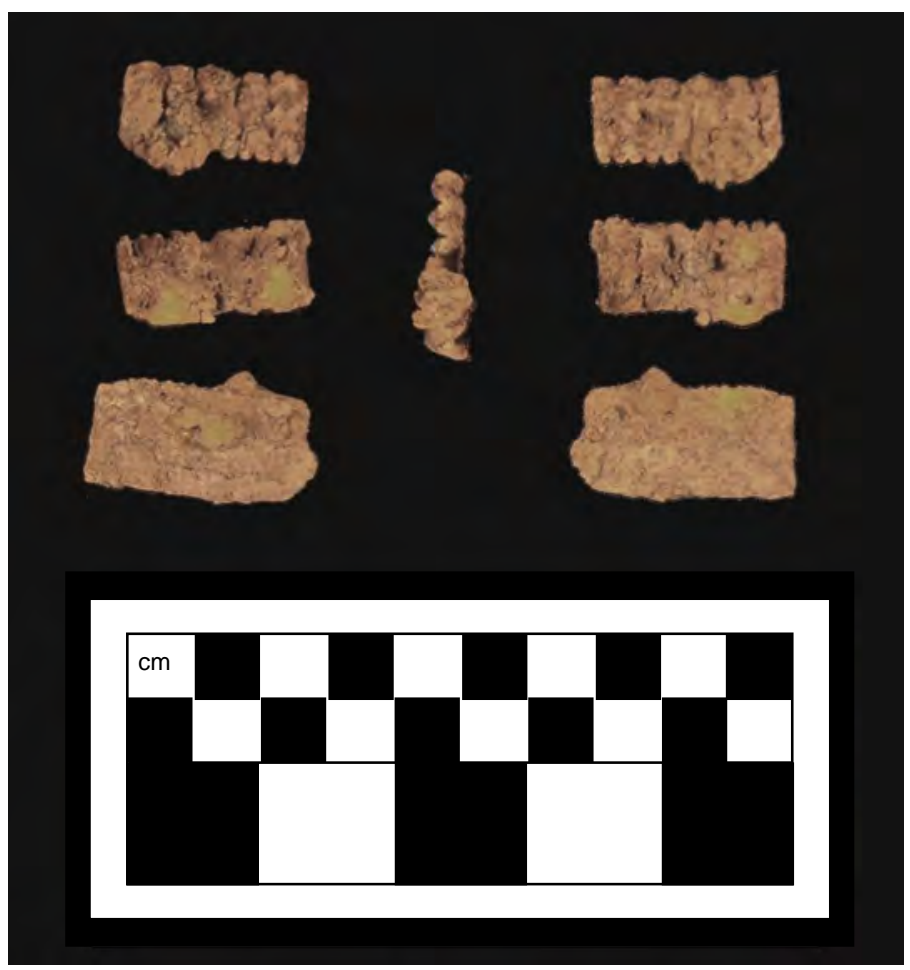

Figure 84. Corrugated fasteners.

Corrugated fasteners were identified in four burials (Burials 6, 7, 17, and 19) as well as in the disturbed area in New Home Cemetery (see Appendix A). At least three general size varieties are present: a roughly $2-\mathrm{x}-1-\mathrm{cm}$ variety, a roughly $1.5-\mathrm{x}-1-\mathrm{cm}$ variety, and a $3-\mathrm{x}-1.4-\mathrm{cm}$ variety. All size variants appear to be of the same construction. They have also been recovered in at least nine historic cemetery excavations in Texas, Alabama, Kentucky, Arkansas, and West Virginia, with associated interment dates ranging from 1896 until 1956 (see Appendix D).

\section{Top Fasteners}

Top fasteners are a form of complex burial container lid closure consisting of eight pieces in a full set-two foot plates, two foot hooks, two head body plates, as well as a left and a right spring. The springs and foot hooks are positioned along the sides of the lid, the springs toward the head, and the foot hooks toward the foot end of the burial container. The head and foot body plates are positioned on the sides of the burial container itself in locations to match with the elements secured to the lid. The foot hooks insert into holes in the foot plates and recesses cut into the top of the side wall below the plates. When the lid is secured, the spring hooks insert in the same manner as the foot hooks, but the level end of the spring either fits onto a projecting fin on the base plate or descends into a recess cut into the top of the side wall, thus limiting horizontal movement of the lid. 


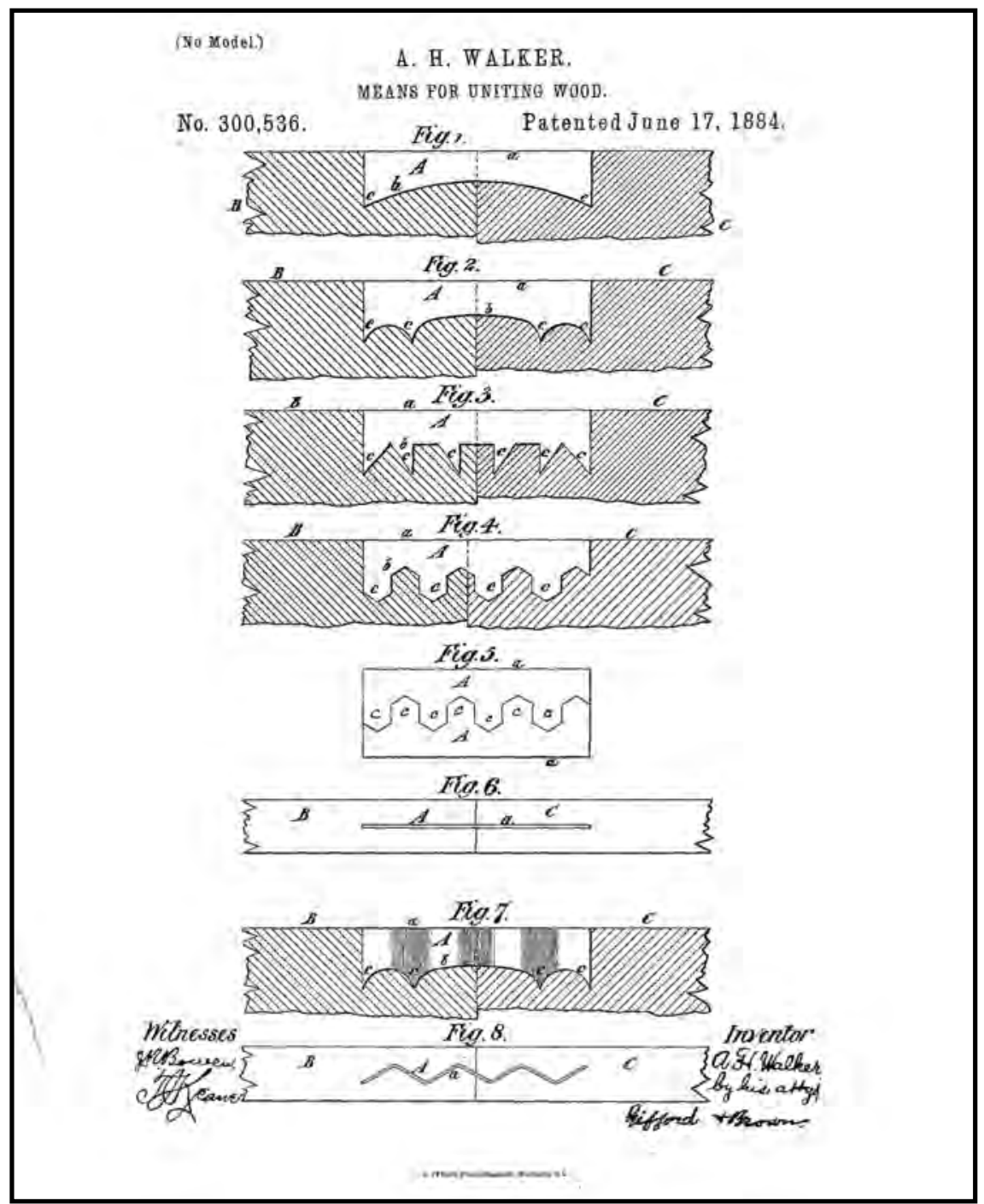

Figure 85. U.S. Utility Patent No. 300536 assigned in 1884 to A. H. Walker for a corrugated fastener.

The first known patent for a top fastener (U.S. Utility Patent No. 377325) was granted to William J. Noble on January 31, 1888 (Figure 87), with a second soon following in May of the same year (U.S. Utility Patent No. 383235). Although these were the first known patents to be issued, it was not the first top fastener for a patent application that was filed. Noble's patent was not granted until April 16, 1889, but William A. Sparks of Rochester, New York, submitted his application for a "coffin-fastener" (U.S. Utility Patent No. 401663) (Figure 88) on November 4, 1886, well before the Noble applications were filed. Modern-day caskets do not use top fasteners, but it is not currently known when top fasteners fell out of favor. The ca. 1920s-1930s Langenau Mfg. Company (Cleveland, Ohio) internal specialty hardware catalog is the only extant period catalog that has been shown to illustrate such fasteners, so they were used at least through the 1930s and likely later in time. 


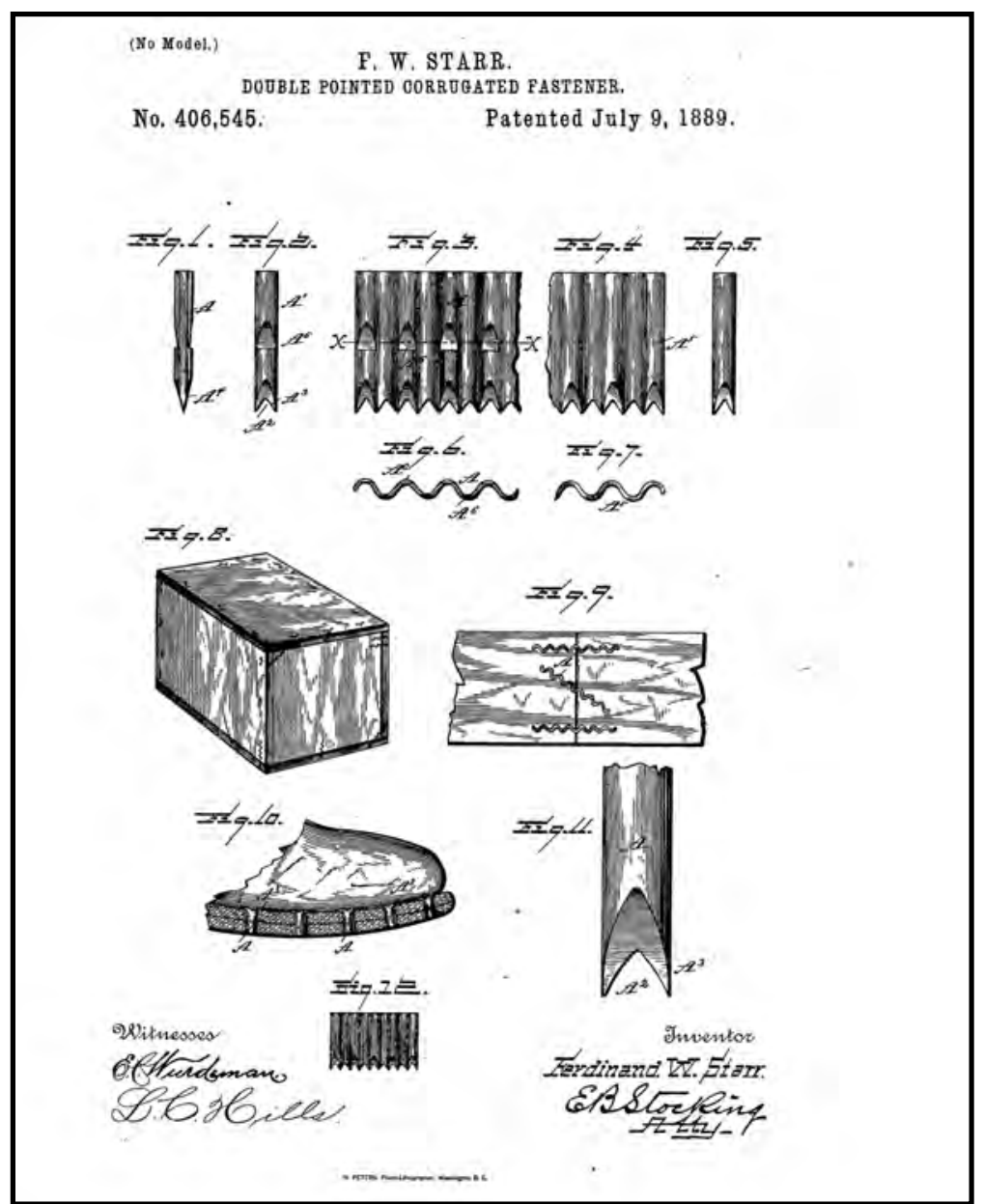

Figure 86. U.S. Utility Patent No. 406,545 assigned in 1889 to Ferdinand W. Starr for a corrugated fastener.

\section{Top Fastener Type 1}

Top Fastener Type 1 is represented by five artifacts recovered from two burials (Burials 2 and 9) in New Home Cemetery (see Appendix A). In Burial 2, only one foot hook and one foot plate (Figure 89) were located. The foot hook is gently curved, and the plate is secured with three screws and is roughly circular with a triangular notch on the side under the tip of the hook. The base plate is rectangular with a wide flute running $3 \mathrm{~cm}$ toward the center, with the last $0.96 \mathrm{~cm}$ of the fluted area consisting of a gently terminating hole. The top fastener set is more complete in Burial 9, with one spring fragment, one head body plate, one foot hook, and one foot plate being recovered. Only the hook segment and neck of the spring (Figure 90) are present, so it is not possible to determine on which side of the burial container it was mounted. The hook segment is circular, but one side is broken off, and two of the original three screws remain. The sides of neck are relatively parallel. The head body plate and foot plate are similarly designed. 


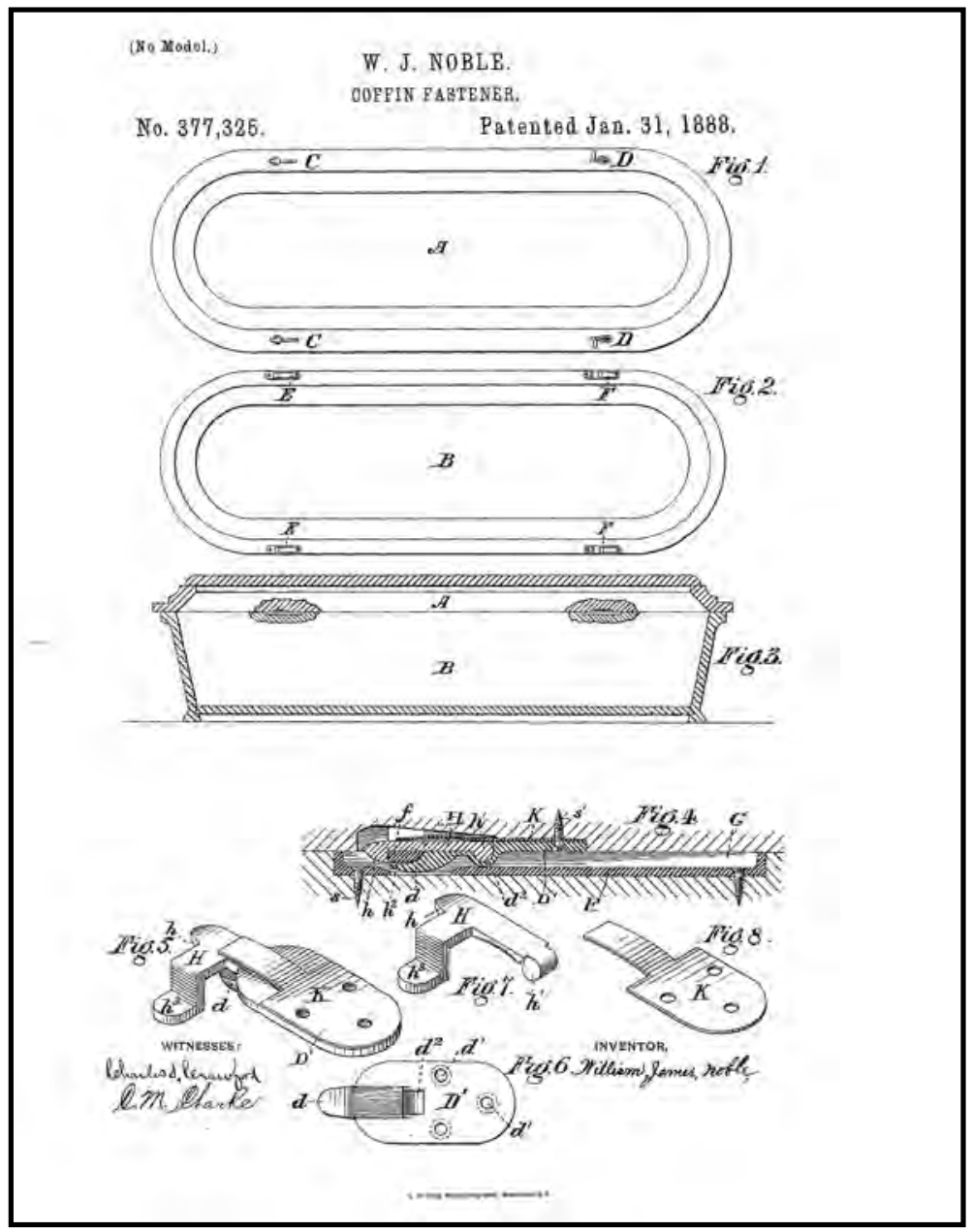

Figure 87. U.S. Utility Patent No. 377325 assigned in 1888 to William J. Noble for a coffin fastener.

Top Fastener Type 1 has only been identified in one mortuary catalog, the ca. 1920s-1930s catalog from the Langenau Mfg. Company (Figure 91). There is no known exact patent match to this variety of top fastener, but it is very similar to the fastener illustrated in Sparks's patent (see Figure 88). A similar top fastener has also been reported in five historic cemetery excavations: two in Georgia, and one each in Alabama, Texas, and Ontario, Canada. With the exception of the burials from Canada, which were not dated very tightly, all of the burials containing top fasteners date between 1900 and the 1930s (see Appendix D).

\section{Dowels}

Dowels are a versatile and esoteric hardware item that could have been used for a variety of purposes within the casket. Few have been reported in the archaeological literature, so information from contextual relationships is scant. Additionally, only the 1920s-1930s Langenau Mfg. Company catalog is known to illustrate dowels. Given their form, they could have been used as stops or catches in securing the casket lid or in a similar capacity with other hardware in construction of the burial container. 


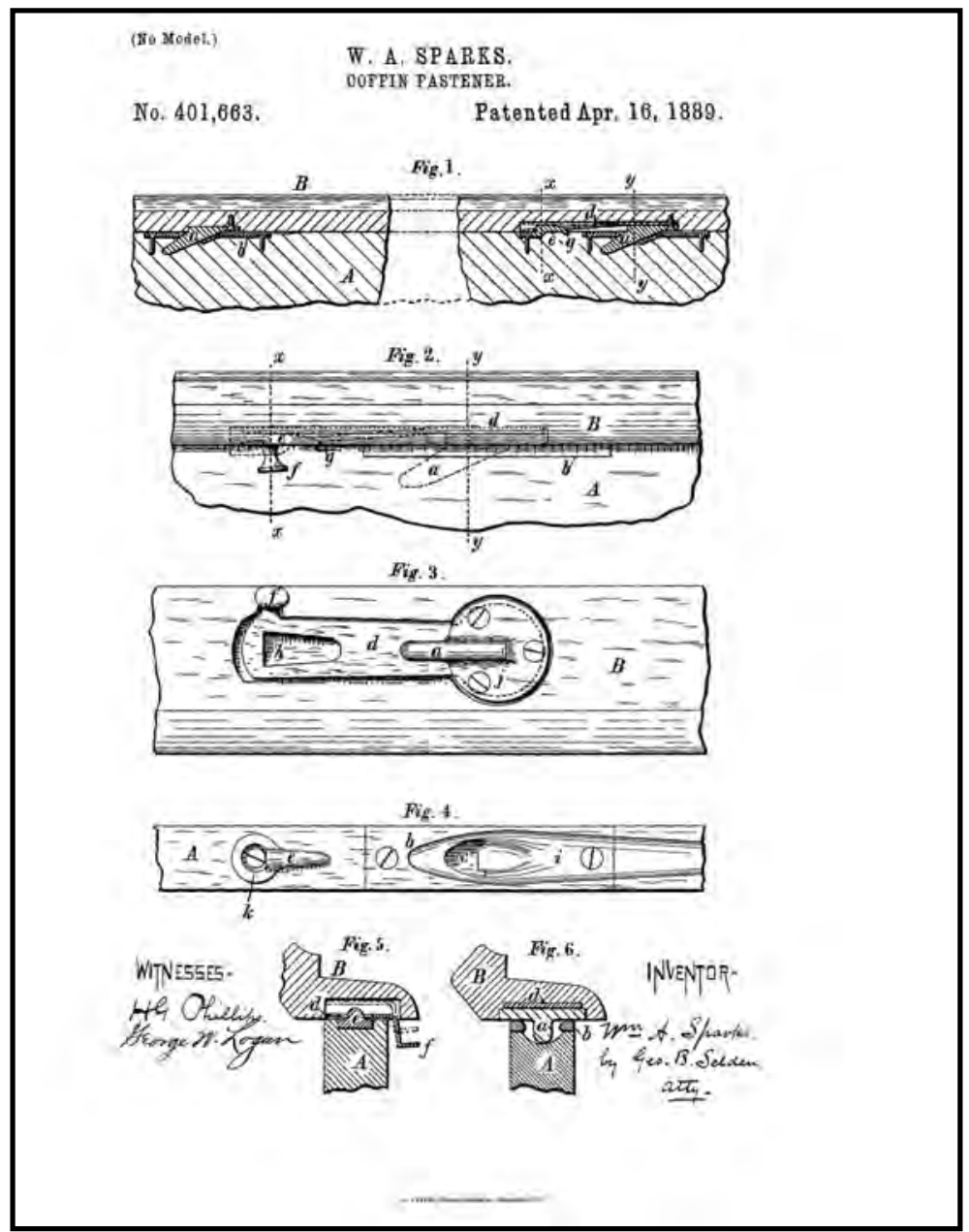

Figure 88. U.S. Utility Patent No. 401663 assigned in 1889 to William A. Sparks for a coffin fastener.

\section{Dowel Type 1}

Dowel Type 1 (Figure 92) is associated Stop Hinge Escutcheon type 7 and is represented by one artifact in Burial 19 in New Home Cemetery (see Appendix A). It is unclear, however, why it was in association with a stop hinge escutcheon. The base plate has two screw holes and rounded corners. The raised arm is shrouded in ferrous corrosion. The visible segment appears to be similar to Dowel No. 9 illustrated on page 30 of the 1920s-1930s Langenau Mfg. Company catalog (Figure 93). No patent records have been located that match this type of hardware, but a similar item has been recovered from one cemetery excavation in Texas from burials dating between 1907 and 1908 (see Appendix D).

\section{Dowel Type 2}

Dowel Type 2 (Figure 94) is represented by two artifacts found in Burials 2 and 9 in New Home Cemetery (see Appendix A). The base plate has two screw holes and squared corners. The raised arm is curved slightly outward and has squared corners. No patent records for this type of dowel 


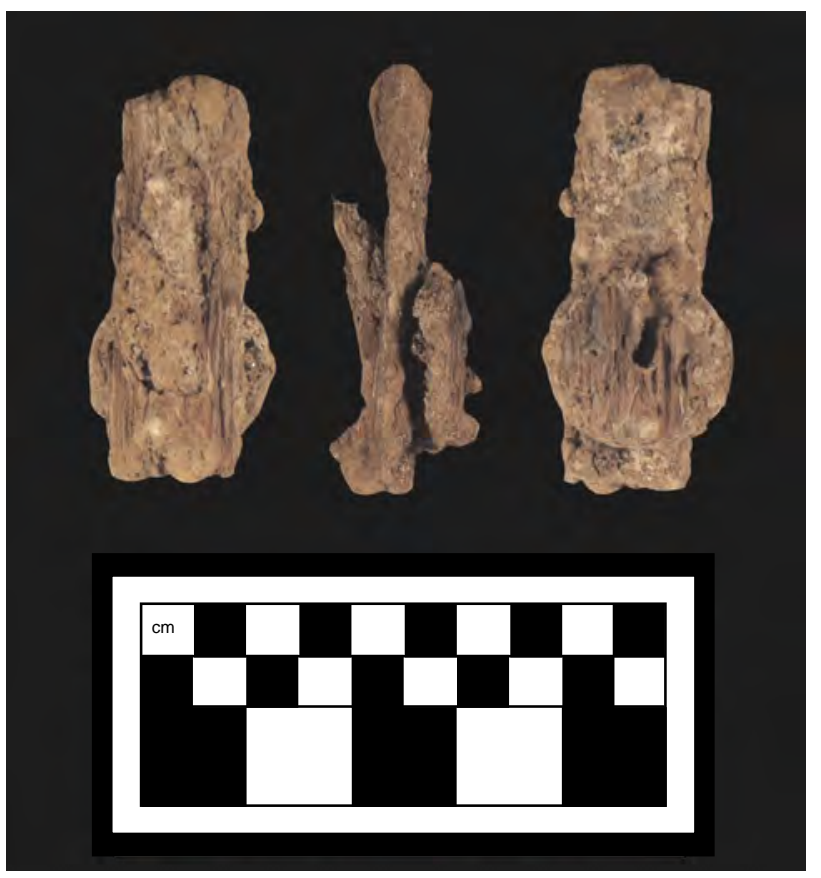

Figure 89. Top Fastener Type 1—foot hook and plate.

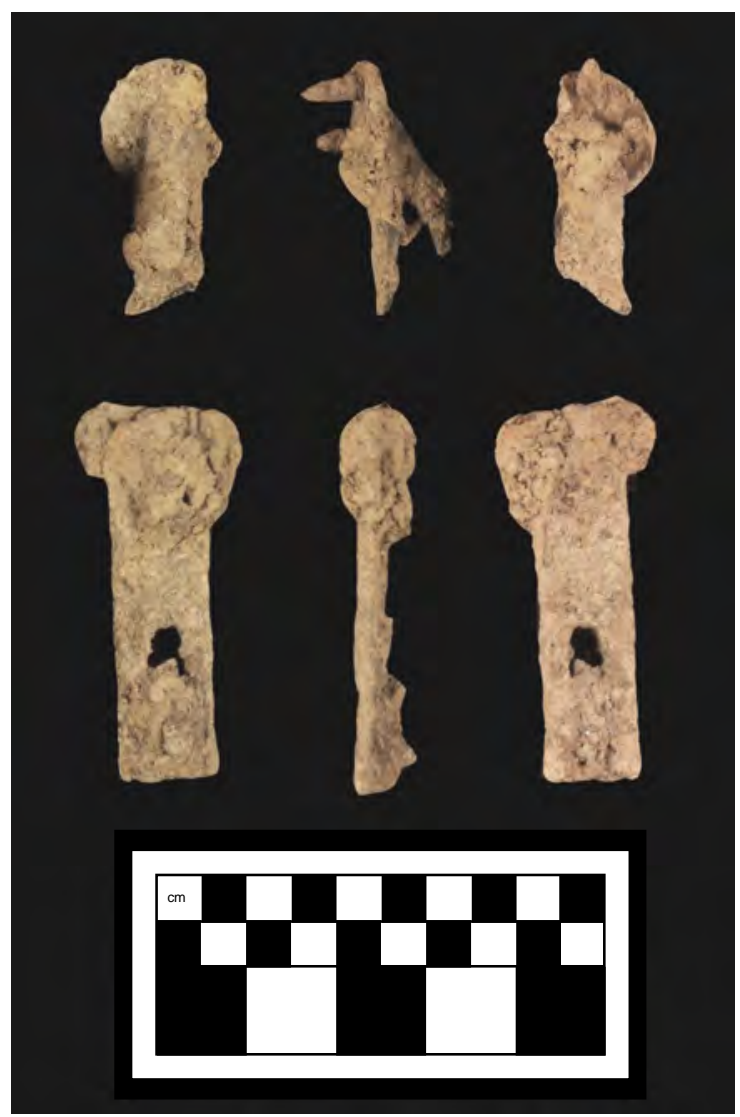

Figure 90. Top Fastener Type 1—spring and head plate. 


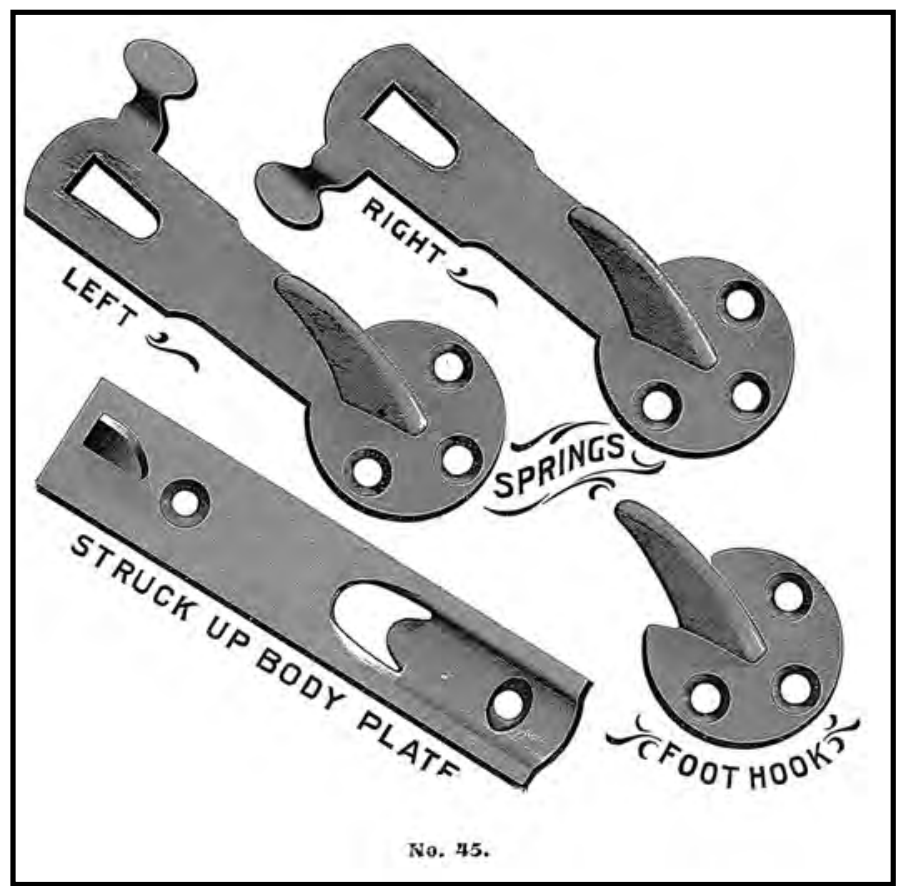

Figure 91. Top Fastener No. 45, which is similar to Top Fastener Type 1, as illustrated on page 77 of the 1920s-1930s Langenau Manufacturing Company catalog.

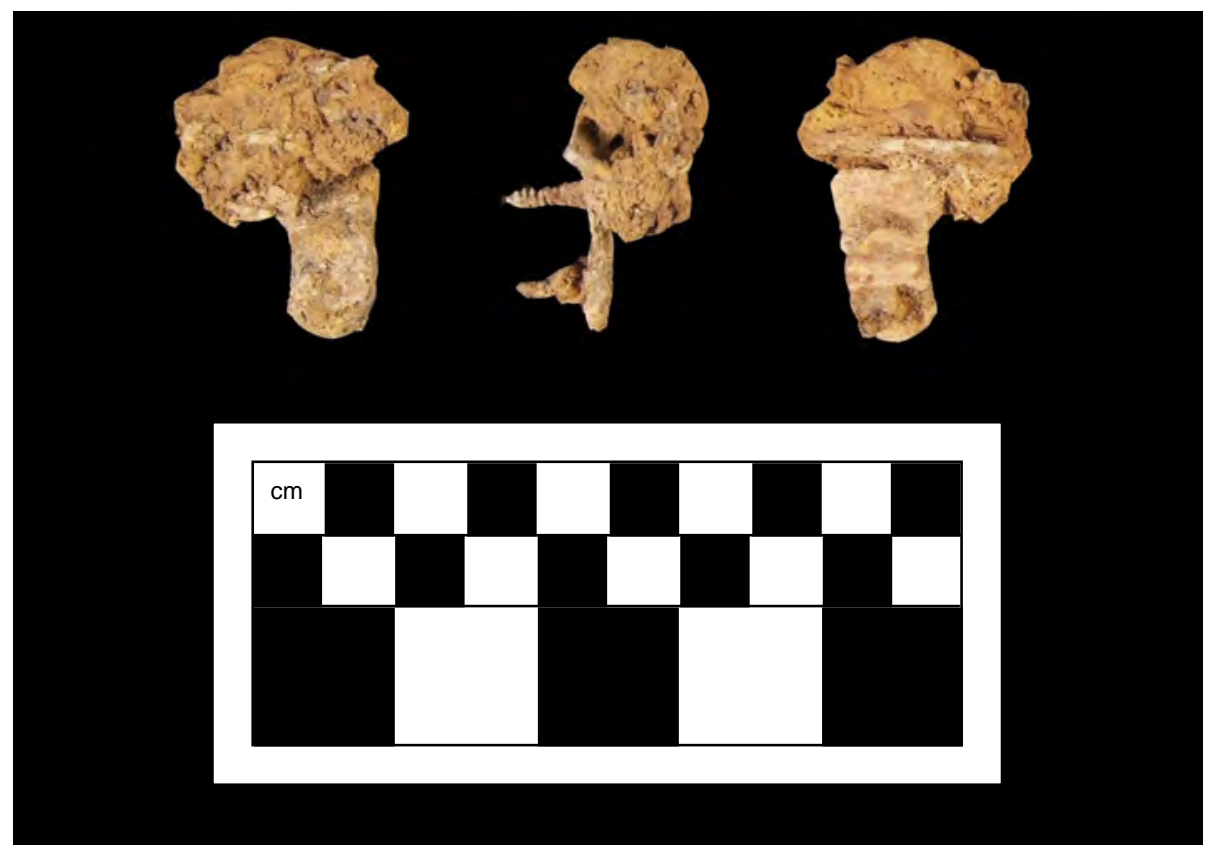

Figure 92. Dowel Type 1 pictured with Stop Hinge Escutcheon Type. 


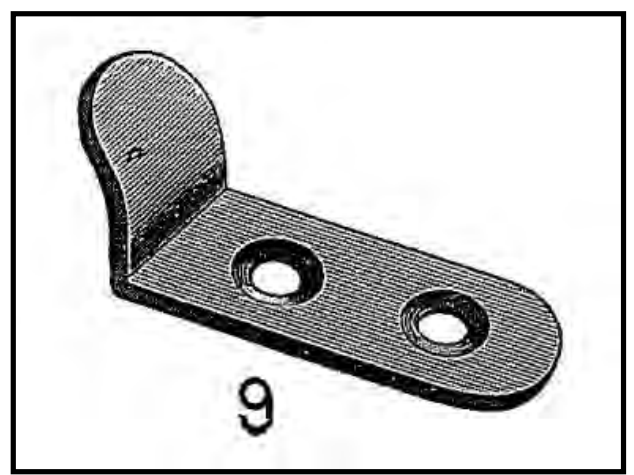

Figure 93. Dowel No. 9, which is a similar match to Dowel Type 1, as illustrated on page 30 of the 1920s-1930s Langenau Manufacturing Company catalog.

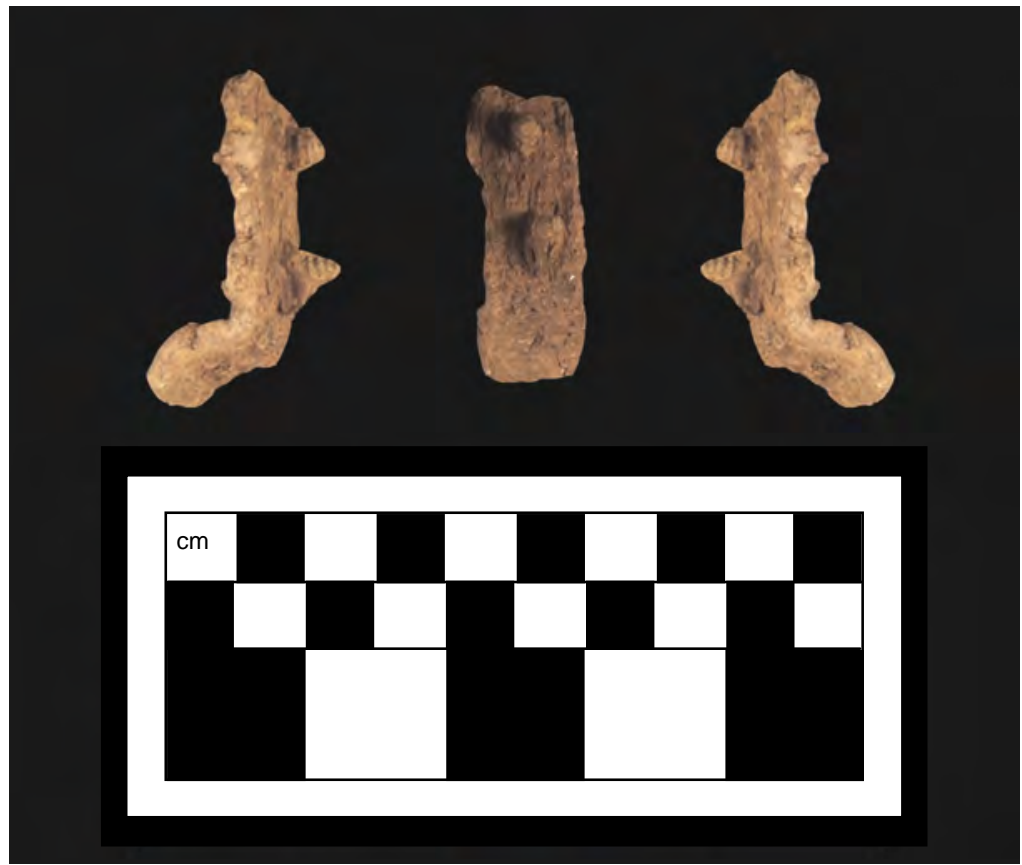

Figure 94. Dowel Type 2.

have been located, and it has not appeared in any known cemetery excavations. It does, however, match Dowel No. 174 (Figure 95) illustrated on page 33 of the 1920s-1930s Langenau Mfg. Company catalog (see Appendix D).

\section{Catch Assemblies}

Catches are small complex ferrous fastening devices that were used to secure the lid of a burial container or a viewing window cover. Figure 96 is the drawing page from a 1953 patent (U.S. Utility Patent No. 2634997) for a catch. This is an admittedly recent piece of hardware, but it does show a fine exploded view of the components of a standard catch. "Fig 1" in the patent (see Figure 96) shows a vertical section through the casket and lid so that the catch can be seen; "Fig 


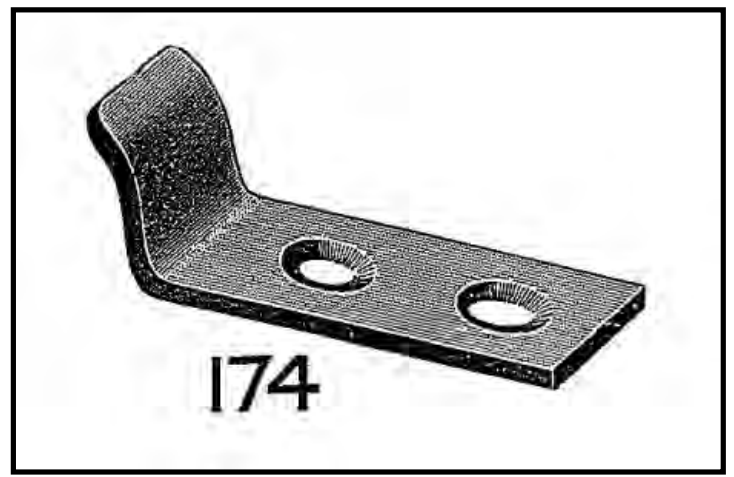

Figure 95. Dowel No. 174, which is a match to Dowel Type 2, as illustrated on page 33 of the 1920s-1930s Langenau Manufacturing Company catalog.

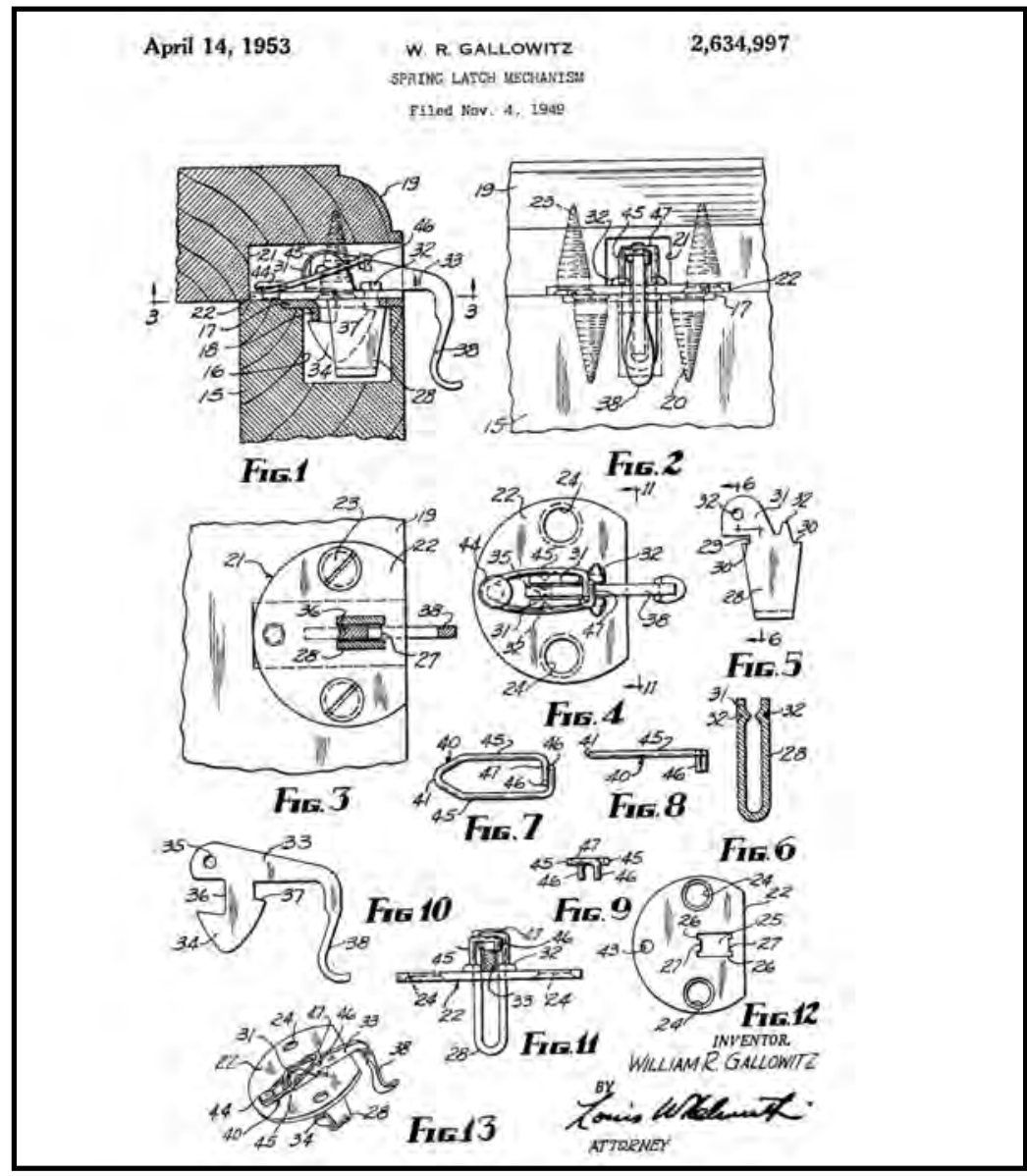

Figure 96. U.S. Utility Patent No. 2634997 assigned to William R. Gallowitz for a spring latch mechanism. 
2" is a front view of the catch; "Fig 3" is a bottom plan view of the catch; "Fig 4" is a top plan view; "Fig 5" is a side view of the "latch guard and mounting ears"; "Fig 6" is a front view of the same; "Figs 7, 8, and 9" are top, side, and end views of the wire spring; "Fig 10" is a side view of the lever plate; "Fig 11" is a front view of the base plate with latch guard and mounting ears; "Fig 12" is a bottom plan view of the base plate; "Fig 13" is the completed catch. The only major difference between this more modern spring and older examples is that most of the earlier devices used thin arched metal plates for the spring rather than wire. Finally, catches must be paired with a matching escutcheon through which the latch guard would pass and the latch would hold when the lid is closed. Catch escutcheons were interchangeable to a certain degree in terms of size and shape, depending on the construction of the burial container and the function and type of catch being used.

The first patent (U.S. Utility Patent No. 275503) for a catch was issued to W. C. Langenau of Cleveland, Ohio, on April 10, 1883 (Figure 97). It was a simple design consisting of a rectangular base plate with latch guard, and a lever connected to the latch plate. Langenau followed with a second similar patent (U.S. Utility Patent No. 281277) in July of the same year. From these early patents, numerous varieties were patented throughout the late nineteenth and early twentieth centuries. As evidenced by the 1953 patent discussed above, catches were still important pieces of hardware in the casket manufacturing industry into the 1950s. It is not currently known whether these same types of catches are used in present-day caskets, or if their popularity has waned at some point since the 1950s.

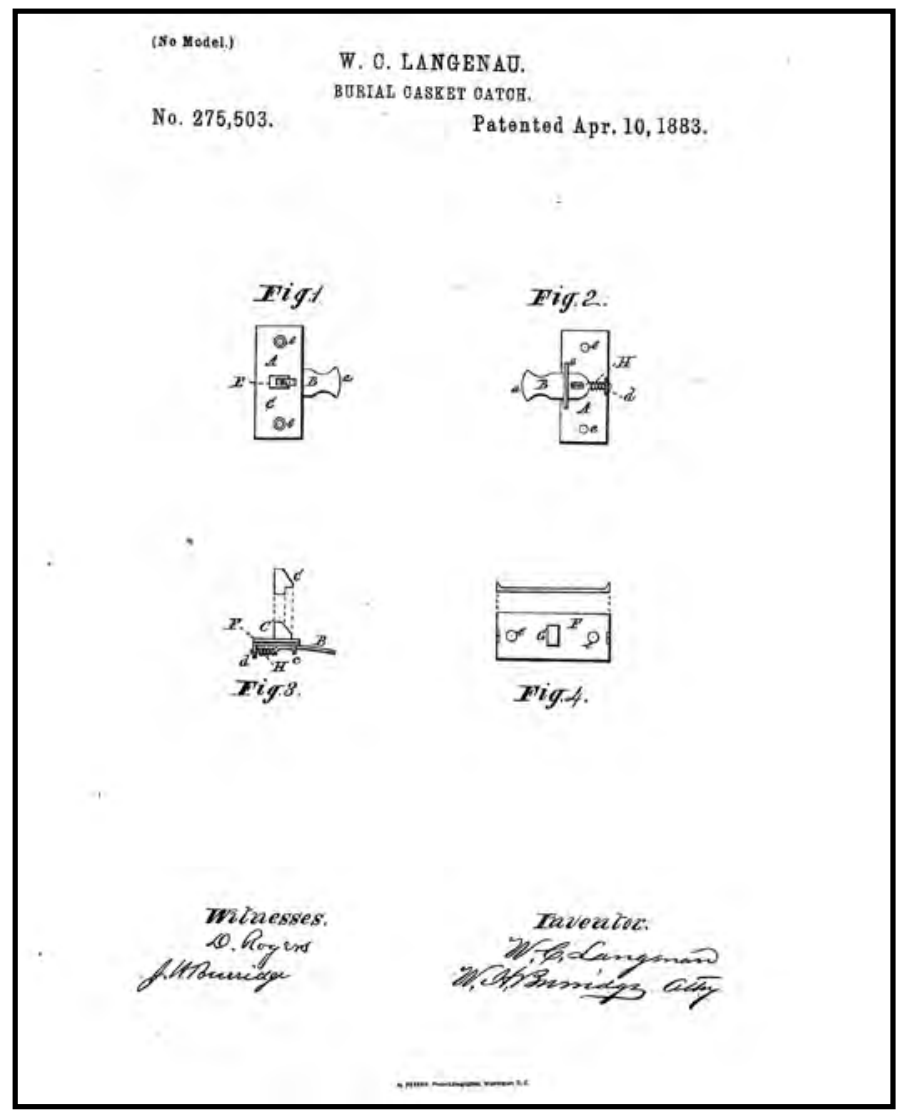

Figure 97. U.S. Utility Patent No. 275,503 assigned in 1883 to W. C. Langenau for a burial casket catch. 


\section{Catch Type 1}

Catch Type 1 (Figure 98) is associated with the 4.5-x-1.5-cm size variant of Catch Escutcheon Type 1 and is represented by three artifacts in two burials (Burials 13 and 14) in New Home Cemetery (see Appendix A). This catch is made of iron. The base plate has two screw holes and is circular with one side clipped. The latch guard is narrow and gently rounded at the top. The lever plate is thin and curved, like a snake's head raised upward, in the same direction as the latch guard. The lever is kept in play by an arched metal spring plate on the bottom of the catch.

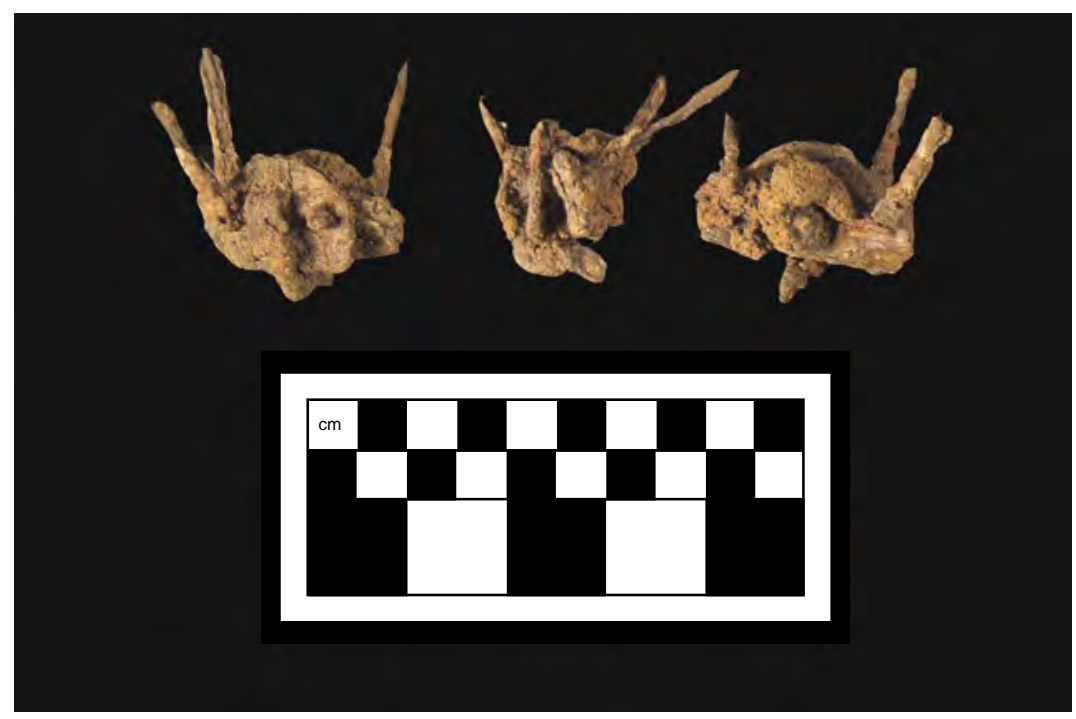

Figure 98. Catch Type 1 and Catch Escutcheon Type 1.

Catch Type 1 has not been identified in any of the historic cemetery excavations used as comparisons in this study, but it has been identified as Catch No. 114 used on burial containers with plush work in the ca. 1920s-1930s Langenau Mfg. Company catalog (Figure 99; see Appendix D). The illustration for Catch No. 114 reveals that this catch was produced by Langenau Mfg. Company of Cleveland, Ohio, which also held the patent for the hardware dated November 26, 1889. A search of patent records revealed that on this date, W. C. Langenau was issued a patent (U.S. Utility Patent No. 416002) for a "coffin fastener" (Figure 100). The catch illustrated in the patent is clearly not the same as Catch Type 1. It more resembles the earlier 1883 Langenau catches. The fact that Catch No. 114 in the later catalog bears this date suggests that it is the catch assembly itself that was the point of interest in the patent and that variation in form was unimportant. Though it is dated to the 1889 patent in the catalog, Catch Type 1 is very similar to that depicted on the illustration page of the U.S. Utility Patent No. 793199, which was issued to Samuel Kelly on June 27, 1905. Though perhaps not an exact match, it is likely an early antecedent of Catch Type 1. This would suggest that Catch Type 1 dates to no earlier than 1905. 


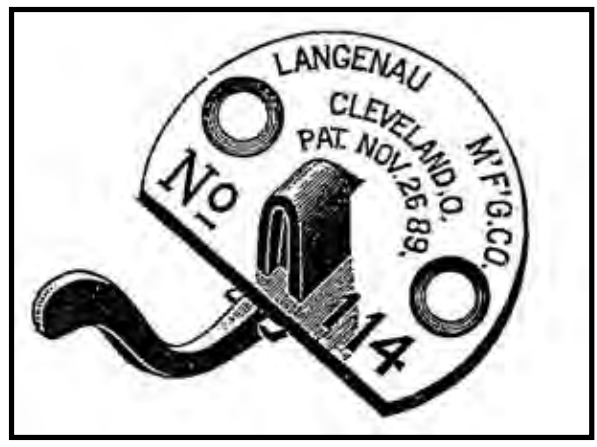

Figure 99. Catch No. 114, which is similar to Catch Type 1, as illustrated on page 8 of the 1920s-1930s Langenau Manufacturing Company catalog.

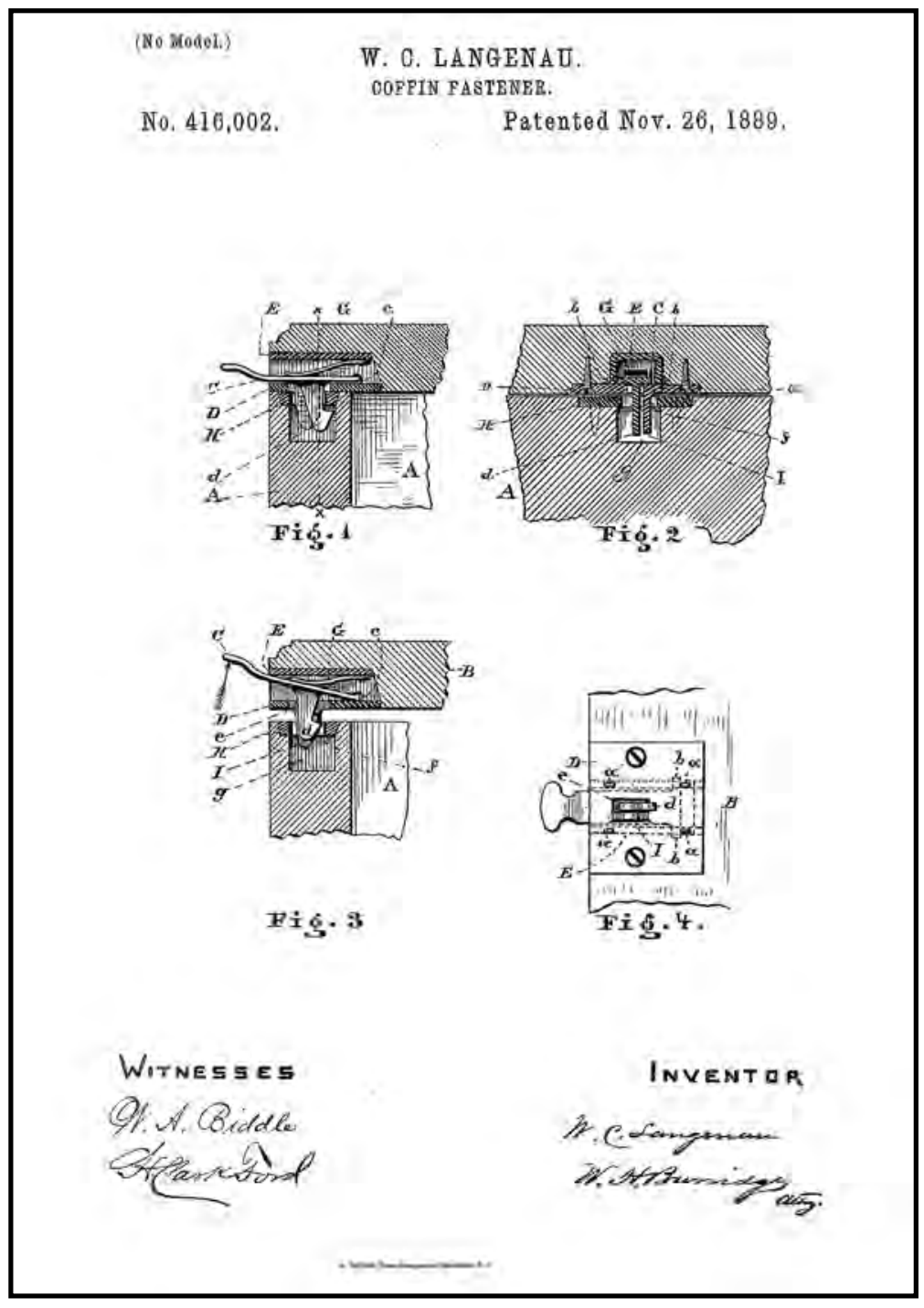

Figure 100. U.S. Utility Patent No. 416002 assigned in 1889 to W. C. Langenau for a coffin fastener. 


\section{Catch Type 2}

Catch Type 2 (Figure 101) is associated with the 3-x-1-cm- and 7-x-2-cm-size variant of Catch Escutcheon Type 1 and is represented by two artifacts in two burials (Burials 2 and 14) in New Home Cemetery (see Appendix A). This catch is made of iron. The base plate has two screw holes and is circular with one side clipped. The latch guard is narrow and gently rounded at the top. The lever plate is thin but broadens out and morphs into a flat T-shaped head. The lever is depressed, extending in the opposite direction from the latch guard. The lever is kept in play by an arched metal spring plate on the bottom of the catch.

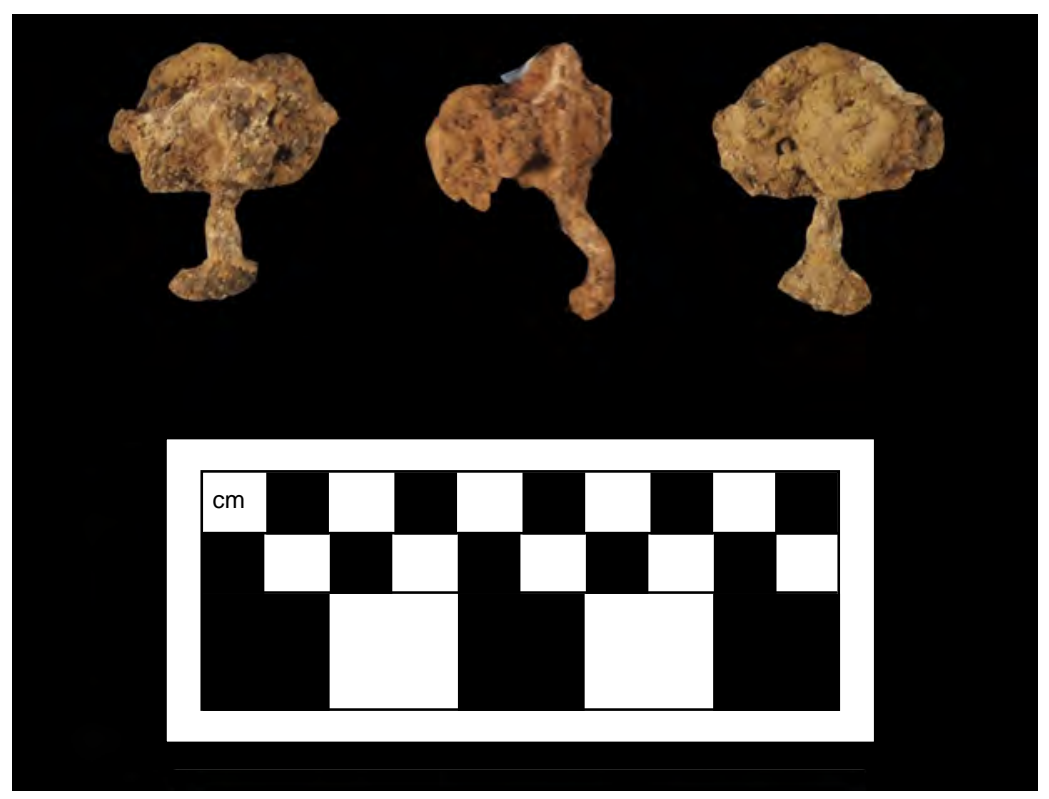

Figure 101. Catch Type 2 and Catch Escutcheon Type 1.

Catch Type 2 has been identified as Catch No. 169 in the ca. 1920s-1930s Langenau Mfg. Company catalog (Figure 102; see Appendix D). The catches were designed for use on burial containers with cloth work; the depressed levers were shaped specifically to conform to recessed top moldings. The illustration for Catch No. 169 also bears the November 26, 1889, Langenau patent date, but as with Catch Type 1, it does not resemble the catch in the patent. No other patents for catches bearing a resemblance to Catch Type 2 were located. It was however, recovered from one historic cemetery excavation in Texas with burial dates between 1907 and 1932.

\section{Catch Type 3}

Catch Type 3 (Figure 103) is associated with the 4.5-x-1.5-cm-size variant of Catch Escutcheon Type 1 and is represented by two artifacts in two burials (Burials 3 and 12) in New Home Cemetery (see Appendix A). This catch is made of iron. The base plate has two screw holes and is circular with one side clipped. The latch guard is narrow and gently rounded at the top. The lever plate is thin but broadens out and morphs into a flat T-shaped head. The lever is extended outward directly in line with the plate, though the head is slightly raised. The lever is kept in play by an arched metal spring plate on the bottom of the catch. 


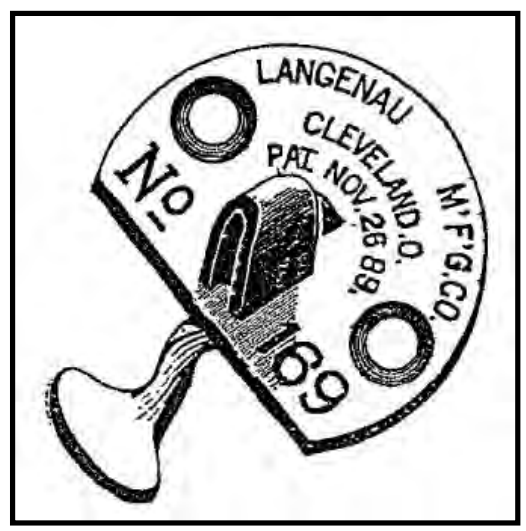

Figure 102. Catch No. 169, which is similar to Catch Type 2, as illustrated on page 20 of the 1920s-1930s Langenau Manufacturing Company catalog.

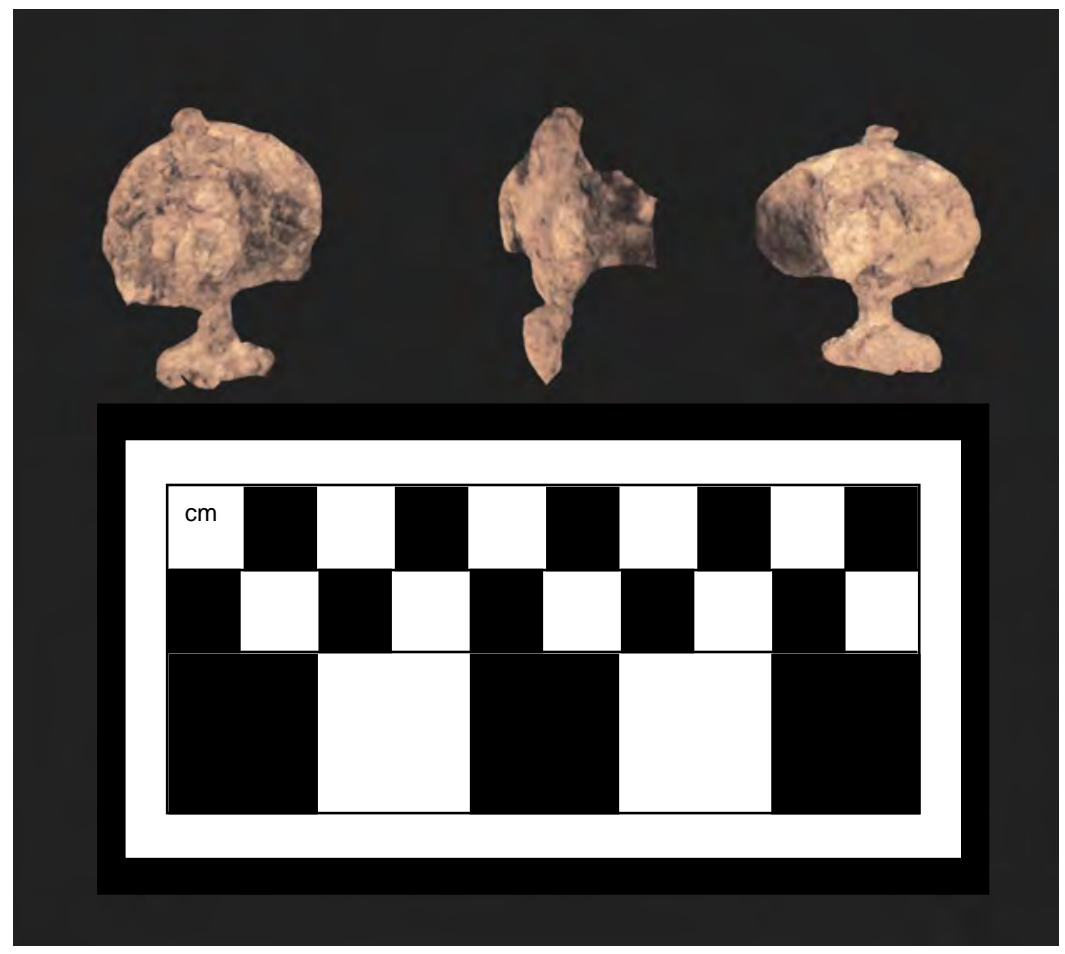

Figure 103. Catch Type 3 and Catch Escutcheon Type 2.

Catch Type 3 has been identified as Catch No. B2 in the ca. 1920s-1930s Langenau Mfg. Company catalog (Figure 104; see Appendix D). The catches were designed for use on burial containers with cloth or plush work. No other patents for catches bearing a resemblance to Catch Type 2 were located. It was, however, recovered from two historic cemetery excavations, one in Texas with burial dates between 1907 and 1932, and the other in Georgia with burial dates between 1921 and 1943. 


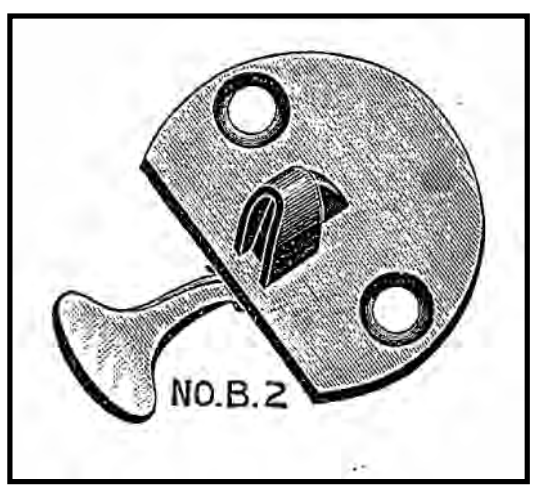

Figure 104. Catch No. B2, which is similar to Catch Type 3, as illustrated on page 10 of the 1920s-1930s Langenau Manufacturing Company catalog.

\section{Catch Type 4}

Catch Type 4 (Figure 105) is associated with the 4-x-2-cm-size variant of Catch Escutcheon Type 1 and is represented by at least one artifact in Coffin 5 in New Home Cemetery (see Appendix A). This catch is made of iron. The base plate has two screw holes and is rectangular in shape. The latch guard and latch appear to have been broken off, but there is a large corroded mass with a squared top on the opposite side of the plate. In its current position, the lever appears to extend straight out from the plate, but a curved portion of the lever arm appears partially in the rusted mass, suggesting that it is indeed curved.

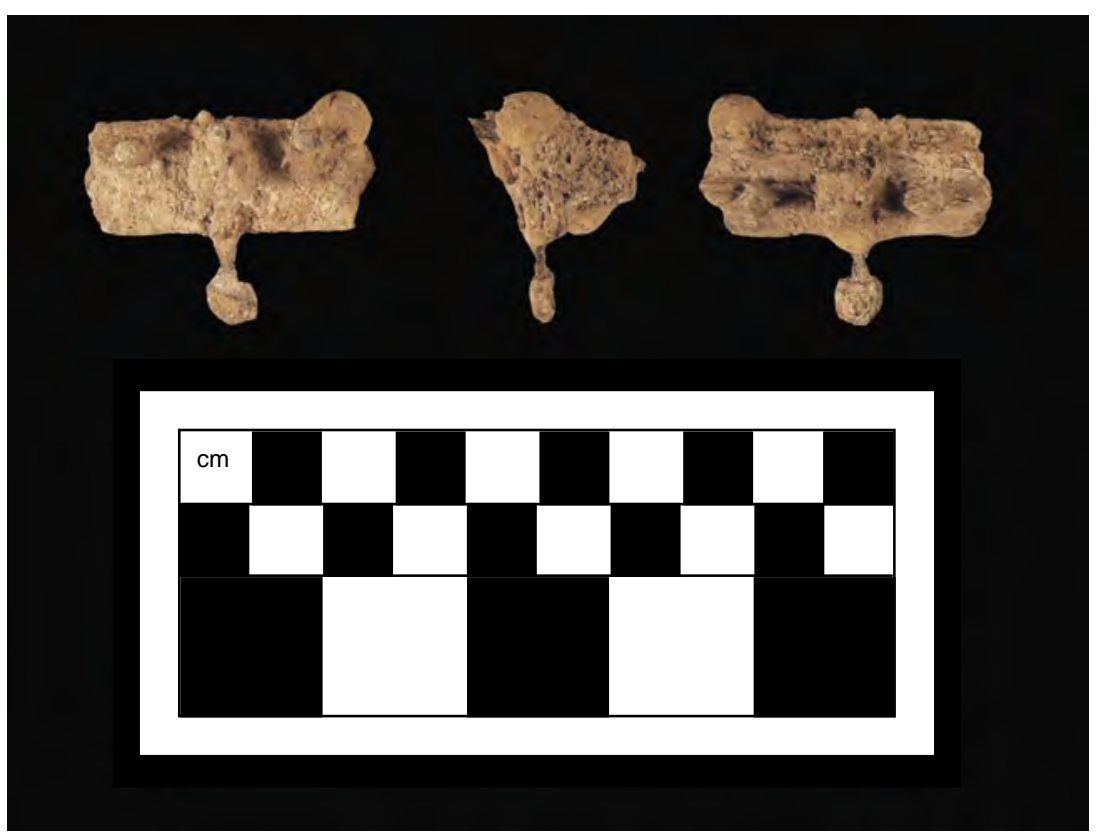

Figure 105. Catch Type 4 and Catch Escutcheon Type 1. 
Catch Type 4 has not been identified in any period catalogs or historic cemetery excavations. It does bear a striking resemblance to the catch illustrated in U.S. Utility Patent No. 2031302 (Figure 106) issued to W. L. Clark on February 18, 1936 (see Appendix D). Clark's catch uses a coiled spring within a squared housing extension instead of the arched spring plate as was common in other catches. It is perhaps this spring and spring housing that is contained within the rusted mass. The possible late introduction of this catch in 1936 is supported by the fact that it does not appear in the ca. 1920s-1930s Langenau catalog.

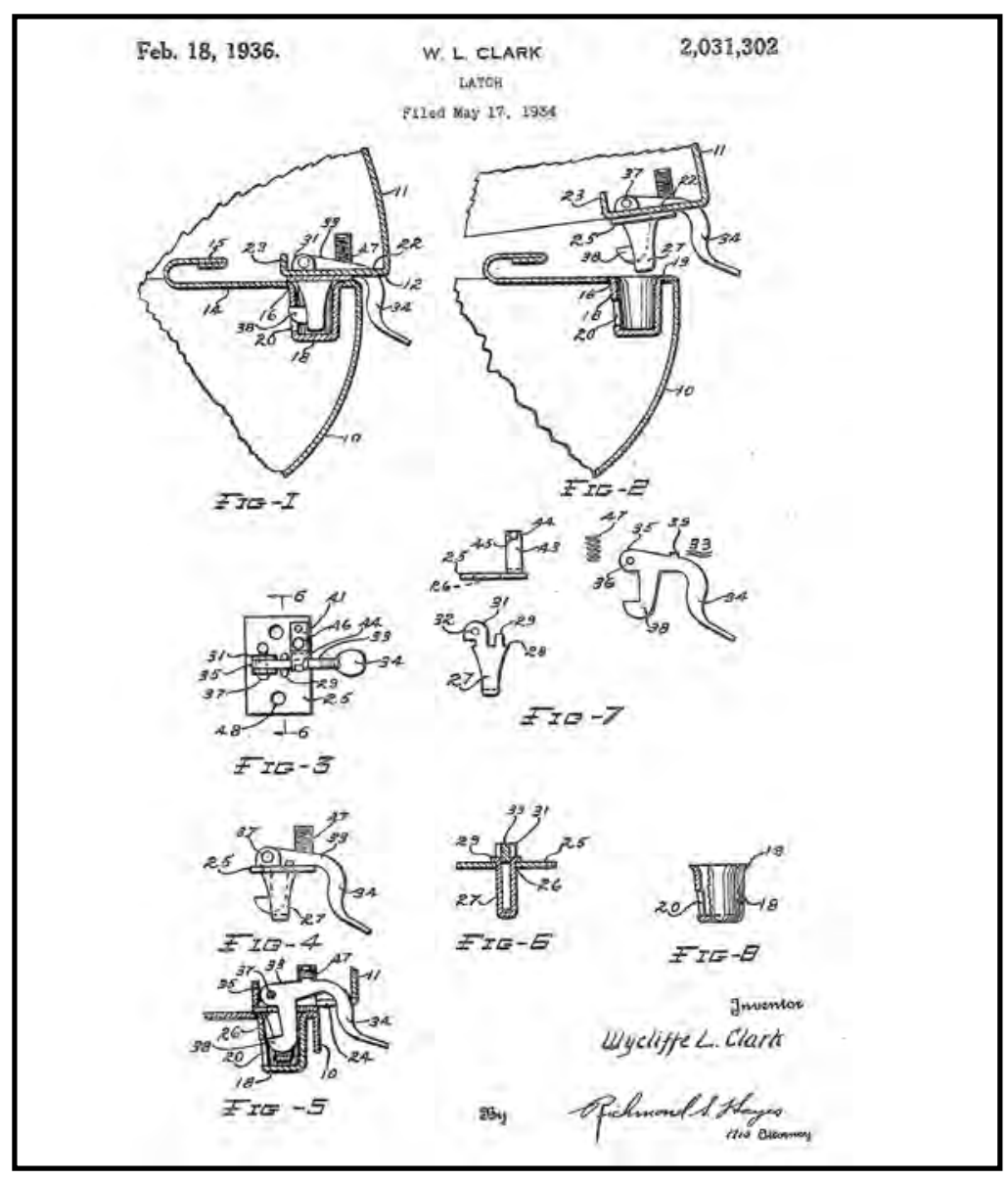

Figure 106. U.S. Utility Patent No. 2031302 assigned in 1936 to W. L. Clark for a spring catch.

\section{Catch Type 5}

Catch Type 5 (Figure 107) is associated with the 4-x-2-cm-size variant of Catch Escutcheon Type 1 and is represented by one artifact in Burial 3 in New Home Cemetery (see Appendix A). This catch is made of iron. The base plate has two screw holes and is rectangular in shape. The latch guard is narrow and gently rounded at the top. The lever plate is thin but flattens out into a teardrop-shaped head. The lever is slightly depressed, extending in the opposite direction as the latch guard. The mechanism for keeping the lever in play is not apparent. 


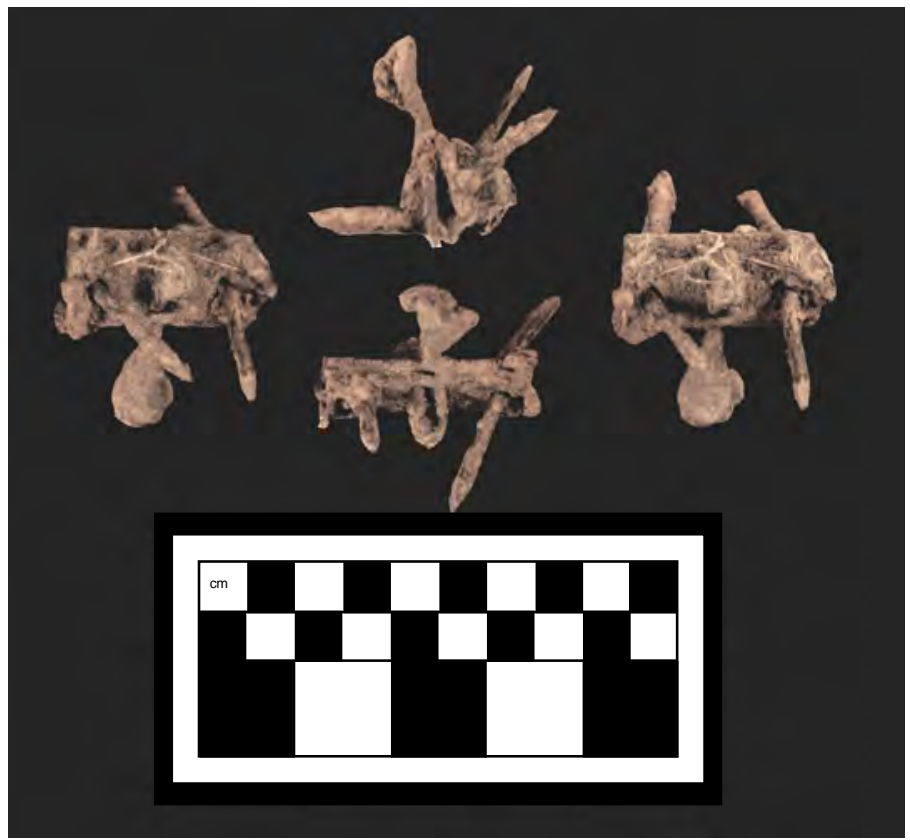

Figure 107. Catch Type 5 and Catch Escutcheon Type 1.

Catch Type 5 has not been identified in any period catalogs or historic cemetery excavations. Additionally, no exact patent matches have been located. One patent for a catch has been identified with this same teardrop-shaped head; U.S. Utility Patent No. 2504716 was issued to Donald Morand on April 18, 1950 (Figure 108). Catch Type 5 has a rectangular base plate whereas Morand's catch has a one-sided clipped plate. Also, Morand's lever head is depressed to a greater degree than is the lever for Catch Type 5. The mechanism for Morand's spring appears to be a small bent wire spring contained completely within the ear mounting, which is why it might not be evident through the ferrous corrosion on Catch Type 5 . The possible late introduction of this catch in 1950 is supported by the fact that it does not appear in the ca. 1920s1930s Langenau catalog.

\section{Catch Type 6}

Catch Type 6 is a catchall category for those artifacts that are fragments or corroded to the degree that a specific type cannot be determined. The four artifacts in this group are associated with the 4-x-1.5-cm- and a 3.23-x-2.85-cm-size variant of Catch Escutcheon Type 1 and were located in three burials (Burials 9 and 18, as well as Coffin 5) in New Home Cemetery (see Appendix A). These catches are made of iron. The base plate has two screw holes and is generally circular with one side clipped. There is heavy corrosion on all pieces, and none still possess their lever arms. In one instance (Figure 109) (Burial 9), the arched metal spring is evident through corrosion and is shared by Catch Types 1, 2, and 3 and many others depicted in the ca. 1920s-1930s Langenau catalog. 


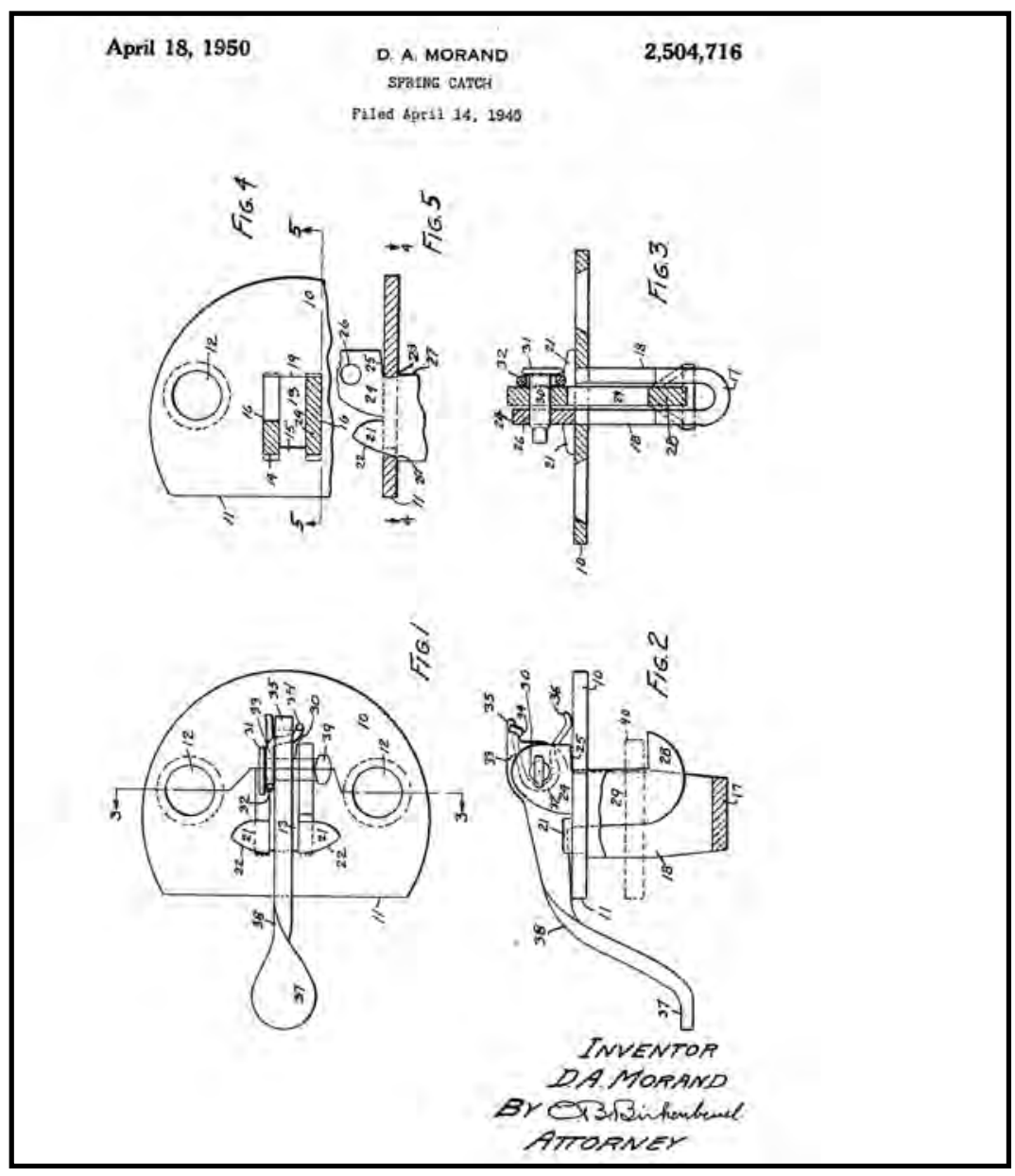

Figure 108. U.S. Utility Patent No. 2,504,716 assigned in 1950 to D. A. Morand for a spring catch.

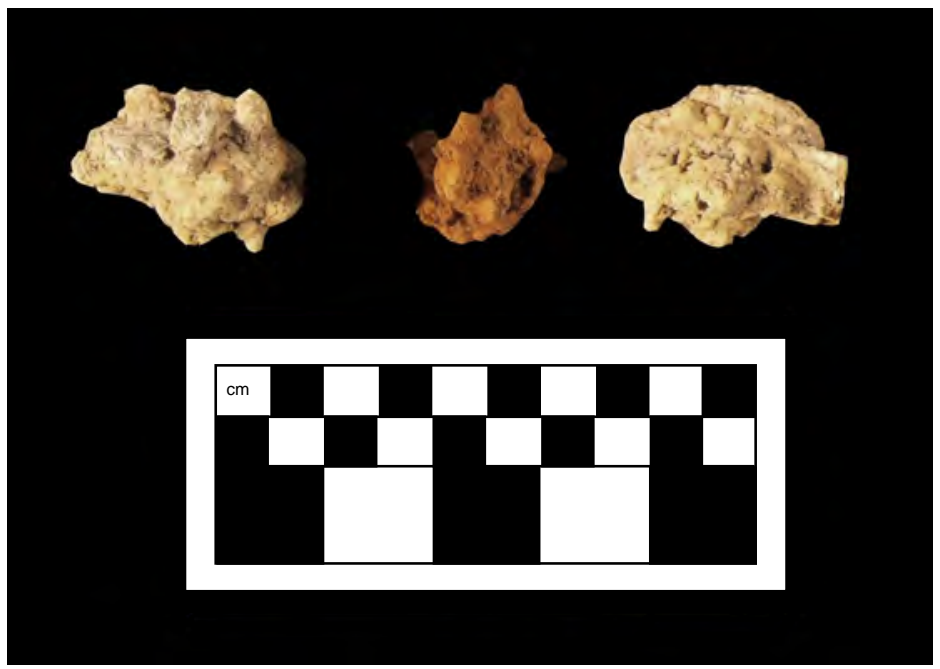

Figure 109. Catch Type 6. 


\section{Catch Escutcheon Type 1}

Catch Escutcheon Type 1 (see figures for any of the above catches except Catch Type 3) is represented by 12 artifacts from eight burials (Burials 2, 3, 9, 12, 13, 14, and 18, as well as Coffin 5) in New Home Cemetery. These are rectangular escutcheons used to match with various sorts of catches for plush or cloth work. This is a catchall category for all rectangular escutcheons recovered in association with Catch Types 1-6 (see Appendix A). These escutcheons are made of iron and have two screw holes. There are a number of size variants included in this escutcheon type: $7-\mathrm{x}-2 \mathrm{~cm}, 4.5-\mathrm{x}-1.5 \mathrm{~cm}, 4-\mathrm{x}-2 \mathrm{~cm}, 4-\mathrm{x}-1.5 \mathrm{~cm}, 3-\mathrm{x}-1 \mathrm{~cm}$, and 3.23-x-2.85 cm. No specific patents are known to exist for this escutcheon type, and it is not clear whether these have specifically been recovered in historic cemetery excavations (though it is extremely likely). Many types of rectangular escutcheons that are interchangeable with Catch Types 1-3 specifically are advertised in the ca. 1920s-1930s Langenau catalog (Figure 110). The New Home Cemetery rectangular catch escutcheons could match to any one of the illustrated escutcheons, but because no side points were observed on the archaeological examples, it is possible that most are size variants of Langenau No. 21 escutcheon.

\section{Catch Escutcheon Type 2}

Catch Escutcheon Type 2 refers to an escutcheon that is generally circular with one side clipped. It is made of iron and has two screw holes and a rectangular central hole. Only one such escutcheon was identified in New Home Cemetery, Burial 3, in association with Catch Type 3 (see Appendix A). There are no specific patents known to exist for this escutcheon type, but it does appear as No. 30 (Figure 111) in the ca. 1920s-1930s Langenau Manufacturing Company catalog (see Appendix D).

\section{Separable Stop Hinge Assemblies}

Separable stop hinges are those devices whereby the arm of the hinge plate is inserted into a recess below a matching escutcheon that can hold the tip of the arm when in the open position (Figure 112). The principal advantage of the separable stop hinge was that when closed the hinge would work as a fastener preventing movement of the lid, and when open, the lid could be secured but also quickly removed from the casket body simply by offering sufficient lifting force so as to release the hinge arm from the escutcheon.

The earliest patent that could be located that illustrates a version of a stop hinge was U.S. Patent 382160, which was granted to Edward and Britain Holmes of Buffalo, New York, on May 1, 1888 (Figure 113). The inventors admit knowledge of other "hook-catches in combination with slotted plates adapted to receive them" being used previously for hinging casket lids, so the history of stop hinges most assuredly predates this patent.

\section{Stop Hinge Type 1}

Stop Hinge Type 1 (Figure 114) is represented by one artifact from Burial 19 in New Home Cemetery (see Appendix A). This hinge is associated with Stop Hinge Escutcheon Type 1. The rectangular base plate of Stop hinge Type 1 has four screw holes that are positioned near each 


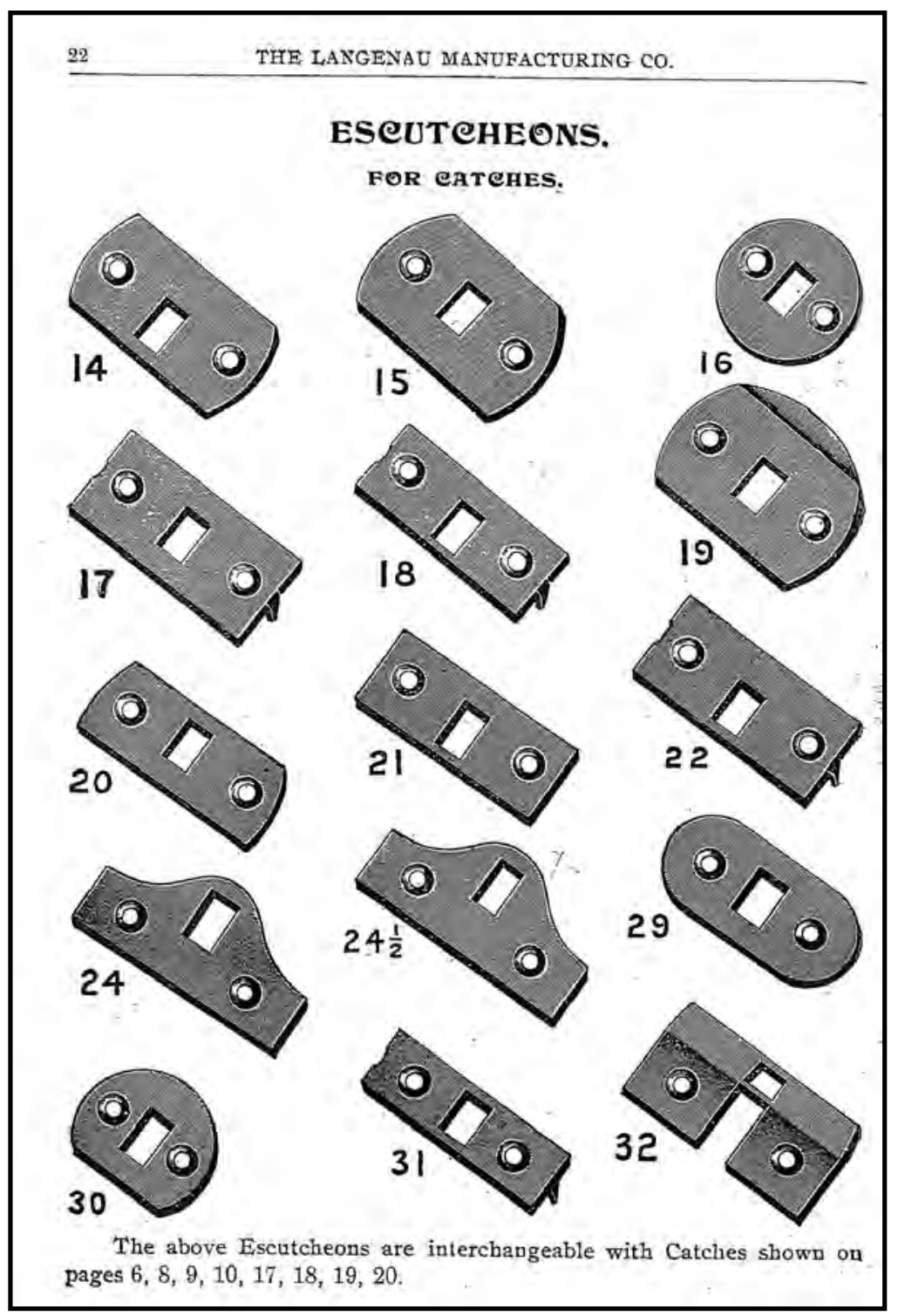

Figure 110. Page 22 of the 1920s-1930s Langenau Manufacturing Company catalog showing rectangular escutcheons similar to Catch Escutcheon Type 1.

corner. The hinge arm extends from the center of the plate with an undulating curve on one edge and a sharp step midway up the other side. It eventually ends at a finger-like terminus over the edge of the base plate.

No patent specific to this type of stop hinge was located. It has, however, been recovered in one cemetery excavation in Georgia in a burial dated to ca. 1921 (see Appendix D). Additionally, it appears as No. 341/2 in the ca. 1920s-1930s Langenau Mfg. Company catalog (Figure 115). The Langenau catalog reveals that this type of hinge was used for hinging heavy tops to shells. 


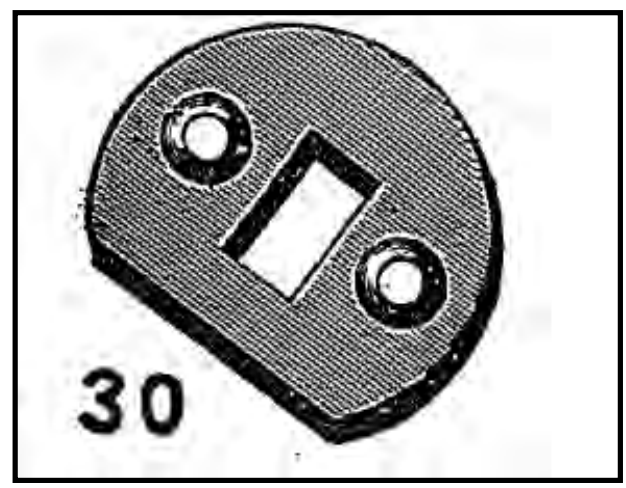

Figure 111. Escutcheon No. 30, which is similar to Catch Escutcheon Type 2, as illustrated on page 22 of the 1920s1930s Langenau Manufacturing Company catalog.

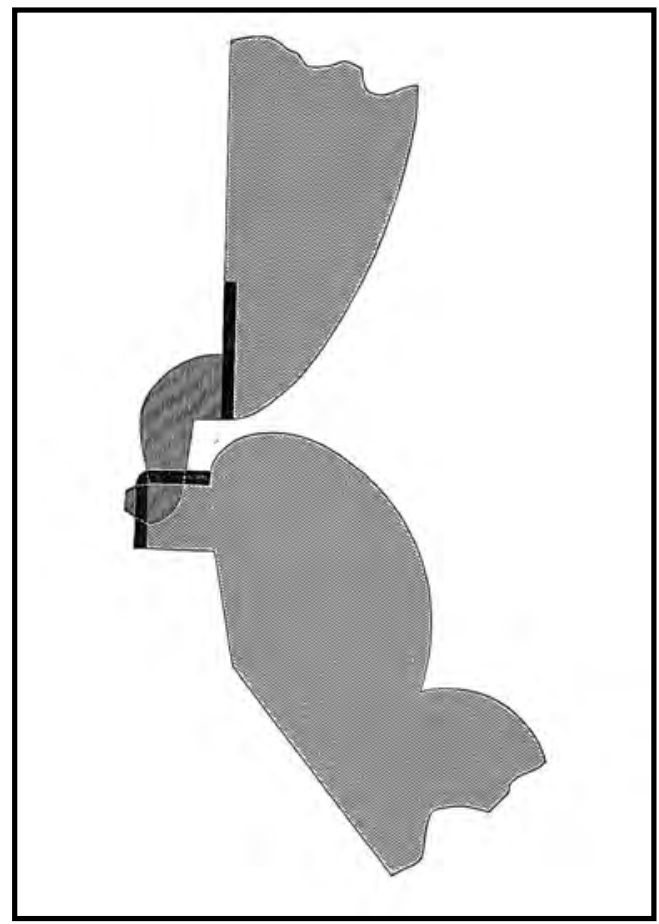

Figure 112. Illustration of a separable stop hinge assembly on page 65 of the 1920s-1930s Langenau Manufacturing Company catalog.

\section{Stop Hinge Type 2}

Stop Hinge Type 2 is represented by four artifacts from four burials (Burials 8, 13, 18, and 19) in New Home Cemetery (see Appendix A). This hinge is associated with Stop Hinge Escutcheon Type 1 in Burials 13 and 19 (Figure 116), and is associated with Stop Hinge Escutcheon Type 2 in Burials 8 and 18 (Figure 117). This hinge is nearly identical in shape to Stop Hinge Type 1 with one minor difference: two of the screw holes are placed at the corners but the other two are offset toward the centerline. 
(IIO Xodel.) E. \& B. HOLMES.
PABTENER FOR BURIAL OASKETS.

No. 382,160. Patented May 1, 1888 .

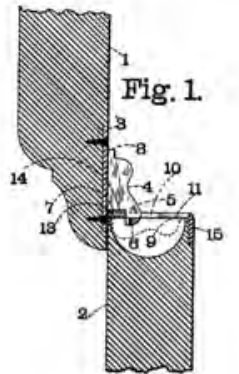

Fig. 2.

Fig. 3.

Fig. 4.

${ }^{2} \overbrace{}^{2}=0$

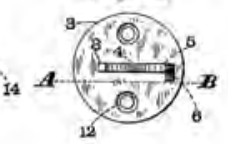

Fig. 5.

$\frac{a+1 .}{i}$
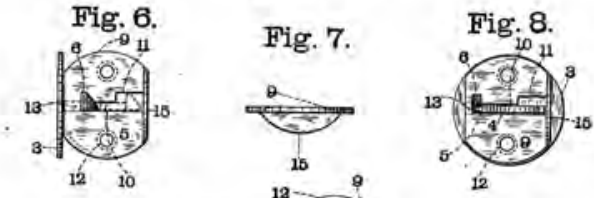

Fig. 9 ,

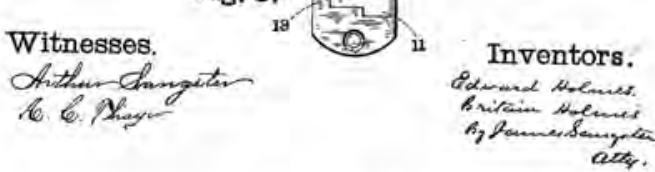

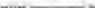

Figure 113. U.S. Utility Patent No. 382160 assigned in 1888to Edward and Britain Holmes for a burial casket fastener.
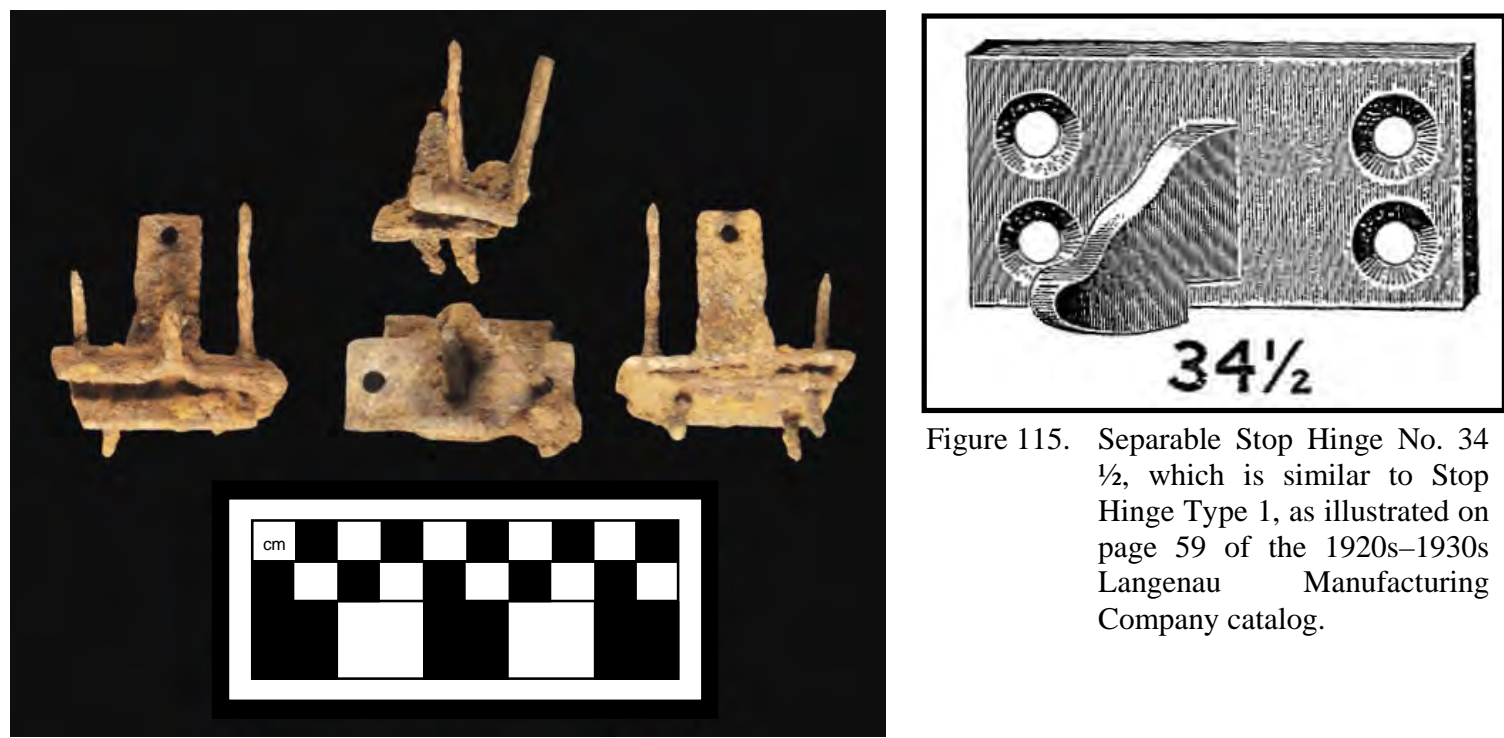

Figure 115. Separable Stop Hinge No. 34 $1 / 2$, which is similar to Stop Hinge Type 1, as illustrated on page 59 of the 1920s-1930s Langenau Manufacturing Company catalog.

Figure 114. Stop Hinge Type 1 and Stop Hinge Escutcheon Type 1. 


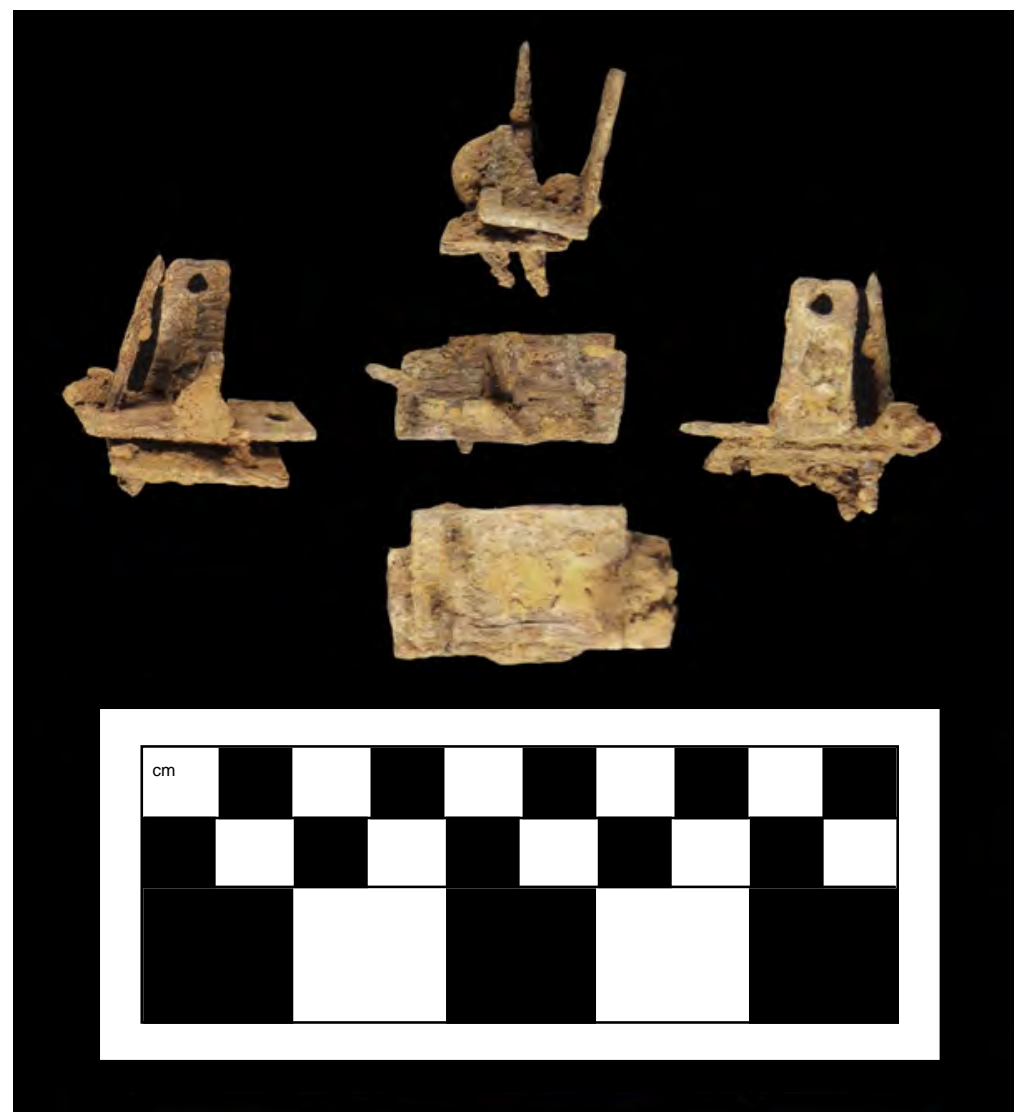

Figure 116. Stop Hinge Type 2 and Stop Hinge Escutcheon Type 1.

No patent specific to this type of stop hinge was located, nor has it been identified in any historic cemetery excavation. It does, however, appear as No. 34 in the ca. 1920s-1930s Langenau Mfg. Company catalog (Figure 118) (see Appendix D). Much like Stop Hinge Type 1, this hinge would have also been used for hinging heavy tops to shells.

\section{Stop Hinge Type 3}

Stop Hinge Type 3 (Figure 119) is represented by one artifact from Burial 17 in New Home Cemetery (see Appendix A). This hinge is associated with Stop Hinge Escutcheon Type 3. The base plate of Stop Hinge Type 3 is generally circular with one side clipped, and it has two screw holes at each side along the clipped margin. The hinge arm extends from the center between the two screws. It has an undulating curve on one edge and a sharp step midway up the other side. The tip of the arm is broken, but it should terminate roughly above the center of the base plate.

No patent specific to this type of stop hinge was located, nor has it been identified in any historic cemetery excavation. It does, however, appear as No. 214 in the ca. 1920s-1930s Langenau Mfg. Company catalog (Figure 120; see Appendix D). Langenau reveals that this type of hinge should be used for "hinging panels to top moldings and top moldings to shells." 

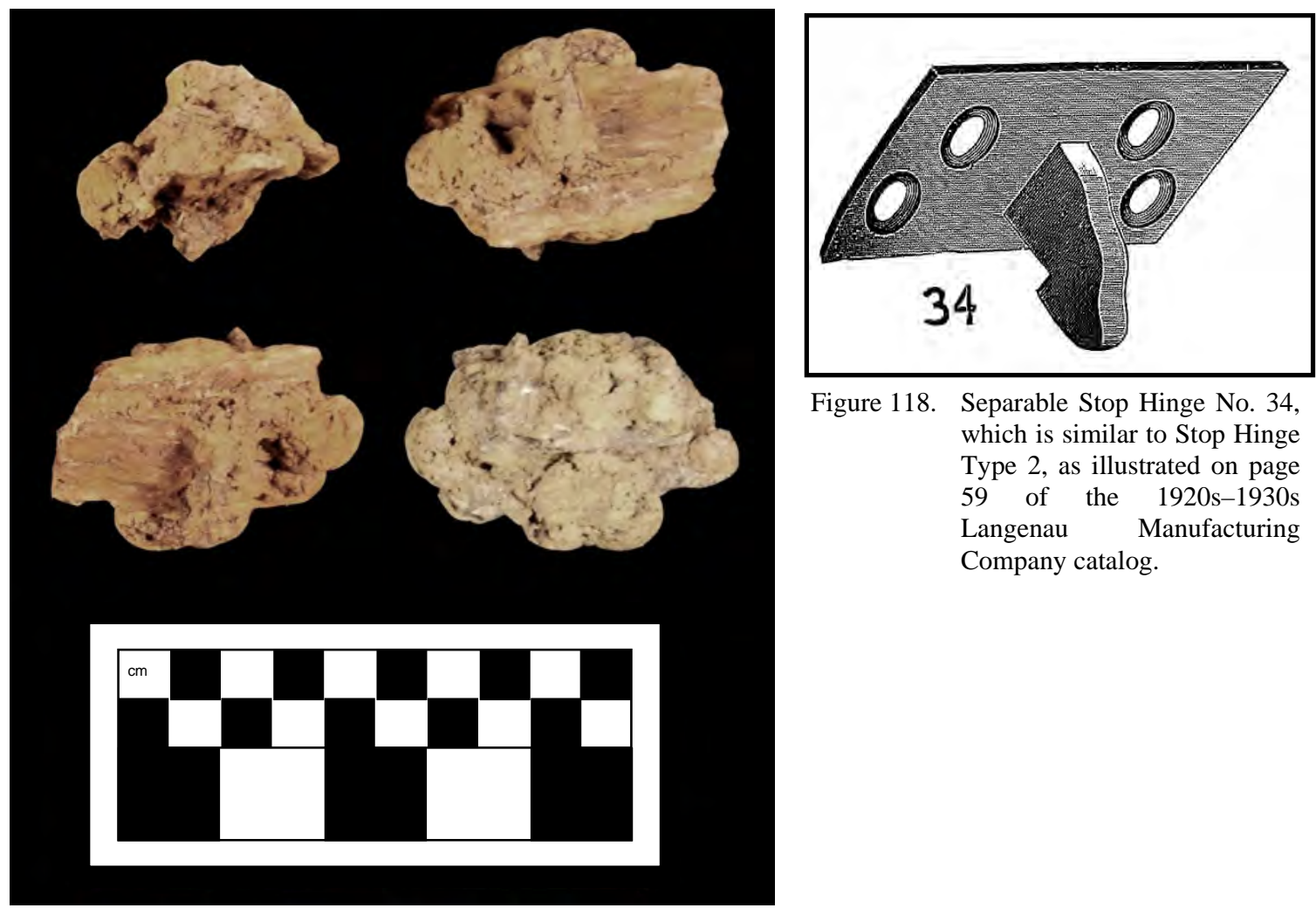

Figure 118. Separable Stop Hinge No. 34, which is similar to Stop Hinge Type 2, as illustrated on page 59 of the 1920s-1930s Langenau Manufacturing Company catalog.

Figure 117. Stop Hinge Type 2 and Stop Hinge Escutcheon Type 2.
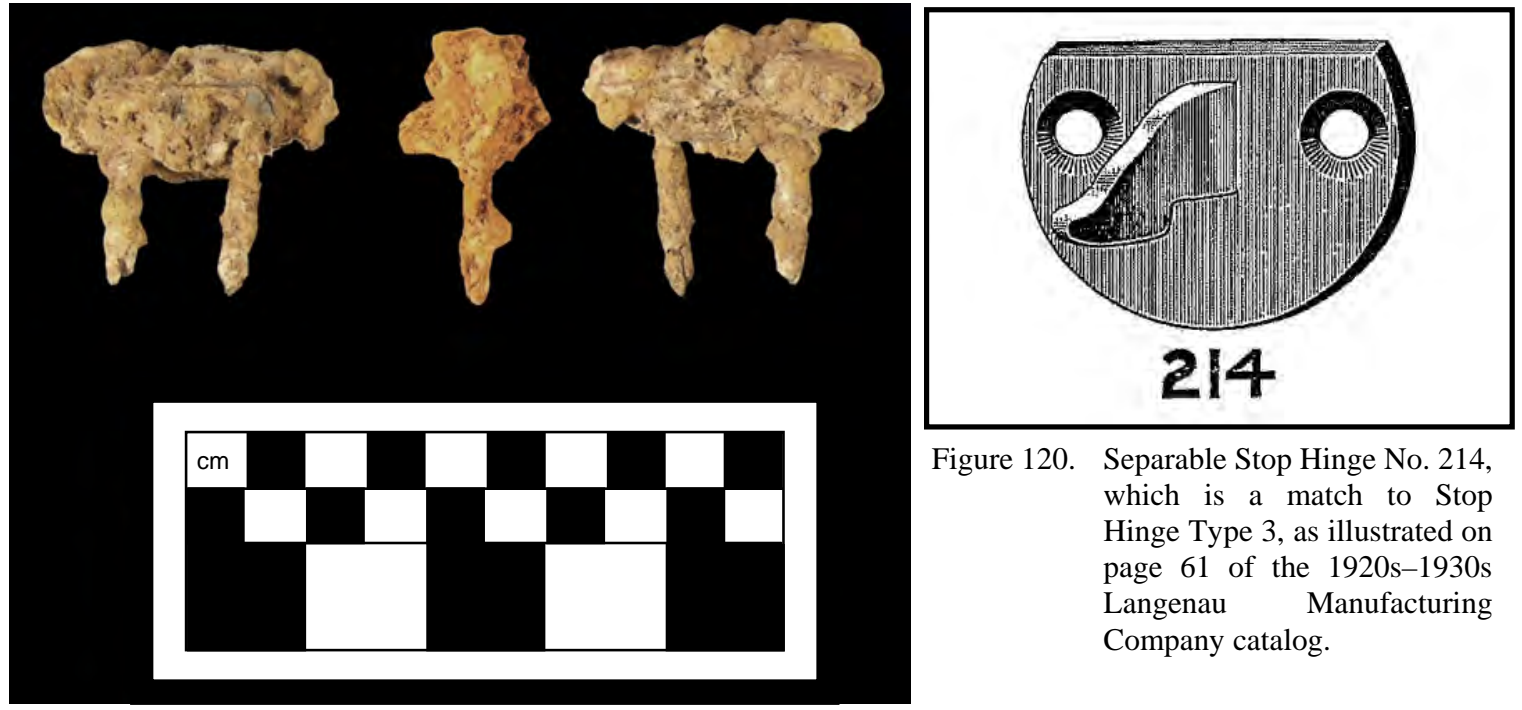

Figure 120. Separable Stop Hinge No. 214, which is a match to Stop Hinge Type 3, as illustrated on page 61 of the 1920s-1930s Langenau Manufacturing Company catalog.

Figure 119. Stop Hinge Type 3 and Stop Hinge Escutcheon Type 3. 


\section{Stop Hinge Type 4}

Stop Hinge Type 4 (Figure 121) is represented by one artifact from Burial 14 in New Home Cemetery (see Appendix A). This hinge is associated with Stop Hinge Escutcheon Type 4. The base plate of Stop Hinge Type 3 is generally circular with one side clipped; it has three screw holes with two positioned at each side of the plate parallel to the clipped edge, and the other hole placed on the side opposite the clipped edge. The hinge arm extends from the center between the three screw holes. It has an undulating curve on one edge and a sharp step midway up the other side. The arm ends in a finger-like projection terminating beyond the clipped edge of the base plate.

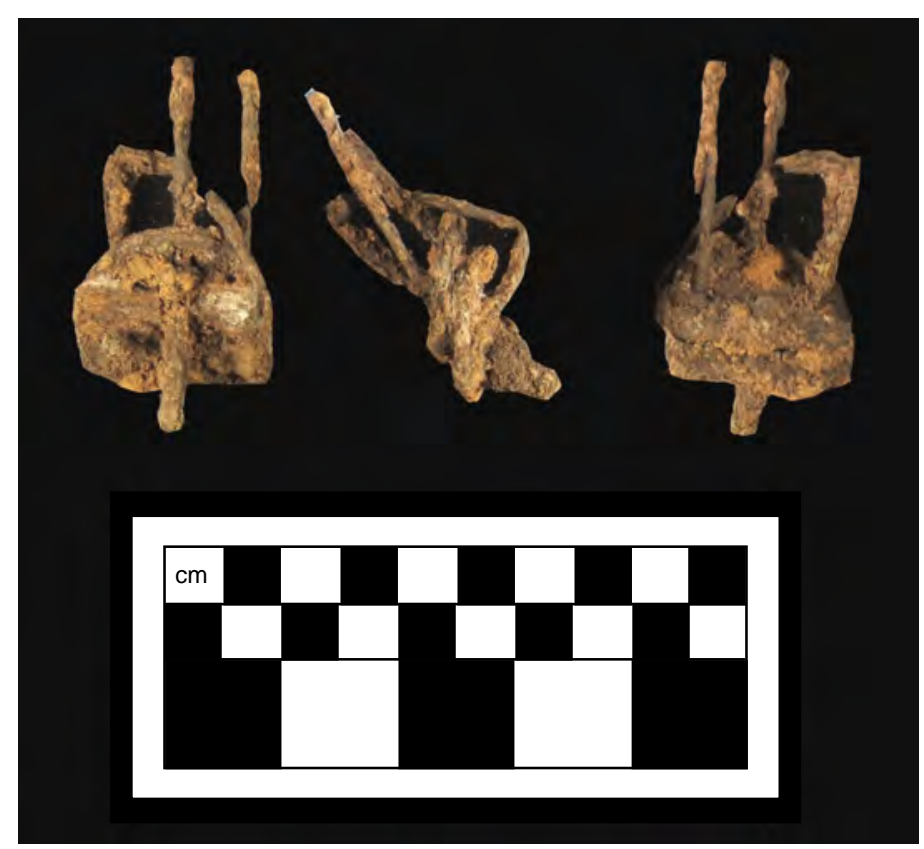

Figure 121. Stop Hinge Type 4 and Stop Hinge Escutcheon Type 4.

No patent specific to this type of stop hinge was located, nor has it been identified in any historic cemetery excavation. It does, however, appear as No. 148 in the ca. 1920s-1930s Langenau Mfg. Company catalog (Figure 122; see Appendix D). Langenau reveals that this type of hinge should be used for "hinging panels to top moldings and top moldings to shells."

\section{Stop Hinge Type 5}

Stop Hinge Type 5 is represented by two artifacts from Burial 18 in New Home Cemetery (see Appendix A). This hinge is associated with Stop Hinge Escutcheon Type 5 (Figure 123) and Stop Hinge Escutcheon Type 6 (Figure 124). The base plate of Stop Hinge Type 5 is rectangular with three screw holes, one at each end, and one in the middle near the top edge. The hinge arm extends from the lower edge of the middle screw hole and sharply descends. It looks like a bent leg attached to the base plate just above the knee. The hinge arm terminates in a "foot" (if the leg analogy continues) that has a rounded bottom. The front of the leg is gently curved until it sharply turns to form the foot, whereas the back of the leg is straight until it curves to form the bottom of the foot. 


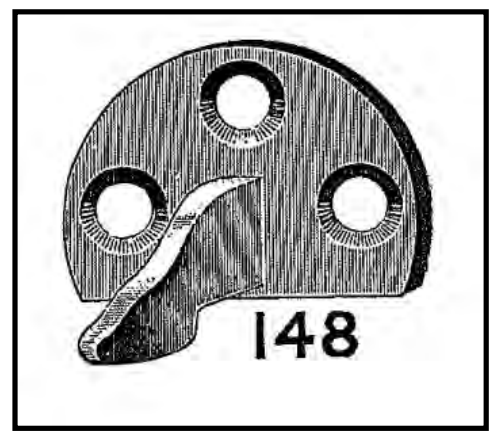

Figure 122. Separable Stop Hinge No. 148, which is a match to Stop Hinge Type 4, as illustrated on page 61 of the 1920s-1930s Langenau Manufacturing Company catalog.

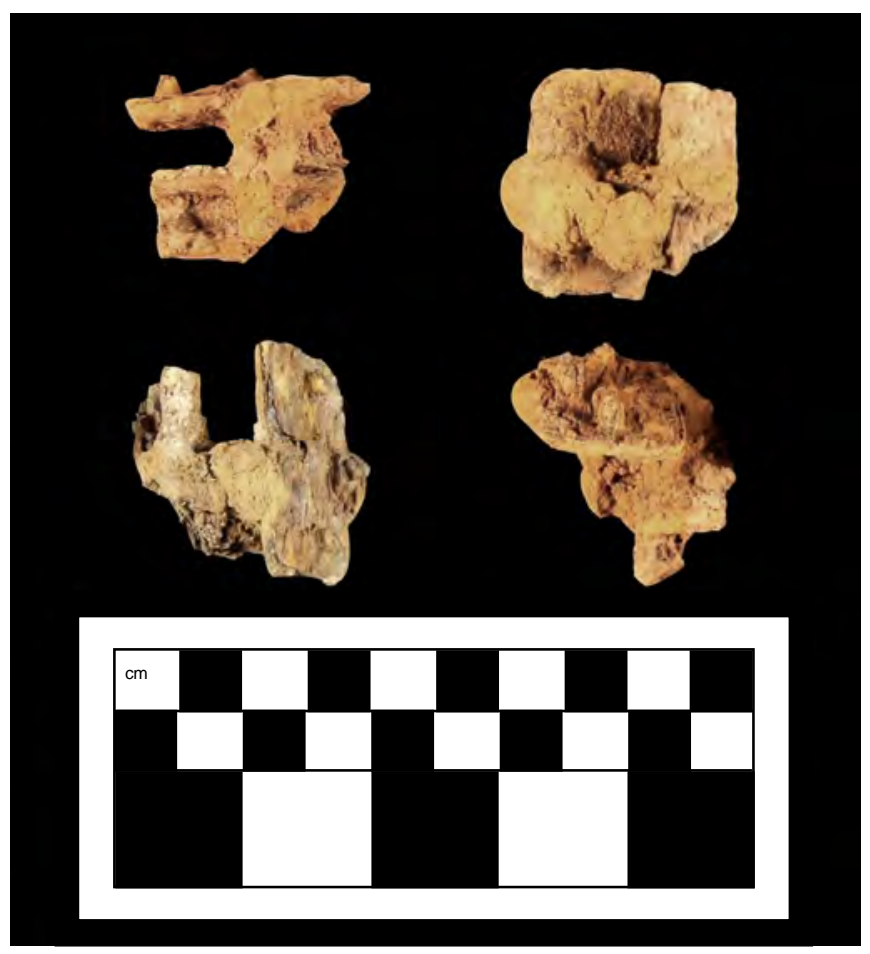

Figure 123. Stop Hinge Type 5 and Stop Hinge Escutcheon Type 5.

No patent specific to this type of stop hinge was located, nor has it been identified in any historic cemetery excavation. It does, however, appear as No. 33 in the ca. 1920s-1930s Langenau Mfg. Company catalog (Figure 125; see Appendix D). Langenau states that this type of hinge should be used for "hinging heavy panels to recessed top moldings." 

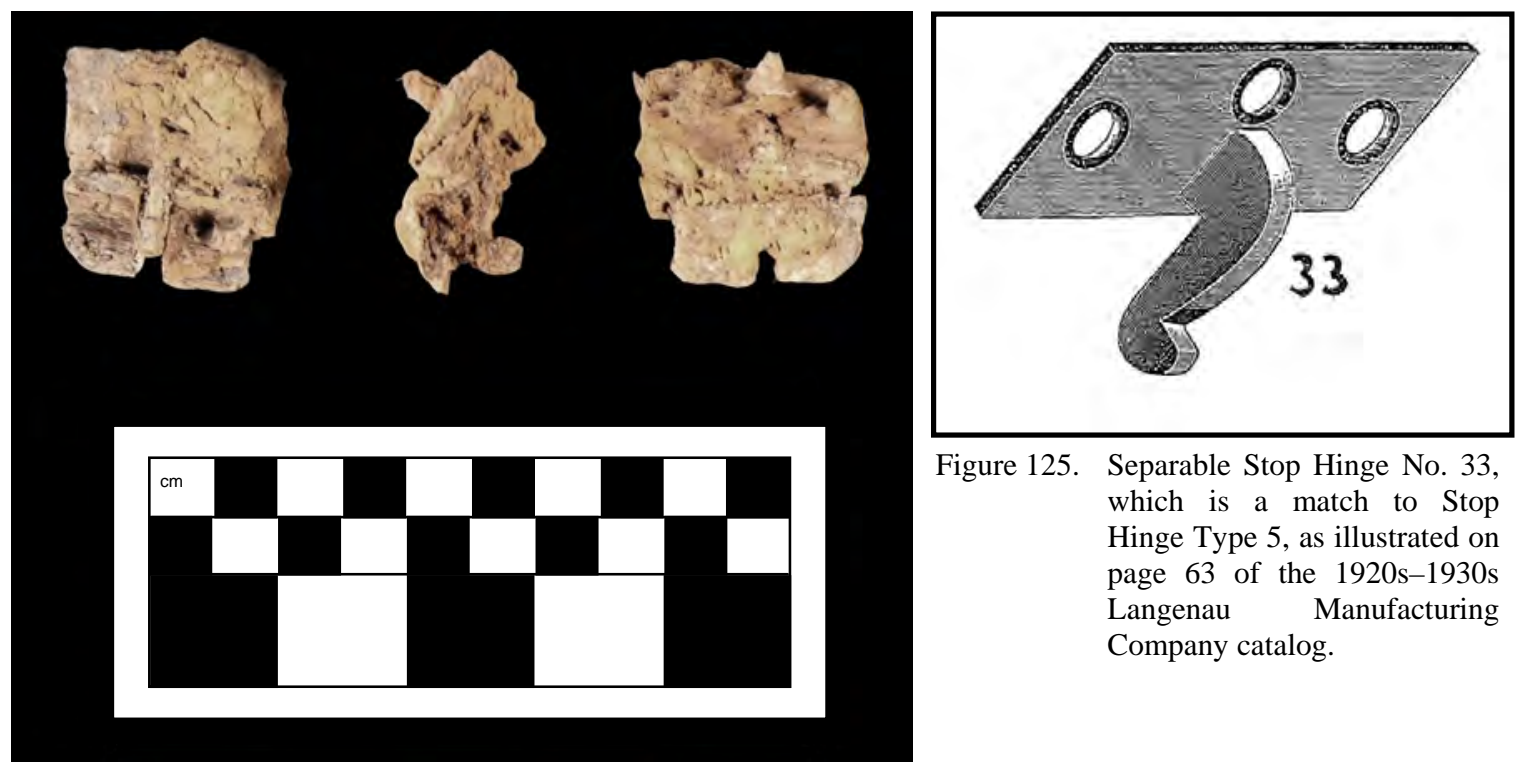

Figure 125. Separable Stop Hinge No. 33, which is a match to Stop Hinge Type 5, as illustrated on page 63 of the 1920s-1930s Langenau Manufacturing Company catalog.

Figure 124. Stop Hinge Type 5 and Stop Hinge Escutcheon Type 6.

\section{Stop Hinge Type 6}

Stop Hinge Type 6 (Figure 126) is represented by three artifacts from Burial 5 in New Home Cemetery (see Appendix A). This hinge is associated with Stop Hinge Escutcheon Type 3. The base plate of Stop Hinge Type 3 is generally circular with two parallel clipped sides, and it has two screw holes at each side along the curved margin. The hinge arm extends from the center between the two screws. It has an undulating curve on one edge and a sharp step midway up the other side. The arm terminates in a finger-like projection beyond the clipped margin of the base plate.

No patent specific to this type of stop hinge was located, nor has it been identified in any historic cemetery excavation. It does, however, appear as No. 28 in the ca. 1920s-1930s Langenau Mfg. Company catalog (Figure 127; see Appendix D). Langenau reveals that this type of hinge should be used for "hinging panels to top moldings and top moldings to shells."

\section{Stop Hinge Escutcheon Type 1}

Stop Hinge Escutcheon Type 1 is represented by three artifacts from two burials (Burials 13 and 19) in New Home Cemetery (see Appendix A). It is associated with Stop Hinge Type 2 in Burial 13, and Stop Hinge Types 1 and 2 in Burial 19. These are ferrous, rectangular, long arm, angled brackets with the base segment containing two screw holes at each end and the arm containing one screw hole. It also contains a central rectangular hole in the base plate and an arched segment at the end of the central hole opposite the arm of the bracket. 

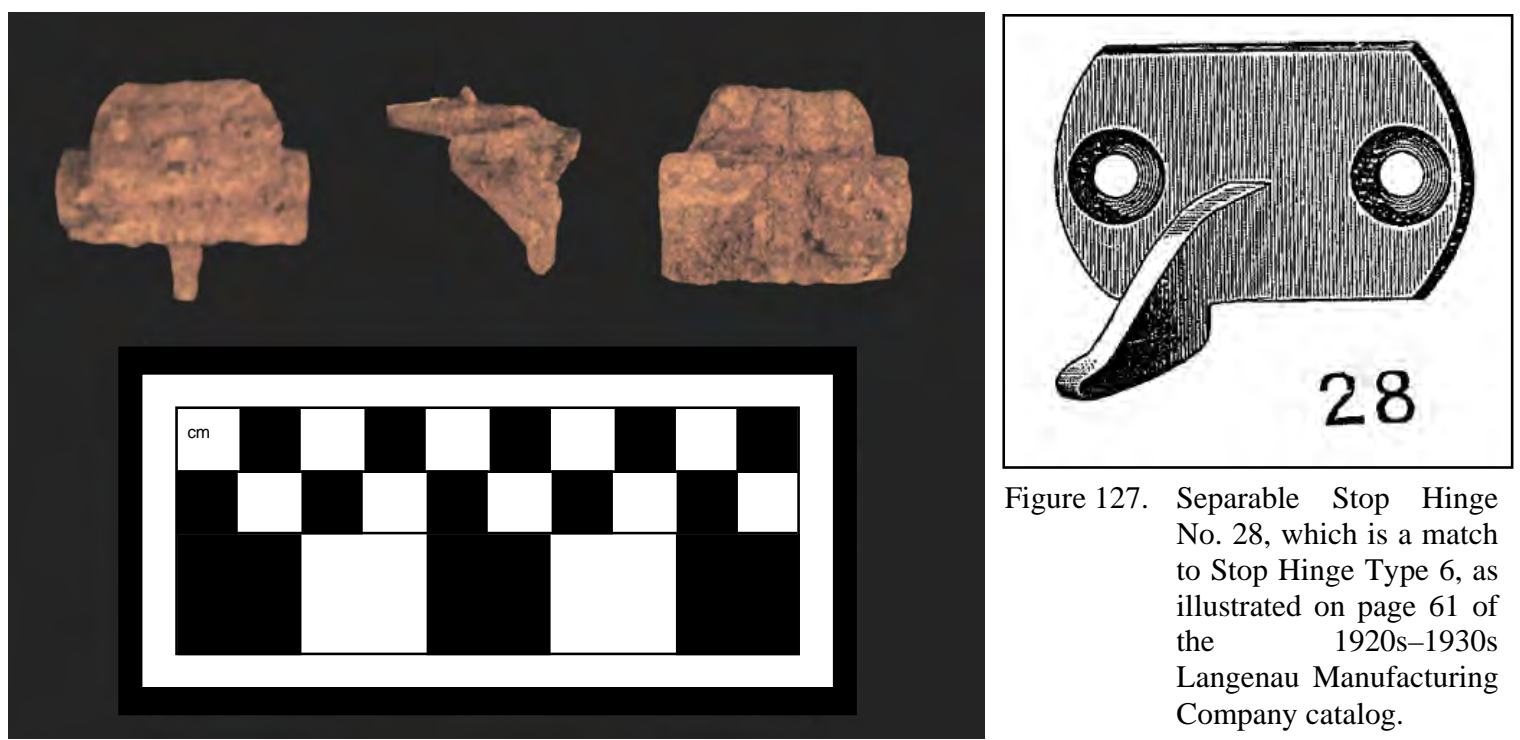

Figure 127. Separable Stop Hinge No. 28, which is a match to Stop Hinge Type 6, as illustrated on page 61 of the 1920s-1930s Langenau Manufacturing Company catalog.

Figure 126. Stop Hinge Type 6 and Stop Hinge Escutcheon Type 3.

No patent specific to this type of escutcheon was located. It has, however, been recovered in one cemetery excavation in Georgia in a burial dated to ca. 1921 (see Appendix D). Additionally, it appears as No. 27 in the ca. 1920s-1930s Langenau Mfg. Company catalog (Figure 128). The Langenau catalog reveals that this type of escutcheon was interchangeable with the hinge used for hinging heavy tops to shells $13 / 16$-inch thick.

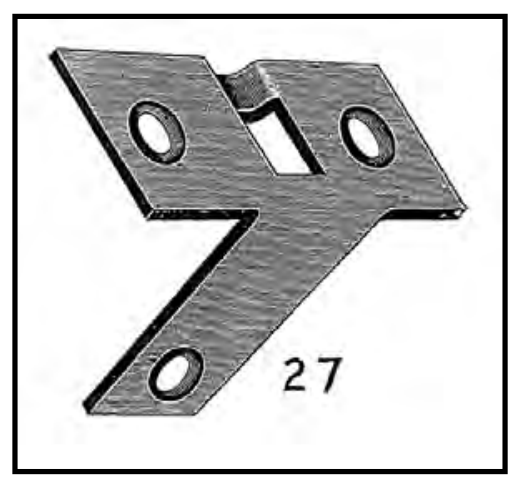

Figure 128. Escutcheon No. 27, which is similar to Stop Hinge Escutcheon Type 1, as illustrated on page 60 of the 1920s-1930s Langenau Manufacturing Company catalog.

\section{Stop Hinge Escutcheon Type 2}

Stop Hinge Escutcheon Type 2 (see Figure 117) is represented by two artifacts from two burials (Burials 8 and 18) in New Home Cemetery (see Appendix A). They are associated with Stop Hinge Type 2. These are ferrous, rectangular escutcheons containing two screw holes at each end of the plate. They also contain a central rectangular hole in the base plate and an arched segment at one end of the central hole. 
No patent specific to this type of escutcheon was located, and it has not been reported as being recovered from any historic cemetery excavations (see Appendix D). It does appear as No. 24 in the ca. 1920s-1930s Langenau Mfg. Company catalog (Figure 129). The Langenau catalog reveals that this type of escutcheon was interchangeable with hinges used for hinging heavy tops to shells ${ }^{13} / 16$-inch thick.

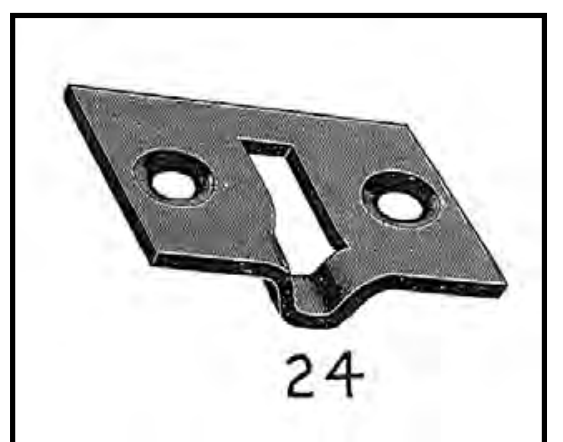

Figure 129. Escutcheon No. 24, which is similar to Stop Hinge Escutcheon Type 2, as illustrated on page 60 of the 1920s-1930s Langenau Manufacturing Company catalog.

\section{Stop Hinge Escutcheon Type 3}

Stop Hinge Escutcheon Type 3 is represented by four artifacts from two burials (Burials 5 and 17) in New Home Cemetery (see Appendix A). They are associated with Stop Hinge Type 6 in Burial 5 and Stop Hinge Type 3 in Burial 17. These are ferrous, rectangular escutcheons containing two screw holes at each end and a central rectangular hole that appears to have a small protrusion descending slightly below plate level on one end of the central hole.

No patent specific to this type of escutcheon was located, and it has not been recovered in any historic cemetery excavations. It does, however appear as No. 2 or 3 in the ca. 1920s-1930s Langenau Mfg. Company catalog (Figure 130). Small differences in dimension seem to distinguish these two numbered pieces, but the differences are slight. The Langenau catalog reveals that this type of escutcheon was interchangeable with hinges used for "hinging panels to top moldings and top moldings to shells” $11 / 16$-inch wide for No. 2 and 5/8-inch wide for No. 3.
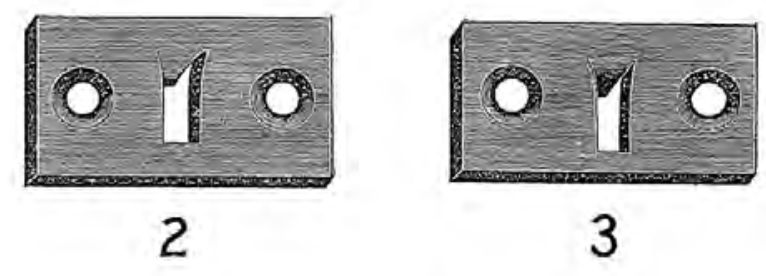

Figure 130. Escutcheon Nos. 2 and 3, which are similar to Stop Hinge Escutcheon Type 3, as illustrated on page 62 of the 1920s-1930s Langenau Manufacturing Company catalog. 


\section{Stop Hinge Escutcheon Type 4}

Stop Hinge Escutcheon Type 4 (see Figures 121 and 122) is represented by one artifact from Burial 14 in New Home Cemetery (see Appendix A). It is associated with Stop Hinge Type 4. It is a ferrous, rectangular escutcheon containing two screw holes at each end and a central rectangular hole. There is also a short arm extending perpendicularly from the base plate at the middle of one long edge.

No patent specific to this type of escutcheon was located, and it has not been recovered in any historic cemetery excavations. It does, however, appear as No. 4 or 5 in the ca. 1920s-1930s Langenau Mfg. Company catalog (Figure 131). Small differences in dimension seem to distinguish these two numbered pieces, but the differences are slight. The Langenau catalog reveals that this type of escutcheon was interchangeable with hinges used for "hinging panels to top moldings and top moldings to shells” 11/16-inch wide for No. 4 and $9 / 16$-inch wide for No. 5.

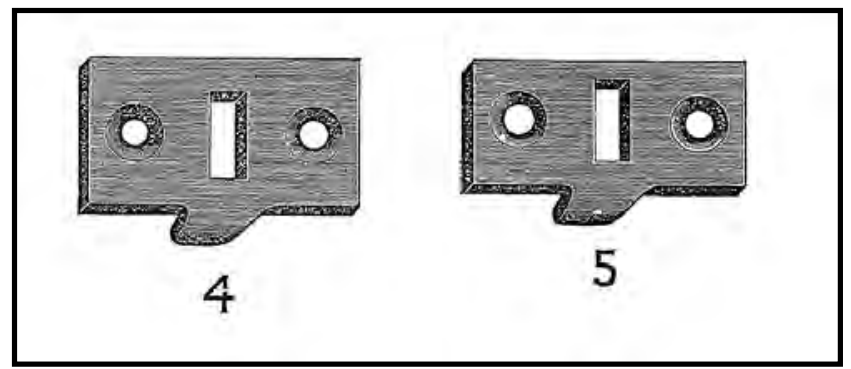

Figure 131. Escutcheon Nos. 4 and 5, which are similar to Stop Hinge Escutcheon Type 4, as illustrated on page 62 of the 1920s-1930s Langenau Manufacturing Company catalog.

\section{Stop Hinge Escutcheon Type 5}

Stop Hinge Escutcheon Type 5 (see Figure 123) is represented by one artifact from Burial 18 in New Home Cemetery (see Appendix A). It is associated with Stop Hinge Type 5. It is a ferrous, rectangular, angle bracket containing two screw holes plus a small central square hole on one face and a long rectangular hole running perpendicular to the long axis of the other face. There appears to be a triangular protrusion near the middle of the edge of this face extending outward along the same plane as the plate.

No patent specific to this type of escutcheon was located, and it has not been recovered in any historic cemetery excavations. It does, however, appear as No. 26 in the ca. 1920s-1930s Langenau Mfg. Company catalog (Figure 132). The Langenau catalog reveals that this type of escutcheon was interchangeable with hinges used for "hinging heavy panels to recessed top moldings” with ledges $1 / 2$-inch wide and $1 / 2$-inch thick.

\section{Stop Hinge Escutcheon Type 6}

Stop Hinge Escutcheon Type 6 (see Figure 124) is represented by one artifact from Burial 18 in New Home Cemetery (see Appendix A). It is associated with Stop Hinge Type 5. It is a ferrous, rectangular, angle bracket containing two screw holes plus a definite rectangular cleave on one 


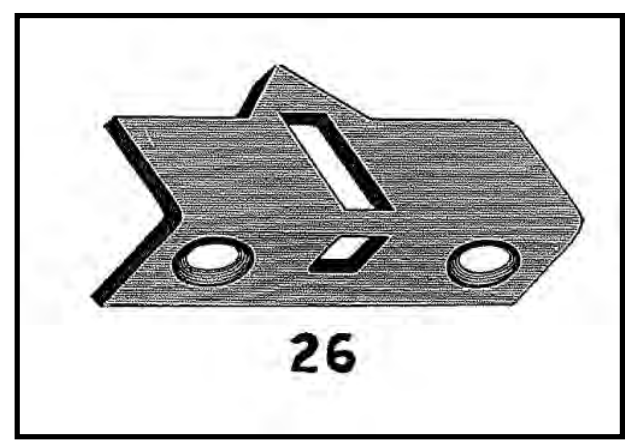

Figure 132. Escutcheon No. 26, which is similar to Stop Hinge Escutcheon Type 5, as illustrated on page 64 of the 1920s-1930s Langenau Manufacturing Company catalog.

face and a long rectangular hole running perpendicular to the long axis of the other face. There appears to be a triangular protrusion extending outward from one corner along the same plane as the plate, but there is heavy corrosion over the remainder of the artifact.

No patent specific to this type of escutcheon was located, and it has not been recovered in any historic cemetery excavations. It does, however, appear very similar to No. 29 in the ca. 1920s1930s Langenau Mfg. Company catalog (Figure 133), except for the fact that Langenau No. 29 appears to be slightly bent in the illustration. The Langenau catalog reveals that this type of escutcheon was interchangeable with hinges used for "hinging heavy panels to recessed top moldings."

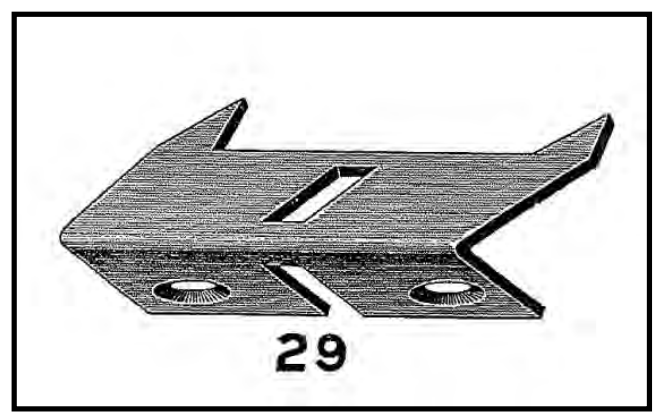

Figure 133. Escutcheon No. 29, which is similar to Stop Hinge Escutcheon Type 6, as illustrated on page 64 of the 1920s-1930s Langenau Manufacturing Company catalog.

\section{Stop Hinge Escutcheon Type 7}

Stop Hinge Escutcheon Type 7 (see Figure 92) is represented by one artifact from Burial 19 in New Home Cemetery (see Appendix A). It is oddly associated with Dowel Type 1 rather than with a Stop Hinge. It is a ferrous, rectangular, angle bracket containing two screw holes plus a small central square hole on one face and a long rectangular hole running perpendicular to the long axis of the other face. There appear to be triangular protrusions extending outward from the corners of this plate along the same plane. 
No patent specific to this type of escutcheon was located, and it has not been recovered in any historic cemetery excavations. It does, however, appear as No. 25 in the ca. 1920s-1930s Langenau Mfg. Company catalog (Figure 134). The Langenau catalog reveals that this type of escutcheon was interchangeable with hinges used for "hinging heavy panels to recessed top moldings" with ledges $11 / 16$-inch wide and $1 / 2$-inch thick.

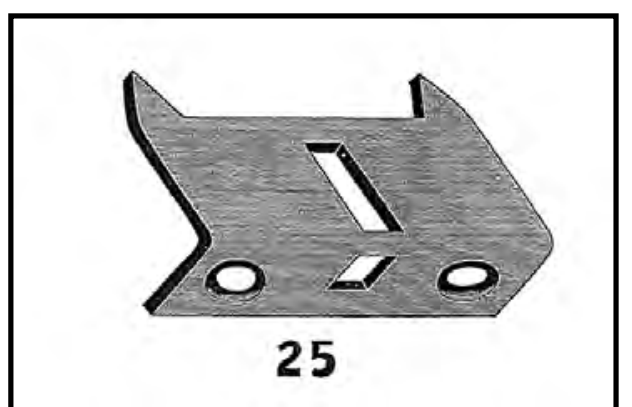

Figure 134. Escutcheon No. 25, which is similar to Stop Hinge Escutcheon Type 7, as illustrated on page 64 of the 1920s-1930s Langenau Manufacturing Company catalog.

\section{Butt Hinges}

The butt hinge is a form of hinge where the two plates of the hinge are held together and pivot around a pin. The butt hinge is a simple and widely used piece of hardware by various craft specialists and industries. This form of hinge has been used in mortuary contexts since the 1700s (Davidson 1999:541). Decorative butt hinges began to disappear from mainstream mortuary hardware catalogs in the 1870s, but plain style butt hinges, like those depicted in the 1980 [1865] Russell \& Erwin Manufacturing Company general catalog (Figure 135), continue to be used on certain burial containers to the present.

\section{Hinge Type 1}

Hinge Type 1 (Figure 136) is a butt type hinge represented by seven artifacts recovered from three burials (Burials 4, 10, and 11) as well as the disturbed area in New Home Cemetery (see Appendix A). It is made of iron, and the sides are different sizes. The smaller of the plates has three screw holes arranged in a triangular pattern pointing toward the hinge articulation. The larger plate has two screw holes at the corners and a lengthwise oval hole extending toward the center of the plate. The pin housing appears to be completely on one face, which means the hinge could lie flat against a surface. There are no specific patents known to exist for this hinge type, and it has not been identified specifically in any hardware catalog or cemetery excavation.

\section{Hinge Type 2}

Hinge Type 2 (Figure 137) is a butt type hinge represented by one artifact recovered from Burial 13 in New Home Cemetery (see Appendix A). It is made of iron, and the sides have rounded corners and are different sizes. The smaller of the plates has two screw holes, and the larger has three screw holes with the point of the triangle pointing away from center. The pin housing 


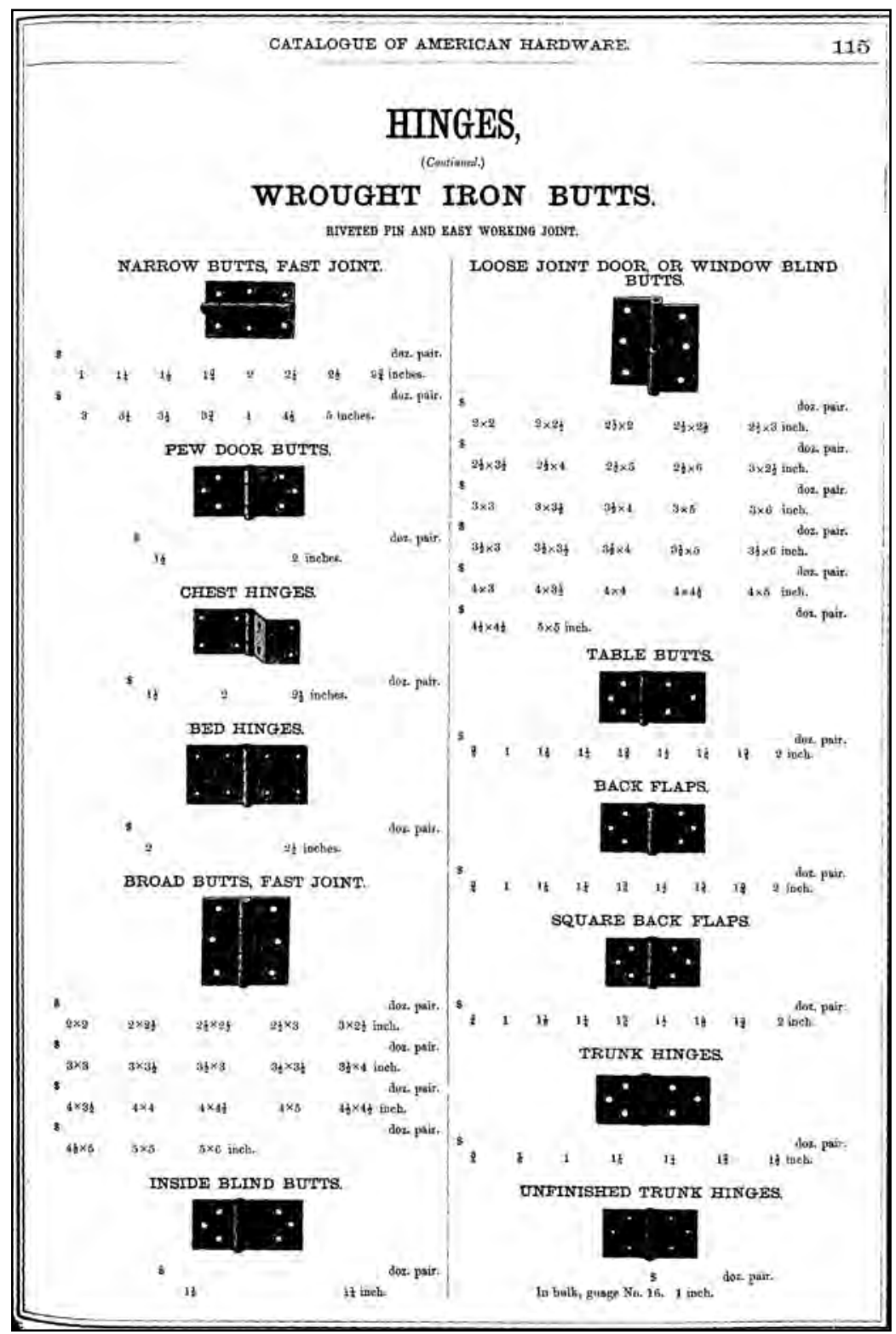

Figure 135. Selection of hinges offered for sale on page 115 of the 1865 Russell \& Erwin Manufacturing Company general catalog.

appears to be completely on one face, which means the hinge could lie flat against a surface. There are no specific patents known to exist for this hinge type, and it has not been identified specifically in any hardware catalog or cemetery excavation.

\section{Supports}

The term "support" (also known as an arm brace by some in the industry) is a general term referring to any number of elongated solid or hinged devices used to help prop open the lid of a casket, preventing it from closing or overextending. Little is known about the history of these 


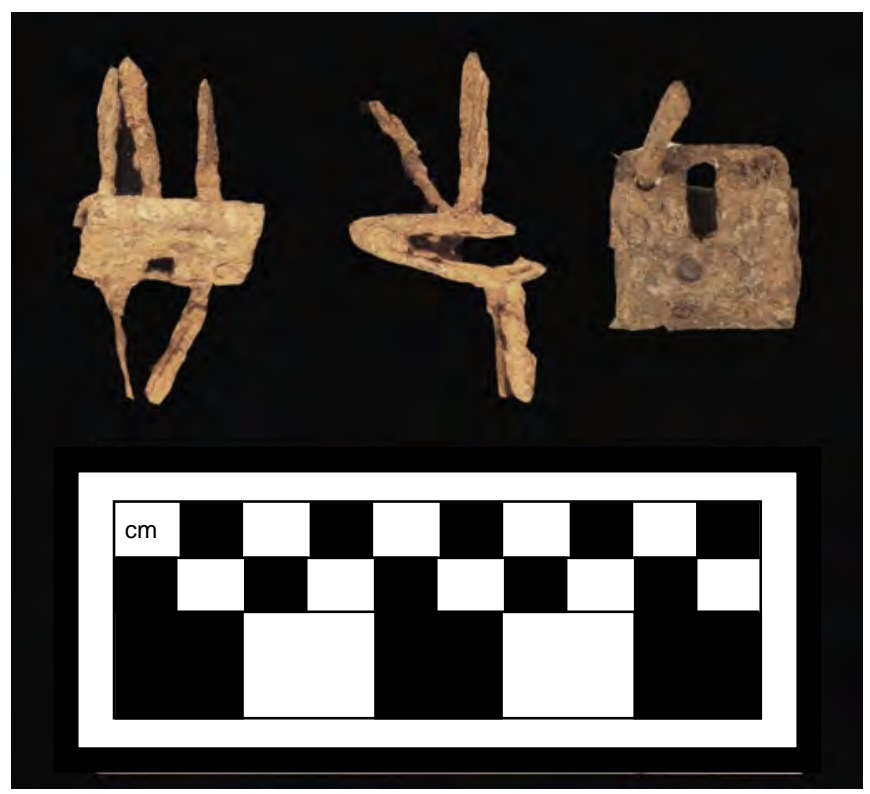

Figure 136. Hinge Type 1.

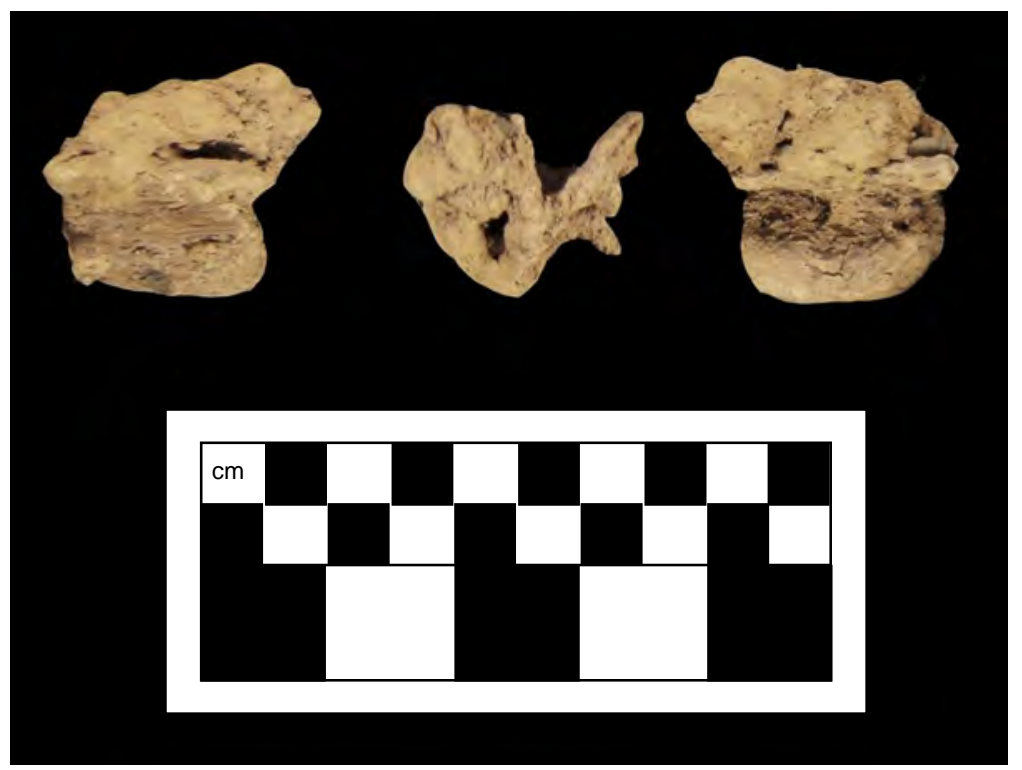

Figure 137. Hinge Type 2.

devices because no early patents specific to supports have been located. It stands to reason that when hinged caskets became more popular in the 1880s (and possibly as early as the 1870s) that supports would have been common installations. They commonly appeared in casket catalogs from the first decades of the 1900s. Figure 138 shows one type of support being used on a casket with a metallic lining sold by the John Marsellus Casket Company sometime between the 1910s and 1920s. Supports are still produced and used for burial containers today. 


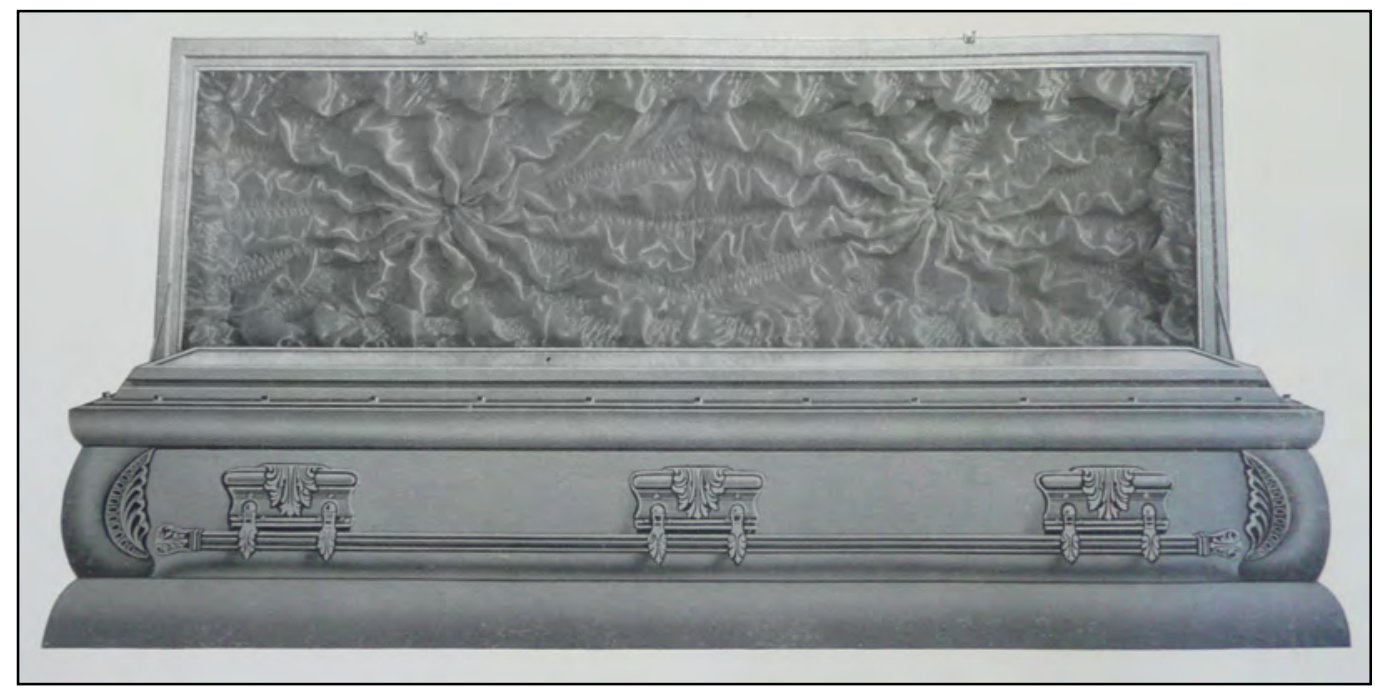

Figure 138. Casket exhibiting supports on page 91 of a 1910-1920 John Marsellus Casket Company catalog.

\section{Support Type 1}

Support Type 1 (Figure 139) is a ferrous, complex support represented by one complete support from Coffin 3, two fragments from Burial 3, and one fragment from Burial 13. Field photographs also revealed that at least one complete support arm had been present on the casket identified as Coffin 4 at the time of excavation (see Appendix A). This support type is made up of two support arms joined together at a circular riveted joint. There is a squared shoulder on each arm below the joint that enables the hinge to avoid overextending. On the opposite ends of each are screw holes that are stepped down in comparison to the remainder of the arm. The screw hole ends of the arms are also rounded.

This artifact type has not been located in patent records, but it has been identified in one known period mortuary hardware catalogs, the ca. 1920s-1930s Langenau Mfg. Company catalog (Figure 140). It has also been recovered from one cemetery excavation in Georgia in a burial dated 1943 (see Appendix D). It is unclear when this particular support entered the market or if it is still produced, but from available information it was in production at least between the 1920s and the 1940s.

\section{Support Type 2}

Support Type 2 refers to an elongated, ferrous metal strap with rounded corners, an eye screw attached to one end, and one or two large (Figure 141) or small (Figure 142) notches at the opposite end. Support Type 2 consists of eight fragments of this type of support arm distributed amongst six burials (Burials 5, 6, 14, 18, and 19, as well as Coffin 5) in New Home Cemetery (see Appendix A). 


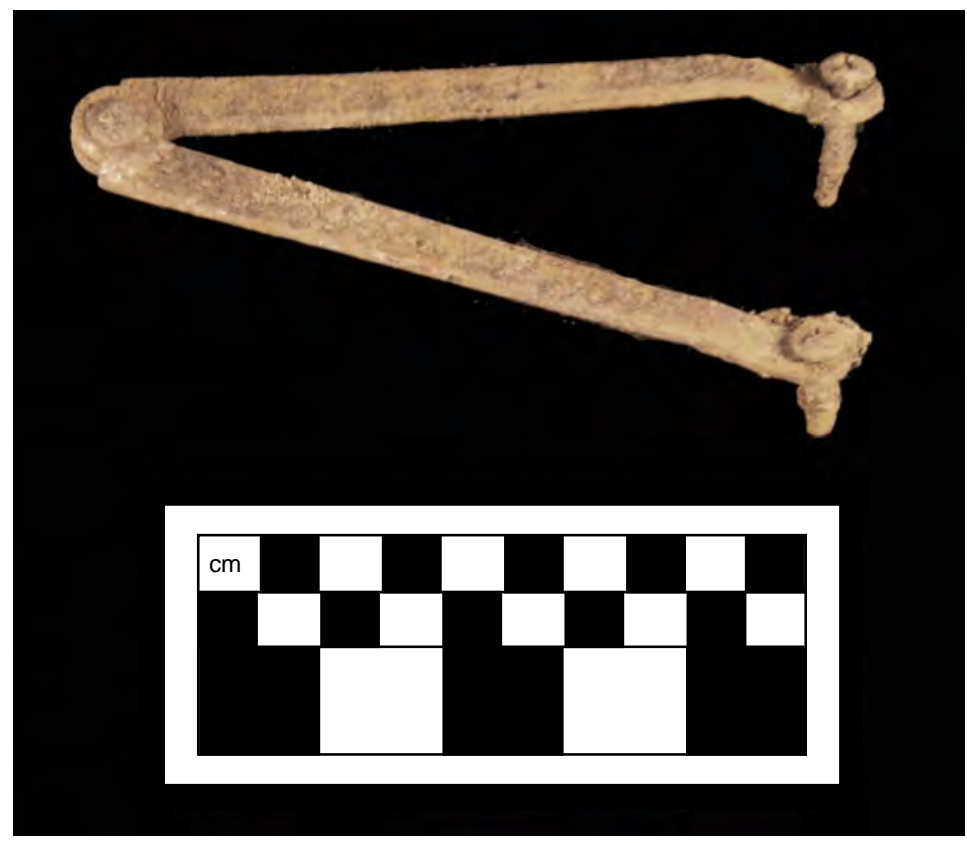

Figure 139. Support Type 1-hinged support.

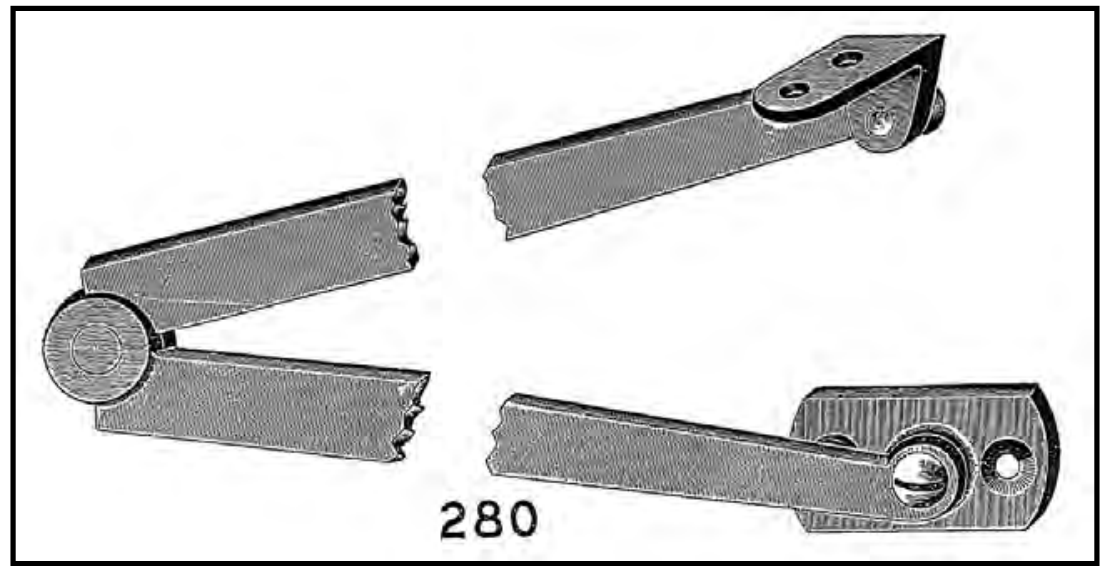

Figure 140. Support No. 280 illustrated on page 69 of the 1920s-1930s Langenau Manufacturing Company catalog that is a match to Support Type 1 .

This artifact type has not been identified in any known period mortuary hardware catalogs, though a fragment of a ferrous strap with an eye screw was recovered from an 1877 burial excavated in Elmbank Cemetery, Ontario, Canada (see Appendix D). Although it is not an exact match to this support type, U.S. Utility Patent No. 1510557 issued to Robert Paden in 1924 illustrates a support arm with a similar eye screw attachment and three notches on the opposite end for adjustable support when a peg was placed within the notch. Dating this support type is difficult, because eye screws have been produced since at least the mid-nineteenth century. The simple support strap would also be easy to produce at any time during the nineteenth and twentieth centuries, meaning it could have a wide date range. The only limiting factor would be that before hinged lid caskets were produced support arms would not have been necessary. 


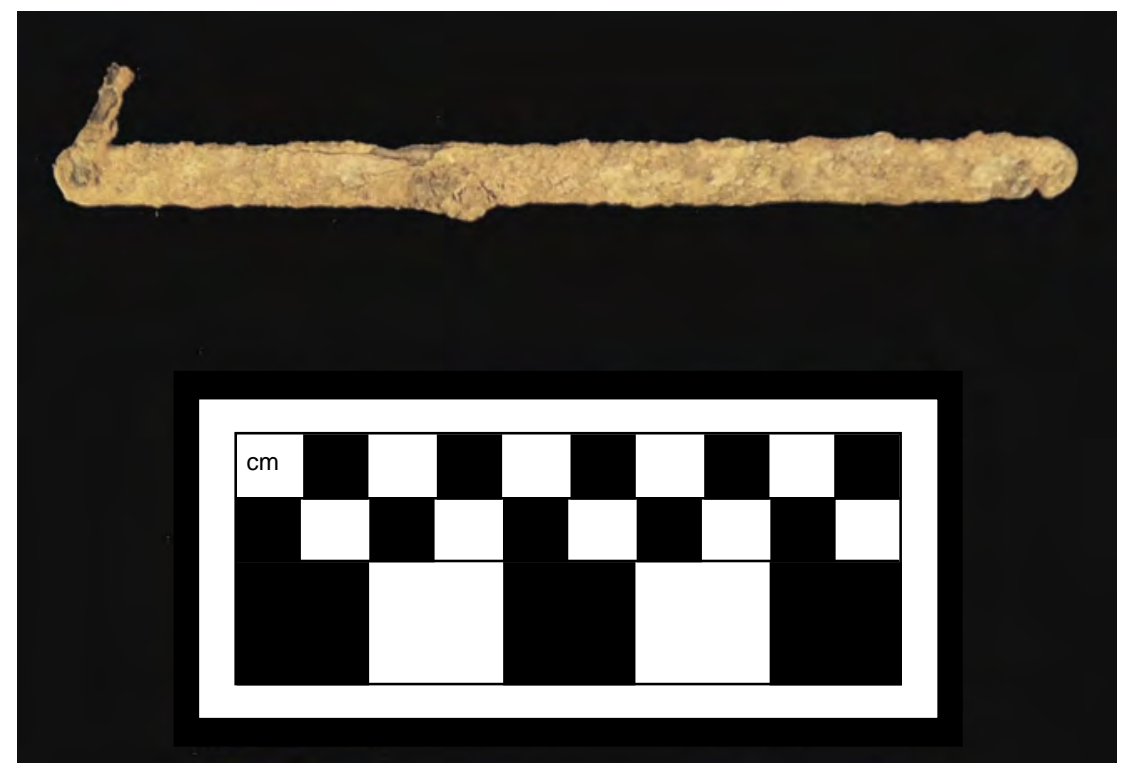

Figure 141. Support Type 2-eye hook support with small hook.

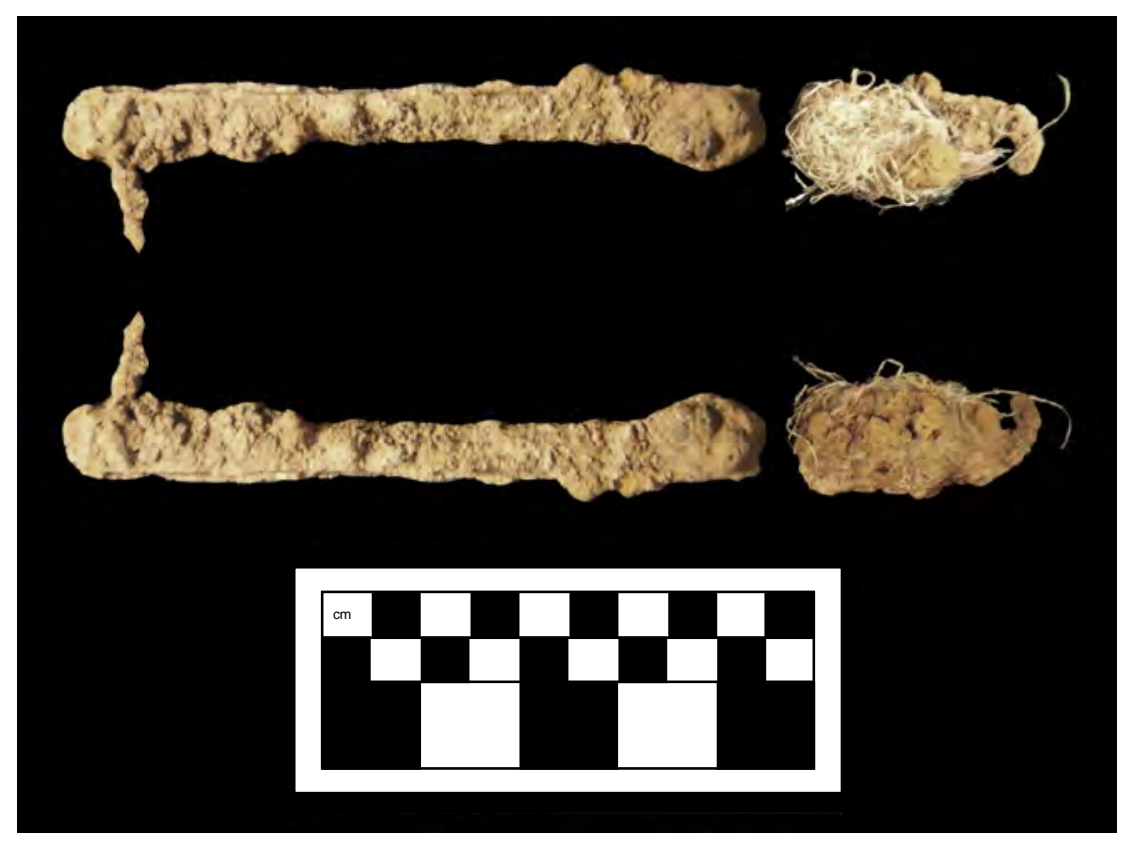

Figure 142. Support Type 2-eye hook support with large hook.

\section{Lining Tacks}

The primary function of lining tacks was to affix cloth lining, either within the interior of the burial container or covering to the exterior of the container, or sometimes the tacks were arranged in a decorative pattern on the surface of the container. These tacks are typically small, with an iron shank and head (flat iron, domed iron, domed brass, domed china, or domed lead/white metal; Figure 143). Often the iron shank deteriorates or becomes detached and only the heads remain (Davidson 1999). 


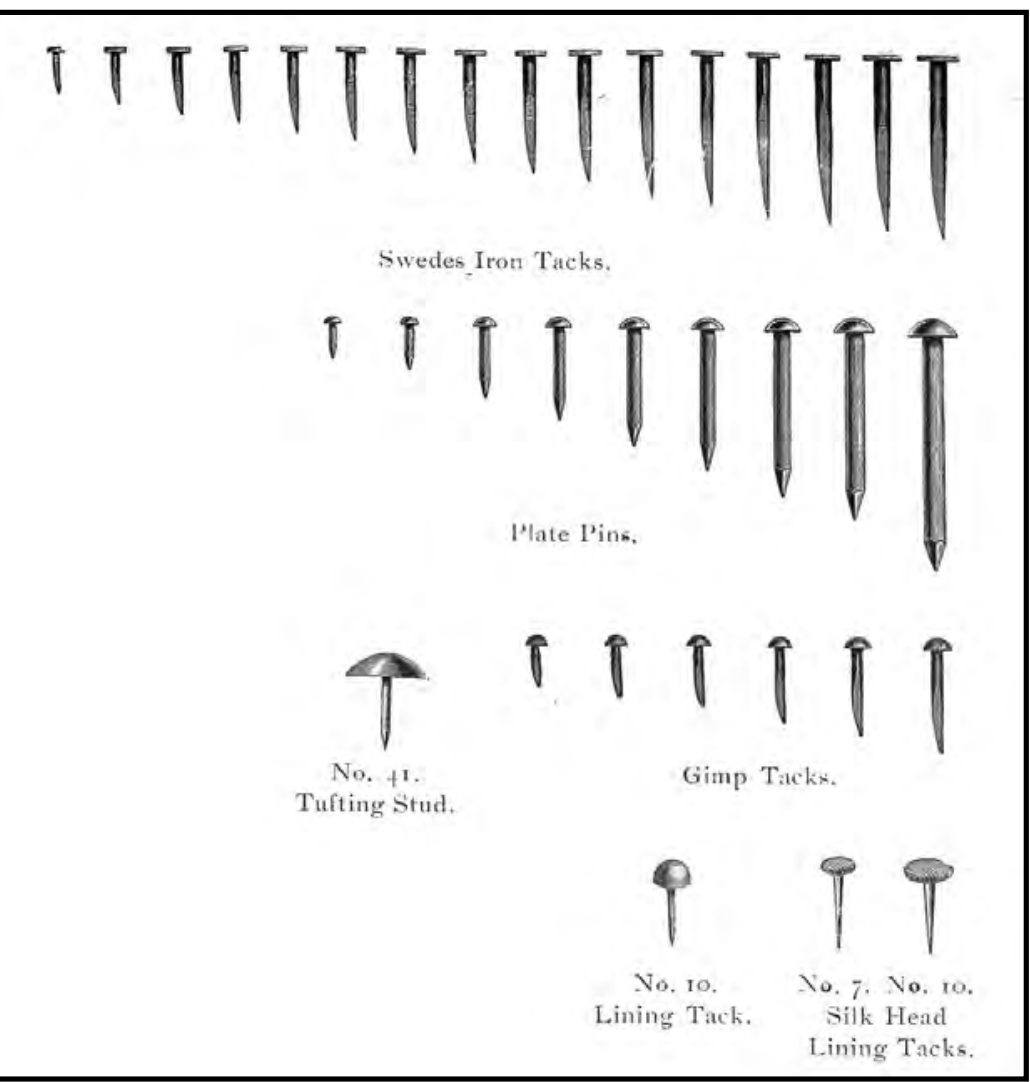

Figure 143. Selection of lining tacks illustrated on page 105 of the 1901 Gate City Coffin Company catalog.

Since the presence or absence of lining or cloth covering is a basic economic indicator, the ability to identify lining tacks is important. Linings do not typically preserve except when lying in association with cuprous hardware, and even then it is difficult to distinguish cloth lining from clothing remnants; therefore, the recovery of lining tacks provides a more concrete indicator (Davidson 2004:418).

\section{Lining Tack Type 1}

Lining Tack Type 1 is a simple flat-headed ferrous lining tack represented by 20 artifacts recovered from seven burials (Burials 5, 7, 9, 14, 16, and 19, as well as Coffin 5) in New Home Cemetery (see Appendix A). This is a ubiquitous form of lining tack and is present in 20 trade catalogs dating between 1865 and 1912. It is unknown when these tacks were first marketed, but they likely have a very long history intimately connected with the history of the nail. In most archaeological cemetery excavation reports, limited attention is given to lining tacks, particularly simple iron tacks that are often mistaken for nail fragments; therefore, it is very difficult to determine with certainty the frequency with which this type of tack appears in the archaeological record. It has been specifically recorded in at least six cemetery excavation reports, however, dating between 1832 and 1907. 


\section{External Burial Container Elements}

External burial container elements, as with internal burial container elements, should be given equal weight in analyses of historic cemetery artifact collections. External elements can be seen by mourners during the funeral and therefore play a key role in social perception of the event, and by extension the social and economic status of the deceased and the deceased's family (Pye 2007). In addition, changes in the type of external elements can reflect changes in consumer culture, or social and religious ideology. Identification of changes in patterns of external elements can also provide important clues to chronology (Davidson 1999).

\section{Handles}

Mainfort and Davidson (2006:122-123) state that the earliest burial container handles were either made specifically for mortuary contexts (citing Rauschenberg 1990:43-44) or were utilitarian furniture hardware employed in a mortuary setting. Utilitarian handles have been noted in a burial in Delaware dating to 1780-1820 (DeCunzo et al. 1992:199). This practice is also noted in nineteenth-century Tucson, Arizona (Pye 2010a), and likely in other locales where necessity or scarce resources required creativity. The use of handles specifically designed for mortuary purposes dates back to at least the eighteenth century. Tharp (1996:81-88) notes that British coffin handles were being imported and advertised for sale in the American colonies as early as 1738.

Handles on burial containers serve multiple functions. A primary function of handles is to provide a means by which the burial container may be carried to the gravesite from the home or from whatever transportation device was employed to convey the deceased to the burial locale (Davidson 1999:535). Great innovation in material, form, and style (the extent of which cannot yet be quantified) in the nineteenth and early twentieth centuries cannot be explained by the transportation function. Burial container handles may well have served broader social and ideological functions.

\section{Box Handle}

In the 1850s, most burial containers were fashioned by friends, family, or the local cabinetmaker. The Civil War promoted the expansion of transportation networks that in turn supported the growing desire to ship the remains of dead soldiers home for burial. The simple wooden receptacles into which the coffins of the deceased soldiers were placed for transport were referred to as shipping boxes and were often equipped with at least four single-lug box handles (HackerNorton and Trinkley 1984:10). As the years passed, it became increasingly common in the later nineteenth century for people to purchase factory-made coffins and caskets from large producers who also shipped these items in shipping boxes. By the turn of the twentieth century, the purchase of ready-made burial containers, either ordered directly from the manufacturer or through the local funeral director, became the norm.

Shipping boxes, also referred to as outer boxes, were also frequently used as vault boxes. The outer boxes would be placed in the bottom of a grave and the coffin or casket lowered into it (Hacker-Norton and Trinkley 1984:10; Oster et al. 2005:191). After the coffin or casket was lowered into the outer box, the lid would be closed and the grave filled. The box handles of the outer box would therefore enter the archaeological record (Buchner et al. 1999; Rose 1985). 


\section{Handle Type 1}

Handle Type 1 (Figure 144) is represented by one artifact found in association with Coffin 2 grave in New Home Cemetery (see Appendix A). This handle is a single-lug box or shipping container handle and was likely associated with an outer shipping box. It is difficult to say with certainty, however, because the grave was heavily disturbed. The lug is generally rectangular, but the lower portion is constricted to the area within the bail and the lower margin is concave. The lug contains three screw holes, one on each lower corner and one in the top center. The handle is made of iron and it has a hollow back. The bail is attached to the lug via side pins. The bail, too, is hollow-backed, although the grip portion of the bail is complete. A seam runs horizontally along the back of the lower portion of the bail where the metal was folded over to form the grip.

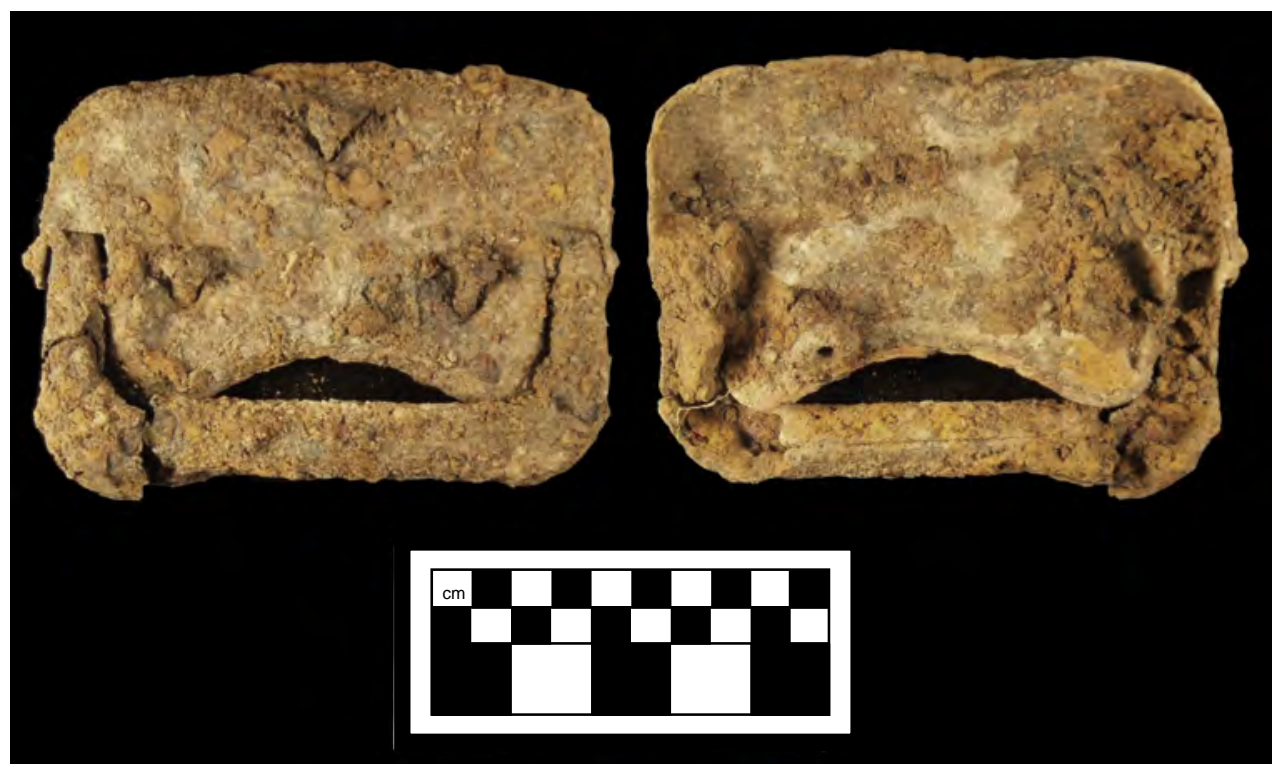

Figure 144. Handle Type 1-box handle.

No definitive matches have been made to patent records, but this same type of box handle has appeared in three period mortuary catalogs ranging in date between 1912 and the 1950s, and appeared in one archaeological collection dating between 1894 and 1926 (see Appendix D). The 1912 Cincinnati Coffin Company catalog was the first known appearance of this box handle (Figure 145). A 1906 Cincinnati Coffin Company catalog was also consulted, and although remarkably similar to the 1912 in most products advertised, Handle Type 1 specifically was not being sold. A survey of other pre-1912 hardware catalogs from other companies that carried box handles also failed to reveal this handle. That would suggest that it entered the market ca. 1910 .

\section{Door/Furniture Handle}

Furniture or door handles were made for use on pieces of wooden furniture, such as chests-ofdrawers, curio cabinets, and table drawers, or for the interior doors of homes, out buildings, or even furniture (Ormsbee 1951, 1952). They were sold throughout the late nineteenth and early twentieth centuries by small hardware merchants as well as by large general hardware distributers 


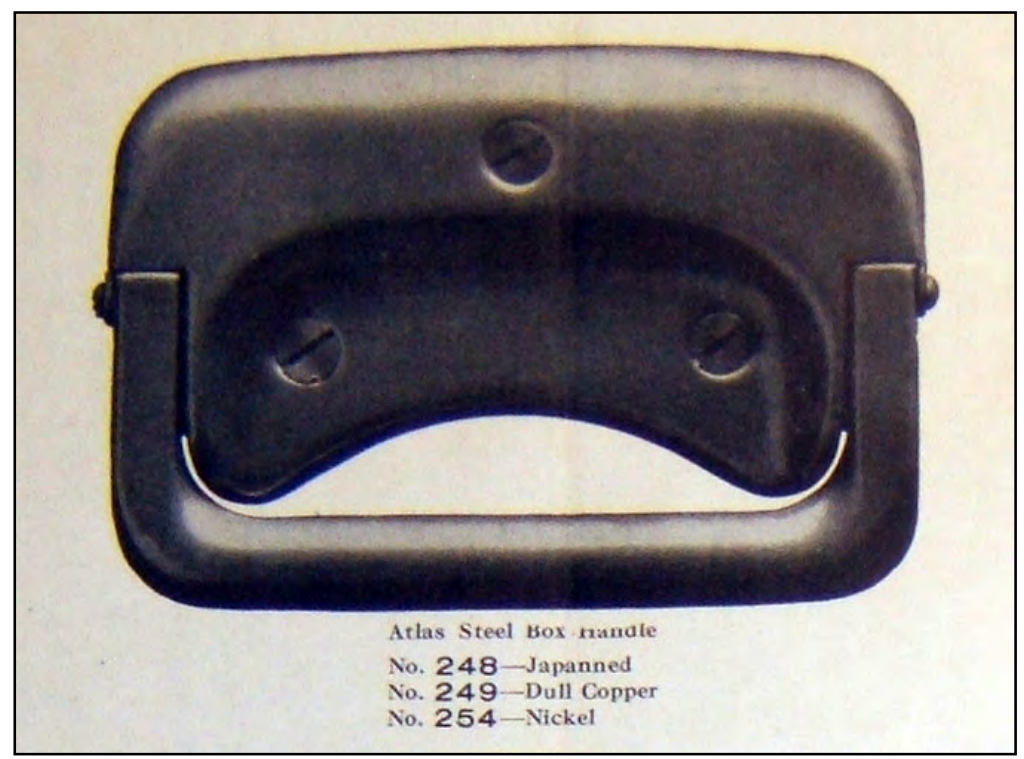

Figure 145. Atlas steel box handle illustrated on page 264 of the 1912 Cincinnati Coffin Company catalog that is a match to Handle Type 1.

such as Russell \& Erwin Manufacturing Company and Sears, Roebuck and Company. These utilitarian hardware elements appear in the archaeological record within historical cemeteries because they were occasionally used in the construction of burial containers.

\section{Handle Type 2}

Handle Type 2 (Figure 146) is represented by one artifact found in disturbed contexts in New Home Cemetery (see Appendix A). This particular door pull is made of cast ferrous metal and has some corrosion present. It has a squat U-shaped form with circular terminations containing one screw hole each. No definitive matches have been made to period mortuary or general hardware catalogs, but the 1980 [1865] Russell \& Erwin Manufacturing Company catalog does depict several varieties of similar door pulls for sale (Figure 147). Because of the simple design and their utilitarian nature, these handles range in date in the U.S. from the colonial period to the present.

\section{Handhold Cover/Grip}

Little is known about these types of products. Most were produced of a thin, stamped tin or copper, but a few were produced from a more substantial iron plate similar to furniture/drawer pulls. They were used to cover handholds or grips on a burial container if those toting locations were unsightly. The stamped varieties would have not served as handles themselves because they would not have been able to withstand the force. 


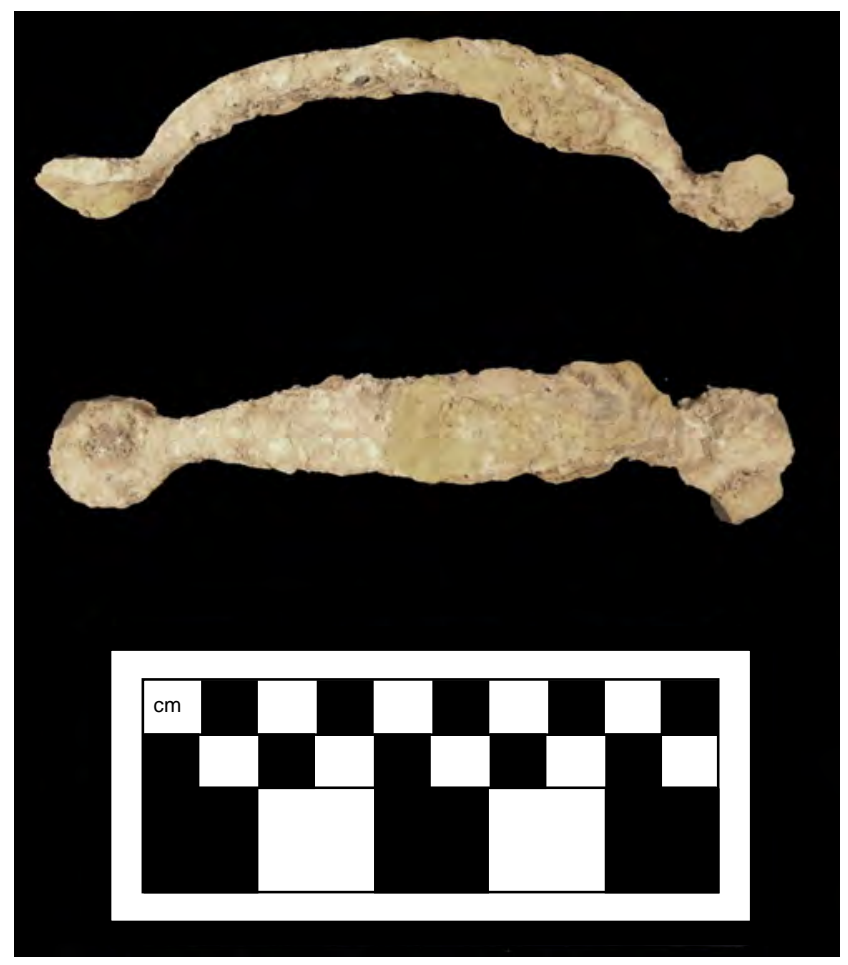

Figure 146. Handle Type 2-furniture/door pull.

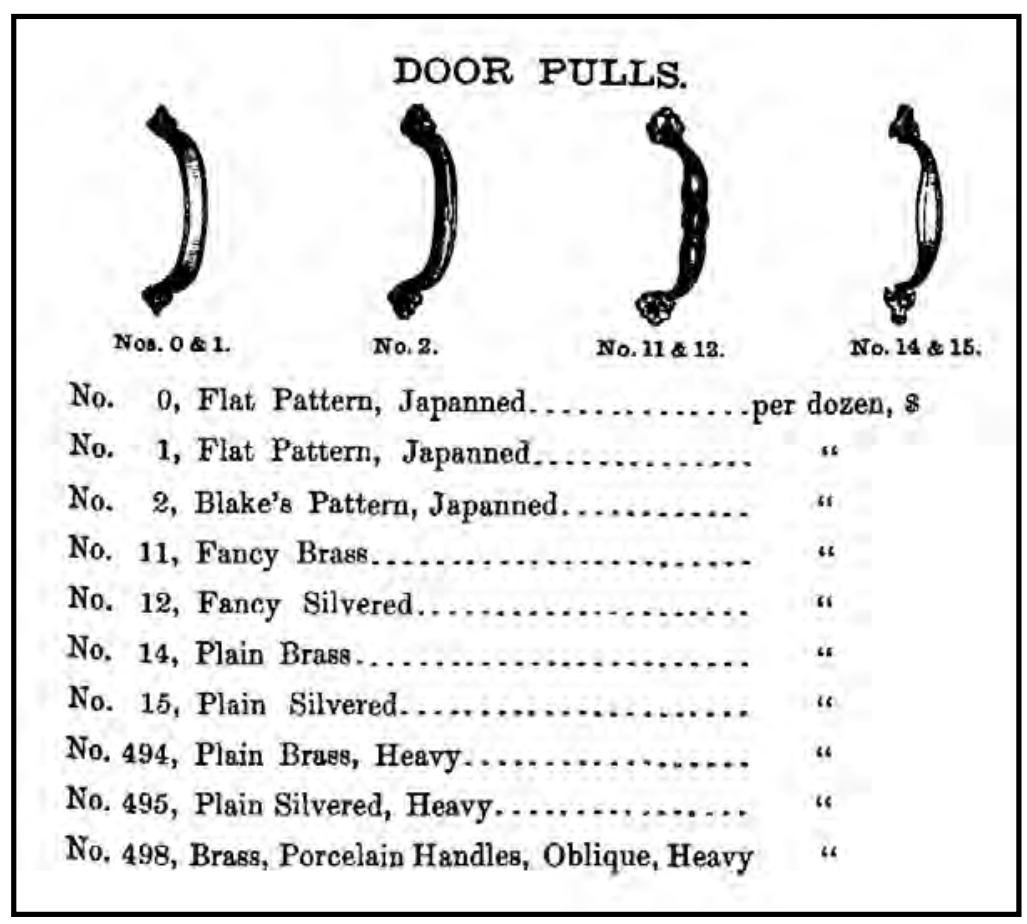

Figure 147. Selection of door pulls advertised on page 143 of the 1865 Russell \& Erwin Manufacturing Company catalog. 
Much like the furniture/door handles discussed above, handhold covers also likely have a wide temporal range in the utilitarian sphere. The first known patent for a handhold/grip specifically designed for use on a casket was U.S. Utility Patent No. 30535, which was granted to Edward R. Sargent on April 11, 1899 (Figure 148).

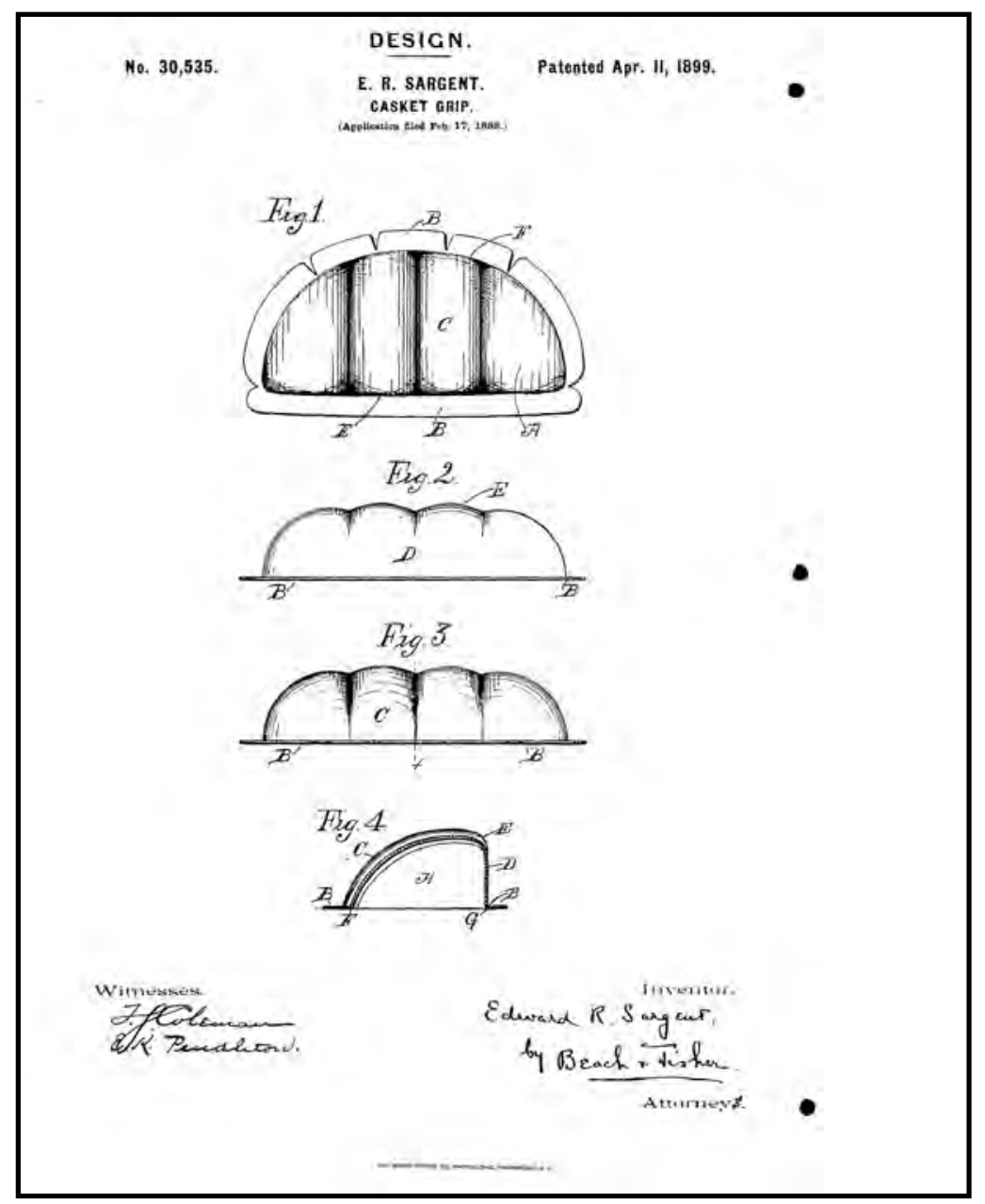

Figure 148. U.S. Design Patent No. 30,535 assigned in 1899 to Edward R. Sargent for a casket grip.

\section{Handle Type 3}

Handle Type 3 (Figure 149) is represented by one handhold cover found in Burial 5 in New Home Cemetery (see Appendix A). This artifact was made of iron and was heavily corroded. That said, it appears to be hollow-backed with a flat bottom edge and a gently arched top margin. No exact patent matches have been located, and it has not been recovered from other cemetery excavations (see Appendix D). It does, however, bear striking resemblance to handhold cover No. 436 as depicted in the ca. 1920s-1930s Langenau Mfg. Company catalog (Figure 150) and Hand Hole Cover No. 36 in the ca. 1921 Sargent \& Company catalog. 


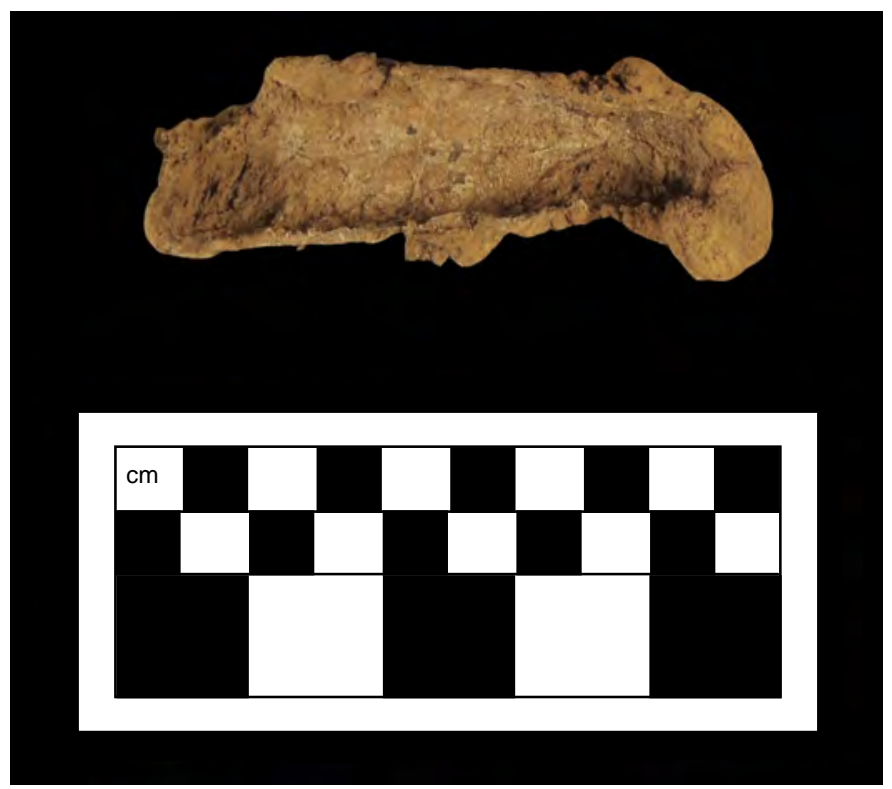

Figure 149. Handle Type 3.

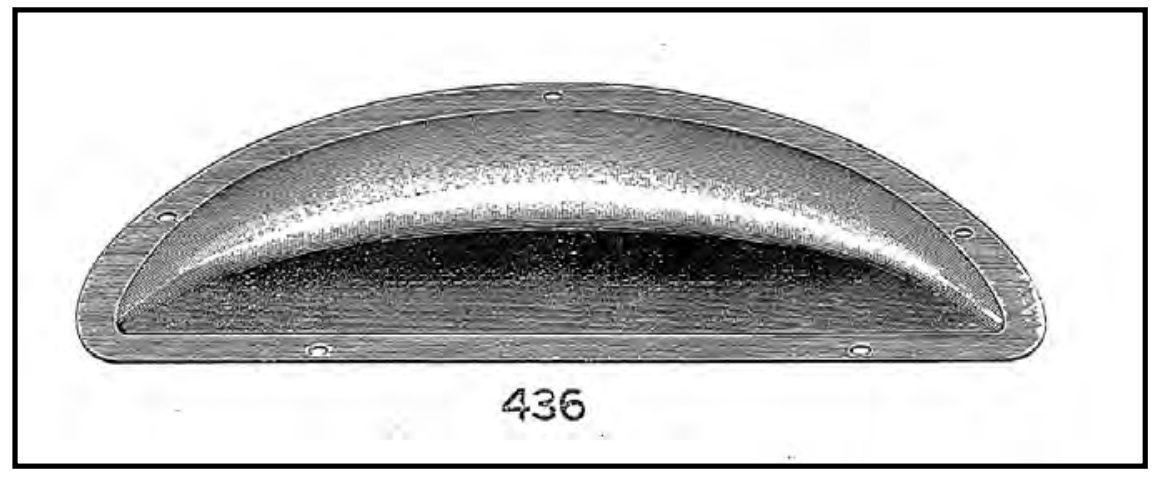

Figure 150. Handle Hold Cover No. 436 as advertised on page 71 in the 1920s-1930s Langenau Manufacturing Company catalog that is similar to Handle Type 3.

\section{Double-Lug, Swing-Bail Handle}

The double-lug, swing-bail handle is one variant of the general swing-bail form. It is composed of three elements: two lugs, which are affixed via screws or occasionally handle nails to the side of the coffin, and the bail, which forms the gripping portion of the handle. The bail is mounted into the lugs by the insertion of two metal pins (of iron or steel wire) at each end. Davidson (1999, 2004:407) reports that swing-bail handles have been in production since the eighteenth century. Their prominence did not wane until short-bar and later the extended-bar handles became more popular, but the form has never entirely disappeared. 


\section{Handle Type 4}

Handle Type 4 (Figure 151) is a double-lug, swing-bail handle represented by five fragmented handles from Burial 7 in New Home Cemetery (see Appendix A). These items appear to have been made of stamped sheet steel and are heavily corroded. It is unclear if they were plated. The handles consisted of two lugs reminiscent of cupcakes turned on their sides. The top of the cupcake is mounded and gently undulating. Below the mound is a constructed shoulder that tapers to a relatively flat bottom. No design pattern is evident through the corrosion. Much like the bail of Handle Type 1, the bail of Handle Type 4 is hollow-backed except for the central segment, which has a rounded, swelled grip. No design pattern can be discerned from the bail either.

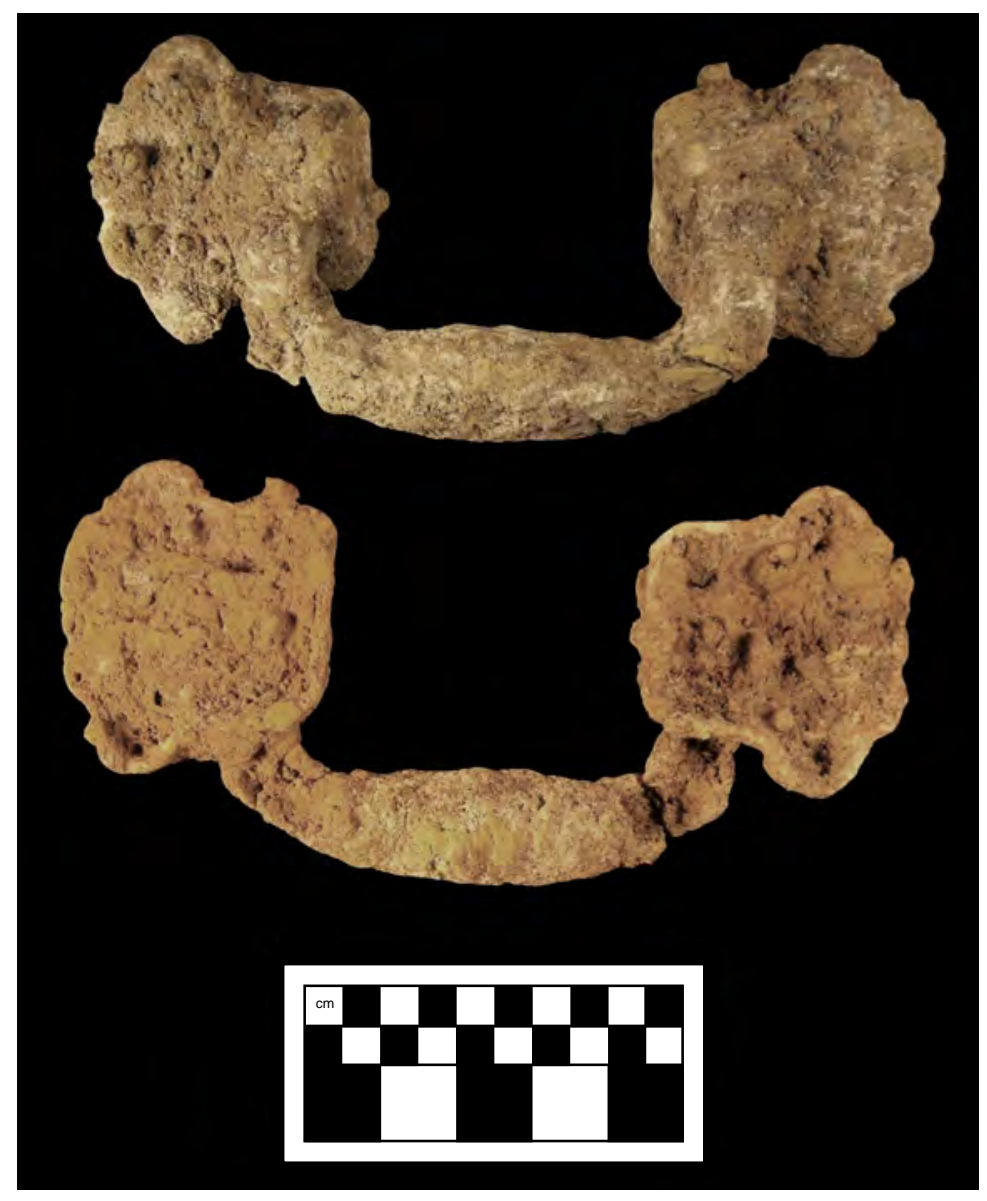

Figure 151. Handle Type 4-double-lug swing-bail.

No known design patents were located for this handle type, and it is not known to have been recovered in any cemetery excavations to date. Although no design elements can be observed, a handle with the same general form was identified in a ca. 1890 Cincinnati Coffin Company (1890b) catalog (Figure 152) (see Appendix D). Because other handles of similar construction also began to appear in that time period, it is reasonable to assume an approximate date of 1890 


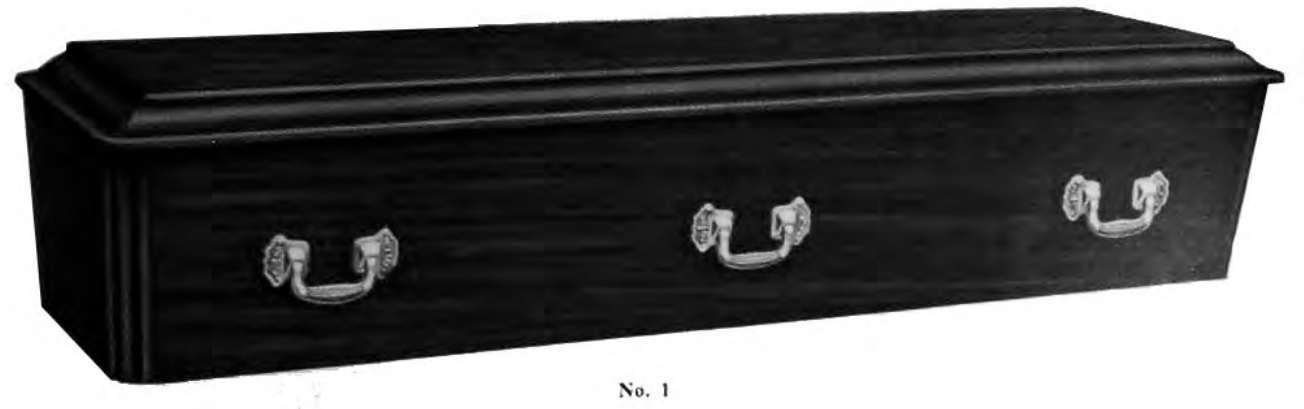

1 Nucre. Mahogany stain. Varnished. Cut top.

6-3 size only

Figure 152. Commonwealth. Illustration of Paramount Casket No. 1 exhibiting handles similar to Handle Type 4 on page 100 of a ca. 1890s Cincinnati Coffin Company (1890 b) catalog.

for this handle type. The fact that there are no other appearances of this handle in the literature is perhaps important. Two other comprehensive hardware catalogs from the Cincinnati Coffin Company (1906 and 1912) were available for comparisons at the time of this analysis. Neither of these catalogs contained the handle in question. It is possible, therefore, that the handle was dropped from production before the printing of the 1906 catalog.

\section{Handle Type 5}

Handle Type 5 (Figure 153) consists of a double-lug, swing-bail handle represented by fragments of four handles from Burial 16 in New Home Cemetery (see Appendix A). These items were made of stamped sheet steel and are heavily corroded. The outside edge of the lug is broad with rounded corners and a small point at the terminus. The top edge forms three gently sloping terraces before reaching the midline at the interior of the lug. The bail is U-shaped and hollowbacked except for the complete parallel-sided grip. The heavy corrosion has made observation of any design elements difficult, but there does appear to be a short ridge descending down the side arms of the bail. Additionally, a large floral tendril or leaf pattern extends vertically from the midline in the upper outside quadrant of the lug.

Based on the general form and observed stylistic elements, Handle Type 5 is a likely match to handle No. 2105 illustrated in the ca. 1921 Sargent \& Company catalog (Figure 154). Exact matches to this handle type were also located in one cemetery excavation in Tennessee from burials dating between 1899 and 1933 (see Appendix D). On August 11, 1914, Edward R. Sargent was granted U.S. Utility Patent No. 1106731 for a handle with this exact general form 


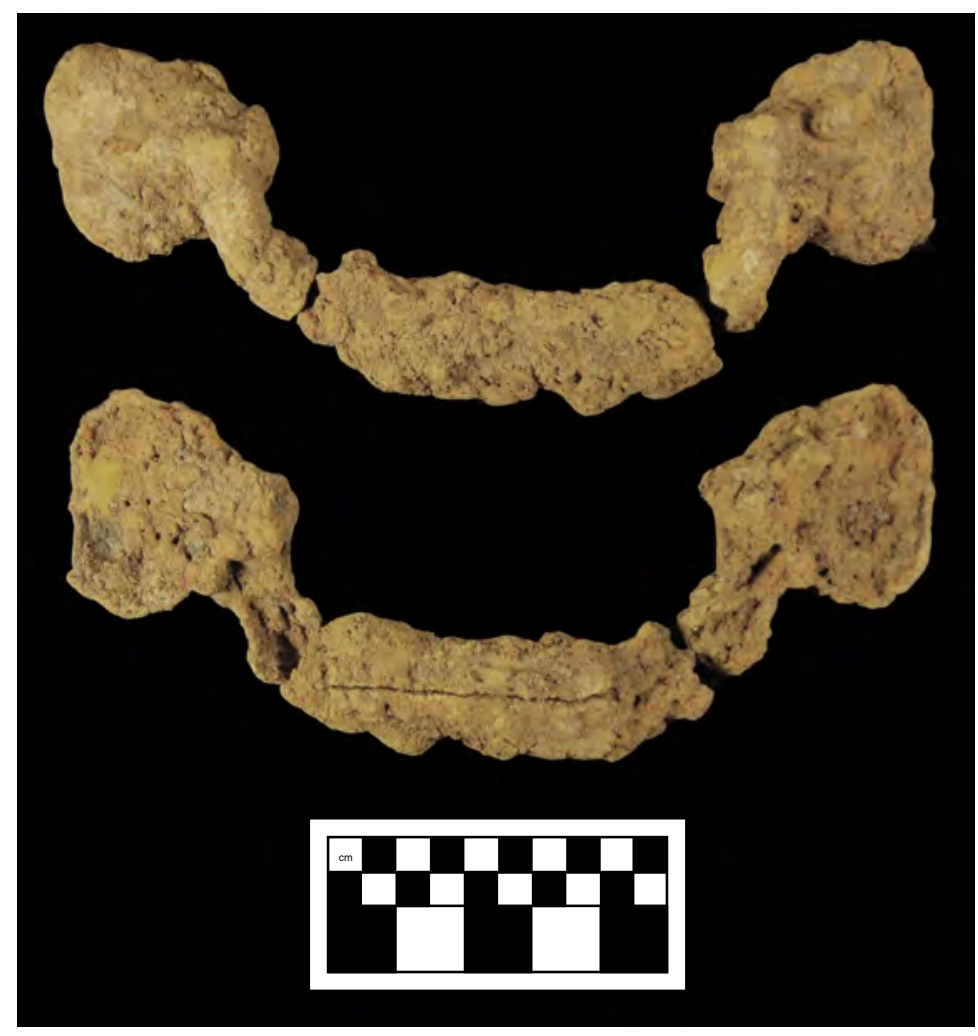

Figure 153. Handle Type 5-double-lug swing-bail.

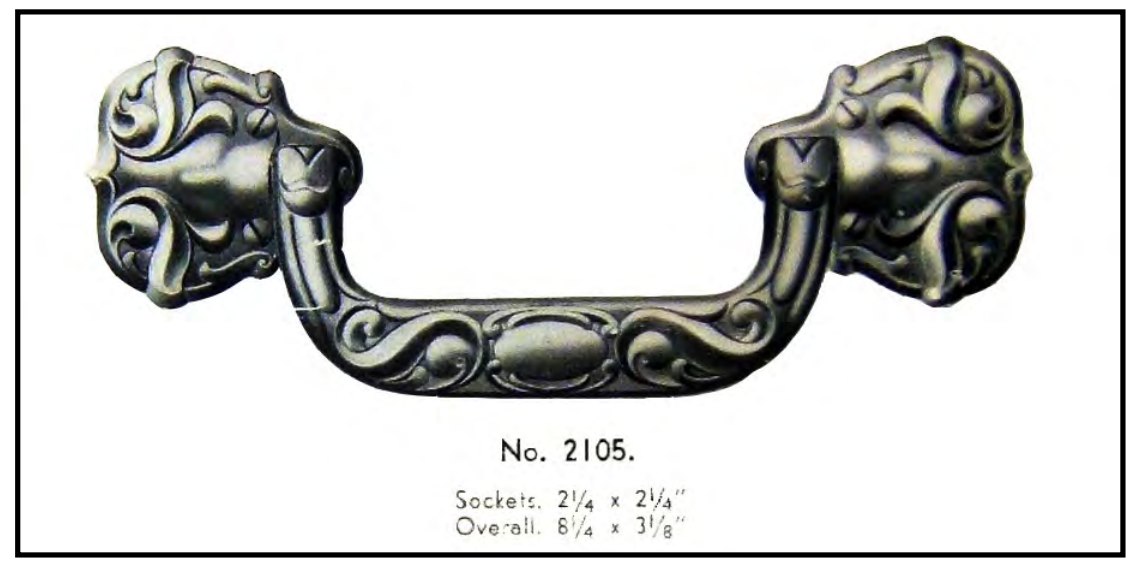

Figure 154. Swing-bail handle No. 2105 illustrated on page 106 of the 1921 Sargent \& Company catalog that is similar to Handle Type 5.

(Figures 155 and 156). The object of the patent was the creation of a light and cheap handle of unusual strength. This was attained by applying small reinforcing parts of hard metal at the points of special strain such as the attachment between the lugs and the bail pins, as well as at the point of attachment between the lug and the burial container. The available information on the presence and absence of this handle form in the period catalogs and archaeological literature presently supports a time range of 1914 to ca. 1935. 


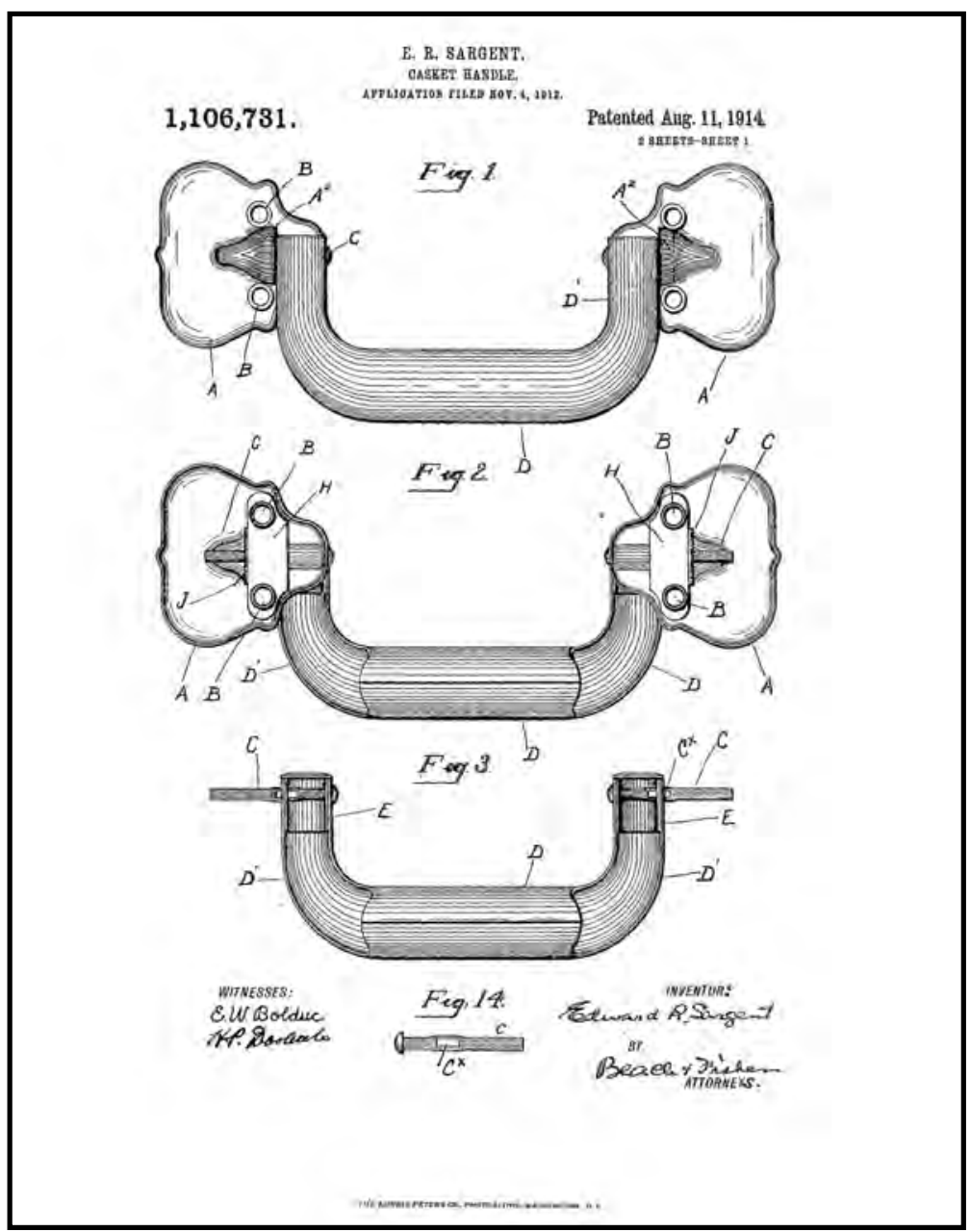

Figure 155. Drawing page 1 of U.S. Utility Patent No. 1,106,731 assigned in 1914 to Edward R. Sargent for a casket handle.

\section{Double-Lug, Short-Bar Handle}

The double-lug, short-bar handle is a variant of the short-bar concept, the history of which has been outlined by Mainfort and Davidson (2006:122-128). This variety is more complex than earlier swing-bail forms and can consist of up to nine parts: two lugs, two arms, two pins, a bar/tube, and two tips. The earliest patent dates for elements of the short-bar handle appear in 1866, with numerous stylistic variants (e.g., C. Strong's 1869 Coffin Handle, U.S. Utility Patent No. 97,827; Figure 157) being patented through the 1870s and 1880s (Mainfort and Davidson 2006:125-126). Based on period trade catalogs available for current study, it is evident that early form, short-bar handles were for sale in 1871, as advertised in the $1871 \mathrm{H}$. E. Taylor \& Co. illustrated catalog. These handles grew in popularity in the 1880s and made up a fair majority of the handles available in catalogs through the early twentieth century. 


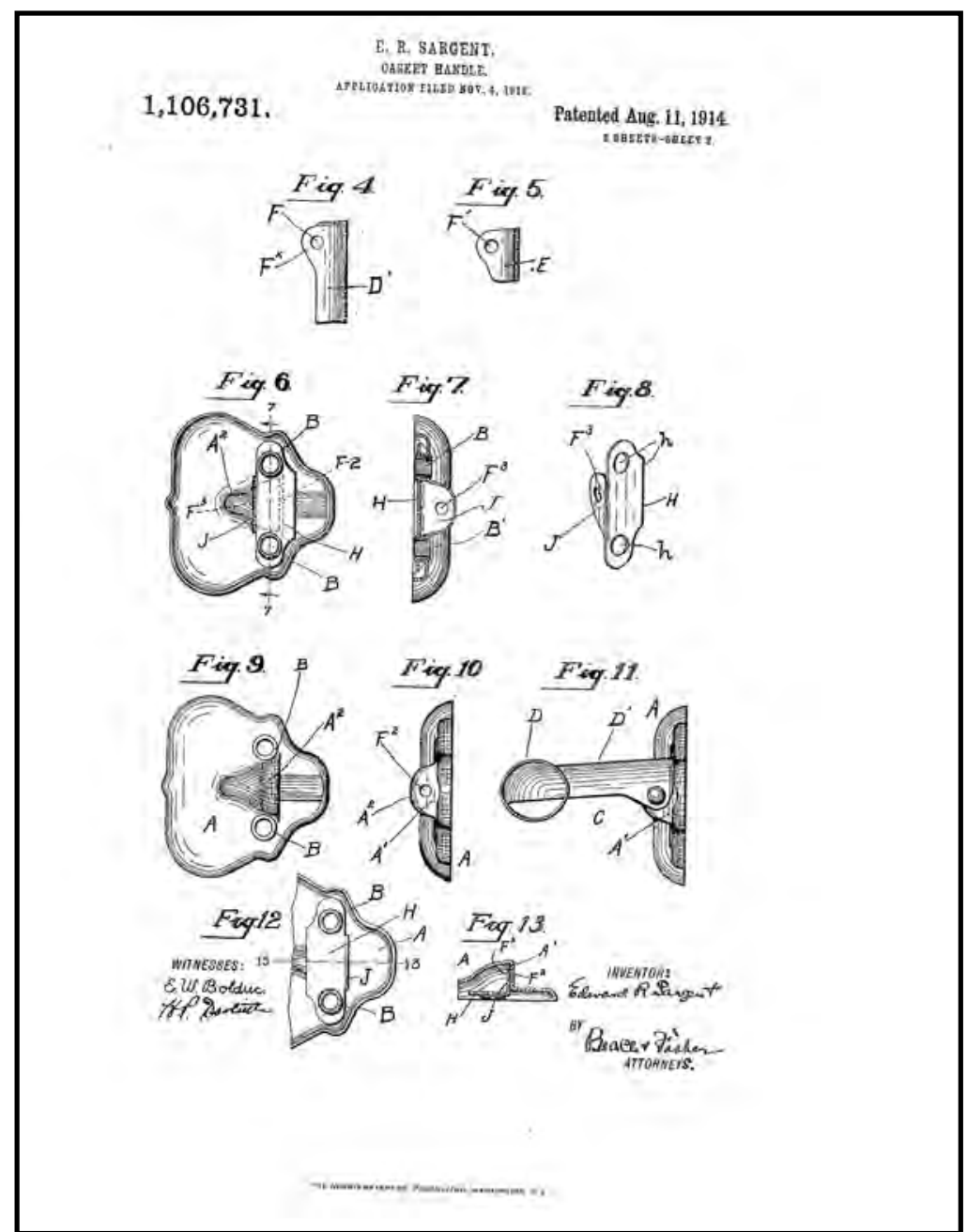

Figure 156. Drawing page 2 of U.S. Utility Patent No. 1,106,731 assigned in 1914 to Edward R. Sargent for a casket handle.

\section{Handle Type 6}

Handle Type 6 (Figure 158) is represented by a minimum of six double-lug, short-bar handles recovered from Burial 2 in New Home Cemetery (see Appendix A). The lugs of this handle are shaped like fiddles. There are no apparent designs on the surface of the lugs. The end segments of the bar are circular, and the caps are domed with a raised ring at the base. The bar has a swelled octagonal grip. All recovered examples are fragmented and corroded to various degrees.

The earliest known patent depicting a swell-bar variety of a short-bar handle was granted on January 20, 1891, to M. Bremer (U.S. Utility Patent No. 444973) (Figure 159). Swell-bars grew in popularity in the 1890s and became very common in catalogs of the first decade of the twentieth century. Swell-bars were far less common in the 1940s, but the occasional piece does appear in catalogs into the 1950s. 


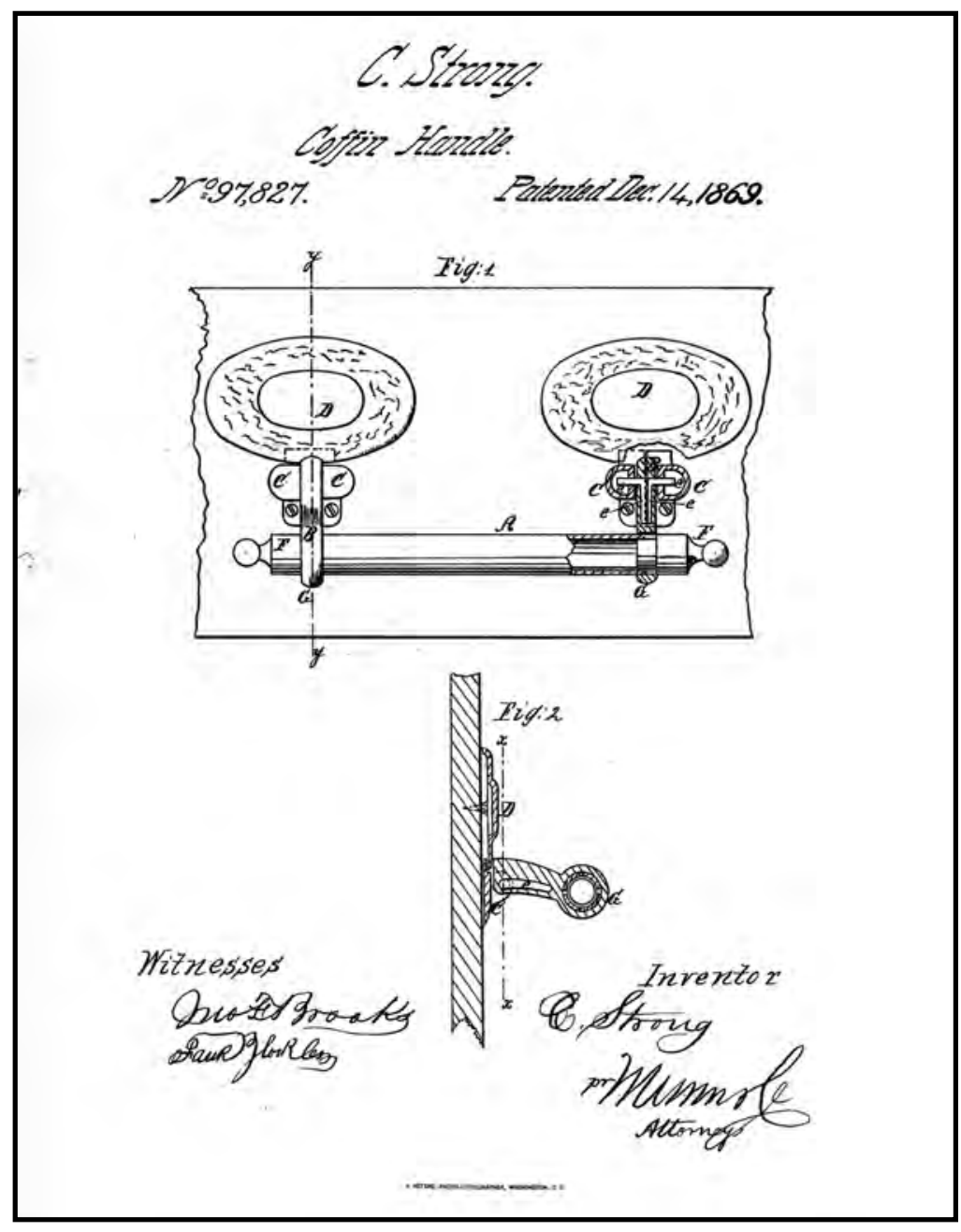

Figure 157. U.S. Utility Patent No. 97827 assigned in 1869 to C. Strong for a coffin handle with early components of a short-bar handle.

On July 19, 1910, U.S. Utility Patent No. 964562 was granted to Edward R. Sargent for a casket handle that matches the form of Handle Type 6 (Figure 160). This handle quickly grew in distribution, appearing in at least eight mortuary merchandise catalogs between ca. 1910 and ca. 1935 (Figure 161) (see Appendix D). Three of those eight catalogs are from the Boyertown Burial Casket Company, but examination of the 1936 Boyertown catalog failed to show this handle in the product line. It is possible, then, to apply a tentative production range between 1910 and 1935. That would be a simple conclusion, but a handle similar to this one has also been recovered from one other cemetery excavation, in Georgia from a burial dated to 1943. If the above production range is accurate, this burial may be explained by the sale lag similar to that noted by Hacker-Norton and Trinkley (1984) in their work with the A. L. Calhoun General Store hardware collection. 


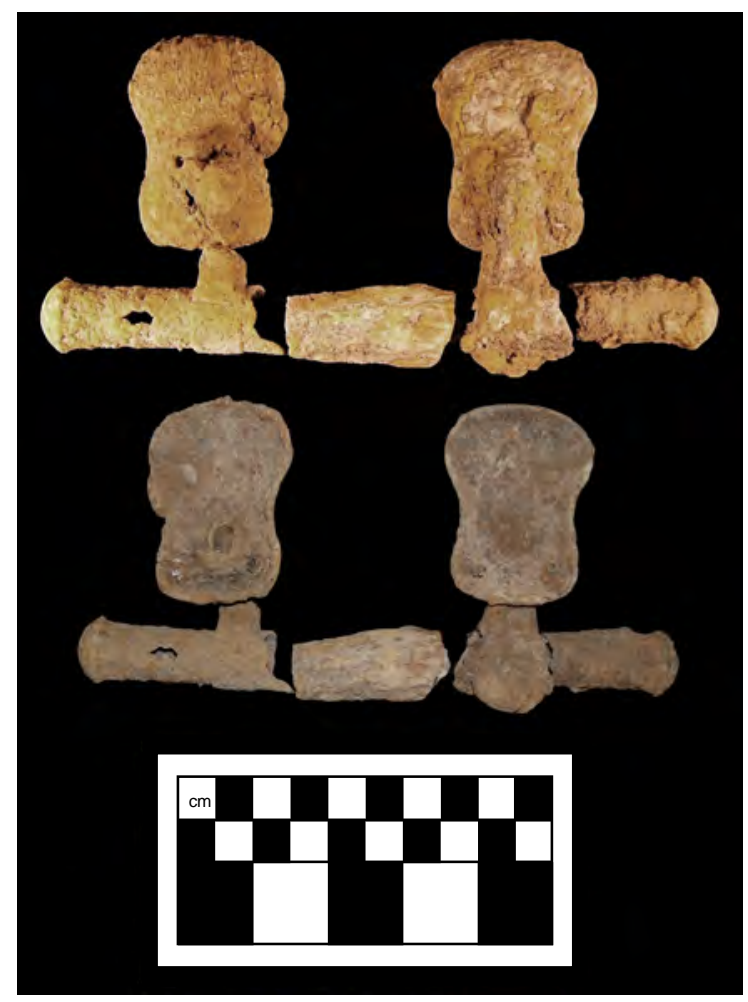

Figure 158. Handle Type 6-double-lug, short-bar handle with an octagonal swell.

\section{Single-Lug, Short-Bar Handles}

The single-lug, short-bar handle is a variant on the short-bar concept, which could employ a single lug with a single arm (sometimes known as drop handles), or more commonly a generally rectangular lug with two attached arms. Davidson (1999:540) notes that this is a "rarer form than the more common double lug short bar types" with a conceivable introduction date around the same time as the double-lug, short-bar handles ca. 1870. A cursory examination by Davidson (1999:540) of period hardware suggests these single-lug, short-bar forms did not increase in popularity until the 1890s. The double-arm lug variety did become popular in the 1890s, and its rise to greater prominence is closely tied to the increased marketing and development of the extension bar (to be discussed later), which used exactly the same components: a bar of variable length, interchangeable lugs, interchangeable arms, and end caps or finials. Single-lug, short bars would often be matched in style with extension bars and placed at both the head and foot ends of the burial container. This practice is still common in the industry today.

Too little information is currently synthesized about the development of the plethora of lug designs to make this element temporally diagnostic beyond the ca. 1890 introduction. Using the shape of the bar as a diagnostic characteristic, however, is a simpler task and can be used in some cases to roughly date certain handle types. The 1947 catalog of the Parsons Casket Hardware Company was very considerate in its inclusion of a fairly comprehensive guide to sizes and styles of bars offered for sale with their handles (Figures 162 and 163). This guide sheds light on the variety of handle shapes that were used in the late 1940s. 


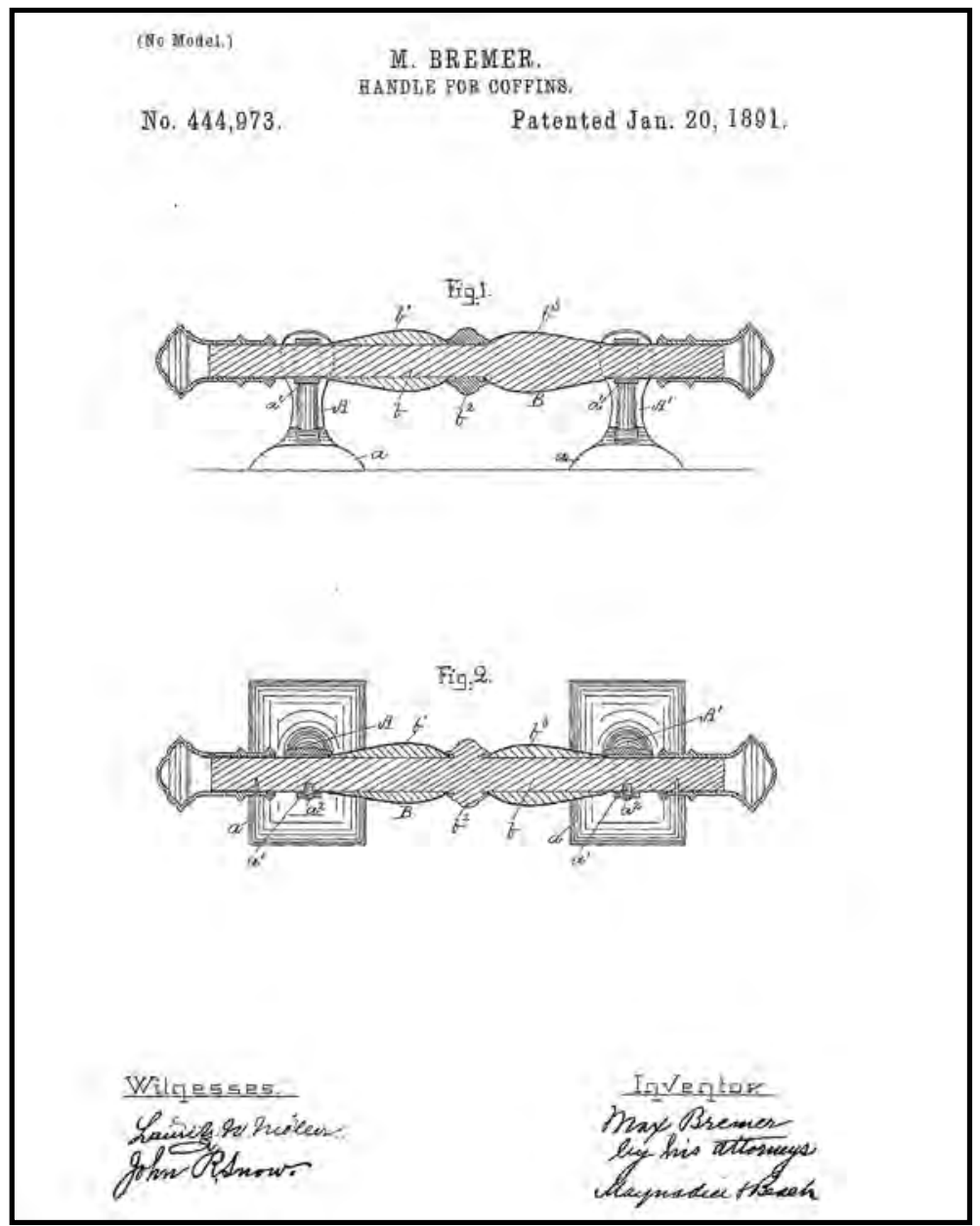

Figure 159. U.S. Utility Patent No. 444973 assigned in 1891 to Max Bremer for a coffin handle exhibiting a swell-bar form.

A survey of hardware and burial container catalogs from the 1940s backward in time reveals several general trends in handle shape. From the 1890s until the 1920s, most of the bars used for double-lug and single-lug short-bars as well as for extension handles were variations of round (including the octagonal) and square, sometimes with the addition of swell-bar elements. In the 1920s, swell-bars and round rope designs lost momentum, but the cloverleaf and oval cloverleaf handles emerged. It was not until the 1940s that other prominent bar shapes, such as the Triple Grove and the Fancy Reeded Oval Bar, began to appear in catalogs. The above chronological outline is far from precise, but does represent an initial attempt at organizing the increasingly standardized handle variants of the twentieth century.

\section{Handle Type 7}

Handle Type 7 (Figure 164) is represented by six single-lug, short-bar handles, five of which were recovered from Burial 5 and the other recovered in backfill in the east side toward the north end of the New Home Cemetery excavation area (see Appendix A). Although the last piece was found in disturbed context, the fact that five identical handles were recovered from only one burial suggests that the lone handle belongs to Burial 5 as well. 


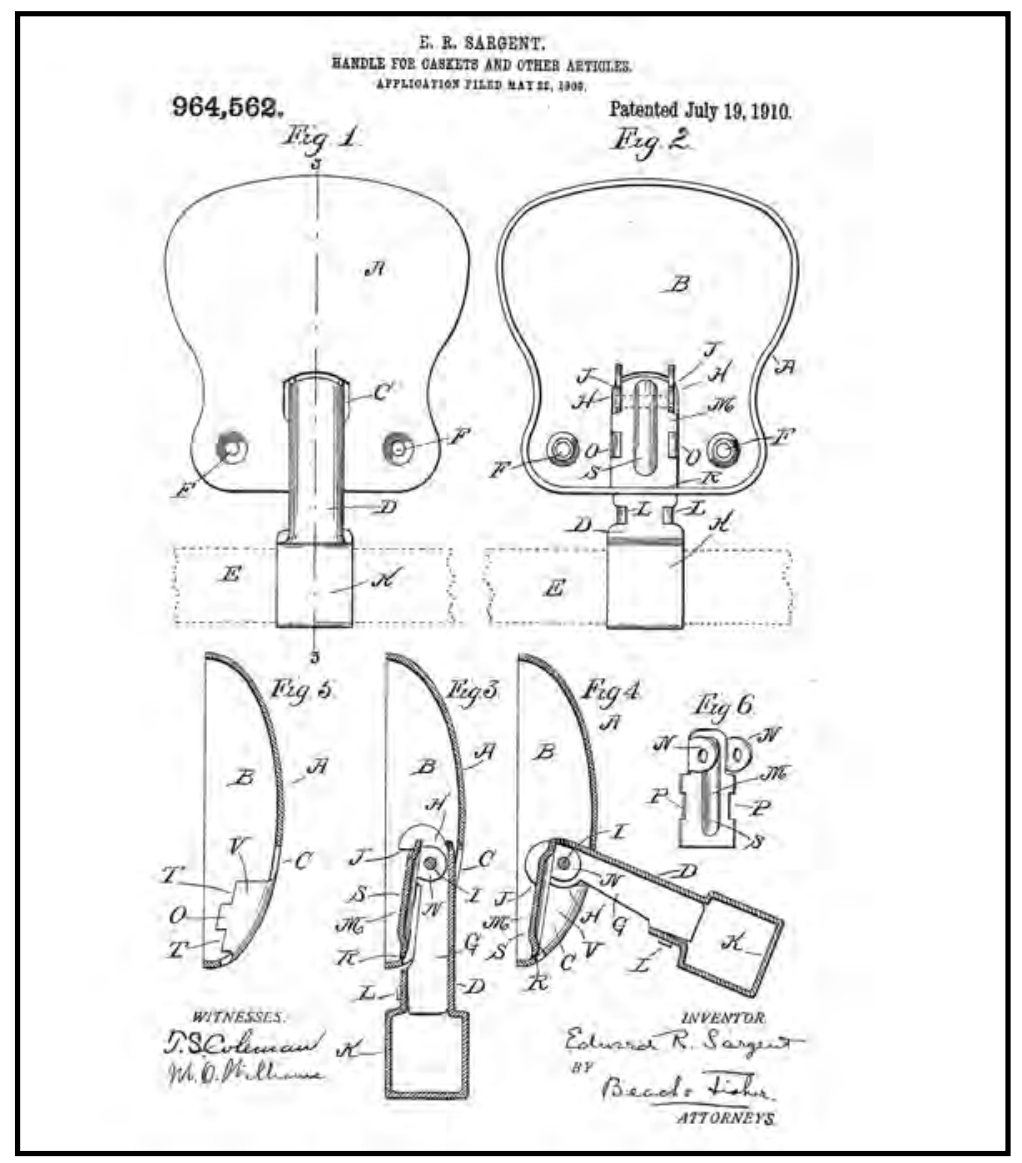

Figure 160. U.S. Utility Patent No.964562 assigned to Edward R. Sargent in 1910 for a casket handle.

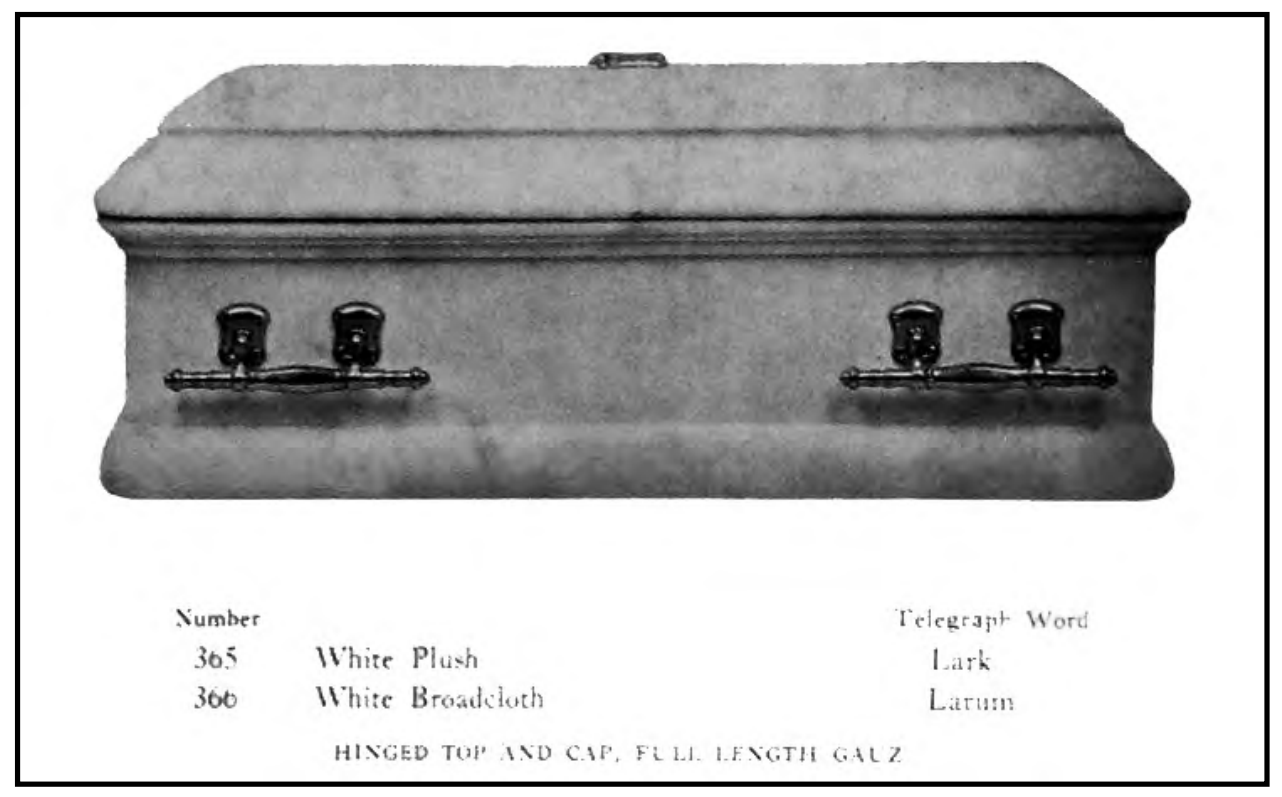

Figure 161. Casket exhibiting a short-bar handle, similar to Handle Type 6, on page 30 of the ca. 1910 Dallas Coffin Company catalog. 


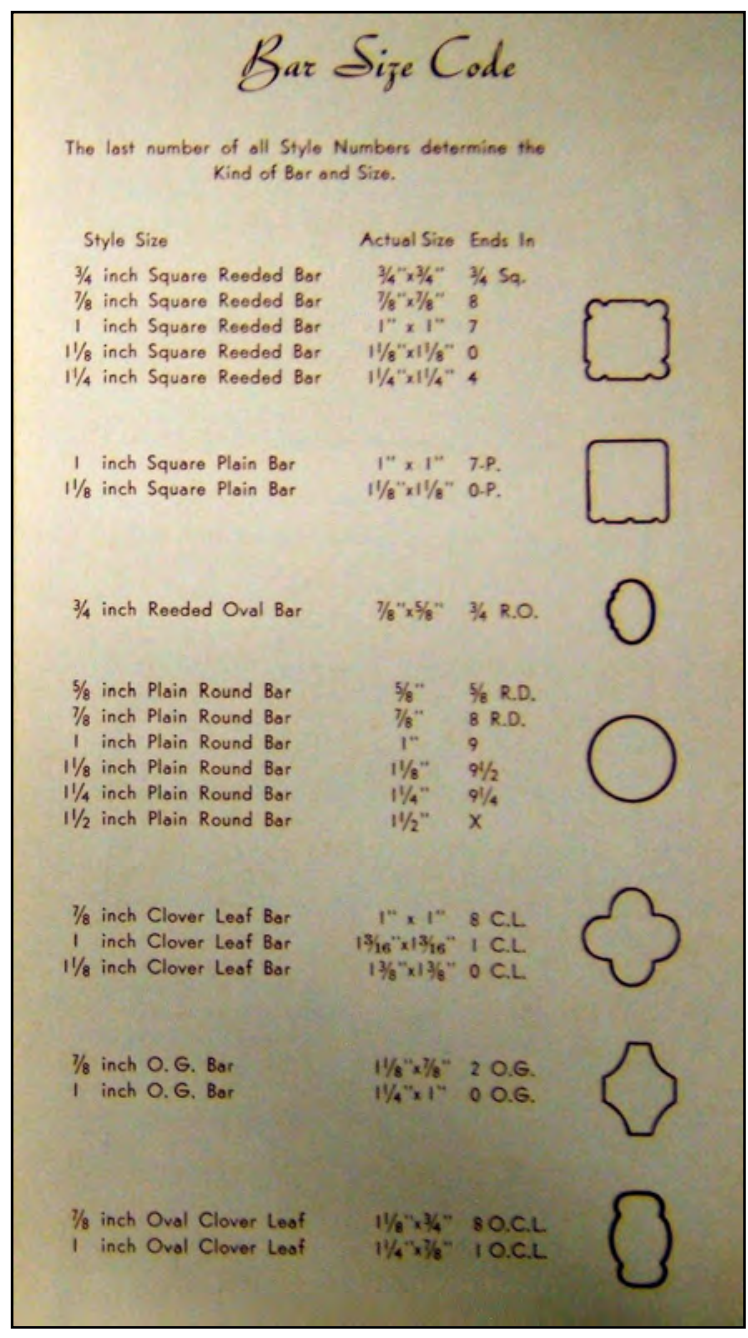

Figure 162. Parsons Casket Hardware Company 1947 Bar Size Code page 1.

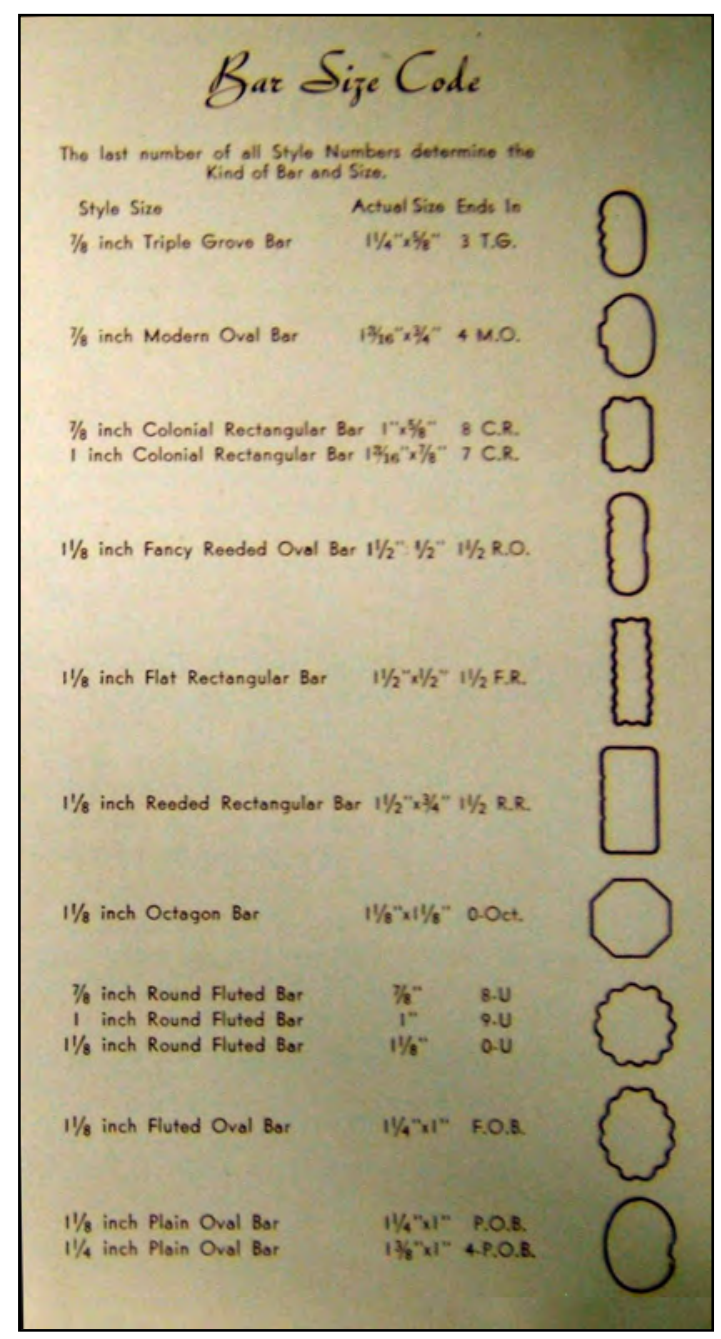

Figure 163. Parsons Casket Hardware Company 1947 Bar Size Code page 2.

Except for the disturbed-area piece, all handles are relatively intact and were made of silver electroplated steel. The bar is a 1-inch Clover Leaf Bar, which places the possible origin of this handle in the 1920s. The end cap is shaped like a flared fan with a narrow border around the perimeter. The front and back segments of the cloverleaf pattern on the handle continue onto the end cap design until reaching a slightly more bulbous termination. The lug is rectangular with rounded corners and very slightly elevated shoulders and a pronounced tympanum. There is a narrow lipping around the margin of the lug. Ascending from the lower center of the lug is what possibly could be a floral bloom of some kind with at least five segments. There are also one or two arches moving over the arm attachments from the bottom corners toward the bottom center. The lug has four screw holes, one on each side of each arm. The arms are joined to the lugs with a trunnion joint. The arms are rounded and bulbous at the top, constrict slightly and then flare out again upon reaching the handle bar. The arm material wraps around the handle and inserts under two side prongs on the back of the arms. Handle Type 7 does not match any known patents, catalogs, or archaeologically excavated mortuary artifacts (see Appendix D). 


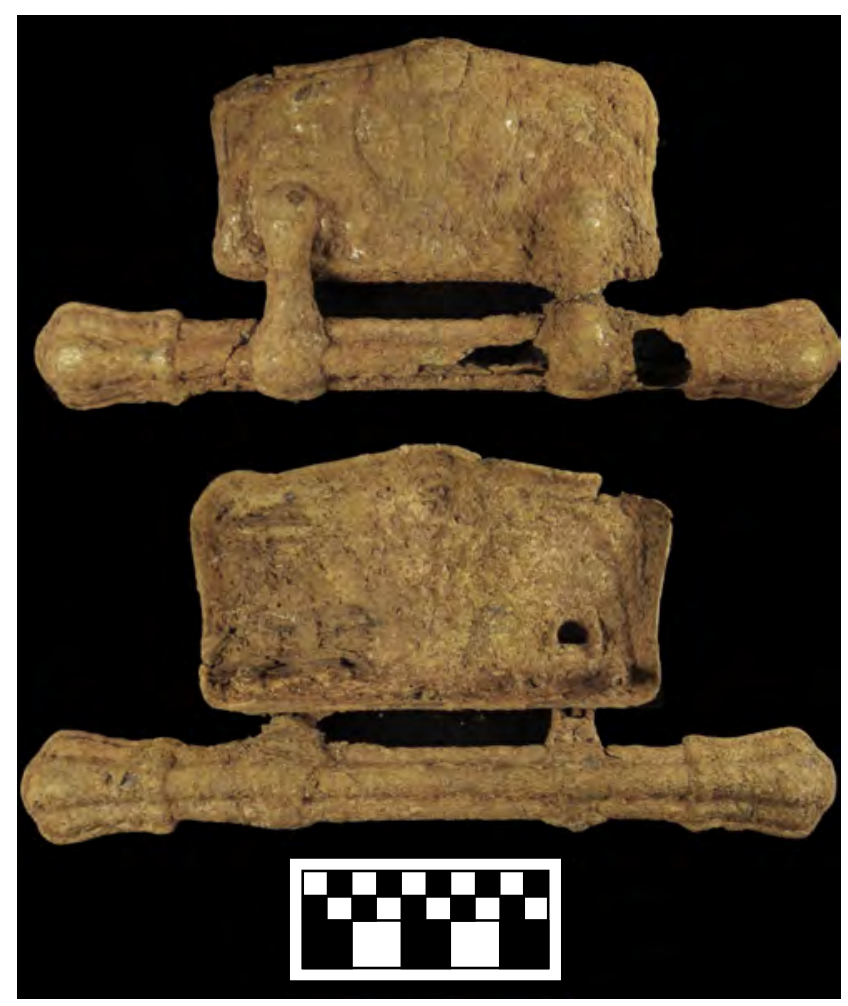

Figure 164. Handle Type 7-single-lug, short-bar handle.

\section{Handle Type 8}

Handle Type 8 is represented by three single-lug, short-bar handles, two of which were recovered with materials from Burial 12 and the other collected in Burial 15 in New Home Cemetery (see Appendix A). The two collected from Burial 12 (Figure 165) are heavily corroded and are stuck together. Because they are stuck together and were apparently stacked at one time suggests that they were not in their original location if they had been used on a burial container. The fact that Burial 12 already has a full set of another type of handle further supports this deduction. It is possible they were originally associated with Burial 15 as well.

All handles are heavily corroded and are of silver or gold electroplated steel. The bar is a $1 \frac{1 / 8-}{8}$ inch Clover Leaf Bar, which places the possible origin of this handle in the 1920s. The end cap is shaped like a flared crown with a small point extending from the apex of the crown. There is a narrow border around the perimeter of the end cap, as well as a wide collar. The lug is rectangular with rounded corners. In the center top edge sits a semicircular platform half the width of the lug. From this platform descend two nested semicircles of increasing size. Extending downward from between the second and third circular platforms is a series of four parallel ridges that travel to the bottom edge of the lug. The lug has three screw holes: one on each of the bottom corners, and the third in the center slightly higher than the other two. The arm attachment type is unclear due to corrosion, as is the attachment type of the arm to the handle bar. The arms are narrow at the top, taper toward the handle, and then flare out upon reaching the handle bar. Handle Type 8 does not match any known patents, catalogs, or archaeologically excavated mortuary artifacts (see Appendix D). 


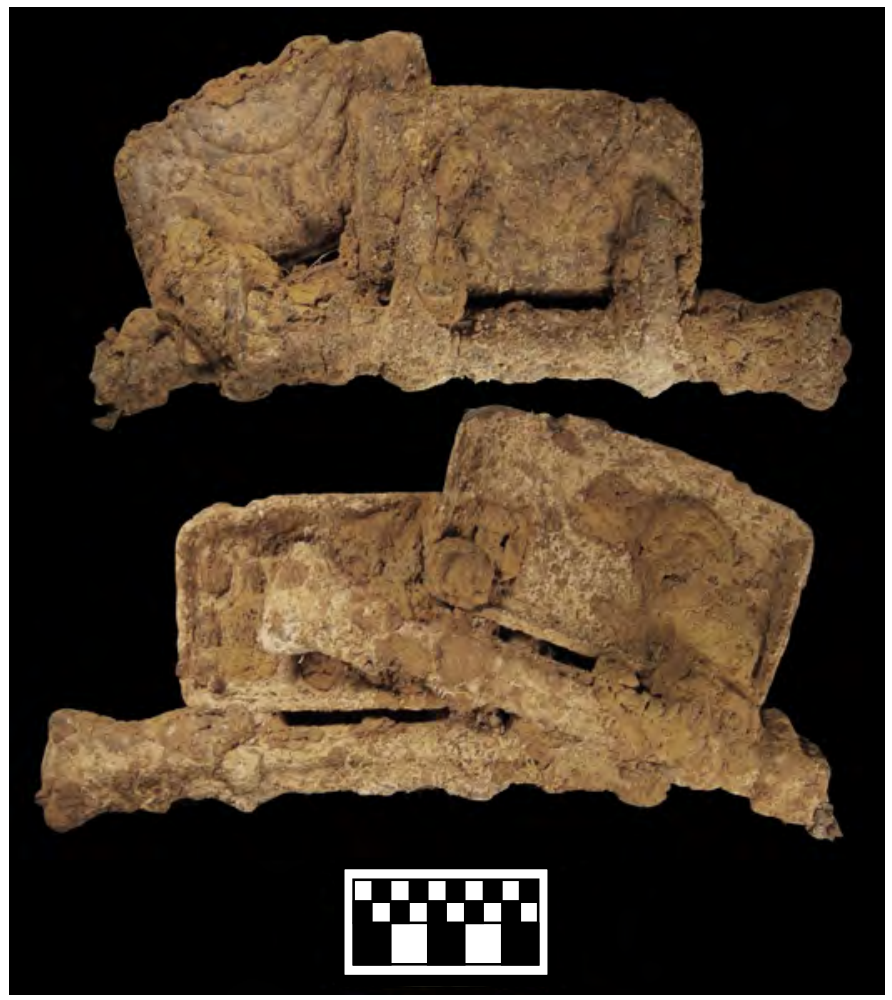

Figure 165. Handle Type 8-single-lug, short-bar handle.

\section{Handle Type 9}

Handle Type 9 (Figure 166) is represented by six single-lug, short-bar handles, five of which were recovered from Burial 19 and the other recovered in backfill adjacent to Burial 19 in New Home Cemetery (see Appendix A). Because of its proximity, the last handle undoubtedly came from Burial 19 also. All handle lugs, arms, and bars are of silver electroplated steel, whereas the finials are of an antimonial lead.

The bar is a $7 / 8$-inch Triple Grove Bar, which places the possible origin of this handle in the 1940s. The finial is shaped like the pommel of a sword with a wide collar, after which the triple grove pattern continues for a short distance before the sides flare out to a raised and slightly curved ring. The finial terminates with a gentle dome. The finial was secured to the inner wooden core of the handle via two small pins or nails placed on the back of the handle. The finial has a silver finish with a black shadowing below the curved ring.

The lug is rectangular with uniform rounded corners and a narrow lipping around the side and bottom margins. The top margin has three tiers as the margin approaches the center, which reflects the three slightly shorter and increasingly wider stacked rectangles that run vertically from the top to bottom of the lug. The lug has four screw holes, one on each side of each arm. The arms are joined to the lugs with a trunnion joint. The arms are rounded at the top with a gently expanding taper to the bottom, which is the widest part. The arm material wraps around the handle and inserts under two side prongs on the back of the arms. Vertical lines also appear on the face of the arms mimicking the taper. Handle Type 9 does not match any known patents, catalogs, or archaeologically excavated mortuary artifacts (see Appendix D). 


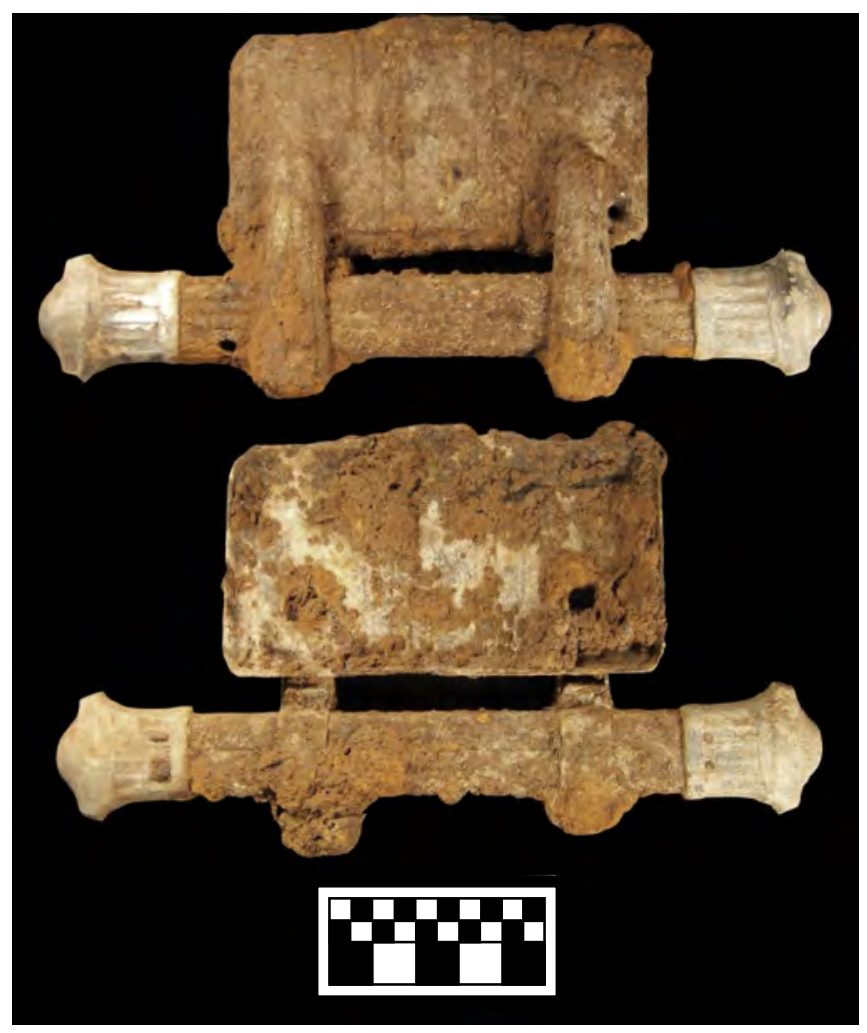

Figure 166. Handle Type 9—single-lug, short-bar handle.

\section{Handle Type 10}

Handle Type 10 (Figure 167) is represented by five intact lugs with arms, one unassociated arm, 12 finials, and 28 bar fragments. Based on the above artifacts, there would have originally been six of these types of handles present in Burial 9 in New Home Cemetery (see Appendix A). The lugs, arms, and bars are of steel, but the finials are of an antimonial lead.

The bar is a $1 \frac{1}{8}$-inch Square Reeded Bar, which places the possible origin of this handle in the 1890s. The finials are square at the base with a narrow lip and widen out to a gently squared and faceted dome at the top. The front and top faces of the finial has a rectangular recess with a double-arched side toward the finial end, which is mirrored a second time below the edge of the dome. The bottom and back faces of the finial share this final double-arched line, but instead of the recessed area, there is a single incised line that runs from the point where the two arches meet to the lip of the finial. There appears to be a cream- to greenish-tinged paint or finish with a black shadowing on these finials. The finial was attached to the wooden core of the handle via a small pin or nail that was placed on the back of the piece.

The lug is generally rectangular with rounded corners with the bottom being widest, the sides slightly concave, and the top rounded. The corrosion is too heavy to tell whether there are any design patterns on the lugs. The lug has four screw holes, one on each side of each arm. The arms attachments are hidden by corrosion. There are heavy corrosion and wood grains present on the arms (suggesting an outer box), so it is difficult to determine the form of the arms as well, but 


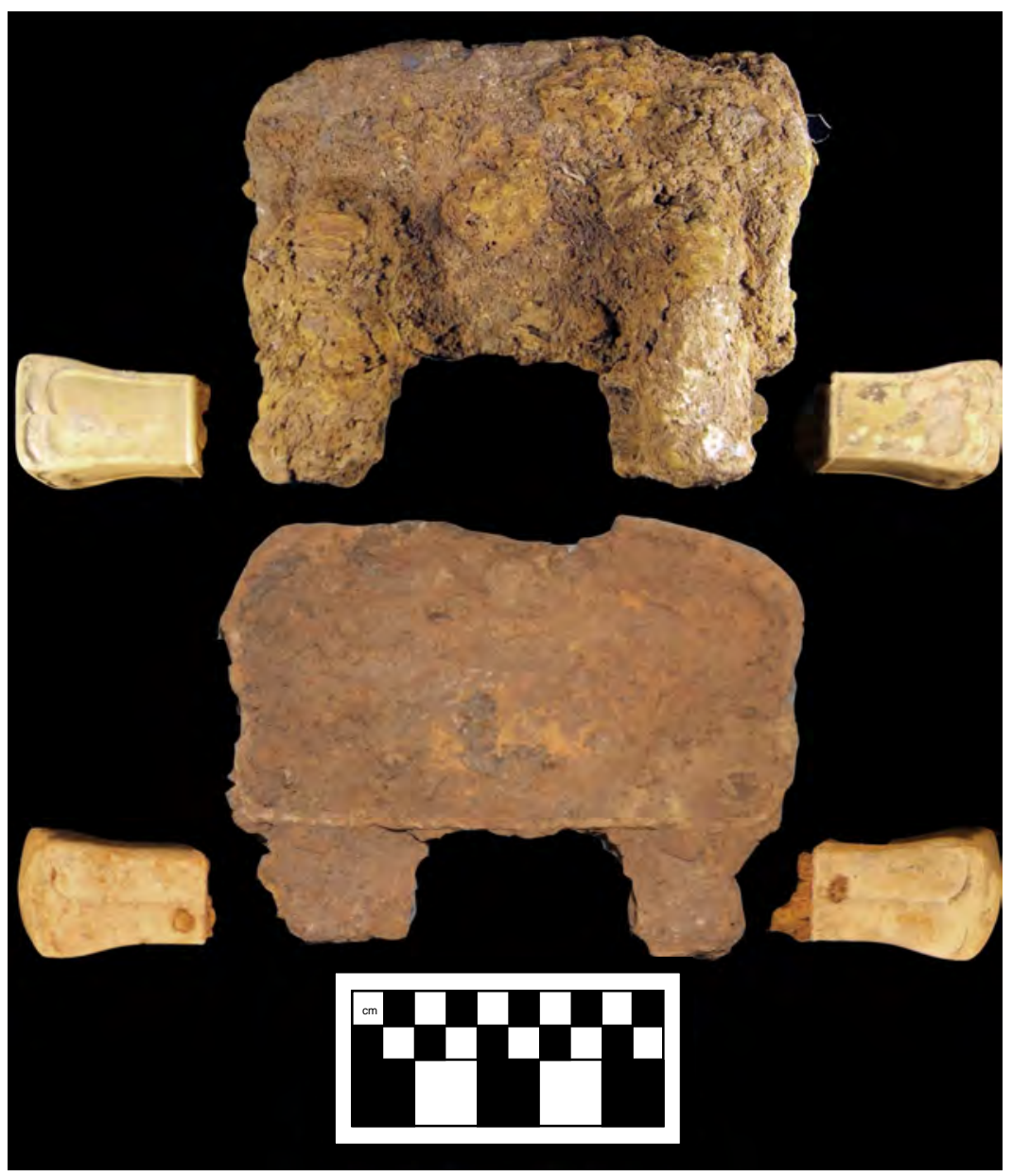

Figure 167. Handle Type 10—single-lug, short-bar handle.

they generally appear to be narrow and rounded at the top tapering to the handle bar. The exact attachment method of the arm to the handle is uncertain. Handle Type 10 does not match any known patents, catalogs or archaeologically excavated mortuary artifacts (see Appendix D).

\section{Swing-Bar Extension Handle}

Extension handles are a type of handle that generally extends the length of the burial container. Figure 168 shows a composite of the variety of extension handles offered for sale in the 1906 Cincinnati Coffin Company catalog. Included are three primary types, all of which have a widely variable component structure: (1) Single-Arm Lug Extension handles typically consist of four lugs with one arm each, the bar/tube, and two end caps or finials; (2) Faux Double-Lug, ShortBar Extension handles typically have six lugs with one arm each, an extension bar with three grip segments (in this case swelled) around which the lugs are positioned, and finally two end caps or finials; (3) Double-Arm Lug Extensions handles most often consist of three plaque type lugs each with two arms, an extension bar, and two end caps or finials. 


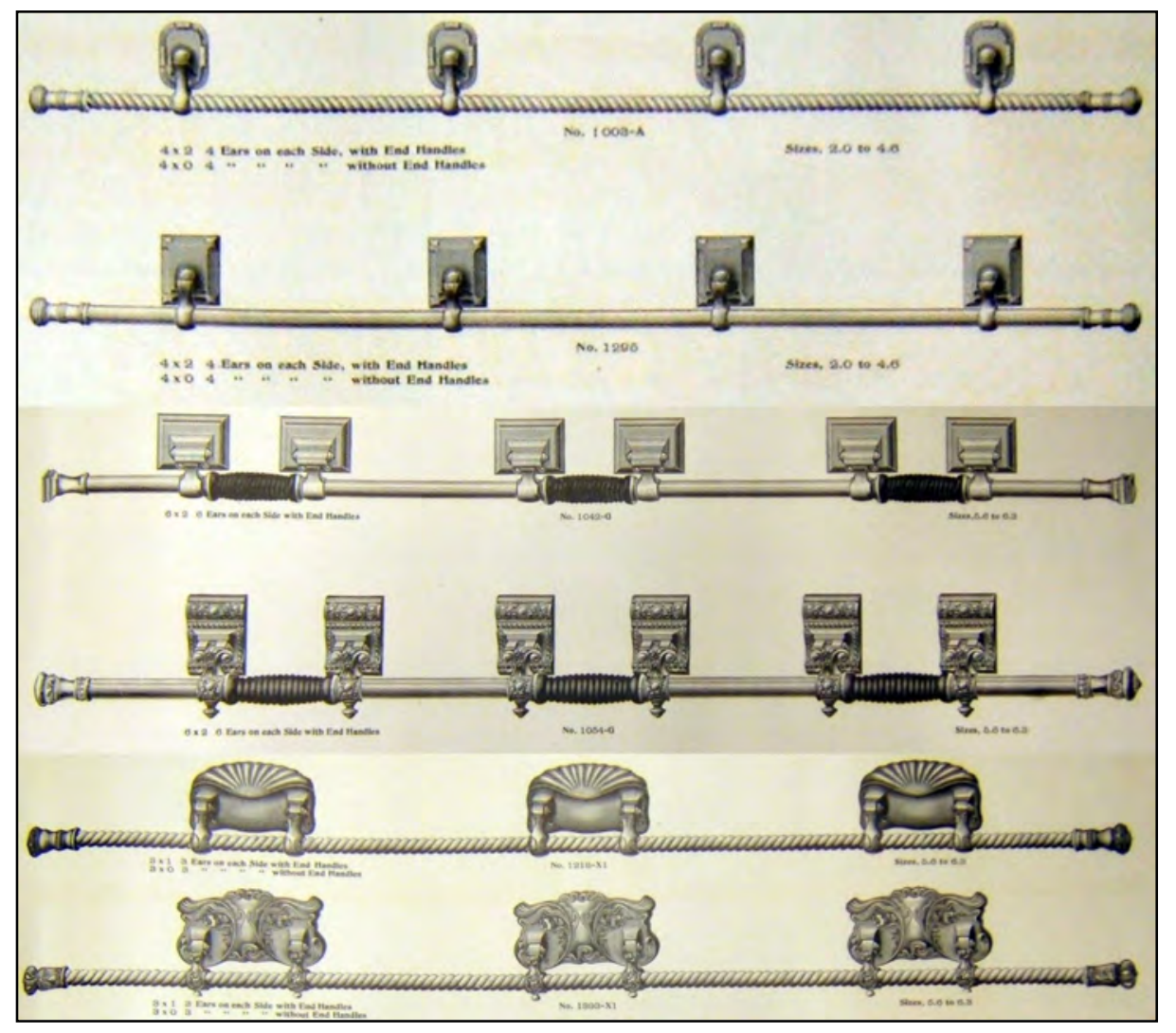

Figure 168. Examples of types of extension handles offered for sale in the 1906 catalog of the Cincinnati Coffin Company.

Extension handles became more prevalent in mortuary merchandise catalogs after the turn of the twentieth century and came to dominate the funeral industry as the century passed. A survey of approximately 200 casket advertising cards dating to the late 1990s from the Batesville and Aurora casket companies revealed only a few caskets that were not outfitted with extension handles (these were hardwood caskets with wooden stationary grips). It would be a mistake, however, to think 1900 marked a date of origin just because the popularity and sale of extension handles increased. The turn of the century instead marked a drastic change in materials used for manufacturing casket hardware and production techniques, which made extension handles less expensive.

The true history of extension handles began as far back as 1865, when a casket fitted with what appears to be a stationary extension bar secured by three lugs appeared in the illustrated price list of the Crane, Breed \& Company (also appearing in the 1867 Crane, Breed \& Company price list) (Figure 169). This piece might have been more ornamental than functional, but it does introduce several important elements in the evolution of extension handles, including the extended bar and symmetrical and/or interchangeable lugs. The first labeled mention of extension bars appears alongside illustrations of handles on page 18 of the 1876 catalog supplement from the Meriden Britannia Company, as well as on page 3 of the June 1876 price list of the Cincinnati Coffin Company. Extension handles continued to appear infrequently in catalogs throughout the 1880s, including the 1885 catalog from the Stein Manufacturing Company (Figure 170). Notice that these early extension handles were almost always of the single-arm lug variety, with three lugs appearing on the casket. 


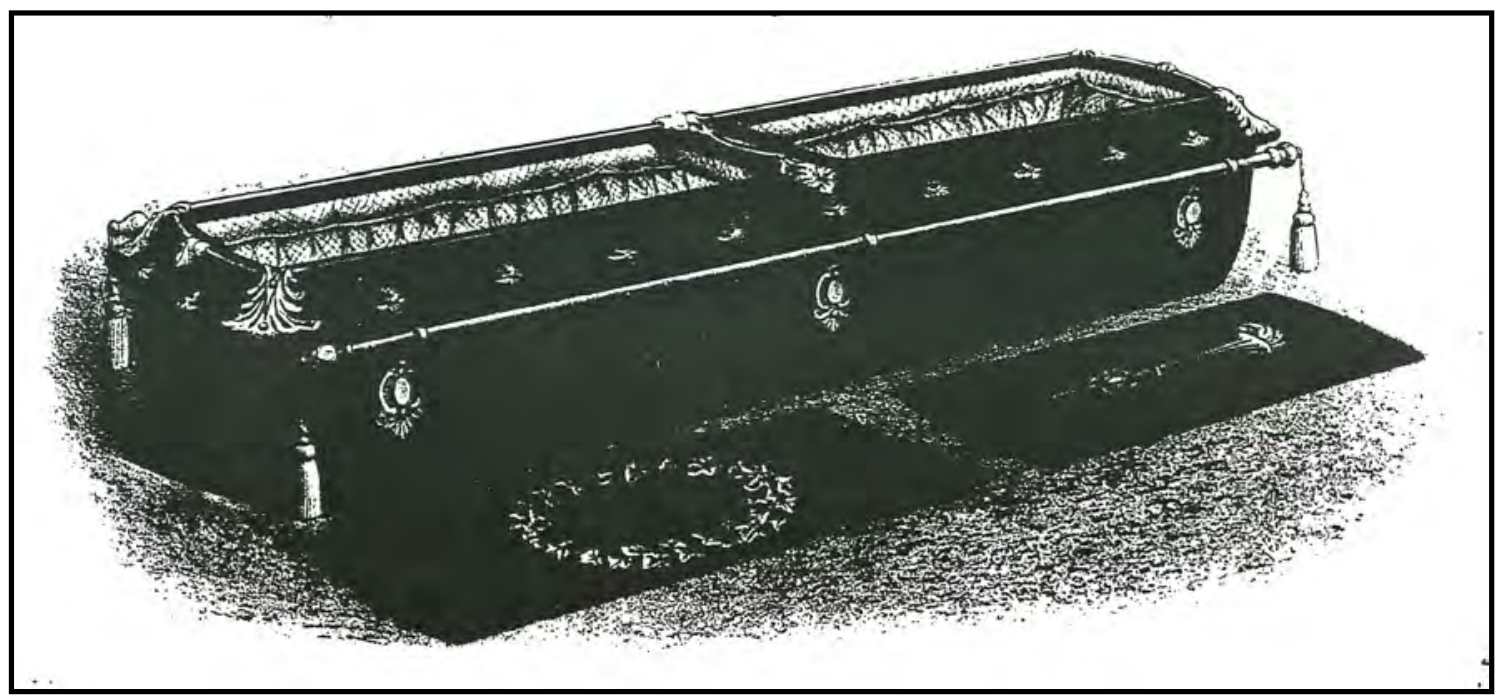

Figure 169. Early example of an extension bar handle illustrated on page 12 of the 1867 Crane, Breed \& Company catalog.

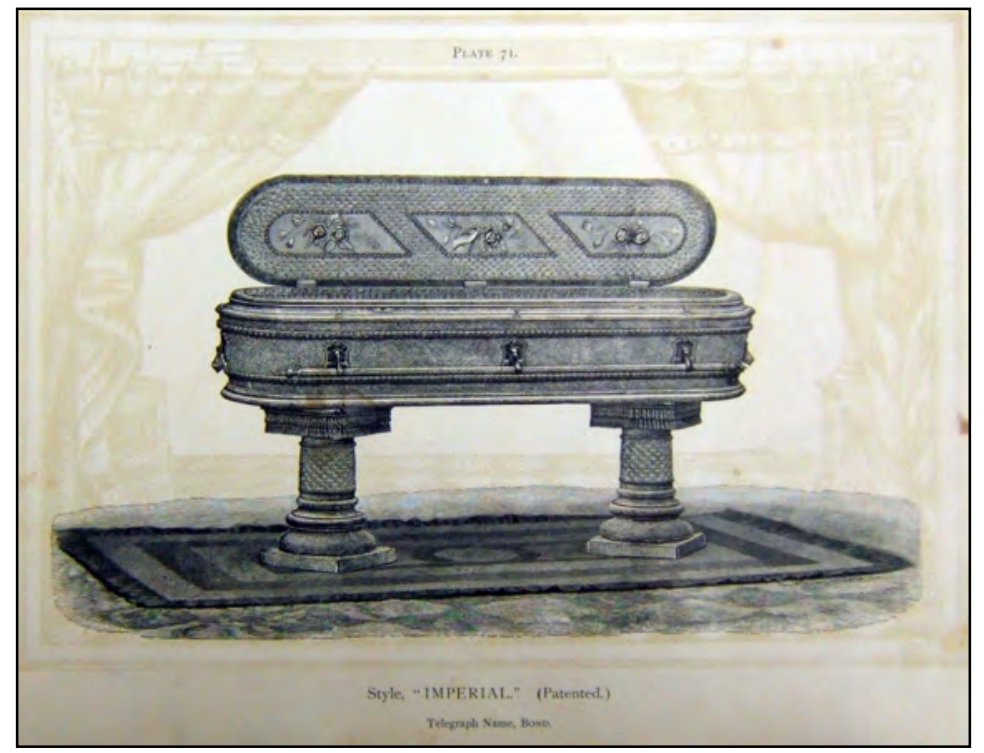

Figure 170. Casket with swing-bar extension handle advertised on page 13 of the 1885 Stein Manufacturing Company catalog.

Although no patents were located to document their development, the double-arm lug extension bars seem to have appeared ca. 1890. This type of handle appears in an advertisement in the 1893 July-August issue of the National Casket Company Bulletin (Figure 171). It is this type of handle that became most popular in the twentieth century. 


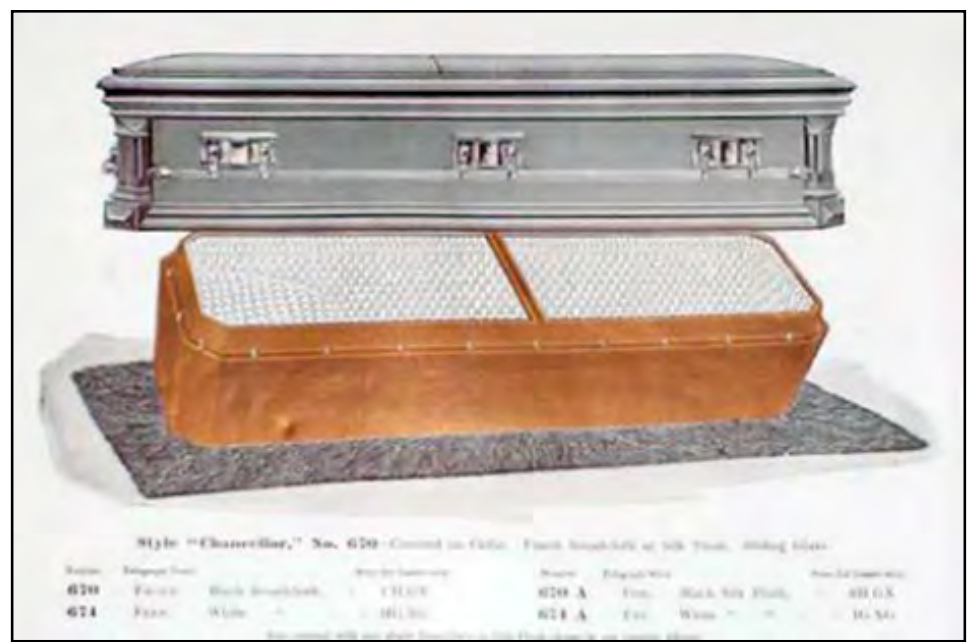

Figure 171. Early example of double-arm lug swing-bar extension handles on casket advertised on page 19 of the July-August 1893 National Casket Company Bulletin.

\section{Handle Type 11}

Handle Type 11 (Figures 172 and 173) is represented by three double-arm lugs, six associated arms, and one extension bar with two end caps. All pieces are of silver electroplated steel and all were recovered from Burial 11 in New Home Cemetery (see Appendix A). The bar is a 1-inch Clover Leaf Bar, which places the possible origin of this handle in the 1920s. The end cap is wider than the bar itself and continues the cloverleaf style with constricting layers of stacked domes.

The lug is rectangular with rounded corners, slightly elevated shoulders, and a tympanum. There is a raised ridge running along the bottom margin, up the sides, and around the shoulders. Before the tympanum begins to slope upward, the ridge curves sharply downward on each side and creates a fan like design in the central portion of the lug. There are subsequent lines mimicking this curve within the fan. The lug has three screw holes, one on each bottom corner, and one slightly higher on the midline. The arms are joined to the lugs with a trunnion joint. The sides of the arms are roughly parallel before reaching the handle, at which point they flare out considerably before wrapping around the handle and inserting under two side prongs on the back of the arms. No design elements appear on the surface of the arms. Handle Type 11 does not match any known patents, catalogs or archaeologically excavated mortuary artifacts (see Appendix D).

\section{Handle Type 12}

Handle Type 12 is represented by five segments of ferrous metal handle bar, one segment of handle bar with an end cap (Figure 174), and one double-arm lug with one attached handle bar segment (Figure 175). All pieces were found in disturbed contexts in New Home Cemetery (see Appendix A). The bar is a $7 / 8$-inch Triple Grove Bar, which places the possible origin of this handle in the 1940s. The end cap is wider than the bar itself, but it also matches the triple grove style. 


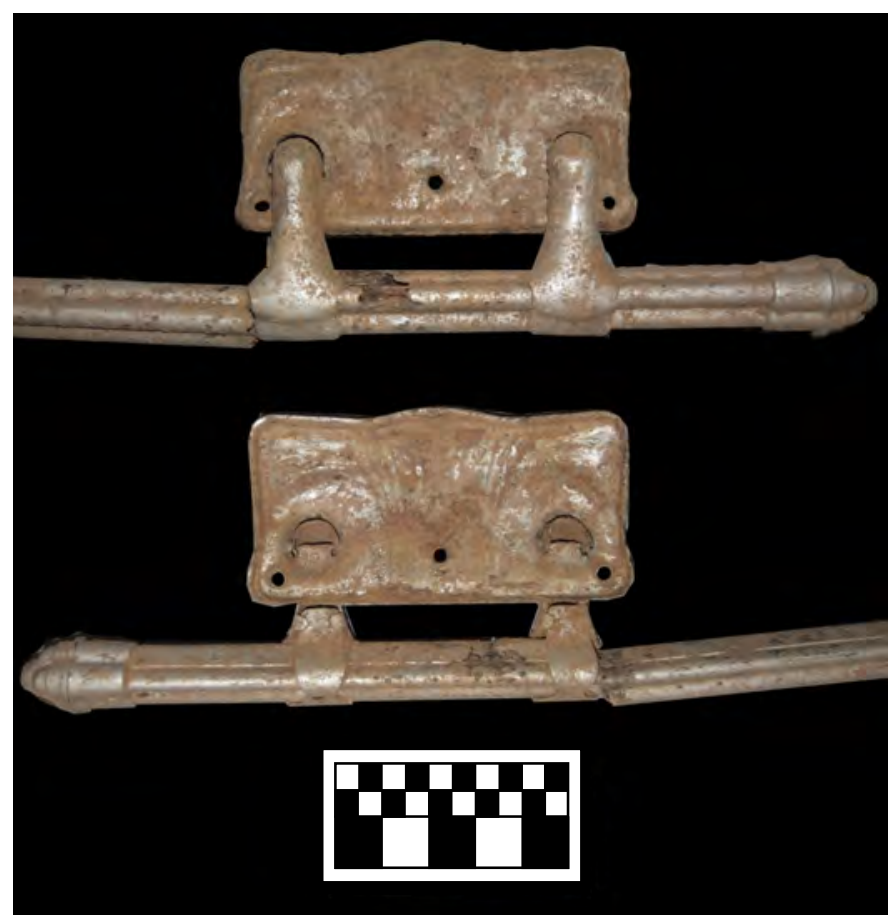

Figure 172. Close-up of Handle Type 11 lug, bar, and end cap.

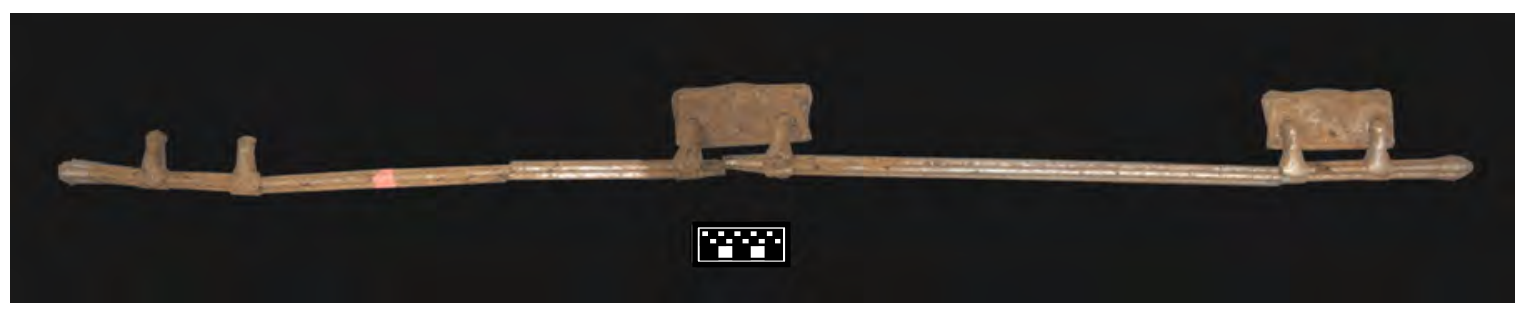

Figure 173. Handle Type 11—double-arm lug swing-bar extension.

The lug is rectangular with rounded corners. There is a fair amount of corrosion, but it appears that a central platform runs from top to bottom with vertical parallel lines decorating it. At least two parallel lines run from the sides and meet the central platform toward the top of the lug. The lug was secured with four screws, two flanking each arm. The arms are gently tapered from top to bottom and appear to grasp the handle bar. No decorative elements are readily apparent on the surface of the arms. From the one visible arm attachment location, it seems that the arms attached at a trunnion joint. Handle Type 12 does not match any known patents, catalogs, or archaeologically excavated mortuary artifacts (see Appendix D). 


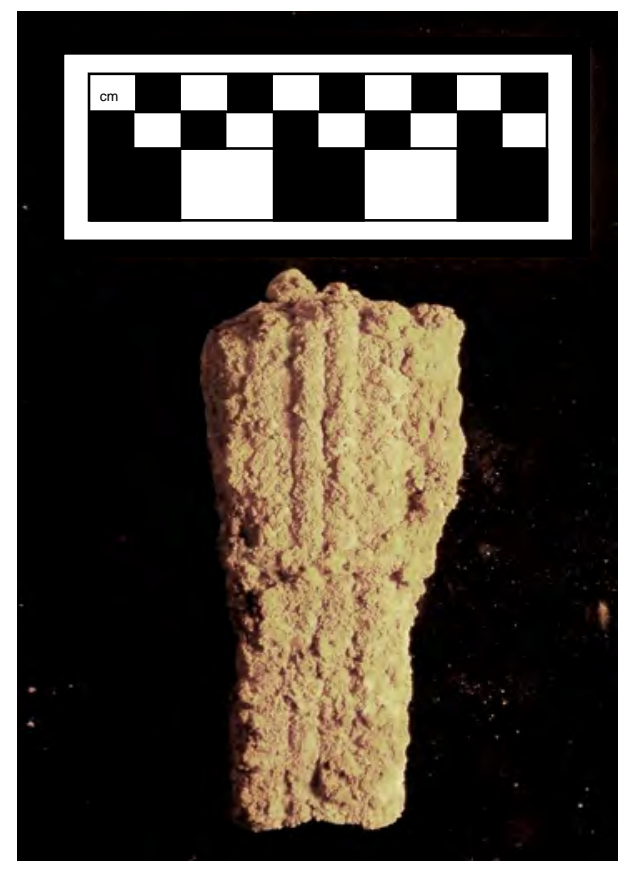

Figure 174. Handle Type 12—swing-bar extension, bar segment and end cap.

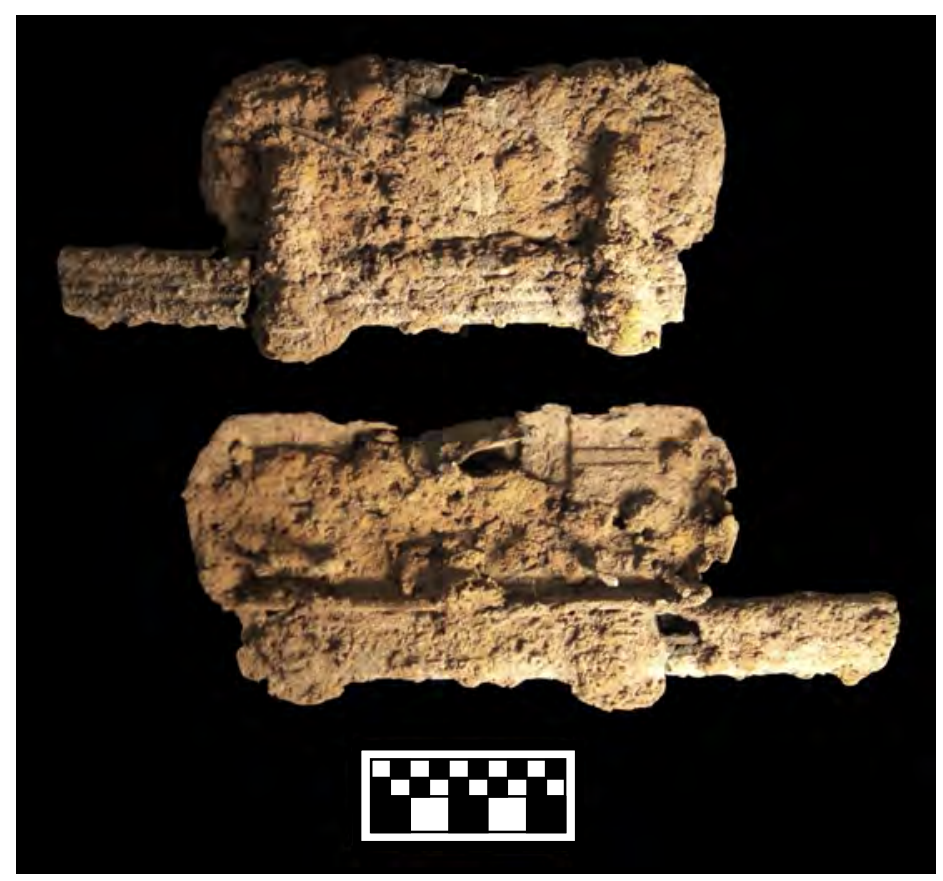

Figure 175. Handle Type 12-swing-bar extension, double-arm lug and bar segment. 


\section{Handle Type 13}

Handle Type 13 (Figures 176 and 177) is represented by two extension handles composed of three double-arm lugs, six associated arms, and one extension bar, with two finials each. The lugs, arms, and bar are of steel, whereas the finials are of an antimonial lead. Very little of the bar actually remains intact, but there were more than 450 fragments of the bar collected from excavations. All handle components were recovered from Burial 18 in New Home Cemetery (see Appendix A).

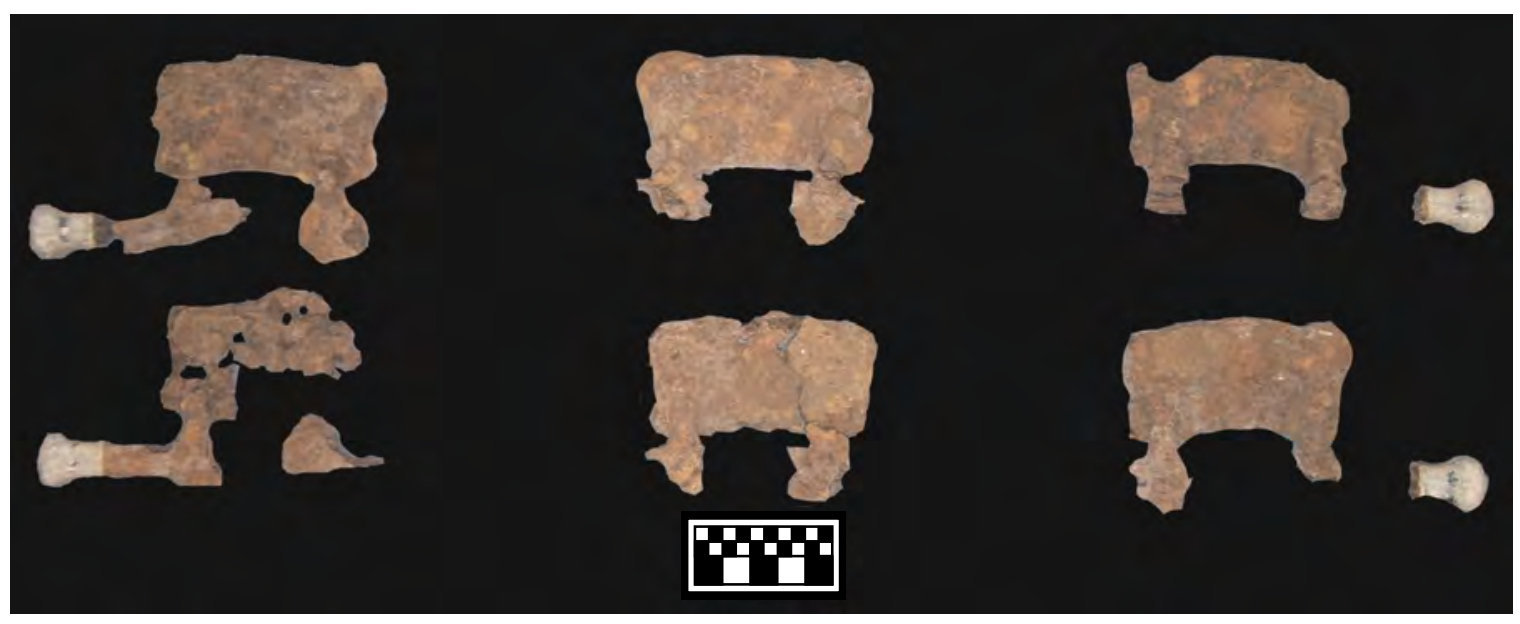

Figure 176. Handle Type 13—double-arm lug swing-bar extension.

The bar is a 1-inch Clover Leaf Bar, which places the possible origin of this handle in the 1920s. The finials are shaped such that each quadrant of the Clover Leaf Bar appears to continue to a rounded termination and another dome is blossoming from the center. The finials are attached to the bar by a small pin or screw driven through the back of the finial and into the handle's wooden core. The lug is generally rectangular with rounded corners and looks somewhat like a banner, with the midsection curved at both the top and bottom. It has slightly elevated shoulders, but no specific design elements can be discerned through the heavy corrosion. The lug has four screw holes, one on each side of each arm. The arms are attached to the lug via a trunnion joint. The sides of the arms are roughly parallel before reaching the handle, at which point they flare out considerably as it reaches the handle. The exact attachment method of the arm to the handle is uncertain. Handle Type 13 does not match any known patents, catalogs or archaeologically excavated mortuary artifacts (see Appendix D).

\section{Handle Type 14}

Handle Type 14 (Figures 178 and 179) is represented by two extension handles consisting of only five of the required six double-arm lugs, only nine associated arms, four finials, and greater than 100 fragments of the extension bar. The lugs, arms, and bar are of steel, and the finials are of an antimonial lead. All handle components were recovered from Burial 13 in New Home Cemetery (see Appendix A). 


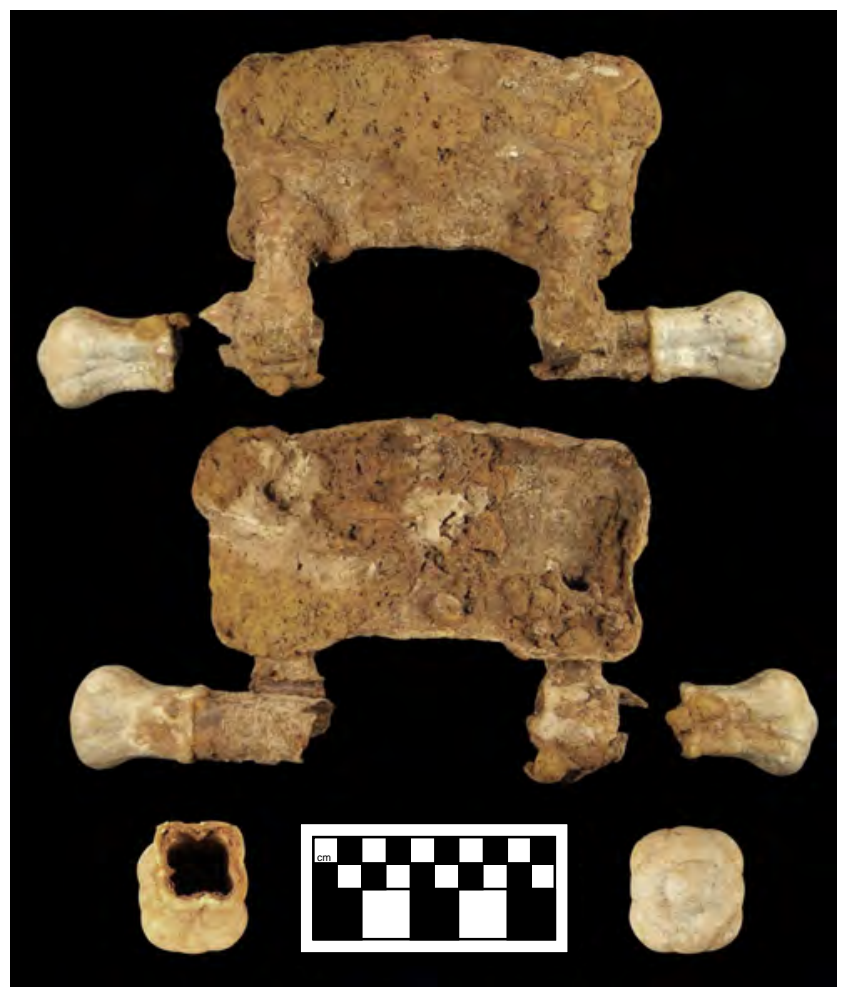

Figure 177. Close-up of Handle Type 13 lug, bar segment, and finials.

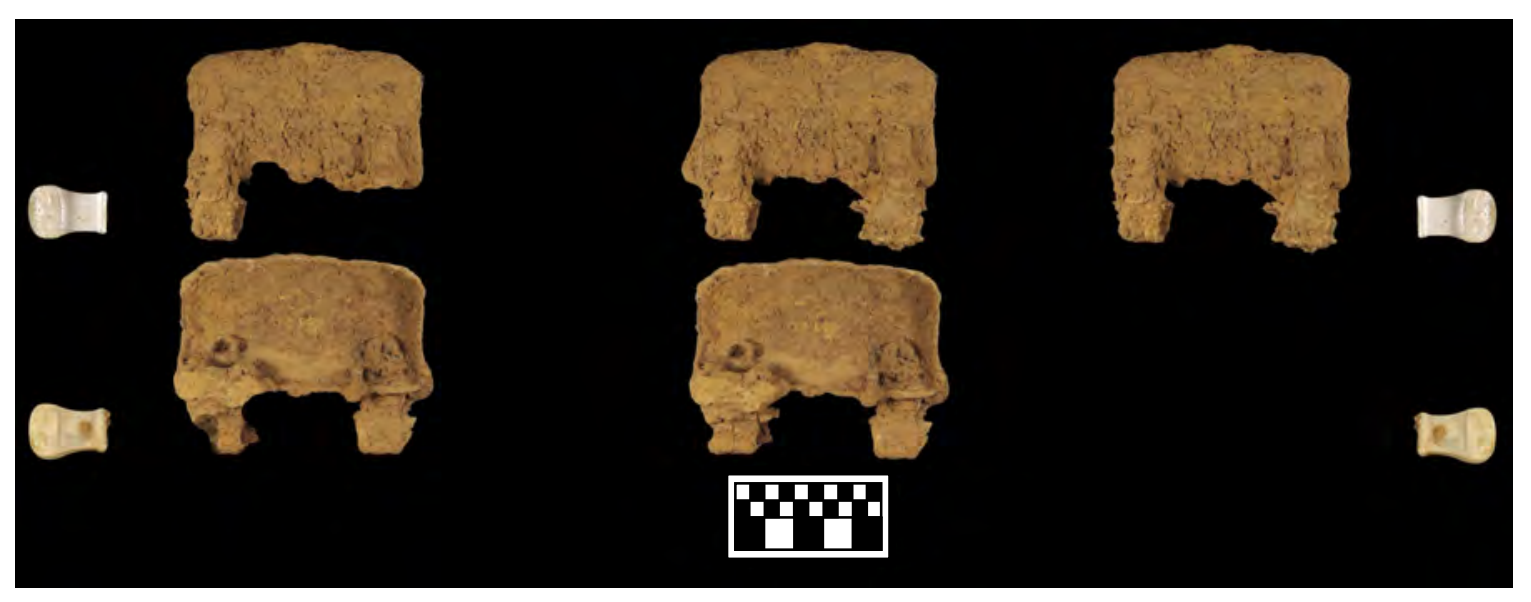

Figure 178. Handle Type 14—double-arm lug swing-bar extension.

The bar is a 3/4-inch Square Reeded Bar, which places the possible origin of this handle in the 1890s. The finials are square at the base with a narrow lip and widen out to a gently squared and faceted dome at the top. There is a raised decorative banner at the head of the finial on each side. The lower line is gently curved from one side to the other; the top line has a sharper curve coming from each side and meeting in a point in the middle. The finials are attached to the bar by a small pin or screw driven through the back of the finial and into the handle's wooden core. The lug is generally rectangular with rounded corners with the bottom being widest, the sides slightly concave, and the top rounded. There appear to be three small bumps along the top at the center, 


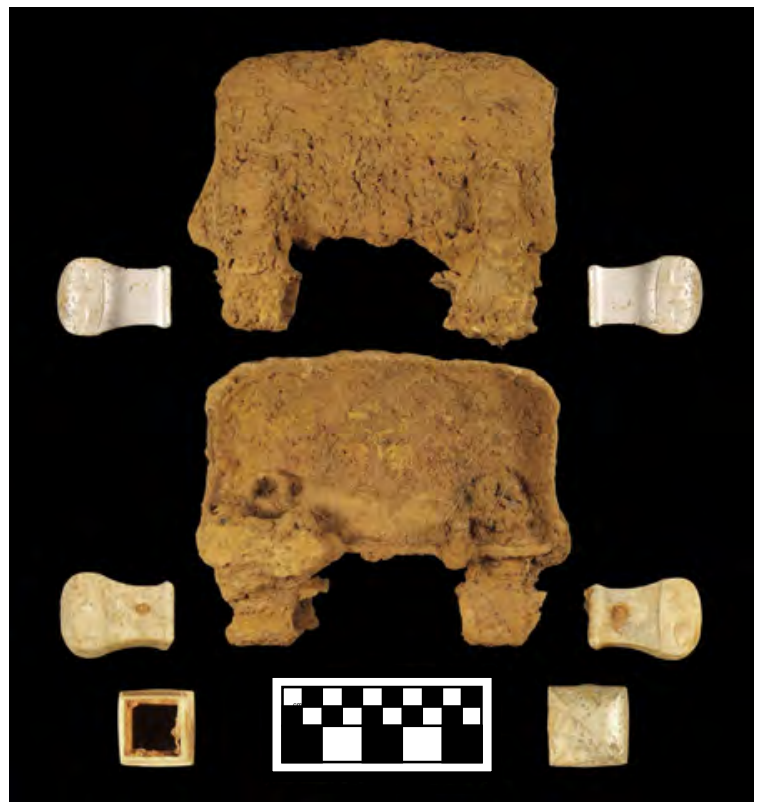

Figure 179. Close-up of Handle Type 14 lug and finials.

which could suggest decorative elements, but the corrosion is too heavy to tell for certain. The lug has four screw holes, one on each side of each arm. The arms are attached to the lug via a trunnion joint. The sides of the arms are roughly parallel before reaching the handle, at which point they flare out considerably. The exact attachment method of the arm to the handle is uncertain. Handle Type 14 does not match any known patents, catalogs or archaeologically excavated mortuary artifacts (see Appendix D).

\section{Handle Type 15}

Handle Type 15 (Figures 180 and 181) is represented by seven segments of two extension bars with double-arm lugs. All pieces are heavily corroded but were probably made from an electroplated steel and all were recovered from Coffin 5 in New Home Cemetery (see Appendix A).

The bar is a $7 / 8$-inch Oval Clover Leaf Bar, which places the possible origin of this handle in the 1920s. The end cap is corroded to the extent that the type cannot be described accurately. The lug is shaped like a squat loaf with rounded corners, a constricted midline, and a slightly raised plateau at the top. No specific stylistic elements can be discerned through the corrosion on the lug. The lug has three screw holes: one on each bottom corner, and one slightly higher on the midline. The arms are joined to the lugs with a trunnion joint. The sides of the arms are gently tapered with the widest point being at the bottom. The attachment of the arm to the handle is different from all other handles recovered. The end of the arm material that wraps around the handle bar is shaped like a "T," which is inserted under two vertical prongs reaching down from the back of the arm. No design elements appear on the surface of the arms. Handle Type 15 does not match any known patents, catalogs, or archaeologically excavated mortuary artifacts (see Appendix D). 


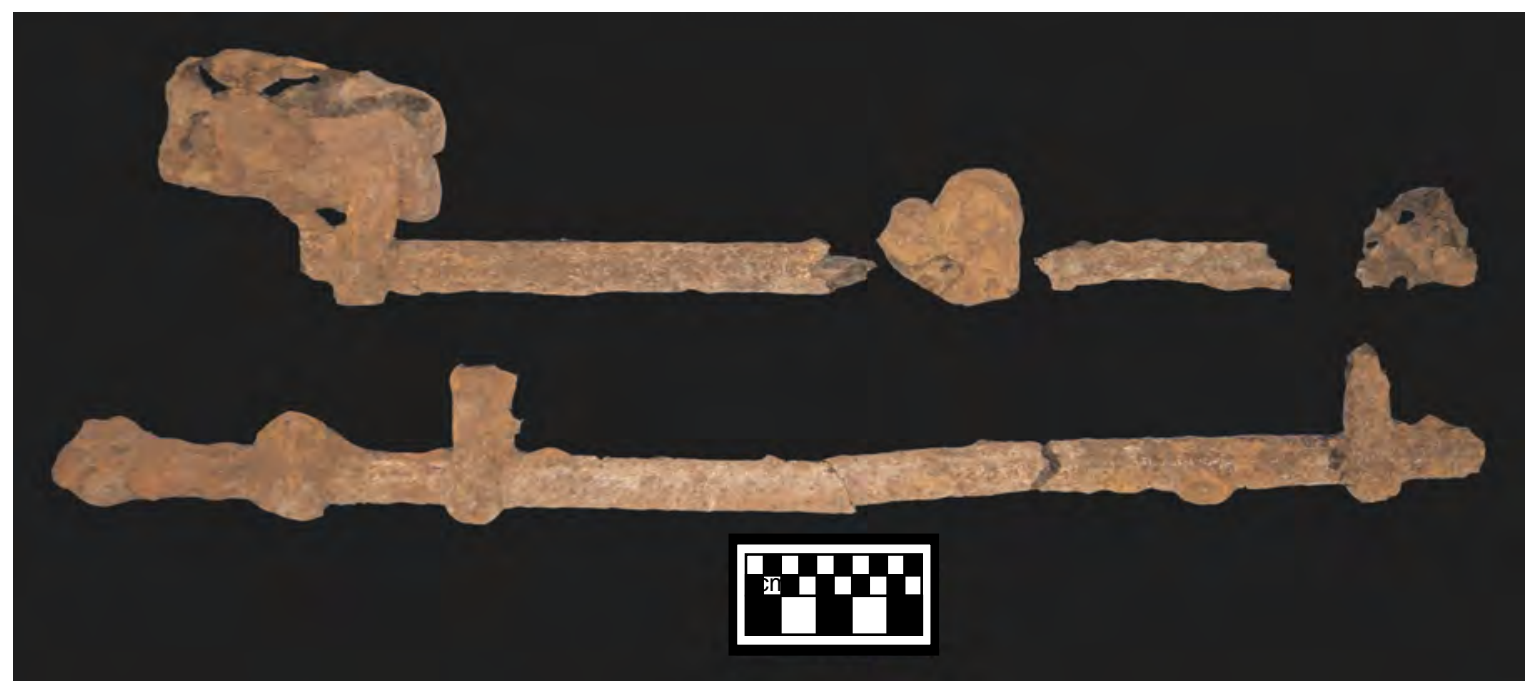

Figure 180. Handle Type 15-double-arm lug swing-bar extension.

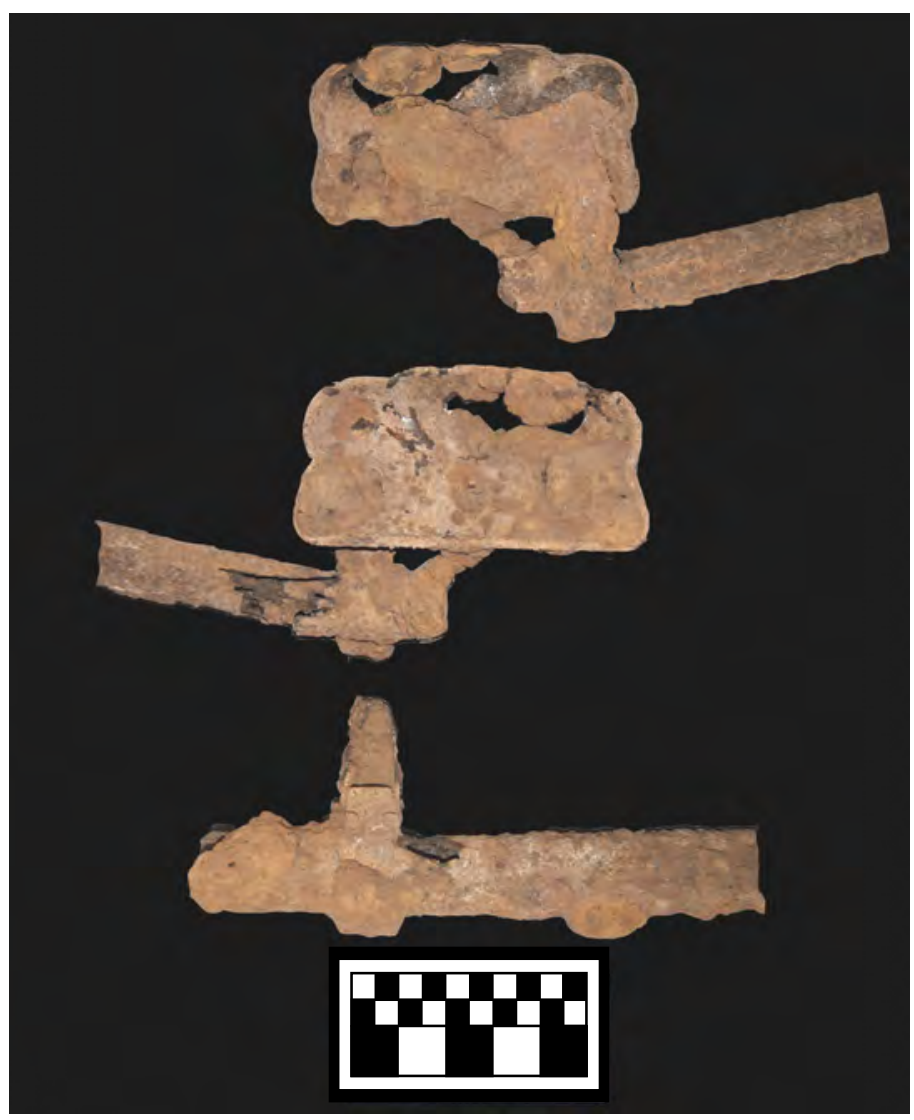

Figure 181. Close-up of Handle Type 15 lug and bar segment. 


\section{Handle Type 16}

Handle Type 16 (Figure 182) is represented by two right sections of extension bars including two double-arm lugs, four associated arms and two segments of handle bar and two associated end caps, as well as one segment of undifferentiated handle bar and a left end cap segment. All pieces are of silver electroplated steel, and all except the last mentioned were recovered from Burial 12 in New Home Cemetery. The last piece was found while scraping deeper in the eastern section where Burials 12 and 15 had been located (see Appendix A).

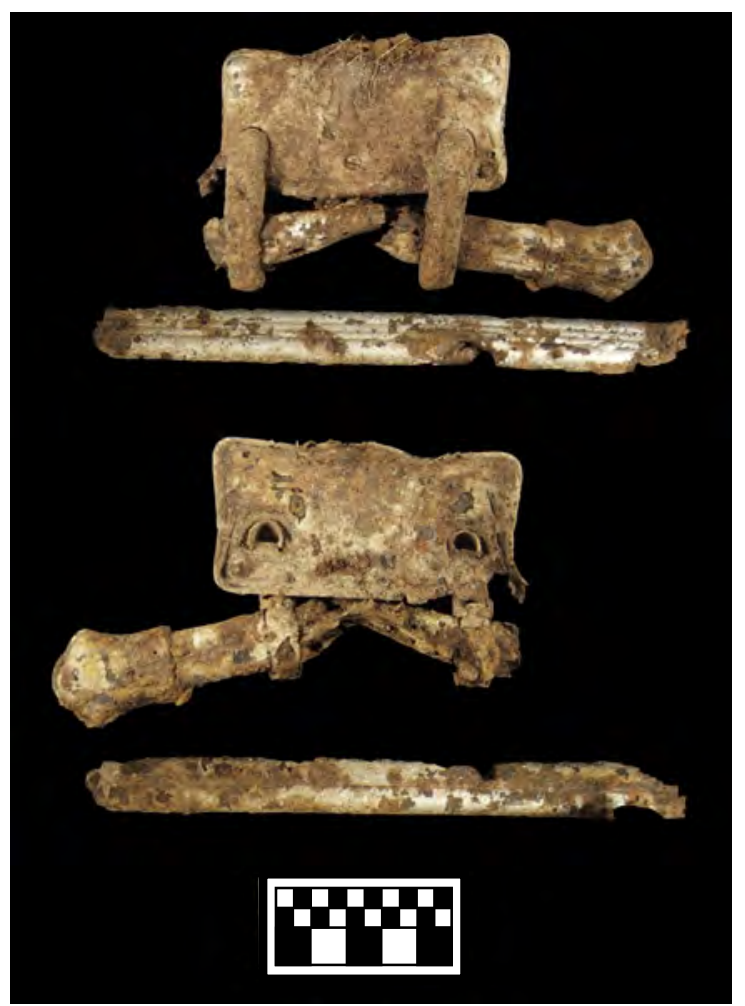

Figure 182. Handle Type 16-double-arm swing-bar extension.

The bar is a $7 / 8$-inch Triple Grove Bar, which places the possible origin of this handle in the 1940s. The end cap is shaped like a flared fan with a narrow border around the perimeter. The lug is rectangular with rounded corners and slightly elevated shoulders and a tympanum. There is a narrow lipping around the margin of the lug. There is a raised ridge running along the bottom margin, up the sides, and around the shoulders. Before the tympanum begins to slope upward, the ridge curves sharply downward on each side and creates a fan-like design in the central portion of the lug. From the top of the tympanum, a floral three-leaf fan descends toward the middle of the lug. There are also one or two arches moving over the arm attachments from the bottom corners toward the bottom center. The lug has three screw holes: one on each bottom corner, and one slightly higher on the midline. The arms are joined to the lugs with a trunnion joint. The sides of the arms are gently tapered with the bottom being wider than the top. The arm material wraps around the handle and inserts under two side prongs on the back of the arms. There appear to be simple straight lines running vertically along the length of the arms. 
Handle Type 16 does not match any known patents or archaeologically excavated mortuary artifacts (see Appendix D), but does bear a resemblance to modern Aurora Casket Company Octagon Cloth Covered No. 119 (Figure 183) depicted on a 1999 advertising card in the author's collection. It is possible that this modern version is a stylistic descendant of the handle recovered in New Home Cemetery.

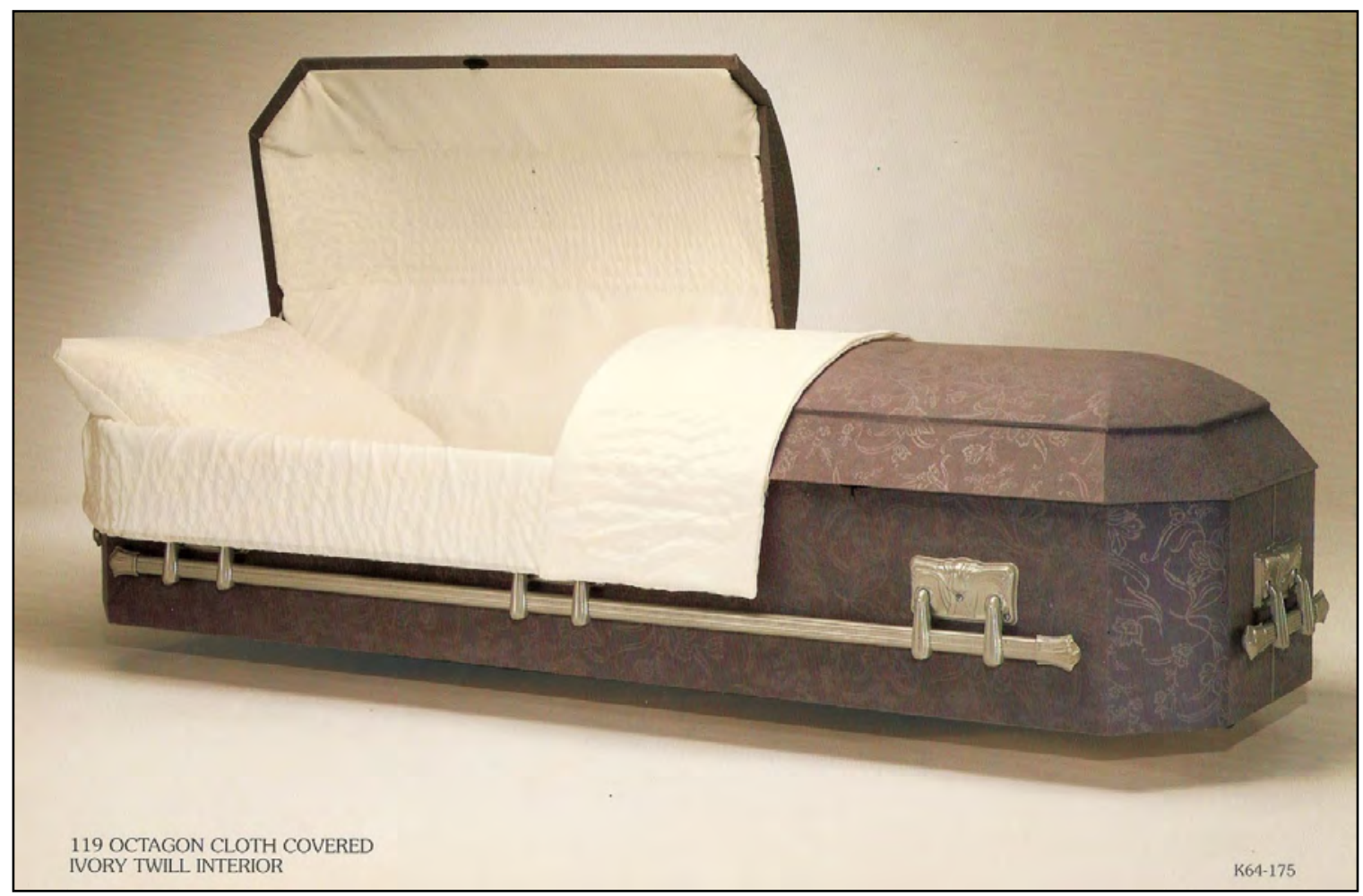

Figure 183. Cloth-covered casket from a 1999 advertising card produced by Aurora Casket Company displaying a swing-bar extension handle similar to Handle Type 16.

\section{Handle Type 17}

Handle Type 17 (Figures 184 and 185) is represented by fragments of two extension handles, including four double-arm lugs and two bar segments, one with a right side end cap, and the other with a left side end cap from Burial 9; and one lug with an associated bar segment recovered from Burial 10 in New Home Cemetery (see Appendix A). The appearance of this handle in Burial 9 is somewhat perplexing. Burial 9 has another full set of short-bar handles (Handle Type 10). Evidence on those handles suggests that there was an outer box in this burial. It is conceivable though somewhat unconventional that the outer box might have been outfitted with extension Handle Type 17. 


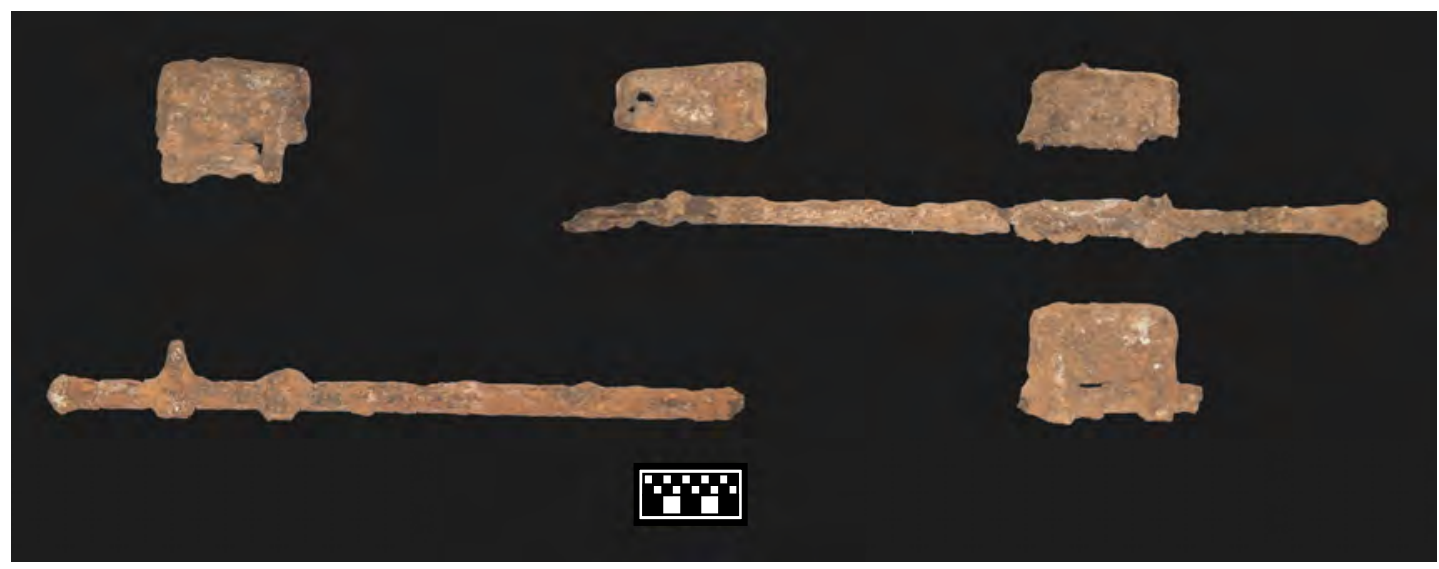

Figure 184. Handle Type 17—double-arm lug swing-bar extension.

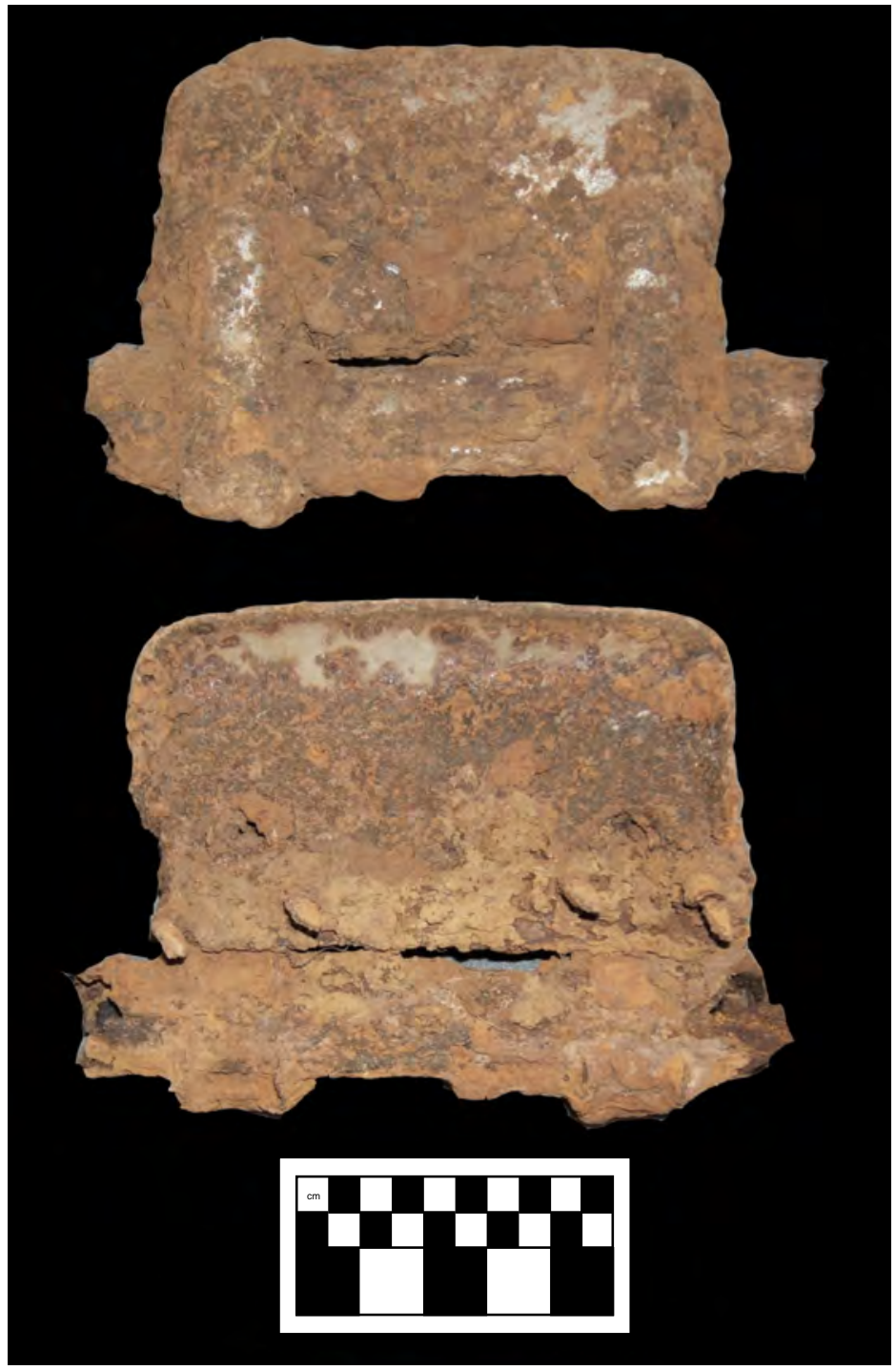

Figure 185. Close-up of Handle Type 17 lug and bar segment. 
All components of Handle Type 17 are of heavily corroded silver electroplated steel. The bar is a 1-inch Oval Clover Leaf Bar, which places the possible origin of this handle in the 1920s. The end cap is a flared continuation of the cloverleaf pattern. The lugs are rectangular with rounded corners and slightly concave sides. No specific stylistic elements are evident on the surface of the lugs. The lug has four screw holes, two flanking each arm. The arms are joined to the lugs with a trunnion joint. The arms are narrowest at the top and gently taper to wider toward the handle. The arms grasp the handle with four protrusions on the back of the arms. Handle Type 17 does not match any known patents, catalogs, or archaeologically excavated mortuary artifacts (see Appendix D).

\section{Handle Type 18}

Handle Type 18 (Figures 186 and 187) is represented by fragments of two full extension handles, each with three rectangular double-arm lugs, and two end caps (though one cap is broken off). All pieces are of heavily corroded silver electroplated steel, and all were recovered from Burial 17 in New Home Cemetery (see Appendix A). The bar is a $7 / 8$-inch Clover Leaf Bar, which places the possible origin of this handle in the 1920s. The end cap is a flared continuation of the cloverleaf pattern.

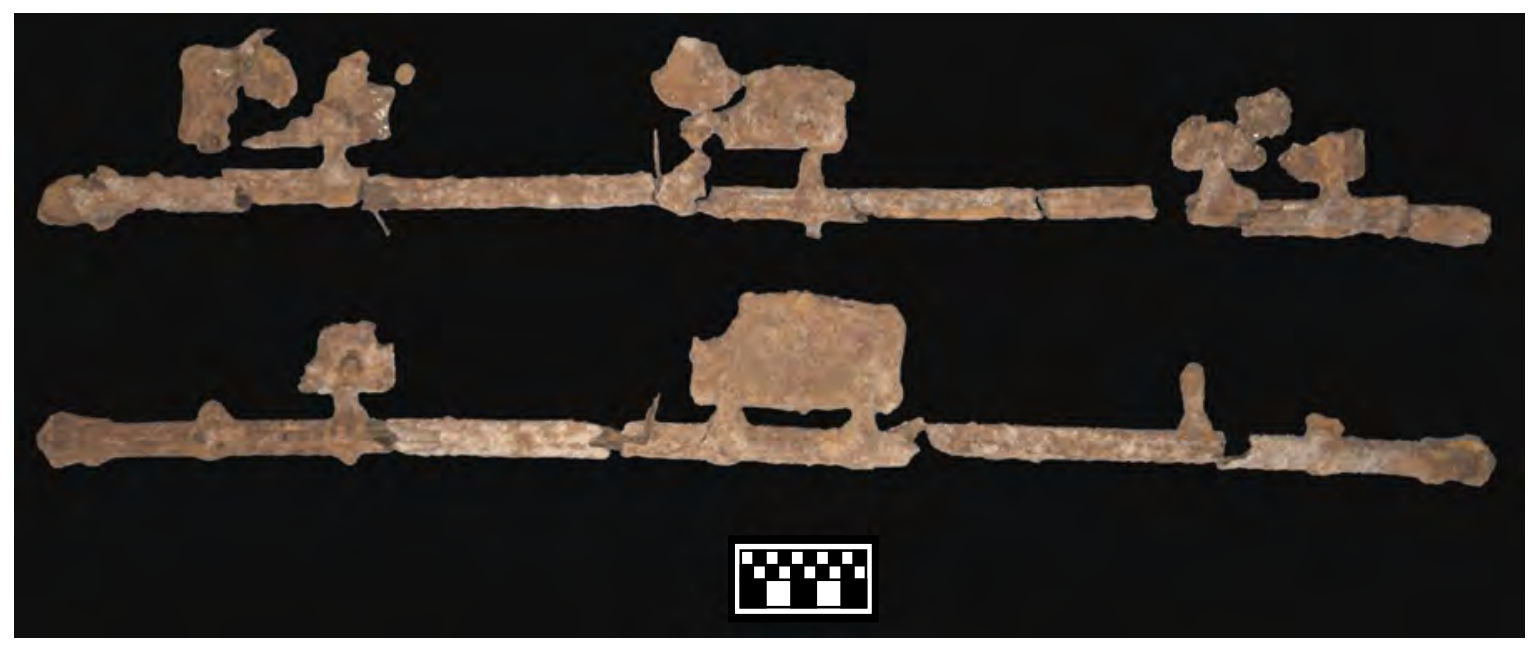

Figure 186. Handle Type 18-double-arm lug swing-bar extension.

The lugs are rectangular with rounded corners and slightly elevated shoulders, gently sloping tympanum, and a slight incurve on the sides. No specific stylistic elements could be observed through the corrosion, although there does seem to be some linear pattern running vertically down the center of the lug. The lug has four screw holes, two flanking each arm. The arms are joined to the lugs with a trunnion joint. The sides of the arms are roughly parallel before reaching the handle, at which point they flare out considerably before wrapping around the handle and inserting under two side prongs on the back of the arms. No design elements appear on the surface of the arms. Handle Type 18 does not match any known patents, catalogs, or archaeologically excavated mortuary artifacts (see Appendix D). 


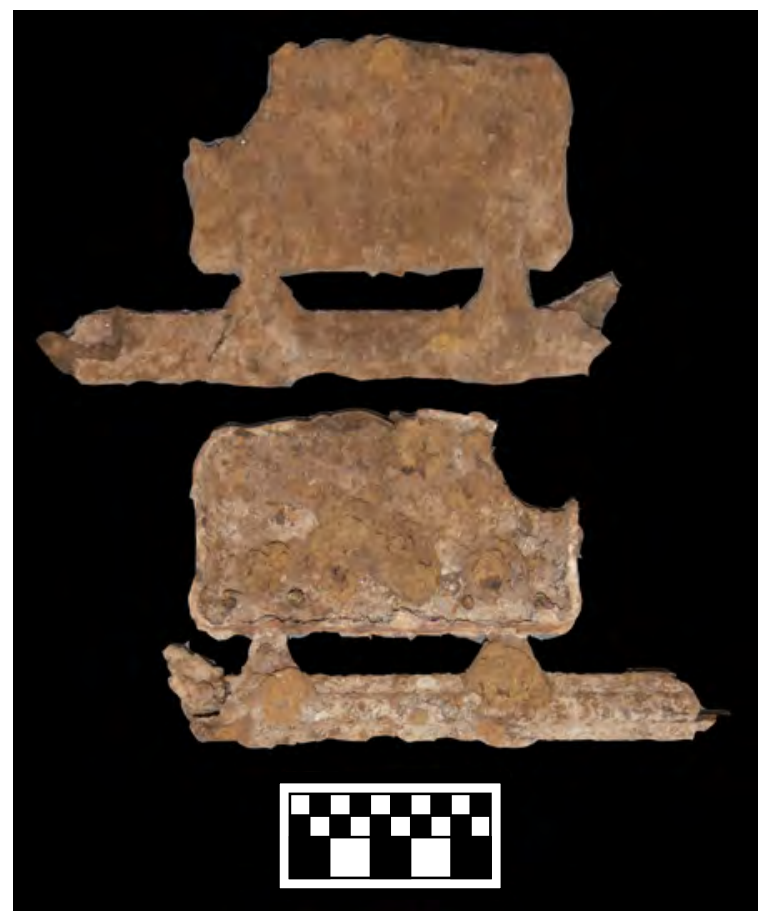

Figure 187. Close-up of Handle Type 18 lug and bar segment.

\section{Stationary Bar Extension Handles}

The origin of stationary extension handles is more elusive than swing-bar extension handles. Though relatively uncommon, burial container handles labeled as stationary were mentioned in catalogs as early as the 1866 P. \& F. Corbin price list. It is likely these would have been similar in appearance to swing-bail handles, or possibly would have been like the seemingly stationary ornament bar from the 1865 and 1867 Crane, Breed \& Company price lists (see Figure 169). Additionally, several caskets illustrated in the 1903 price list of the National Casket Company depicted hardwood caskets with carved wooden stationary extension handles (Figure 188).

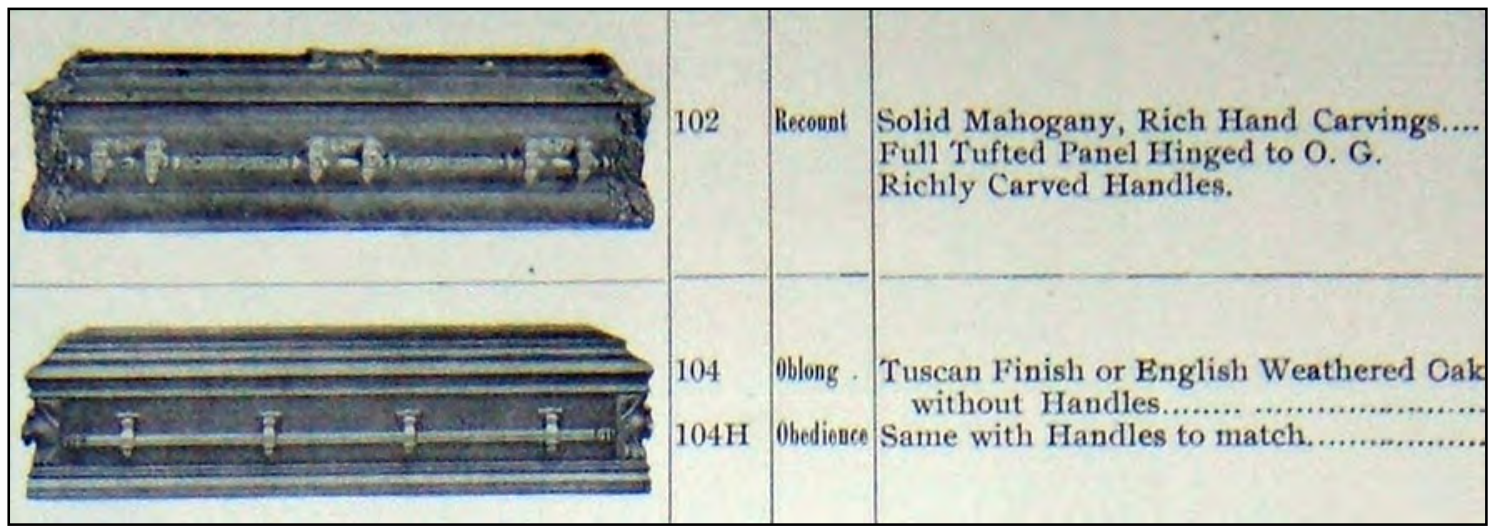

Figure 188. Carved hardwood stationary extension handles mounted on caskets offered for sale on page 40 of the 1903 National Casket Company illustrated price list. 
No patents for stationary extension bars themselves or components of stationary extension bars were found to date before the granting of U.S. Design Patent 104148 to Robert L. Randall of the Mortuary Supply Company, Inc., on April 20, 1937. Earlier patents are sure to exist because this is a design and not utility patent, which means that the form should already have been in production. A search of catalogs from this era revealed that indeed the first known appearance of modern stationary extension bars was in the 1936 Boyertown Burial Casket Company Catalog "L” (Figure 189).

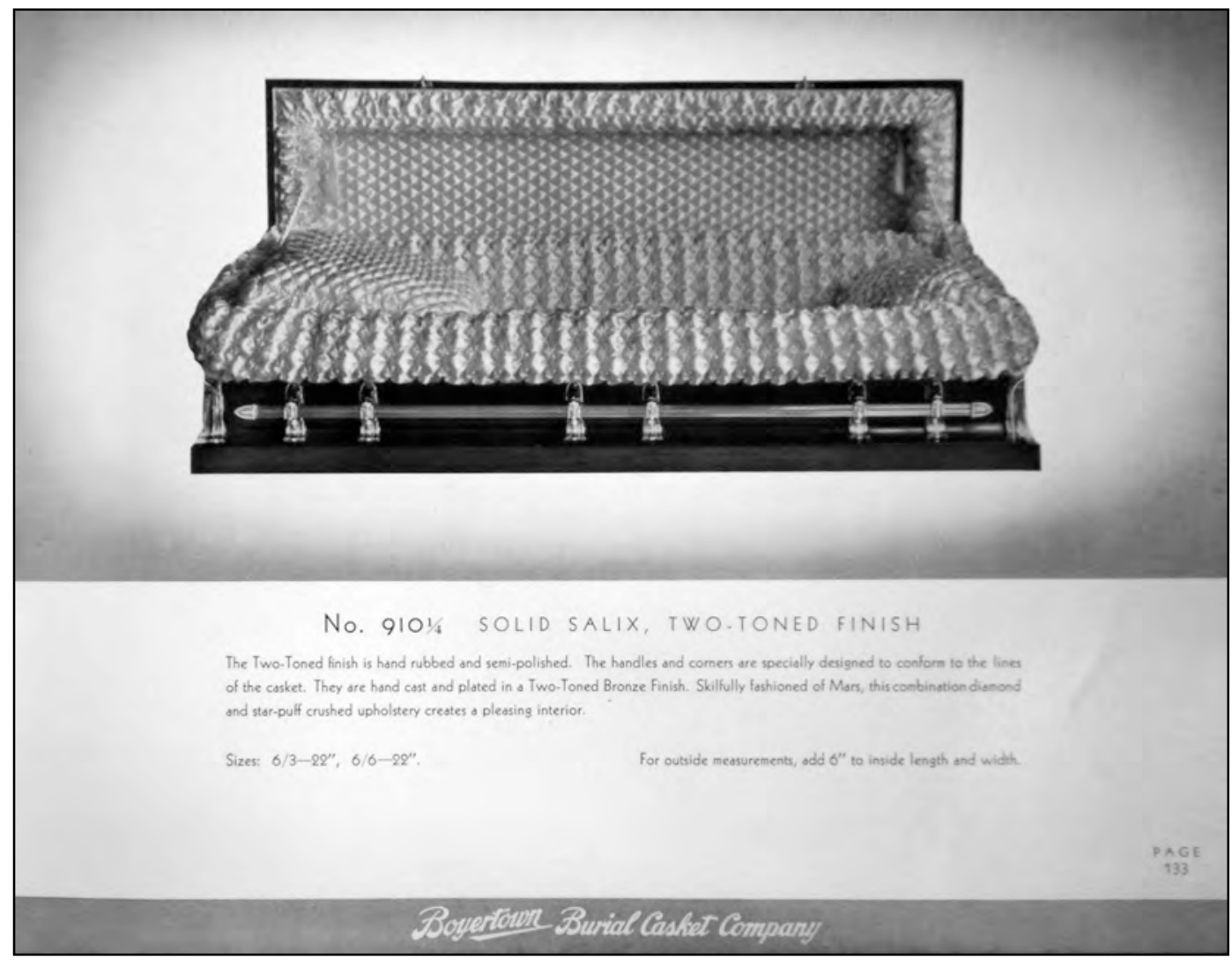

Figure 189. Modern stationary extension bar shown on page 133 in the 1936 Boyertown Burial Casket Company Catalog "L".

\section{Handle Type 19}

Handle Type 19 is a stationary extension handle represented by seven segments of handle bar, six complete or fragmented small side braces (Figure 190), as well as two fragments of large corner braces (Figure 191). These artifacts were recovered from disturbed contexts in New Home Cemetery (see Appendix A). The steel, silver electroplated bar is a 1/1/8-inch Fancy Reeded Oval design. Three of the bar segments are articulated with side braces, which are made of a clear plastic that has been airbrushed on the back with a fine silver paint. The center of the brace is made up of four nested elongated arches. Flanking the largest arch is a squared, top shoulder, which curves slightly inward at the side before jutting out again to another similar shoulder. Below the second shoulder is a rounded screw hole extension. From front to back, the brace is narrowest at the top and curves gently outward to meet the widest point at the bottom. 


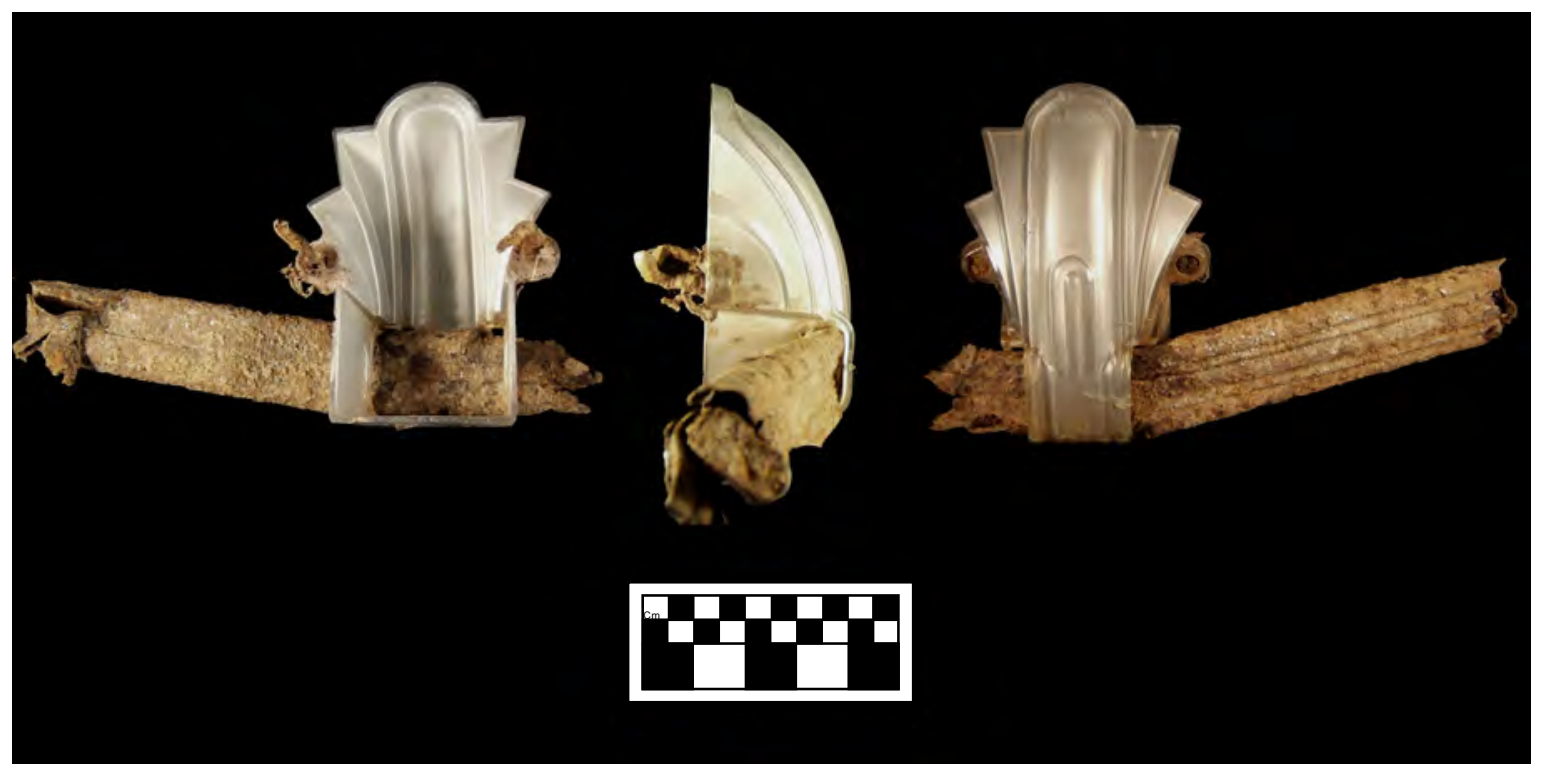

Figure 190. Handle Type 19—stationary extension side brace and bar segment.

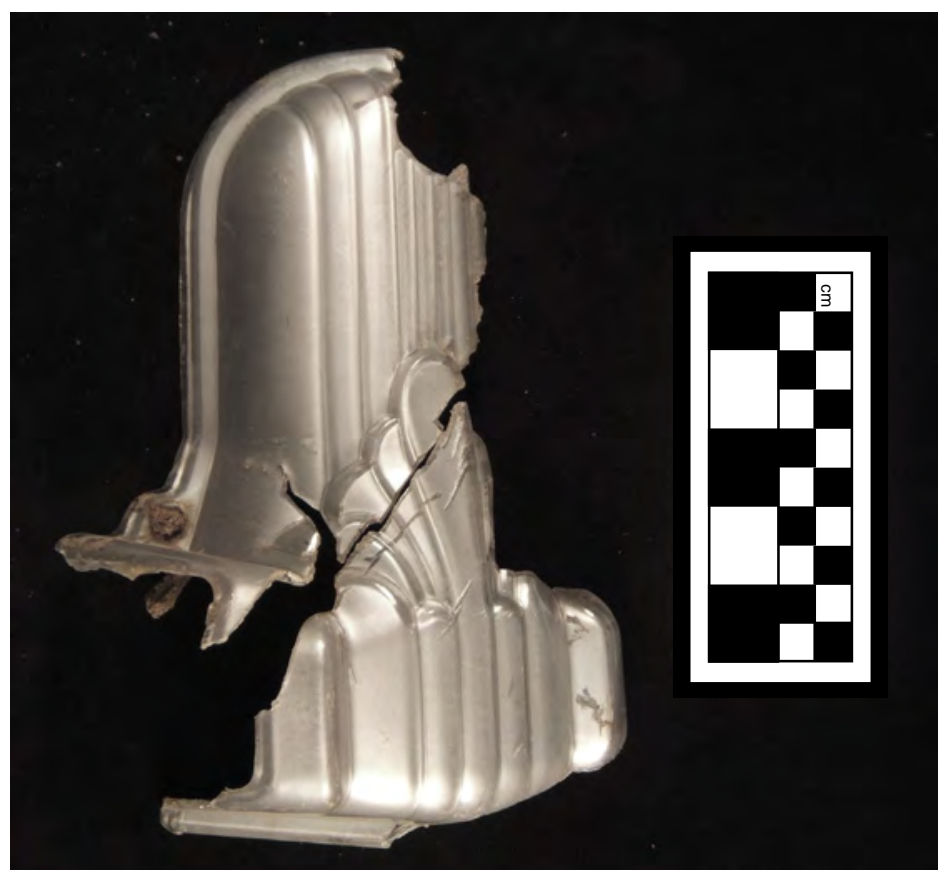

Figure 191. Handle Type 19—stationary extension corner brace.

Neither of the large corner braces was complete. Although they match the material type of the small braces, their style differs somewhat. The main body of the central segment is an elongated arch; however, there are at least six parallel ridges that descend from the top until they meet a fanned series of seven curved ridges appearing like a peacock's tail. The fanned ridges continue to the bottom. Most of the areas of articulation with the handle are broken, but they appear to be generally squared. One screw hole would have been placed on each side within the joint between the squared lower area and the central arch. 
The bar is a standard extension bar form appearing in the literature since the 1940s, but the plastic braces are somewhat anomalous. Because most of the casket companies do not want to extensively promote low end items, or expound on low quality materials, there have been no references found to plastic "hardware" in the available literature. This makes it difficult to accurately place the introduction date of this handle type. The modern "atomizing” airbrush was invented by Charles Burdick in 1892 (U.S. Utility Patent No. 474157), so that characteristic does not narrow down the time range. The clear plastic appears to be a relatively modern material, however, so it likely does not date earlier than the 1950s.

No known patents or archaeological matches are known to exist, but at least the side braces have been identified in one casket advertising card from the Aurora Casket Company dating to 1999 (see Appendix D). Figure 192 shows the casket in question with identical side braces. Both the handle bar and the corner braces are different. The ad does not provide full descriptions of the materials used, noting that there is an ivory crepe interior and a silver embossed doeskin covering. The hardware is not mentioned, but considering the body of this cloth-covered casket is likely made of a cheap fiber-board, it is possible that this is indeed the same sort of plastic handles.

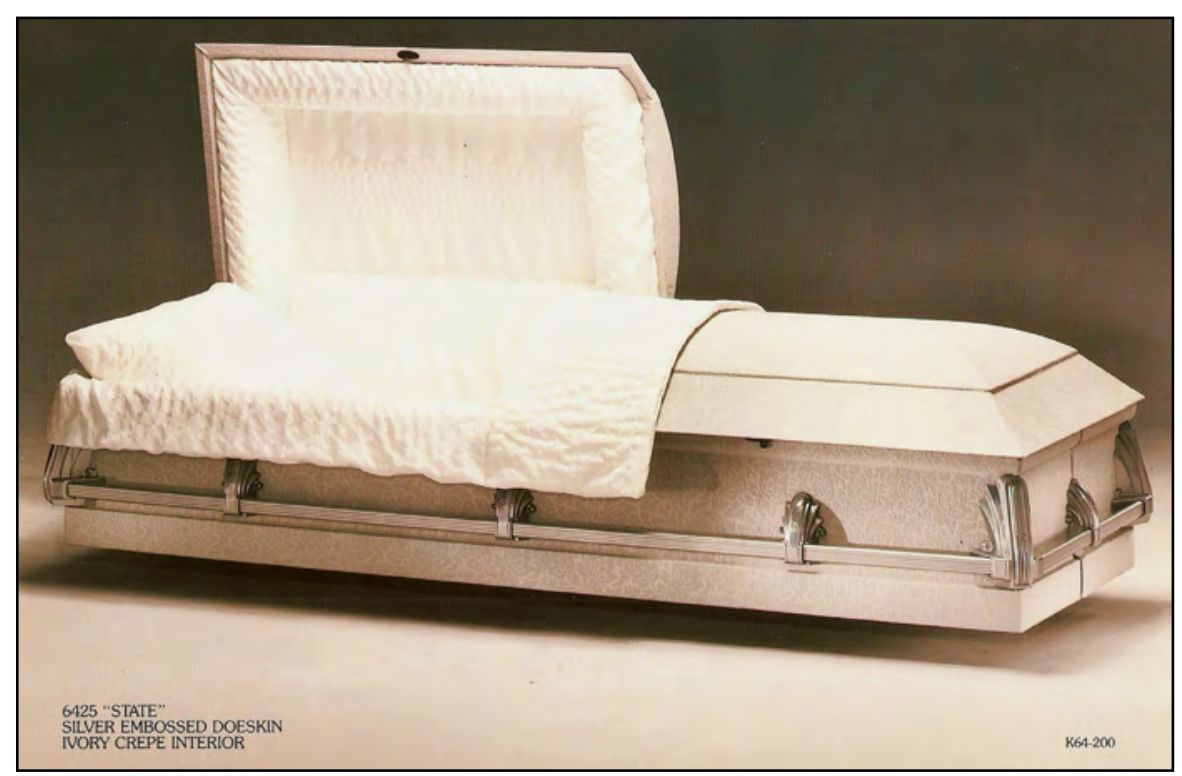

Figure 192. Cloth-covered casket from a 1999 advertising card produced by Aurora Casket Company displaying a stationary extension handle similar to Handle Type 19.

\section{Handle Type 20}

Handle Type 20 is a stationary extension handle represented by three stamped ferrous metal side braces with associated electroplated steel handle bar segments (Figure 193) and two corner supports also with associated handle bar segments (Figure 194). These items were recovered from Coffin 1 in New Home Cemetery (see Appendix A). 


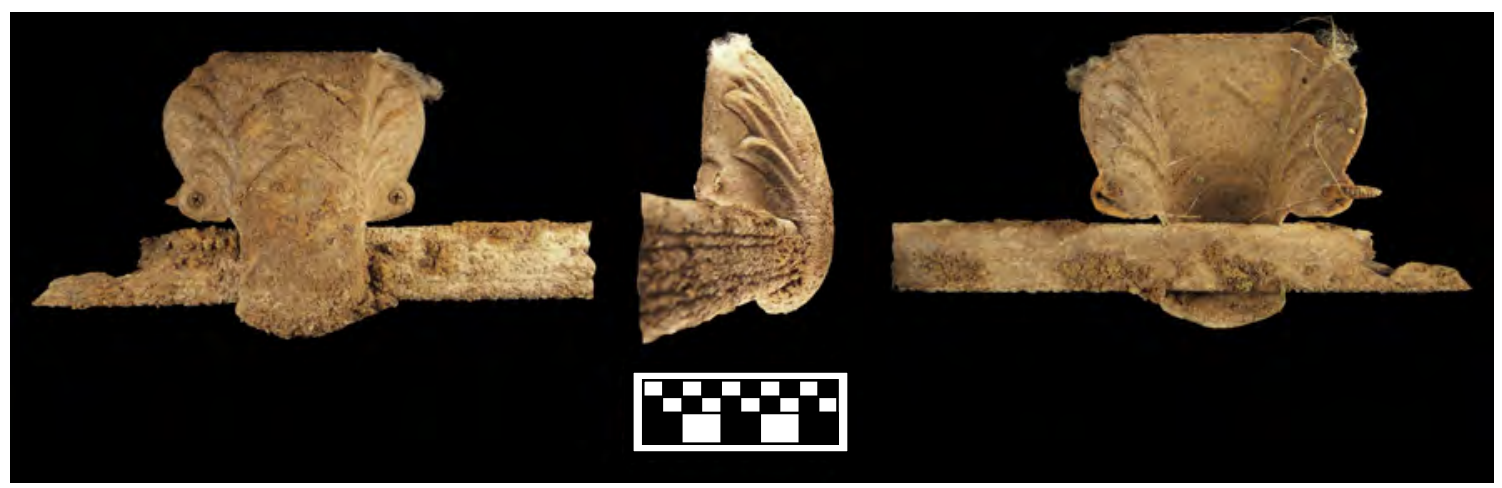

Figure 193. Handle Type 20—stationary extension side brace and bar segment.

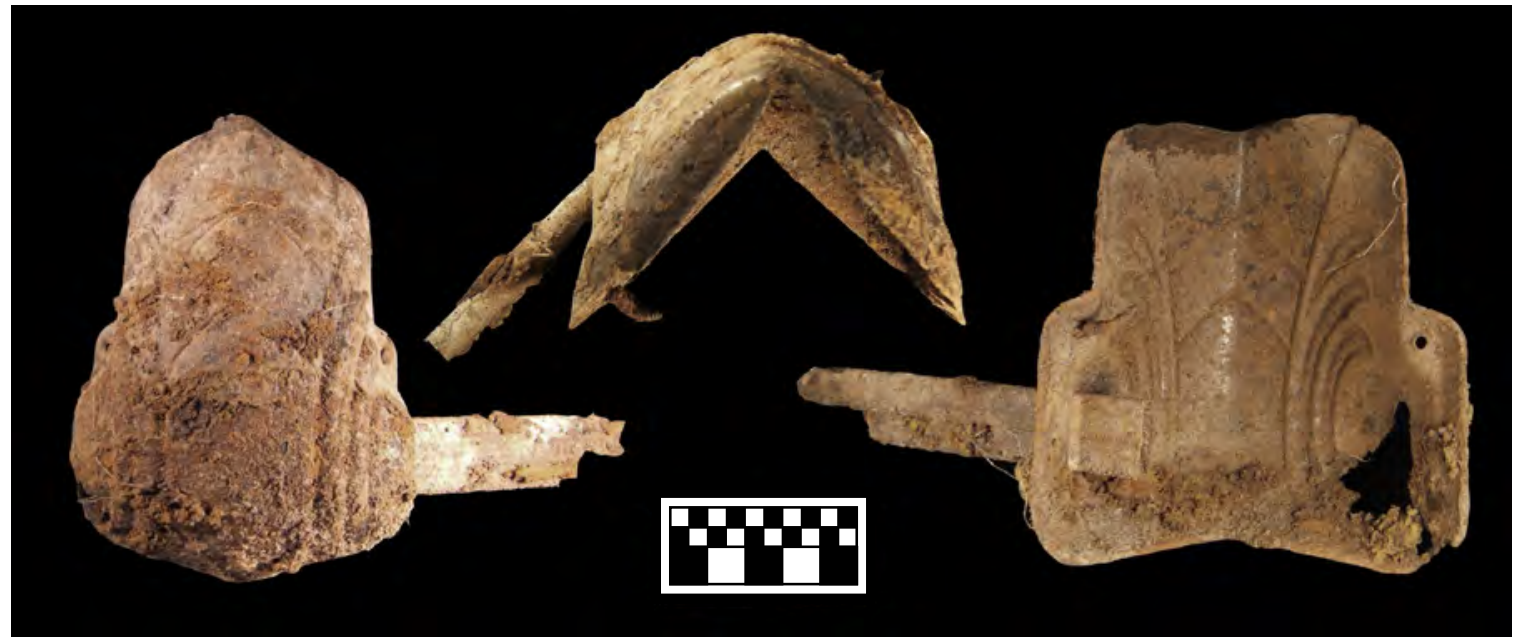

Figure 194. Handle Type 20—stationary extension corner brace.

The side braces are narrow at the bottom where they grasp the handle bar with four little projections rather than wrapping around it. The section of the brace that holds the bar tapers out slightly as it travels upward. From the top corners, a brim or hood similar to that of a cobra descends down the side before reaching a small hole where screws attach the piece to the burial container. Two curved "W" designs appear on the face of the brace with the central point falling in the midline, and the lower points falling on each side of the central column and two lines extending onto the hood from this point forming three long bladed leaves. The three side braces were secured using Phillips head screws, which dates this burial to after 1936 . The bar is a 11/8inch Fancy Reeded Oval Bar, which did not appear in the catalogs until the 1940s. The braces appear to be matched to this tall type of bar, so it is likely they also do not date prior to the 1940s. The corners are only minimally corroded. They share the same design pattern as the side braces with a central column, flanked by three long bladed leaves and "W" patterns running across the upper section. The bottoms of the corners are widest, and the tops are somewhat narrowed. Two screw holes appear on shoulders that extend outward just beside the tip of the lowest leaf. Handle Type 20 does not match any known patents, catalogs, or archaeologically excavated mortuary artifacts (see Appendix D). 


\section{Handle Type 21}

Handle Type 21 (Figures 195 and 196) is represented by four stamped ferrous metal side supports and 53 pieces of a heavily fragmented handle bar. These items were recovered from Burial 4 in New Home Cemetery (see Appendix A). Although heavily fragmented, the bar appears to be a $7 / 8$-inch Triple Grove Bar. The braces are rectangular at their backs and secured with two screws, one at the top and one at the bottom. The lower half of the brace is the widest and thickest, bearing horizontal parallel lines. The back plate remains the same width, but the front section narrows slightly for another $4 \mathrm{~cm}$ of horizontal lines. The remaining upper section narrows further and contains vertical parallel lines. Handle Type 21 does not match any known patents, catalogs, or archaeologically excavated mortuary artifacts (see Appendix D).

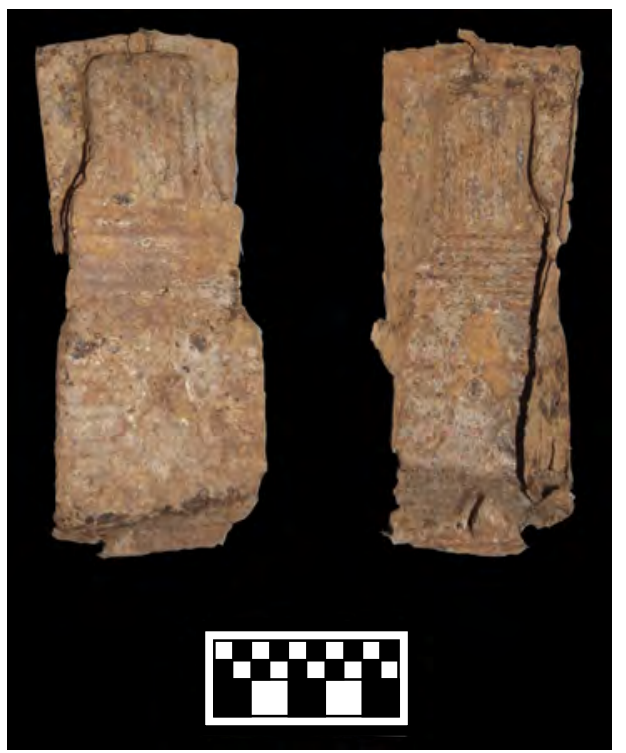

Figure 195. Handle Type 21—stationary extension handle side braces and bar fragments.

Handle Type 22

Handle Type 22 (Figure 197) is represented by one stamped ferrous metal corner support with a short segment of a crushed and twisted handle bar recovered in a disturbed area with materials from Coffin 2 in New Home Cemetery (see Appendix A). The exact type of bar that was inserted into this corner support is not clear because it is corroded and distorted. The corner is corroded somewhat, but is in fine shape. It looks like a singular forward curving horn helmet with a feathered plume rising up from a central archway on the front of the piece. A rectangular platform extends backward from the edge of the front arch past the handle bar articulation hole and beyond. Much of the margin is broken, and no attachment points are present though it is likely that the corner was attached using at least two screws. Handle Type 22 does not match any known patents, catalogs, or archaeologically excavated mortuary artifacts (see Appendix D). 


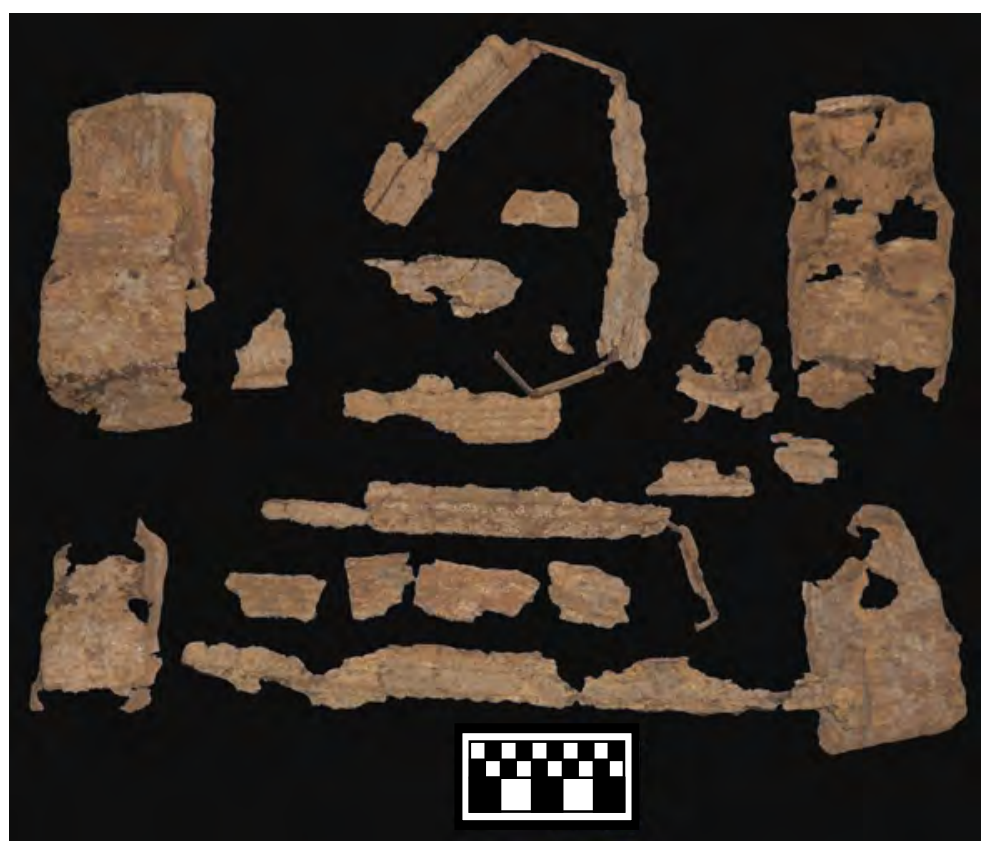

Figure 196. Handle Type 21—stationary extension side brace.

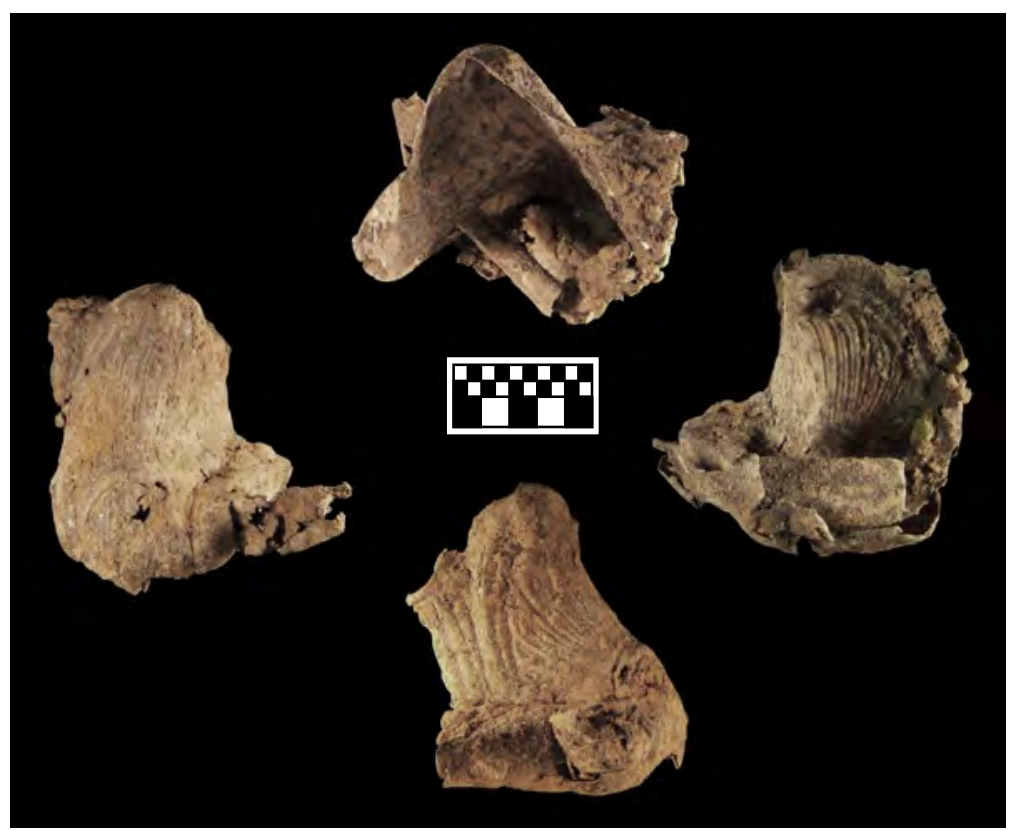

Figure 197. Handle Type 22—stationary extension corner brace.

\section{Handle Type 23}

Handle Type 23 is represented by at least one side brace and one segment of handle bar recovered from Coffin 4 in New Home Cemetery (see Appendix A). This particular handle was not available for lab analysis and was not documented in the field. There exists only one field 
photograph that shows this handle (Figure 198), and that image is not exceptionally clear. Both the side brace and handle bar are likely made of steel, and the bar appears to be a form that is wider than it is thick, but the exact type cannot be determined. The side brace is shaped like a fan and is mildly similar to the side brace from Handle Type 19. From front to back, the brace is narrowest at the top and curves gently outward to meet the widest point at the bottom and is squared where it articulates with the handle bar.

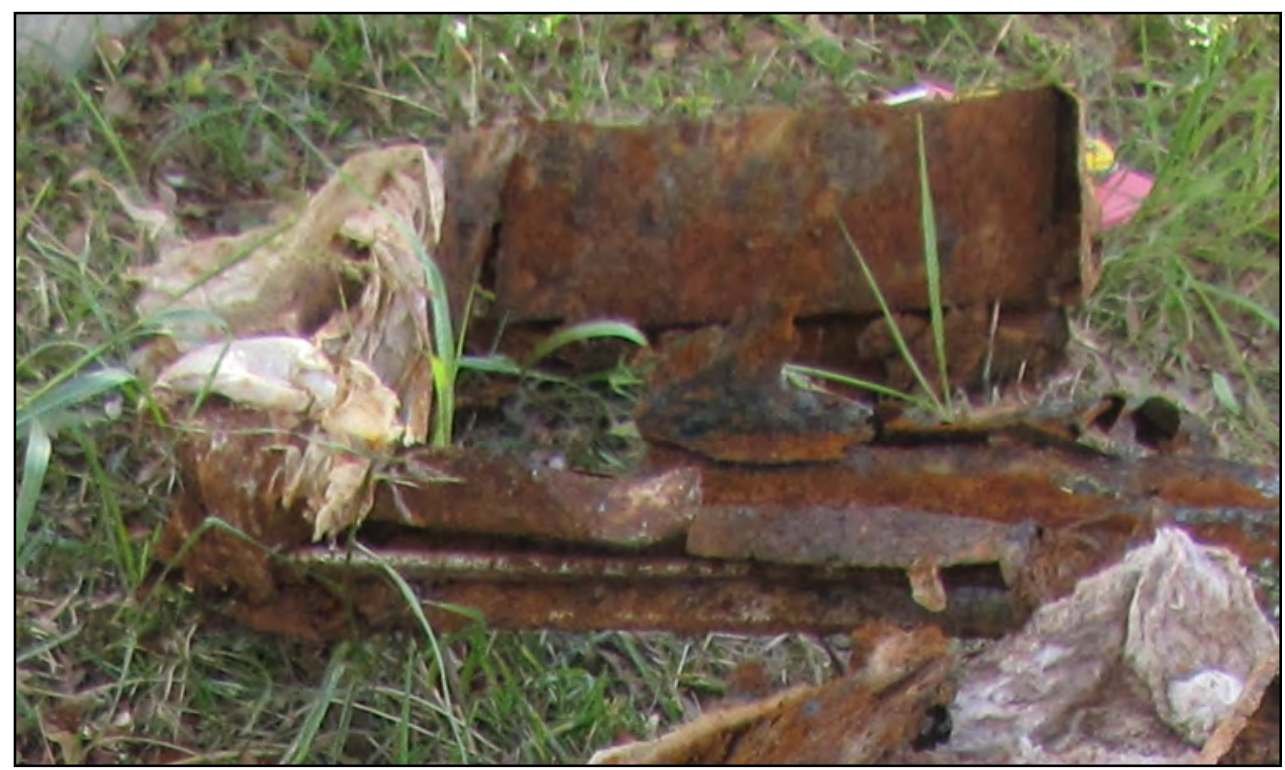

Figure 198. Handle Type 23—stationary extension handle from Coffin 4 shown in field photo.

Due to the lack of observation, it is difficult to determine whether Handle Type 23 matches any known patents or archaeologically excavated mortuary artifacts, but it does not appear to (see Appendix D). It does, however, look very similar to the handles utilized on the 1998 Batesville Casket Company product called "New Pointe Triton Grey" (see Figure 74). It is not known when this particular handle type entered the market, but stationary extension bars were not popular until the late 1930s, and Coffin 4 itself could not have been interred prior to the 1960s.

\section{Unclassifiable Handles}

The unclassifiable handle category is a catchall group consisting of eight handle types for which there was not enough of the handle to determine whether or not each was a short bar or an extension bar. In some instances, a lug is present, but not enough of the bar is present to tell whether it extends beyond a reasonable length from the arm to be classified as a short bar. In addition, there are cases where only an end cap and a short segment of the handle bar are present, placing that item in either the short bar or extension bar categories. 


\section{Handle Type 24}

Handle Type 24 (Figure 199) is represented by one stamped ferrous metal double-arm handle lug recovered from disturbed contexts in New Home Cemetery (see Appendix A). This lug is shaped like a squat loaf widest at the bottom, slightly constricted in the middle, and rounded on top. There is a raised ridge running around the perimeter of the lug. Additionally, a half circle platform is at the apex of the lug. This lug has three screw holes, one at each bottom corner and one in the center. It appears to have trunnion arm mounts. Handle Type 24 does not match any known patents, catalogs, or archaeologically excavated mortuary artifacts (see Appendix D).

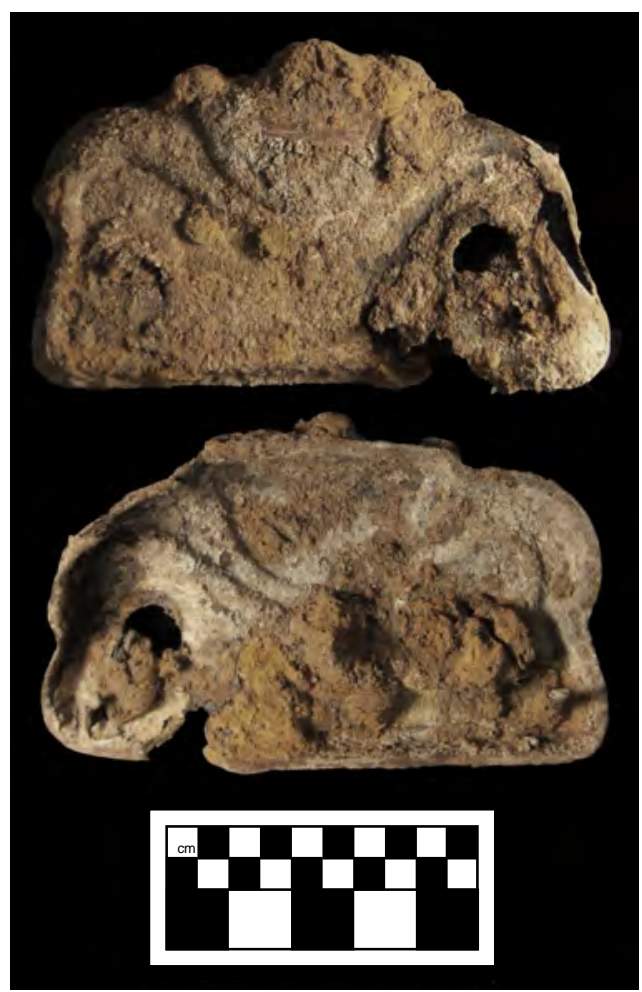

Figure 199. Handle Type 24—unknown form, double-arm lug.

\section{Handle Type 25}

Handle Type 25 (Figure 200) is represented three antimonial lead finials, four lug fragments, two arm fragments, and nine bar fragments with wood grains evident on the interior. These items were recovered from Burial 8 in New Home Cemetery (see Appendix A). The finials are cylindrical with a raised collar at the base, a parallel-sided shaft extending up to a raised floral motif band. On top of the floral band is a circle of small raised dots below a gentle dome. There is a mold seam that bisects the finial, and there is evidence of a small rusted pin or nail that once held the finial to the wooden core of the bar. Both the arm and bar fragments indicate that the bar was a $5 / 8$-inch plain round bar. The lug fragments and presence of two arm fragments suggest that it was a rectangular double-arm lug. 


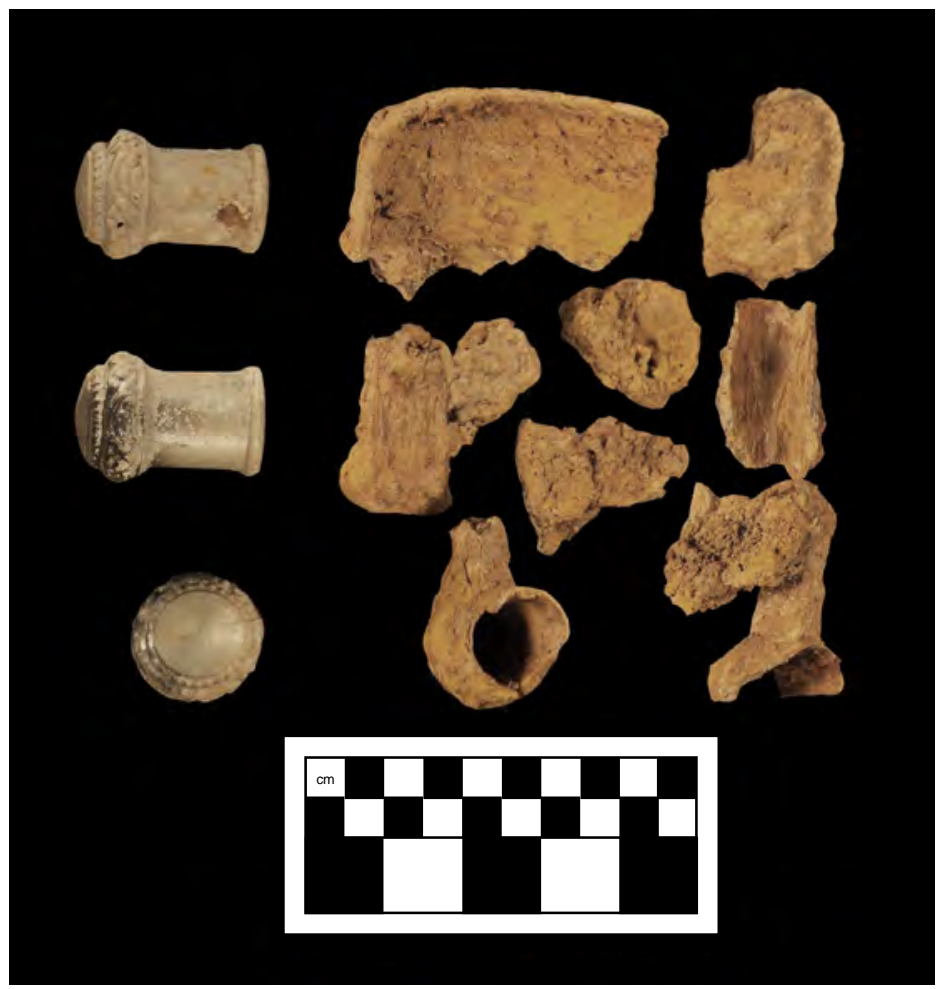

Figure 200. Handle Type 25-unknown form, finials, and lug fragments.

Since relatively little remains of this handle set, it is not possible to definitively state whether this represents an extension handle or a short bar handle. The finials are a match to those appearing with one handle in the ca. 1904 Gate City Coffin Company catalog (Figure 201), but based on the lug fragments, the remainder of the illustrated handle in the catalog does not match. No patent or archaeologically excavated mortuary artifact matches were located for this particular handle type (see Appendix D).

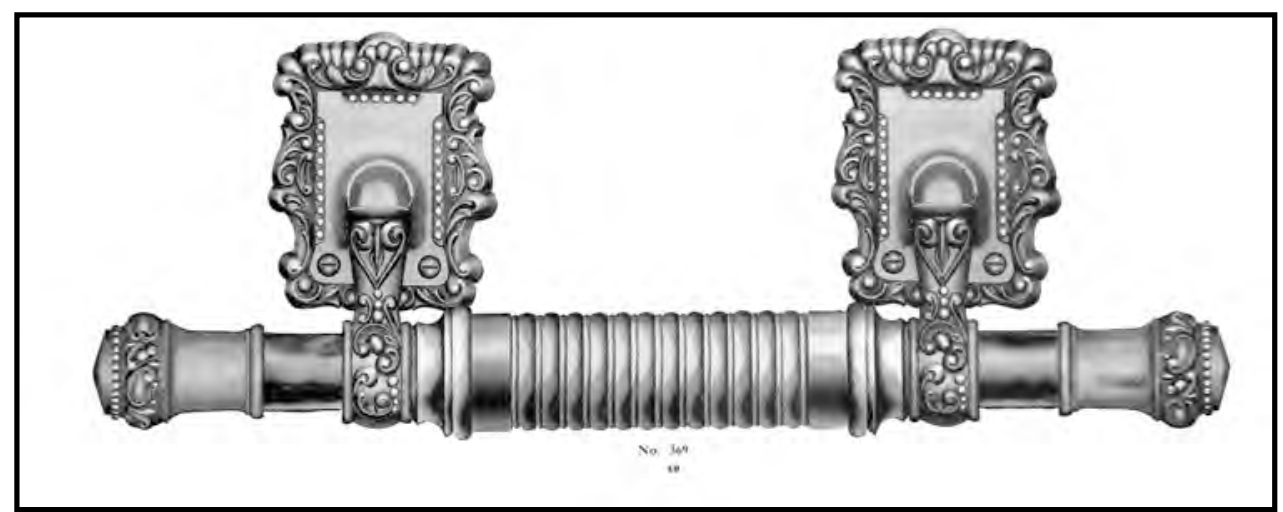

Figure 201. Short-bar handle No. 369 with finials matching those of Handle Type 25 illustrated in the ca. 1904 Gate City Coffin Company. 


\section{Handle Type 26}

Handle Type 26 (Figure 202) is represented by one stamped, ferrous metal, single-arm lug recovered from Burial 3 in New Home Cemetery (see Appendix A). This lug is shaped like an elongated scalloped shell. The top portion of the arm is sitting in the trunnion housing. The lug was secured with two screws, one on each side of the arm. The lug is heavily corroded. Handle Type 26 does not match any known patents, catalogs, or archaeologically excavated mortuary artifacts (see Appendix D).

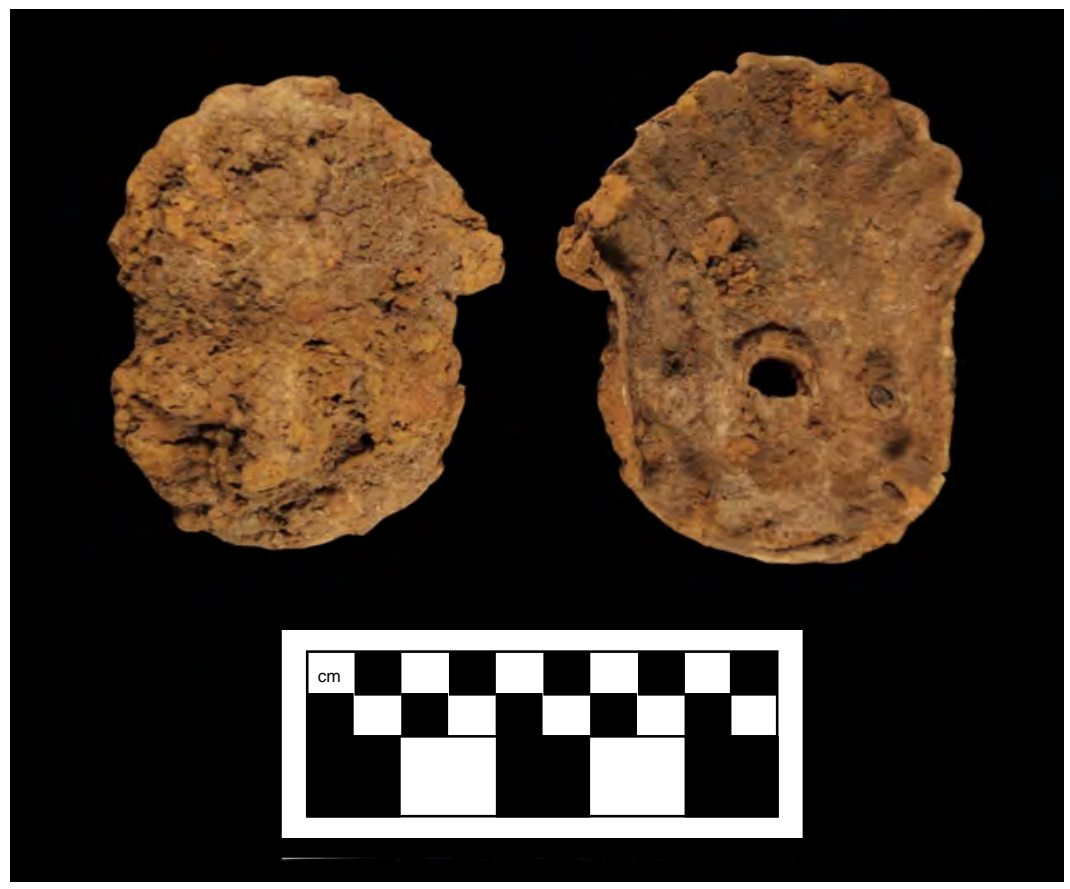

Figure 202. Handle Type 26-unknown form, single-arm lug.

\section{Handle Type 27}

Handle Type 27 (Figure 203) is represented by eight, stamped, ferrous metal, single-arm lugs with attached arms recovered from Burial 14 in New Home Cemetery (see Appendix A). This lug is generally shaped like a fiddle except the lower edge is flattened and the upper edge is more pointed. There is a wide, elongated ridge with a semicircular termination that descends from the top of the lug to the top of the arm. This ridge is flanked by two lines on each side that veer toward the bottom corners when the ridge ends. They shortly meet scroll patterns at the lower quadrants. The tops of the scrolls curl outward, but the lower ends of the scrolls curl inward toward the arms. The arms are tapered, widening toward the end, which terminates with a gently curved point in the center similar in style to the ridge on the lug. The arm appears to have grasped the handle bar rather than wrapping around it. There was also one segment of $7 / 8$-inch Oval Clover Leaf Bar with one flared flat-bodied oval cloverleaf end cap present in Burial 14. The surface shows evidence of silver- or gold-colored electroplating, and the end cap has a central pin that extends all the way through and secured it to the handle's wooden core. It is possible that this bar segment was associated with the lugs described here. Handle Type 27 does not match any known patents, catalogs, or archaeologically excavated mortuary artifacts (see Appendix D). 


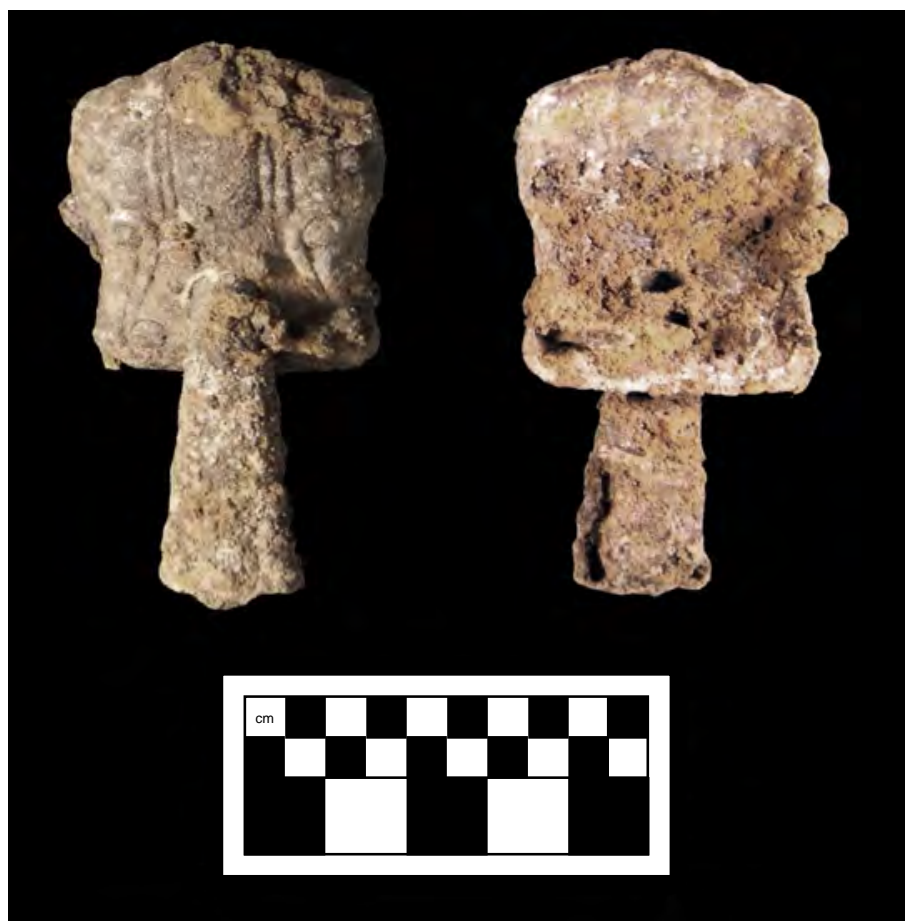

Figure 203. Handle Type 27—unknown form, single-arm lug.

\section{Handle Type 28}

Handle Type 28 (Figure 204) is represented by five ferrous metal bar fragments and one segment of bar with an attached antimonial lead finial recovered from disturbed contexts in New Home Cemetery (see Appendix A). The finial is designed like a crown-shaped floret in the front but a scallop in the back; there is a small pin or nail in the center back of the finial that was used to secure the piece to the wooden core of the bar, which is a $7 / 8$-inch cloverleaf bar. Handle Type 28 does not match any known patents, catalogs, or archaeologically excavated mortuary artifacts (see Appendix D).

\section{Plaques}

Plaques, also known as coffin plates, refer to machine-stamped or cast metal plates that would have been attached to the lid of a burial container (over the thorax or hips) during a funeral, thus playing an important role in funeral pomp and ceremony (Davidson 1999:548; Gordon 2003:1). Plaques were produced from a variety of metals, including various copper, tin, zinc, or lead-based alloys such as white metal, Britannia metal, pewter, brass, and bronze (Davidson 1999:548; Mainfort and Davidson 2006:151).

Plaques are one of the earliest forms of mortuary hardware. They appear in two English sample books from 1797 (Figure 205), which are the earliest mortuary hardware catalogs that have been located to date (see Appendix D). Many of these early forms of coffin hardware were produced in Britain and then were exported to the U.S. prior to the establishment of the U.S. funeral product industry. The extra cost of the importation along with the fact that most of these early forms of hardware were actually made of silver rather than cheaper materials made them less 


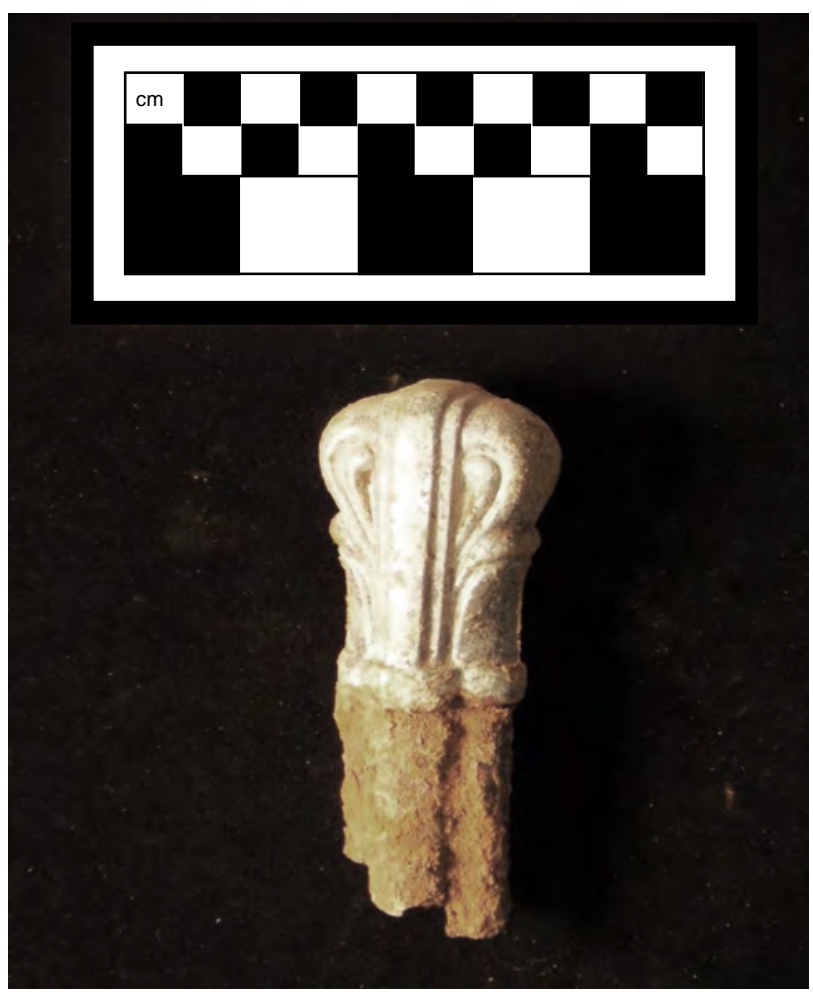

Figure 204. Handle Type 28—unknown form, finial and segment of bar.

accessible to the masses in the eighteenth and early nineteenth centuries. The iron coffin handles recovered from the eighteenth-century African Burial Ground in New York (Perry et al. 2006), as well as the stamped tin coffin handle plates from the early nineteenth century Tenth Street First African Baptist Church Cemetery (Crist et al. 1996) in Philadelphia represent exceptions to this general pattern (Springate 2011).

In their early period of use, plaques generally were blanks upon which the name and personal information of the deceased could be hand-engraved or painted (Pike and Armstrong 1980:149150). Although hand-engraved blanks persisted through the nineteenth century, it became much more common to purchase factory stamped or engraved plates exhibiting common nineteenthcentury phrases or sentiments, such as "At Rest," "Our Darling," "Rest in Peace," etc. (Mainfort and Davidson 2006:151).

At the end of the funeral, most plaques were probably buried with the deceased to identify their remains if disturbed at a later date. However, during the nineteenth and early twentieth centuries, it was also fashionable for some Americans to remove plaques after the funeral but before committal and keep them as mementos of the deceased (Gordon 2003).

\section{Plaque Type 1}

Plaque Type 1 (Figure 206) is represented by one rectangular plaque from Burial 18 in New Home Cemetery (see Appendix A). It was attached to three slats of coffin wood originally via two escutcheon pins, one on each side of the plaque. It was made of a stamped metal alloy, 


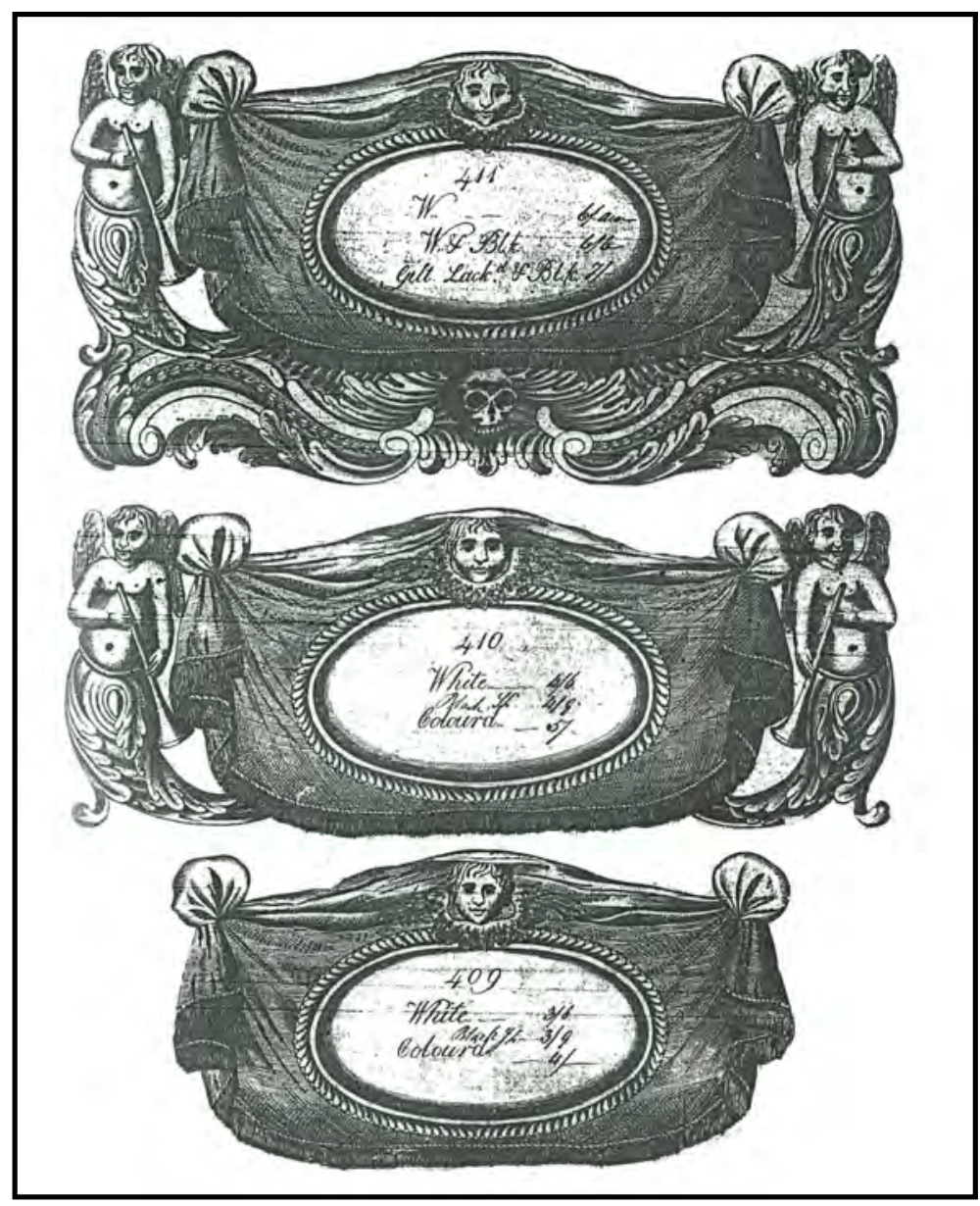

Figure 205. Examples of early “coffin plates” from ca. 1797 anonymous English hardware sample books.

possibly Britannia metal, and has silver electroplating. White, balanced-weave fabric is adhering to the reverse face of the plaque, indicating that the burial container had been cloth-covered.

The plate appears have had a border that covered a narrow strip of the plate and a wider portion of the surrounding wood. The plate itself appears to be plain, with the exception of embossed lettering in the center that reads "At Rest" in Old English Text MT type font. "At Rest" was a common phrase found on plaques in the late nineteenth- and early twentieth-century mortuary hardware catalogs, but no exact match for this item was located in patent records, catalogs, or cemetery excavation reports. Since no clean historical illustration could be found, this plaque has been reconstructed based on composite catalog information and physical evidence on the artifact (Figure 207). The exact font used on this Plaque Type 1 matches that which appears on page 75 of the ca. 1935 Victor Casket Hardware Company catalog. There is a great deal of variation in the appearance of fonts, even within the same general font category, and this exact font does not show up in earlier catalogs. That could mean that it was developed as a stylistic variation sometime in the early 1930s. The rope border that has been added to the composite image was borrowed from one plaque found in the 1912 Cincinnati Coffin Company catalog. Rope borders were commonly included with nineteenth- and early twentieth-century coffin plaques; the use of a perishable material could explain why the border is missing in this case. 


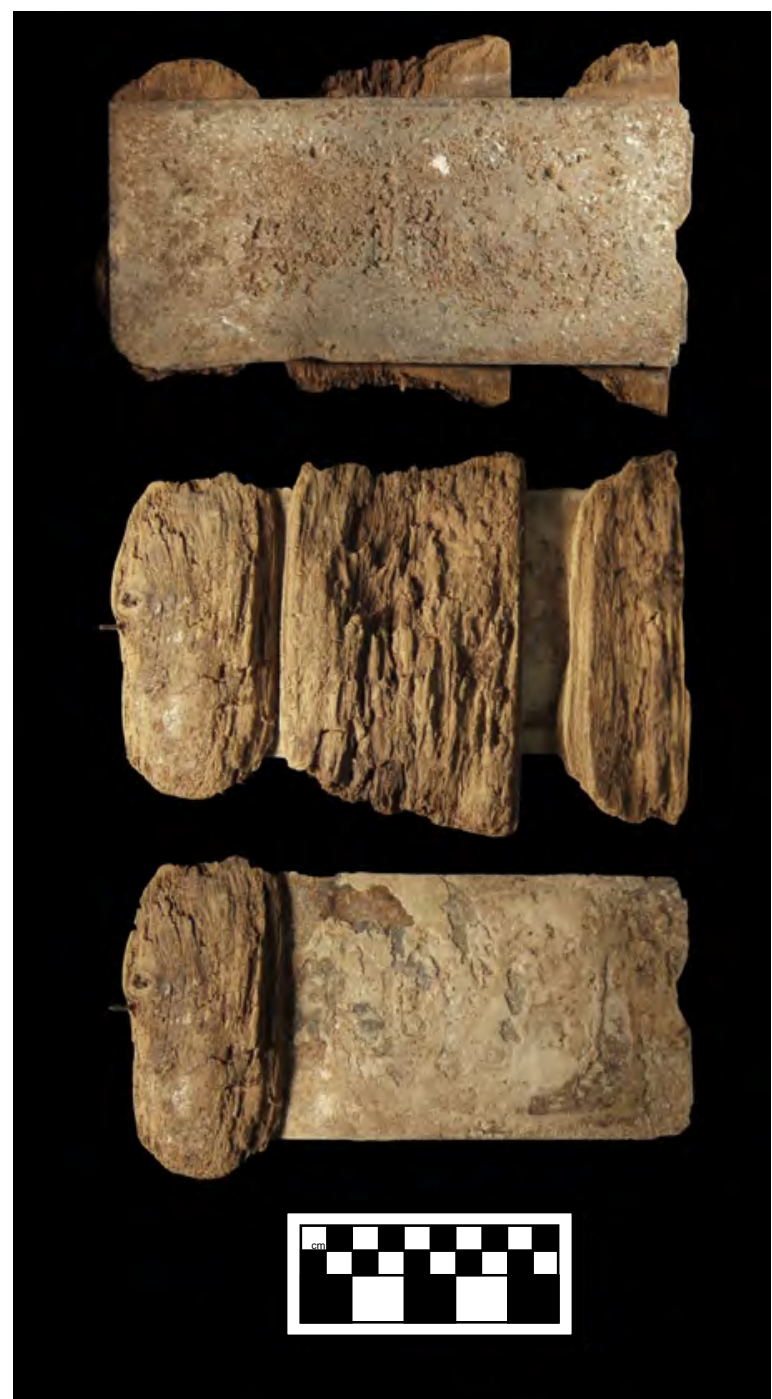

Figure 206. Plaque Type 1—“At Rest” plaque with wood recovered from Burial 18.

\section{Plaque Type 2}

Plaque Type 2 (Figure 208) is represented by 20 fragments of one ferrous metal plate recovered from across the hips of the individual interred in Burial 16 in New Home Cemetery (see Appendix A). This plate is generally rectangular; however, it appears to have raised shoulder corners and possibly an elevated design element on the upper margin of the plaque. There is no evidence of embossed or engraved lettering on the face of this plate. Due to the fragmentary nature of this plaque, it is not possible to definitively match it to any patent records, catalogs, or cemetery excavation reports. 


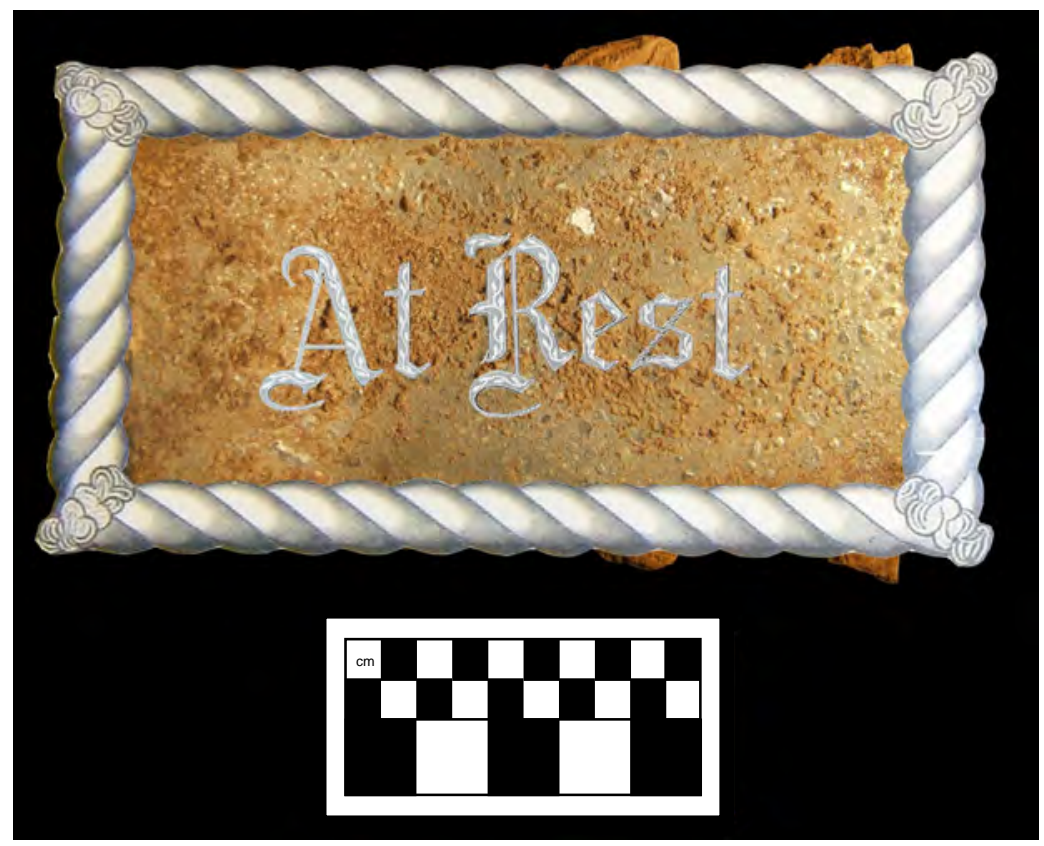

Figure 207. Possible reconstruction of the Plaque Type 1 original appearance based on examples of plaques advertised for sale in early twentieth-century catalogs.

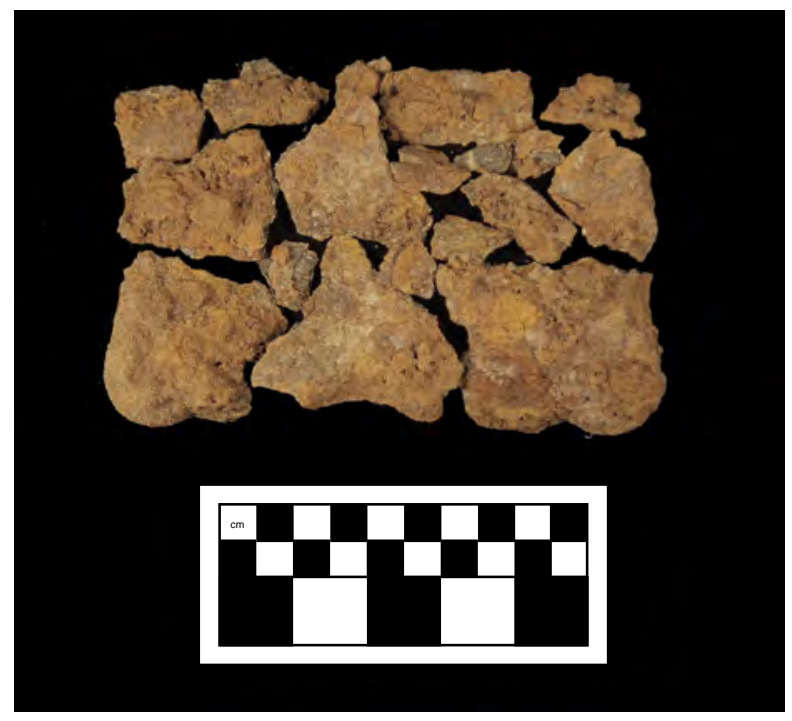

Figure 208. Plaque Type 2.

\section{Plaque Type 3}

Plaque Type 3 (Figure 209) is represented by 20 fragments of one ferrous metal plate recovered from Burial 13 in New Home Cemetery (see Appendix A). This plate likely was rectangular with gently rounded corners, but not enough of the plate is present to effectively reconstruct its overall size. It was made of a ferrous metal and is heavily corroded. There is no evidence of embossed or engraved lettering on the face of this plate, but there are numerous wood grains on both faces, 


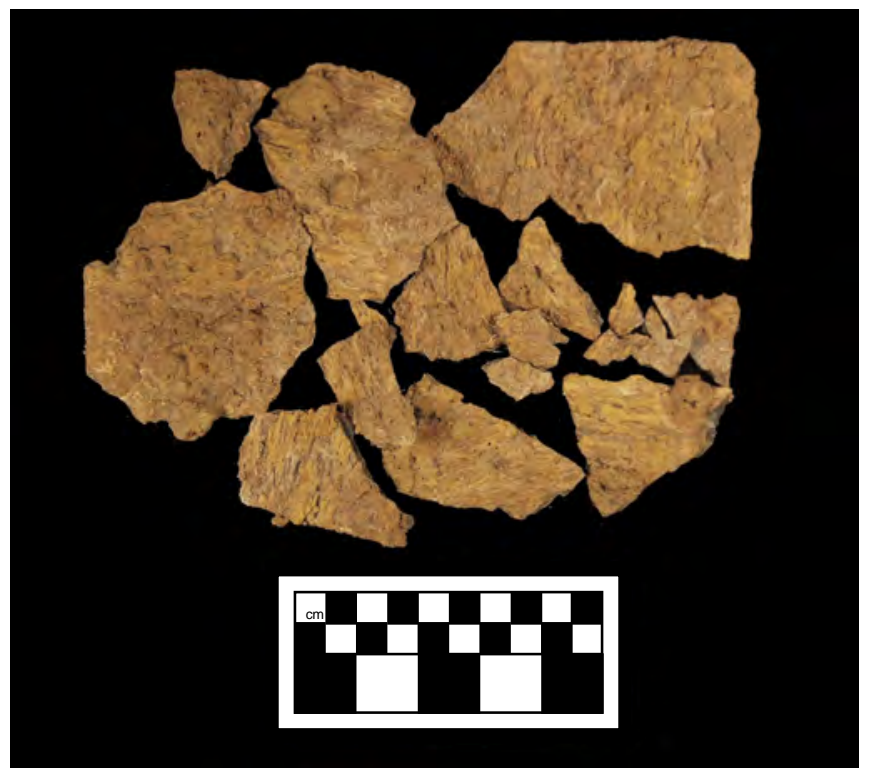

Figure 209. Plaque Type 3.

suggesting that possibly there had been an outer box in this burial. Due to the fragmentary and simple nature of this plaque, it is not possible to definitively match it to any patent records, catalogs, or cemetery excavation reports.

\section{Plaque Type 4}

Plaque Type 4 (Figure 210) is represented by 15 fragments of one ferrous metal plate recovered from Burial 2 in New Home Cemetery (see Appendix A). This plate is similar to Plaque Type 3 in that it is rectangular with gently rounded corners and is of a ferrous metal; however, this plaque has a slightly white patina even though the piece is heavily rusted. There is no evidence of embossed or engraved lettering on the face of this plate. Due to the fragmentary and simple nature of this plaque, it is not possible to definitively match it to any patent records, catalogs, or cemetery excavation reports.

\section{Thumbscrews}

Thumbscrews evolved out of earlier forms of coffin screws with the first identified iteration appearing in an 1859 patent issued to Mr. H. Marshall for an innovative type of rectangular metal casket (U.S. Utility Patent No. 25659). The flat-bodied forms, like those recovered from the New Home Cemetery excavations, first appeared with an 1874 patent issued to W. M. Smith (U.S. Utility Patent 7797) for a flat-bodied, urn-shaped design. These new types of thumbscrews were soon on the market, appearing in catalogs in 1875. Thumbscrews are great temporal diagnostics because continued advancements and variations in designs yielded further registered patents up to 1884. Moreover, companies and individuals continued to produce new forms (though few were patented) even into the twentieth century (Mainfort and Davidson 2006:133-134). Due to the increased use of complex lid fasteners, such as the ones already discussed in this analysis, the popularity of thumbscrews as lid fasteners declined in the first two decades of the twentieth 


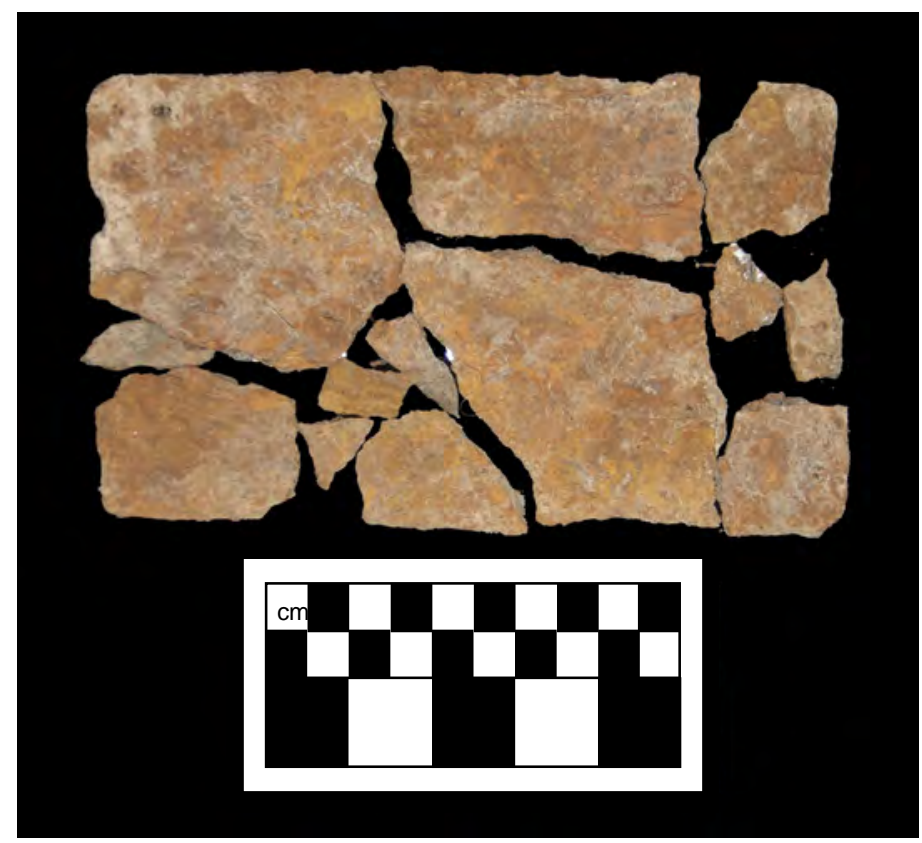

Figure 210. Plaque Type 4.

century. As evidenced by the presence of only two forms of thumbscrews in the 1959 Victor Casket Hardware Company catalog, a greatly reduced and simplified selection of thumbscrews was sold up to the 1960s, mostly as outer box fasteners.

\section{Thumbscrew Type 1}

Thumbscrew Type 1 (Figure 211) is associated with Escutcheon Type 1 and is represented by seven artifacts in Burial 16 in New Home Cemetery (see Appendix A). The white metal finial of this thumbscrew is shaped like a broad fan with a tapered shoulder. The rays of the fan converge at the center, which features an embossed rectangle, before they fan back out to a lesser extend below the center. Two vine-like tendrils flank the bottom of this fan bundle and extend upward toward the center. The cylindrical base appears to be the sepal of a flower, from which the finial emerges.

No matches to this thumbscrew have been located in patent records, but it has been matched to nine period catalogs (Figure 212) and artifacts from four archaeological cemetery excavations. The catalogs range in date from ca. 1900 to 1912, and the cemeteries, which were located in Tennessee, Alabama, Arkansas, and Texas, range in date from 1893 until 1933 (see Appendix D).

\section{Thumbscrew Type 2}

Thumbscrew Type 2 (Figure 213) is associated with Escutcheon Type 2 and is represented by one artifact from Burial 7 in New Home Cemetery (see Appendix A). It consists of a flat-bodied head with a tri-lobed crown motif, with floral tendrils curling inward along each side margin at the lobes, and two lines extending to the bottom corners from the apex of the crown. Below the crown are a constricted neck and a raised cylindrical base. 


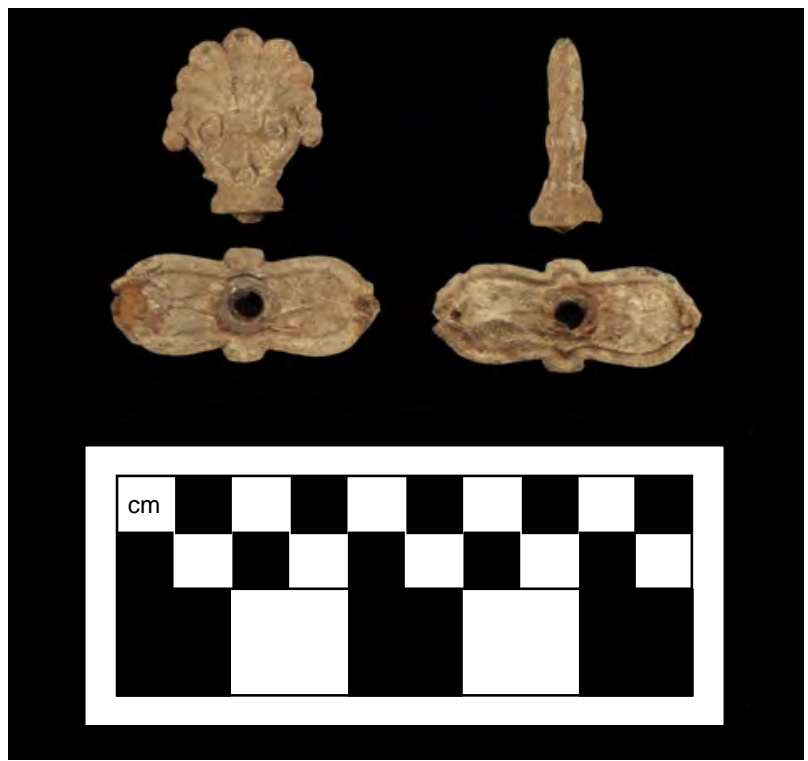

Figure 211. Thumbscrew Type 1 and Thumbscrew Escutcheon Type 1.

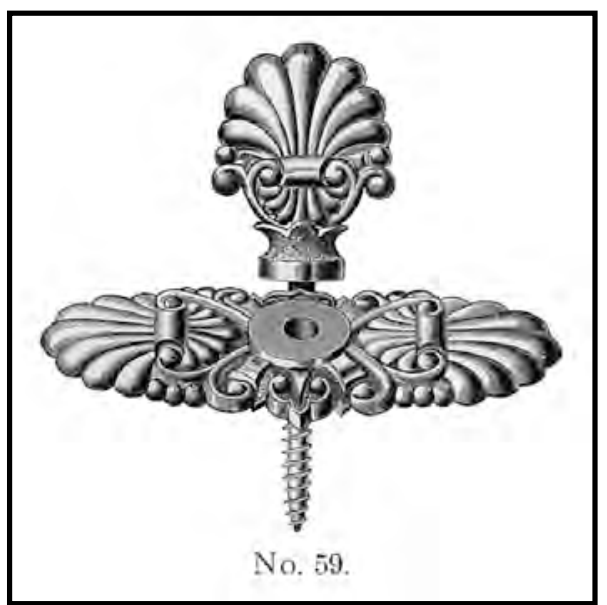

Figure 212. Thumbscrew No. 59 illustrated on page 100 of the 1901 Gate City Coffin Company catalog.

Exact matches have been not been located in any patent records or period trade catalogs. This screw has, however, been identified in the archaeological excavations of two historic cemeteries, one in Georgia with burials dating between 1875 and 1930, and the other in Arkansas from a burial dating between 1890 and 1927 (see Appendix D). This time window brackets the range of common use for thumbscrews in general and therefore does not provide much aid in determining a tighter chronology.

\section{Thumbscrew Escutcheons}

The term "escutcheon" refers to decorative screw plates with a central hole through which a thumbscrew can pass for mounting. These accessories were first known to have been illustrated on page 331 of the 1980 [1865] Russell \& Erwin Manufacturing Company hardware catalog. These early varieties consisted largely of simple diamond-shaped forms. It was not until the 


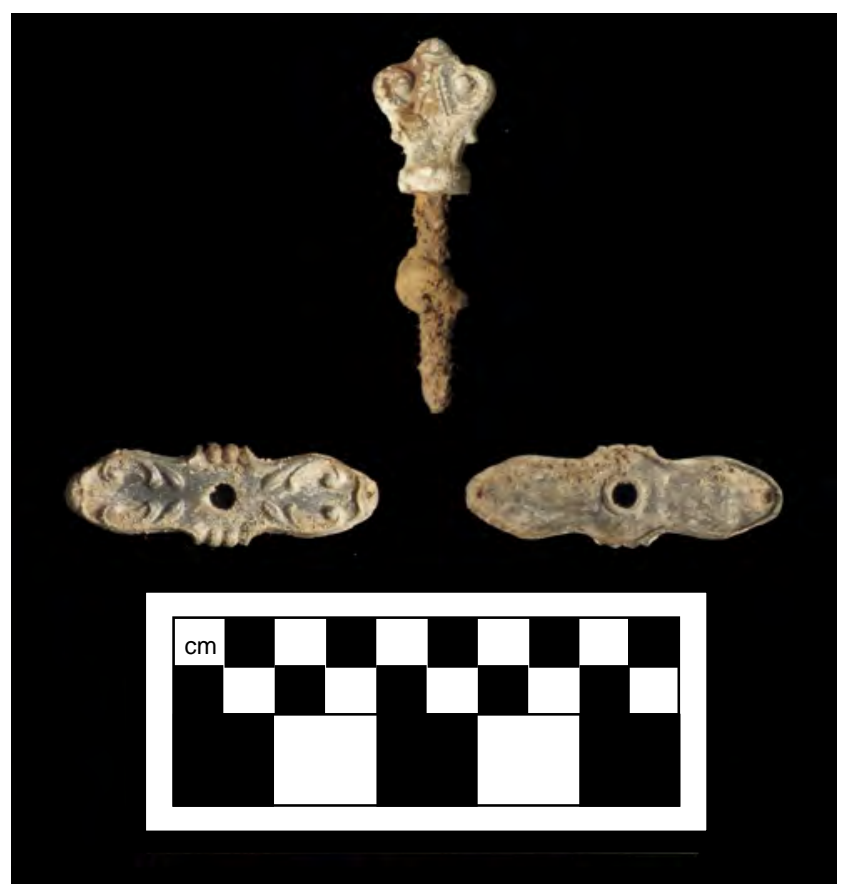

Figure 213. Thumbscrew Type 2 and Thumbscrew Escutcheon Type 2.

widespread introduction of thumbscrews in the 1870s that escutcheon designs began to evolve in turn, so that they could be sold with thumbscrews as matched sets. This type of artifact has a broad temporal range of approximately 1865-1920s (Mainfort and Davidson 2006:147).

\section{Thumbscrew Escutcheon Type 1}

Thumbscrew Escutcheon Type 1 (see Figure 211) is represented by six artifacts from Burial 16 in New Home Cemetery (see Appendix A). This escutcheon is associated with Thumbscrew Type 1 and shares minimal stylistic elements with this thumbscrew. The fact that it is not paired with Thumbscrew Type 1 in any of the catalog appearances of that item lends support to the notion that they are not a matched set. The plate has a central hole around which is a raised band. From the center extend two leaf-like elements that make up the body of the plate. The leaves terminate in what appear to be immature floral buds. Two rectangular protrusions extend along the side margins flanking the center hole, and a raised ridge runs along the sides from the center out to the buds.

This artifact type does not appear in any patent records, period trade catalogs, or archaeological historic cemetery excavations. It has, however, been recorded as present in the historic coffin hardware collection recovered from the A. L. Calhoun General Store in South Carolina, which dated between 1894 and 1926 (Hacker-Norton and Trinkley 1984; see Appendix D). 


\section{Thumbscrew Escutcheon Type 2}

Thumbscrew Escutcheon Type 2 (see Figure 213) is represented by one artifact recovered in Burial 7 in New Home Cemetery (see Appendix A) and is associated with Thumbscrew Type 2. It shares minimal stylistic elements with its associated thumbscrew; thus, in concert with the fact that no catalog matches have been located, it is not clear whether these originally came in a set. The plate has a central hole from which extends two leaf-like elements that make up the body of the plate. The leaves have two inward facing floral tendrils running lengthwise along the piece. At the point where the leaves join along the side margins are what appear to be floral buds flanking the center hole. No matches have been located in period trade catalogs, patent records, or historic cemetery excavations; therefore, a date range of 1875-1920 can be applied based on the general time frame for the manufacture of the associated thumbscrew (see Appendix D).

\section{Embalming Paraphernalia}

The variety of goods marketed for use in the embalming trade is nearly as expansive as the various types of hardware used in the construction and decoration of burial containers. In this typology, "embalming paraphernalia" is used to refer to any chemical, tool, device, or object that might have been used by embalmers in the preparation of the body for burial.

\section{Embalming Paraphernalia Type 1}

Embalming Paraphernalia Type 1 (Figure 214) is represented by two artifacts recovered in Burial 10 in New Home Cemetery (see Appendix A). It is a small cream-colored plastic object with a circular face with two central holes and a threaded funnel-shaped base.

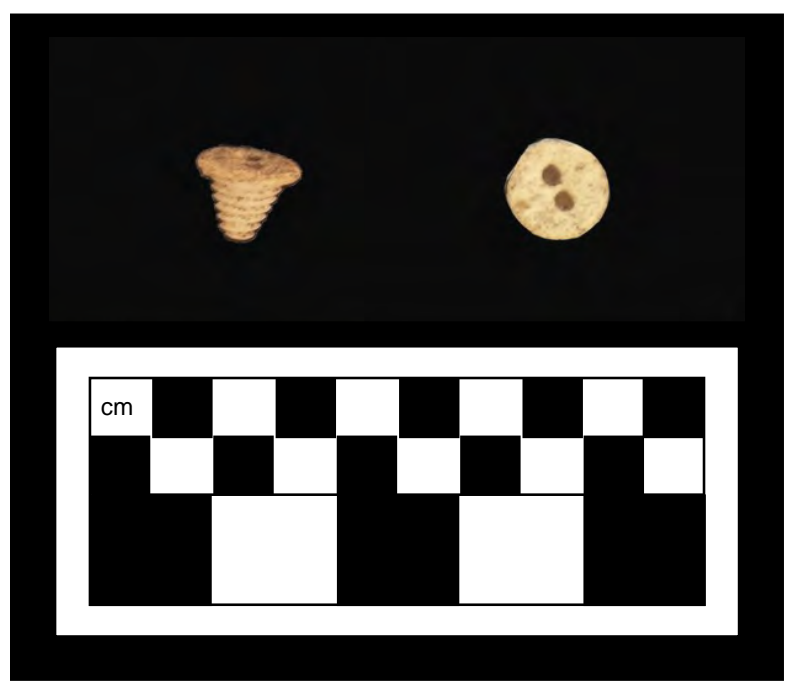

Figure 214. Embalming Paraphernalia Type 1—trocar button. 
This artifact is known as a trocar button and is an embalming appliance used to plug a body cavity incision left during the embalming process. The trocar button's namesake is the trocar, a pointed, tubular instrument patented in 1888 (U.S. Patent 387454) used to puncture the body cavity and convey embalming liquids. Interestingly, incisions were typically sutured prior to the invention of the more efficient trocar plug, which was patented by Arthur V. Cullen in 1948 (U.S. Patent 243381; Figure 215). This definite late introduction date makes this type of artifact a good temporal indicator when recovered from burials.

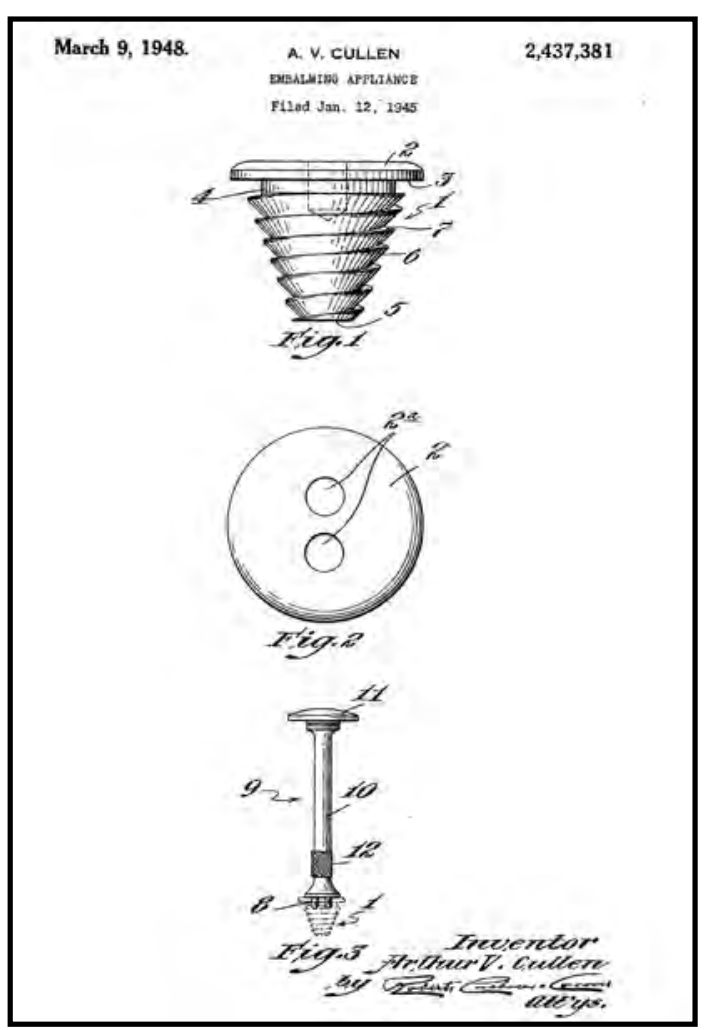

Figure 215. U.S. Utility Patent No. 2437381 assigned in 1948 to Arthur V. Cullen for an embalming appliance.

Trocar buttons were immediately introduced into the market after the patent was issued. This item was recovered in one burial dated to 1951 during the Texas State Cemetery relocation (Dockall et al. 1996:159). Additionally, this type of trocar button was advertised for sale in the 1950s catalog of Royal Bond, Inc. (Figure 216). In that catalog, these items were selling for $\$ 6.85$ per gross, and their applicators, which look like a small screwdriver with two round prongs, were sold for $\$ 1.50$ each. Trocar buttons of this type are still manufactured and appear in two other catalogs from 2002 and 2010 (see Appendix D). 


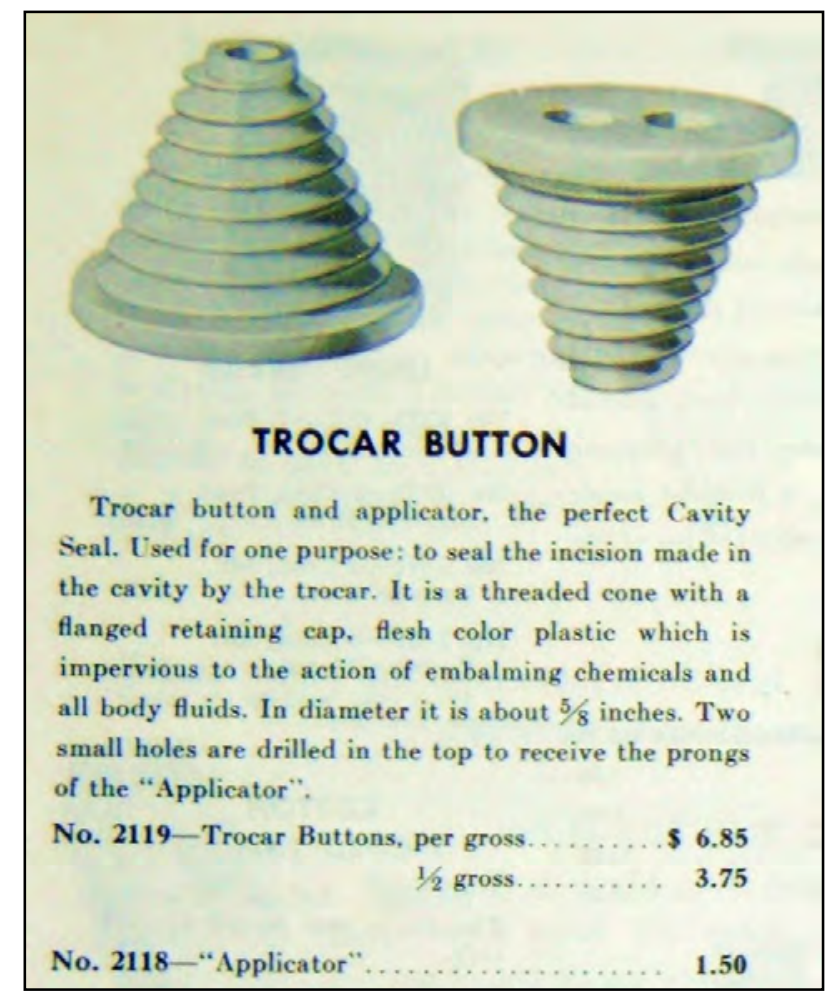

Figure 216. Trocar Button No. 2119 illustrated on page 49 of the ca. 1950s Royal Bond, Inc., catalog.

\section{Embalming Paraphernalia Type 2}

Embalming Paraphernalia Type 2 (Figure 217a) is represented by one artifact recovered in Coffin 4 in New Home Cemetery (see Appendix A). This artifact is a high-density polyethylene bottle that once contained approximately 20 ounces of an embalming fluid called C-45 Cavity Chemical, which was produced by General Funeral Home Supply in Huffman, Texas. This bottle was likely discarded in the casket by the funeral home and was found along with five other bottles (Embalming Paraphernalia Types 3-7) in a plastic bag at the foot of the casket. Discarded bottles have sometimes been used under the body or under the lining to prop up the body, to fill empty space in the foot end if the deceased was too short for the container, or to deter movement of the body.

Cavity chemicals are typically injected into the thoracic and abdominal cavities permeating the viscera, disinfecting the body, destroying any viruses or bacteria that may be present, halting fermentation and putrefaction, and converting albuminous content to a "resinous state of preservation" (Eckels ca. 1939:13). One bottle generally would be diluted with water according to manufacturer instructions in an embalming fluid machine and then injected into the body cavities. Cavity chemicals advertised in the 2002 Kelco Supply Company (2002a) catalog note that in instances where the deceased was suffering from pneumonia, peritonitis, uremia, or other conditions affecting the cavities, one bottle should be used in both the thoracic and abdominal cavities. The fact that two bottles of cavity fluid were recovered from Coffin 4 in New Home Cemetery could indicate such an occurrence, or the funeral home might just have been discarding materials not specifically used for this specific deceased individual. 


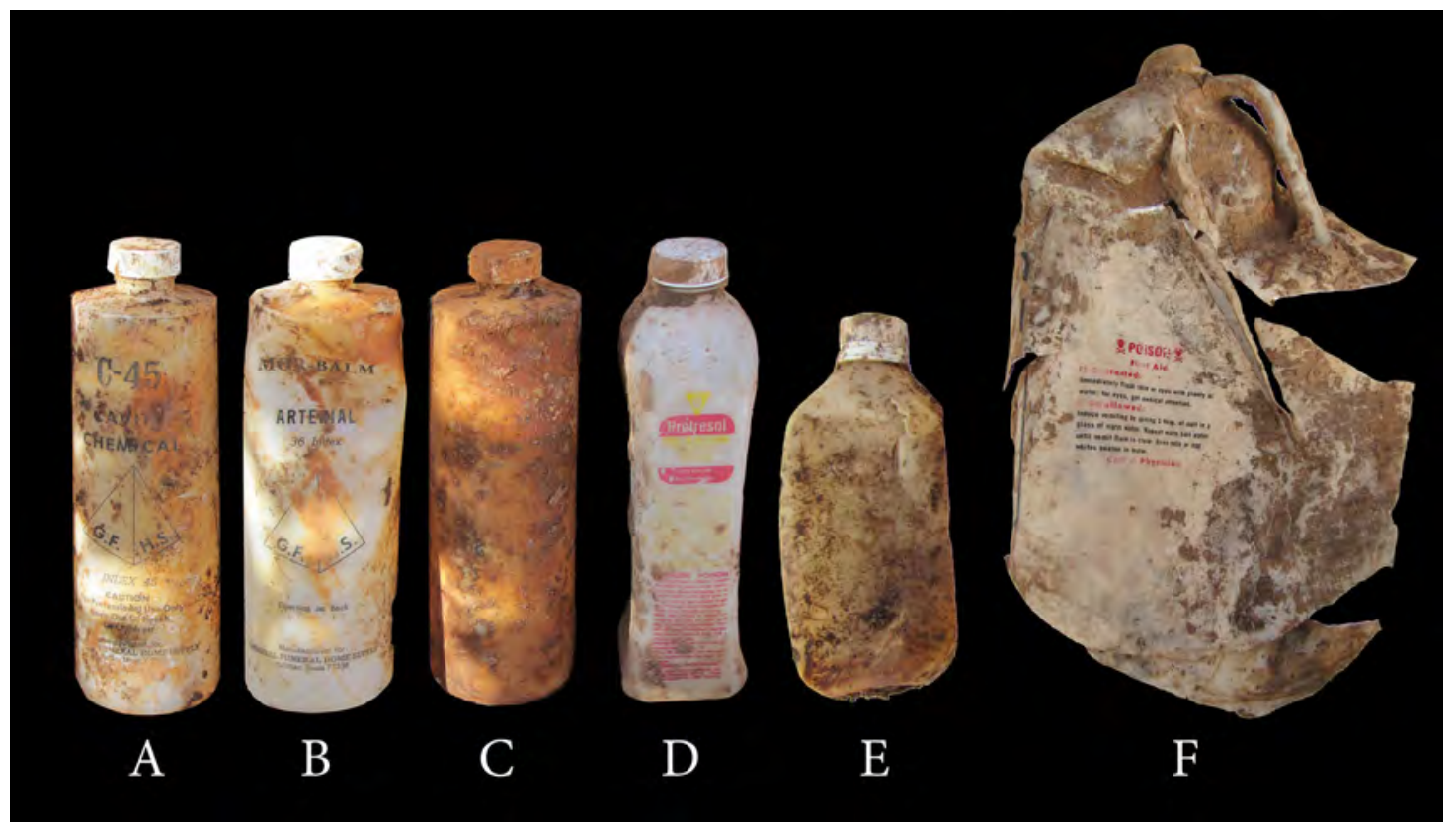

Figure 217. Embalming Paraphernalia Types 2-7-plastic embalming fluid bottles from Coffin 4; bottle A shows Type 2 C-45 Cavity Chemical.

Each company that produced embalming fluids seems to have had its own individual recipes for certain broad categories, and even variations within these categories. The 2002 Kelco Supply Company catalog, for instance, has four different types of cavity fluids; most modern embalming fluids are made of five components: alcohols, humectants, anticoagulants, surfactants, and formaldehyde (Kelco Supply Company 2002a:A7). Alcohols are used in conjunction with the formaldehyde to aid in disinfecting and preserving tissue. Humectants are additives that enhance tissue corpulence and pliability by lessening the resultant dehydration caused by the formaldehyde. Anticoagulants (sequestering agents) work to deactivate clotting agents in blood and promote vascular drainage. Surfactants (wetting agents) serve as lubricants in the vascular system while also helping to promote diffusion of formaldehyde gases and other embalming fluid components through breaking down of the surface tension between the blood vessel walls and surrounding tissue cells (Kelco Supply Company 2002a:A4-A5).

Formaldehyde was first used in the embalming of a human corpse in 1899 and had replaced embalming fluids containing the salts of heavy metals (i.e., arsenic, lead, and mercury) by the years 1906-1910 (Bedino 2003:2616). Formaldehyde is the primary preservative and disinfectant in modern embalming fluids, and the relative strength of a given embalming fluid is measured by something called "index." Index refers to a percentage calculated by determining the number of grams of formaldehyde gas dissolved in 100 milliliters of water (Kelco Supply Company 2002a:A3).

No product safety data sheets have been located for General Funeral Home Supply, so it is not possible to say for sure what the percentages were of all chemical components used in the C-45 Cavity Chemical. The bottle does indicate that the index was 45, so that means it was 45 percent formaldehyde. An index of 45 suggests this cavity chemical was very strong as this is even beyond the "High Index" classification in the 2002 Kelco Supply Company (2002a) catalog. 
No patent information or catalogs have been located that specifically mention this particular cavity fluid, so it is not known when the chemical entered the market, and it has not been reported in other cemetery excavations (see Appendix D). The product came in a plastic bottle, which is an extremely important time diagnostic. In merchandise catalogs of the 1950s, embalming fluids were still being sold in glass bottles. In fact, it was not until the early 1960s that methods of producing high-density polyethylene made plastic more cost effective than glass in commercial industries. Therefore, this bottle cannot date to before the early 1960s. Though it is likely that this bottle was left in the casket by the funeral company previously contracted to relocate the remains, it is also possible that the bottle was introduced at the time of that disinterment.

\section{Embalming Paraphernalia Type 3}

Embalming Paraphernalia Type 3 (see Figure 217b) is represented by one artifact recovered in Coffin 4 in New Home Cemetery (see Appendix A). This artifact is a high-density polyethylene bottle that once contained approximately 20 ounces of an embalming fluid called Mor-Balm Arterial, which was produced by General Funeral Home Supply in Huffman, Texas. This bottle was likely discarded in the casket by the funeral home and was found along with five other bottles (Embalming Paraphernalia Types 2, 4-7) in a plastic bag within the casket.

As the name implies, arterial fluid is generally injected into the arteries after drainage of the blood. It acts to harden and preserve the vessel walls and hold features in proper position, thus preventing distention of the neck and sagging of the facial muscles (Royal Bond, Inc., ca. 1950:19). From the arteries, the fluid is forced into every remote part of the vascular system halting decay throughout the body and preserving the appearance of naturally firm yet relaxed body tissue. The bottle recovered from New Home Cemetery suggests that Mor-Balm had a formaldehyde index of 36, which is very high for an arterial fluid. Most of the arterial fluids in the 2002 Kelco Supply Company (2002a) catalog had indices in the low 20s.

No patent information or catalogs have been located that specifically mention this particular arterial fluid, so it is not known when the chemical entered the market, and it has not been reported in other cemetery excavations (see Appendix D). The product came in a plastic bottle, which is an extremely important time diagnostic. As mentioned previously, this bottle cannot date to before the early 1960s. Though it is likely that this bottle was left in the casket by the funeral company previously contracted to relocate the remains, it is also possible that the bottle was introduced at the time of that disinterment.

\section{Embalming Paraphernalia Type 4}

Embalming Paraphernalia Type 4 (see Figure 217c) is represented by one artifact recovered in Coffin 4 in New Home Cemetery (see Appendix A). This artifact is a high-density polyethylene bottle that once contained approximately 20 ounces of an unknown embalming fluid. The bottle was coated with soil and discolored to the point that the writing on the label could not be discerned from the field photographs. Enough of the label could be observed to note that it was produced by General Funeral Home Supply in Huffman, Texas, and that it was not the same chemical as the previous two discussed. This bottle was likely left in the casket by the funeral home and was found along with five other bottles (Embalming Paraphernalia Types 2-3, 5-7) in a plastic bag within the casket. The product came in a plastic bottle, which is an extremely 
important time diagnostic. As mentioned previously, this bottle cannot date to before the early 1960s. Though it is likely that this bottle was left in the casket by the funeral company previously contracted to relocate the remains, it is also possible that the bottle was introduced at the time of that disinterment.

\section{Embalming Paraphernalia Type 5}

Embalming Paraphernalia Type 5 (see Figure 217d) is represented by one artifact recovered in Coffin 4 in New Home Cemetery (see Appendix A). This artifact is a high-density polyethylene bottle that once contained 16 ounces of an embalming fluid called Pretresol Pre-Injection, which was produced by L. H. Kellogg Chemical Company of Minneapolis, Minnesota. This bottle was likely discarded in the casket by the funeral home and was found along with five other bottles (Embalming Paraphernalia Types 2-4, 6-7) in a plastic bag within the casket.

Pretresol is a pre-injection fluid that is composed of 41 percent methanol and 5 percent formaldehyde (Kelco Supply Company 2002a:54 Directory F). Pre-injection fluid is forced into the vascular system where it relieves vascular congestion through the dilution of blood and the expansion of vessel walls to allow for easier flow of fluids (Eckels ca. 1939:15; Royal Bond, Inc., ca. 1950:26). By removing dark and heavy clots of blood, it reduces the likelihood that the body will have discoloration, and also clears blockages so that arterial fluids will have an even distribution for disinfection and preservation purposes. Additionally, pre-injection fluid acts to reoxygenate tissue cells, which promotes a life-like coloration (Royal Bond, Inc., ca. 1950:26).

No patent information has been located that specifically mentions this particular pre-injection fluid, and it has not been reported in other cemetery excavations. Pretresol was, however, being sold in the 2002 Kelco Supply Company (2002a) catalog, and it continues to be produced today (see Appendix D). The L. H. Kellogg Chemical Company, which manufactures Pretresol, is also the parent company of Kelco Supply Company and was first founded in 1941 (Star Tribune 2009). As discussed above, however, it is known that plastic bottles did not come into common industrial use until the early 1960s. Though it is likely that this bottle was left in the casket by the funeral company previously contracted to relocate the remains, it is also possible that the bottle was introduced at the time of that disinterment.

\section{Embalming Paraphernalia Type 6}

Embalming Paraphernalia Type 6 (see Figure 217e) is represented by one artifact recovered in Coffin 4 in New Home Cemetery (see Appendix A). This artifact is a polyethylene bottle that once contained approximately one pint an unknown fluid, likely an embalming fluid. No label or writing can be seen in the field photographs of this artifact. This bottle was likely left in the casket by the funeral home and was found along with five other embalming fluid bottles (Embalming Paraphernalia Types 2-5, 7) in a plastic bag within the casket. As mentioned previously, this bottle cannot date to before the early 1960s. Though it is likely that this bottle was left in the casket by the funeral company previously contracted to relocate the remains, it is also possible that the bottle was introduced at the time of that disinterment. 


\section{Embalming Paraphernalia Type 7}

Embalming Paraphernalia Type 7 (see Figure 217f) is represented by one artifact recovered in Coffin 4 in New Home Cemetery (see Appendix A). This artifact is a polyethylene bottle that once contained approximately one gallon of an unknown poisonous fluid, likely an embalming fluid. No brand name or manufacturing company labels can be seen in the field photographs of this artifact; however, a warning label has survived. This bottle was likely left in the casket by the funeral home and was found along with five other embalming fluid bottles (Embalming Paraphernalia Types 2-6) in a plastic bag within the casket. As mentioned previously, this bottle cannot date to before the early 1960s. Though it is likely that this bottle was left in the casket by the funeral company previously contracted to relocate the remains, it is also possible that the bottle was introduced at the time of that disinterment.

\section{Embalming Paraphernalia Type 8}

Embalming Paraphernalia Type 8 (Figure 218, right) is represented by two artifacts recovered from Burial 10 in New Home Cemetery (see Appendix A). They are small, flesh-colored, spheroidal celluloid shells that conform to the shape of the eye (being either circular or ovoid). These devices are known as eye caps and are typically placed under the eyelids of a corpse by a mortician. The outer surface of the shell is marked with numerous triangular perforations. The perforations create triangular prongs that grip the interior of the lid and usually work along with some type of adhesive to keep the eyes closed.

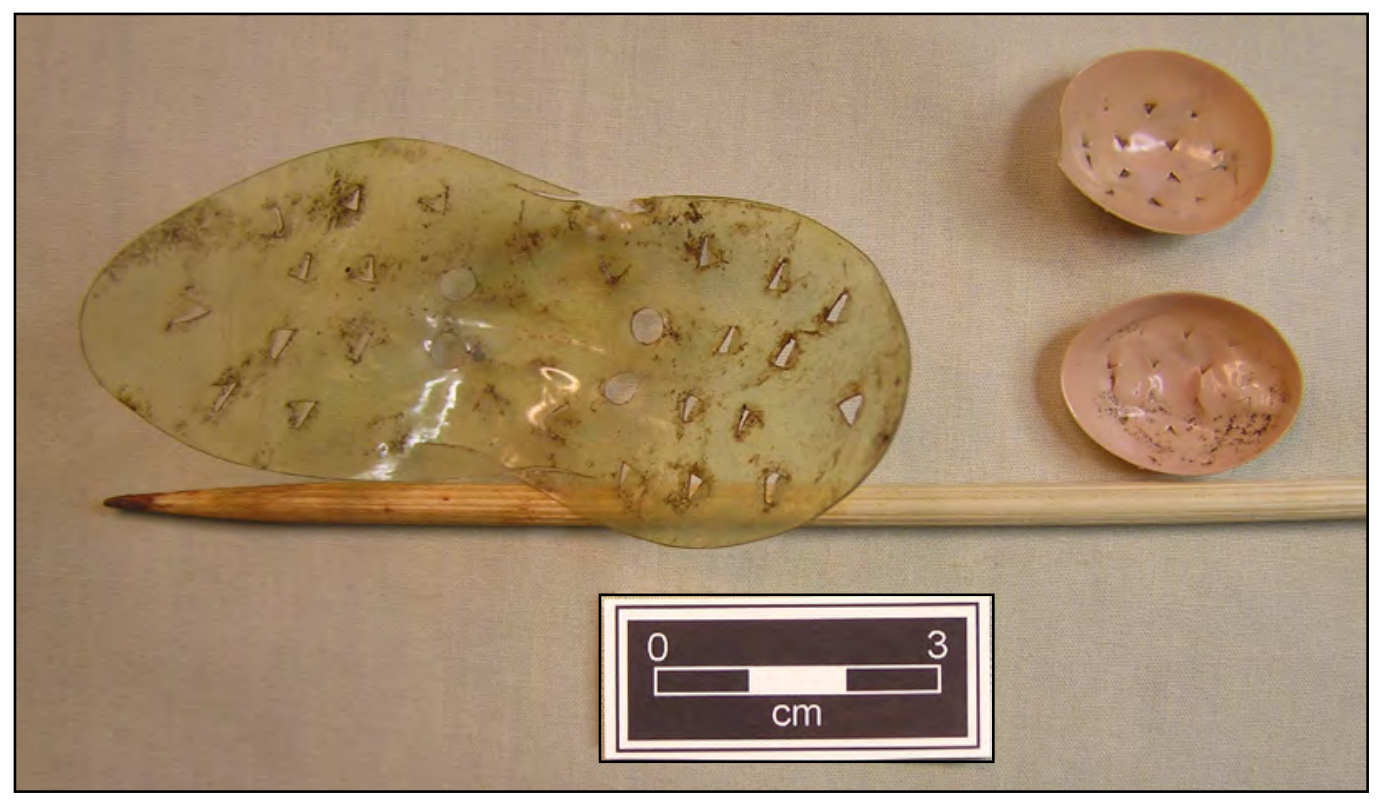

Figure 218. Embalming Paraphernalia Type 8 (eye caps, right) and Type 9 (mouth former, left). 
The earliest known patent for an eye cap was issued to Hamilton Morgan in 1912 (U.S. Patent 1016083). Morgan's Eye Cap was quickly introduced into the market and has appeared in embalming and undertaker supply catalogs through to the present day with little variation in form (Figure 219). The eye caps recovered from Burial 10 in New Home Cemetery appear to be examples of ovoid-shaped Morgan's Eye Caps. At this time, a systematic search of the literature has not been conducted to ascertain whether this type of eye cap has been recovered from other twentieth century archaeologically relocated burials.

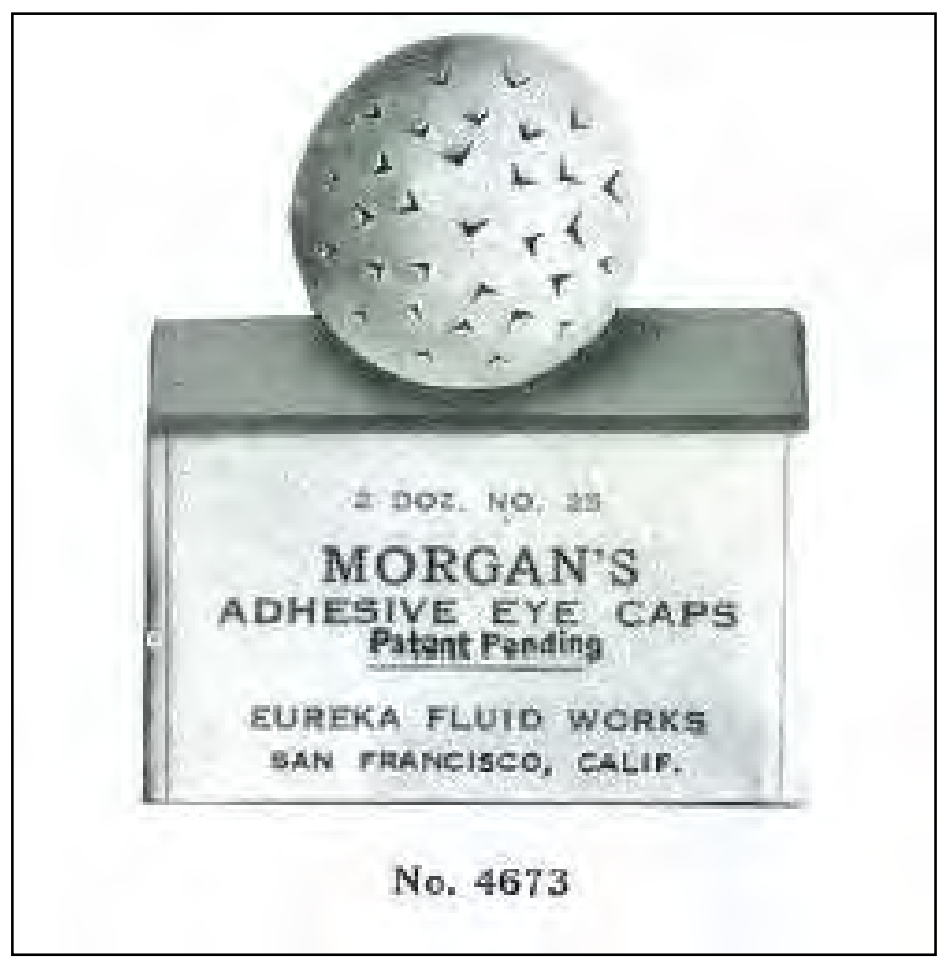

Figure 219. Morgan’s Eye Cap as illustrated on page 216 of the 1940 Dominion Manufacturers, Ltd., catalog.

\section{Embalming Paraphernalia Type 9}

Embalming Paraphernalia Type 9 (see Figure 218, left) is represented by one artifact recovered from Burial 10 in New Home Cemetery (see Appendix A). It is a transparent celluloid or vinyl shell which conforms to the natural expression of the mouth and dentition. This device is known generally as a "mouth former," and specifically as a "Natural Expression Mouth Former." It is typically placed under the lips of a corpse by a mortician. The shell is marked with numerous triangular perforations using the same logic and technique as the eye caps discussed previously. The perforations create triangular prongs that grip the interior of the lips to keep the lips closed. Additionally, four small circular holes, two on each side of the mid-line, are used for ports to inject chemicals into the mouth and to guide the wire or string closures of the jaws. 
The earliest known patent for a mouth closing device was granted to R. Garvey in 1930 (U.S. Patent 1775718), but this invention was nothing more than a method for using twisted wires to secure the jaws. In 1939, U.S. Patent 2162612 was granted to A. Ebert for a "Lip Closing Appliance for Corpse." This device consisted of a metal plate with prongs on one surface that would secure the interior of the lips and prevent opening of the mouth. This patent was the first to use the phrase "natural expression" in the description of the function of the device. It was not until 1963, however, that the so-named "Natural Expression Former" was patented by inventor Charles W. Rector (U.S. Patent 3103052). An improvement to the original patent was granted in 1965 (U.S. Patent 3195215), and it is this exact device that was located in Burial 10 at New Home Cemetery (Figure 220).

Even though there were a number of mouth closing devices patented in the 1930s and 1940s, of which only a couple were mentioned above, none of them performed in such a way as to make them popular products. In fact, mouth formers or other similar mouth closure devices were not even carried by period supply companies. Granted the comparative collection of catalogs has a gap in the late 1940s and 1950s, but the earliest mouth formers appear in the 1963 Dodge Chemical Company catalog. The improved "Natural Expression Former" hit the market quickly after its patent was granted, appearing in the 1966 Superior Funeral Supply Company catalog (Figure 221).

This information suggests a very late date of interment (>1965) for Burial 10 in New Home Cemetery. There are very few cemeteries with burials dating from such a late period that are contained within the comparative sample (Appendix E). No other archaeological examples are known to exist.

\section{Miscellaneous Mortuary Artifacts}

The miscellaneous artifact category is a catchall group for items that either cannot be reasonably identified based on condition or lack of historical information, or those items for which the inclusion within the burial is somewhat anomalous. Some of these artifacts (i.e., bolts) could possibly be placed in other categories already discussed, such as the internal construction elements, but for the reasons mentioned above, they have been placed here.

\section{Miscellaneous Artifact Type 1}

Miscellaneous Artifact Type 1 (Figure 222) consists of 13 loose pieces of yellowish brown and reddish brown foam, as well as several other small fragments adhering to the backs of burial container handle lugs, on the tips of screws attached to handles, or on other hardware. Foam was recovered from seven burials (Coffins 1, 3 and 4, as well as Burials 4, 10, 11, and 12) in New Home Cemetery (see Appendix A).

Because this foam was recovered from the backs of handles, it is possible that the foam was used to pad the backs of hardware so as to not scuff the side of the burial container, or to secure a tighter fit. The foam was also found occasionally on the ends of clinched handle screws that would suggest that the padding also could have been within the burial container. Louis G. Kregel was issued a patent in 1890 (U.S. Patent 430964) for padding for burial caskets (Figure 223). In this patent, Kregel describes padding that should be cut into a certain design so as to effectively 


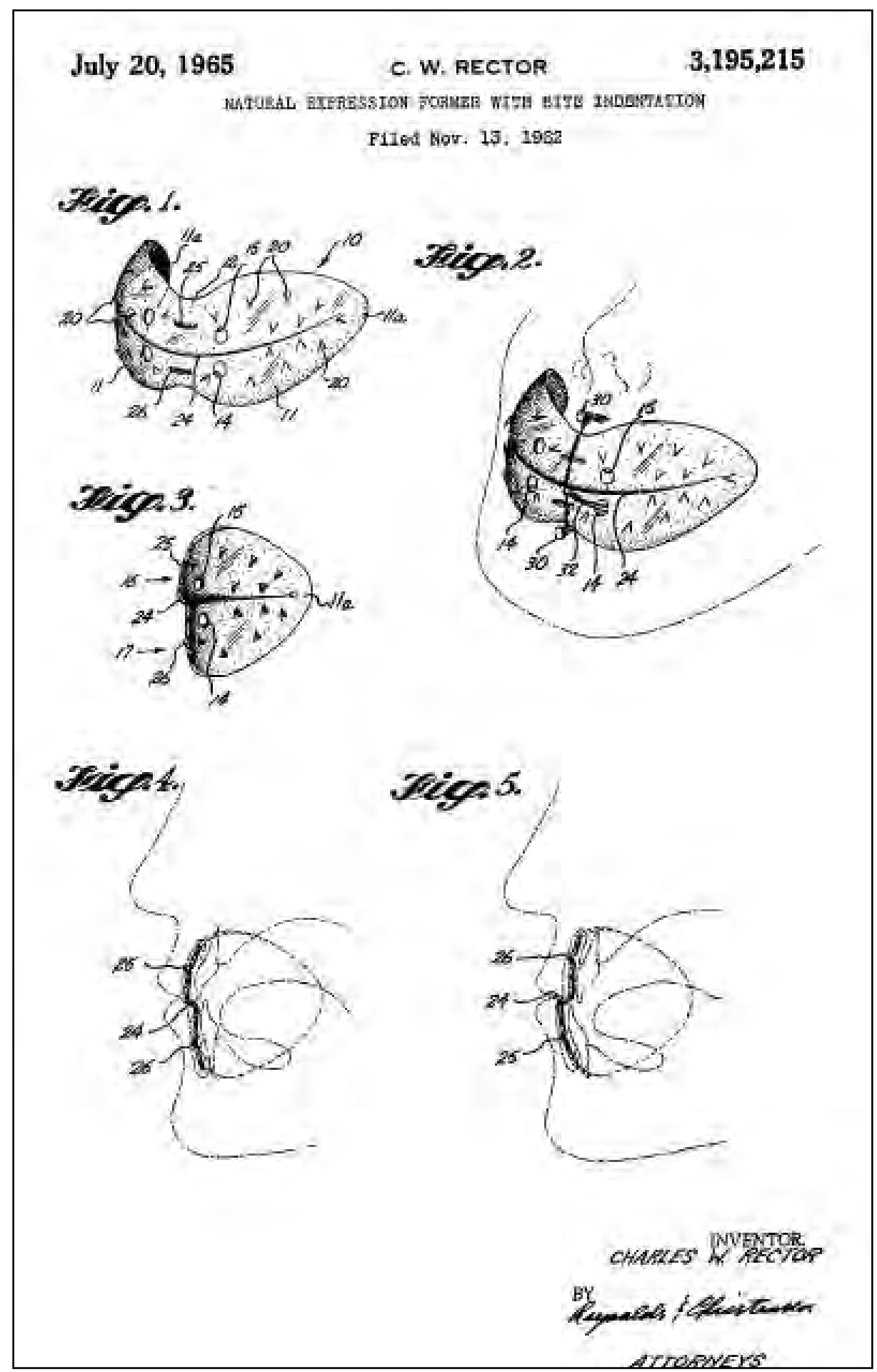

Figure 220. U.S. Utility Patent No. 3195215 assigned in 1965 to Charles W. Rector for the "Natural Expression Former." 


\section{NATURAL \\ EXPRESSION \\ VINYL \\ MOUTH FORMERS

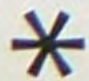

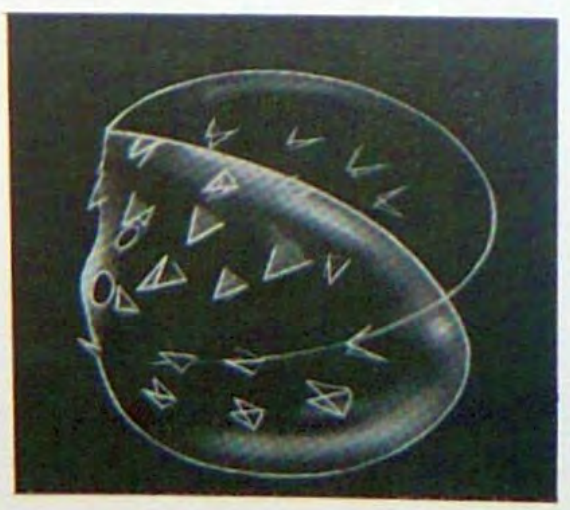

This unique device applies the proven merits of the eye cap to the closure and contouring problems of the mouth. This similarity lies in the natural expression former's ability to gain strength from recurved surfaces, yet utilizing extremely thin resilient material. This device offers more value per dollar combined with more positive control of facial expression and contours. It serves better because it is tough yet resilient. Pre-conformed of .010 thick flexible clear vinyl with sharp, self-impaling spurs readily trimmed to size and perforated for free air movement. Impervious to chemical action. Has bite indicator guide lines. HAS APERATURES FOR TWISTED NEEDLE INJECTOR WIRES.
No. B33C/3086 Vinyl Mouth Formers
2 Dozen $\$ 9.80$
6 Dozen 29.00
12 Dozen 57.00

Figure 221. Natural Expression Vinyl Mouth Former illustrated on page 185 of the 1966 Superior Funeral Supply Company catalog.

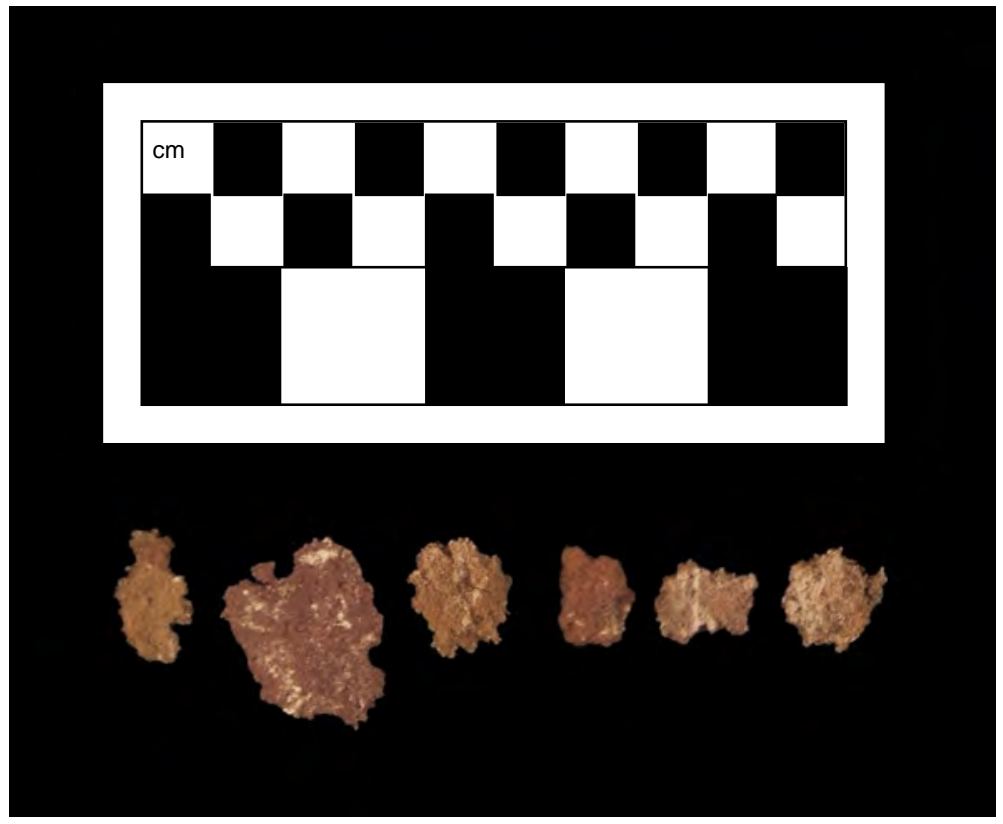

Figure 222. Miscellaneous Artifact Type 1-foam from caskets. 


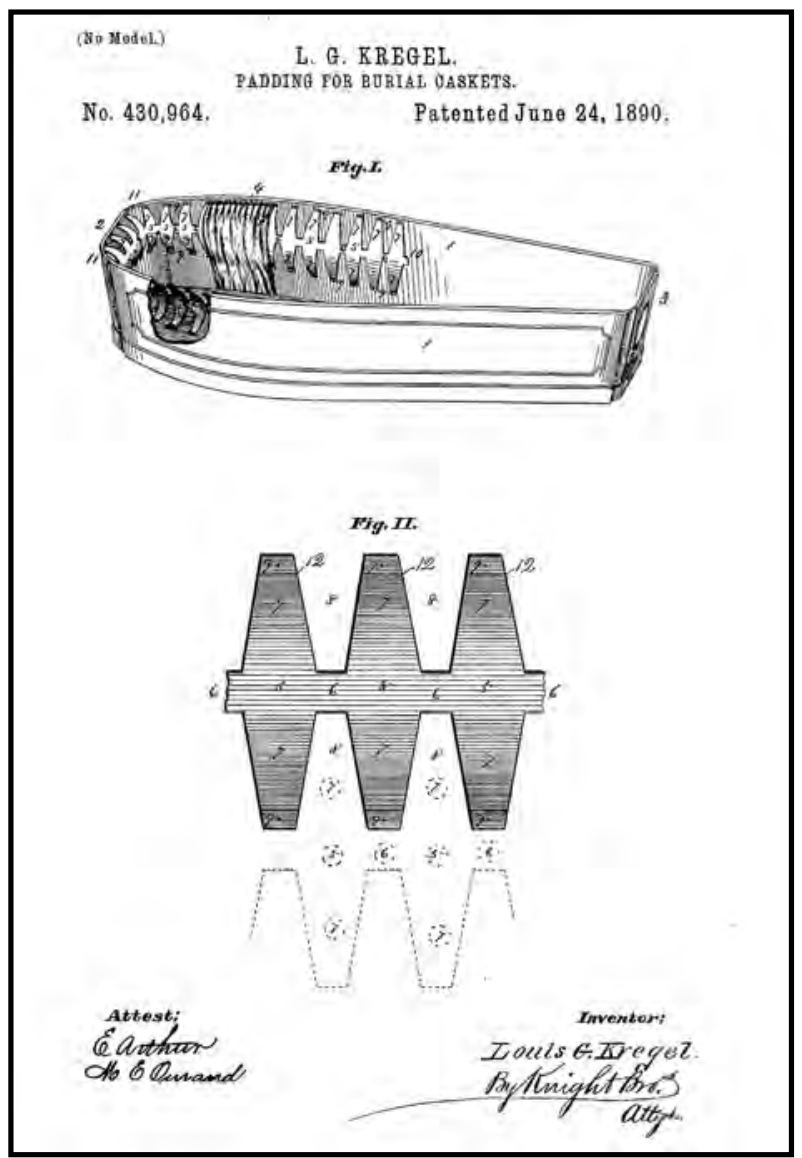

Figure 223. U.S. Utility Patent No. 430964 assigned in 1890 to Louis G. Kregel for burial casket padding.

maintain proper fullness of the head lining. Though Kregel did not confine himself to any particular type of padding material, it is reasonable to assume that the foam recovered at New Home Cemetery could have served such a function. This was confirmed by the fact that large strips of padding were found on the interior side walls of Coffin 4.

\section{Miscellaneous Artifact Type 2}

Miscellaneous Artifact Type 2 (Figure 224) is represented by portions of fibrous netting with rectangular holes formed from flat, thin strands. It was recovered from Burial 4 in New Home Cemetery; field photographs suggest that Coffins 1,3 , and 4 also contained this type of netting, but it was not recorded or collected for analysis (see Appendix A). There were white cotton fibers adhering to some portions of the netting from Burial 4.

Figure 225 shows that at least in Coffin 4 this netting was attached to the full upper margin of the casket and dropped down along the interior of the side walls. Because only this one view of the netting in Coffin 4 is available in field images and no further notes were recorded by excavators, the netting's exact function is unclear. It is likely that is would have been used to help secure various sorts of padding below the cloth lining, which would have deterred the body from shifting during transport. Alternatively, it could have been secured to the sides of the burial container and acted as a cradle or hammock for the body and again deter movement. 


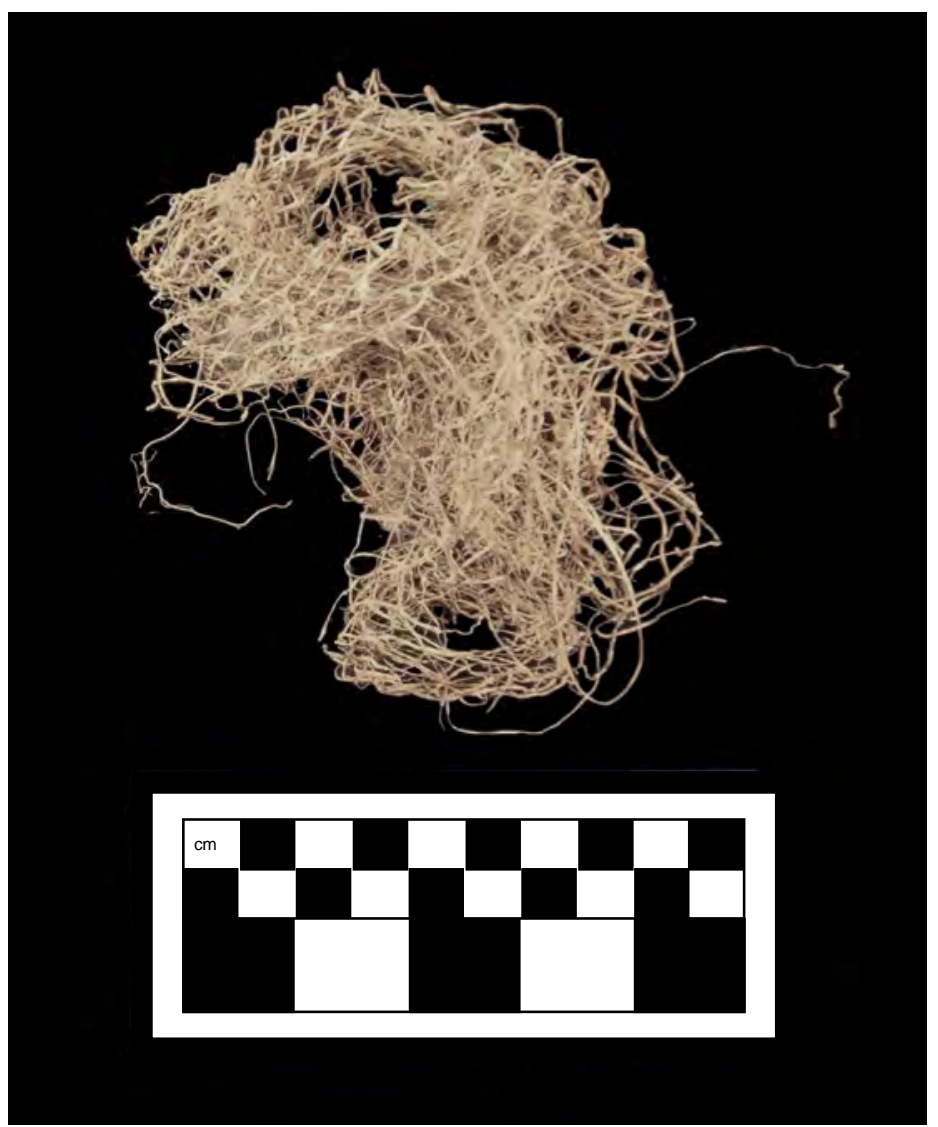

Figure 224. Miscellaneous Artifact Type 2-netting.

\section{Miscellaneous Artifact Type 3}

Miscellaneous Artifact Type 3 (Figure 226) is represented by three fragments of one rectangular ferrous metal plate recovered from the Coffin 4 in the disturbed area of the New Home Cemetery excavations (see Appendix A). This is a large 8-x-3-inch plate with a curved furrow running the length of the plate along the center. It appears to be a housing plate of some kind, such as for a large sliding bolt or some such element. The function of this object is unclear, but archaeologists note that it was used in the construction of a wooden enclosure that surrounded both Coffins 3 and 4 together.

\section{Miscellaneous Artifact Type 4}

Miscellaneous Artifact Type 4 (Figure 227) is represented by one 61/4-inch-long rounded headed spike recovered from Coffin 1 area in the disturbed area in New Home Cemetery (see Appendix A). Although this artifact was recovered with other mortuary items, its exact context is unknown; therefore, it is not possible to determine its exact function. It could have been used in some capacity in the burial container. It could also have been placed within the grave fill for symbolic or religious purposes, or it might just be an incidental inclusion. 


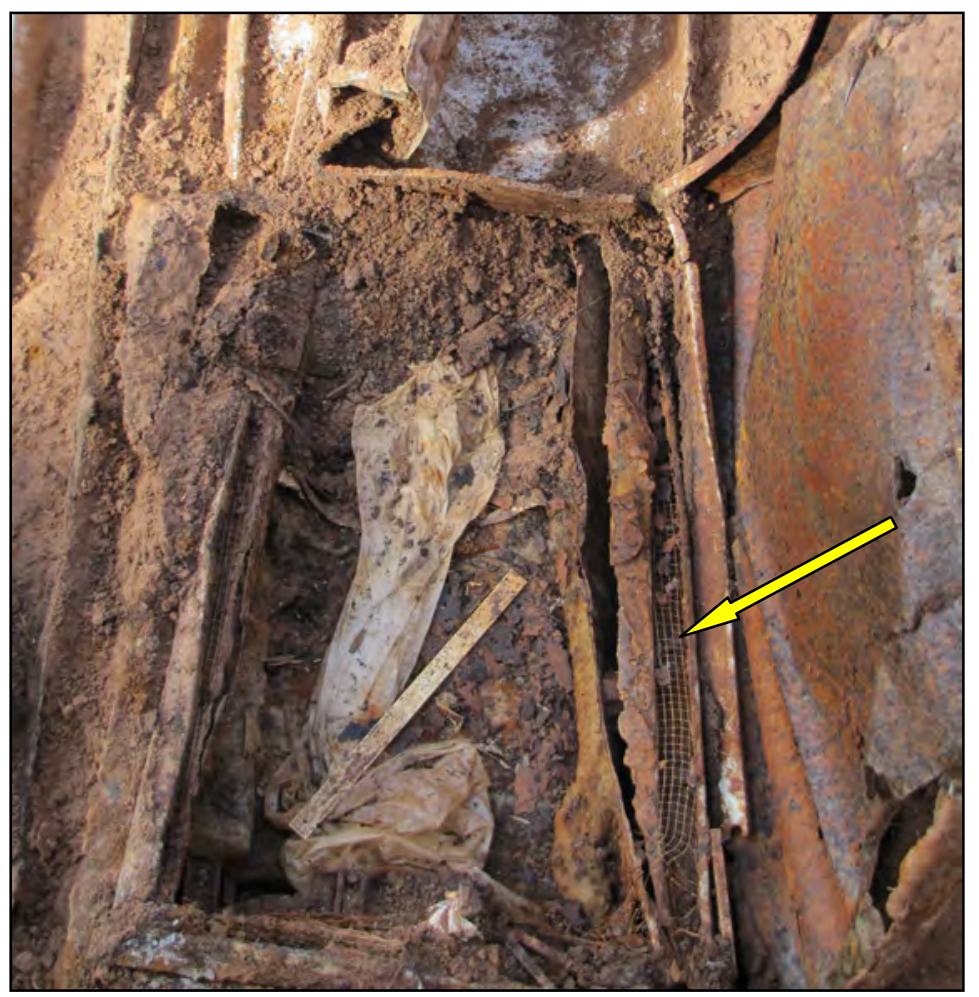

Figure 225. Field image of Coffin 4 showing netting (Miscellaneous Artifact Type 2) in situ (see arrow).

\section{Miscellaneous Artifact Type 5}

Miscellaneous Artifact Type 5 (Figure 228) is represented by two decorative items collected from the general disturbed area on the site, as well as in surface collections in soil scraped from the south section of the ROW (see Appendix A). They appear to be made of a Britannia metal or some other type of cast alloy with silver and gold colored electroplating. Stylistically, they look like flower buds with a squared bottom and clusters of leaves in the middle. On the back of the pieces are two small circular mold marks that look similar to the pontil marks on glass bottles. Although these artifacts were discovered from disturbed contexts, they do appear to share many qualities with other mortuary artifacts described previously. Their exact function is unknown, however. They do not have any type of nail or screw hole, but the curves along the side and the little protrusions on each bottom side could have been used to secure it to a handle bar or a similar element.

\section{Miscellaneous Artifact Type 6}

Miscellaneous Artifact Type 6 (Figure 229) is represented by one item recovered from disturbed contexts within New Home Cemetery (see Appendix A). It is a thin strip of ferrous metal with rounded corners and a screw at both ends. It is bent over forward at one end just after the screw hole. The piece is then bent nearly 85 degrees upward at the middle. This piece was originally thought to be some sort of glass stop like No. 8 from page 49 of the 1920s-1930s Langenau Mfg. Company catalog (Figure 230). However, the Langenau glass stop is not bent like Miscellaneous 


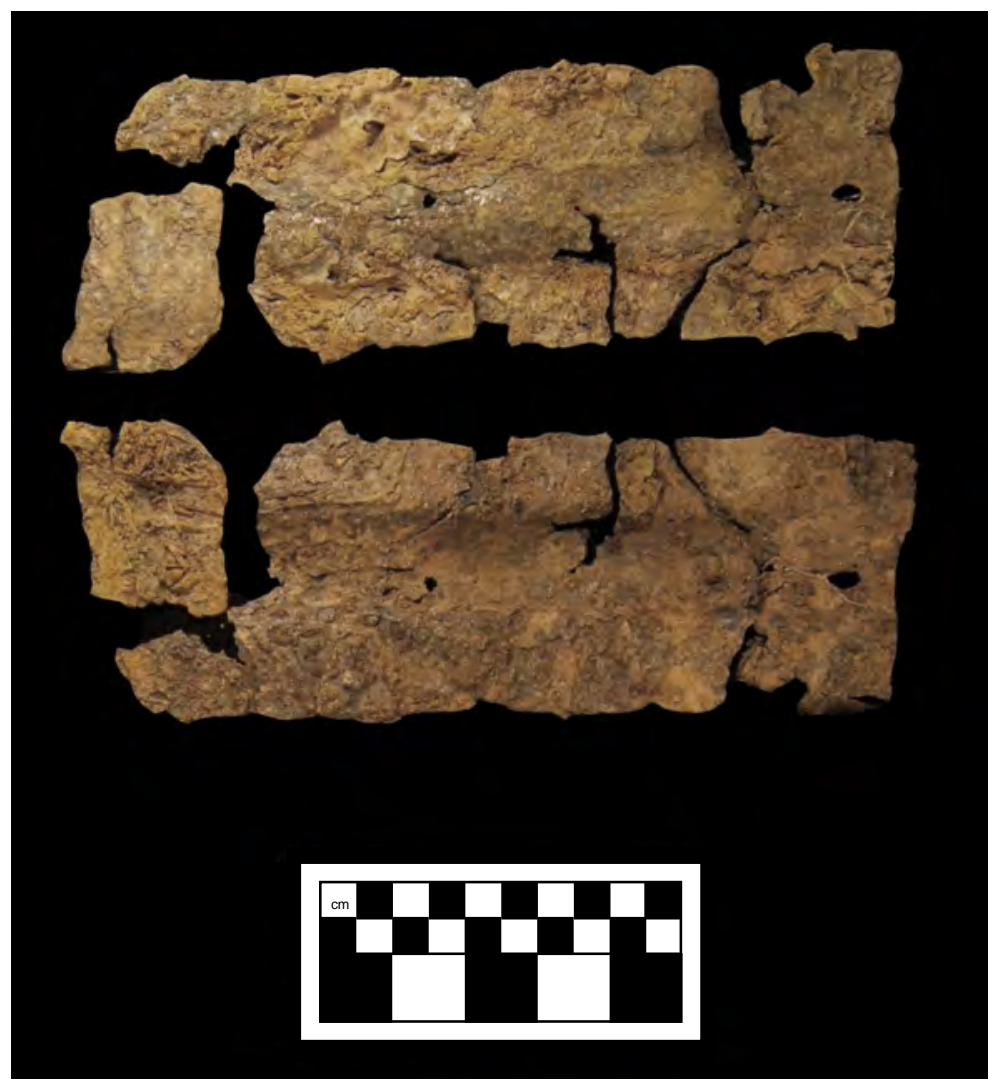

Figure 226. Miscellaneous Artifact Type 3—unidentified housing plate.

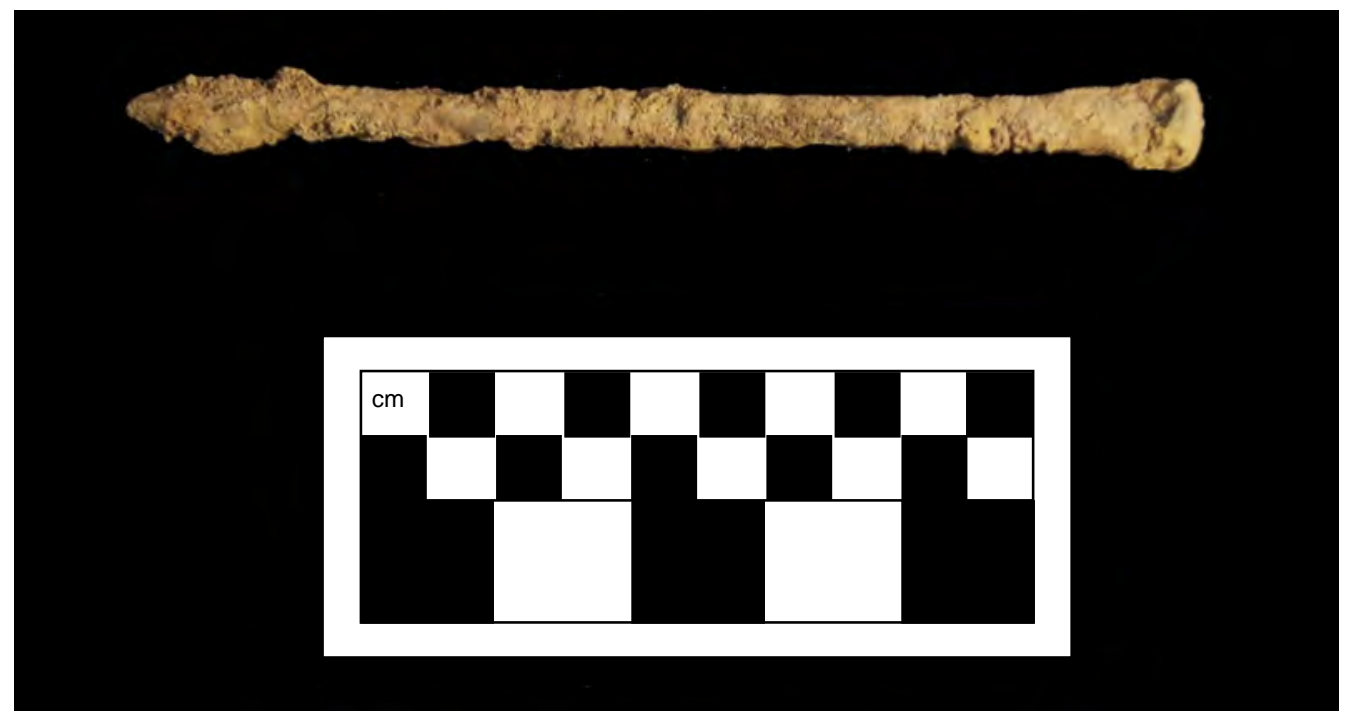

Figure 227. Miscellaneous Artifact Type 4—spike. 


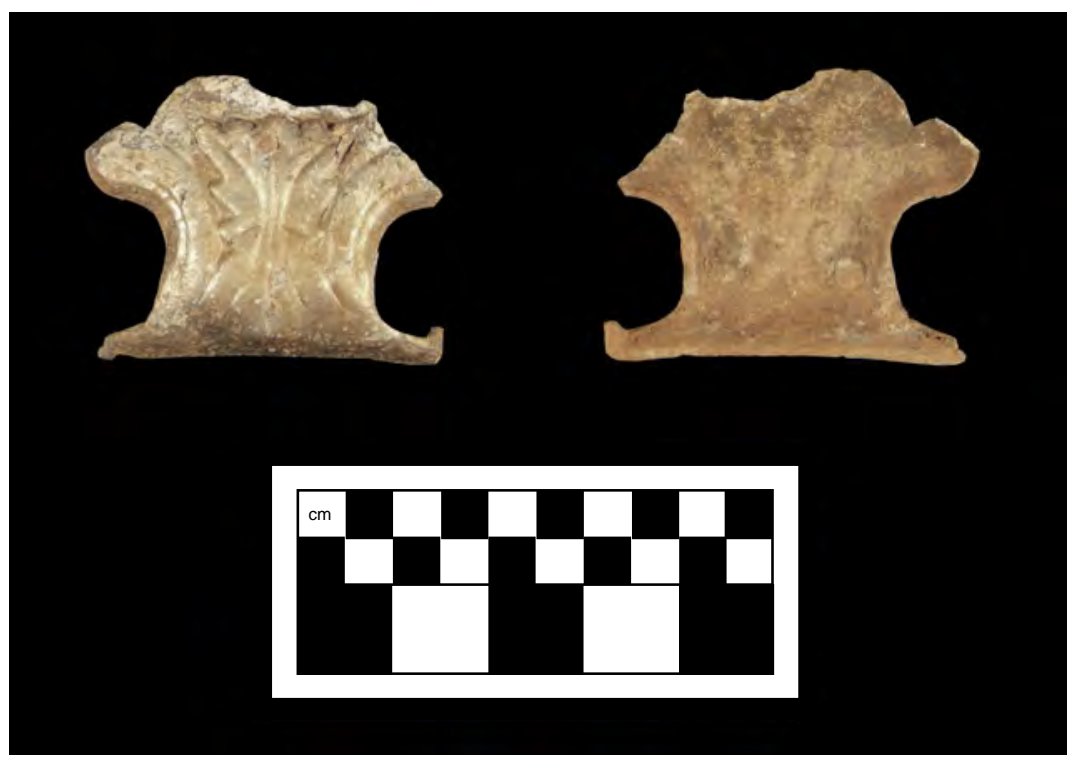

Figure 228. Miscellaneous Artifact Type 5-unidentified casket hardware.

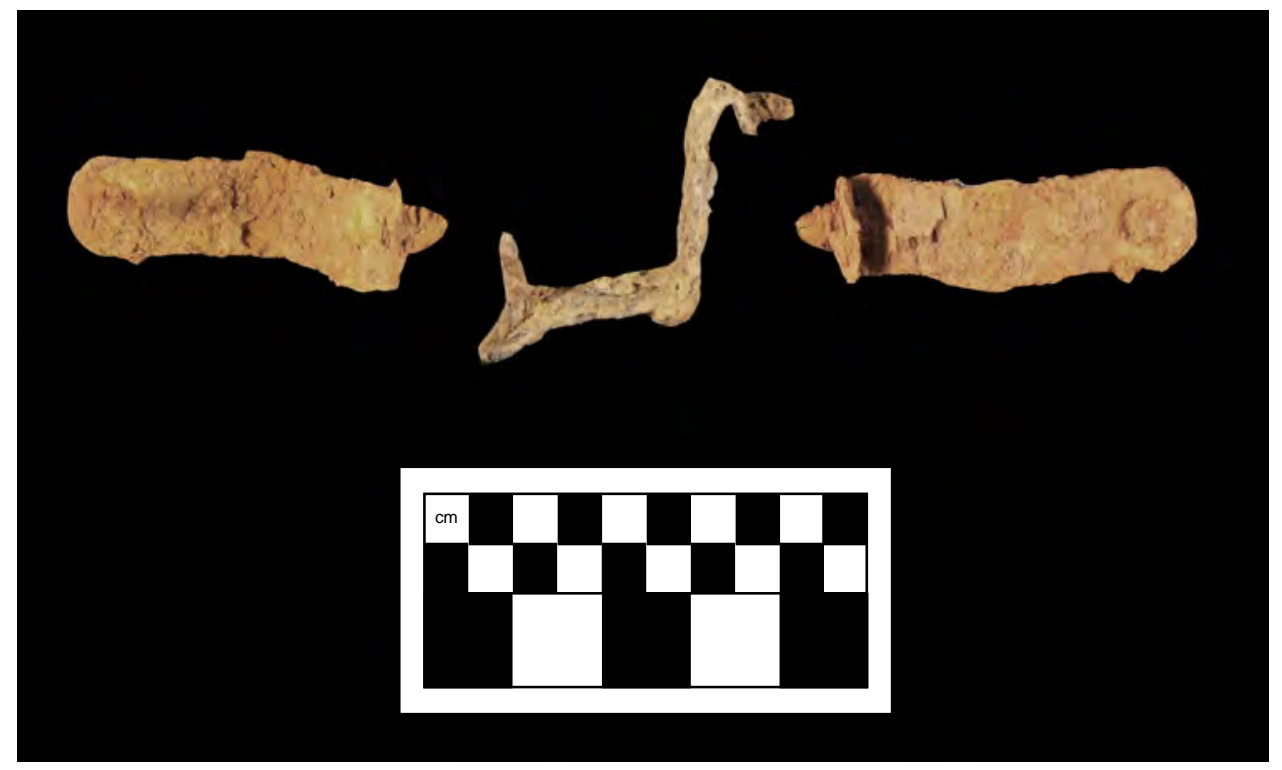

Figure 229. Miscellaneous Artifact Type 6-unidentified hardware.

Artifact Type 6; it has two screw holes at the lower end instead of just one, and its corners are squared instead of rounded. Finally, the idea of it being a glass stop is less likely because no glass viewing windows were recovered from New Home Cemetery burial excavations. 


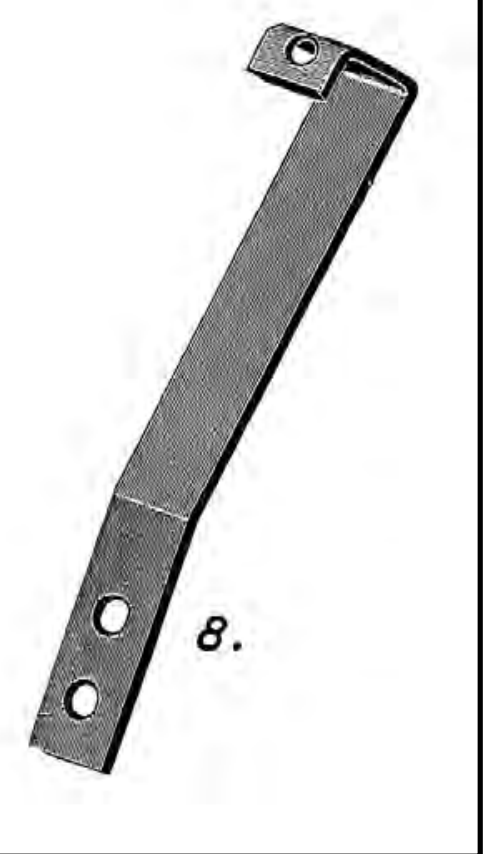

Figure 230. Glass Stop No. 8 illustrated on page 49 of the 1920s-1930s Langenau Manufacturing Company catalog.

\section{Miscellaneous Artifact Type 7}

Miscellaneous Artifact Type 7 (Figure 231) is represented by one item recovered from Burial 12 in New Home Cemetery (see Appendix A). It is a thin strip of ferrous metal, rounded and bent forward, and rounded at one end with a constricted neck and a flattened end with rounded corners at the other terminus. Two incised lines appear to parallel the sides. This piece was originally thought to be some sort of dowel, but it does not appear to have been affixed with any screws or nails. It is very similar in appearance to the end portion of the glass stop (also referred to as a Viewing Window Latch in Mainfort and Davidson 2006:165) depicted in Charles A. Conklin's 1891 U.S. Patent 456401; however, it does not appear to be broken. Additionally, the glass stop in the patent does not have incised lines. Finally, it is not likely to be a portion of a glass stop because no glass viewing windows were recovered from New Home Cemetery burial excavations.

\section{Miscellaneous Artifact Type 8}

Miscellaneous Artifact Type 8 (Figure 232) is represented by one dome-headed bolt with a square nut recovered from Burial 6 in New Home Cemetery (see Appendix A). This is a large 3-inch bolt with a $1 / 4$-inch-diameter shaft. This bolt is similar to one sold in the 1980 [1865] Russell \& Erwin Manufacturing Company catalog (Figure 233). Although this artifact was recovered with items from the burial, its exact context is unknown; therefore, it is not possible to determine its exact function. It could have been used in some capacity in the burial container or in the lowering device at the time of the funeral. It is also possible that it was an incidental inclusion. 


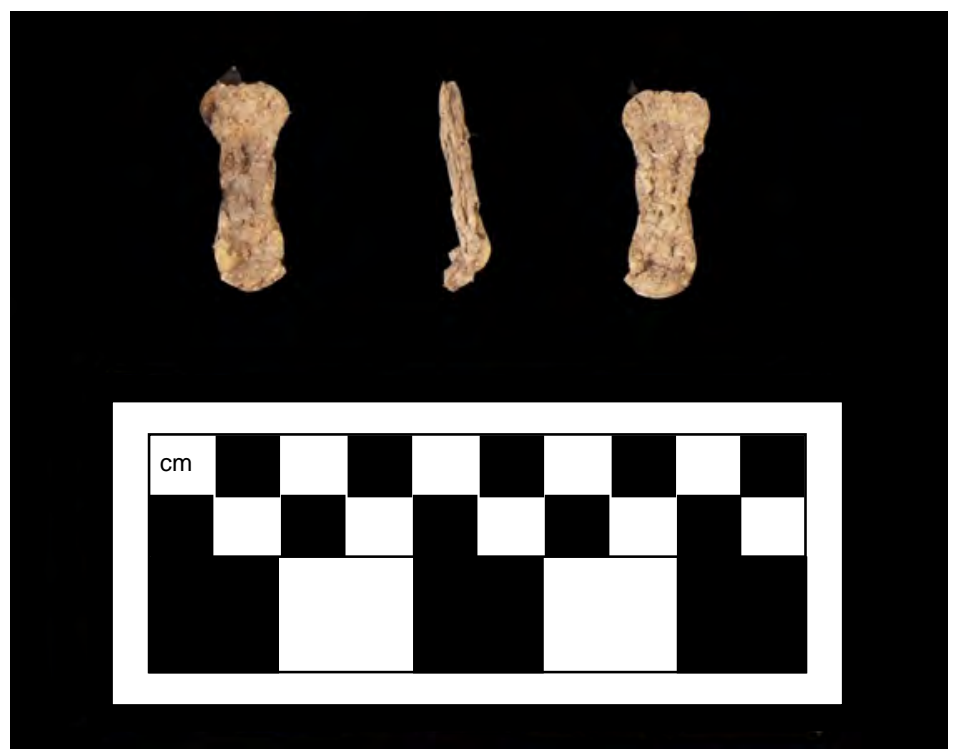

Figure 231. Miscellaneous Artifact Type 7—unknown ferrous artifact.

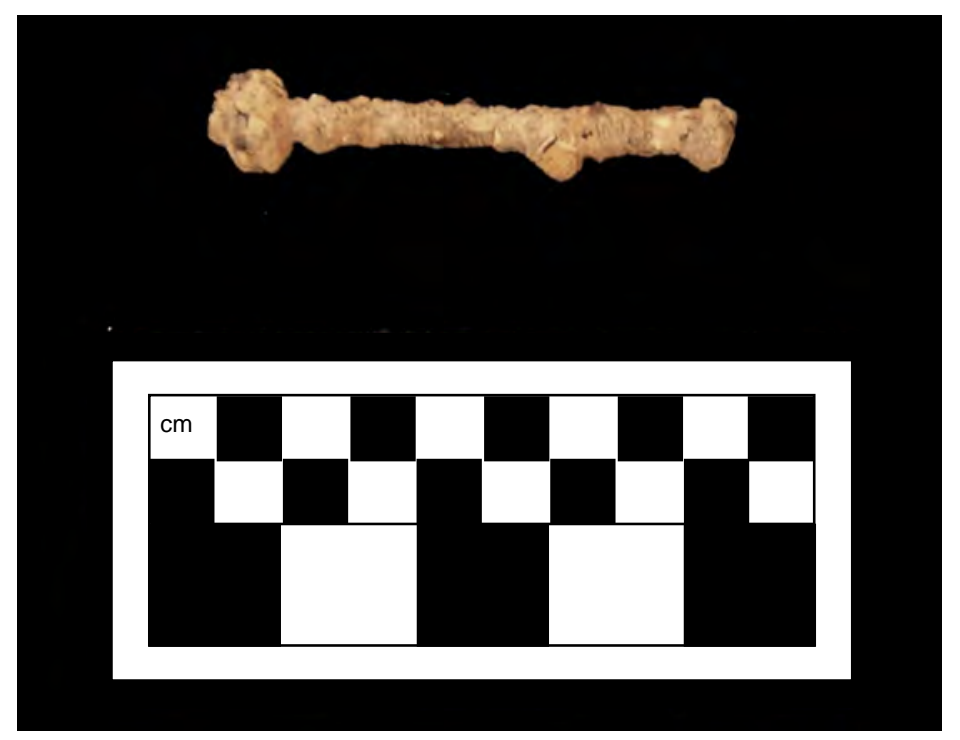

Figure 232. Miscellenaous Artifact Type 8-dome-headed bolt with square nut.

\section{Miscellaneous Artifact Type 9}

Miscellaneous Artifact Type 9 (Figure 234) is represented by one square-headed bolt recovered from surface collections in New Home Cemetery (see Appendix A). This is a large 5-inch bolt with a $1 \frac{1}{2}$-inch-wide head. Although it possibly was used in some capacity in a burial container or in the device used to lower a casket into a grave, it is more likely some type of carriage bolt or railroad bolt. Though it might not be directly associated with the burial container or devices employed at the funeral, it might have been intentionally placed within the burial because it held special meaning or significance to the family or community involved. Alternatively, its inclusion in the cemetery fill might have been incidental. 


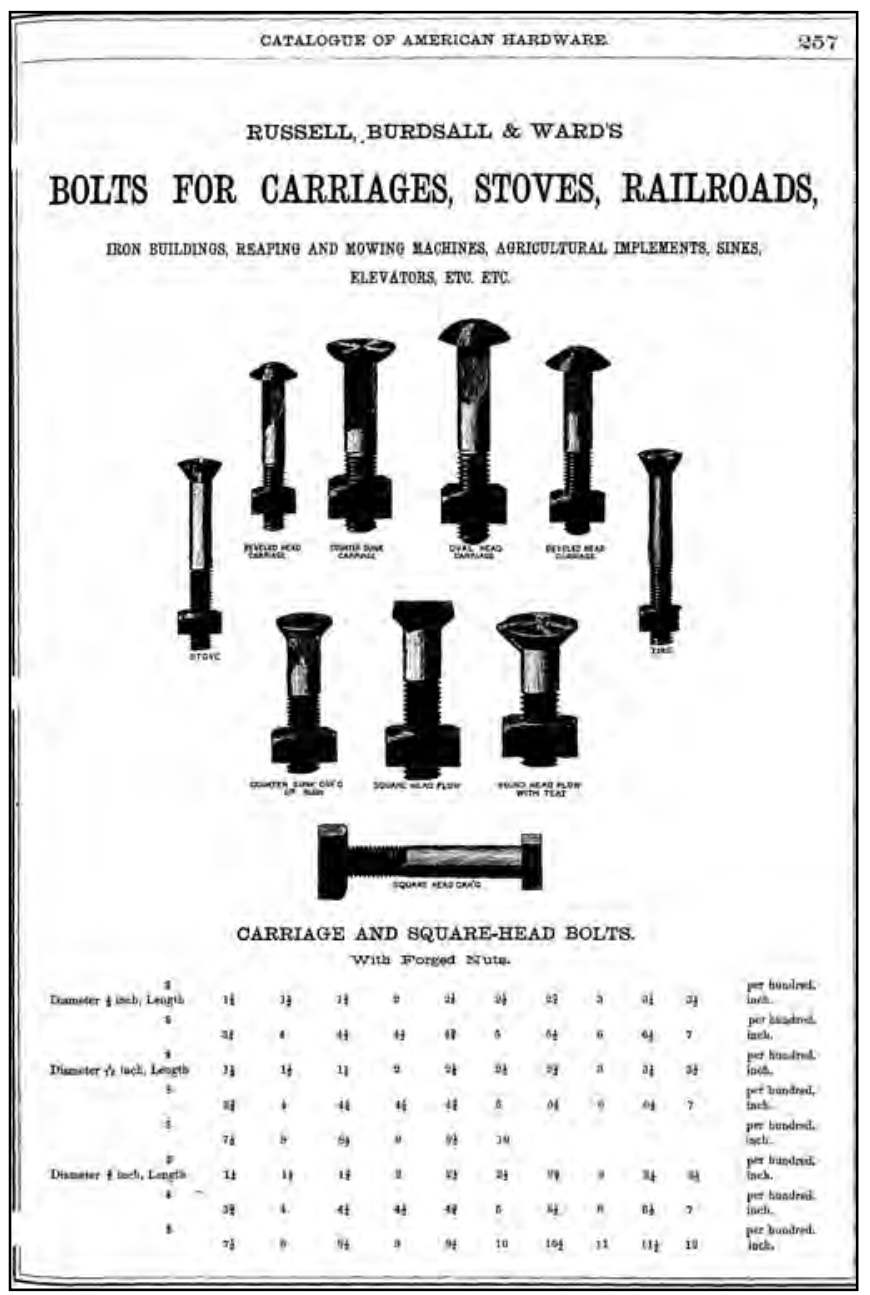

Figure 233. Assortment of bolts offered for sale on page 257 of the 1865 Russell \& Erwin Manufacturing Company general catalog.

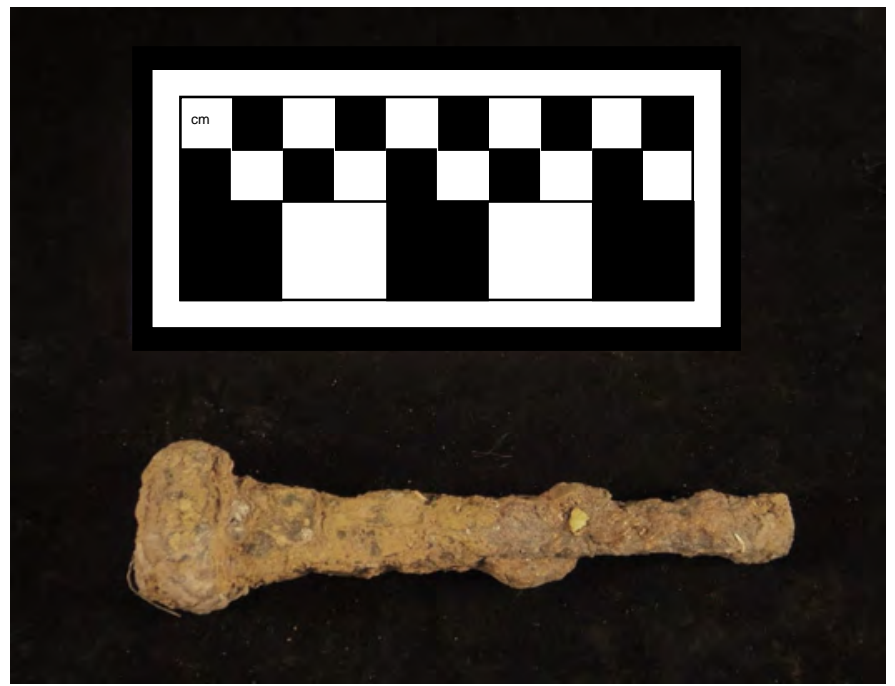

Figure 234. Miscellaneous Artifact Type 9—square-headed bolt. 


\section{Miscellaneous Artifact Type 10}

Miscellaneous Artifact Type 10 (Figure 235) consists of six strands of ferrous metal wire recovered from three burials (Burials 3, 7, and 9) as well as from the disturbed area at New Home Cemetery. Also included within this artifact type category are three segments of single strand, wide barb, barbed wire; and one segment of twisted double strand, narrow barb, barbed wire recovered from Burial 9 fill (see Appendix A). Archaeologists observed that it appeared that the barbed wire (along with amaryllis lily bulbs) was forming some type of boundary around the burial itself. Barring the possibility that barbed wire was included as a meaningful object associated with burial, barbed wire might find its way onto a cemetery site through incidental discard or loss. The plain wire can also be explained in this fashion, or it too might have served some mortuary function. Wire could have been used in floral arrangements to be held by the deceased or to be placed on the casket, as was the case in many of the burials recovered during excavations of the nineteenth-century Alameda-Stone Cemetery in downtown Tucson, Arizona (Heilen and Gray 2010).

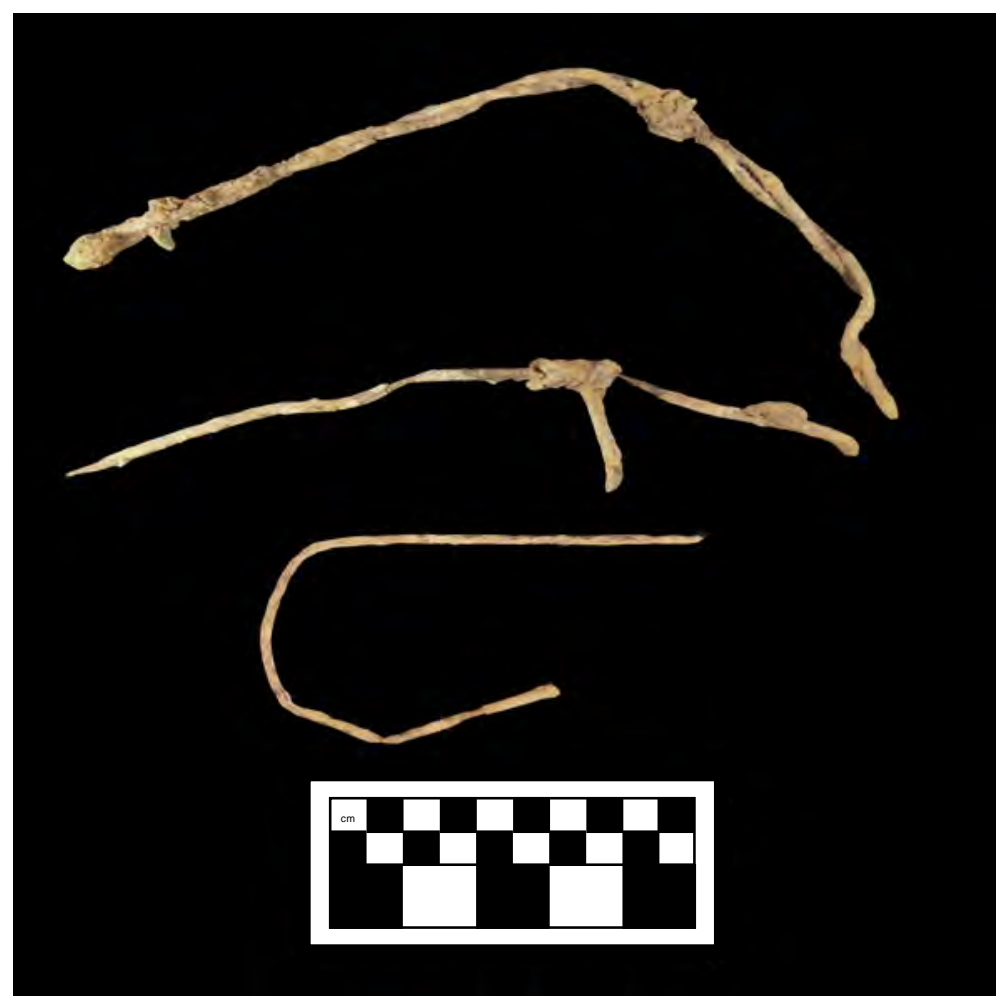

Figure 235. Miscellaneous Artifact Type 10-barbed wire and plain wire.

\section{PERSONAL ARTIFACT DESCRIPTIONS}

In addition to the mortuary hardware and embalming supplies recovered from the New Home Cemetery burials, limited numbers of personal items were found in association with the interments. These items would have been parts of the clothing in which the individuals were buried, pieces of jewelry that individuals were wearing at the time of burial, or other grooming or personal items included in the burial container prior to interment. 


\section{Clothing}

Although fabric did not survive in most of the burials, small remnants of clothing were recovered from Burials 5, 10, and 17 and Coffin 4, as well as an isolated item from the disturbed area.

Leah Simpson, Burial 5, retained some articles of clothing. A pair of nylon panty hose and a small metal lingerie adjuster were recovered from the burial. Leah Simpson was interred with these white or beige nylons, which consisted of a synthetic and elastic blend of cloth that formed the panty portion of the undergarment. The panty hose were torn during the excavation but still remained in good condition. No maker's tag could be identified on the garment. Leah Simpson was buried without shoes.

The individual from Burial 10 was interred in a man's suit. The burial clothing consisted of matching jacket and pants, a tie and tie tack, a button-up white dress shirt, a sleeveless undershirt, cotton underwear with an elastic waistband, and socks. No shoes were recovered from this burial. Much of the clothing was of synthetic material, thus allowing preservation. The jacket and pants were of a beige polyester blend, and the button-up white shirt and undershirt were also of a synthetic polyester blend, all well preserved. White plastic four-holed buttons were recovered from the dress shirt. The socks were of a black synthetic material and well preserved. The elastic waistband of the underwear was recovered; however, the cotton portion of the underwear contained no synthetic elements and was not preserved. The synthetic fiber blend tie was rusty orange in color with dark green symbols. The iron tie tack was highly corroded. Although considering the excellent preservation of the clothing, no maker's tags could be identified from the items.

Burial 17 had been previously removed by the funeral home in 2004 but some remains were left behind, along with a small fragment of clothing. A beige-colored nylon scrap of material was found covering the lower arm bones. The item of cloth was fragmentary, and no additional information could be gained from the item.

What little that was recovered from Coffin 4 included some articles of clothing that were left behind when the funeral home disinterred the remains in 2004. A man's suit jacket and button-up white dress shirt were recovered from the coffin (Figure 236). The jacket was a smoky blue polyester blend with a maker's tag that may read "Jerry Leonard.” A Jerry Leonard big and tall men's clothing store existed in the midwestern United States and especially in the Houston area from approximately the mid-twentieth century. However, the maker's tag is somewhat difficult to read and is only speculation. The button-up white dress shirt also consisted of a synthetic blend of fabric and was well preserved. However, no maker's tag could be identified on this item of clothing. Both items of clothing were covered with rust from the metal coffin in which the body was interred.

A lingerie strap was recovered as an isolated find in the disturbed area of the cemetery. The strap was of synthetic materials with a plastic-like strap adjuster. Since no burial could be associated with this fragment of clothing, no additional information could be gleaned. 


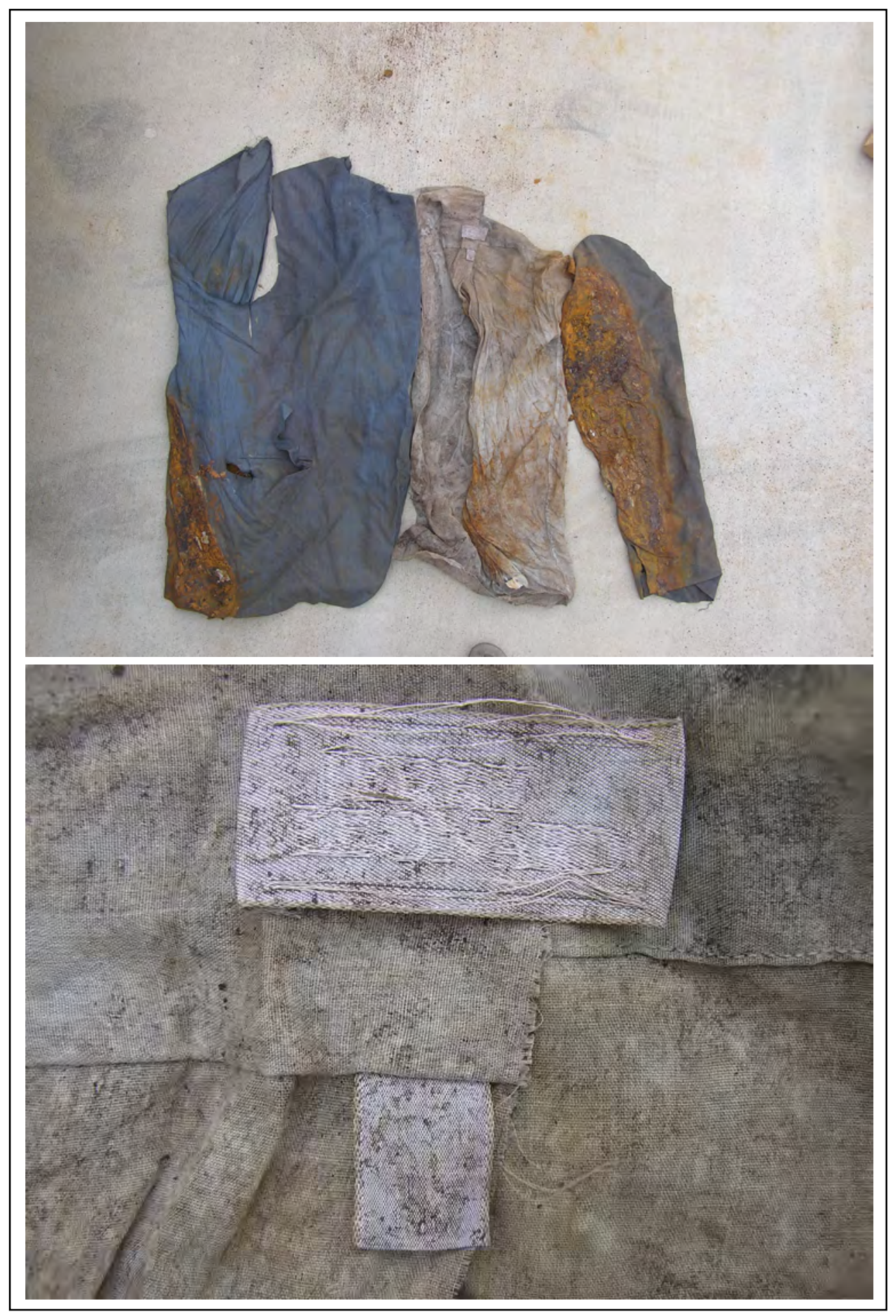

Figure 236. Coffin 4: (top) man's blue suit jacket (left) and suit sleeve (right) with white shirt (center); (bottom) close-up of maker's tag possibly reading "Jerry Leonard." 


\section{Buttons}

The majority of the buttons recovered from burials were shell buttons (Figures 237 and 238). The manufacture of shell buttons in the United States began in the 1850s, but it was not until 1891 that the shell button industry became established when John Boepple began producing buttons from shells found in the Mississippi River (Claasen 1994:1, 4, 80). Shell buttons were recovered from Burials 2, 6, 7, 14, and 18 (see Figures 18, 231, and 232). Both 2-hole and 4-hole sewthrough buttons were identified; most were very plain. Two 2-hole buttons found in Burial 18 (see Figure 238) contained a diamond-shaped inset in the center that contained the holes. A single 2-hole shell button and two solid shell buttons with rounded tops were found in Burial 14; these buttons were fastened to clothing by small iron wire shanks (see Figure 238). Sizes ranged from $1 \mathrm{~cm}$ to $2 \mathrm{~cm}$ in diameter, and all of the shell buttons recovered were in a fragile condition.

Other buttons recovered included composition, modern plastic, and cloth-covered buttons. Composition buttons are those composed of a mixture of substances (Hughes and Lester 1991:55; Luscomb 1992:46), but the term is sometimes used for early forms of plastic and rubber. Burials 4 and 17 contained a brown 4-hole sew-through button measuring 1.8 and $2 \mathrm{~cm}$. respectively. The button from Burial 4 (see Figure 18) is a polished plastic button that also had a soft "burnished" appearance on the front, whereas the button from Burial 17 (see Figure 238) appeared to be a hard rubber composite. A small (1.1-cm diameter), 4-hole white button with a deep inset was also found in this burial. Seven small 4-hole buttons were recovered from Burial 10. These buttons are plastic and have a high gloss, pearlized appearance. Two of these buttons measured $1 \mathrm{~cm}$ in diameter with the remaining measuring $1.2 \mathrm{~cm}$. Burial 12 contained a single white, glossy, plastic, 4-hole button with a very shallow inset measuring $1.2 \mathrm{~cm}$ in diameter. A single cloth-covered button was identified in Burial 2 (see Figure 237). The cloth is of synthetic manufacture and is a light color, perhaps off-white or beige, but is stained so the actual color is uncertain. The 3-cm-diameter button disc under the cloth appears to be of a noncorrosive metal. A yellowish, 2-hole plastic button was recovered from Burial 3. This button has a recessed "moat" between the edge and the center.

\section{Lingerie Parts}

A single metal lingerie slide was recovered from Burial 5 (see Figure 237). This double-slot slide or buckle is the type that is found on brassiere and slip straps and was introduced to modern lingerie in 1913 (Panati 1987:341). Also recovered from the overburden was a plastic lingerie slide still attached to the white or off-white fabric strap originally associated with it. This strap and slide likely came from one of the burials which had been previously disinterred by a local funeral home and became separated from the burial when removed.

\section{Safety Pin}

One safety pin was recovered from Burial 9 (see Figure 237). Only the head or shield and the pointed part of the pin shaft were identified. The shield appears to be composed of some cuprous material, and the shaft is corroded iron. This particular type was not identified. 


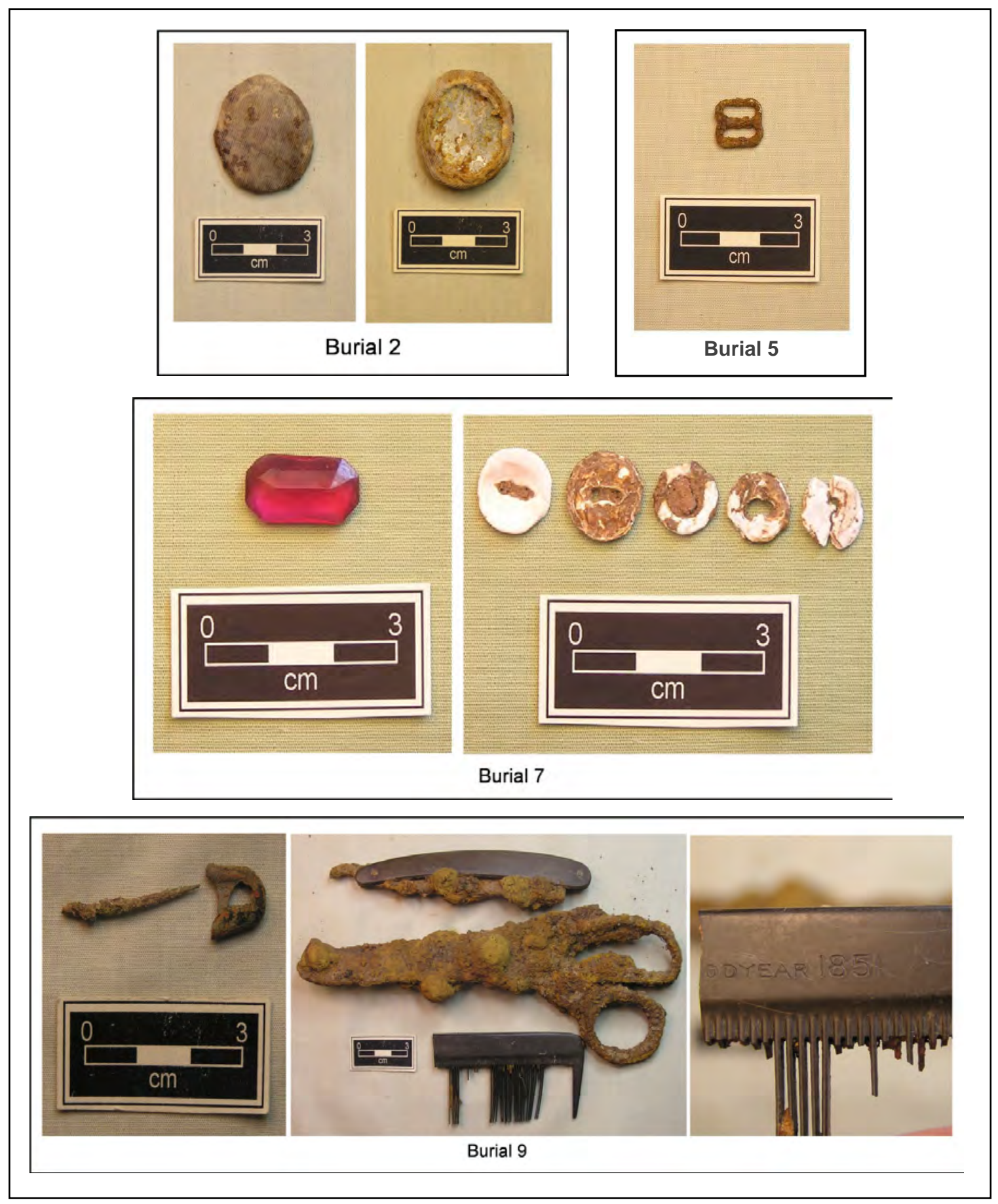

Figure 237. Personal items recovered from Burials 2, 5, 7, and 9 at New Home Cemetery. 


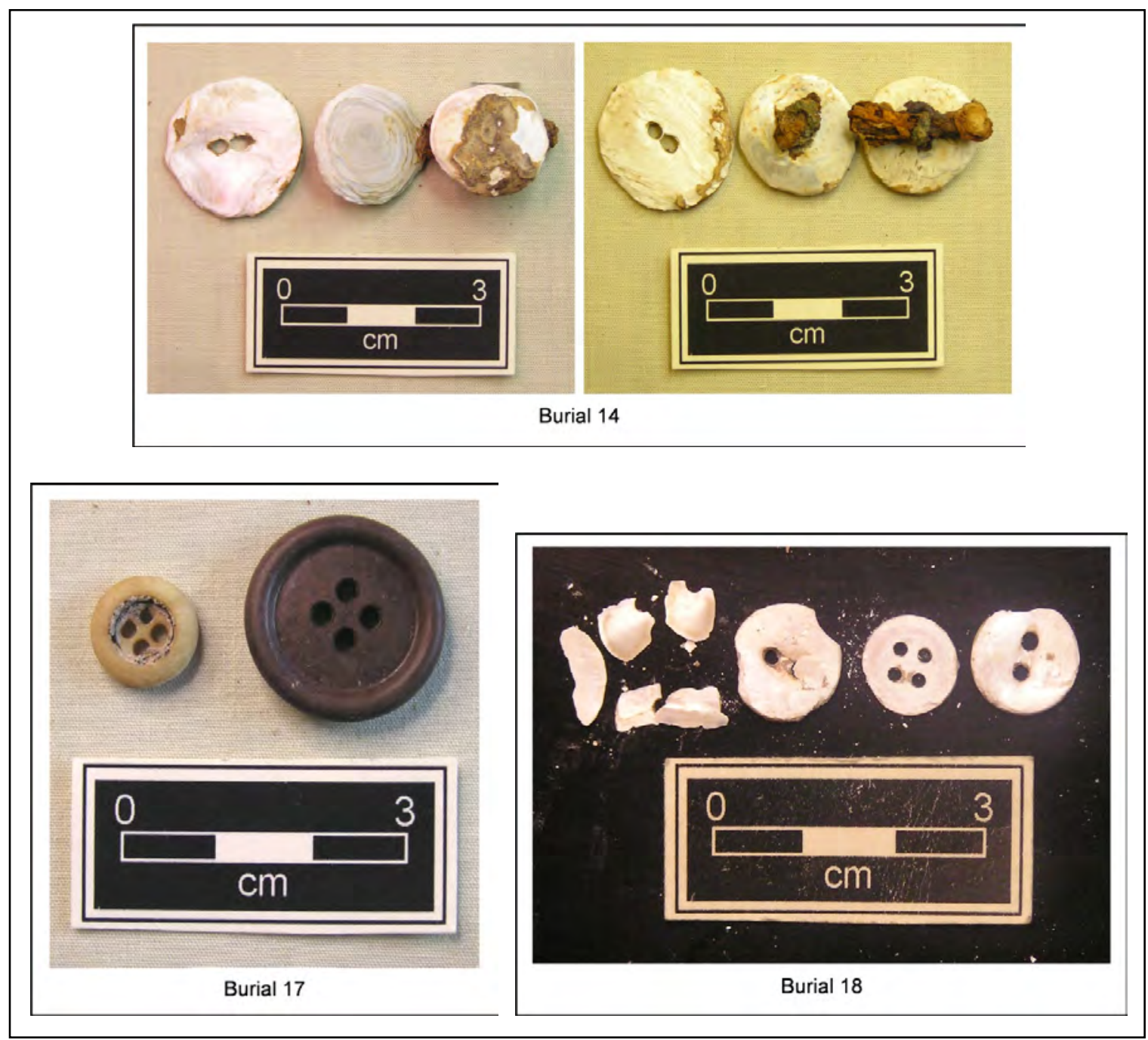

Figure 238. Personal items recovered from Burials 14, 17, and 18 at New Home Cemetery.

\section{Grooming Items}

Three specific grooming items were recovered from Burial 9: a straight razor, a pair of scissors, and a comb (see Figure 237). The blade of the straight razor is of iron and completely corroded shut within the black celluloid handle. The pins holding the handle and blade were made of brass or some cuprous metal. The scissors are also of iron and were completely rusted shut, though the finger grips, blades, and connecting screw were discernible. The black hard rubber comb fragment contains closely spaced teeth and a partial mark. The mark “. . . ODYEAR 1851" is probably "GOODYEAR" and "1851" likely a style or model number (see Figure 237: Burial 9, right frame). A small fragment of a black comb missing the teeth was also recovered from Burial 2 (see Figure 17). This comb appears to be of a hard rubber or a composite material. 


\section{Jewelry}

A red, faceted glass, "gem” was found in Burial 7 (see Figure 237). It measures $1.8 \mathrm{~cm}$ in length and $1 \mathrm{~cm}$ in width. This gem was found among hand bones and was likely set in a cuprous ring setting because the finger bones were green. 


\title{
CHAPTER 6 BIOARCHAEOLOGICAL INTERPRETATION
}

\author{
by Mary Cassandra Hill and Jeremy W. Pye
}

\section{AFRICAN AND AFRICAN-AMERICAN BURIAL PRACTICES AND THE SOUTHERN FOLK TRADITION}

... at last the grave, save for its rawness, resembled any other marked off without order about the barren plot by shards of pottery and broken bottles and old brick and other objects insignificant to sight but actually of a profound meaning and fatal to touch, which no white man could have read [Faulkner 1942:131-132]

Over the past five decades, extensive attention has been focused on aspects of African and African American belief systems and ritual behaviors. Deetz (1977), Jamieson (1995), Thompson (1984), and Vlach (1978) are only a few that bring attention to these studies. Deetz (1977:212, 251-252) referred to "africanisms" [sic] as survivals of collective existence material culture that, "will continue to be a major component of the work of historical archaeologists in the years to come. As it [African American archaeology] does, we will sharpen our approaches to the excavated material, to ensure that we recover the maximum degree of understanding” (Deetz 1977:251). Jamieson (1995) in particular emphasized that reducing the extensive variation seen in African mortuary customs to the same few primarily West African societies, over and over again in the overwhelming majority of reports, does great disservice to the rich mosaic of panAfrican culture. Therefore, it is up to individual scholars working in a region to conduct historical research in an attempt to understand point of origin from which Africans were brought, and therefore what types of cultural practices might be carried over and blended in this new context.

For the past 20 years or so, cultural geographers have been investigating the relationship between cultural geography and burial customs. Cultural geography is defined as a subfield within human geography and is "the study of cultural products and norms and their variations across and relations to spaces and places. It focuses on describing and analyzing the ways language, religion, economy, government and other cultural phenomena vary or remain constant, from one place to another and on explaining how humans function spatially" (Cultural Geography 2011; Jordan-Bychkov et al. 1994). 
Cultural geographer Gregory Jeane (1989) observed many items that he felt were common among rural, southern cemeteries for what he described as the Upland South cemetery type. These typically have the following defining criteria: locations on elevated areas such as hilltops; relatively small size; graves marked with local, natural stones, rather than commercial-grade tombstones; introduced vegetation such as cedar trees, palms, yucca, or magnolias; and a sense of "cultural piety" in which the community and/or families of the deceased who are interred maintain the upkeep of the cemeteries, rather than paying an outsider to cut the grass and trim the bushes.

In reviewing the work of cultural geographers Terry Jordan (1982) and Robert Thompson (1984) for his thesis research, Bruner (1998) stated that the Anglo-American model for southern folk cemeteries (see also Southern Folk Cemetery 2011) identifies four traits indicative of the AngloAmerican burial tradition in Texas:

1. Graves [are] marked with a tombstone, with the marker typically having the name and dates of birth and death. The marker also may have an indication of familial relations of the deceased. If iconography is displayed on the marker, it is associated with Anglo-American traditions.

2. Graves are aligned in an east/west direction with the feet of the interred individual to the east.

3. The wife of the deceased person will be buried on the left side of the husband (possibly reflecting the Christian scripture of Eve being created from a left rib of Adam).

4. Graves in Anglo-American cemeteries usually are arranged in fairly uniform columns or rows [Bruner 1998:2].

For African-American cemeteries, the model derived from the work of Thompson's (1984) study of African-American art forms, supplemented by Jordan's (1982) observations on cemeteries in Texas, has five identifying characteristics (Angelfire 2011; Bruner 1998):

1. The presence of surface offerings on the graves that have a variety of forms (objects that were last used by the deceased, favored objects of the deceased, lamps, pipes of all kinds, vehicle effigies, and seashells)

2. Bottle trees: glass shards and bottles hung from the branches of trees in and around the cemetery

3. Cemeteries that are located near waterways

4. Graves in family clusters spread throughout the cemetery, with the grave orientations not always being east/west; and

5. Trees or other plants intentionally seeded or transplanted on or beside the graves

Some of these grossly generalized practices of African-Americans are similar to those of AngloAmericans, and some are quite different. These differences are evident archaeologically, which is why historic cemeteries are extremely valuable for their contributions to population demography and studies of cultural heritage. Methods for the archaeological investigation of historic cemeteries vary due to several factors that include cemetery location, vegetation, soil compaction, and the possible presence of materials that may be a problem for public health concern (Kellan 1998), and are greatly influenced by budgetary and time constraints. Examination of undocumented cemetery samples literally enables the occupants to speak from the grave about their lives and social conditions for specific time periods. Because archaeology is a comparative science, it is useful to possess knowledge of the many other African American cemeteries that have unfortunately been disturbed through the years. Comparison allows the individual voices to contribute to dialogue. 


\section{Comparative Cemeteries}

When reviewing research on historic African diasporic cemetery studies, the most frequently cited works include New York African Burial Ground, Cedar Grove Cemetery in Arkansas, and Freedman's Cemetery in Dallas, Texas. Others that may be of comparative value to this study are included in the discussion that follows. Although only a selection of important projects will be highlighted here, Appendix E contains a listing of many other projects of interest to the historical mortuary archaeologist.

\section{New York African Burial Ground}

In 1991, while excavating the foundation for the construction of a federal courthouse in the Wall Street district of Manhattan, workers uncovered the remains of something that was believed to have been long destroyed by urban construction. Labeled on early maps of the colony of New Amsterdam as the "Negroes Burial Ground" [sic], the excavations eventually uncovered more than 400 human graves of what is one of the earliest and most extensive cemeteries of enslaved Africans and other people of color who survived the Middle Passage and worked in servitude in the construction of the city of New York. Historic documents indicate that as many as 10,000 to 20,000 individuals may have been interred from the late seventeenth through the eighteenth centuries in the five areas in lower Manhattan Island that had been appropriated for discriminatory exclusion from the general population of the colony. The cemetery was not restricted to Africans and other people of color. Anyone who was not a member of the Dutch Reformed Church, those who were prisoners or were convicted of crimes and were subsequently executed, Native Americans, and even Hessian mercenary soldiers were interred in the burial ground.

The archaeological excavations and subsequent laboratory analyses of human remains and artifacts took over 10 years to complete and were fraught with controversy. The reports of the project (one of the most extensive ever funded by the U.S. General Services Administration [2012]) are published on line and available to the public. The New York African Burial Ground project was completed by a multicultural research team at Howard University in Washington, D.C., and was featured in a series of four documentaries on A\&E television.

The history of the cemetery goes back to the early development of the colony when it was under Dutch rule. At that time, the Dutch West India Company, a provisional arm of the Netherlands government, was heavily involved with international colonial trade. The colony of New Amsterdam was dependent primarily on a labor force of indentured servitude. This was because the company was unsuccessful in attracting large numbers of Dutch colonists. The ones who came were entrepreneurs who were more interested in trading and making their fortunes than in permanent settlement. However, as the colony grew, the company became more and more involved in the Atlantic slave trade. Although the labor force was primarily enslaved Africans, people of color were brought to New Amsterdam from all parts of the world (for example, India, Asia, and New Guinea). When England took over the colony in the early 1700s, it was renamed New York. New York imported huge numbers of enslaved Africans. Men and women were put to work on the construction of the fort and other public works projects such as municipal roads and buildings, servicing ships at the docks, clearing tracts of land for settlement and cultivating crops, transport of goods from docks to warehouses and to markets, and from farms to markets where they assisted vendors on the streets. Women were employed as domestics in households 
and businesses, nursemaids, cooks, street vendors, laundresses, seamstresses, and agricultural workers. Men were used in heavy manual labor, particularly in construction, blacksmithing, and as stevedores in all aspects of general life. Children of all ages also were an integral part of the labor force.

Analyses of the remains of the individuals from New York African Burial Ground showed that their habitual activities of literally being worked to death left indelible marks on their skeletons. Extreme enthesopathy (pathology of the attachments of muscles to bones) was common. In addition, pathology of the spinal column and joints of the arms and legs indicated behavior associated with heavy lifting and carrying. This was expressed not only as arthritis, but also as fractures of the cranial base and elements of the spinal column. Subadult mortality was high (please refer to African Burial Ground 2011; Blakey 1998; Hill et al. 1995; Kellan 1998; Mack et al. 1995 for extensive discussions of the New York African Burial Ground project).

\section{Cedar Grove Cemetery}

Cedar Grove Cemetery, in southwest Arkansas, a rural cemetery associated with a church, dated from the late 1800s to the first two decades of the 1900s. Archaeological features and artifacts confirmed that the cemetery was in use during that time period. Eighty historic graves were excavated in 1980 as part of a salvage project in which the U.S. Army Corps of Engineers constructed a revetment on privately owned land. Historic and prehistoric graves were impacted by the project. Working under exceedingly limited time constraints, the project was among the first large-scale mitigations involving African-American remains. Reviews of historic documents and census records indicated that the people interred in the cemetery were emancipated plantation workers, tenant farmers, sharecroppers, and their descendants. The researchers utilized methods of analysis that would later become part of the repertoire for the Standards volume. Their results of the dental and skeletal analyses showed that the population sample from rural Arkansas was under considerable social and physiological stress (Rose 1985). Ninety percent of the individuals had evidence of at least one form of skeletal lesion. Nutritional stress and infectious disease were cited as the most critical environmental stressors. The report also reviewed socio-political issues relevant to the study, and documented African-American burial practices.

\section{Freedman's Cemetery}

Freedman's Cemetery, Dallas, Texas, is the largest African-derived historic cemetery sample that has so far been excavated in the United States. With 1,157 burials, it represents a monumental work in historic cemetery analyses, taking years of cooperative efforts on the parts of all involved. As reported by Peter et al. (2000), the cemetery was a municipal burial ground for the urban Black community for over 30 years (1869 to ca. 1907). It represents African Americans from all socioeconomic divisions. The First African Baptist Church cemetery in Philadelphia (Parrington and Roberts 1990) is a similar study sample, but from a northeastern U.S. location. Since it covers such a varied assemblage of individuals, it is difficult to make comparisons between urban and rural citizens. The volumes provide excellent reviews of mortuary customs and funerary hardware for the turn of the nineteenth century. The biological information supports census data for that time period as well. Relevant information concerning the health and quality of life for emancipated Africans during Post-Reconstruction Texas also has been discussed through examination of these individuals (Condon et al. 1998; Tiné 2000). 


\section{Phillip's Memorial Cemetery}

Phillip's Memorial Cemetery, Texas City, Texas, was a salvage project for the recovery of remains that had been left behind when human graves were disinterred and relocated in 1927. It was a rural African American cemetery in rural Galveston County. Fifty-three interments were excavated, resulting in the complete or partial remains of 43 individuals. The report indicates that disturbance other than disinterment activities (such as rodents) occurred. Rodent activity is a common occurrence for historic and prehistoric graves. The recovery was under very restricted time constraints, leading to prioritization of analyses that could be accomplished in a short period of time. The report (Dockall, Powell, and Steele 1996) reviews various aspects of the recovery process, funerary material culture, and biological analyses following the Standards (Buikstra and Ubelaker 1994).

\section{Pioneer Cemetery}

A portion of Pioneer Cemetery, Houston, Texas, was excavated in the process of making improvements to State Highway 332 in Brazoria County. It was a predominantly African American burial area from the late 1800s to the early 1900s, and archaeological investigations adjacent to the cemetery had shown that several unmarked graves were within the proposed ROW. Six potential graves were identified; three were disinterred, analyzed, and repatriated. Two were determined not to be within the ROW, and one was determined not to have been a burial. The report by Tiné and Boyd (2003) documented all of the activities of the project, including the excavation, analyses of the human remains and funerary artifacts, and repatriation ceremony. The remains were those of an infant, a younger woman, and an older woman. The report includes reviews of various biological indicators of stress for African Americans at the turn of the nineteenth century, as well as mortuary practices and funerary hardware. An additional 12 burials were impacted by subsequent road construction in 2008 and 2009. Based on hardware analysis conducted by Pye (2011), these burials likely dated between 1888 and 1900 .

\section{Third New City Cemetery}

Third New City Cemetery, Houston, Texas, was a predominantly African American cemetery that was in use from the late 1800s until approximately 1910. The remains of 355 individuals were recovered during excavations that were undertaken for the redevelopment of the Allen Parkway Village public housing project in downtown Houston (Foster and Nance 2002). Archival and historical research revealed that though the cemetery was predominantly African American, there possibly also were burials of patients from a hospital dating from 1850 that occupied the property prior to the location of the Third New City Cemetery there in 1880. As with Freedman's Cemetery, the individuals were from many various socioeconomic classes. Analyses of the remains reported that the population sample was relatively healthy. The report includes extensive documentation of the ca. World War II structures that were demolished as part of the revitalization of the area, along with the review of the skeletal biology information. 


\section{New Home Cemetery Burials in Context}

\section{Cemeteries Located in Groves of Trees}

It is not unusual to find African American cemeteries, much like New Home Cemetery, in natural groves of trees (Yalom 2008:115). Trees often were used in the custom of hanging bottles from limbs or placing bottles over the ends of branches. "Islands" of trees in an otherwise open area often are the only physical suggestion that there may have been a cemetery located in that spot (Bybee 2003b; Deetz 1977). Bottle trees are one of several traits attributed to African beliefs in spirits that reside at the cemetery (Southern Memorial Association 2003-2011; Thompson 1984). Dirt from the graves is often placed in the bottles (Southern Memorial Association 2003-2011). Similarly, cloth pouches containing coins and/or pierced coins were hung from the branches of trees.

\section{Grave Orientation}

All of the burials that were excavated by the Geo-Marine crew in New Home Cemetery were supine and oriented east/west, with the crania west, facing east. This is in complete agreement with the blending of southern folk cemeteries and African American folk cemeteries described by Jordan (1982), Vlach (1978), and Yalom (2008). Interestingly, however, the New Home Cemetery in its current configuration, with some areas oriented east/west in rows and other areas in small clusters in varied orientations, conforms more to the "Chaos" model that epitomizes African American graveyards (Jamieson 1995). However, even as early as colonial times, burials from New York African Burial Ground were in coffins oriented east/west with heads west. The other comparative cemeteries also had the same east/west orientation.

\section{Funerary Markers}

It is not uncommon for graves of African-Americans in the nineteenth and early twentieth centuries to be marked with mass-produced tombstones, home-made concrete markers, fieldstones, wooden markers, or seashells (Yalom 2008:115). In earlier times, markers in rural cemeteries were fashioned from materials, such as wood, that disintegrated over time (Jordan 1982; Yalom 2008). For most of the cemeteries used as archaeological comparatives, no formal aboveground markers were present. A fragment of slate, which may have been used as a marker, was recovered from Pioneer Cemetery (Tiné and Boyd 2003).

The grave markers at New Home Cemetery display an interesting array of ethnic/folk elements and contemporary, mass-produced headstones. These range from small crosses fashioned from white PVC pipe, to hand-shaped/hand-labeled markers, to modern, commercially engraved, gray granite headstones (Figure 239; see Figures 3, 5, 7, and 8). The markers that Geo-Marine recovered from the excavation area included fragments of slate that were possibly a personal offering or a slate tablet (possibly the woman buried in Burial 11 had been a teacher) used as a marker; several types of mass-produced temporary grave markers (all provided by a funeral home); and a fragment of cross-shaped white PVC pipe. These markers, such as the temporary marker shown in Figure 239, reflect more modest means of identification, suggesting limited resources for burial. Although there was no evidence of formal markers, there may have been some in the past. 


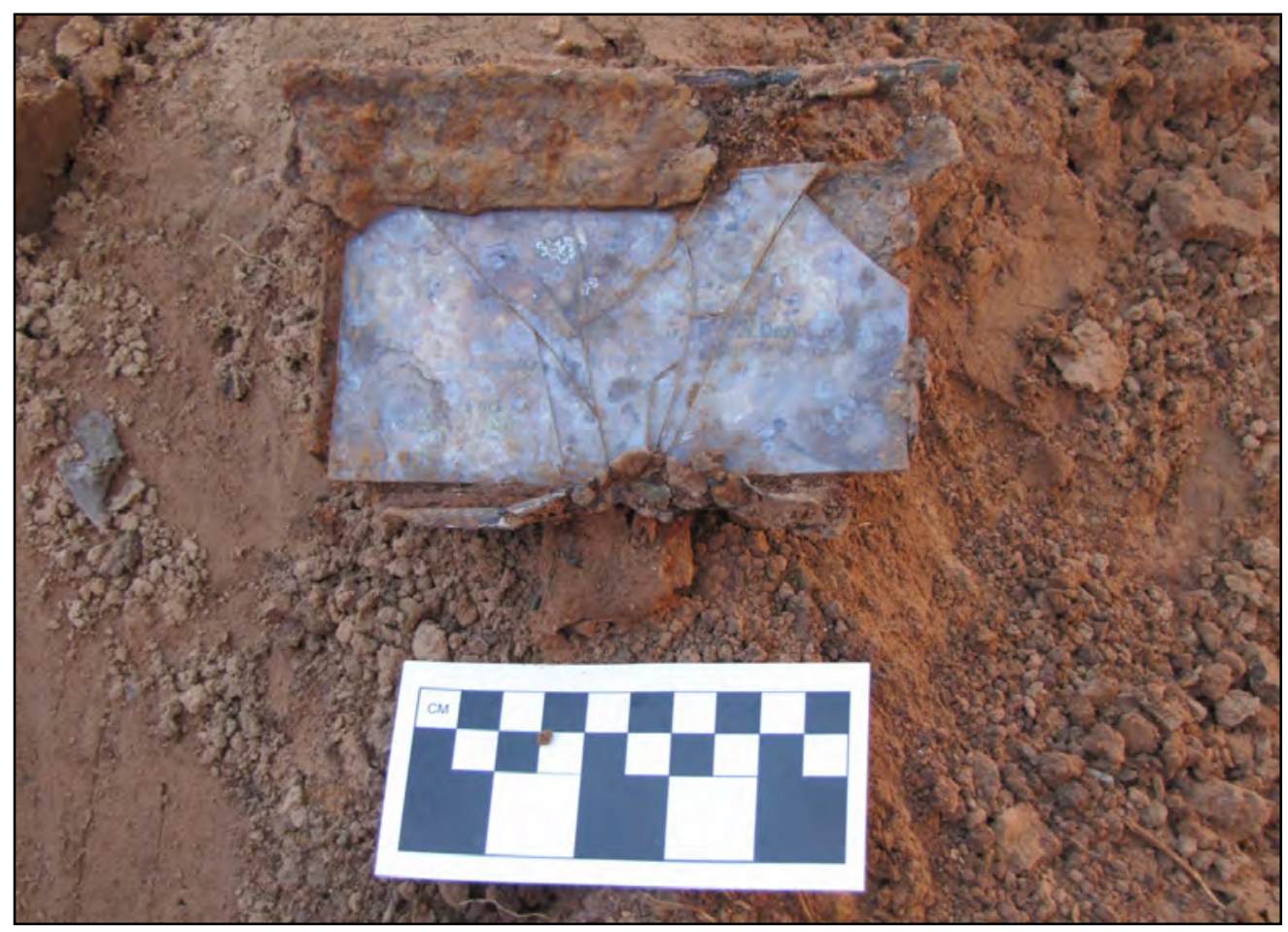

Figure 239. Temporary marker (Marker Type 1) associated with Burial 6.

\section{Surface Artifacts}

Many practices and beliefs are said to have been carried over from "traditional African" habits of decorating the grave. Coins, white shells and pebbles, white flowers, bottles and bottle trees, medicine bottles, mirrors (usually broken), blue glass, and metal items such as iron pipes, horseshoes, iron spikes, metal from automobiles, or iron bed frames are all found in African and African American cemetery contexts. Dishes, bottles, and jars were often broken in order to release their spirits and prevent the souls of the dead from returning in search of them. Medicine bottles inserted upside down on top of the grave allowed for the continuance of the medical regime followed during life. It was said that one could see ancestral spirits in the bright reflections of sunlight on mirror fragments and other shiny objects. White shells and pebbles represented the world of the dead, which is underwater. Bed frames symbolized a peaceful resting place in order to keep the soul from wandering. Iron pipes driven vertically into graves allowed the souls of the dead to communicate with the living. Coins scattered around the surfaces of cemeteries and placed into the hands and pockets of the deceased were the deceased person's contribution to the coffers of the community of the dead (Angelfire 2011; Heege 1998; History of African American Death 2011; Jeane 1989; Jordan 1982; Southern Memorial Association 2003-2011; Thompson 1984; Vlach 1978; and Yalom 2008). One should keep in mind though that these practices reflect a hodgepodge of unique African tribal practices creolized and even reinvented in the New World.

Artifacts recovered from the overburden at New Home Cemetery included the following: fragments of brick, whole bricks, fragments of concrete, segments of concrete curbing (also called coping), individual shells, a mason jar, fragments of broken glass, fragments of necks and bases of bottles, animal bones, ceramic tiles, fragments of ceramic plates and saucers, a coffee 
sieve, a roll of movie film, a fragment of a vinyl phonograph, wooden fencing slats, a horseshoe/muleshoe, fragments of slate, an Indian-head penny, and a large iron bolt that was similar to the ones from the metal coffins that also were recovered. Taken out of the context of an African-American Southern Folk Cemetery, these items might be classified as random debris, refuse, or even junk or garbage. Though some items might very well be unrelated to the cemetery because of heavy historical disturbance within the project area, assuming that all items are refuse would be hasty. As was previously discussed, some of these same types of artifacts were commonly used to decorate the surface of graves in African-American cemeteries (Jordan 1982; Vlach 1978; Yalom 2008).

A muleshoe, for example, was found turned upside down and driven into the ground at Pioneer Cemetery. Individual coins and/or pierced coins were reported at Cedar Grove Cemetery, Third New City Cemetery, Freedman's Cemetery, and Pioneer Cemetery; a penny was recovered from the general overburden at New Home Cemetery. Individual shells also were recovered at New Home Cemetery, Freedman's Cemetery, and Pioneer Cemetery. Burial 9 at New Home Cemetery had fragments of barbed wire and amaryllis lily bulbs along the sides of the casket, indicating that it had been spatially separated and lovingly maintained. Spatially distinct graves are more a phenomenon of the Southern Folk Cemetery tradition (Deetz 1977; Jeane 1989; Jordan 1982; Yalom 2008). Large fragments of concrete curbing also were recovered at New Home Cemetery from the overburden near Coffin 1 (Figure 240). Curbing (sometimes called "coping” by commercial funeral homes) is used to delineate personal space either for individuals or for family plots within cemeteries, and is used sometimes in African-American cemeteries (Jordan 1982; Yalom 2008), although such materials are not present in the comparative samples mentioned above.

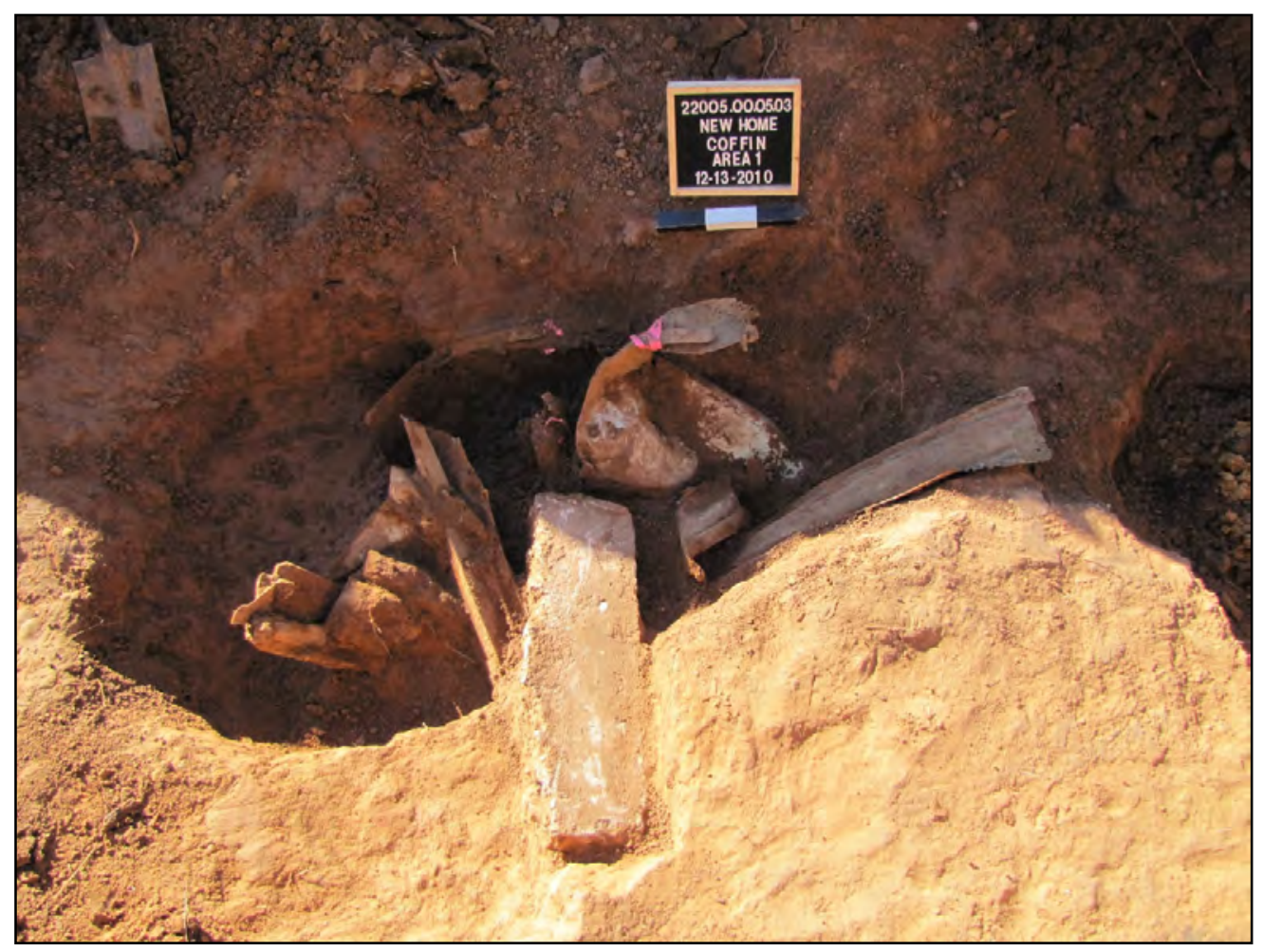

Figure 240. Mangled casket (Coffin 1) and fragment of concrete curbing. 


\section{Burial Containers and Hardware}

It can be argued that the most important event of a funeral is the viewing of the remains. During slavery, African-Americans held wakes that lasted throughout the night on the day of death, prior to burial the following day, in order to keep a vigil over the deceased (Ellis 1914). This practice was made even more popular after the development of chemical arterial embalming during the Civil War (Habenstein and Lamers 1955, 1960). The focus of the viewing was not just the body, however, but rather the burial container and its adornments.

The New Home Cemetery collection contained mostly simple wooden burial containers, which did not survive in the archaeological record. Therefore it is not possible to make many assumptions or observations about their aesthetic quality or craftsmanship. A sizeable collection of mortuary hardware was recovered, including 28 handle types, two thumbscrew types, two thumbscrew escutcheon types, four casket plaques, 28 types of complex internal casket hardware (including multiple types of hinges, catches, top fasteners, and escutcheons), as well as other construction hardware such as cut and wire nails, lining tacks, slotted and Phillips head gimlet screws, staples, corrugated fasteners, and joining plates. Cloth adhering to the back of a plaque from Burial 18 suggests that the casket had been cloth-covered. Lastly, at least one type of metal casket was also recovered, which would have made quite an impression on the funeral scene compared with the wooden caskets used in other burials.

A comparison to other archaeologically recovered cemetery samples mentioned above is difficult because most of the burials at New Home Cemetery are not contemporaneous. The analyzed artifacts suggest that the 24 interments at New Home most likely occurred between 1895 and the 1960s. Burials in the New York African Burial Ground, for example, took place 100 to 200 years earlier. For comparative cemeteries that do overlap with the New Home timeline, similar types of hardware do appear. New Home Cemetery is not unique in regard to artifact types.

\section{Personal Items}

Personal items that are buried with an individual indicate great sensitivity on the part of those participating in the burial ceremony. The items not only reflect their importance to the deceased, but they frequently reflect belief systems as well, such as a belief in the afterlife in which those items will continue to be needed by the deceased. Additionally, by placing special items with the deceased, the soul will be at rest and will not wander in search of those items (Jordan 1982; Thompson 1984; Vlach 1978; Yalom 2008). Personal items were not commonly included in New Home interments. One individual had professional-grade scissors, a straight razor, and comb, perhaps an indication of aspiring to be a barber or that he just took great pride in his apperance. Another individual had a ring on the left hand, and Leah Simpson (Burial 5) had a brass stain on the side of her cranium that may have been an ornamental hair comb and brass stains that may have been earrings. All of the comparative cemeteries reported a similar paucity of personal items included in the burial containers.

\section{Burial Clothing}

Studies of colonial-period African-American cemeteries, such as New York African Burial Ground, revealed that it was a common practice to wrap the corpse in plain cotton or linen fabric and use shroud pins to secure the wrappings. Tiné and Boyd (2003:32) state that the buttons from 
Pioneer Cemetery probably represented closures for burial gowns or robes for the individuals, which were common in the nineteenth century. In the twentieth century, more and more people were buried in fine suits or gowns that were worn in life rather than in special burial garments. Everyday forms of buttons, hook and eye closures, and even snaps or zippers would therefore be expected. To date, no one except Davidson (2006) has made a systematic attempt to use period garment catalogs in an effort to describe and effectively "reconstruct" clothing from excavated burials.

The severe time schedule for data recovery at New Home Cemetery did not allow for extensive analysis and description of burial garments, nor did it allow for screening of burial fill. The recovery of very small items, such as hook and eye closures, would have been exceedingly difficult. However, very small buttons were recovered from several individuals, as well as a small lingerie buckle and satin lingerie strap with buckle (Burial 5). Archaeologists recovered complete, nearly complete, or fragmentary shirts, pants, jackets, undershirts, socks, stockings, and a tie from Burials 5 and 10, as well as Coffin 4. With the exception of the stockings, all of the items mentioned are indicative of the types of clothing that were likely worn by the men in Sugar Land.

The following items were conspicuous in their absence at New Home Cemetery: shoes, belts/buckles, and buckles for suspenders. Even if the shoe leather had deteriorated, there was no evidence of shoe buttons, hooks, or eyelets (for laces). Shoes are not part of the burial protocol for African Americans, although a single shoe sometimes was placed on top of the casket (Jamieson 1995; Parler 1962; Puckett 1926; Vlach 1978). There was no evidence of individuals being buried wearing shoes at Phillips Memorial Cemetery (Dockall et al. 1996:21), although shoes have been recovered in many other cemeteries. It is possible that individuals were buried wearing no shoes, but they could also have been wearing special burial slippers, which were sold in numerous supply catalogs of the period and would have been supplied by the funeral home.

\section{Chronology of New Home Cemetery Burials}

The 24 burials recovered from the largely twentieth-century New Home Cemetery revealed a sizeable sample of mortuary artifacts, which have been described in detail in Chapter 5 of this report (see also Appendix A). A brief or more detailed history of the many artifact forms has also been provided when possible, but assigning dates to unmarked burials involves a synthesis of the available information and a proper interpretation. Davidson (2006:203) notes, "assigning dates of interment to unmarked burials is not like estimating a date range for an occupational midden or a structure," because burials are discrete features associated with events that often took place over the course of several hours. Barring postdepositional disturbance, the contents of graves have remained sealed since interment until recovery by the archaeologist (Rowe 1962). They therefore have great potential to provide a reliable chronological range.

The first step in establishing this chronology is determining the terminus post quem (TPQ), or the "limit after which," that refers to the date of introduction of a certain product. This can be tracked either through the identification of patent records or less reliably through a survey of a representative collection of merchandise catalogs. Because burials are discrete events, the TPQ would be the earliest production date of the most recent artifact within the burial (Davidson 2006:203). 
Establishing a terminus ante quem (TAQ), or "limit before which," is a much more difficult and less precise endeavor (Deetz 1996; Mainfort and Davidson 2006:203; Noël-Hume 1991:11). TAQs can often be based on knowledge about the decline in production or popularity of certain artifacts, a marked absence of certain artifact types in catalogs of companies that had previously carried the items, and historical information about the use-life of a given cemetery. Though burials sometimes can take place after an official closing of a cemetery, the historical information about the cemetery use-life tends to provide the most reliable TAQ for burials that cannot be more tightly dated.

There are a number of interrelated explanations why artifact production dates are sometimes not reliable indicators of TAQs. Forms of outmoded hardware could have been used for longer periods of time because they would have been available through local general stores or undertakers who had accumulated a back-stock of merchandise over the years they were in business. This idea was proposed by Hacker-Norton and Trinkley (1984) in their study of the A. L. Calhoun collection, which consisted of late nineteenth-century mortuary hardware that had been curated in a rural South Carolina general store and would have potentially been available for sale as late as the 1920s. Similarly, various types of clothing, buttons, glassware, tableware, and other personal objects could have been curated by families long after their introduction and decline in market popularity.

It is very reasonable to assume that rural shopkeepers and undertakers would have accumulated back-stocks, but it is also possible that these individuals were purchasing already outmoded merchandise from regional catalogs, traveling company salesmen, jobbers, or general hardware catalogs that continued to carry earlier forms of hardware, like coffin screws and tacks, well into the twentieth century (Davidson 2006:141; Pye 2011; Tiné and Boyd 2003:31). General hardware catalogs tended to sell very limited numbers of styles and types of products, often restricted to only a few pages as compared to the mainstream hardware catalogs that could contain upward of 200 pages of hardware. The observation that hardware might have been obtained through second-hand or wholesale distributors is significant because general hardware or general merchandise catalogs were crucial links to the national, capitalist, consumer market in rural areas of the United States.

Table 2 presents an interpretation of the burial chronologies of the 24 burials recovered during excavations at New Home Cemetery. The rows list the individual burial features, and the columns represent the various selected types of mortuary artifacts from the New Home collection, as well as the TPQs and TAQs of said artifacts when that information was known. There is a relatively even distribution of TPQ dates from 1895 through the 1960s. Although some burials could be more tightly dated by the addition of a TAQ based on the presence or absence of certain artifacts in merchandise catalogs, the continued use of some type of products in the modern funeral industry complicated the task for the majority of these burials.

Some perspective on the possible end of interments in the disturbed portion of the cemetery comes from the fact that on July 17, 1950, a deed passed between the New Home Mission Baptist Church and the state of Texas granting a portion of the church's original land holdings for the proposed building of FM 1464. The recovered materials, particularly a temporary marker dated 1954 found in association with Burial 5, plastic embalming fluid bottles in Coffin 4, and embalming paraphernalia in Burial 10 suggest that all interments did not cease. Using 1950 as an end date for this portion of the cemetery is therefore not reliable. The presence of five metallic caskets (Coffins 1, 2, 3, 4, and Burial 4) reflect the shift toward this type of product as a funeral 
industry standard, which intensified in the 1950s. Coffins 3, 4, and Burial 10 each contain artifacts that would have been introduced in the 1960s. These burials likely are no younger than the 1960s because later in the twentieth century, more and more cemeteries throughout the country began to require concrete or metal vaults for burial. No such vaults were reported in New Home Cemetery, though there were indications that inclusive wooden vaults (e.g., shipping boxes or outer boxes) were used in at least five burials (Burials 2, 13, 16, as well as Coffins 1 and 2).

Figure 241 shows a visual interpretation of sequence of burials in the impacted area of New Home Cemetery. In this figure burials have been roughly assigned temporal categories: 18951920, 1920-1940, 1940-1960, and after 1960. It appears that the earliest burials (Burials 1, 2, 7, 8, 9, and 16), dating between 1895 and 1920 occur in the western row in the southern portion of the project area. Three burials (Burials 13, 18, and 19) in this row date to the second temporal period (1920-1940). All burials in the eastern row of the southern portion of the project area appear to date significantly later (1940-1960: Burial 3, Coffins 1 and 2; post-1960: Coffins 3 and 4) suggesting that perhaps the boundary of the cemetery was perhaps pushed between these periods of use due to the original laying of FM 1464. The cluster of burials in the middle of the project area is less distinct and ordered and date between the 1920s (Burials 6, 14, 15, 17; Burial 12 and Coffin 5), and the late 1960s (Burial 10). Burial 12, which dates between 1940 and 1950, was found partially atop Burial 15, which therefore aids in the limiting of the temporal estimate for the interment of Burial 15. Burial 10, which appears to be the latest burial impacted, was found under the current fence line of New Home Cemetery suggesting the fence was placed more recently. Finally, the northernmost cluster of burials (Burials 4, 5, and 11) date as a group between the late 1930s and the early 1950s.

\section{Quality of Life: Health and Socioeconomics at New Home Cemetery}

The human skeleton is dynamic throughout the life course, continually growing and developing, forming and remodeling bone tissue, as well as developing and wearing down teeth. Some of the processes that control these dynamic qualities of the skeleton are coded in biology, but many can be affected by circumstances of life, such as health and lifestyle (narrowly defined by Larsen and Walker [2010] as activity and workload). In their analysis of a skeletal sample from Dickson Mounds, Goodman and Armelagos (1989) suggest that the environment acts as a baseline for all factors affecting health, whereas cultural adaptations serve as a buffer. Culture, however, often produces its own set of stressors, often related to urbanization, warfare, poor education, poor sanitation, overcrowding, limited access to resources, etc. (Goodman et al. 1988). In other words, health and evidence of skeletal stress are largely a reflection of factors related to socioeconomics and overall quality of life (Davidson et al. 2002).

\section{Demography and Skeletal Analysis}

Demography of historic cemetery samples is a primary source of controversy. In order to even consider constructing life tables and mortality tables, a researcher has to assume that the population was stable and that the sample is a valid representation of the entire population. The most serious criticism of cemetery demography tables (morbidity and mortality) is that they use longitudinal data (individuals who died over an extended period of time) to represent crosssectional data (all individuals who died at a single moment in time) (Bocquet-Appel and Masset 1982, 1996). Milner et al. (2000:467) state that though this is a valid concern, the critiques have 
Table 2

Burial Chronology Based on Mortuary Artifacts

\begin{tabular}{|c|c|c|c|c|c|c|c|c|c|c|c|c|c|c|}
\hline Feature & $\begin{array}{l}\text { Marker } \\
\text { Type }\end{array}$ & $\begin{array}{l}\text { Plaque } \\
\text { Type }\end{array}$ & Handle Type & $\begin{array}{l}\text { Thumbscrew } \\
\text { Type }\end{array}$ & $\begin{array}{l}\text { Thumbscrew } \\
\text { Escutcheon } \\
\text { Type }\end{array}$ & $\begin{array}{l}\text { Top } \\
\text { Fastener } \\
\text { Type } \\
\end{array}$ & Catch Type & Butt Hinge Type & $\begin{array}{l}\text { Stop Hinge } \\
\text { Type }\end{array}$ & Screw Type & Nail Type & $\begin{array}{l}\text { Other Diagnostic Artifacts } \\
\text { or Notes }\end{array}$ & $\begin{array}{l}\text { Burial Container } \\
\text { Type }\end{array}$ & $\begin{array}{l}\text { Interment Date } \\
\text { (Range) }\end{array}$ \\
\hline Coffin 1 & - & - & 20 (1940-?) & - & - & - & - & - & - & Phillips (1936-present) & Wire (1895-present) & - & Metallic casket & $>1940$ \\
\hline Burial 1 & - & - & - & - & - & - & - & - & - & - & Wire (1895-present) & - & Casket & $>1895$ \\
\hline Coffin 2 & - & - & $\begin{array}{l}1 \text { (ca. 1910- } \\
\text { present); } \\
22(1940-?)\end{array}$ & - & - & - & - & - & - & $\begin{array}{l}\text { Slotted or unknown } \\
\text { (1846-present) }\end{array}$ & Wire (1895-present) & - & Casket & $>1940$ \\
\hline Burial 2 & - & $4(?)$ & 6 (1910-1940s) & - & - & 1 (1889-?) & 2 (1889-?) & - & - & $\begin{array}{l}\text { Slotted or unknown } \\
\text { (1846-present) }\end{array}$ & Wire (1895-present) & - & Casket & 1910-1940s \\
\hline Coffin 3 & - & - & 23 (1940-?) & - & - & - & - & - & - & - & Wire (1895-present) & $\begin{array}{l}\text { Support 1 (1920s-?); } \\
\text { buried at the same time as } \\
\text { coffin } 4\end{array}$ & Metallic casket & $>1960$ \\
\hline Burial 3 & - & - & $26(1900-?)$ & - & - & - & $\begin{array}{l}3 \text { (1889-?); } \\
5 \text { (1950-?) }\end{array}$ & - & - & Phillips (1936-present) & Wire (1895-present) & Support 1 (1920s-?) & Casket & $>1950$ \\
\hline Coffin 4 & - & - & $23(1940-?$ & - & - & - & - & - & - & - & Wire (1895-present) & $\begin{array}{l}\text { Support } 1 \text { (1920s-?); } \\
\text { embalming chemicals } \\
\text { (1960s-present); buried at } \\
\text { same time as coffin } 3\end{array}$ & Metallic casket & $>1960$ \\
\hline Burial 4 & - & - & $21(1940-?)$ & - & - & - & - & 1 (1700-present) & - & $\begin{array}{l}\text { Slotted or unknown } \\
\text { (1846-present) }\end{array}$ & Wire (1895-present) & & Metallic casket & $>1940$ \\
\hline Coffin 5 & - & - & $\begin{array}{l}3 \text { (1899-?); } \\
15 \text { (1920-?) }\end{array}$ & - & - & - & $\begin{array}{l}4 \text { (1936-?); } \\
6 \text { (1883-?) }\end{array}$ & - & - & $\begin{array}{l}\text { Slotted or unknown } \\
\text { (1846-present) }\end{array}$ & Wire (1895-present) & Support 2 (1865-present) & Casket & $>1936$ \\
\hline Burial 5 & $\begin{array}{l}3(1954 \text { ID } \\
\text { tag) }\end{array}$ & - & 7 (1920-?) & - & - & - & - & - & $6(1888-?)$ & $\begin{array}{l}\text { Slotted or unknown } \\
\text { (1846-present) }\end{array}$ & Wire (1895-present) & Support 2 (1865-present) & Casket & 1954 \\
\hline Burial 6 & $\begin{array}{r}1 \text { (1926- } \\
\text { present) }\end{array}$ & - & - & - & - & - & - & - & - & $\begin{array}{l}\text { Slotted or unknown } \\
\text { (1846-present) }\end{array}$ & Wire (1895-present) & $\begin{array}{l}\text { Support } 2 \text { (1865-present; } \\
\text { corrugated fasteners } \\
\text { (1890-present) }\end{array}$ & Casket & $>1926$ \\
\hline Burial 7 & - & - & $4(1890-1905)$ & $\begin{array}{l}2(1875- \\
1920)\end{array}$ & $\begin{array}{c}2(1875- \\
1920)\end{array}$ & - & - & - & - & $\begin{array}{l}\text { Slotted or unknown } \\
\text { (1846-present) }\end{array}$ & Wire (1895-present) & $\begin{array}{l}\text { Corrugated fasteners } \\
\text { (1890-present) }\end{array}$ & Casket & 1895-1905 \\
\hline Burial 8 & - & - & 25 (1900-?) & - & - & - & - & - & 2 (1888-?) & $\begin{array}{l}\text { Slotted or unknown } \\
\text { (1846-present) }\end{array}$ & Wire (1895-present) & - & Casket & $>1900$ \\
\hline Burial 9 & - & - & $\begin{array}{l}10 \text { (1890-?); } \\
17 \text { (1920-?) }\end{array}$ & - & - & 1 (1889-?) & 6 (1883-?) & - & - & $\begin{array}{l}\text { Slotted or unknown } \\
\text { (1846-present) }\end{array}$ & Wire (1895-present) & - & Casket & $>1895$ \\
\hline Burial 10 & - & - & 17 (1920-?) & - & - & - & - & 1 (1700-present) & - & $\begin{array}{l}\text { Slotted or unknown } \\
\text { (1846-present) }\end{array}$ & Wire (1895-present) & $\begin{array}{l}\text { Trocar button (1948- } \\
\text { present); morgan's eye } \\
\text { cap (1911-present); } \\
\text { Natural expression mouth } \\
\text { former (1963-present) }\end{array}$ & Casket & $>1965$ \\
\hline Burial 11 & $\begin{array}{l}1(1926- \\
\text { present } \\
4(1926- \\
\text { present) }\end{array}$ & - & $11(1920-?)$ & - & - & - & - & 1 (1700-present) & - & Phillips (1936-present) & Wire (1895-present) & - & Casket & $>1936$ \\
\hline
\end{tabular}


Table 2 (cont'd)

\begin{tabular}{|c|c|c|c|c|c|c|c|c|c|c|c|c|c|c|}
\hline Feature & $\begin{array}{l}\text { Marker } \\
\text { Type }\end{array}$ & $\begin{array}{l}\text { Plaque } \\
\text { Type }\end{array}$ & Handle Type & $\begin{array}{l}\text { Thumbscrew } \\
\text { Type }\end{array}$ & $\begin{array}{l}\text { Thumbscrew } \\
\text { Escutcheon } \\
\text { Type }\end{array}$ & $\begin{array}{l}\text { Top } \\
\text { Fastener } \\
\text { Type }\end{array}$ & Catch Type & Butt Hinge Type & $\begin{array}{l}\text { Stop Hinge } \\
\text { Type }\end{array}$ & Screw Type & Nail Type & $\begin{array}{l}\text { Other Diagnostic Artifacts } \\
\text { or Notes }\end{array}$ & $\begin{array}{l}\text { Burial Container } \\
\text { Type }\end{array}$ & $\begin{array}{l}\text { Interment Date } \\
\text { (Range) }\end{array}$ \\
\hline Burial 12 & $\begin{array}{l}2(1927- \\
1950)\end{array}$ & - & $\begin{array}{l}8(\mathbf{1 9 2 0 - ? ) * ;} \\
16(1940- \\
\text { present) }\end{array}$ & - & - & - & 3 (1889-?) & - & - & Phillips (1936-present) & Wire (1895-present) & - & Casket & 1940-1950 \\
\hline Burial 13 & - & $3(?)$ & 14 (1890-?) & - & - & - & 1 (1889-?) & 2 (1700-present) & 2 (1888-?) & $\begin{array}{l}\text { Slotted or unknown } \\
\text { (1846-present) }\end{array}$ & Wire (1895-present) & Support 1 (1920s-?) & Casket & $>1920$ \\
\hline Burial 14 & $\begin{array}{l}1 \text { or } 2 ? \\
\text { (1926- } \\
\text { present) }\end{array}$ & - & 27 (1920-?) & - & - & - & $\begin{array}{l}1 \text { (1889-?); } \\
2 \text { (1889-?) }\end{array}$ & - & 4 (1888-?) & $\begin{array}{l}\text { Slotted or unknown } \\
\text { (1846-present) }\end{array}$ & Wire (1895-present) & Support 2 (1865-present) & Casket & $>1926$ \\
\hline Burial 15 & - & - & 8 (1920-?) & - & - & - & - & - & - & $\begin{array}{l}\text { Slotted or unknown } \\
\text { (1846-present) }\end{array}$ & Wire (1895-present) & $\begin{array}{l}\text { Found partially beneath } \\
\text { burial } 12\end{array}$ & Casket & 1920-1940 \\
\hline Burial 16 & - & $2(?)$ & 5 (1914-1935) & $\begin{array}{c}1(1890- \\
1920)\end{array}$ & $\begin{array}{l}1(1894- \\
1926)\end{array}$ & - & - & - & - & $\begin{array}{l}\text { Slotted or unknown } \\
\text { (1846-present) }\end{array}$ & $\begin{array}{l}\text { Wire and cut (1895- } \\
\text { present) }\end{array}$ & - & $\begin{array}{l}\text { Hexangonal } \\
\text { coffin }\end{array}$ & 1914-1926 \\
\hline Burial 17 & - & - & 18 (1920-?) & - & - & - & - & - & 3 (1888-?) & $\begin{array}{l}\text { Slotted or unknown } \\
\text { (1846-present) }\end{array}$ & Wire (1895-present) & $\begin{array}{l}\text { Corrugated fasteners } \\
\text { (1890-present) }\end{array}$ & Casket & $>1920$ \\
\hline Burial 18 & - & $1(>1930$ ?) & $13(1920-?)$ & - & - & - & 6 (1883-?) & - & $\begin{array}{l}2 \text { (1888-?; } \\
5 \text { (1888-?) }\end{array}$ & $\begin{array}{l}\text { Slotted or unknown } \\
\text { (1846-present) }\end{array}$ & Wire (1895-present) & Support 2 (1865-present) & Casket & $>1930$ \\
\hline Burial 19 & $\begin{array}{l}3 \text { or } 4 \text { ? } \\
\text { (1926- } \\
\text { present) }\end{array}$ & - & 9 (1940-?) & - & - & - & - & - & $\begin{array}{l}1 \text { (1888-?); } \\
2 \text { (1888-?) }\end{array}$ & $\begin{array}{l}\text { Slotted or unknown } \\
\text { (1846-present) }\end{array}$ & $\begin{array}{l}\text { Wire and cut (1895- } \\
\text { present) }\end{array}$ & $\begin{array}{l}\text { Support } 2 \text { (1865-present); } \\
\text { corrugated fasteners } \\
\text { (1890-present) }\end{array}$ & Casket & $>1926$ \\
\hline $\begin{array}{l}\text { Disturbed } \\
\text { Provenience/ } \\
\text { Surface } \\
\text { Collection* }\end{array}$ & $\begin{array}{c}1 \text { (1926- } \\
3 \text { (1937- } \\
\text { present); } \\
5 \text { (1950- } \\
\text { present) }\end{array}$ & - & $\begin{array}{l}2(1700- \\
\text { present); } \\
12(1940 s-?) ; \\
19(1950 s- \\
\text { present); } \\
24(1900-?) ; \\
28(1920-?)\end{array}$ & - & 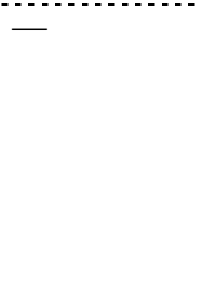 & - & - & 1 (1700-present) & & $\begin{array}{l}\text { Slotted or unknown } \\
\text { (1846-present) }\end{array}$ & $\begin{array}{l}\text { Wire and cut (1895- } \\
\text { present) }\end{array}$ & $\begin{array}{c}\text { Corrugated fasteners } \\
\text { (1890-present) }\end{array}$ & & $\begin{array}{l}\text { Disturbed } \\
\text { context } \\
\text { (1895- } \\
\text { present) }\end{array}$ \\
\hline
\end{tabular}




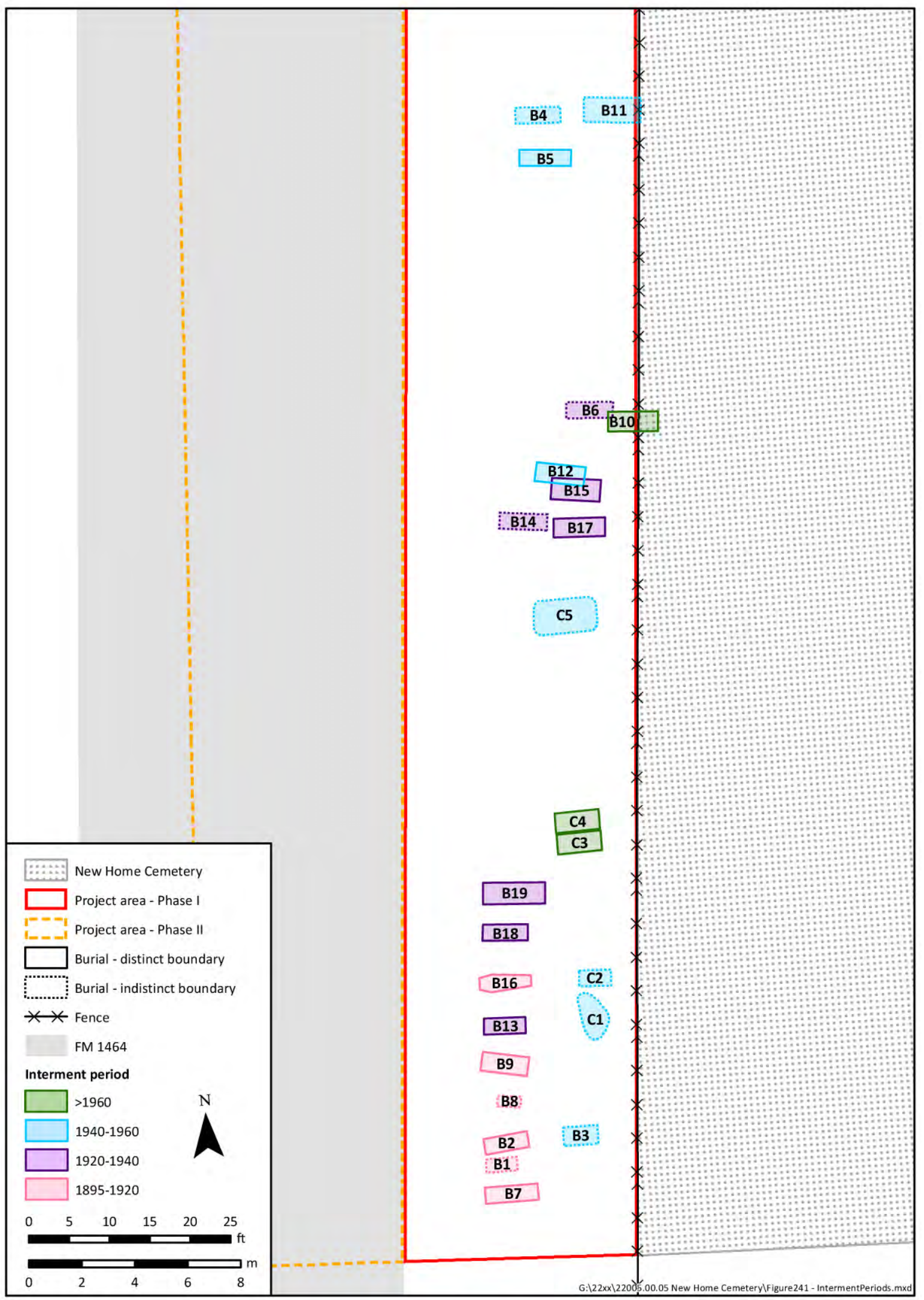

Figure 241. Plan map of New Home Cemetery project area showing chronology of burials (CSJ 1415-02-032). 
focused on methodological issues, "leaving largely unaddressed the theoretical questions that inspire us to look at skeletons in the first place. ... Y Yet much more attention should be directed toward what we can learn from skeletons that cannot be learned from other sources, particularly archaeological remains and historical documents.”

Cemetery samples may not be representative of the larger populations; they are the ones who died, and they represent completely different cohorts of a time continuum. There often were rapid changes in environmental conditions. Certainly, that was the case for Sugar Land. In just 50 years, the means of production changed from slavery, to government-controlled slavery (convict labor) and tenant farming, to model company town (welfare capitalism).

The individuals from New Home are a very small sample. Yet, they can be compared to the reference studies in several respects. For example, Rose and Santeford (1985:144-145) note that the Cedar Grove individuals reflected an obvious genetic admixture of Native American and American Black [sic] in southwest Arkansas, and that this indicated some interesting problems for future research with the skeletal nonmetric and dental data. In his closing remarks concerning future work, Rose even suggested that he would have to review the anthropometrics, because there were problems in the discriminant function analyses. Several individuals were categorized as Caucasian.

There does not seem to be any doubt about the historical fact of genetic admixture between Native Americans and African derived Blacks both free and slave. First of all many southern slaves were imported by way of the Caribbean Islands where originally both Blacks and Indians were kept as slaves with considerable opportunity for interbreeding. Within the American south Blacks and Indians frequently intermarried, while many American Indian tribes owned large numbers of slaves, especially the "Five Civilized Tribes" of the American Southeast (Porter 1932). When these tribes were relocated to the Indian Territories they brought their slaves with them. Additionally, the Indian Territories served as an excellent hiding place for runaway slaves [Rose and Santeford 1985:145].

Although there was only time for macroscopic assessment of skeletal and dental traits supplemented with basic anthropometrics, the New Home Cemetery individuals obviously exhibit traits that are indicative of ethnic/racial admixture. The uncomplimentary description cited in The City of Sugar Land (Arcadia Publishing 2010:23, 26) that referred to a mixture of different genetic affinities certainly corroborates these observations. Dental traits for Carabelli's cusp (European), shoveling (Native American), and Uto-Aztecan premolar (Native American) are a few of the observations for New Home. This does not say that those individuals were not African American, but it does indicate that there was genetic admixture.

Even though there was undeniable hardship experienced by all of the African American cemetery samples, the expression of nutritional stress through metabolic indicators (porotic hyperostosis and cribra orbitalia) varied. All of the samples had some individuals who had these pathologies, but for the Texas cemeteries, the skeletal expressions of anemias were much more moderate than expected. Lesions that are diagnostic of anemias are indicative of childhood stress. The fact that these lesions are all very moderate and healed in the Texas samples is indicative of populations that are successful in coping with nutritional stress.

There was no evidence of any notable infection in the New Home Cemetery individuals. Small, benign, button osteomas (bone growths) were noted for a couple of people, one individual had an ossified mass in the pelvis, and two individuals had evidence of probable soft tissue masses (possible goiter?) that notably altered the shape of the jaw. 
Not surprisingly, all of the comparative cemetery samples had evidence of hard manual labor. Individuals from New York African Burial Ground exhibited the suite of pathologies that define extreme axial loading. These include fracture of the cranial base, burst fracture (Jefferson fracture) of the first cervical vertebra, microfractures of the spinous processes and pedicles of elements throughout the spinal column, enthesopathy and syndesmopathy of muscles and tendons associated with power lifting and carrying behaviors, and extreme arthritic changes in most joints of the spine. Several individuals from New Home Cemetery exhibit pathology that is exactly the same as that found in New York African Burial Ground.

The following information is derived from Kennedy (1989). Markers of occupational stress have a very lengthy history that began as early as the 1400s to 1500s in military and trade medicine. Anatomists recorded specific types of injuries that occurred to individuals who engaged in occupations that required the use of specific tools and weapons and repetitive behaviors. Two early studies were from Charles Thackrah (1795-1833), who described "trade diseases" including the skeletal conditions of pelvic deformation and spinal scoliosis (S-shaped deformity) that were characteristic of weavers who sat for long periods of time at their looms, and the work of William Lane (1856-1943) who wrote a treatise on the severe skeletal injury and deformity in individuals who worked as stevedores in the docks and warehouses of London. A large body of research is devoted to the physical and physiological changes that occur in response to different repetitive activities and behaviors.

For the purposes of the New Home Cemetery study, the cranial base fractures and burst fractures of the first cervical vertebra are particularly important. Medical texts cite the mechanism of injury as axial loading to the top of the head. There are three basic types of cranial base fractures: transverse (also called "hinge"), longitudinal, and ring. Ring fracture of the cranial base occurs when a sudden jolt tightens the foramen magnum down onto the spinal column, in a similar manner to tightening an axe head down onto the handle by pounding the base of the handle on a hard surface (Spitz 1980:148). Prior to the development of CT and MRI scans, basilar fractures were exceedingly difficult to diagnose in a standard X-ray. Once the fracture occurs, the individual can live for several days or weeks before complications occur as a consequence of infection or neural injury (Rogers and Hendrix 1992). Symptoms may include dark circles around the eyes or behind the ears due to pooling of blood, and leakage of cerebrospinal fluid from the nose and ears (Basilar Skull Fracture 2011; Skull Fracture 2011).

"Jefferson" fracture of the first cervical vertebra is a fracture of the ring, essentially a burst fracture. C1 is shaped like a large, open ring, and the cranial base rests on top of it. It is the only vertebra that does not have a centrum. The knob (also called the dens or odontoid process) for C2 rests inside the ring and is held in place by the very strong transverse ligament. The ring and knob allow humans to hold their heads up and freely rotate them from side to side. This interarticulation is critical for the balance of the head in different positional behaviors. Medical texts cite the mechanism of injury (burst fracture of C1) as axial loading to the top of the head (Rockwood and Green 1984; Rogers and Hendrix 1992; Wilson 1982). Postmortem dissections of African men who worked as stevedores offloading sacks of mealie meal list the C1 fracture as the one (of all the cervical vertebrae) which is less likely to be a debilitating (i.e., paraplegia or quadriplegia) or mortal injury. This is because the cross-sectional area of the spinal canal is the largest at this location and the least likely to injure the spinal cord if fragmented (Levy 1968). However, risk of injury and death increased with the increase of size and/or weight of the bags (that tend to shift in transit) and the increase in the distance required to carry the load. An individual who is assisted with the loading also is less likely to sustain severe injury than if he tries to load it himself. Usually, the bag is lifted by four men (one on each corner) and the porter then walks underneath the load and it is positioned on top of his head for transport (Levy 1968). 
Load-bearing stress also has been described by Bridges (1994), Kennedy (1989), Lai and Lovell (1992), and Lane (1885). Lane (the anatomist and surgeon mentioned earlier in the discussion; 1885) and Bridges (1994) describe arthritic pathology of the vertebral column. Lai and Lovell (1992) examined individuals believed to have been Les Voyageurs in Canada, and described several joint complexes, including the shoulder girdle and chest.

A lifetime of habitual behavior can leave an indelible mark on the human skeleton. Some occupations are more detrimental because they exceed the anatomical and physiological endurance limits of the bones and joints.

\section{Society and Access to Health Care}

Two individuals at New Home Cemetery had gold-capped teeth, indicating that they not only had access to dental care, but had the means to pay for more expensive materials. Others at New Home were edentulous, typical of poor dental health and advanced age. Evidence of professional dental care for some individuals was noted at Cedar Grove, Freedman's, Third New Cemetery, and Phillips Memorial cemeteries.

Markers for poor dental health, as well as episodic general stress indicators that are recorded in the dental tissues (enamel hypoplasia and hypocalcification), were observed in all of the comparative cemetery samples. Although conjectural, it is possible that proximity to Sugar Land, either directly or indirectly, contributed to the poor dental health of the New Home individuals. Sugar cane is an inexpensive, readily available source of energy. However, chewing the fibrous canes and ingesting the sugary juice quickly wears down the enamel surfaces, exposing the secondary dentin and pulp cavities of teeth, rendering them more susceptible to decay and eventual loss through antemortem abscessing and evulsion.

The fact that several individuals lived well into advanced age (regardless of poor dental care) attests that living conditions, while categorized by hard work, were such that people were able to survive beyond what might have been expected for individuals who were otherwise marginalized at the turn of the nineteenth century and into the twentieth century. Compensation for sharecroppers and tenant farmers supposedly included housing, rations, medicine, a garden plot, and wages or a portion of the crop (Roark 1977:113-114; Watkins 1985:9). The welfare capitalism model for company town Sugar Land provided similar amenities (Arcadia Publishing 2010).

Yet, there were problems with hazardous work conditions and interpersonal violence. Rural agriculture incurred a high level of occupational risks in relation to the pulling, pushing, and lifting by heavy manual labor; the proximity to large (often unpredictable) animals; and the use of machinery, especially in the decades prior to OSHA standards.

Sugar planting, harvesting, and processing is tiring, hot, dangerous work and requires a large number of workers whose work habits must be intensely coordinated and controlled. . . . Yet, it was not unusual for slaves to be injured or crushed when trapped and pulled into the rollers as they fed stalks into the mill or tried to untangle stalks from flywheels and gears. ... the heat in the sugar houses was so intense that slaves were rotated out after four hours, their limbs swollen from the heat and humidity. ... [sugar cakes] had to be broken up with shovels, picks, and crowbars [West 2011:2]. 
Social disenfranchisement and institutionalized racism often resulted in open expressions of desperation in the form of interpersonal violence. Evidence of trauma related to interpersonal violence (fractures and weapon use) was reported for New York African Burial Ground, Cedar Grove, Freedman's, and Phillips Memorial cemeteries. A fracture of the fifth metacarpal (that may have been a Boxer's fracture) was reported for the Third New City cemetery sample. New Home Cemetery had an individual (Burial 18) who had multiple traumas, including a severe injury to the left shoulder that greatly resembled a healed gunshot wound. However, this individual may have been a veteran; and without the assistance of radiography, the differential diagnosis of gunshot trauma is problematic. Three of the New Home individuals (Burials 16, 18, 19) had Pond fractures (small, depressed, blunt force trauma to the cranial vault). Table 3 is a summary of the skeletal pathology observed among the New Home Cemetery burials:

Table 3

New Home Skeletal Pathology Summary

Burial Number Observed Pathology *

1a $\quad$ Metabolic distress

2 Endocranial pressure; cranial base fx; axial loading; metabolic stress

3 Possible Jefferson $\mathrm{fx}^{* *}$ /axial loading of cervical spine

$5 \quad$ Spontaneous hip fx with subsequent trauma; metabolic stress; hyperostosis frontalis interna

6 Severe axial loading; Jefferson fx; "Weaver’s Bottom”

$7 \quad$ Axial loading; flail chest

$9 \quad$ Metabolic stress; severe axial loading; severe trauma to left side of face in childhood; severe "Weaver's Bottom"; healed Jefferson fx

10 Axial loading; pillastering of femora (power lift); spondylolisthesis

11 Osteopenia

12 Cranial base fx; Jefferson fx; axial loading

13 Osteoporosis/osteopenia; endocranial pressure; metabolic stress; axial loading; workrelated arthropathy; possible goiter?; kneeling

14 "Weaver’s Bottom”; arthritis

$16 \quad$ Pond fx; endocranial pressure; axial loading; clay shoveler’s fx; work-related arthropathy

17 Axial loading

18 Pond fx; axial loading; severe trauma to left shoulder + left lower leg \& foot; osteopenia

19 Pond fx; axial loading; clay shoveler’s fx; "Weaver’s Bottom”; ossified mass in right pelvis

C. 1 Axial loading

C. 5 Osteoporosis/osteopenia

*Note: Does not include dental pathology

$* * \mathrm{fx}=$ fracture 


\section{Burial Socioeconomics and Social Support}

As mentioned above in the section on burial chronology, the New Home Cemetery burials date between 1895 and the late 1960s. There are no artifacts that seem to be truly out of place temporally (a mixing of the old and the new), which means that the families of the deceased were able to participate in contemporary consumer practices. If the community had been relying on outdated, and therefore cheaper, hardware from the backstock of a general store, there could be mismatched sets of hardware on burial containers and the total costs of burial would likely be reduced. Such a reduced cost would be more attractive to a clientele of lesser means. The majority of New Home Cemetery burials do not show evidence of having mismatched sets of hardware. The few that do have multiple types of handles, for example, or missing elements of a set can be explained based on history of disturbances or loss from poor preservation.

All of the burial containers and associated mortuary artifacts at New Home Cemetery, however, would not have been considered high-end even in that period. Burial 18, for instance, contained a cloth-covered casket indicated by the presence of a white cotton cloth adhering to the back of the associated plaque. Cloth-coverings are used often to disguise a burial container with shoddy construction or one produced from a low quality material (Pye 2010a). The inclusion of several metallic caskets would indicate a greater expenditure than wooden burial containers if these burials dated to the nineteenth century when metallic caskets were less commonly used. As previously stated, however, by the mid-twentieth century, metallic caskets were nearly ubiquitous, with fine hardwood caskets often fetching a greater price than metallic. The presence of mostly steel handles is also another indicator of a lower expenditure compared with carved wooden handles or handles made of a higher quality of metal.

Because many of the exact styles of mortuary artifacts from New Home Cemetery could not be identified, or price lists were not available from the certain companies or the certain time periods, it is not possible to create a specific cost profile for each burial at this time. It has also not been firmly established in the literature if there was a standard markup in price as goods traveled from the wholesale hardware or casket dealer, to the funeral director, and then to the consumer. It is certain that a markup did take place, but any responsible cost calculations must take markups into account when painting a picture of burial container or funeral expenditure.

Churches, social organizations, and benevolent societies were an integral part of the social environment at the turn of the nineteenth century, and continue to play major roles of social and spiritual support today. Watkins (1985:12), writing about the Cedar Grove community, noted that many of these organizations provided monetary support in the form of burial insurance. The dues for membership would help cover the expenses incurred for appropriate burial. In a manner similar to the Woodmen of the World, social organizations such as the Prince Hall Masons provided individuals with social contacts who were willing to provide many types of assistance (Prince Hall 1996; Prince Hall Freemasonry 2011; Woodmen of the World 2011). Lodges also provided payment for hospitalization and/or health care. It is very common for churches and other social organizations to take up monetary collections or donations for individuals or families who are experiencing hardship, or to provide comfort in the form of assistance in domestic tasks, bringing food to the sick or infirm, and making repairs to homes, etc. Although thorough historical research has not been conducted into the role of various organizations on the social support of funeral financing in Sugar Land, at least one tombstone set in the undisturbed portion of New Home Cemetery reveals that the deceased was a member of the Freemason Society (Figure 242). 


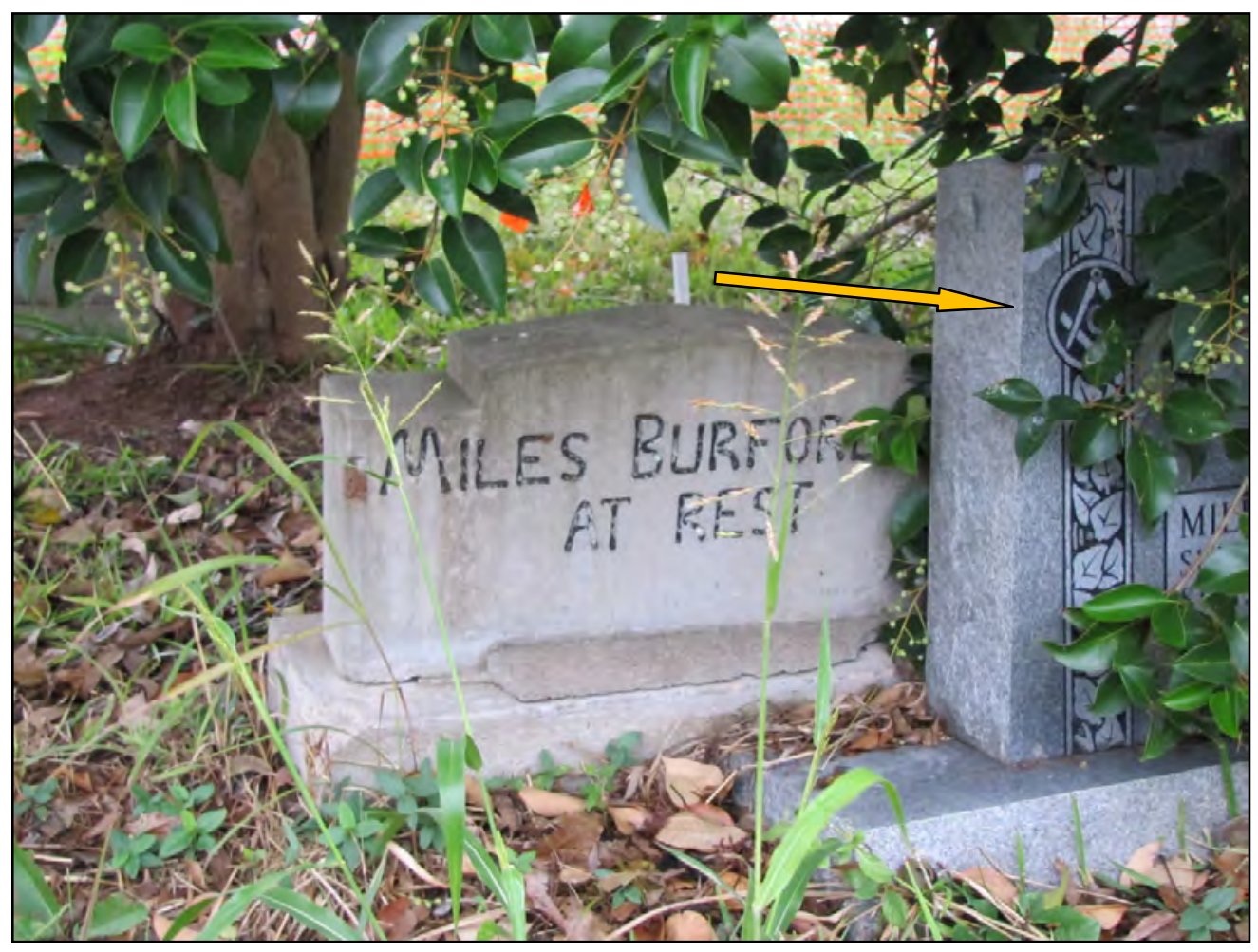

Figure 242. Tombstones in New Home Cemetery (note Masonic symbol on stone at right). 



\section{CHAPTER 7 SUMMARY \\ by Mary Cassandra Hill and Jeremy W. Pye}

Bioarchaeology can be simplistically defined as the contextual analysis of human populations from archaeological sites (Buikstra 1977). It uses a combination of archaeology and skeletal biology to address questions about how people lived in the past. Through an archaeological lens, researchers attempt to focus on the production of individual "osteobiographies," and population biocultural adaptation (Beck 2006). Attention is directed toward skeletal remains because bioarchaeologists feel that human remains are a unique source of information about the responses past populations have had in the face of challenges of past social and natural environments (Walker 2008). When coupled with thorough analysis and contextualization of mortuary artifacts, studies of human remains are promising avenues of research.

Human remains and funerary materials, including metal caskets, were encountered during site testing procedures for Phase II survey in an area of Sugar Land, Harris, County, Texas, which had been part of the original New Home Cemetery. It was necessary to determine the extent to which those remains, plus any other identified remains, were intact. This was accomplished through controlled excavation by professionally trained bioarchaeologists. Second, biological profiles were assessed for each of the individuals and these were compared to similar cemetery population samples. Third, mortuary artifacts were analyzed to determine what they might be able to reveal about chronology of interments and economic expenditure. Lastly, all remains were reinterred in a respectful manner in a new location within the existing cemetery.

Previous chapters of this work have described several general classes of mortuary artifacts and specific descriptions of those deceased individuals represented by skeletal remains in New Home Cemetery burial excavations. A sizeable collection of mortuary-specific artifacts was recovered, including at least one type of metal casket, five types of grave markers, 28 handle types, two thumbscrew types, two thumbscrew escutcheon types, four casket plaques, 28 types of complex internal casket hardware (including multiple types of hinges, catches, top fasteners, and escutcheons), nine items referred to as embalming paraphernalia, as well as other construction hardware such as cut and wire nails, lining tacks, slotted and Phillips head gimlet screws, staples, corrugated fasteners, and joining plates. Personal artifacts, such as a straight razor, scissors, artificial flowers, articles of clothing or clothing fasteners, were also recovered. The analyzed artifacts suggest that the 24 interments in question most likely occurred between 1895 and the late 1960s. 
Sugar Land, Texas, has a notably rich history of growth and development that began with one of the founding fathers of the State. The people who were the labor force came from very diverse cultural backgrounds, but they shared one thing in common: hard work. Analyses of the biological and funerary remains afford the first opportunity to describe the lives and lifestyles of individuals who were possibly members of the working class during the extreme late nineteenth and early to mid-twentieth centuries in Sugar Land. The examinations of these individuals and their mortuary contexts revealed that there was creolization of the people as well as their burial customs.

This report cannot even scratch the surface of such a broad and rich anthropological topic. Death and burial are among the most important and meaningful cultural and spiritual events in a community; it is vital that scholars are able to understand the context of mortuary practice. The job of mortuary archaeologists and historical bioarchaeologists, therefore, is to try to put enough of the pieces back together from the analysis of material and skeletal remains to shed light on the articulations of the larger puzzle - the social, religious and ideological interrelationships of past communities. 


\section{REFERENCES CITED AND SOURCES CONSULTED}

Abbott, James T.

2001 Houston Area Geoarchaeology: A Framework for Archaeological Investigation, Interpretation, and Cultural Resource Management in the Houston Highway District. Archaeological and Environmental Consultants. Submitted to Texas Department of Transportation, Environmental Affairs Division, Archaeological Studies Program Report 27.

Abernathy Casket Company

ca. 1930 Casket Catalogue "H." Abernathy Casket Company, Kansas City, Missouri.

Adams, D.

2006 "New Home Cemetery being restored by group.” Fort Bend Herald, Sunday, July 16, 2006.

Adams, W. H.

2002 Machine Cut Nails and Wire Nails: American Production and Use for Dating 19thCentury and Early-20th-Century Sites. Historical Archaeology 36(4):66-88.

African American Funeral and Burial Practices

2011 "African American Funeral and Burial Practices." Electronic document http://www.babyboomercaretaker.com/funeral/burial/African-American-FuneralAnd-Burial-Practices.html, accessed 3/19/2011.

African Burial Ground

2011 "The African Burial Ground. Return to the Past to Build the Future.” History. Electronic document http://www.africanburialground.gov/ABG_History.htm, accessed 5/24/2011.

American Steel Grave Vault Company

1975 The Perfect Symbol of Enduring Trust, Confidence, and Lasting Protection. American Steel Grave Vault Company, Galion, Ohio. 
[Advertising Cards]. American Steel Grave Vault Company, Galion, Ohio.

Angelfire

"Elements Found in Texas Folk Cemeteries." Electronic document http://www.angelfire.com/tx5/texasczech/Grave\%20Markers/Elements.htm, accessed 5/29/11.

Anonymous

ca. 1797 [book of coffin plates, handles, ornaments, etc.]. Unknown company, England.

Anonymous

$1922 \quad$ Price List (August 1, 1922). Unknown company, United States.

Arcadia Publishing

2010 The City of Sugar Land. Images of America Series. Arcadia Publishing, Charleston, South Carolina.

Archaeological Services, Inc.

1992 Archaeological Mitigation of the Cheyne Cemetery, Part Lot 14, Concession 1, E.H.S., Toronto Township, City of Brampton, Regional Municipality of Peel. Archaeological Services, Inc., Toronto, Ontario, Canada. Submitted to Corporation of the City of Brampton, Ontario, Canada.

ca. 2000 Archaeological Report on the Salvage Recovery of Human Remains at Trinity Anglican Church Cemetery, 79 Victoria Street, Town of Aurora, Regional Municipality of York, Ontario. Archaeological Services, Inc., Toronto, Ontario, Canada.

Archaeological Services, Inc., and Gary Warrick

2005 Archaeological Investigation of the Pea Hill Site, City of Hamilton. Archaeological Services, Inc., Burlington, Ontario. Prepared for the Red Hill Valley Project, city of Hamilton and the Grand Council of the Haudenosaunee.

Atkinson, J. R., and Kenneth R. Turner

1987 The Blackburn Cemetery: An Abandoned Burial Site on the Old Natchez Trace in Maury County, Tennessee. Southeast Archaeological Center, National Park Service, Tallahassee, Florida.

Atlantic Coffin \& Casket Company

1918 Catalogue "B," Wholesale dealers in coffin \& casket hardware, linings. . . . Atlantic Coffin \& Casket Company, Rose Hill, North Carolina.

Atz, L.

2005 Crawford Cemetery Relocation, Gwinnett County, Georgia. Brockington and Associates, Inc., Atlanta, Georgia. Prepared for Cheeley Investment, Duluth, Georgia. 
Atz, L., and W. Weaver

2006 Relocation of the Roughton/Browne Cemetery, Sandersville, Washington County, Georgia. Project FLF 540(29). Brockington and Associates, Inc., Atlanta, Georgia. Prepared for PBS\&J, Atlanta, Georgia.

Aurora Casket Company, Inc.

ca. 1999 [Catalog of Advertising Cards]. Aurora Casket Company, Inc., Aurora, Indiana.

1999 [Price List]. Aurora Casket Company, Inc., Aurora, Indiana.

Baackes, $\mathrm{M}$.

1896 The History of the American Wire Nail Industry. The Iron Age 57:105-106.

Basilar Skull Fracture

2011 "Basilar skull fracture." Electronic Document http://en.wikipedia.org/wiki/ Basilar_skull_fracture, accessed 6/15/2011.

Bass, W. M.

1987 Human Osteology. A Laboratory and Field Manual. 3rd edition. Special Publication No. 2. Missouri Archaeological Society, Columbia, Missouri.

Bastis, K.

2006 Health, Wealth, and Available Material: The Bioarchaeology of the Bulkeley Tomb in Colchester, Connecticut. Masters thesis, University of Connecticut.

Batesville Casket Company

1997 [Catalog of Advertising Cards]. Batesville Casket Company, Batesville, Indiana.

1998 [Catalog of Advertising Cards]. Batesville Casket Company, Batesville, Indiana.

Beaty, J., and L. Atz

2002 Relocation of an Abandoned Cemetery in Augusta, Richmond County, Georgia. Project-NH-117-1(114). Brockington and Associates, Inc., Atlanta, Georgia. Prepared for the Georgia Department of Transportation, Atlanta, Georgia.

Beck, L. A.

2006 Kidder, Hooton, Pecos, and the Birth of Bioarchaeology. In Bioarchaelogy: The Contextual Analysis of Human Remains, edited by J. E. Buikstra, and Lane Beck, pp. 83-94. Academic Press, Burlington, MA.

Bedino, J. H.

2003 Embalming Chemistry: Glutaraldehyde Versus Formaldehyde. Champion Expanding Encyclopedia of Mortuary Practices 649:2614-2632.

Belknap, W. B., \& Company

1895 Importers and Jobbers of Hardware. Belknap, W. B., \& Company, Louisville, Kentucky. 
1901 Catalogue No. 29 (general hardware with coffin trimmings). W. B. Belknap \& Company, Louisville, Kentucky.

Bell, E. L.

1987 The Historical Archaeology of Mortuary Behavior at a Nineteenth-Century Almshouse Burial Ground. Master's thesis, Boston University, Boston, Massachusetts.

1990 The Historical Archaeology of Mortuary Behavior: Coffin Hardware from Uxbridge, Massachusetts. Historical Archaeology 24(3):54-78.

Benson, D. L.

1983 A Taxonomy for Square Cut Nails. In The Conference on Historic Site Archaeology, edited by S. South, pp. 123-152, Vol. 15. University of South Carolina, Columbia, South Carolina.

Berg, R. E.

1990 An Investigation of Burials at the Scisson Family Cemetery in Gregory County, South Dakota. South Dakota Archaeology 14:36-92.

Beynon (Landers), D. E.

1989 Remember Me As You Pass By: Excavation of the Voegtly Cemetery. A 19th Century German-American Churchyard in Pittsburgh, Pennsylvania. 3 vols. GAI Consultants, Inc., Monroeville, Pennsylvania. Report to the Pennsylvania Department of Transportation.

Bird, M. C.

2000 Final Report: Phase III Mitigation of the Burying Ground at Vandaworker's Corners (11-L-572), Village of Deer Park, Ela Township, Lake County, Illinois. Cultural Resource Management Report No. 811. Midwest Archaeological Research Services, Inc., Harvard, Illinois. Prepared for Mr. Lonnie Chafin, Northern Illinois Conference of the United Methodist Church, Chicago, Illinois.

Bird, M. C., A. L. Grauer, and J. T. Kuttruff

2003 Data Recovery at the Thurston Cemetery within the County Right-of-Way, York Township, DuPage County, Illinois. Midwest Archaeological Research Services, Inc., Harvard, Illinois. Prepared for Chuck Tokarshi, DuPage County Division of Transportation, Wheaton, Illinois.

Blakey, M. L.

1998 "The New York African Burial Ground Project: An Examination of Enslaved Lives, a Construction of Ancestral Ties.” Electronic document http://www.huarchivesnet.howard.edu/0008huarnet/blakey1.htm, accessed $5 / 24 / 2011$.

Blakely, R. L., and L. A. Beck

1982 Bioarchaeology in the Urban Context. In Archaeology of Urban America: The Search for Pattern and Process, edited by R. S. Dickens, Jr., pp. 175-207. Academic Press, New York, New York. 
Bliss-Holbrook Company, Inc.

ca. 1905 Catalogue of Coffin Studs, Plates and Ornaments Mfg. by Bliss-Holbrook Company, Inc. Bliss-Holbrook Company, Inc., Attleboro, Massachusetts.

Bocquet-Appel, J-P., and C. Masset

1982 Farewell to paleodemography. Journal of Human Evolution 11:321-333.

1996 Paleodemography: expectancy and false hope. American Journal of Physical Anthropology 99:571-583.

Boen, R. M., and C. Taft

1999 39MN7 Howard Cemetery, Accession Number 99-103. Draft, Burial Report \#1999-

11. South Dakota State Historical Society, Archaeological Research Center, Rapid City, South Dakota.

Boyertown Burial Casket Company

n.d. $\quad$ "The Pure Cast Iron Alloyed with Copper Casket no. 5." Boyertown Burial Casket Company, Boyertown, Pennsylvania.

n.d. $(<1910) \quad$ [Catalogue]. Boyertown Burial Casket Company, Boyertown, Pennsylvania.

1919 Catalogue “I.” Boyertown Burial Casket Company, Boyertown, Pennsylvania.

1919 Revised Price List of Pedestals Made of Brass Tubing (November 17, 1919). Boyertown Burial Casket Company, Boyertown, Pennsylvania.

ca. 1923 Catalogue “J.” Boyertown Burial Casket Company, Boyertown, Pennsylvania.

1923 Metal Catalogue, Number 4. Boyertown Burial Casket Company, Boyertown, Pennsylvania.

1927 Catalog “K.” Boyertown Burial Casket Company, Boyertown, Pennsylvania.

1935 Substitution of Volume and Quantity Discount Plan to Dollar Volume Basis. (Brochure; effective March 1, 1935). Boyertown Burial Casket Company, Boyertown, Pennsylvania.

1936 Catalog "L" Hardwood and Covered Caskets. Boyertown Burial Casket Company, Boyertown, Pennsylvania.

1936 Catalog “LX” Supplement to Catalog “L.” Boyertown Burial Casket Company, Boyertown, Pennsylvania.

1938 Catalog “L-16,” 16-Guage Metal Caskets. Boyertown Burial Casket Company, Boyertown, Pennsylvania.

1940 Price List Applying to Hardwood \& Covered Caskets, Also Metal Caskets, Catalogs " $L$," " $L X$," and " $L-16$ " (effective February 19, 1940). Boyertown Burial Casket Company, Boyertown, Pennsylvania. 
Boyertown Casket Company

ca. 1897 [Catalogue]. Boyertown Casket Co., Boyertown, Pennsylvania.

ca. 1900 [Catalogue]. Boyertown Casket Co., Boyertown, Pennsylvania.

Bradle, M. R., J. S. Belew, and S. M. Wallace

2002 Archaeological Survey of the Connally I.S.D. School Tract and Cemetery Relocation, McLennan County, Texas. Report of Investigations Number 26. American Archaeology Group, Inc., Lampasas, Texas. Prepared for Connally Independent School District, Waco, Texas.

Braley, C. O.

1992 Archaeological and Archival Investigations at the Deepstep A.M.E. Church Cemetery, Washington County, Georgia. Southeastern Archaeological Services, Inc., Athens, Georgia. Prepared for Engelhard Corporation, Pigments and Additives Division, Gordon, Georgia.

Braley, C. O., and W. G. Moffat

1995 Report. Archaeological and Historical Investigations of Redfield Cemetery, Jones County, Georgia. Vol. I. Southeastern Archaeological Services, Inc., Athens, Georgia. Prepared for the Macon Water Authority, Macon, Georgia.

Brauner, D. R., and P. C. Jenkins

1980 Archeological Recovery of Historic Burials within the Applegate Lake Project Area, Jackson County, Oregon. Department of Anthropology, Oregon State University, Corvallis, Oregon. Prepared for the U.S. Army Corp of Engineers, Portland District.

Bridges, P. S.

1994 Vertebral arthritis and physical activities in the prehistoric southeastern United States. American Journal of Physical Anthropology 93:83-93.

Brock, J., and S. J. Schwartz

1991 A Little Slice of Heaven: Investigations at Rincon Cemetery, Prado Basin, California. Historical Archaeology 25(3):78-90.

Broehm, C. J., D. K. Boyd, and M. D. Freeman

2004 Archaeological Excavation and Reburial of Historic Graves in the Oscar Abstein Cemetery (41HR976), Harris County, Texas. Reports of Investigations, Number 140. Prewitt and Associates, Inc., Austin, Texas. Submitted to Texas Department of Transportation, Environmental Affairs Division, Archaeological Studies Program, Report 64, Austin, Texas.

Brown, M. R., and J. W. Dunn

1975 A Guide to the Grading of United States Coins. $6^{\text {th }}$ edition. Western Publishing Company, Inc., Racine, Wisconsin. 
Bromberg, F. W., S. J. Shepard, B. H. Magid, P. J. Cressey, T. Dennée, and B. K. Means

2000 "To Find Rest From All Trouble": The Archaeology of the Quaker Burying Ground, Alexandria, Virginia. Alexandria Archaeology, Office of Historic Alexandria, Alexandria, Virginia.

Bruner, D.

1998 "Levi Jordan Plantation. Hidden Power: Burial Practices from an AfricanAmerican Slave and Tenant Community.” Electronic Document http://www.webarchaeology.com/html/BrunrSHA.htm, accessed 7/3/2011.

Buchner, C. A., E. Breitburg, C. Williams, and E. A. Williams

1999 At Rest, Again: The Ridley Graveyard (40WM208) Archaeological Relocation Project, Williamson County, Tennessee. Panamerican Consultants, Inc., Memphis, Tennessee. Submitted to Gresham Smith and Partners, Nashville, Tennessee, and Tennessee Department of Transportation, Environmental Planning Office, Nashville, Tennessee.

Buffalo Burial Vault Works

ca. 1950 Vaults By Buffalo. Buffalo Burial Vault Works. Buffalo, New York.

Buikstra, J. E.

1977 Biocultural Dimensions of Archaeological Study: A Regional Perspective. In Biocultural Adaptation in Prehistoric America. R. Blakely, editor. pp. 67-84. University of Georgia Press, Athens, Georgia .

Buikstra, J. E., and D. H. Ubelaker

1994 Standards for Data Collection from Human Skeletal Remains. Research Series No. 44. Arkansas Archeological Survey, Fayetteville, Arkansas.

Buikstra, J., J. A. O’Gorman, and C. Sutton

2000 Never Anything So Solemn: An Archeological, Biological, and Historical Investigation of the Nineteenth-Century Grafton Cemetery. Kampsville Studies in Archeology and History No. 3. Center for American Archeology, Kampsville, Illinois.

Burgess, L., D. Owsley, and J. Imlay

2007 Death, Wealth and Ornament: Coffin Hardware from Congressional Cemetery. Paper presented at the Society for Historical Archaeology 40th Annual Conference on Historical and Underwater Archaeology, January 10-14, 2007. Williamsburg, Virginia.

Burnston, S. A., and R. A. Thomas

1981 Archaeological Data Recovery at Catoctin Furnace Cemetery, Frederick County, Maryland. Mid-Atlantic Archaeological Research, Inc., Newark, Delaware. Submitted to Orr and Son, Consulting Archaeologists for Maryland Department of Transportation. 
Burton, J. F., J. D. Haines, and M. M. Farrell

2001 "I Rei To": Archaeological Investigations at the Manzanar Relocation Center Cemetery, Manzanar National Historic Site, California. Publications in Anthropology 79. Western Archeological and Conservation Center, National Park Service, U.S. Department of the Interior.

Bybee, A. D.

2002 Bioanthropological Investigations of the Reynolds Cemetery (46Ka349) in Kanawha County, West Virginia. Cultural Recourses Analysts, Inc., Hurricane, West Virginia. Prepared for Dr. Robert F. Maslowski, U.S. Army Corp of Engineers, Huntington District, West Virginia.

2003a "Risky business: potential hazards in the archaeological investigation of historic cemeteries.” Paper presented at the $5^{\text {th }}$ Annual Council for West Virginia Archeology Spring Workshop. Electronic document http://crai-ky.com/ education/reports-cem-hazards.html, accessed 2/10/2011.

2003b "Bioanthropological Investigations of Historic Cemeteries: What can we learn from biological, cultural, and mortuary remains?” Paper presented at the $5^{\text {th }}$ Annual Council for West Virginia Archeology Spring Workshop. Electronic document http://www.crai-ky.com/reports-bioanthropology.html, accessed 2/19/2011.

2003c Bioanthropological Investigations of the Burning Spring Branch Cemetery (46Ka142) in Kanawha County, West Virginia. Cultural Resource Analysts, Inc., Hurricane, West Virginia. Prepared for Dr. Robert F. Maslowski, U.S. Army Corps of Engineers, Huntington District, West Virginia.

2003d Bioanthropological Investigations of a 19th Century Cemetery (15CP61) in Campbell County, Kentucky. Cultural Resource Analysts, Inc., Lexington, Kentucky. Prepared for Mr. Doug Lambert, Palmer Engineering, Winchester, Kentucky.

2003e Bioarchaeological Investigations of Unmarked Graves at the Samuel Robinson and Upper Prater Cemeteries (15PI190 and 15PI191), Pike County, Kentucky (Item No. 12-263.00). Cultural Resource Analysts, Inc., Lexington, Kentucky. Prepared for Mr. Doug Lambert, Palmer Engineering, Winchester, Kentucky.

2004 Old Branham (15Fd94): Bioarchaeological Investigations of an Historic Cemetery, Floyd County, Kentucky (Item No. 12-301.00). Cultural Resource Analysts, Inc., Lexington, Kentucky. Prepared for Mr. David Waldner, P. E., Director, Kentucky Transportation Cabinet, Division of Environmental Analysis, Frankfort, Kentucky.

2007a Bioarchaeological Investigations of the Evans Cemetery (46MD62), McDowell County, West Virginia. Contract Publication Series WV07-03. Cultural Resource Analysts, Inc., Hurricane, West Virginia. Prepared for Mr. Timothy B. Sedosky, Potesta \& Associates, Inc., Charleston, West Virginia. 
2007b Bioarchaeological Investigations of the Alderson-Jackson Cemetery (15JS174), Jessamine County, Kentucky. Cultural Resource Analysts, Inc., Lexington, Kentucky. Prepared for Matt Steen, Development Coordinator, Bellerive Development Company, Nicholasville, Kentucky.

2007c Bioarchaeological Investigations of an Unmarked Grave at the Rudy Cemetery (15JF675), Jefferson County, Kentucky (Item No. 5-28.10). Cultural Resource Analysts, Inc., Lexington, Kentucky. Prepared for Mr. David Waldner, P. E., Kentucky Transportation Cabinet, Division of Environmental Analysis, Frankfort, Kentucky.

Bybee, A. D., and M. Richmond

2003 Data Recovery at a Nineteenth Century Cemetery (15Mm137) in Montgomery County, Kentucky (Item No. 7-320.00). Cultural Resource Analysts, Inc., Lexington, Kentucky. Prepared for Mr. Doug Lambert, Palmer Engineering, Winchester, Kentucky.

Cande, $\mathrm{K}$.

1995 The Ozarks as Destination: Phase III Archeological Investigations at the Lambert Farmstead (3CW647) and Phase II Testing at the Dement Farmstead and Cemetery (3CW685), Crawford County, Arkansas. Final Report, Project Nos. 829 and 864. Arkansas Archeological Survey, Fayetteville. Submitted to Arkansas Highway and Transportation Department.

Carr, W. S., \& Company

ca. 1890 Price list of Undertakers Hardware, W. S. Carr \& Co, Manufacturers. W. S. Carr \& Company, Baltimore, Maryland.

Casket

1919 Casket [trade magazine]. April 11, 1919.

Central Burial Casket Company

n.d. (>1919) Catalogue "A”-High Grade Burial Caskets. Central Burial Casket Company, Guthrie, Oklahoma.

Champion Company

n.d. A Lasting Tribute . . Champion Metal Burial Vaults.” Champion Company, Springfield, Ohio.

Chappell, Chase, Maxwell \& Company

1884 Illustrated Catalogue: Cloth, Velvet-covered, and Wood Finished Burial Caskets. Chappell, Chase, Maxwell \& Company, Oneida, New York.

Chattanooga Coffin \& Casket Company

1905 Wholesale Pricelist of Undertaker's Hardware, Embalming Instruments and Sundry Supplies. Chattanooga Coffin \& Casket Company, Chattanooga, Tennessee. 
1905 Catalogue No. 4. Illustrated Catalogue of Undertakers' Hardware, Embalming Instruments and Sundry Supplies. Chattanooga Coffin \& Casket Company, Chattanooga, Tennessee.

\section{Chicago Coffin Company \\ 1896 Pocket Catalogue. Chicago Coffin Company, Chicago, Illinois.}

Cincinnati Coffin Company

1876 Reduced Wholesale Price List (June 1, 1876). Cincinnati Coffin Company, Cincinnati, Ohio.

1880 Ninth Illustrated Catalogue. Cincinnati Coffin Company, Cincinnati, Ohio.

1881 Tenth Illustrated Catalogue. Cincinnati Coffin Company, Cincinnati, Ohio.

1881 Reduced Wholesale Price List (January 1881). Cincinnati Coffin Company, Cincinnati, Ohio.

1881 Supplementary Price List (July 1881). Cincinnati Coffin Company, Cincinnati, Ohio.

1882 Eleventh Annual Illustrated Catalogue. Cincinnati Coffin Company, Cincinnati, Ohio.

1882 Wholesale Price List (January 1882). Cincinnati Coffin Company, Cincinnati, Ohio.

1883 Illustrated Catalogue. Cincinnati Coffin Company, Cincinnati, Ohio.

1883 Price List (March 1, 1883). Cincinnati Coffin Company, Cincinnati, Ohio.

ca. 1890a Illustrated Casket Catalogue “D6.” Cincinnati Coffin Company, Cincinnati, Ohio.

ca. 1890b Catalogue “D7”-Paramount. Cincinnati Coffin Company, Cincinnati, Ohio.

1903 Revised Prices List of Metal Linings (June 25, 1903). Cincinnati Coffin Company, Cincinnati, Ohio.

1903 Revised Prices on Children's Caskets (June 25, 1903). Cincinnati Coffin Company, Cincinnati, Ohio.

1905 Revised Hardware Price Book, for Use in Connection with Casket Hardware Catalogue "F.3" and June Supplement (December 12, 1905). Cincinnati Coffin Company, Cincinnati, Ohio.

1906 Catalogue “F.4,” Illustrating Casket Hardware and Sundry Undertakers' Supplies. Cincinnati Coffin Company, Cincinnati, Ohio. 
1912 Casket Hardware Catalogue F5. Cincinnati Coffin Company, Cincinnati, Ohio.

Claasen, C.

1994 Washboards, Pigtoes, and Muckets: History of Musseling in the Mississippi Watershed. Historical Archaeology 28(2).

Clark Grave Vault Company

ca. 1950 “And Most Important of All ... ” Clark Grave Vault Company, Columbus, Ohio.

1961 Price Card (Effective November 4, 1961). Clark Grave Vault Company, Columbus, Ohio.

Cleveland Burial Case Company

1882 Illustrated Catalogue of Wood, Cloth Covered and Metallic Caskets. Cleveland Burial Case Company, Cleveland, Ohio.

ca. 1880 [hardware catalogue]. Cleveland Burial Case Company, Cleveland, Ohio.

ca. 1890 Illustrated Catalogue of Undertakers' Silver Plated Hardware, Casket and Coffin Handles, Name Plates, Thumbscrews, Thumbscrew Plated, Tack, Ornaments, Escutcheons and Hardware Sundries, Robes, Linings, and Undertakers' Dry Goods. Cleveland Burial Case Company, Cleveland, Ohio.

1920 Price List-Pedestals and Candelabras (April 1, 1920). Cleveland Burial Case Company, Cleveland, Ohio.

Cobb, D. E.

1999 "Such a Lonely Place?" An Archaeological and Osteological Investigation of the Brunson-Sisson Cemetery (11WI874), Will County, Illinois. Illinois State Museum Society, Contract Archaeology Program, Technical Report No. 98-1264-26. Illinois State Museum, Quaternary Studies Program. Submitted to Material Service Corporation, Lyons, Illinois.

\section{Columbus Coffin Company}

1882 Illustrated Catalogue Wood \& Cloth Covered Coffins \& Caskets, Undertakers Hardware. Columbus Coffin Company, Columbus, Ohio.

Condon, C. G., J. L. Becker, H. J. Edgar, J. M. Davidson, J. R. Hoffman, P. Kalima, D. Kysar, S. Moorehead, V. M. Owens, and K. Condon

1998 Freedman's Cemetery: Site 41DL316, Dallas, Texas, Assessments of Sex, Age at Death, Stature, and Date of Interment for Excavated Burials. Report No. 9. Archaeology Studies Program, Environmental Affairs Division, Texas Department of Transportation, Austin, Texas.

Connolly, T. J., Christopher L. Ruiz, Jeanne McLaughlin, Guy L. Tasa, and Elizabeth Kallenbach 2008 Rediscovery and Recovery of the Stevens Family Pioneer Cemetery at River Bend, Springfield, Oregon. Museum Report 2008-058. Museum of Natural and Cultural History and State Museum of Anthropology, University of Oregon, Eugene, Oregon. Prepared for PeaceHealth Sacred Heart Medical Center at River Bend. 
2010 The Archaeology of a Pioneer Family Cemetery in Western Oregon, 1854-1879. Historical Archaeology 44(4):28-45.

Constantine Casket Company

n.d. (ca. 1890s) Illustrated and Descriptive Catalogue of Fine Cloth Covered Burial Cases. Constantine Casket Company, Constantine, Michigan.

Cooper, J. H., A. L. Tiné, M. Prior, C. M. Clow, D. Shanabrook, and E. Salo

2000 Cultural Resources and Bioarchaeological Investigations at the Dallas Convention Center and Pioneer Cemetery, Dallas, Texas. Miscellaneous Reports of Investigations Number 205. Geo-Marine, Inc., Plano, Texas. Prepared for HKS, Inc., Dallas, Texas.

Corbin, P. \& F.

1859 Illustrated Catalogue and Price List. P. \&. F. Corbin, New Britain, Connecticut.

1866 Price List, Manufacturers of Wrought Cast Brass . . Hinges, Coffin Trimmings . . . P. \& F. Corbin, New Britain, Connecticut.

Cordova, K. J., and N. J. Anderson

2010 FM 1464 at New Home Cemetery between Stratford Creek Drive and Oyster Creek, CSJ 1415-02-032. PBS\&J. Submitted to the Texas Department of Transportation.

Costello, J. G.

1991 Excavation of Burials from the Madam Felix/Hettick Cemetery, CA-CAL-1122H. Foothill Resource Associates, Mokelumne Hill, California. Prepared for Meridan Gold Company, Copperpolis, California.

Craig, J., and C. S. Larsen

1993 Life and Death on the Illinois Prairie: Archaeological and Osteological Investigations at the Cross Cemetery, Springfield, Illinois. Hanson Engineers, Inc., Springfield, Illinois. Prepared for Roosevelt National, Springfield, Illinois.

Crane, Breed \& Company

1858 Fisk's \& Crane's Patent Metallic Burial Cases and Caskets- "Air-Tight and Indestructible for Protecting and Preserving the Dead." Crane, Breed \& Company, Cincinnati, Ohio.

1865 Wholesale Prices of Plain Cases, Crane’s Metallic Burial Casket, etc. Crane, Breed \& Company, Cincinnati, Ohio.

1867 Wholesale Pricelist of Patent Metallic Burial Cases and Caskets, Hearses, Name Plates, etc. Crane, Breed \& Company, Cincinnati, Ohio.

1875 Illustrated Catalogue and Price List of Patent Metallic Burial Cases and Caskets. Crane, Breed \& Company, Cincinnati, Ohio.

1877 Illustrated Catalogue of Undertakers’ Goods. Crane, Breed \& Company, Cincinnati, Ohio. 
1895 Illustrated Catalogue of Hearses and Trimmings. Crane, Breed \& Company, Cincinnati, Ohio.

1910 Catalogue “D,” Burial Garments, Robes, Casket Linings, Pillow and Lining Sets, Door Crapes. Crane \& Breed Mfg. Co., Cincinnati, Ohio.

Crawford, K.

2003 Archaeological Investigations of St. Peter's Anglican Church Property, 240 College Street, Cobourg, County of Northumberland, Ontario. Archaeological Services, Inc., Toronto, Ontario, Canada. Prepared for AE VOS Corporation, Toronto, Ontario, Canada; Anglican Diocese of Toronto, Toronto, Ontario, Canada; and Mr. Michael D’Mello, Registrar, Cemeteries Section, Ministry of Consumer and Business Services, Toronto, Ontario, Canada.

Crawford, K., A. Hutcheson, E. MacDonald, B. Nahri, D. Robertson, and R. Williamson

2008 Ontario Cemeteries Act Site Investigation, the Old Don Jail Burial Area, Proposed Draft Plan of Subdivision, Part of Lot 15, Concession 1 from the Bay, Geographic Township of York and Part of Block U, Don Improvement Plan, City of Toronto. Archaeological Services, Inc., Toronto, Ontario, Canada. Prepared for Bridgepoint Health, Toronto, Ontario, Canada, and Cemeteries Regulation Unit, Ministry of Small Business and Consumer Services, Toronto, Ontario, Canada.

Crawfordsville Casket Company

ca. 1900 Illustrated Catalogue No. 9 of Coffins, Caskets, Dry Goods, Hardware, etc. Crawfordsville Casket Company, Crawfordsville, Indiana.

Crescent Sales

2009 Funeral Link 12(11).

2010 Funeral Link 13(11).

2011 Funeral Link 14(1).

Crist, T. A. J., W. H. Henry, J. W. Joseph, R. H. Pitts, W. P. Catts, A. Caton, A. Washburn, and S. Norris

2000 With Death Came Liberty: The Archaeology and History of the Sam Goode Cemetery, Mecklenburg County, Virginia. Technical Report No. 747. Contract DACW21-99-D-0004, Delivery Order 0005. New South Associates, Stone Mountain, Georgia. Submitted to the U.S. Army Corps of Engineers, Savannah District, Georgia.

Crist, T. A. J., R. H. Pitts, A. Washburn, J. P. McCarthy, and D. G. Roberts

1996 "A Distinct Church of the Lord Jesus": The History, Archaeology, and Physical Anthropology of the Tenth Street First African Baptist Church Cemetery, Philadelphia, Pennsylvania. Site Number 36PH72. Vine Expressway (I-676), L.R. 67045. ER\# 82-0133-101-W. John Milner Associates, Inc., Philadelphia, Pennsylvania. Prepared for Gaudet \& O’Brien Associates/Urban Engineers, Inc., Philadelphia, Pennsylvania, and Department of Transportation, Commonwealth of Pennsylvania, St. Davids, Pennsylvania. 
Crow, M. S.

2004 Mortuary Practice in Sociohistorical and Archaeological Contexts: Texas, 18211870. Master’s thesis. Texas A\&M University, College Station, Texas.

Cultural Geography

2011 “Cultural Geography.” Electronic document http://en.wikipedia.org/wiki/ Cultural_geography, accessed 6/4/2011.

Cultural Resources, Inc.

2002 Results of Excavation of a Human Burial at Site 44SK481 Located at the Former Nansemond Ordnance Station, Suffolk, Virginia. Cultural Resources, Inc., Fredericksburg, Virginia. Prepared for the U.S. Army Corps of Engineers, Norfolk District.

Dallas Coffin Company

ca. 1910 Catalogue “G.” Dallas Coffin Company, Dallas, Texas.

Dase, A. E.

2004 Hell-Hole on the Brazos: A Historic Resources Study of Central State Farm, Fort Bend County, Texas. Technical Reports Number 70. Prewitt and Associates Inc., Austin, Texas. Submitted to Berg-Oliver Associates, Inc.

Davidson, J. M.

1999 Freedman's Cemetery (1869-1907): A Chronological Reconstruction of an Excavated African-American Burial Ground, Dallas, Texas. Master's thesis, Department of Anthropology, University of Arkansas, Fayetteville, Arkansas.

2004 Mediating Race and Class Through the Death Experience: Power Relations and Resistance Strategies of an African-American Community, Dallas, Texas (18691907). Ph.D. dissertation, The University of Texas, Austin, Texas. University Microfilm International, Ann Arbor, Michigan.

2006 Material Culture, Chronology, and Socioeconomics. In Two Historic Cemeteries in Crawford County, Arkansas, edited by R. C. Mainfort, Jr., and J. M. Davidson, pp. 97-218. Research Series No. 62. Arkansas Archeological Survey, Fayetteville, Arkansas.

Davidson, James M., Jerome C. Rose, Myron P. Gutmann, Michael R. Haines, Keith Condon, and Cindy Condon

2002 The Quality of African-American Life in the Old Southwest near the Turn of the Twentieth Century. In The Backbone of History: Health and Nutrition in the Western Hemisphere, edited by R. H. Steckel and Jerome C. Rose, pp. 226-277. Cambridge University Press, Cambridge. 
DeCunzo, L., A. Hoseth, J. Hodny, J. E. Jamison, W. P. Catts, and D. C. Bachman

1992 Final Archaeological Investigations at the John Darrach Store Site, Delaware Route 6-Woodland Beach Road, Smyrna Section, Delaware Route 1 Corridor, Kent County, Delaware. Delaware Department of Transportation Archaeology Series No. 93. University of Delaware, Department of Anthropology, Center for Archaeological Research. Submitted to U.S. Department of Transportation, Federal Highway Administration, and Delaware Department of State, Division of Historical and Cultural Affairs, Bureau of Archaeology and Historic Preservation. Prepared for Delaware Department of Transportation, Division of Highways, Location and Environmental Studies Office.

Deetz, J.

1977 In Small Things Forgotten. An Archaeology of Early American Life. Anchor Books/Random House, Inc., New York.

1996 In Small Things Forgotten. Revised expanded edition. Anchor Books, New York, New York.

Deming, J., M. M. Almy, J. S. Matthews, and S. H. Koski

1993 Report on the Excavation of 8LL1758 as Contained Within the Proposed U.S. 41 Business Right-of-Way on Fowler Street Between First and Second Streets in the City of Fort Myers, Lee County, Florida. Archaeological Consultants, Inc., Sarasota, Florida. Prepared for Florida Department of Transportation, District One, Bartow, Florida.

Dockall, H. D., J. F. Powell, and D. G. Steele

1996 Home Hereafter: An Archaeological and Bioarchaeological Analysis of an Historic African-American Cemetery (41GV125). Reports of Investigations No. 5. Center for Environmental Archaeology, Texas A\&M University, College Station, Texas.

Dockall, H. D., D. K. Boyd, M. D. Freeman, R. L. Garza, K. E. Stork, K. W. Kibler, and J. E. Baker

1996 Confederate Veterans At Rest: Archaeological and Bioarchaeological Investigations at the Texas State Cemetery, Travis County, Texas. Reports of Investigations Number 107. Prewitt and Associates, Inc., Austin, Texas. Submitted to Texas Parks and Wildlife Department, Austin, Texas.

Doddridge, John E., Inc.

n.d. [Casket Advertising Cards]. John E. Doddridge, Inc., Richmond, Indiana.

Dodge Chemical Company

1963 Catalogue of Embalming Chemicals, Cosmetics, Instruments, and Supplies. Dodge Chemical Company, Boston, Massachusetts.

1963 Supplementary Price List (October 1, 1963). Dodge Chemical Company, Boston, Massachusetts. 
Doherty, J. L. (editor)

1981 Women at Work. 153 Photographs by Lewis W. Hine. George Eastman House, Rochester, in association with Dover Publications, Inc., New York.

Dominion Manufacturers, Ltd.

n.d. (post-1906) Catalogue No. 30 Illustrating Casket Hardware Designed \& Manufactured by ... Dominion Manufacturers, Ltd., Toronto, Ontario, Canada.

1940 Dominion Equipment and Sundries. Dominion Manufacturers, Ltd., Toronto, Ontario, Canada.

Dottridge Brothers, Ltd.

n.d. $\quad$ Price List of Finished Coffins, List No. 1058. Dottridge Brothers, Ltd., London, England.

n.d. Wholesale Coffin List. Dottridge Brothers, Ltd., London, England.

n.d. Wholesale Coffin Furniture \& Drapery List [Price List]. Dottridge Brothers, Ltd., London, England.

n.d. Wholesale Coffin Furniture \& Drapery List. Dottridge Brothers, Ltd. London, England.

1965 Coffin Furniture. Dottridge Brothers, Ltd., London, England.

Durfee Embalming Fluid Company

1900 [Catalog] Durfee Embalming Fluid Company, Grand Rapids, Michigan.

n.d. Cemetery Tents and Accessories. Durfee Embalming Fluid Company, Grand Rapids, Michigan

Earls, A. C., C. R. Lintz, G. W. Gill, P. L. O’Neill, and W. N. Trierweiler

1991 Investigations of Historic Cemeteries at O. H. Ivie Reservoir, Coleman, Concho, and Runnells Counties, Texas. Technical Report No. 403. Mariah Associates, Austin, Texas.

Eckels, H. S., \& Company

1903 Eckels' Undertakers' Directory, Reference Book and Shipping Guide. H. S. Eckels \& Company, Philadelphia, Pennsylvania.

ca. 1939 Professional Supplies for the Mortician: Catalogue of Preservatives, Disinfectants, Antiseptics, Cosmetics, Instruments, Leather Goods, Operating Room Equipment, and Mortuary Equipment. H. S. Eckels \& Company, Philadelphia, Pennsylvania.

ca. 2008 Impressions. H. S. Eckels \& Company, Philadelphia, Pennsylvania. 
Edgerton, C. E.

1897 The Wire-Nail Association of 1895-1896. Political Science Quarterly 12(2):246272.

Edwards, D. M., L. Hager, and R. Jackson

2005 Former Sacramento County Hospital Burial Ground Excavation, University of California Davis Medical Center, Radiation Oncology Expansion Project. Pacific Legacy, Inc., Cameron Park, California. Prepared for the University of California Davis Medical Center, Sacramento, California.

Edwards, J. D., and T. Wells

1993 Historic Louisiana Nails: Aids to the Dating of Old Buildings. The Fred B. Kniffen Cultural Resources Laboratory Monographs Series No. 2. Geoscience Publications. Department of Geography \& Anthropology, Louisiana State University.

Elia, R. J., and A. B. Wesolowsky (editors)

1991 Archaeological Excavations at the Uxbridge Almshouse Burial Ground in Uxbridge, Massachusetts. BAR International Series 564. Tempus Reparatum: Archaeological and Historical Associates Limited, Oxford, England.

Elliott, D. T., and R. F. Elliott

1989 Bethany Cemetery, Effingham County, Georgia. Ebenzer Archaeological Report Series No. 2. Elliott and Elliott, Athens, Georgia.

Ellis, George Washington

1914 Negro Culture in West Africa. The Neale Publishing Co., New York, New York.

Epstein, S. M.

1981 A Coffin Nail From the Slave Cemetery at Catoctin, Maryland. MASCA (Museum Applied Science Center for Archaeology) Journal 1(7):208-210.

Espenshade, C. T.

2004 Historic Grave Removal and Reinterment: Final Report-Clarion County, Pennsylvania, Shippenville Borough, S.R. 0322, Section 370, Shippenville Intersection Improvement Project. Skelly and Loy, Inc., Monroeville, Pennsylvania. Prepared for Pennsylvania Department of Transportation, Engineering District 10-0.

Ezell, R., and C. A. Huston

2006a Archaeological Removal of Historic Burials at the Williams-Green Cemetery (44CU134) on the Continental 181 Fund, LLC Tract in Culpeper County, Virginia. ECS Mid-Atlantic, LCC, Fredericksburg, Virginia. Prepared for Ms. Sara Schrank, Continental Properties Company, Inc., Continental 181 Fund, LLC, Menomonee Falls, Wisconsin.

2006b Archaeological Removal of Five Historic Burials from Site $44 S T 00613$ at the Quantico Corporate Center Tract, Stafford County, Virginia. ECS Mid-Atlantic, LLC, Fredericksburg, Virginia. Prepared for Mr. Samer Shalaby, Development Consulting Services, PLC, Fredericksburg, Virginia. 
Farrell, J. J.

1980 Inventing the American Way of Death, 1830-1920. Temple University Press, Philadelphia, Pennsylvania.

Faulkner, W.

1942 Go Down Moses. First Vintage International Edition 1990. Random House, Inc., New York.

Ferguson, B. H.

1983 Final Report on the McGee Creek Cemetery Relocations, Atoka County, Oklahoma. Bureau of Reclamation, McGee Creek Project, Farris, Oklahoma.

Ferguson, B. H., S. K. Ireland, G. A. Agogino, and R. Holloway

1993 "And they laid them to rest in the little plot beside the Pecos": Final Report on the Relocation of the Old Seven Rivers Cemetery, Eddy County, New Mexico, Vol 1. Soil Conservation Service, Fort Worth, Texas. Denver Office of the Bureau of Reclamation, Denver, Colorado.

Fontana, B. L.

1965 The Tale of a Nail: On the Ethnological Interpretation of Historic Artifacts. The Florida Anthropologist 18(3, Part 2):85-101.

Fontana, B. L., and J. C. Greenleaf

1962 Johnny Ward’s Ranch: A Study in Historic Archaeology. Kiva 29(1-2):1-115.

Foster, E., and L. A. Nance (editors)

2002 Archaeological Investigation Report: Allen Parkway Village, 41HR886, Houston, Harris County, Texas. PBS\&J, Austin, Texas. Prepared for the Housing Authority of the City of Houston, Texas.

Fox, A. A.

1984 A Study of Five Historic Cemeteries at Choke Canyon Reservoir, Live Oak and McMullen Counties, Texas. Choke Canyon Series, Vol. 9. Center for Archaeological Research, The University of Texas, San Antonio, Texas.

Freedom Casket Company

1921 Revised Price List Applying to Catalogue “E” (May 2, 1921). Freedom Casket Company, Freedom, Pennsylvania.

1923 Revised Price List Applying to Catalogue “E” (February 26, 1923). Freedom Casket Company, Freedom, Pennsylvania.

Frigid Fluid Company

2010 Embalming Catalog. Frigid Fluid Company, Northlake, Illinois.

2010 Funeral Home Catalog. Frigid Fluid Company, Northlake, Illinois.

2010 Flower Vases Offered by Frigid Fluid Co. Frigid Fluid Company, Northlake, Illinois. 
Gadus, E. F., J. E. Baker, and A. E. Dase

2002 "A mother left to mourn": Archaeological and Historical Investigations at a Nineteenth-Century Family Cemetery at the Jewett Mine, Freestone County, Texas. Reports of Investigations Number 136. Prewitt and Associates, Inc., Austin, Texas. Submitted to Northwestern Resources Company, Jewett, Texas.

Gardner, J.

2009 Union Bethel AME Church Cemetery Relocation. Letter Report. Brockington and Associates, Inc., Jacksonville, Florida. Prepared for Mr. John D. Stephens, Stephens MDS LP, College Park, GA.

Garner, B., I. Miklavcic, and T. Brennan

2001 The Kniseley Family Cemetery, Lots 27 and 28, Concession 3, Elm Street, City of Port Colborne, Ontario. Archaeological Services, Inc., Toronto, Ontario. Submitted to the Registrar of Cemeteries, Ministry of Consumer \& Commercial Relations, Toronto, Ontario.

Garrow, P. H.

1985 A Preliminary Seriation of Coffin Hardware Forms in Nineteenth and Twentieth Century Georgia. Early Georgia 15(1-2):19-45.

1990 Archaeological Investigations of the Sandy Creek Cemetery, Lot 31, Block "B," Sandy Creek Estates, Clark County, Georgia. Garrow and Associates, Atlanta, Georgia.

Garrow, P. H., and S. A. Symes

1987 The Big Lazer Creek Unmarked Cemetery: A Multidisciplinary Investigation. Garrow and Associates, Atlanta, Georgia.

Garrow, P. H., S. A. Symes, and H. W. Case

1985 Physical Anthropology and Archaeological Investigations of the Nancy Creek Primitive Baptist Church Cemetery, Chamblee, Georgia. Garrow and Associates, Inc., Atlanta, Georgia. Prepared for Parsons, Brinckerhoff, Quade \& Douglas, Inc./ Tudor Engineering Company, MARTA N760/Cemetery Contract No. EX3-2.

Gate City Coffin Company

1901 Catalogue F. Gate City Coffin Company, Atlanta, Georgia.

ca. 1904 Catalogue No. G, Illustrating Coffin and Casket Hardware and Undertakers' Supplies, Embalming Instruments, etc. Gate City Coffin Company, Atlanta, Georgia.

Globe Casket Manufacturing Company

n.d. $\quad$ Illustrated Catalogue of Cloth Covered Burial Caskets. Globe Casket Mfg. Co., Kalamazoo, Michigan.

ca. 1935 Casket Catalogue No. 12. Globe Casket Mfg. Co., Kalamazoo, Michigan. 
Goldstein, L. G., and J. E. Buikstra

2004 A Nineteenth-Century Rural Irish Cemetery in McDonough County, Illinois. In An Upper Great Lakes Archaeological Odyssey: Essays in Honor of Charles E. Cleland, edited by W. A. Lovis, pp. 43-63. Cranbrook Institute of Science, Wayne State University Press.

Goodman, A. H., and G. J. Armelagos

1989 Infant and Childhood Morbidity and Mortality Risks in Archaeological Populations. World Archaeology 21(2):225-243.

Goodman, A. H., R. B. Thomas, A. C. Swedlund, and G. J. Armelagos

1988 Biocultural Perspectives on Stress in Prehistoric, Historical, and Contemporary Population Research. Yearbook of Physical Anthropology 31:169-202.

Gordon, W. A.

2003 Coffin Plates and Competitive Display. Ph.D. dissertation, Department of Anthropology, Arizona State University, Tuscon, Arizona. University Microfilms International, Ann Arbor, Michigan.

Gresham, T. H., and G. Martin

1999 Archaeological Disinterment of the Brassel Cemetery, Jefferson County, Georgia. Southeastern Archaeological Services, Inc., Athens, Georgia. Prepared for the Georgia Department of Transportation, Office of Environment/Location, Atlanta, Georgia.

Grey, A. E., M. D. Patten, and M. S. Warner

1993 A Preliminary Archaeological Assessment of the Venable Lane Site. University of Virginia, Department of Anthropology. Submitted to the Facilities Planning and Construction Department, Facilities Management, University of Virginia.

Gust, S., A. Diaz, and K. Houck

2006 The Historic Los Angeles Cemetery (CA-LAN-3553) Summary Report, Los Angeles Metro Gold Line Project, East Portal Area, Los Angeles, CA. Cogstone Resources Management Inc., Santa Ana, California. Submitted to UltraSystems Environmental, Inc., Irvine, California, and Metropolitan Transportation Authority, Los Angeles, California.

Haase, Wilbert W. Co.

1954 Wilbert Burial Vaults: The Foremost Name in Burial Vaults. Wilbert W. Haase Co., Forest Park, Illinois.

Habenstein, R. W., and W. M. Lamers

1955 The History of American Funeral Directing. Bulfin Printers, Inc., Milwaukee, Wisconsin.

1960 Funeral Customs the World Over. Buflin Printers, Inc., Milwaukee, Wisconsin.

1985 The History of American Funeral Directing. 2nd revised edition. National Funeral Directors Association, Milwaukee, Wisconsin. 
Hacker, D., and M. Trinkley

2010 Analysis of Remains from Mount Olive Cemetery, Portsmouth, Virginia. Research Contribution No. 535. Chicora Foundation, Inc., Columbia, South Carolina.

Hacker-Norton, D., and M. Trinkley

1984 Remember Man Thou Art Dust: Coffin Hardware of the Early Twentieth Century. Research Series 2. Chicora Foundation, Inc., Columbia, South Carolina.

Halo International Corp.

2008 Unique Funeral Supplies. Halo International Corp., Twinsburg, Ohio.

Hamilton Casket Company, Inc.

ca. 1940 [Casket Advertising Sheet]. Hamilton Casket Company, Inc., Chicago, Illinois.

Hamilton, Lemmon, Arnold \& Company

1879 June 7, 1879, Price List (Coffin Hardware). Hamilton, Lemmon, Arnold \& Company, Allegheny City, Pennsylvania, and Pittsburgh, Pennsylvania.

1880 Reduced Price List; Price List of Hardware and Trimmings (June 23, 1880). Hamilton, Lemmon, Arnold \& Company, Pittsburgh, Pennsylvania, and Allegheny, Pennsylvania.

1881 Revised Price List of the Excelsior Coffin and Casket Works (March 8, 1881). Hamilton, Lemmon, Arnold \& Company, Pittsburgh, Pennsylvania, and Allegheny, Pennsylvania.

1882 Illustrated Catalogue of Varnished and Cloth Covered Burial Cases and Caskets. Hamilton, Lemmon, Arnold \& Company, Pittsburgh, Pennsylvania, and Allegheny, Pennsylvania.

1882 Excelsior Coffin and Casket Works: Price Lists of Hardware, Robes, Linings, Trimmings, etc. Manufactured by Hamilton, Lemmon, Arnold \& Company (March 1, 1882). Hamilton, Lemmon, Arnold \& Company, Pittsburgh, Pennsylvania, and Allegheny, Pennsylvania.

1884 Excelsior Coffin \& Casket Works Price List of Hardware, Robes, Linings, Trimmings, \&C. Manufactured by Hamilton, Lemmon, Arnold \& Company. Hamilton, Lemmon, Arnold \& Company, Pittsburgh, Pennsylvania, and Allegheny, Pennsylvania.

1884 Revised Price List of Varnished \& Cloth-Covered Burial Cases and Caskets Manufactured by Hamilton, Lemmon, Arnold \& Company, Excelsior Coffin \& Casket Works (January 16, 1884). Hamilton, Lemmon, Arnold \& Company, Pittsburgh, Pennsylvania, and Allegheny, Pennsylvania.

1886 Excelsior Coffin \& Casket Works: Revised Price List of Coffin \& Casket Hardware, Manufactured by Hamilton, Lemmon, Arnold \& Company (November 1, 1886). Hamilton, Lemmon, Arnold \& Company, Pittsburgh, Pennsylvania, and Allegheny, Pennsylvania. 
1886 Excelsior Coffin and Casket Works: Price List of Wrappers, Robes, Linings, Trimmings, \& etc. Manufactured by Hamilton, Lemmon, Arnold \& Company (November 1, 1886). Hamilton, Lemmon, Arnold \& Company, Pittsburgh, Pennsylvania and Allegheny, Pennsylvania.

1887 Price List and Telegraph Key of Varnished and Cloth Covered Burial Cases and Caskets (January 1, 1887). Hamilton, Lemmon, Arnold \& Company, Pittsburgh, Pennsylvania, and Allegheny, Pennsylvania.

1888 Price List of Wrappers, Robes, Linings, Trimmings etc. (February 1, 1888). Hamilton, Lemmon, Arnold \& Company, Pittsburgh, Pennsylvania, and Allegheny, Pennsylvania.

1888 Revised Price List of Coffin \& Casket Hardware (February 1, 1888). Hamilton, Lemmon, Arnold \& Company, Pittsburgh, Pennsylvania, and Allegheny, Pennsylvania.

Harrisburg Burial Case Company

ca. 1885 Illustrated Catalogue of Coffins, Caskets, and Undertakers' Supplies. Harrisburg Burial Case Company, Harrisburg, Pennsylvania.

Hawley Bros. Hardware Company

1884 No. 15 Price List \& Illustrated Catalog of Hardware \& Agricultural Implements. Hawley Bros. Hardware Company, San Francisco, California.

Heege, $\mathrm{K}$.

1998 “They're Pretty, but They're Work”: Shell-Decorated Graves as Community Art. Folklore Forum 29(1):65-98.

Heilen, M., and M. A. Gray (editors)

2010 Deathways and Lifeways in the American Southwest: Tucson's Historic AlamedaStone Cemetery and the Transformation of a Remote Outpost into an Urban City. 3 vols. Statistical Research, Inc., Tucson, Arizona. Submitted to Roger Anyon, Pima County Cultural Resources and Historic Preservation Office, Tucson, Arizona.

Heringer, T. M., Coroner and N. Haywood

1980 Preliminary Report on the Casket and Human Remains from the Site of Old St. Andrew Roman Catholic Cemetery. Wanikan (Newsletter of the Thunder Bay Chapter of the Ontario Archaeological Society) 80(4):7-10.

Hill, F. H. \& Company

ca. 1880 Price List to Accompany Illustrated Catalogue of 1880. F. H. Hill \& Co., Chicago, Illinois.

1881 Illustrated Catalogue of Burial Cases and Caskets, and Undertakers' Supplies. F. H. Hill \& Co., Chicago, Illinois.

1884 Prices of Wood Burial Cases and Caskets (associated with the No. 15 Catalogue) (January 16, 1884). F. H. Hill \& Co., Chicago, Illinois. 
1884 Reduced Prices of Wood Burial Cases and Caskets (associated with the No. 15 Catalogue) (May 20, 1884). ). F. H. Hill \& Co., Chicago, Illinois.

ca. 1923 Bronze, Copper, Steel, Hardwood \& Cloth Covered Caskets, Casket Hardware, Metal Linings, Dry Goods and Vaults. F. H. Hill \& Co., Chicago, Illinois.

Hill, M. C.

2001 Porotic Hyperostosis as an Indicator of Anemia: an Overview of Correlation and Cause. Unpublished Ph.D. dissertation, Department of Anthropology, University of Massachusetts/Amherst.

Hill, M. C., and G. J. Armelagos

1990 Porotic hyperostosis in Past and Present Perspective. In A Life in Science: Papers in Honor of J. Lawrence Angel, edited by J. Buikstra; pp. 52-63. Scientific Papers 6. Center for American Archeology, Kampsville, Illinois.

Hill, M. C., M. L. Blakey, and M. Mack

1995 Women, endurance, enslavement: exceeding the physiological limits. Paper presented at the American Association of Physical Anthropology Annual Meeting, Oakland, California.

Hilliard, J., J. Davidson, and L. Donat

n.d. Archeological Investigation of the Droke Graveyard, 3BE655, Benton County, Arkansas. Manuscript on file at the Arkansas Archeological Survey, Fayetteville, Arkansas.

Hillson, S.

1996 Dental Anthropology. Cambridge University Press, United Kingdom.

History of African American Death

2011 "The History of African American Death: Superstitions, Traditions, and Procedures." Electronic document http://northbysouth.kenyon.edu/1998/death/deathhistory.htm, accessed 6/7/2011.

Hogue, S. H., and J. S. Alvey

2006 Final Report on Archaeological Burial Recovery at Pepper Hill I Cemetery, 22LO998, Lowndes County, Mississippi. Cobb Institute of Archaeology, Mississippi State University, Mississippi State, Mississippi. Prepared for Weyerhaeuser Pulp and Paper Plant, Columbus, Mississippi.

Holland Supply Company

ca. 2006 [Catalog]. Holland Supply Company, Holland, Missouri.

Hughes, E., and M. Lester

1991 The Big Book of Buttons. Boyertown Publishing, Boyertown, Pennsylvania. 
Hutcheson, A., D. Robertson, A. Veilleux, and R. F. Williamson

2008 Cemetery Investigation at the Church of the Assumption of Our Lady, Part of Lots 535 and 536, Village of Bell Ewart, Town of Innisfil, Simcoe County, Ontario. Archaeological Services, Inc., Toronto, Ontario, Canada. Prepared for Culture Programs Unit, Programs and Services Branch, Ontario Ministry of Culture, Toronto, Ontario, Canada.

Hydrol Chemical Company

2010 Funeral Supply Catalog: Hydrol No. 74. Hydrol Chemical Company, Philadelphia, Pennsylvania.

Imperial Casket Company

1935 Price List: Wood Burial Caskets, Metal Caskets, Hardwood Caskets, Vaults, Burial Garments (April 18, 1935). Imperial Casket Company, Kansas City, Missouri

ca. 1935 [Catalog]. Imperial Casket Company, Kansas City, Missouri.

Işcan, M. Y., and K. A. R. Kennedy (editors)

1989 Reconstruction of Life from the Skeleton. Alan R. Liss, Inc., New York.

Işcan, M. Y., S. R. Loth, R. K. Wright

1985 Age estimation from the rib by phase analysis. Journal of Forensic Sciences 30:853863.

Ives \& Allen

1871 Price List and Illustrated Catalogue of Hardware. Ives \& Allen, Montreal, Québec, Canada.

Jaffe, H. L.

1972 Metabolic, Degenerative, and Inflammatory Diseases of Bones and Joints. Lea and Febiger, Philadelphia, Pennsylvania.

Jamieson, R. W.

1995 Material culture and social death: African-American burial practices. Historical Archaeology 29(4):39-58.

Janaway, R.

1998 An Introductory Guide to Textiles from 18th and 19th Century Burials. In Grave Concerns: Death and Burial in England, 1700 to 1850. CBA Research Report No. 113, pp. 17-32. Council for British Archaeology, York, UK.

Jeane, D. G.

1989 The Upland South Folk Cemetery Complex: Some Suggestions of Origin. In Cemeteries and Gravemarkers: Voices of American Culture, edited by R. E. Meyer, pp. 107-136. Utah State University Press, Logan, Utah.

Johnson, J. Oliver, Inc.

1934 Catalog T-34, "Everything for the Cemetery.” J. Oliver Johnson, Inc., Chicago, Illinois. 
Jones, B. C.

1992 Archaeological Evaluation of Lions Club Lot in Cedar Key, Florida: Salvage of Historic Burials and Preservation of Weeden Island (Pasco) Burial Area. Florida Archaeological Reports No. 9. Bureau of Archaeological Research, Division of Historical Resources, Florida Department of State, Tallahassee, Florida.

Jones, J. B., S. C. Pullins, C. Birkett, E. J. Monroe, M. E. Lamb, and W. H. Moore

2006 Archaeological Data Recovery at Site 44PO126 Associated with the Proposed Route 288 Project, Powhatan County, Virginia. William and Mary Center for Archaeological Research, The College of William and Mary, Williamsburg, Virginia. Prepared for Virginia Department of Transportation, Richmond, Virginia, VDOT Project 0288-072-104.

Jordan, T. G.

1982 Texas Graveyards. A Cultural Legacy. The University of Texas Press, Austin, Texas.

Jordan-Bychkov, T. G., M. Domosh, and L. Rowntree

1994 The Human Mosaic: a Thematic Introduction to Cultural Geography. Harper Collins College Publishers, New York.

Joseph, J. W., M. B. Reed, and C. E. Cantley

1991 Agrarian Life, Romantic Death: Archaeological and Historical Testing and Data Recovery for the I-85 Northern Alternative, Spartanburg County, South Carolina. Technical Report 39. New South Associates, Stone Mountain, Georgia. Submitted to the South Carolina Department of Highways and Public Transportation, Columbia, South Carolina.

Jurney, D. H.

1987 Cut and Wire Nails: Functional and Temporal Interpretations. In Historic Buildings, Material Culture, and People of the Prairie Margin, edited by D. H. Jurney, and Randall W. Moir, pp. 83-96. Archaeology Research Program, Institute for the Study of Earth and Man, Southern Methodist University, Dallas, Texas.

Katz, S. R., and M. Haynes

1986 Twixt God and Man: An Historical Study of the Seven Rivers Cemetery. Manuscript on file, Bureau of Reclamation, Denver, Colorado.

Katzenberg, M. A., and S. R. Saunders

2000 Biological Anthropology of the Human Skeleton. Wiley-Liss, New York.

Kelco Supply Company

2002a A Comprehensive Source for the Deathcare Profession (Directories A-E). Kelco Supply Company, Minneapolis, Minnesota.

2002b Directory G—General Supply Price Guide. Kelco Supply Company, Minneapolis, Minnesota. 
Kellan, A.

1998 "Bones Reveal Little-Known Tale of New York Slaves.” Electronic document http://www.cnn.com/TECH/9802/12/t_t/burial.ground/, accessed 5/24/2011.

Kennedy, K. A. R.

1989 Skeletal Markers of Occupational Stress. In Reconstruction of Life from the Skeleton, edited by M. Y. Isçan and K. A. R. Kennedy, pp. 129-160. Alan R. Liss, Inc., New York.

Keystone Coffin \& Casket Works

1875 Illustrated Catalogue and Price List of Coffins and Caskets . . Keystone Coffin \& Casket Works, Alleghany City, Pennsylvania.

King, J. A., and D. H. Ubelaker

1996 Living and Dying on the 17th Century Patuxent Frontier. Maryland Historical Trust Press, Crownsville, Maryland.

King, M. K., and B. P. Miller

1991 Archaeological Investigations, Haul Road Trenching, and Disturbance Documentation of the Piggery Point Burials on Deer Island, Boston, Massachusetts. The Public Archaeology Laboratory, Inc., Pawtucket, Rhode Island. Submitted to Massachusetts Water Resources Authority, Boston, Massachusetts.

Kogon, S. L., and R. G. Mayer

1995 Analysis of Coffin Hardware from Unmarked Burials, Former Weselyan Methodist Church Cemetery, Weston, Ontario. North American Archaeologist 16(2):133-162.

Koch, J. K.

1983 Mortuary Behavior Patterning and Physical Anthropology in Colonial St. Augustine. In Spanish St. Augustine: The Archaeology of a Colonial Creole Community, edited by Kathleen Deagan, pp. 187-227. Academic Press, New York, New York.

Kozin, S. H., and A. C. Berlet

1992 Handbook of Common Orthopedic Fractures. 2nd edition. Medical Surveillance, Inc., West Chester, Pennsylvania.

Lai, P., and N. C. Lovell

1992 Skeletal markers of occupational stress in the fur trade: a case study from Hudson's Bay Company fur trade post. International Journal of Osteoarchaeology 2:221-234.

Lamb, L. J.

ca. 1895 Catalog of Sheet Metal Coffins and Ornaments (with price list). Louis J. Lamb, Attleboro, Massachusetts.

ca. 1895 No. 2 Price List of Sheet Metal Coffin Hardware. Louis J. Lamb, Attleboro, Massachusetts. 
Lane, W. A.

1885 Some Points in the Physiology and Pathology of the Changes Produced by Pressure in the Bony Skeleton of the Trunk and Shoulder Girdle. Guy's Hospital Report 43:321-434.

Lang, K. A.

1984 Coffins and Caskets: Their Contribution to the Archaeological Record. Master's thesis, University of Idaho, Moscow, Idaho.

Langenau Manufacturing Company

1920s-1930s Hardware Specialties. Langenau Mfg. Company, Cleveland, Ohio.

Larsen, C. S., and P. L. Walker

2010 Bioarchaeology: Health, Lifestyle, and Society in Recent Human Evolution. In A Companion to Biological Anthropology, edited by C. S. Larsen, pp. 379-394. WileyBlackwell, West Sussex, UK.

Lawrence, J. W., P. W. Schopp, and R. J. Lore

2009 "They Even Threaten the Sick That They Will Not Be Buried in the Churchyard": Salvage Archaeology of the Raritan-in-the-Hills Cemetery, Somerset County, New Jersey. Historical Archaeology 43(1):93-114.

Lebo, S. A.

1988 An Archaeological and Bioarchaeological Perspective: The Tucker (41DT104) and Sinclair (41DT105) Cemeteries of Delta County, Texas. Institute of Applied Sciences, University of North Texas, Denton, Texas.

Lee, A. B.

2002 Report of Field Investigations for the Relocation of the Hosier Family Cemetery, Butler Township, Montgomery County, Ohio, MOT-75-3.842 (PID19070). Hardlines Design Company, Columbus, Ohio. Submitted to Paul Graham, Assistant Environmental Administrator, Ohio Department of Transportation, Office of Environmental Services, Columbus, Ohio.

LeeDecker, C. H., J. Bloom, I. Wuebber, and M. Pipes

1995 Final Archaeological Excavations at a Late 18th-Century Family Cemetery for the U.S. Route 113 Dualization, Milford to Georgetown, Sussex County, Delaware. DelDOT Archaeology Series No. 134. The Cultural Resource Group, Louis Berger \& Associates, Inc., East Orange, New Jersey, with Karen R. Rosenberg, Department of Anthropology, University of Delaware. Submitted to U.S. Department of Transportation, Federal Highway Administration, and Delaware Department of State, Division of Historical and Cultural Affairs, Bureau of Archaeology and Historic Preservation. Prepared for Delaware Department of Transportation, Division of Planning, Location and Environmental Studies Office. 
LeeDecker, C., J. Shellenhamer, and S. Jacobe

2009 "A Vapor That Appeareth for a Little Time and Then Vanisheth Away": Archaeology of the Wrenn-Hutchison Cemetery, Chantilly, Virginia. Louis Berger Group, Inc., Washington, D.C. Prepared for Commonwealth Centre Investors, LLC, Yardley, Pennsylvania.

Levy, L. F.

1968 Porter’s neck. British Medical Journal 2:16-19.

Lipovitch, D., E. MacDonald, I. Miklavcic, D. Robertson, and R. Williamson

2003 Archaeological Investigations of the Elmbank Church and Cemetery, Former Lot 8, Concession 5, Toronto Township, Peel County, Ontario. Archaeological Services, Inc., Toronto, Ontario, Canada. Prepared for Greater Toronto Airports Authority, Lester B. Pearson International Airport, Toronto AMF, Ontario, Canada.

Little, B. J., K. M. Lanphear, and D. W. Owsley

1992 Mortuary Display and Status in a Nineteenth-Century Anglo-American Cemetery in Manassas, Virginia. American Antiquity 57(3):397-418.

Lockhart, W. L.

1881 Wholesale Price List of Caskets and Coffins. Wm. L. Lockhart, East Cambridge, Massachusetts.

1883 Price List and Telegraph Key of Solid Mahogany and Cloth Covered Caskets as Shown in Advance Plates. Wm. L. Lockhart, East Cambridge, Massachusetts.

Louisville Coffin Company

1893 Price List Wood Burial Cases and Caskets and Cloth Covered Caskets. Louisville Coffin Company, Louisville, Kentucky.

Loveday, A. J., Jr.

1983 The Rise and Decline of the American Cut Nail Industry: A Study of the Interrelationships of Technology, Business Organization, and Management Techniques. Greenwood Press, Westport, Connecticut.

Luscomb, S. C.

1992 The Collector's Encyclopedia of Buttons. Schiffer Publishing, Ltd., West Chester, Pennsylvania.

Mack, M., M. C. Hill, and M. L. Blakey

1995 Preliminary analysis of skeletal remains from the New York African Burial Ground. Paper presented at the American Anthropological Association Annual Meeting, Washington, D.C.

McReynolds, M. J.

1981 Archaeological Investigations at the Laredo Cemetery Site (41WB22), Webb County, Texas. Reports of Investigations, Number 11. Prewitt and Associates, Inc., Austin, Texas. Submitted to the City of Laredo, Engineering Department, Laredo, Texas. 
Magoon, D., L. Norr, D. L. Hutchinson, and C. R. Ewen

2001 An Analysis of Human Skeletal Materials from the Snow Beach Site (8WA52). Southeastern Archaeology 20(1):18-30.

Mainfort, R. C., Jr., and J. M. Davidson (editors)

2006 Two Historic Cemeteries in Crawford County, Arkansas. Research Series No. 62. Arkansas Archeological Survey, Fayetteville, Arkansas.

Manger, E. C., \& Son Company

ca. 1890 Catalogue No. 2. E. C. Manger \& Son Company, Green Bay, Wisconsin.

1920s Casket Catalog C. E. C. Manger \& Son Company, Green Bay, Wisconsin.

Markham \& Strong

1865 Revised Price List of Goods Manufactured by Markham \& Strong, East Hampton, Connecticut. Markham \& Strong, East Hampton, Connecticut.

Marsellus, John, Casket Company

ca. 1910 Caskets of Quality. John Marsellus Casket Company, Syracuse, New York.

ca. 1948 Semi-Centennial Catalog. John Marsellus Casket Company, Syracuse, New York.

Maryland Burial Case Company

1887 Price List-Wood Coffins \& Caskets. Maryland Burial Case Company, Baltimore, Maryland.

Matternes, H. B.

1998a The Cool Branch Cemetery (40HK9). In A Final Report on Archaeological Investigations of Sites 40HK5, 40HK6, 40HK7, and 40HK9, State Route 31 Corridor from Mountain Valley Road, Hancock County, Tennessee, edited by S. Meters, Hugh B. Matternes, C. Bentz, and B. J. Duggan, pp. 130-186. Transportation Research Center, University of Tennessee, Knoxville, Tennessee.

1998b Who Are the People in Cool Branch Cemetery (40HK9)? A Bioanthropological Case Study. Tennessee Anthropologist 23(1 \& 2):73-85.

Matternes, H. B., and K. Serio

2005 1SC320: A Rural 19th Century Cemetery on the Outskirts of Ragland, Alabama, Saint Clair County, Alabama. Technical Report No. 1274. New South Associates, Stone Mountain, Georgia. Submitted to National Cemetery Company, Birmingham, Alabama.

Mechanicstown Burial Case Company

1888 Illustrated Catalogue of Coffins, Caskets, and Undertakers' Supplies, mfg and sold by Mechanicstown Burial Case Company. Mechanicstown Burial Case Company, Mechanicstown, Maryland. 
Meer, R. M.

1990 Report of Investigations of Skeletal Remains Recovered from the Stoltz Site (B0Z0048/198807), Ozaukee County, Wisconsin. State Historical Society of Wisconsin, Burial Site Preservation Program, Madison, Wisconsin.

Meriden Britannia Company

1869 Illustrated and Descriptive Price List of Coffin and Casket Trimmings. Meriden Britannia Company, Meriden, Connecticut.

1876 Second 1876 Supplement of Wm. M. Smith's Original Designs of Casket Trimmings. Meriden Britannia Company, West Meriden, Connecticut.

1880 Illustrated Catalogue of Wm. M. Smith's Fine Silver, Bronze, etc., Casket Trimmings. Meriden Britannia Company, West Meriden, Connecticut.

Merit Company

1954 Metal Caskets (November 15, 1954). Merit Company, Chicago, Illinois.

Michael, R. L.

1974 Cut Nail Manufacture: Southwestern Pennsylvania. Bulletin of the Association for Preservation Technology 6(1):99-108.

Miklavcic, I.

2001 Archaeological Assessment of the South Boundary of St. Paul's Pioneer CemeteryProject P.50.15.2000, Town of Newmarket, Regional Municipality of York, Ontario. Archaeological Services, Inc., Toronto, Ontario, Canada. Prepared for the Corporation of the Town of Newmarket, Ontario, Canada.

Miller, K. A.

1996 Archaeological Investigations at 41TV1696-The Givens Grave Site, Travis County, Texas. Archaeological Report No. 96-57. SWCA, Inc., Environmental Consultants, Austin, Texas. Prepared for Trammell Crow Company.

Miller Bros. \& Company

ca. 1871 Illustrated Catalogue of Coffin Trimmings. Miller Bros. \& Company, Boston, Massachusetts.

Mills, E. S.

1979 Graves in the Gravel: The Unmarked Cemetery of Las Vegas, New MexiCompany. Master's thesis, New Mexico Highlands University, Las Vegas, New MexiCompany.

Milner, G. R., J. W. Wood, and J. L. Boldsen

2000 Paleodemography. In Biological Anthropology of the Human Skeleton, edited by M.

A. Katzenberg and S. R. Saunders, pp. 467-497. Wiley-Liss, New York.

Milwaukee Casket Company

ca. 1911 Casket Catalogue D: Manufacturers \& Jobbers of a Complete Line of . . . Milwaukee Casket Company, Milwaukee, Wisconsin. 
Minnesota Casket Company

1923 Catalogue “B,” Manufacturers of Caskets of Redwood, Resist Decay. Minnesota Casket Company, Minneapolis, Minnesota.

Mitchell Casket Company

ca. 1900 Cloth Covered Caskets. Mitchell Casket Company, Mitchell, Indiana.

Mound Coffin Company

1920 [unnamed]; Contains Caskets, Robes, Linings. Mound Coffin Company, St. Louis, Missouri.

1924 Wholesale Price List (for) Metal, Cloth and Varnished Coffins; Metal Linings; Funeral Dry Goods, Sundries, etc. Mound Coffin Company, St. Louis, Missouri.

Murphy, John \& Company

ca. 1900 [Catalogue]. John Murphy \& Co., Pittsburg, Pennsylvania.

ca. 1900 Price List of Hardware, Robes, Linings, and Undertakers' Supplies. John Murphy \& Co., Pittsburg, Pennsylvania.

1923 Price List Applying to 1922 Catalogue (February 9. 1923). John Murphy \& Co., Pittsburgh, Pennsylvania.

National Casket Company

1893 National Casket Company Bulletin 1(7-8):19.

1896 Complete Price List of Burial Robes, Linings, and Miscellaneous Dry Goods. National Casket Company, New York, New York.

ca. 1896 Supplement to Pocket Edition of Casket Catalogue B. National Casket Company, New York, New York.

1897 Catalogue No. 12, Illustrating Undertakers' Hardware. National Casket Company, New York, New York.

1899 National Casket Company Complete Price List and Telegraph Code Accompanying Pocket Catalogue “D.” National Casket Company, New York, New York.

1903 Illustrated Price List of Caskets and Sundries (October 15, 1903). National Casket Company, New Haven, Connecticut.

1908 Catalogue “L.” National Casket Company, Boston, Massachusetts.

1908 Catalogue “L.” National Casket Company, Pittsburgh, Pennsylvania.

1910 Wholesale Price List for Casket Catalogue "L." National Casket Company, New York, New York.

1911 Catalogue M. National Casket Company, New York, New York. 
1922 Catalogue Q. National Casket Company, Baltimore, Maryland.

1922 Price List Applying to Catalogue “Q” (March 23, 1922). National Casket Company, Pittsburg, Pennsylvania.

1922 [Supplement] New Pine Box Prices (October 30, 1922). National Casket Company, Pittsburgh, Pennsylvania.

1922 Price List Applying to Catalogue “Q” (November 1, 1922). National Casket Company, Pittsburg, Pennsylvania.

1923 Bronze Sarcophagi, Illustrating and Describing Some Ancient Customs, Past Masterpieces and Present Tendencies in the Finest Types of Burial Enclosures. National Casket Company, Albany, New York.

1923 [Supplement] Casket and Outer Box Prices (January 29, 1923). National Casket Company, Pittsburgh, Pennsylvania.

1923 [Supplement] Burial Robes and Lining Prices (February 12, 1923). National Casket Company, Pittsburgh, Pennsylvania.

1923 Price List Applying to Catalogue “Q” (June 11, 1923). National Casket Company, Pittsburg, Pennsylvania.

1923 Price List Applying to Catalogue “Q” (October 1, 1923). National Casket Company, Pittsburg, Pennsylvania.

1924 Price List Applying to Catalogue “Q” (February 23, 1924). National Casket Company, Pittsburg, Pennsylvania.

1924 Price List Applying to Catalogue “Q” (December 1, 1924). National Casket Company, Pittsburg, Pennsylvania.

1925 Dry Goods Price List (April 1, 1925). National Casket Company, Pittsburg, Pennsylvania.

1927 Price List Applying to Catalogue “Q” (December 27, 1927). National Casket Company, Baltimore, Maryland.

1928 Catalog "RC," Covered Caskets. National Casket Company, New York, New York.

1930 [Supplement] New Numbers for Caskets Shown in Metal Casket Catalog RM (December 1, 1930). National Casket Company, Pittsburg, Pennsylvania.

1931 [Supplement] New Numbers for Caskets Shown in Catalog RC and RH (February 10, 1931). National Casket Company, Pittsburg, Pennsylvania.

1931 Price List-Casket Catalogues $R C, R H$, and RM (February 15, 1931). National Casket Company, Pittsburg, Pennsylvania. 
1931 Price List-Casket Catalogues RC, RH, and RM (February 24, 1931). National Casket Company, New York, New York.

1937 Catalog “SMHC," Metal Hardwood, and Cloth Covered Caskets. National Casket Company, Syracuse, New York.

1940 Catalog “T” Metal, Hardwood, and Covered Caskets. National Casket Company, Philadelphia, Pennsylvania.

National Metal Products Company

n.d. [Casket Hardware Advertising Cards] National Metal Products Company, Connersville, Indiana.

Nawrocki, S., K. Bauman, C. Weiler, J. Schultz, M. Williamson, C. Schmidt, L. Judd, H. Thew

1998 Analysis of Human Skeletal Remains from the Historic Euroamerican Rhoads Cemetery (12-Ma-777), Indianapolis, Marion County, Indiana. Special Report No. 2. University of Indianapolis Archaeology \& Forensics Laboratory. Submitted to Duke Realty Investments, Inc., and the Division of Historic Preservation \& Archaeology of the Indiana Department of Natural Resources, Indianapolis, Indiana.

Nelson, L. H.

1963 Nail Chronology as an Aid to Dating Old Buildings. Technical Leaflet No. 15. History News 19(2).

1968 Nail Chronology as an Aid to Dating Old Buildings. Technical Leaflet No. 48. American Association for State and Local History, Nashville, Tennessee.

Noël-Hume, I.

1991 A Guide to Artifacts of Colonial America. (reprint). Vintage Books, New York, New York.

Norris, C. Sidney, \& Company

ca. 1880 Illustrated Catalogue of Coffin Handles and Undertakers' Trimmings. C. Sidney Norris \& Company, Boston, Massachusetts.

ca. 1880 Price List of Coffin Handles and Undertakers' Trimmings. C. Sidney Norris \& Company, Boston, Massachusetts.

Northern Casket Company

2010a Cloth Casket Catalogue. Northern Casket Company, Lindsay, Ontario, Canada.

2010b Enviro-Casket Catalogue. Northern Casket Company, Lindsay, Ontario, Canada.

2010c Rental Casket Catalogue. Northern Casket Company, Lindsay, Ontario, Canada.

2010d Ash Casket Catalogue. Northern Casket Company, Lindsay, Ontario, Canada.

2010e Cherry Casket Catalogue. Northern Casket Company, Lindsay, Ontario, Canada. 
2010f Poplar Casket Catalogue. Northern Casket Company, Lindsay, Ontario, Canada.

2010g Oak Casket Catalogue. Northern Casket Company, Lindsay, Ontario, Canada.

2010h Pine/Maple/Mahogany Casket Catalogue. Northern Casket Company, Lindsay, Ontario, Canada.

2010i Specialty Casket Catalogue. Northern Casket Company, Lindsay, Ontario, Canada.

Oregon Casket Company

ca. 1932 Catalogue “G.” Oregon Casket Company, Portland, Oregon.

Ormsbee, T. H.

1951 Field Guide to Early American Furniture. Bantam Books, New York, New York.

1952 Field Guide to American Victorian Furniture. Bonanza Books, New York, NY.

Orser, C. E., Jr., A. M. Nekola, and J. L. Roark

1987 Exploring the Rustic Life: Multidisciplinary Research at Millwood Plantation, a Large Piedmont Plantation in Abberville County, South Carolina, and Elbert County, Georgia. Russell Papers. Mid-American Research Center, Loyola University of Chicago. Submitted to the National Park Service.

Ortner, D. J., and W. Putschar

1981 Identification of Pathological Conditions in Human Skeletal Remains. Smithsonian Institution Press, Washington, D.C.

Oster, W. J., G. G. Weaver, J. P. Richardson, and J. M. Wyatt

2005 Archaeological and Osteological Investigations of the Providence Baptist Church Cemetery (40SY619), Memphis-Shelby County Airport, Memphis, Shelby County, Tennessee. Weaver \& Associates, LLC, Memphis, Tennessee. Submitted to Memphis-Shelby County Airport Authority, Memphis, Tennessee, and Allen \& Hoshall, Memphis, Tennessee.

Owosso Casket Company

1920 Wholesale Price List (September 20, 1920). Owosso Casket Company, Owosso, Missouri.

1921 Wholesale Price List (April 18, 1921). Owosso Casket Company, Owosso, Missouri.

1921 List of Reduced Prices of Oak, Mohogany, Walnut, Cypress and Other Finished Caskets (September, 20, 1921). Owosso Casket Company, Owosso, Missouri.

Owsley, D. W.

1994 Bioarchaeology on a Battlefield: The Abortive Confederate Campaign in New MexiCompany. Archaeology Notes No. 142. Museum of New Mexico, Office of Archaeological Studies, Santa Fe, New MexiCompany. 
Owsley, D. W., W. F. Hanna, M. L. Richardson, and L. E. Burgess

2003 [2001] Bioarcheological and Geophysical Investigation: The Soldiers Plot, Emmanuel Lutheran Church Cemetery, New Market, Virginia (Site No. 44SH364). ASV Special Publication Number 41. Archeological Society of Virginia. Spectrum Press, Richmond, Virginia.

Owsley, D. W., J. K. Krakker, M. Jacobs, and R. W. Mann

1992 The History and Archaeology of St. James Episcopal Church, Brandy Station, Virginia (Site No. 44CU90). Bookcrafters, Fredricksburg, Virginia.

Owsley, D. W., C. E. Orser, Jr., R. Montgomery, and C. C. Holland

1985 An Archaeological and Physical Anthropological Study of the First Cemetery in New Orleans, Louisiana. Department of Geography and Anthropology, Louisiana State University, Baton Rouge, Louisiana.

Painter, M., A. L. Magennis, C. J. Zier, N. Mitchell, L. B. Conyers, and J. D. Kennedy

2002 Archaeological and Osteological Investigations of Cemetery 2 on the Grounds of the Colorado Mental Health Institute at Pueblo, Pueblo County, Colorado. Centennial Archaeology, Inc., and Colorado State University, Fort Collins, Colorado.

Panati, Charles

1987 Panati’s Extraordinary Origins of Everyday Things. Harper \& Row, New York.

Parler, M.C.

1962 Folk Beliefs from Arkansas: Death and Funeral Customs. Vol. VIII. University of Arkansas, Fayetteville, Arkansas.

Parrington, M., and D. G. Roberts

1990 Demographic, Cultural, and Bioanthropological Aspects of a Nineteenth-Century Free Black Population in Philadelphia, Pennsylvania. In A Life in Science: Papers in Honor of J. Lawrence Angel, J. Buikstra, editor, pp. 138-170. Scientific Papers 6. Center for American Archeology, Kampsville, Illinois.

Parrington, M., D. G. Roberts, S. A. Pinter, and J. C. Wideman

1989 The First African Baptist Church Cemetery: Bioarchaeology, Demography, and Acculturation of Early Nineteenth Century Philadelphia Blacks. 2 vols. John Milner Associates, Inc., Philadelphia, Pennsylvania. Prepared for the Redevelopment Authority of the City of Philadelphia, Pennsylvania.

Parsons Casket Hardware Company

$194749^{\text {th }}$ Annual Edition Catalogue. Parson's Casket Hardware Company, Belvidere, Illinois.

Paxson, Comfort \& Company

ca. 1870 Illustrated Catalogue of Wood and Metallic Burial Caskets, Coffins, Corpse Preservers and Linings. Paxson, Comfort \& Company, Philadelphia, Pennsylvania. 
pre-1878 Illustrated Catalogue of Undertakers' Supplies. Paxson, Comfort \& Company, Philadelphia, Pennsylvania.

1881 Illustrated Catalogue of Undertakers’ Supplies. Philadelphia, Pennsylvania.

1884 Illustrated and Descriptive Catalogue of Wood, Metallic, and Cloth Covered Burial Caskets. Paxson, Comfort \& Company, Philadelphia, Pennsylvania.

1884 Reduced Wholesale Price List, to Accompany Illustrated Catalogue of December 1881. Paxson, Comfort \& Company, Philadelphia, Pennsylvania.

1886 Supplementary Catalogue of Undertakers’ Hardware. Paxson, Comfort \& Company, Philadelphia, Pennsylvania.

1889 Wholesale Price List of Untrimmed Coffins and Caskets for Sale to the Trade Only, by Paxson, Comfort \& Company. Paxson, Comfort \& Company, Philadelphia, Pennsylvania.

ca. 1890 Catalogue of Burial Caskets for Sale to the Trade Only by the Paxson, Comfort \& Company. Paxson, Comfort \& Company, Philadelphia, Pennsylvania.

ca. 1890 Price List of Elegant Emblems Made from Natural Flowers, Also Preserved Flowers and Prepared Italian Wheat Adapted Especially for Funeral Purposes. Paxson, Comfort \& Company, Philadelphia, Pennsylvania.

ca. 1890 Wholesale Pricelist of Burial Robes, Wrappers, Dresses, Suits, Habits, \&c., \&c. Manufactured by Paxson, Comfort \& Company. Paxson, Comfort \& Company , Philadelphia, Pennsylvania.

1893 Illustrated and Descriptive Catalogue of Wood, Metallic, and Cloth Covered Burial Caskets and Coffins. Paxson, Comfort \& Company, Philadelphia, Pennsylvania.

1898 Illustrated and Descriptive Catalogue of Wood, Cloth-covered and Metallic Burial Caskets and Coffins For Sale to the Trade Only by Paxson, Comfort \& Company. Paxson, Comfort \& Company, Philadelphia, Pennsylvania.

Pearce, R. J.

1989 Excavation of the Wise Family Pioneer Cemetery and Homestead, Lot 17, Concession 2, Town of Richmond Hill. Museum of Indian Archaeology, London, and Affiliate of the University of Western Ontario. Submitted to the Glen Group, Don Mills.

Pearson, C. E.

1978 The Rabbit Hill Site: A Late Nineteenth Century Southern Plains Indian Burial at Fort Sill, Oklahoma. Bulletin of the Oklahoma Anthropological Society 27:171-178.

Peck \& Walters Manufacturing Company

1853 Price List . . . Peck \& Walters Mfg. Company, New Britain, Connecticut. 
Peck \& Walters Manufacturing Company and Sargent \& Bros.

1857 Cabinet Maker's Hardware. Peck \& Walters Mfg. Company, and Sargent \& Bros., New Haven, Connecticut.

Perry, W. R., J. Howson, and B. A. Bianco (editors)

2006 New York African Burial Ground, Archaeology Final Report. 4 vols. Howard University, Washington, D.C. Prepared for the United States General Services Administration, Northeastern and Caribbean Region.

Peter, D. E., M. Prior, M. M. Green, and V. G. Clow (editors)

2000 Freedman's Cemetery: A Legacy of a Pioneer Black Community in Dallas, Texas. 2 vols. Special Publications No. 6. Geo-Marine, Inc., Plano, Texas. Submitted to Texas Department of Transportation, Environmental Affairs Division, Archaeology Studies Program, Report No. 21.

Philadelphia Manufacturing Company

1949 Casket Hardware by Philadelphia. Philadelphia Mfg. Company, Philadelphia, Pennsylvania.

ca. 1950 Casket Hardware. Philadelphia Mfg. Company, Philadelphia, Pennsylvania.

Phillips, M. K.

1989 A Revised Chronology of Cut Nails in New England 1790-1820: A Case Study of the Spencer-Pierce-Little House Addition. Master's thesis, Boston University, Boston, Massachusetts.

Pike, M. V., and J. G. Armstrong (editors)

1980 A Time to Mourn: Expressions of Grief in Nineteenth Century America. The Museums at Stony Brook, Stony Brook, New York.

Piper, H. M., and J. G. Piper

1982 Archaeological Excavations at the Quad Block Site, 8-Hi-998, Located at the Site of the Old Fort Brooke Municipal Garage, Tampa, Florida. Piper Archaeological Research, Inc., St. Petersburg, Florida. Prepared for the city of Tampa, Florida.

Pomfret, J.

2003 Disinterment and Relocation of an Abandoned Cemetery in Stewart County, Georgia. GDOT Project No. EDS-27(173). Georgia Department of Transportation, Cultural Resources Division.

Portal to Texas History

2010 Portal to Texas History. Online document available at http://texashistory.unt.edu/

Porter, K. W.

1932 Relations between Negroes and Indians within the Present Limits of the United States. Journal of Negro History 17:287-367. 
Powell, G. S.

2000 Emergency Excavations and Preliminary Analyses of Artifacts and Skeletal Remains from the St. Francis Regis Cemetery, Kansas City, Missouri. CAR Project 1128. Center for Archaeological Research, Southwest Missouri State University, Springfield, Missouri. Prepared for DST Realty, Kansas City, Missouri, and Historic Preservation Program, Missouri Department of Natural Resources, Jefferson City, Missouri.

Powers \& Walker Casket Company

ca. 1896 Catalogue C, High Class Cloth Covered and Varnished Caskets. Powers \& Walker Casket Company, Grand Rapids, Michigan.

Priess, P. J.

1970 Penny Wise, Penny Foolish: The Description of Nail Sizes. Historical Archaeology 3(3):8-9.

1973 Wire Nails in North America. Bulletin of the Association for Preservation Technology 5:87-92.

Prince Hall

1996 "Who is Prince Hall?” Electronic document http://www.mindspring.com/ johnsonx/ whoisph.htm, accessed 3/19/2011.

Prince Hall Freemasonry

2011 "Prince Hall Freemasonry." Electronic document http://wiki.ask.com/ Prince_Hall_Freemasonry, accessed 3/19/2011.

Puckett, N. N.

1926 Folk Beliefs of the Southern Negro. University of North Carolina Press, Chapel Hill, NC.

Pye, J. W.

2007 A Look Through the Viewing Glass: Social Status and Grave Analysis in a 19th Century Kansas Cemetery. Master's Thesis, The University of Arkansas, Fayetteville, Arkansas.

2010a Analysis of Burial Container Construction and Elaboration at the Tucson City Cemetery, Tucson, Arizona. Department of Anthropology, University of Florida, Gainesville, Florida. Prepared for Statistical Research Inc., Tucson, Arizona, and Roger Anyon, Pima County Cultural Resources and Historic Preservation Office, Tucson, Arizona.

2010b Typology and Analysis of Burial Container Hardware Recovered from the Excavation of Rambo Cemetery, Rome, Georgia. University of Florida, Gainesville. Prepared for Brockington and Associates, Inc., Norcross, Georgia. 
2011 Typology and Analysis of Burial Container Hardware Recovered from Excavations in a $19^{\text {th }}$ Century Pioneer Cemetery, Brazoria County, Texas. Department of Anthropology, University of Florida, Gainesville. Prepared for Prewitt and Associates, Inc., Austin, Texas.

Raemsch, C. A., and J. W. Bouchard

2000 The Henry Lehman Family Cemetery: A Unique Contribution to Nineteenth-Century Domestic Archaeology. In Nineteenth and Early Twentieth-Century Domestic Site Archaeology in New York State, edited by J. P. Hart, and Charles L. Fisher. New York State Museum Bulletin, No. 495. The University of the State of New York, New York State Education Department, Albany, New York.

Rauschenberg, B. L.

1990 Coffin Making and Undertaking in Charleston and its Environs, 1705-1820. Journal of Early Southern Decorative Arts 16(1):19-63.

Reichs, K. J.

1998 Forensic Osteology. Advances in the Identification of Human Remains. 2nd edition. Charles C. Thomas, Springfield, Illinois.

Reynolds, M., and E. Kane

2010 Relocation of the Rambo Family Cemetery, Rome, Floyd County, Georgia: Final Report. Brockington and Associates, Inc., Atlanta, Georgia. Prepared for PBS\&J, Atlanta, Georgia.

Richards, P. B.

1997 Unknown Man No. 198: The Archaeology of the Milwaukee County Poor Farm Cemetery. Doctoral dissertation, Department of Anthropology, The University of Wisconsin, Milwaukee, Wisconsin. University Microfilms International, Ann Arbor, Michigan.

Richards, P. B., and M. W. Kastell

1993 Archaeological Excavations at the Almshouse Burial Ground, Milwaukee County Poorhouse, Wauwatosa, Wisconsin. 2 vols. Report of Investigations, Number 333. Great Lakes Archaeological Research Center, Inc., Milwaukee, Wisconsin. Prepared for Milwaukee County Department of Public Works, Professional Services Division, Milwaukee, Wisconsin.

Rinehart, C. J., M. Rupnik, J. L.Tippett, S. Jacobe, S. Fiedel, and E. Voigt

2009 Data Recovery at Guinea Road Cemetery (Site 44Fx1664) Route 236 (Little River Turnpike), Fairfax County, Virginia. VDOT Project:0236-029-120, RW201; PPMS No.:17671; VDHR File No.:2004-0680. The Louis Berger Group, Richmond, Virginia. Prepared for the Virginia Department of Transportation, Fredericksburg, Virginia.

Roark, J. L.

1977 Masters without Slaves. Norton Press, New York. 
Rock Falls Mfg Company

ca. 1890 Columbian Catalogue No. 7. Rock Falls Manufacturing Company, Sterling, Illinois.

Rockwood, C. A., Jr., and D. P. Green

1984 Fractures in Adults. J. B. Lippincott Co., Philadelphia, Pennsylvania.

Rogers, L. F., and R. W. Hendrix

1992 Radiology of Skeletal Trauma. Volume 1. 2nd edition. Churchill Livingstone, New York.

Rose, J. C. (editor)

1983 Cedar Grove Historic Cemetery: A Study in Bio-History. Project No. 528. Arkansas Archaeological Survey, Fayetteville, Arkansas. Prepared for the Department of the Army, U.S. Army Corps of Engineers, New Orleans District, Louisiana. Contract No. DACW 29-81-C-0059, Modification P0002.

1985 Gone to a Better Land: A Biohistory of a Rural Black Cemetery in the PostReconstruction South. Research Series No. 25. Arkansas Archeological Survey, Fayetteville, Arkansas.

Rose, J. C., and L. G. Santeford

1985 Burial Interpretation. In Gone to a Better Land, edited by J. C. Rose. Research Series No. 25. Arkansas Archeological Survey, Fayetteville, Arkansas.

Rowe, J.

1962 Worsaae's Law and the Use of Grave Lots for Archaeological Dating. American Antiquity 28(2):129-137.

Royal Bond, Inc.

ca. 1950 Catalog No. 8. Royal Bond, Inc., St. Louis, Missouri.

Russell \& Erwin Manufacturing Company

1980 [1865] Illustrated Catalogue of American Hardware of the Russell and Erwin Manufacturing Company. Association of Preservation Technology, Manufactory, New Britain, Connecticut.

Sargent \& Company (J. B.)

1861 Hardware Catalog. Sargent \& Company (J.B.), New Britain, Connecticut.

Sargent \& Company

1866 Prices of Hardware. Sargent \& Company, New Haven, Connecticut.

1869 Price List and Illustrated Catalogue. Sargent \& Company, New Haven, Connecticut.

1871 Price List and Illustrated Catalogue of Hardware mfg and for Sale by Sargent \& Company. Sargent \& Company, New Haven, Connecticut. 
1874 Price List and Illustrated Catalogue of Hardware mfg and for Sale by Sargent \& Company. Sargent \& Company, New Haven, Connecticut.

1877 Price List and Illustrated Catalogue of Hardware $\mathrm{mfg}$ and for sale by Sargent \& Company. Sargent \& Company, New Haven, Connecticut.

1888 Price List and Illustrated Catalogue of Hardware mfg and for sale by Sargent \& Company. Sargent \& Company, New Haven, Connecticut.

ca. 1921 Catalogue No. 17: Casket Hardware, Box Hardware, and Miscellaneous Goods used by Casket Manufacturers ... Sargent \& Company, New Haven, Connecticut.

Saunders, S. R., and R. Lazenby

1991 The Links that Bind: The Harvie Family Nineteenth Century Burying Ground. Occasional Papers in Northeastern Archaeology No. 5. Copetown Press, Dundas, Ontario.

Sauter, W.

1888 Wholesale Price List. William Sauter, Baltimore, Maryland.

Schermer, S. J., R. M. Lillie, J. R. Sellars, and M. Ingalls

2006 Investigation of Inadvertent Discovery of Historic Grave at Tallyn's Ranch, at the South Boundary of Site 13DA185, West Des Moines, Dallas County, Iowa. Contract Completion Report No. 1466. Office of the State Archaeologist, The University of Iowa, Iowa City, Iowa.

Schmidt Manufacturing Company

ca. 1905 Catalogue of Coffin Studs, Plates and Ornaments. Schmidt Mfg. Company, Dubuque, Iowa.

ca. 1910 Illustrated Catalogue of Casket Hardware Manufactured by Schmidt Manufacturing Company, Dubuque, Iowa. Schmidt Mfg. Company, Dubuque, Iowa.

ca. 1934 Illustrated Catalog of Casket Hardware, Catalogue No. 29. Schmidt Manufacturing Company, Dubuque, Iowa.

Schwartz, J. H.

1995 Skeleton Keys. An Introduction to Human Skeletal Morphology, Development, and Analysis. Oxford University Press, New York.

Sewell, K., and P. Stanton

2008 Dove Cemetery: Reflections on Cultural Identity at the Edge of Western ExpansionThe Excavation and Interpretation of Dove Cemetery, CA-SLO-1892H, San Lui Obispo County, California. Technical Report 06-55. Statistical Research, Inc., Redlands, California. Prepared for Grant Robbins, Centex Homes, Central Coast Division, San Luis Obispo, California. 
Shogren, M. G., K. R. Turner, and J. C. Perroni

1989 Elko Switch Cemetery: An Archaeological Perspective. Report of Investigations No. 58. Alabama State Museum of Natural History, Division of Archaeology. Prepared for the State of Alabama, Alabama Highway Department, Montgomery, Alabama, and Project I-565, Huntsville, Madison County, Alabama.

Shuler, K. A., E. C. Poplin, and R. Bailey, Jr.

2005 Cemetery Relocation at Site 38CH1648, Johnson Hagood Stadium, The Citadel, Charleston, South Carolina. Brockington and Associates, Inc., Charleston, South Carolina. Prepared for The Citadel, Charleston, South Carolina.

Simmons Hardware Company

1902 Catalogue No. 421, Builder's Hardware. Simmons Hardware Company, Inc., St. Louis, Missouri.

1903 Catalogue No. 443, Builders Hardware (general hardware, with some coffin trimmings). Simmons Hardware Company, Inc., St. Louis, Missouri.

1918 Catalogue No. P, Complete Catalogue. (General hardware, with a page of coffin trimmings). Simmons Hardware Company, Philadelphia, Pennsylvania.

Skull Fracture

2011 “Skull Fracture.” Electronic document http://en.wikipedia.org/wiki/Skull_fracture, accessed 6/29/2011.

Sledzik, P.

2000 Nasty Little Things: Molds, Fungi, and Spores. In Dangerous Places: Health, Safety, and Archaeology, edited by David Poirier and Kenneth Feder, pp. 71-77. Bergin \& Garvey, Westport, Connecticut.

Smits, N. J., and J. Reese

2005 Archaeological Exploration of Multnomah County's Morrison Property at SE 20th and Morrison, Portland, Oregon. Archaeological Investigations Northwest, Inc. Prepared for Multnomah County Facilities and Property Management, Portland, Oregon.

Soil Survey Staff

2010 "Soil Survey Staff, Natural Resources Conservation Service, United States Department of Agriculture.” Soil Survey Geographic (SSURGO) Database for Fort Bend County, Texas. Available online at http://soildatamart.nrcs.usda.gov, accessed October 27/2010.

South, S. A.

1979 The General, the Major, and the Angel: The Discovery of General William Moultrie's Grave. Research Manuscript Series No. 146. Institute of Archeology and Anthropology, University of South Carolina, Columbia, South Carolina. 
Southern Folk Cemetery

2011 "The Southern Folk Cemetery." Electronic document http://southerngraves.i-foundit.net/southernfolkcemetery.html, accessed 5/27/11.

Southern Memorial Association

2003-2011 “African Burial Customs.” Electronic document http://www.gravegarden.org/ abc.htm, accessed 7/3/2011.

Spencer, S. D.

2002 Manslick Road Cemetery, Burial \#34. In unpublished manuscript, Program of Archaeology, University of Louisville, Louisville, Kentucky.

Spitz, W. U.

1980 Blunt Force Injury. In Medicolegal Investigation of Death, edited by W. U. Spitz and R. S. Fisher. 2nd edition. Charles C. Thomas, Springfield, Illinois.

Springate, M. E.

2011 "Coffin Handles from the African Burial Ground, New York City: Notes on Their Source and Context. The African Diaspora Archaeology Network Newsletter June 2011.” Electronic document http://www.diaspora.uiuc.edu/news0611/news0611.html, accessed 6/30/2011.

St. Louis Coffin Company

1901 Souvenir Catalog, No. 20 Illustrating Highest Types of Our Art. St. Louis Coffin Company, St. Louis, Missouri.

ca. 1901 Estimated Undertaker's Selling Prices ... (for) Art Book No. 20. St. Louis Coffin Company, St. Louis, Missouri.

1904 Combined Buying and Selling Price List and Catalogue (may be used in connection with or independent of our Art Catalogue No. 20) (March 1, 1904). St. Louis Coffin Company, St. Louis, Missouri.

1911 Catalog No. 22 (Coffins, Caskets, Vaults, Hearses). St. Louis Coffin Company, St. Louis, Missouri.

1918 The St. Louis Patented Copper-Bearing Steel Caskets (August 1, 1918). St. Louis Coffin Company, St. Louis, Missouri.

\section{Star Tribune}

2009 “Obituary-Leo A. Hodroff.” Star Tribune, November 7-8, 2009. Electronic document http://www.legacy.com/obituaries/startribune/obituary.aspx?n=leo-ahodroff\&pid=135556509, accessed 4/12/2011.

Steele, D. G., and C. A. Bramblett

1988 The Anatomy and Biology of the Human Skeleton. Texas A\&M University Press, College Station, Texas. 
Stein Manufacturing Company

1882 Telegraph Key and Price List for Untrimmed Caskets. Stein Mfg. Company, Rochester, New York.

1883 “The Most Serviceable Invention of the Age: Our 'Patent Fastener' For Casket Tops.” Stein Mfg. Company, Rochester, New York

1885 Supplement to our Book of Designs. Stein Mfg. Company, Rochester, New York.

1887 Revised Price List and Telegraph Key for Stein Mfg. Company, Producer of Fine Funeral Supplies and Textile Covered Caskets (June 1, 1887). Stein Mfg. Company, Rochester, New York.

ca. 1890 [Catalog of Casket Designs]. Stein Mfg. Company, Rochester, New York.

Stolts, Russell \& Company

1880 Illustrated and Descriptive Catalogue of Undertakers' Supplies. Stolts, Russell \& Company, New York, New York.

Strezewski, M.

2003 "Ellen We Miss Thee At Home": Archaeological Investigations at the Michigan City Old Graveyard (12Le348), LaPorte County, Indiana. Reports of Investigations 308. IPFW Archaeological Survey. Indiana University-Purdue University at Fort Wayne, Fort Wayne, Indiana.

Sugar Land, Texas

2011 "Sugar Land, Texas." Electronic document http://en.wikipedia.org/wiki/ Sugar_Land,_Texas?rnd+752029166606021675541746741, accessed 3/23/2011.

Sunbury Coffin and Casket Works

1882 Wholesale Price List of Untrimmed Coffins and Caskets. Sunbury Coffin and Casket Works, Sunbury, Pennsylvania.

1883 Wholesale Price List of Untrimmed Coffins and Caskets. Sunbury Coffin and Casket Works, Sunbury, Pennsylvania.

Sunnyside

1929 Sunnyside. [trade magazine] May 15, 1929.

Superior Funeral Supply Corporation

19661966 Catalog, Superior Funeral Supply Corp. Superior Funeral Supply Corporation, Cleveland, Ohio.

Sutherland, F.

2006 Use, Reuse, and Desecration: Analysis and Interpretation of the Lucy Kimball Mead Tomb, Littleton, Massachusetts. Bachelor's thesis, Boston University, Boston, Massachusetts. 
Swauger, J. L.

1959 An American Burial Technique of the Early 19th Century. Pennsylvania Archaeologist 29(1):38-39.

Taylor, A. J., A. A. Fox, and I. W. Cox

1986 Archaeological Investigations at Morgan Chapel Cemetery (41BP200), A Historic Cemetery in Bastrop County, Texas. Archaeological Survey Report No. 146. Center for Archaeological Research, The University of Texas, San Antonio, Texas.

Taylor, H. E., \& Company

1872 (1871) Illustrated Catalogue of Caskets, Coffins, Shrouds, Trimmings, etc. Taylor \& Company, New York, New York.

1875 Illustrated Catalogue of Undertakers' Sundries. No. 163. H. E. Taylor \& Company, Bowery, New York.

1878 Supplementary List: Undertakers' Sundries. H. E. Taylor \& Company, New York, New York.

1884 Robes and Linings. H. E. Taylor \& Company, Bowery, New York.

Texas Beyond History

2002 "Making Sugar in Nineteenth-Century Texas. Lake Jackson. Plantation History." Texas Beyond History. Electronic Document http://www.texasbeyondhistory.net/ jackson/sugar.html, accessed 6/17/2011.

Thacker Caskets, Inc.

n.d. $\quad$ Salesman Training Module 4: Product Knowledge \& The Thacker Product Line. Thacker Caskets, Inc., Clinton, Maryland.

Tharp, B. W.

1996 "Preserving Their Form and Features": The Role of Coffins in the American Understanding of Death, 1607-1870. Ph.D. dissertation, Department of Anthropology, The College of William and Mary, Williamsburg, Virginia. University Microfilms International, Ann Arbor, Michigan.

Thiel, J. H., and M. M. Margolis

2007 Excavation and Analysis of Burials 13 and 14 from the Court Street Cemetery, AZ BB:13:156 (ASM), Tucson, Pima County, Arizona. Project Report No. 07-136. Desert Archaeology, Inc., Tucson, Arizona. Prepared for Kristi Jenkins, city of Tucson, Department of Urban Planning and Design.

Thompson, R. F.

1984 Flash of the Spirit: African and Afro-American Art and Philosophy. Random House, New York. 
Thoms, A. V.

2001 The Matagorda Cemetery Project: Unmarked Graves and Community Heritage. Technical Report No. 5. Center for Ecological Archaeology, Texas A\&M University, College Station, Texas.

Tiné, A.

2000 Understanding Life and Death through Freedman's Cemetery: a Comparative Bioarchaeological Study of African American Health. In Freedman's Cemetery: a Legacy of a Pioneer Black Community in Dallas, Texas, edited by D. E. Peter, M. Prior, M. M. Green, and V. G. Clow, pp. 461-517. Volume 2. Special Publications No. 6. Geo-Marine, Inc., Plano, TX. Submitted to Texas Department of Transportation, Environmental Affairs Division, Austin; Archeology Studies Program Report No. 21.

Tiné, A. L., and D. K. Boyd

2003 Archeological Excavation and Reburial of Unmarked Historic Graves in the Pioneer Cemetery (41BO202), Brazoria County, Texas. Reports of Investigation No. 139. Prewitt Associates, Inc., Austin, Texas. Submitted to Texas Department of Transportation, Environmental Affairs Division, Archeology Studies Program Report No. 59.

Tiné, A. L., J. Cooper, and M. Wurtz

2002 Archaeological and Bioarchaeological Investigations at Potter's Field/Greenwood Cemetery along Clyde Lane, Dallas, Texas. Miscellaneous Reports of Investigations Number 241. Geo-Marine, Inc., Plano, Texas. Prepared for the city of Dallas, Texas.

Tippet, L., S. Jacobe, and S. Fiedel

2009 Data Recovery at the Lackey Cemetery (Site 44RB0509 and VDHR No. 081-7093) Interstate-81 NB Truck Climbing Lanes, Rockbridge County, Virginia. VDOT Project:00081-081-708, P101; PPMS No.:84363; VDHR File No.:2007-1094. The Louis Berger Group, Inc., Richmond, Virginia. Prepared for the Virginia Department of Transportation, Richmond, Virginia.

Todd, Polluck \& Granger

1876 Tenth Annual Illustrated Catalogue and Wholesale Pricelist of Furniture, Etc., Manufactured and Sold by Todd, Pollock \& Granger. Todd, Polluck \& Granger, Burlington, Iowa.

Trinkley, M., and D. Hacker-Norton

1984 Analysis of Coffin Hardware from 38CH778, Charleston County, South Carolina. Research Series No. 3. Chicora Foundation, Inc., Columbia, South Carolina.

Trinkley, M., D. Hacker, and N. Southerland

2009 Removal of Four Burials from St. Johannes Cemetery, Bensenville, Illinois. Research Contribution No. 519. Chicora Foundation, Inc., Columbia, South Carolina.

2010 Removal of One Burial from St. Johannes Cemetery, Bensenville, Illinois. Research Contribution No. 530. Chicora Foundation, Inc., Columbia, South Carolina. 
Tri-State Industries, LTD.

n.d (1965-1979) Catalog No. 16. Tri-State Industries, Ltd, Bronx, New York.

Trubitt, M. B. D., D. W. Steadman, and A. Grauer

1999 Report of Excavations at Dunning Cemetery No. 2. Cultural Resource Management Report No. 625. Midwest Archaeological Services, Inc., Harvard, Illinois. Prepared for Norwood Builders, Inc.

Trudeau, M. F.

2005 Relocation of the Historic Neal (Big Cove) Cemetery, Madison County, Alabama. Alexander Archaeological Consultants, Inc., Wildwood, Georgia. Prepared for the Alabama Historical Commission, Montgomery, Alabama, and the city of Huntsville, Alabama.

Turner II, C. G., C. R. Nichol, and G. R. Scott

1991 Scoring Procedures for Key Morphological Traits of the Permanent Dentition: the Arizona State University Dental Anthropology System. In Advances in Dental Anthropology, edited by M. A. Kelley and C. S. Larsen, pp. 13-31. Wiley-Liss, New York.

Turpin, S. A., and L. C. Bement.

2002a Relocation of the Craddock Cemetery, 41BP581, Three Oaks Mine, Bastrop County, Texas. Technical Report 17. TAS, Inc., Austin, Texas. Prepared for ALCOA, Inc.

2002b Relocation of the Nisbett and Anderson Cemeteries, 41RT189 and 41RT350, Pit 6, Calvert Mine, Robertson County, Texas. Technical Report 19. TAS, Inc., Austin, Texas. Submitted to Walnut Creek Mining Company.

Ubelaker, D. H.

1995 Historic Cemetery Analysis: Practical Considerations. In Bodies of Evidence: Reconstructing History through Skeletal Analysis, edited by Anne Grauer, pp. 37-48. Wiley-Liss, New York.

Undertakers' Supply Company

ca. 1921 Catalogue No. 2. Undertakers’ Supply Company, Chicago, Illinois.

1923 Catalogue No. 4. Undertakers’ Supply Company, Chicago, Illinois.

ca. 1930s a [Catalogue] Undertakers’ Supply Company, Chicago, Illinois.

ca. 1930s b Red Catalogue No. 13-The Superior Line. Undertakers' Supply Company, Chicago, Illinois.

Union Casket Company

1881 Telephonic \& Telegraphic Key and Wholesale Price List Combined, of Untrimmed Cloth Covered Caskets. Union Casket Company, Boston, Massachusetts. 
United States Casket Company

1922 [Supplement] Notice: Outside Box Prices Reduced (March 20, 1922). United States Casket Company, Pittsburgh, Pennsylvania.

1922 Price List Applying to Catalogue “D” (May 25, 1922). United States Casket Company, Pittsburgh, Pennsylvania.

1922 [Supplement] Price Changes (November 1, 1922). United States Casket Company, Pittsburgh, Pennsylvania.

1922 Price List Applying to Catalogue " $D$ ” (December 1, 1922). United States Casket Company, Pittsburgh, Pennsylvania.

1923 Price List Applying to Catalogue “D” (July 1, 1923). United States Casket Company, Pittsburgh, Pennsylvania.

1923 Price List Applying to Catalogue " $D$ ” (November 1, 1923). United States Casket Company, Pittsburgh, Pennsylvania.

1924 Price List Applying to Catalogue "D" (March 25, 1924). United States Casket Company, Pittsburgh, Pennsylvania.

1925 Price List Applying to Catalogue “D” (June 20, 1925). United States Casket Company, Pittsburgh, Pennsylvania.

U.S. General Services Administration

2012 The African Burial Ground-Introduction to the Electronic Versions of the Final Reports: Introduction to the Electronic Versions of the New York African Burial Ground Scientific Reports. U.S. General Services Administration. Available from http://www.gsa.gov/portal/content/249941.

United States Patent Office

2012 United States Patent Office Online database. (utility and design patents). Electronic document http://www.uspto.gov/patents/process/search/

Veilleux, A., and D. Robertson

2008 Stage 2 Archaeological Assessment, Bridgepoint Health Master Plan, City of Toronto, Ontario. Archaeological Services, Inc., Toronto, Ontario, Canada. Prepared for Bridgepoint Health, Toronto, Ontario, Canada.

Victor Casket Hardware Company

ca. 1935 Casket Hardware, Catalog No. 3. Victor Casket Hardware Company, Galesburg, Illinois.

1956 Catalogue No. 6. Victor Casket Hardware Company, Galesburg, Illinois.

1959 Price List Applying to Catalogue No. 6 (May 20, 1959). Victor Casket Hardware Company, Galesburg, Illinois. 
Vlach, J. M.

1978 Graveyard Decoration. In The Afro-American Tradition in Decorative Arts, pp. 13947. Cleveland Museum of Art, Cleveland, Ohio.

Walker, P. L.

2008 Bioarchaeological Ethics: A Historical Perspective on the Value of Human Remains. In Biological Anthropology and the Human Skeleton, edited by M. A. Katzenberg and S. R. Saunders, pp. 3-40. John Wiley \& Sons, Hoboken, New Jersey.

Walker, R.

1990 The Early History of PVC Pipe. Uni-Bell PVC Pipe News (Summer):1-2.

Walwer, G. F.

1996 Combining Archival and Archeological Research: The Connecticut School for Boys Cemetery. CRM 19(10):8-11.

Ward, T., and M. Graham

1978 The Archaeo-Osteology of Three Historic Cemeteries in Person County, N.C. The Research Laboratories of Anthropology, University of North Carolina, Chapel Hill, North Carolina.

Warfield \& Rohr

ca. 1878 Illustrated Catalogue of Undertakers' Supplies. Warfield \& Rohr, Baltimore, Maryland.

ca. 1878 Wholesale Price List of Undertakers' Supplies. Warfield \& Rohr, Baltimore, Maryland.

ca. 1880 Illustrated and Descriptive Catalogue of Undertakers' Trimmings and Cabinet Hardware. Warfield \& Rohr, Baltimore, Maryland.

1886 Wholesale Price-List of Coffin and Casket Trimmings, Linings, Robes and Wrappers. Warfield \& Rohr, Baltimore, Maryland.

ca. 1890 Illustrated Catalogue of Warfield \& Rohr, Manufacturers and Jobbers of Wood Coffins and Caskets, Cloth Covered Caskets, Metallic Caskets, Air-Tight Zinc and Copper Linings, Etc. Warfield \& Rohr, Baltimore, Maryland.

ca. 1890 Illustrated Catalogue. Warfield \& Rohr, Baltimore, Maryland.

ca. 1890 Wholesale Price List of Untrimmed Wood Coffins and Caskets, Cloth Covered Caskets, Metallic Coffins and Caskets ... Warfield \& Rohr, Baltimore, Maryland.

1893 Revised Price-List of Coffin and Casket Hardware, Robes, Linings, and Undertakers' Sundries (Nov 1, 1893). Warfield \& Rohr, Baltimore, Maryland.

Watkins, B.

1985 Historical Background. In Gone to a Better Land, edited by Jerome Rose. Research Series No. 25. Arkansas Archeological Survey, Fayetteville, Arkansas. 
Wayne, J. L., \& Sons

1874 Illustrated Catalogue of Casket and Coffin Trimmings, Manufactured by the Wayne Hardware Company. J. L. Wayne \& Sons, Cincinnati, Ohio; Chicago, Illinois; and New York, New York.

Wells, T.

1993 Nail Dating: Identifying Chronologically Significant Features on Nails. Master's thesis, Department of Anthropology, Louisiana State University, Baton Rouge, Louisiana.

1998 Nail Chronology: The Use of Technologically Derived Features. Historical Archaeology 32(2):78-99.

West, J. M.

2011 "Sugar and Slavery: Molasses to Rum to Slaves. Slavery in America. History." Electronic document http://slaveryinamerica.org/history/hs_es_sugar.htm, accessed $3 / 23 / 2011$.

White, T. D., and P. Folkens

1991 Human Osteology. Academic Press, Inc., New York.

Wilke, C.

2011 "Sugar Production.” Handbook of Texas Online. Published by the Texas State Historical Association. Electronic document http://www.tshaonline.org/handbook/ online/articles/afs02, accessed 6/17/2011.

Williamson, C.

2005 Sacred Trust: The Voluntary Removal and Reburial of Human Remains from a Historic Cemetery in Louisiana. Master's thesis. Louisiana State University, Baton Rouge, Louisiana.

Wilmarth, W. D., \& Company

1894 Illustrated and Descriptive Catalogue of Coffin and Casket Trimmings. W. D. Wilmarth \& Company, Atlanta, Georgia.

Wilson, J. N.

1982 Watson-Jones Fractures and Joint Injuries. Volume 11. 6th edition. Churchill Livingstone, New York.

Wilson, K. J.

1998a Archaeological Disinterment/Reinterment of the Pine Ridge Cemetery, Butts County, Georgia. TRC Garrow Associates, Inc., Atlanta, Georgia. Submitted to Republic Industries, Winder, Georgia.

1998b Archaeological Disinterment of the Oliver Family Grave Sites, Roanoke, Virginia. TRC Garrow Associates, Inc., Atlanta, Georgia. Submitted to the Office of Economic Development, city of Roanoke, Virginia. 
1998c Archaeological Disinterment of the Turner Cemetery, Marshal County, Mississippi. TRC Garrow Associates, Inc., Atlanta, Georgia. Submitted to Lewis \& Lewis, P.A., Clarksdale, Mississippi.

Wilson, K. J., and J. L. Holland

1998 Archaeological Disinterment of the Fuller Cemetery, Calhoun, Georgia. TRC Garrow Associates, Inc., Atlanta, Georgia.

Wilson, $\mathrm{M}$.

1968 Two Historic Burials in the Three Forks Locale. Bulletin of the Oklahoma Anthropological Society 17:75-86.

Winchell, F., J. C. Rose, and R. W. Moir

1992 Bioanthropological Investigation of Nineteenth Century Burials at Site 41DT105: Cultural Resource Studies for Cooper Lake, Delta County, Texas. Archaeology Research Program, Department of Anthropology, Southern Methodist University, Dallas, Texas. Submitted to U.S. Army Corps of Engineers, Fort Worth District, Texas.

Wood, W. D.

2008 Report on the Disinterment and Relocation of the James and Sarah Barnes Cemetery, Bibb County, Georgia. Southern Research, Historic Preservation Consultants, Inc., Ellerslie, Georgia. Prepared for Macon-Bibb Industrial Authority, Macon, Georgia.

Wood, W. D., K. R. Burns, and S. R. Lee

1986 The Mt. Gilead Cemetery Study: An Example of Biocultural Analysis from Western Georgia. Southeastern Archaeological Services, Inc., Athens, Georgia. Prepared for U.S. Army Infantry Center, Fort Benning, Georgia, and U.S. Army Corps of Engineers, Savannah District, Georgia.

Woodley, P. J.

1992 The Stirrup Court Cemetery Coffin Hardware. Ontario Archaeology 53:45-63.

Woodmen of the World

2011 "Woodmen of the World." Electronic document http://wiki.ask.com/ Woodmen_of_the_World, accessed 3/19/2011.

Yalom, M.

2008 The American Resting Place. Four Hundred Years of History through Our Cemeteries and Burial Grounds. Houghton Mifflin Books, New York.

Young, A. L.

1991 Nailing Down the Pattern in Historical Archaeology. Master's thesis, University of Tennessee, Knoxville, Tennessee.

Zanesville Coffin Company 1880 Illustrated Catalogue. Zanesville Coffin Company, Zanesville, Ohio. 

APPENDIX A

ARTIFACT INVENTORY 



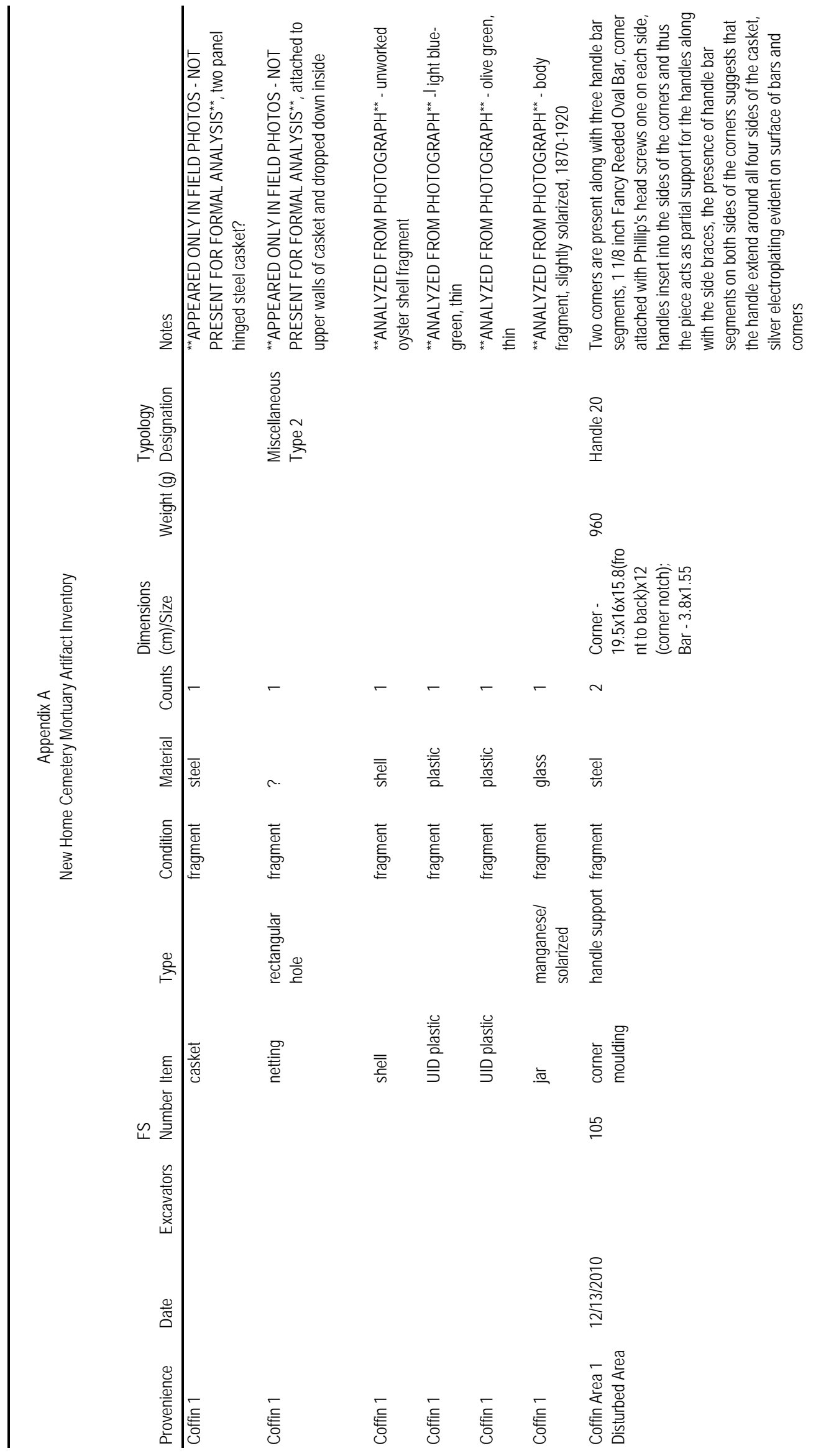




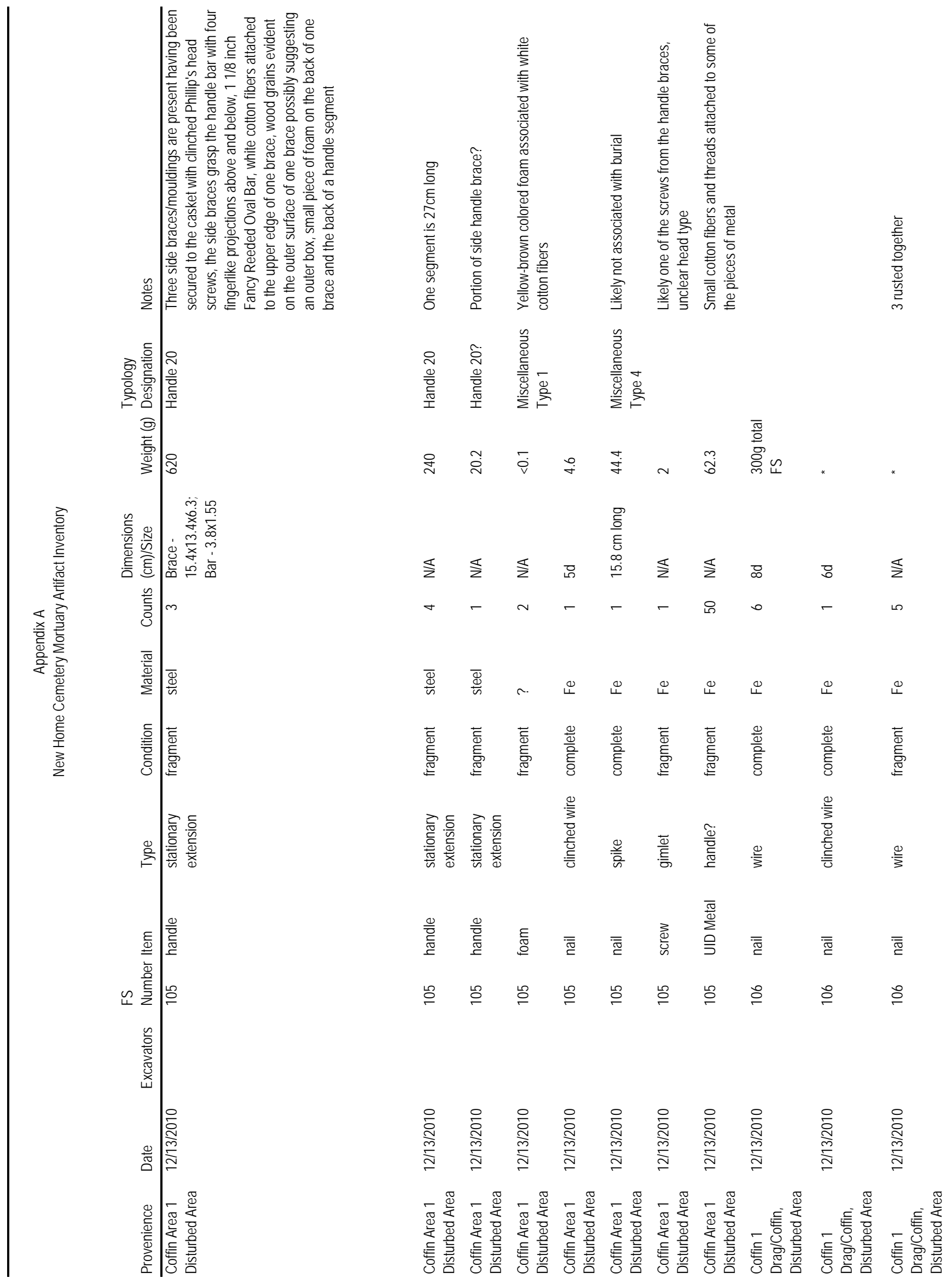




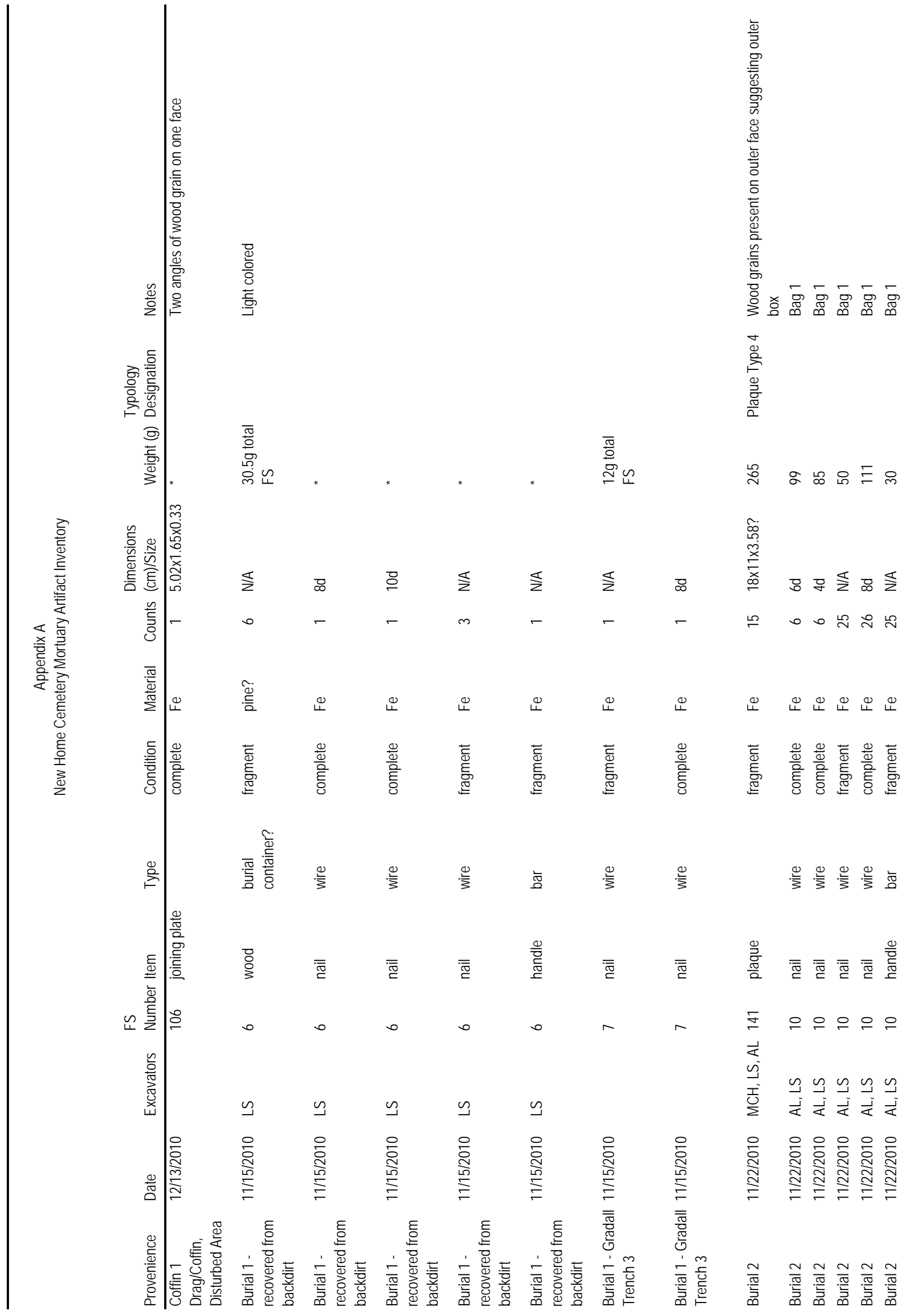




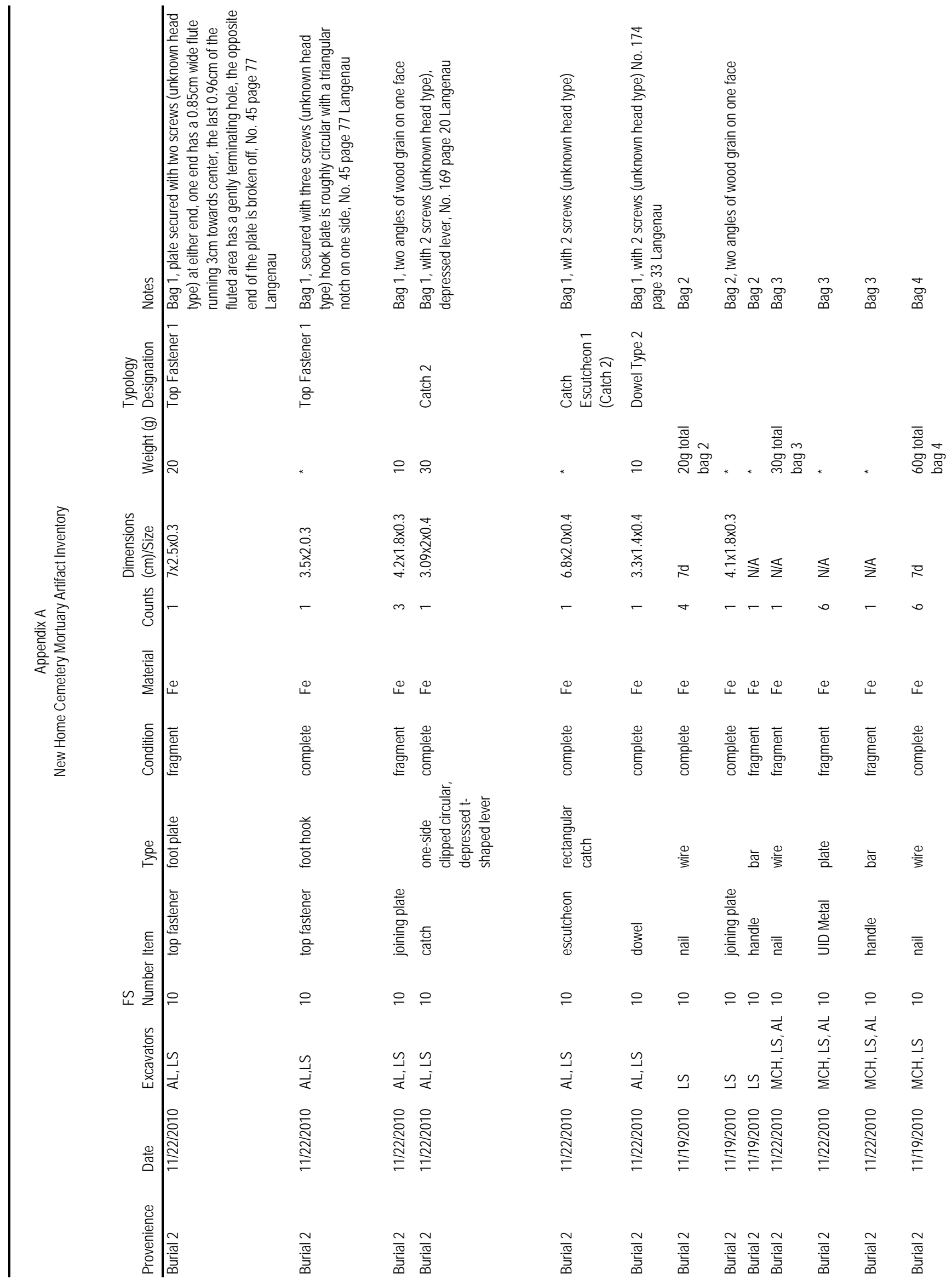




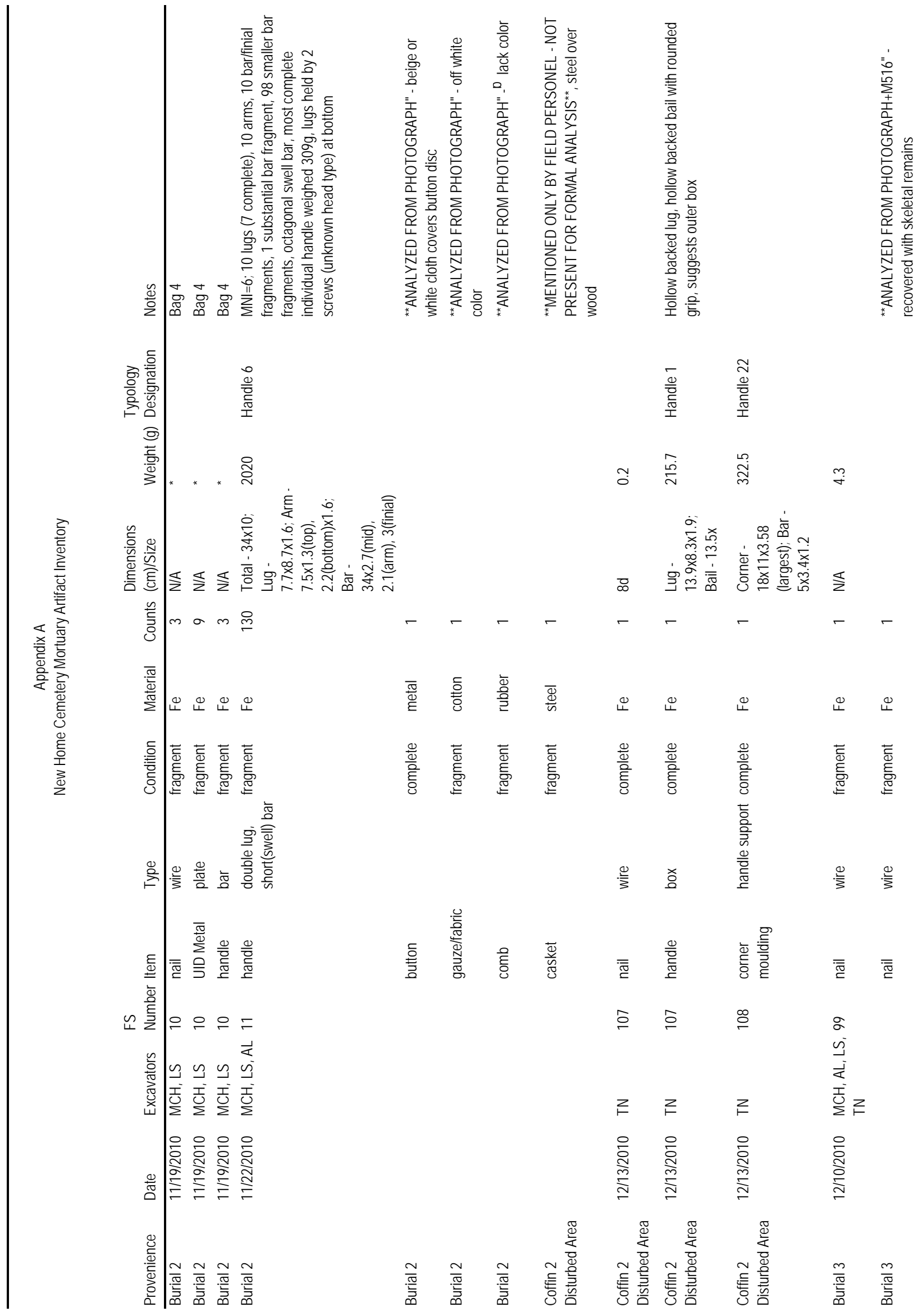




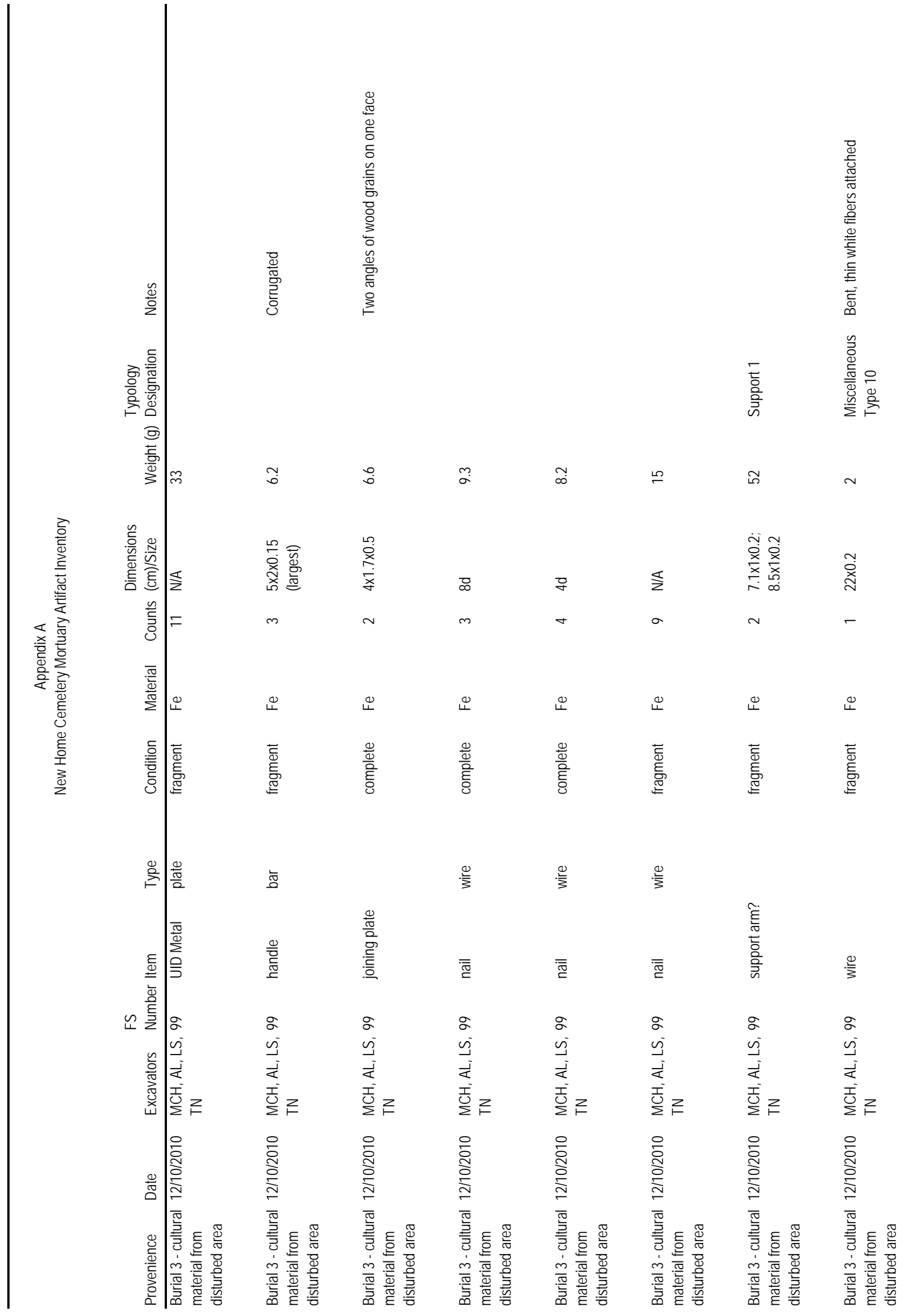




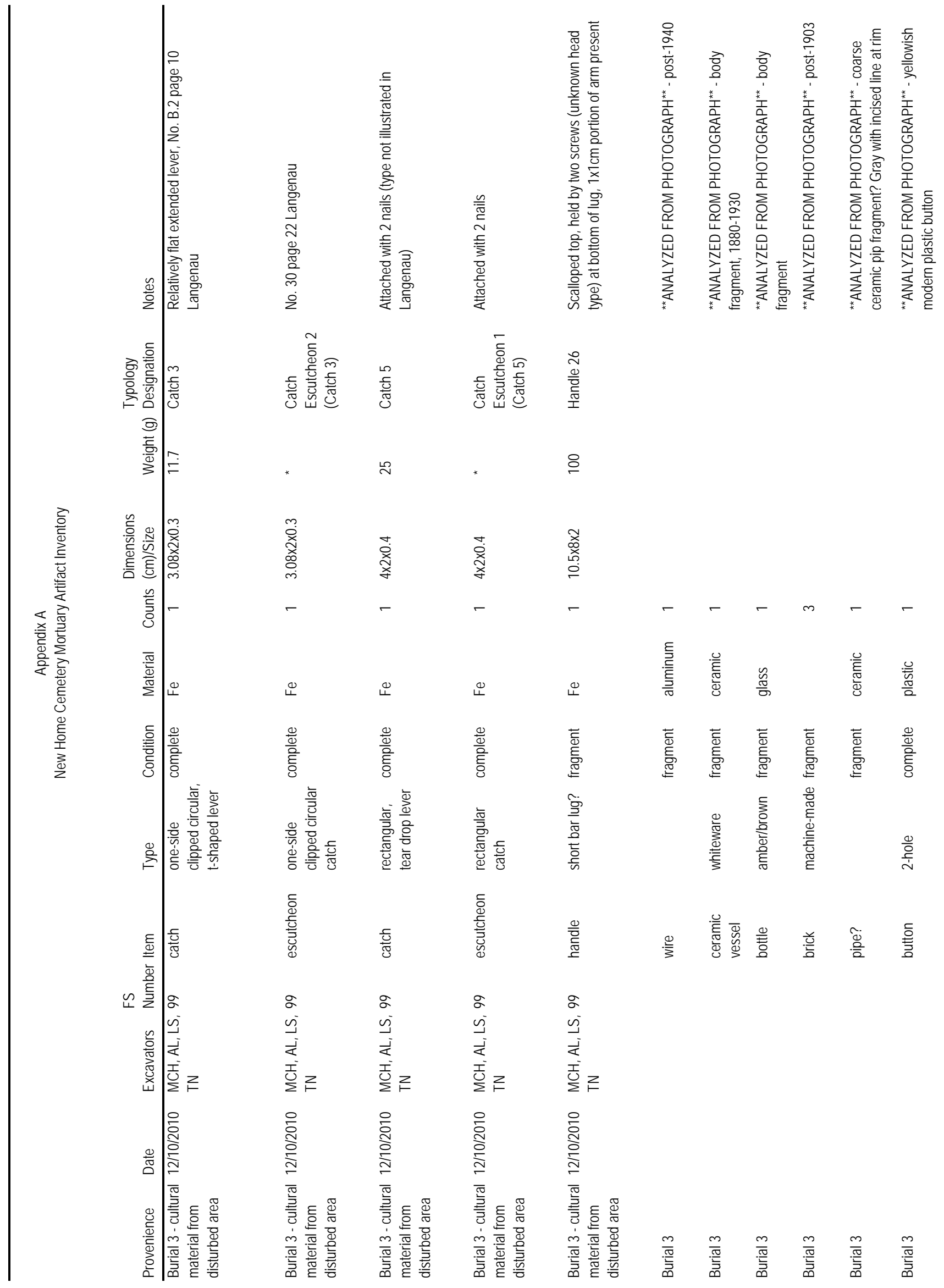




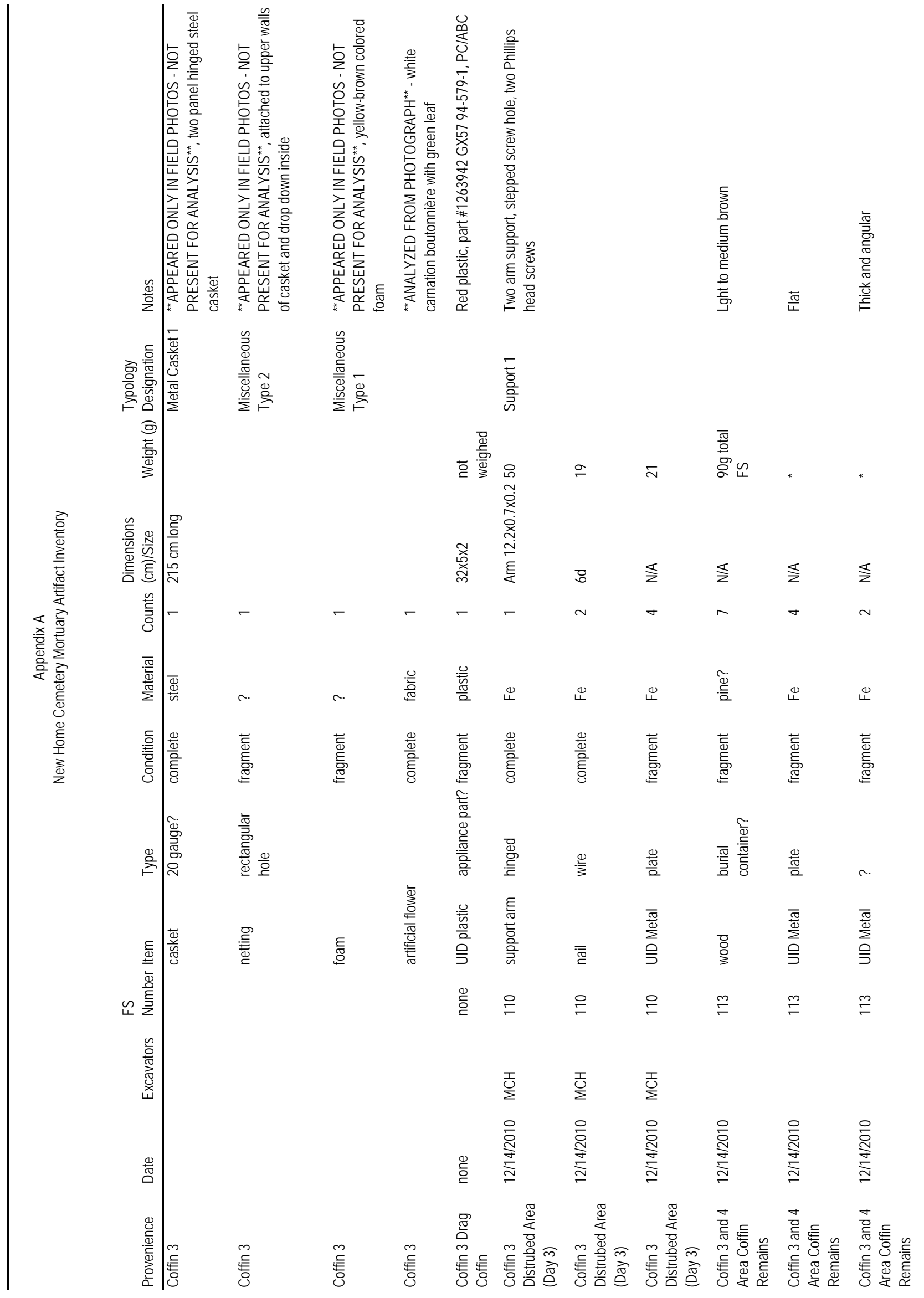




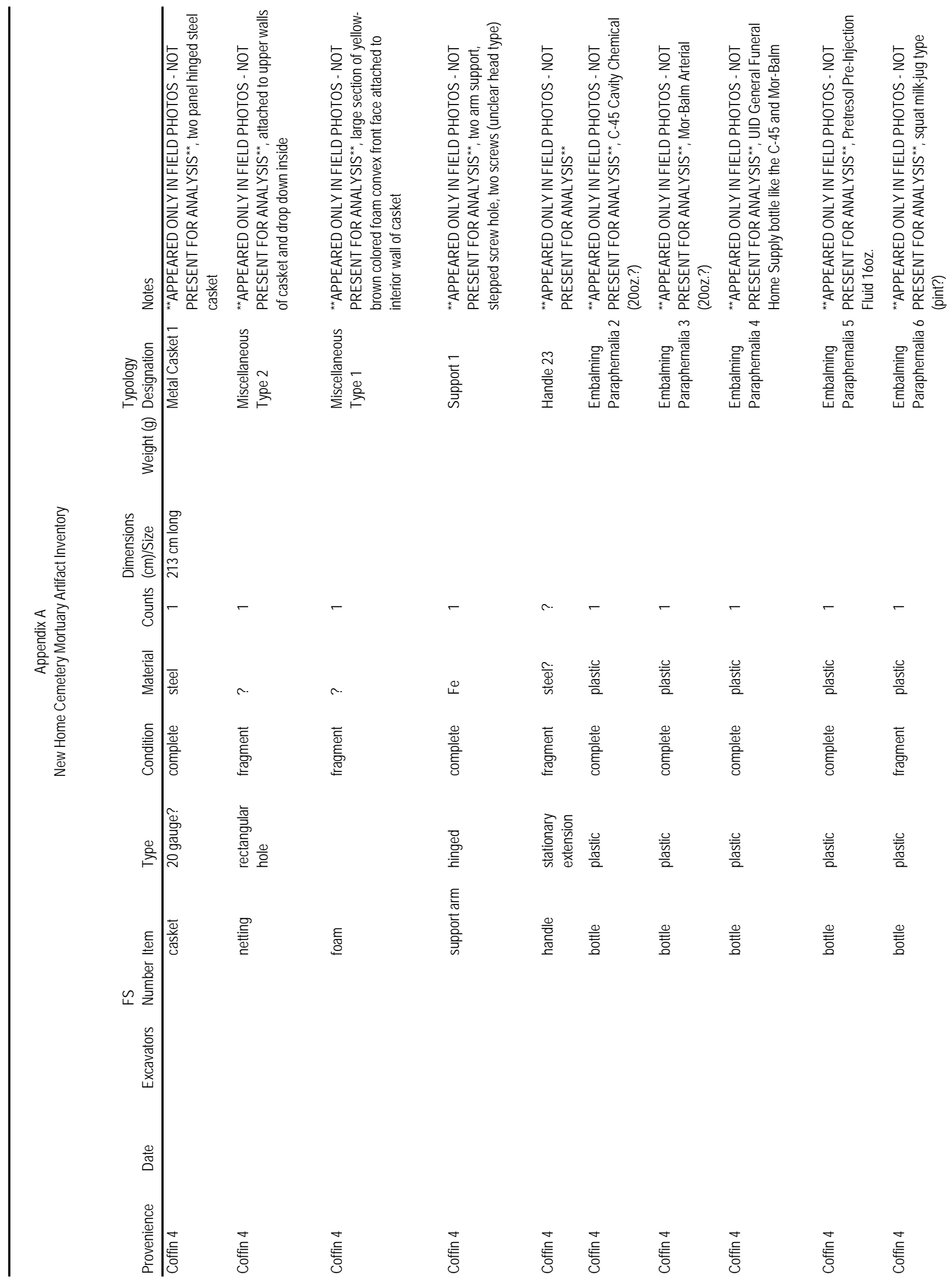




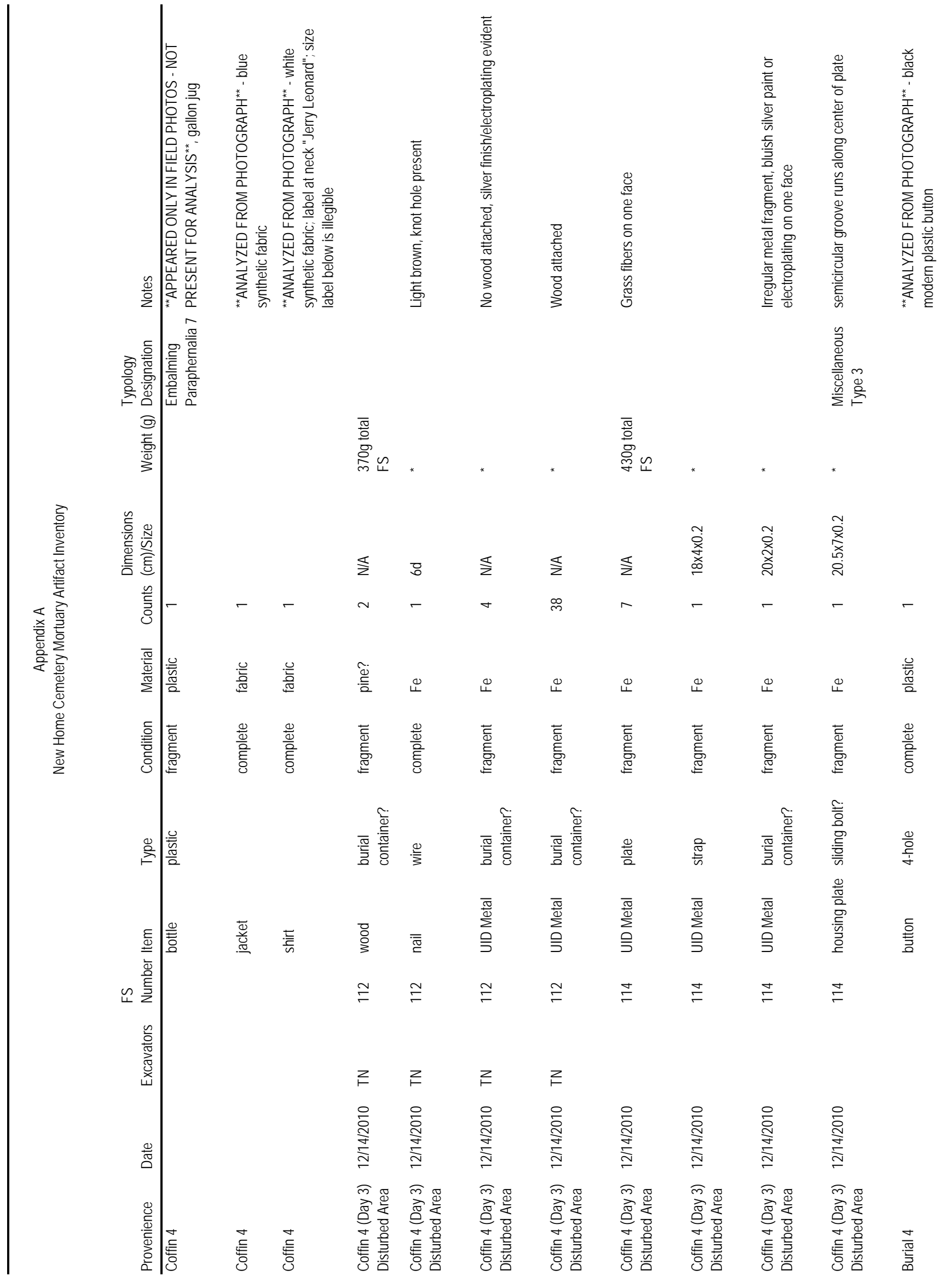




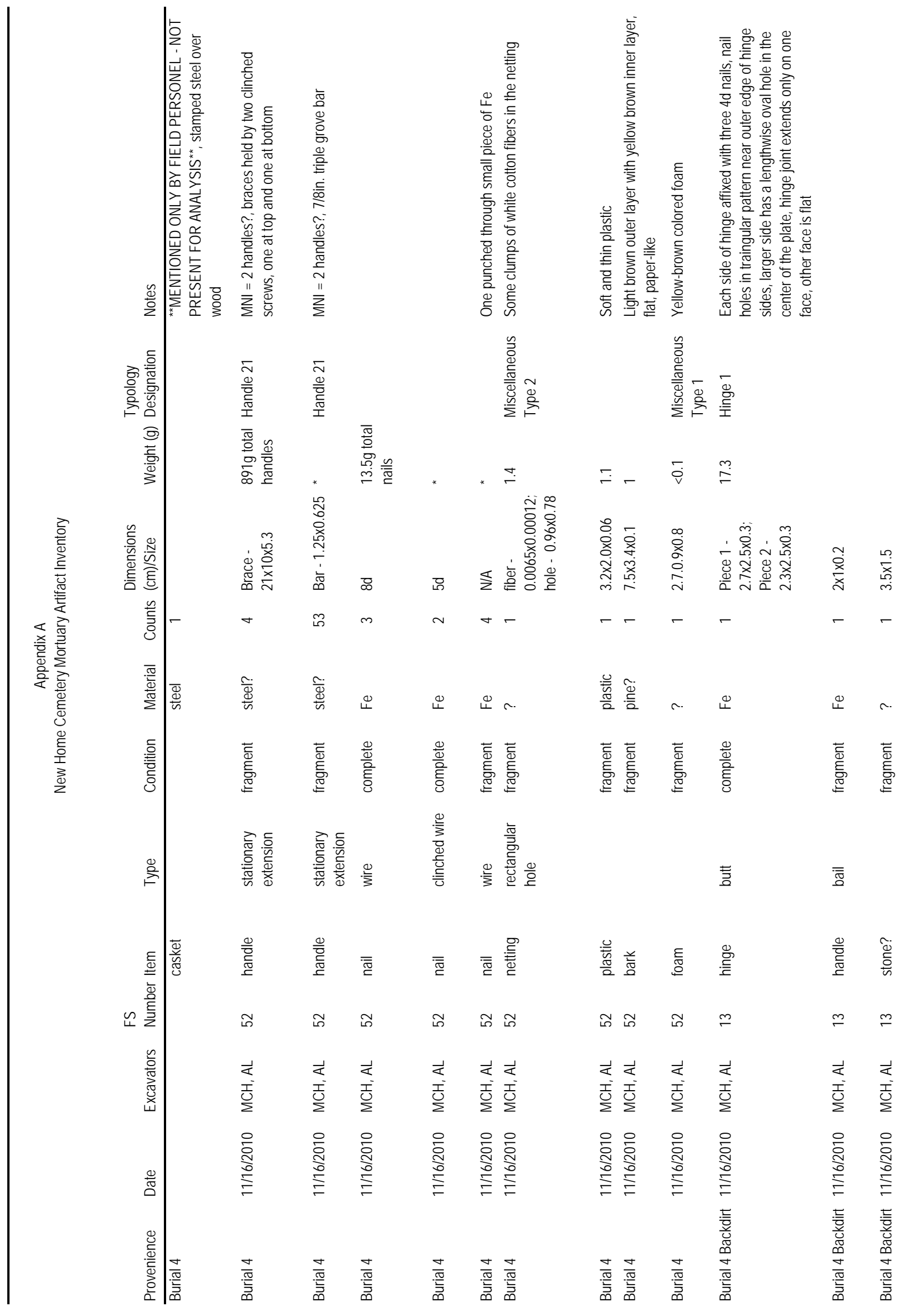




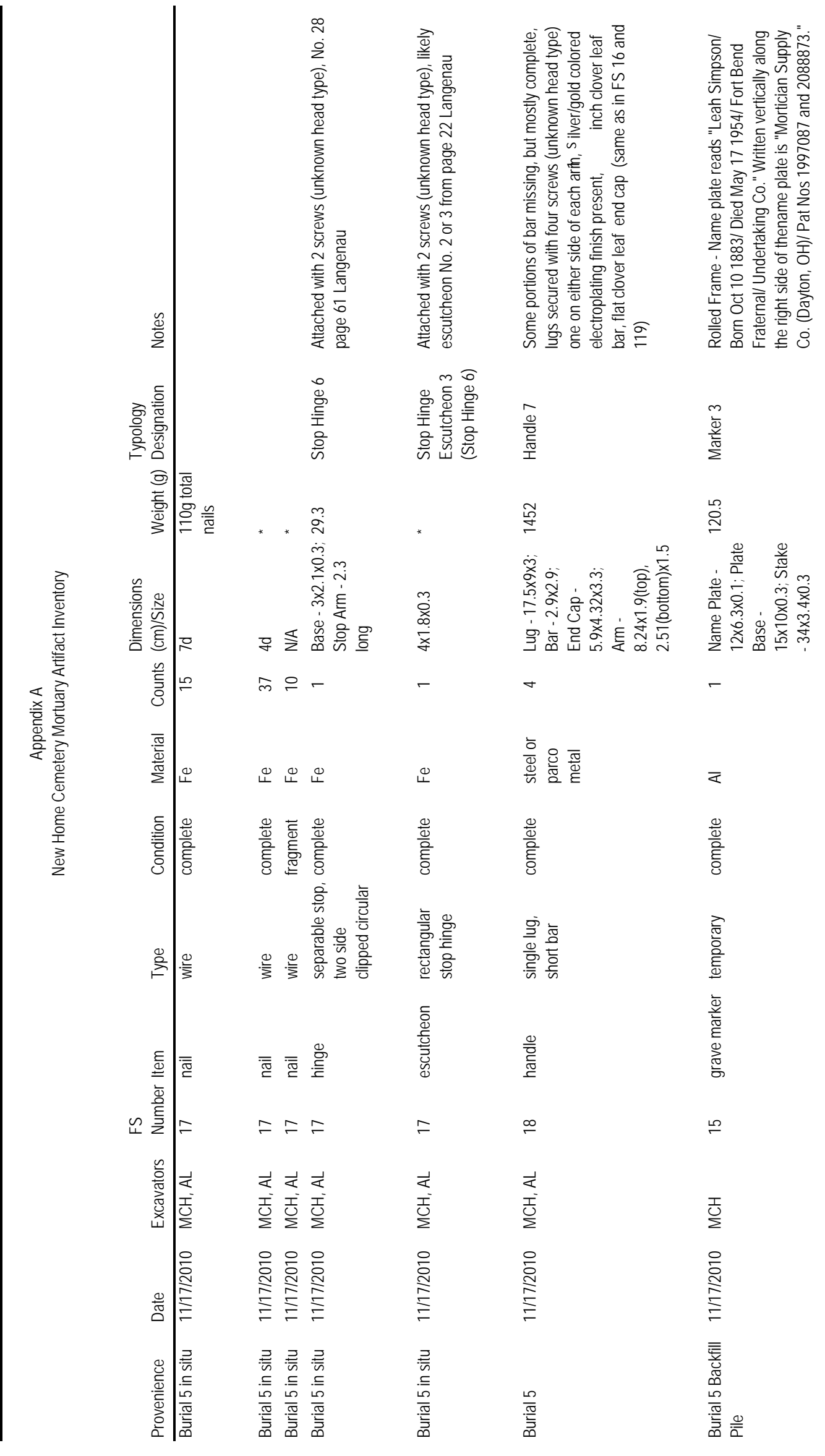




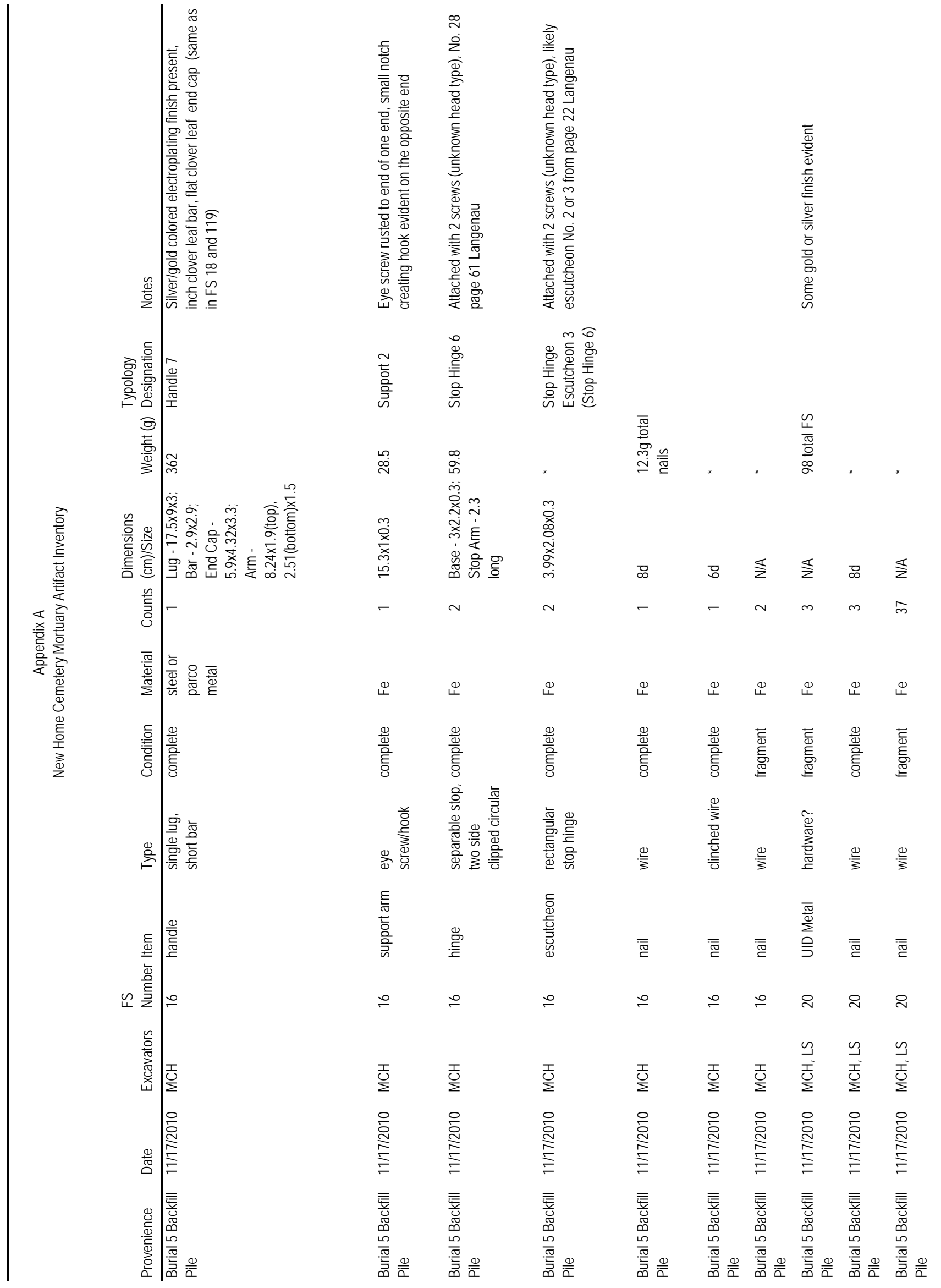




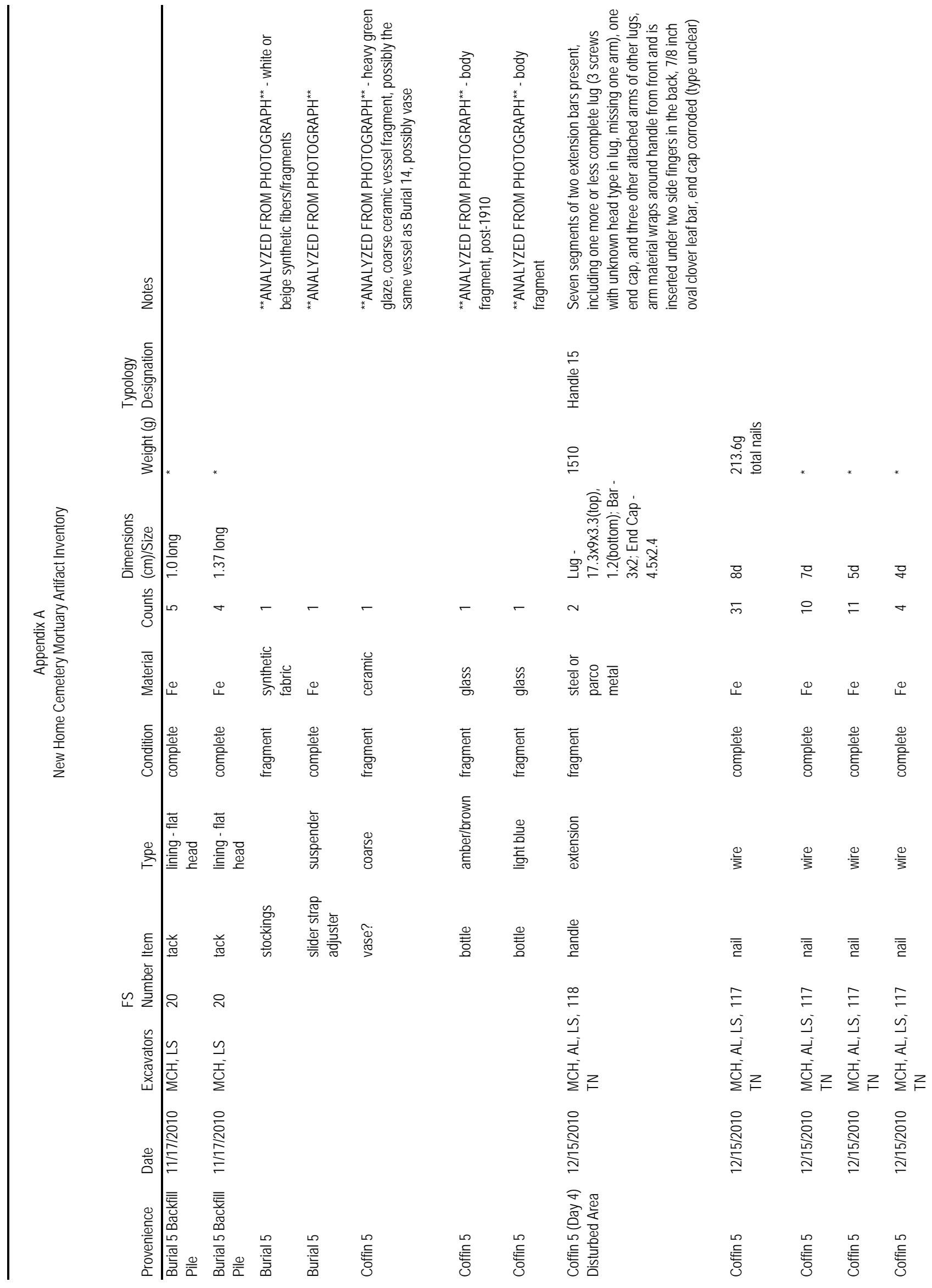




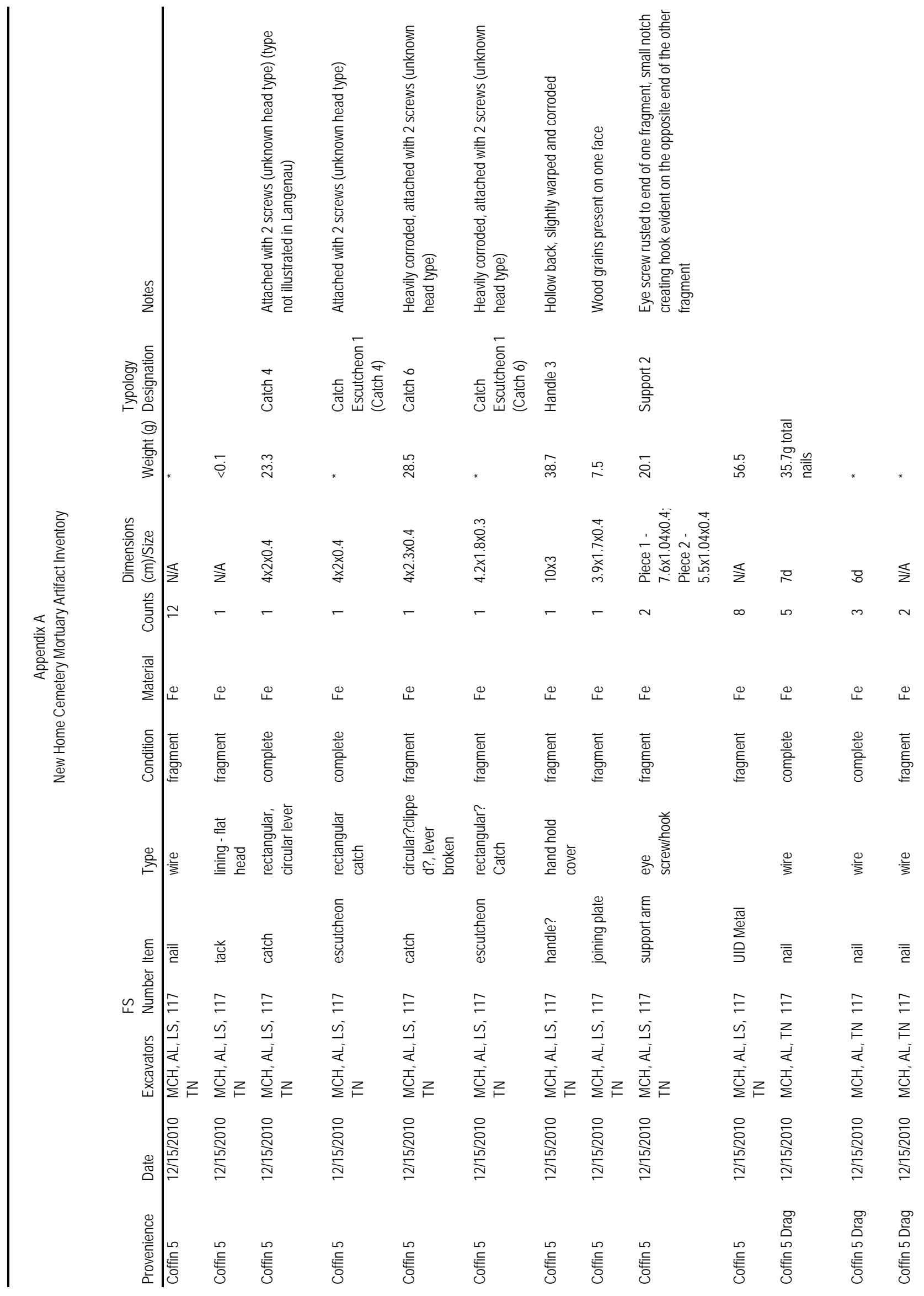




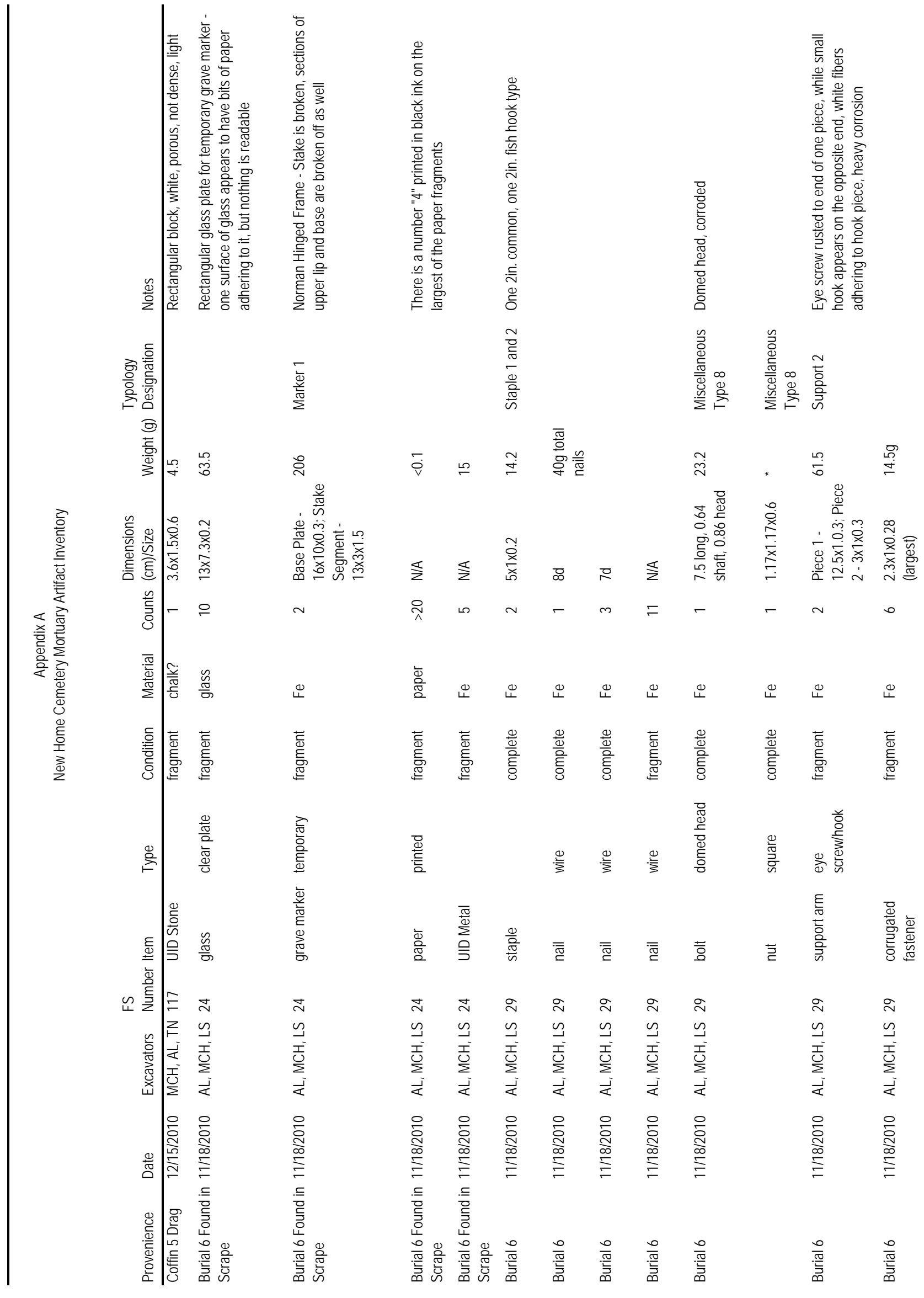




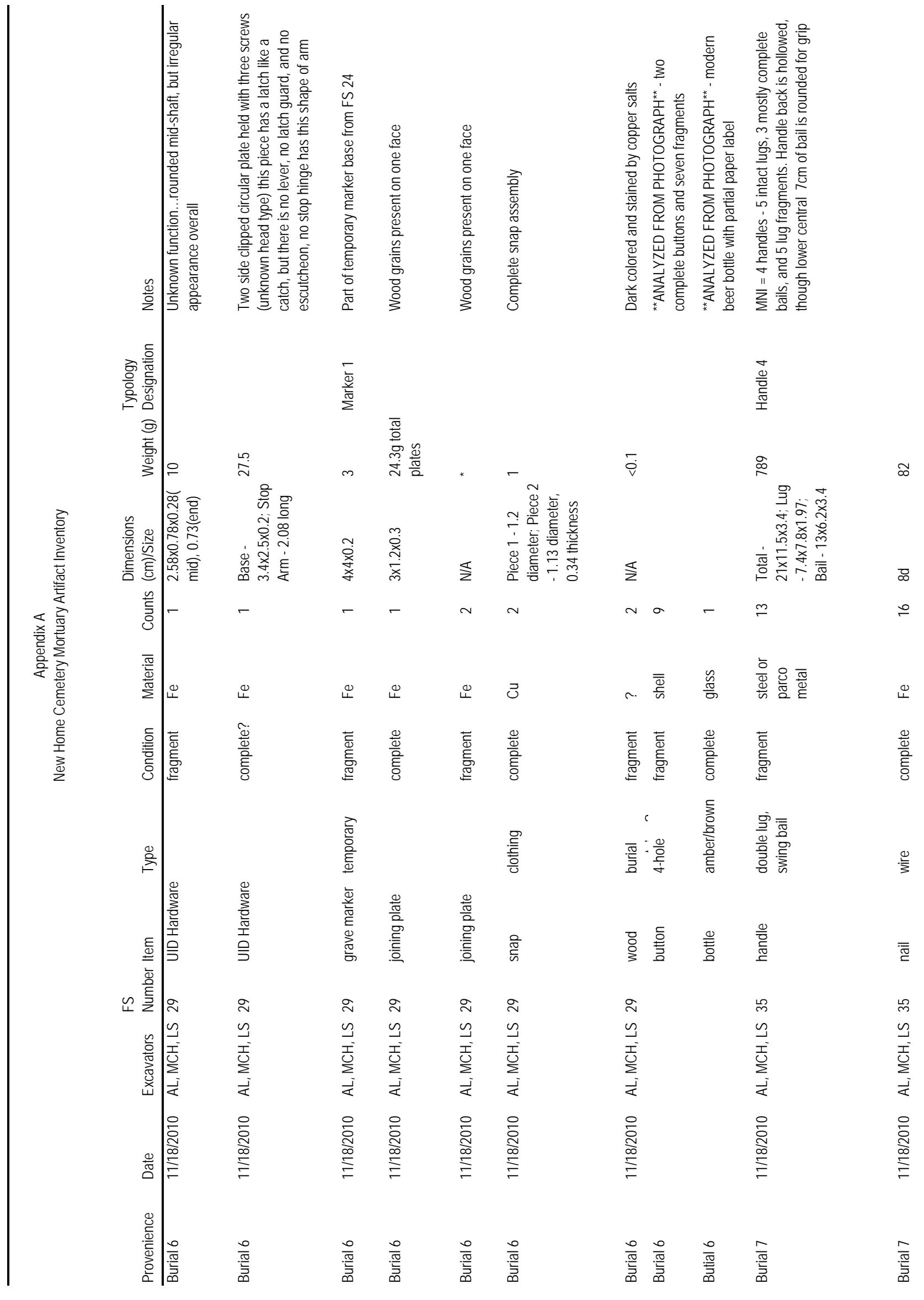




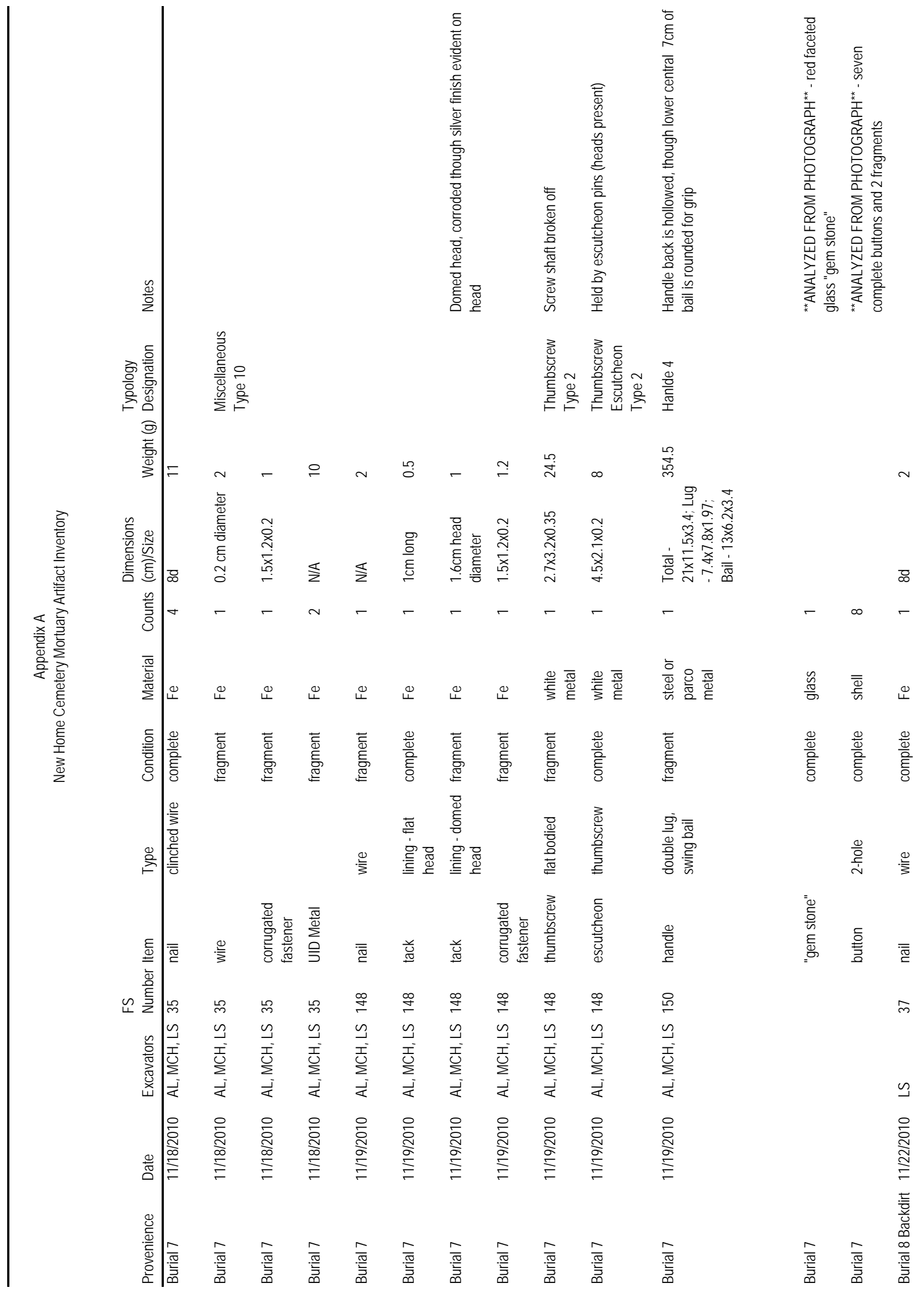




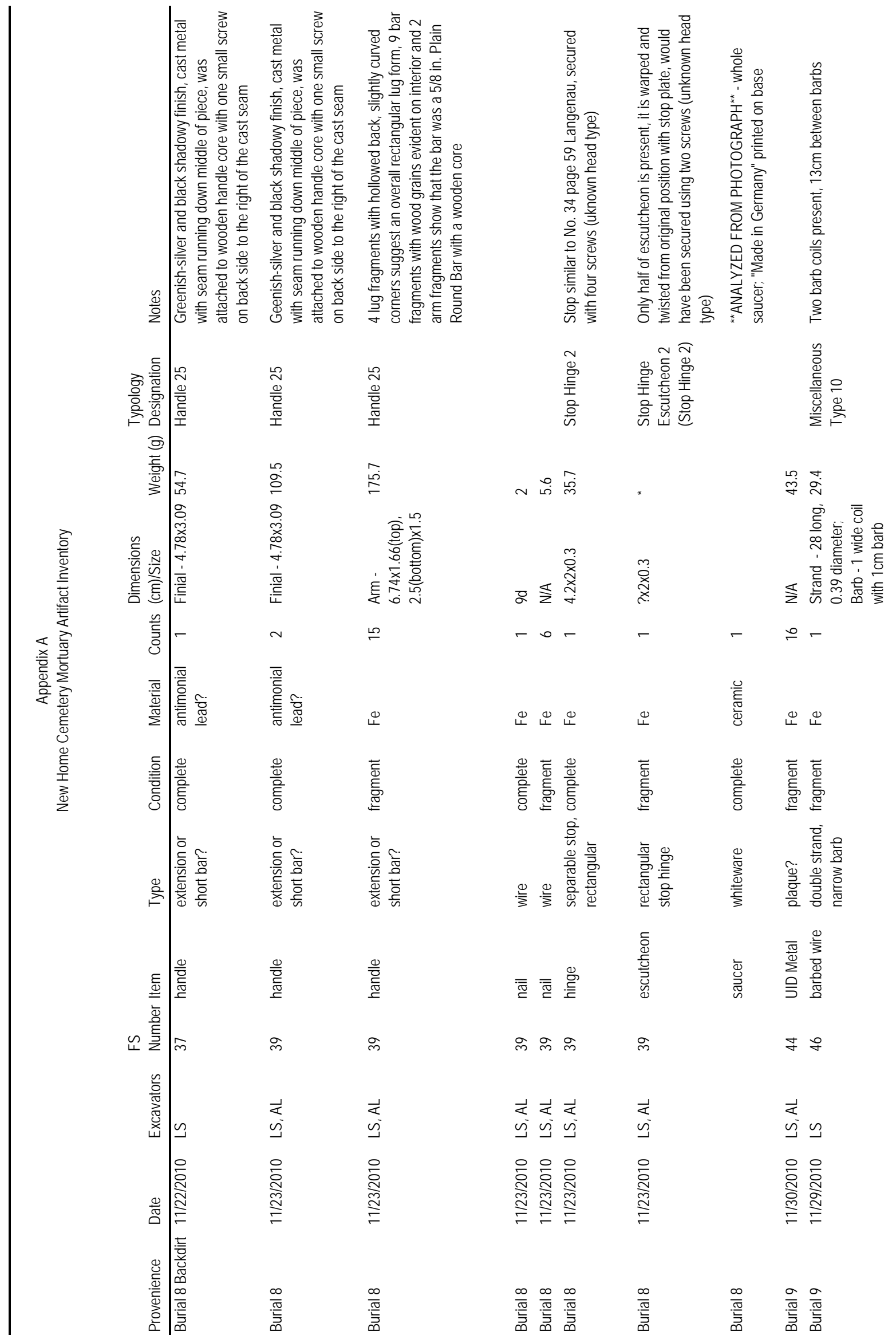




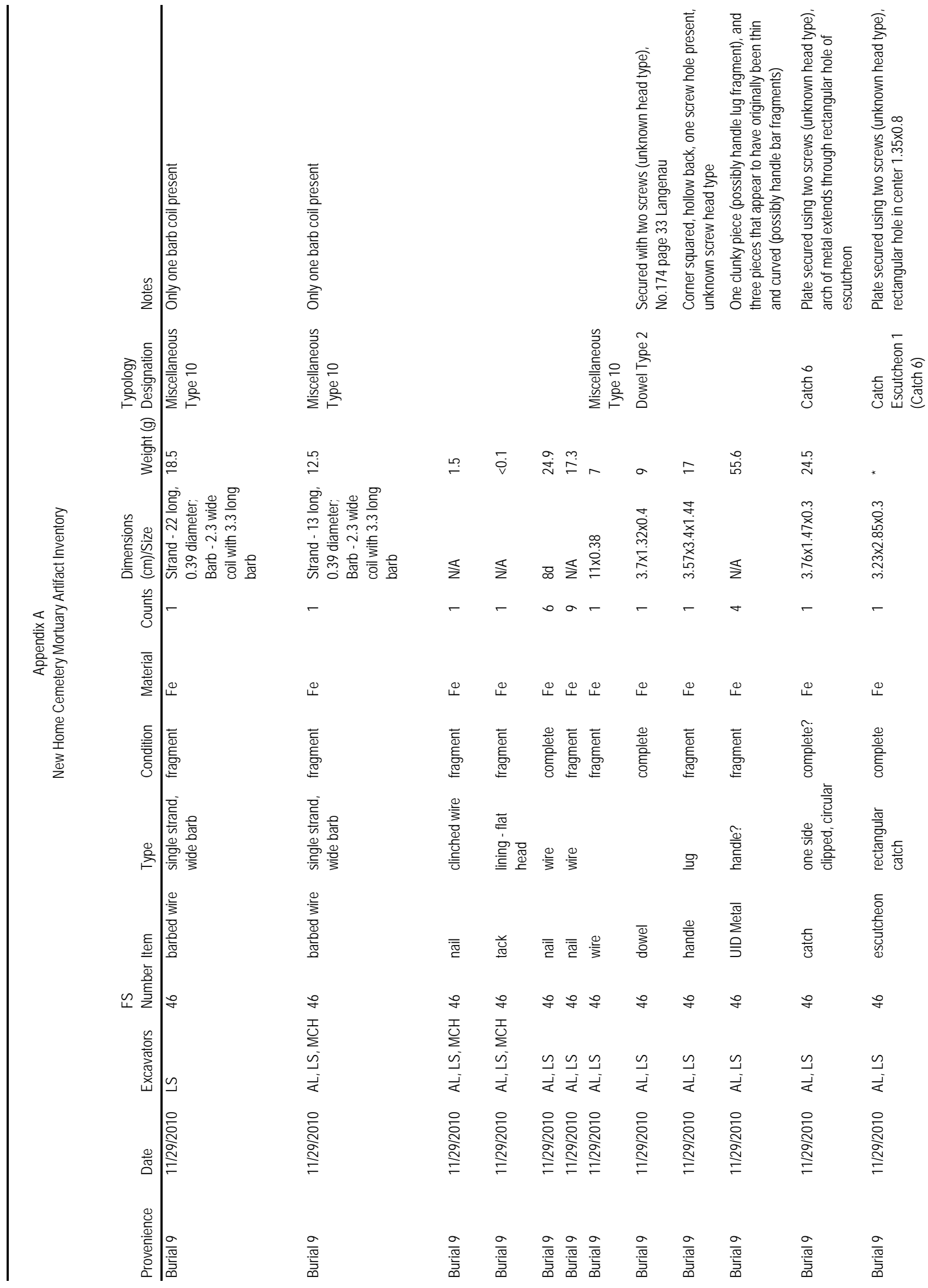




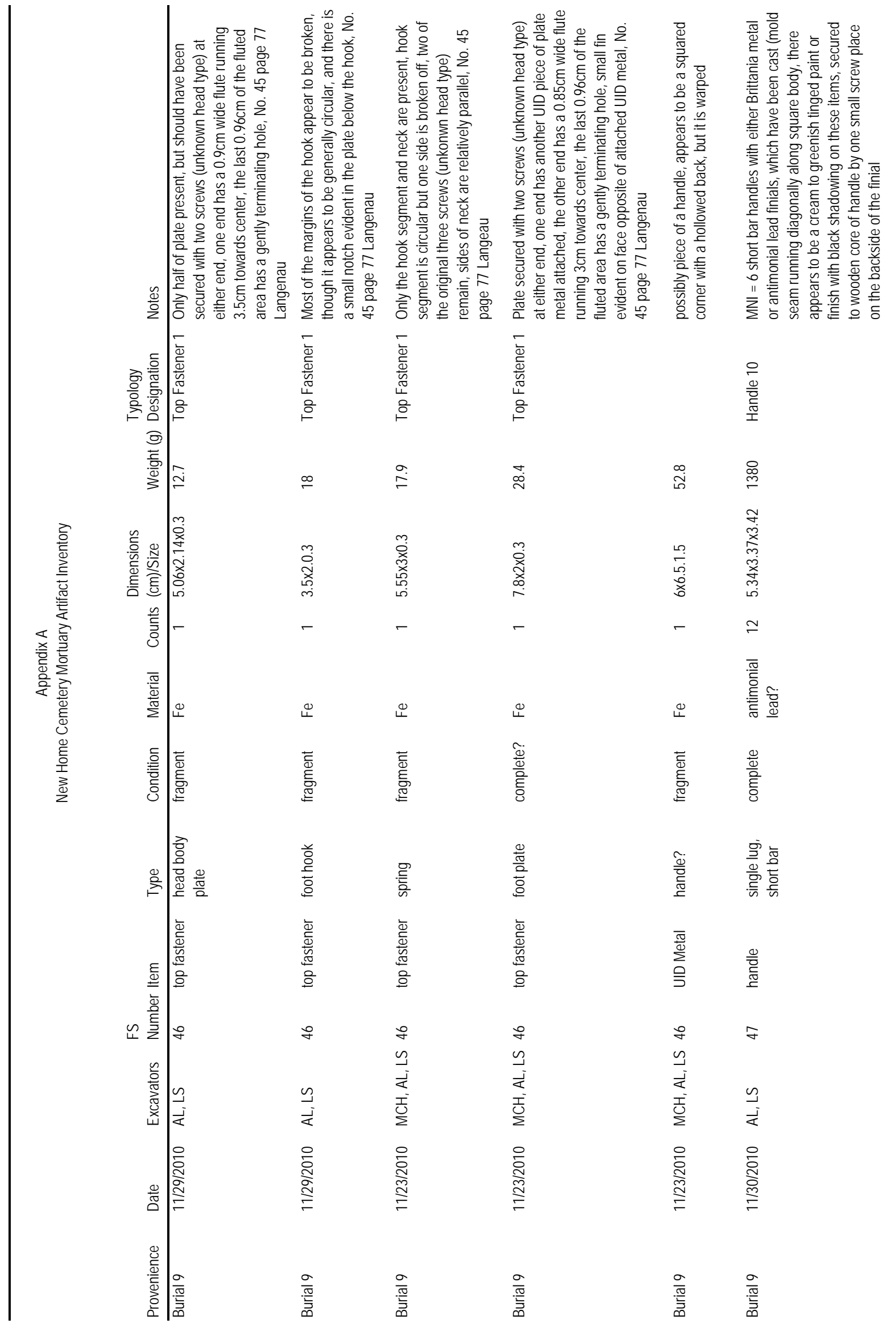




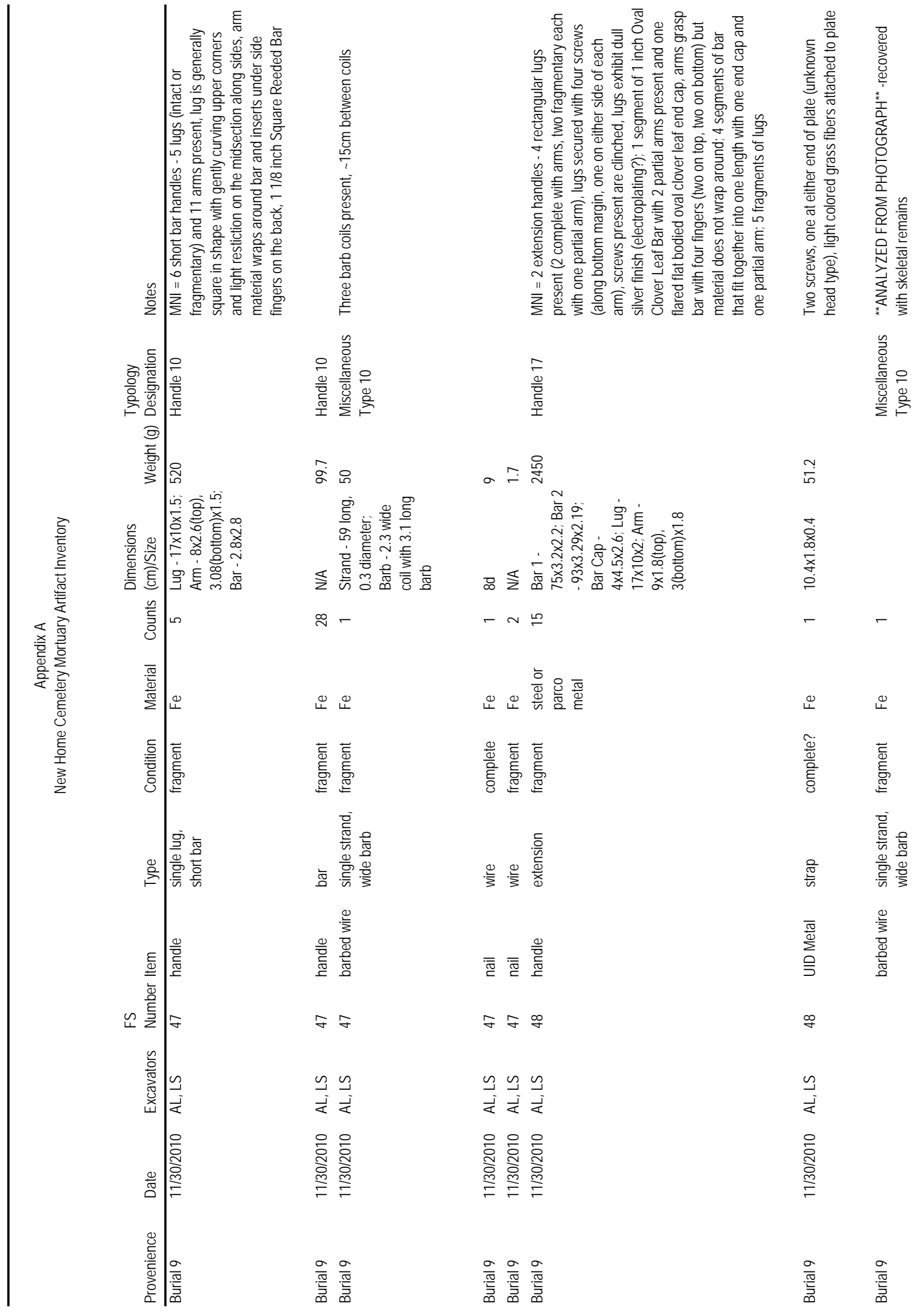




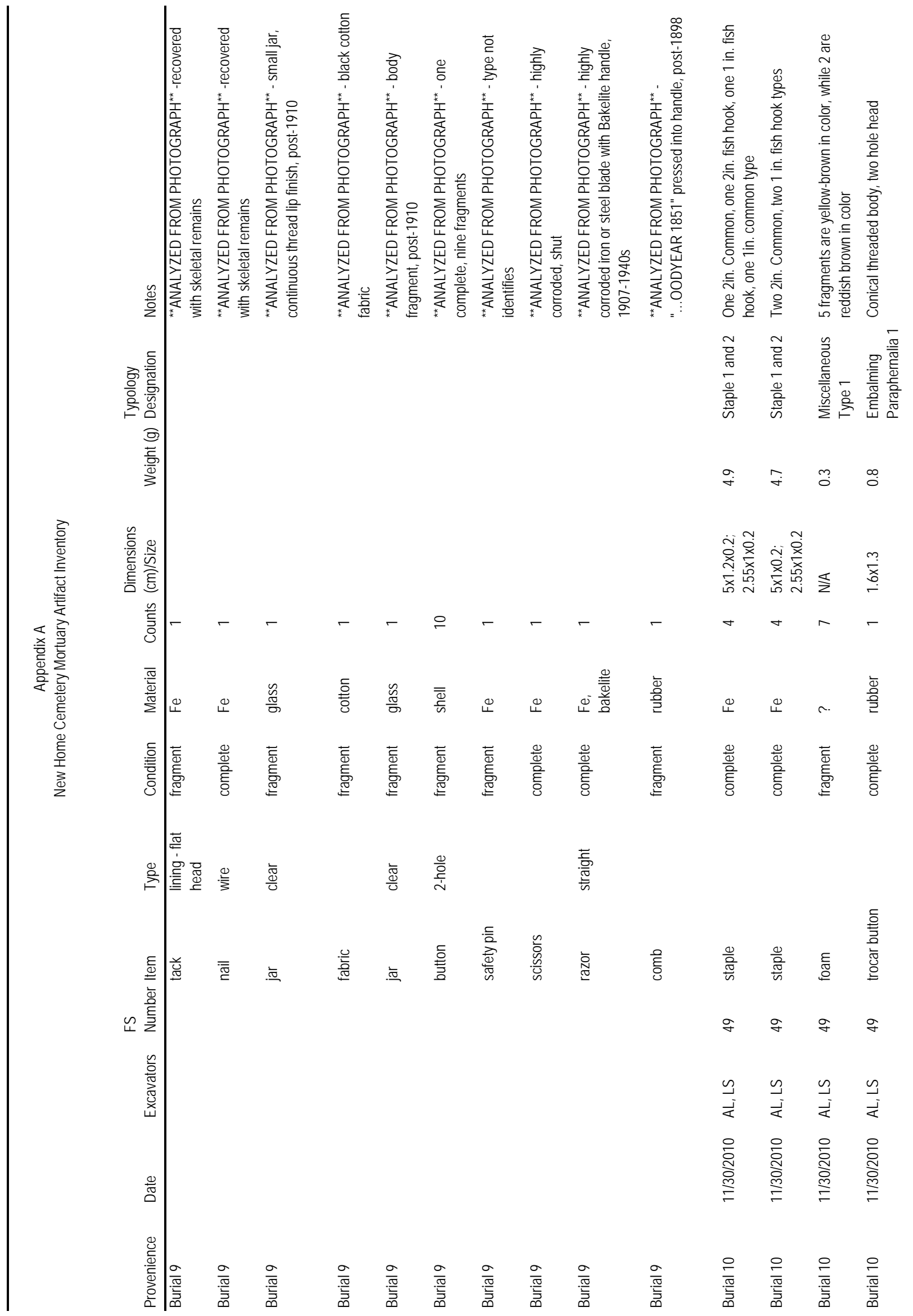




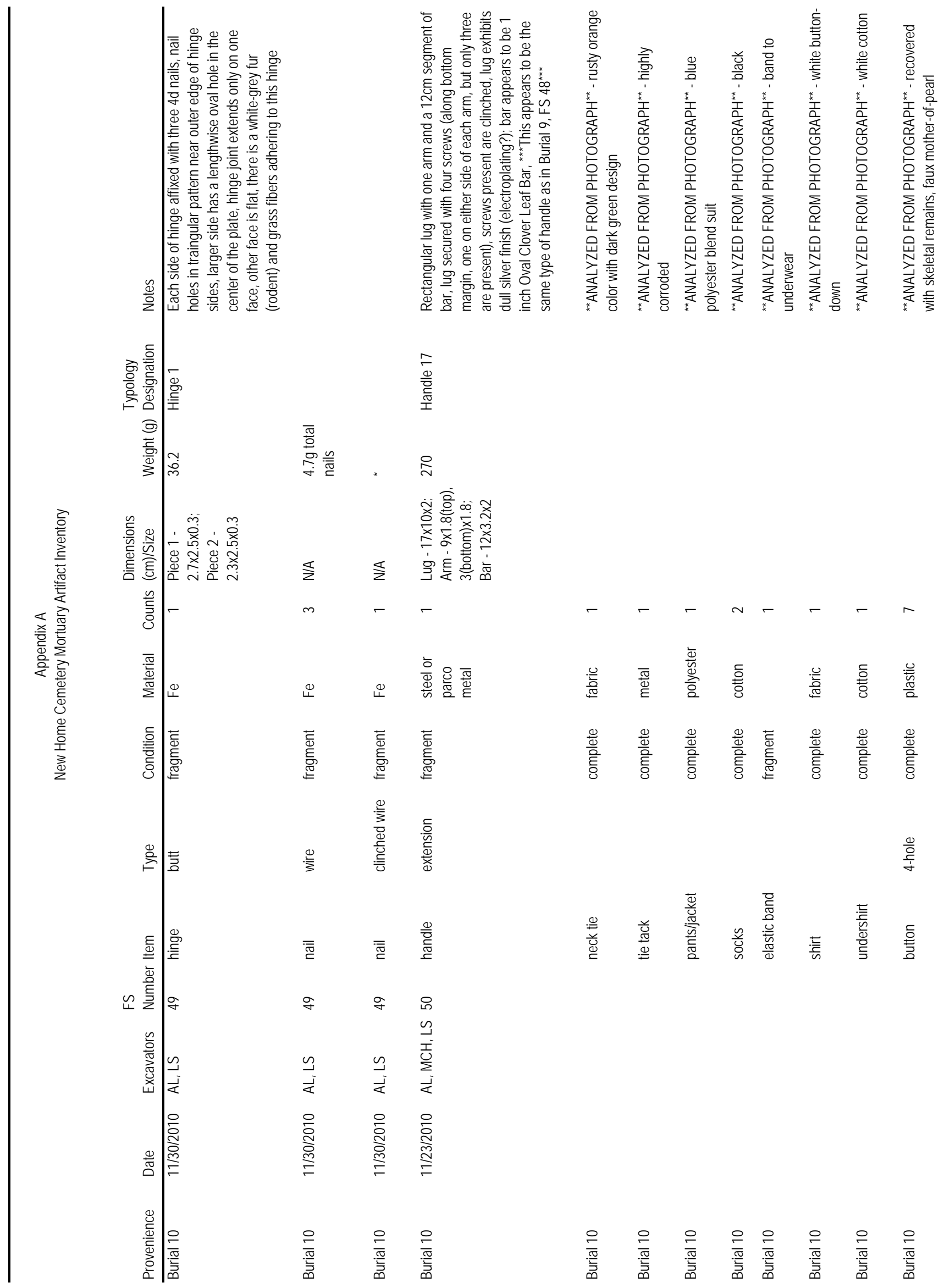




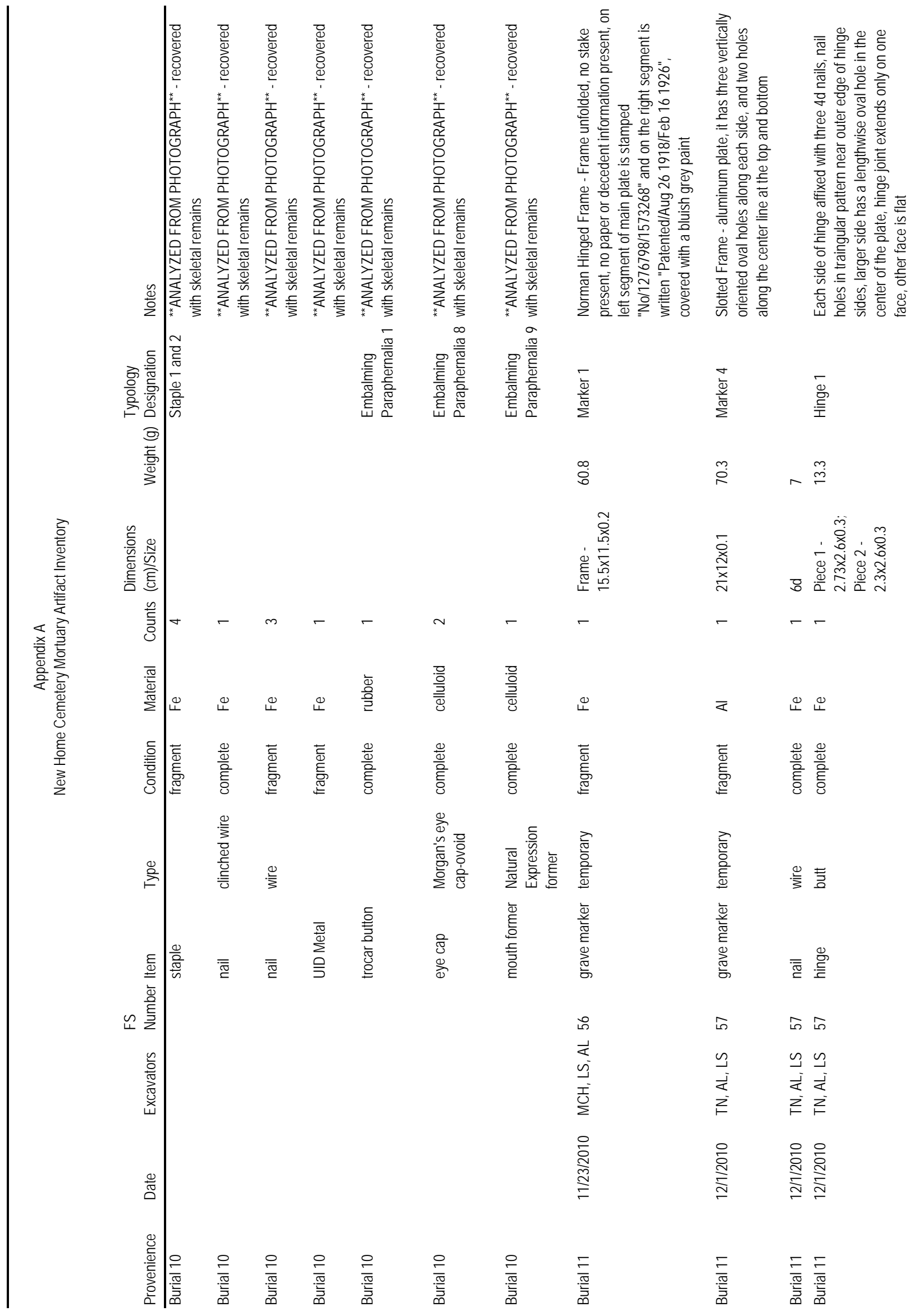




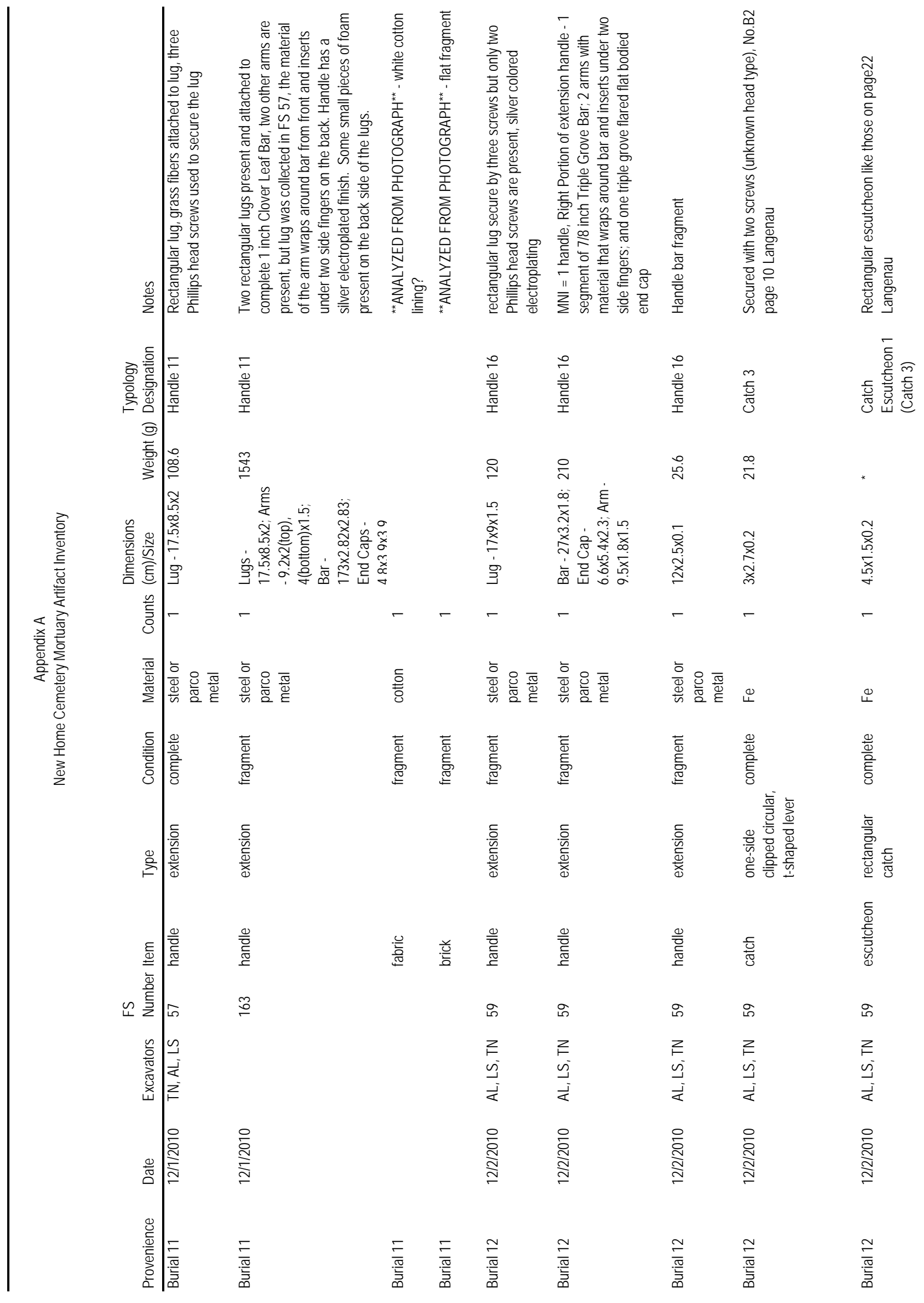




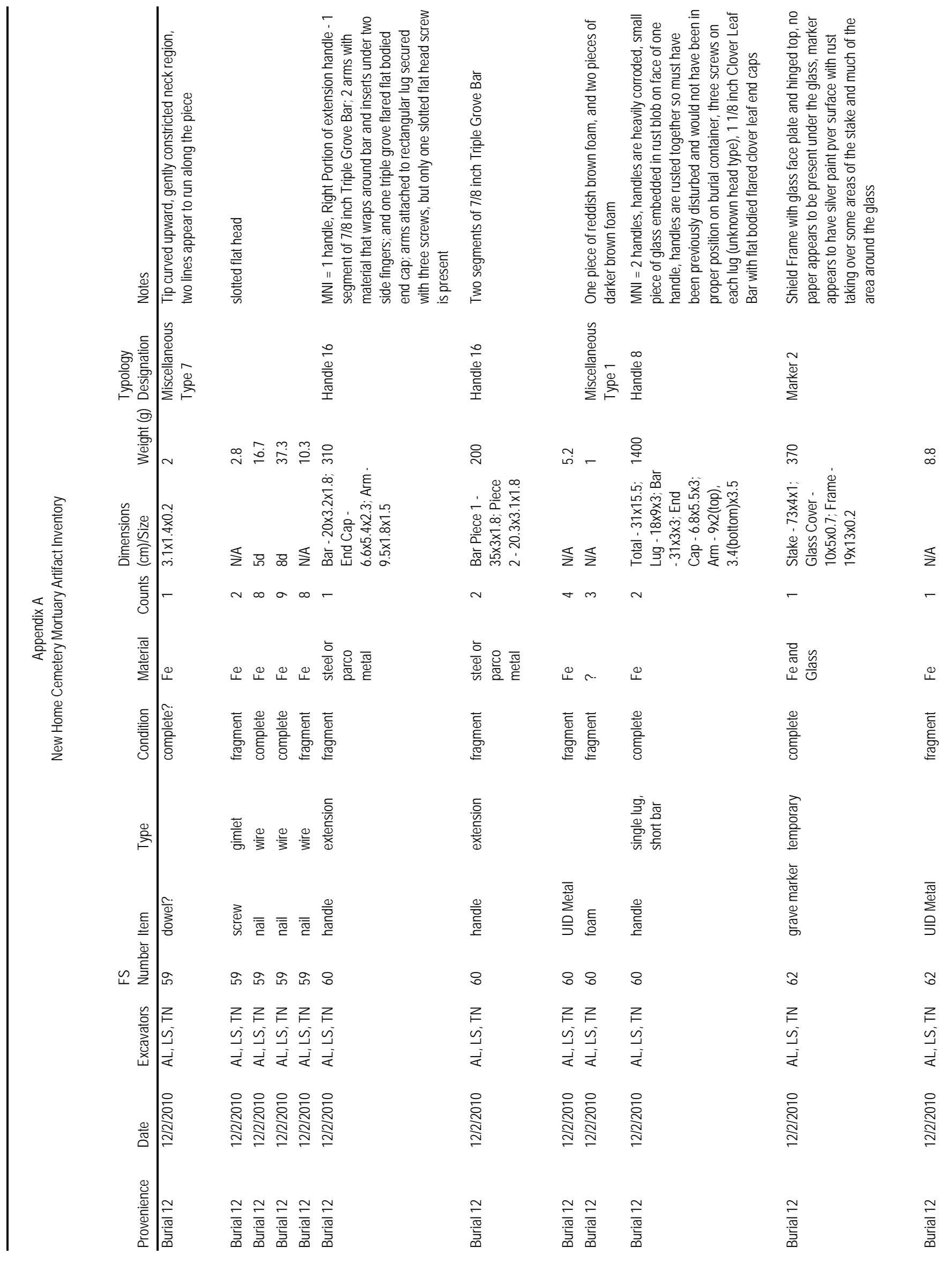




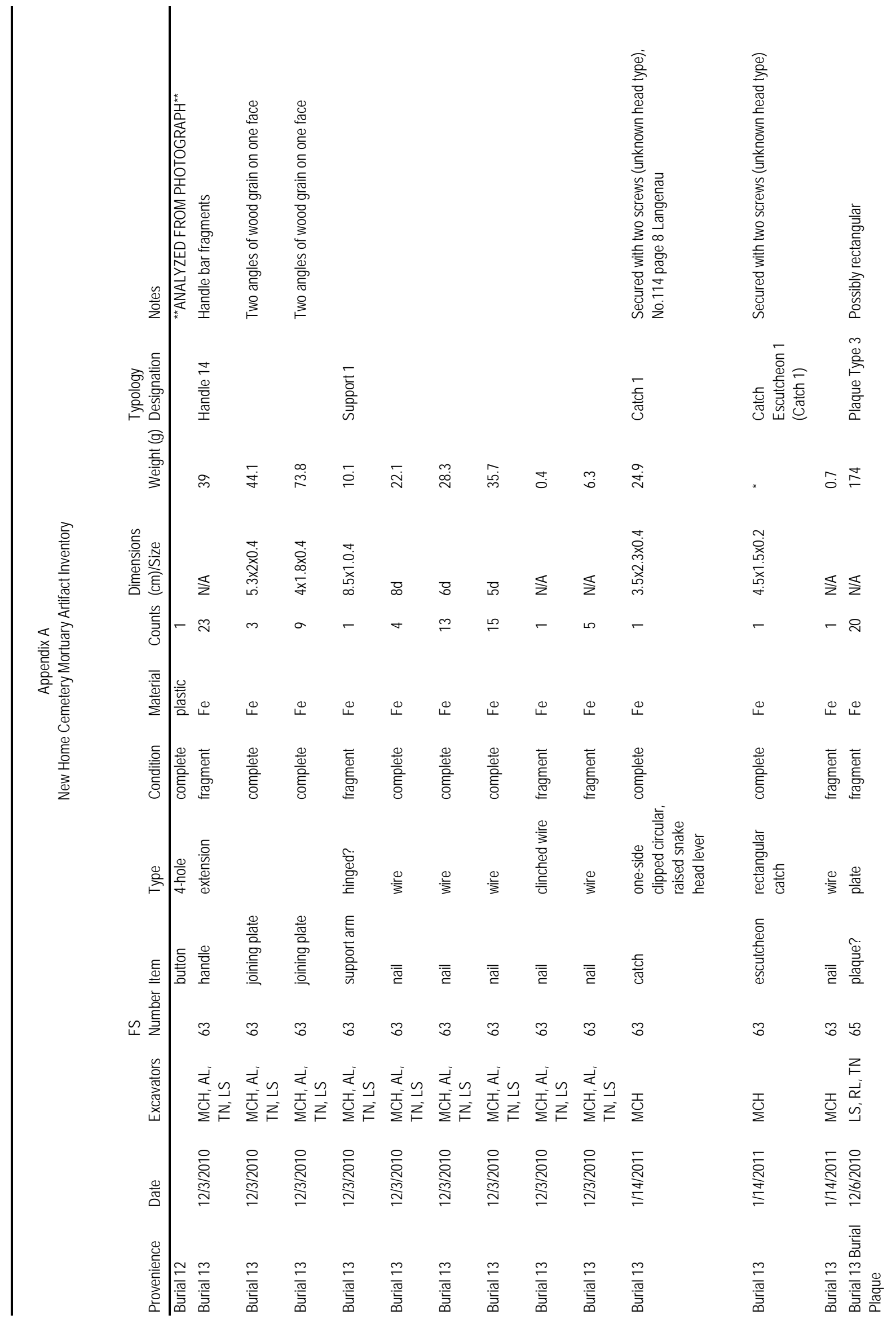




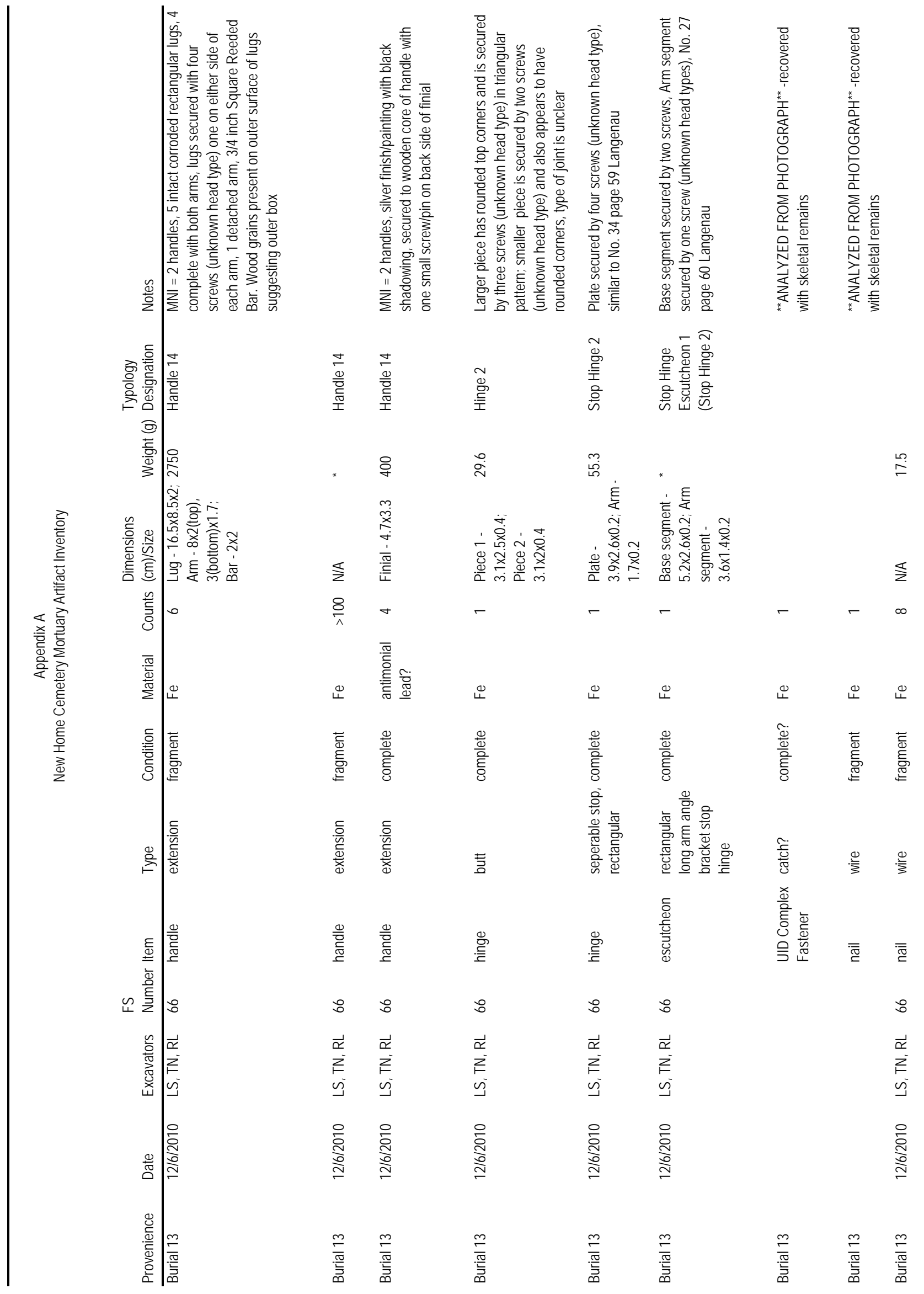




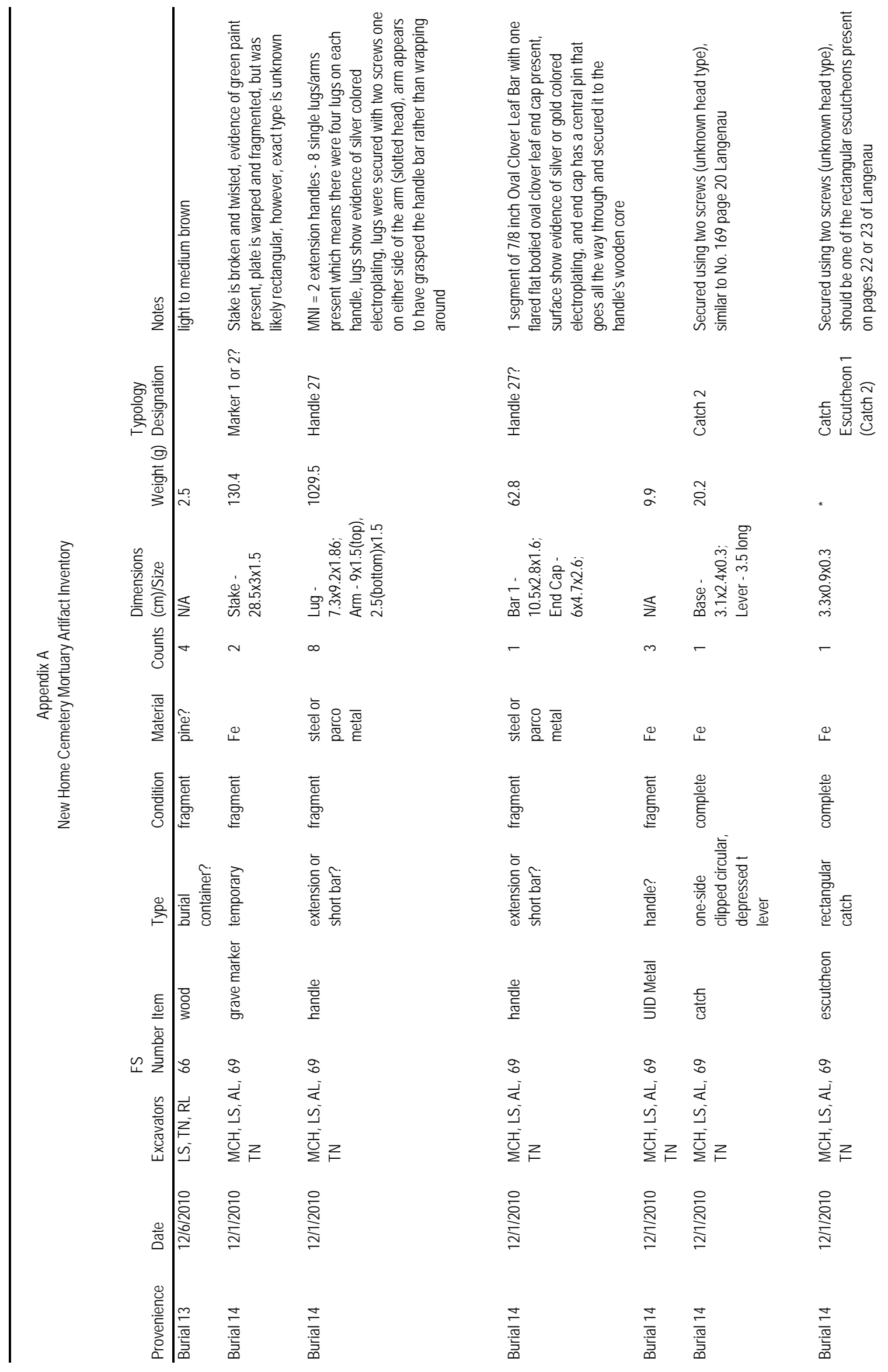




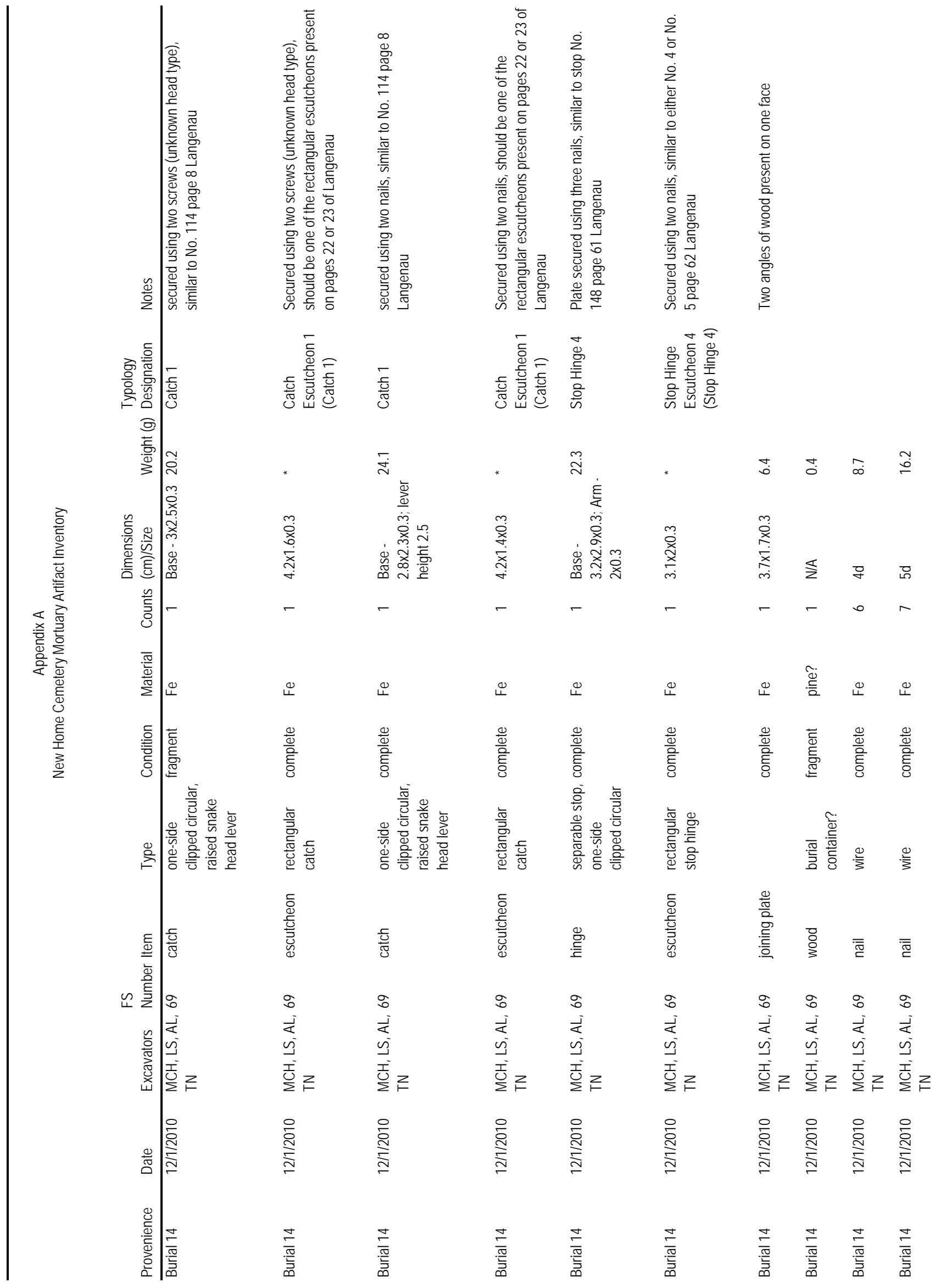




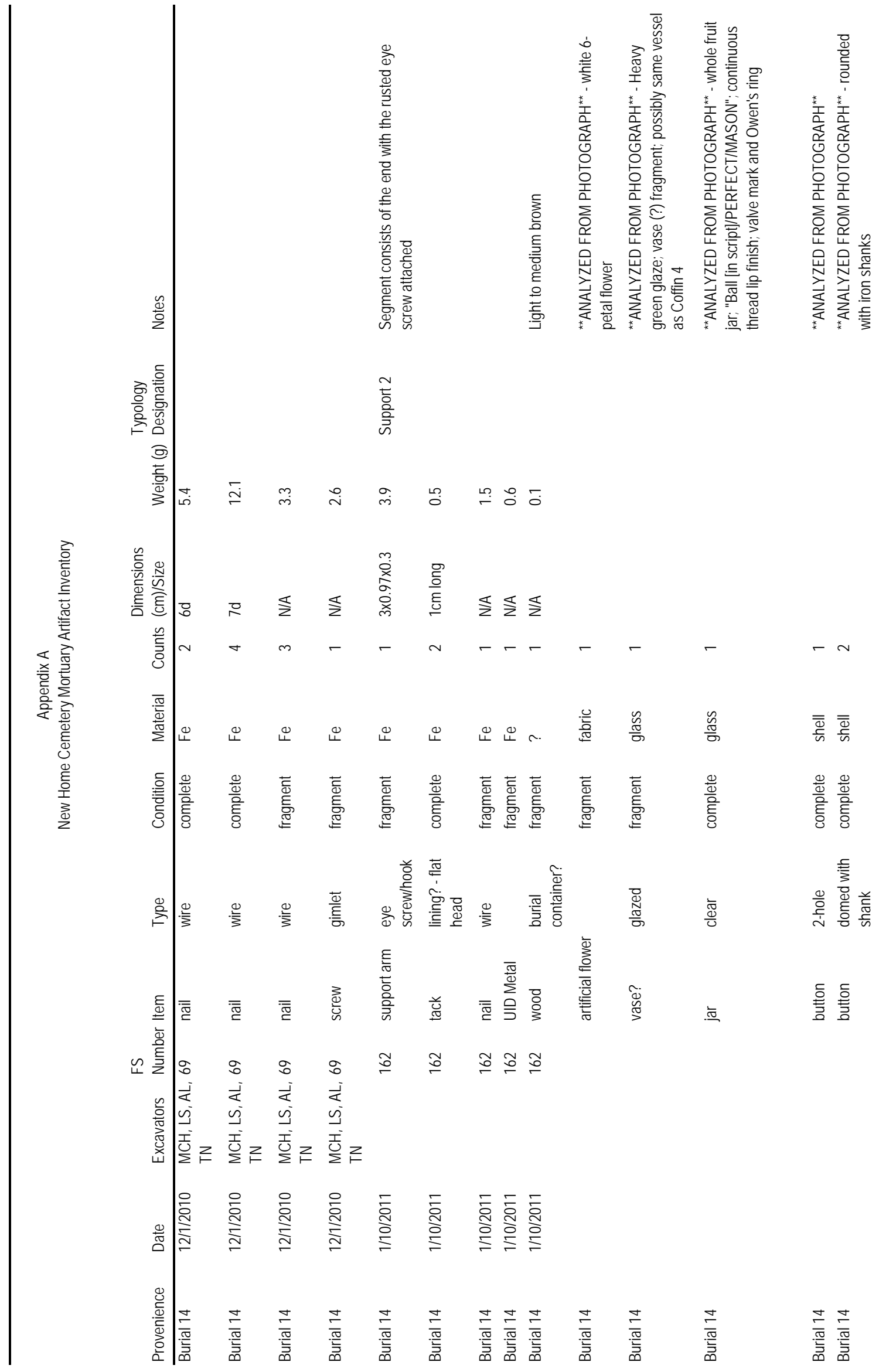




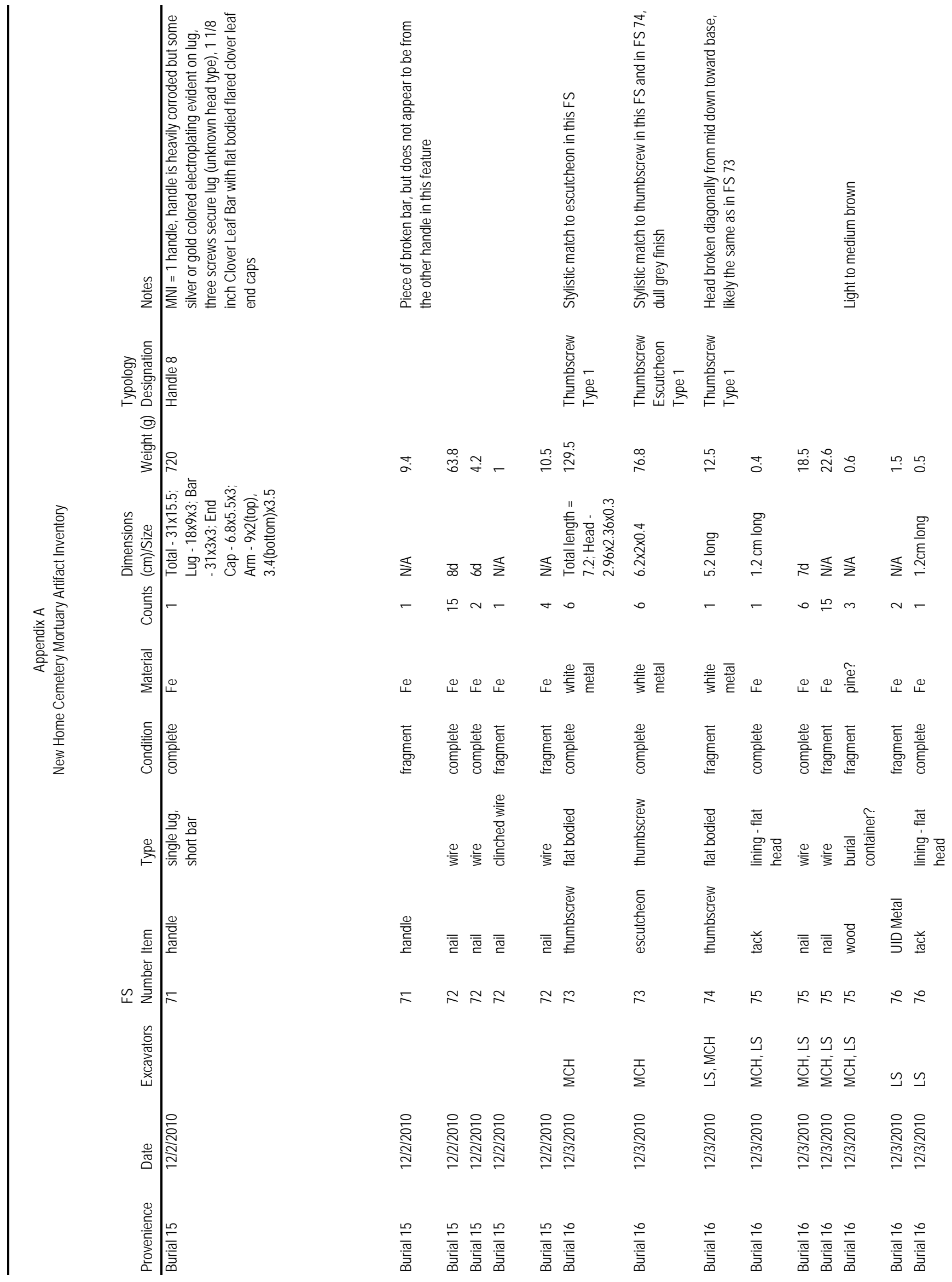




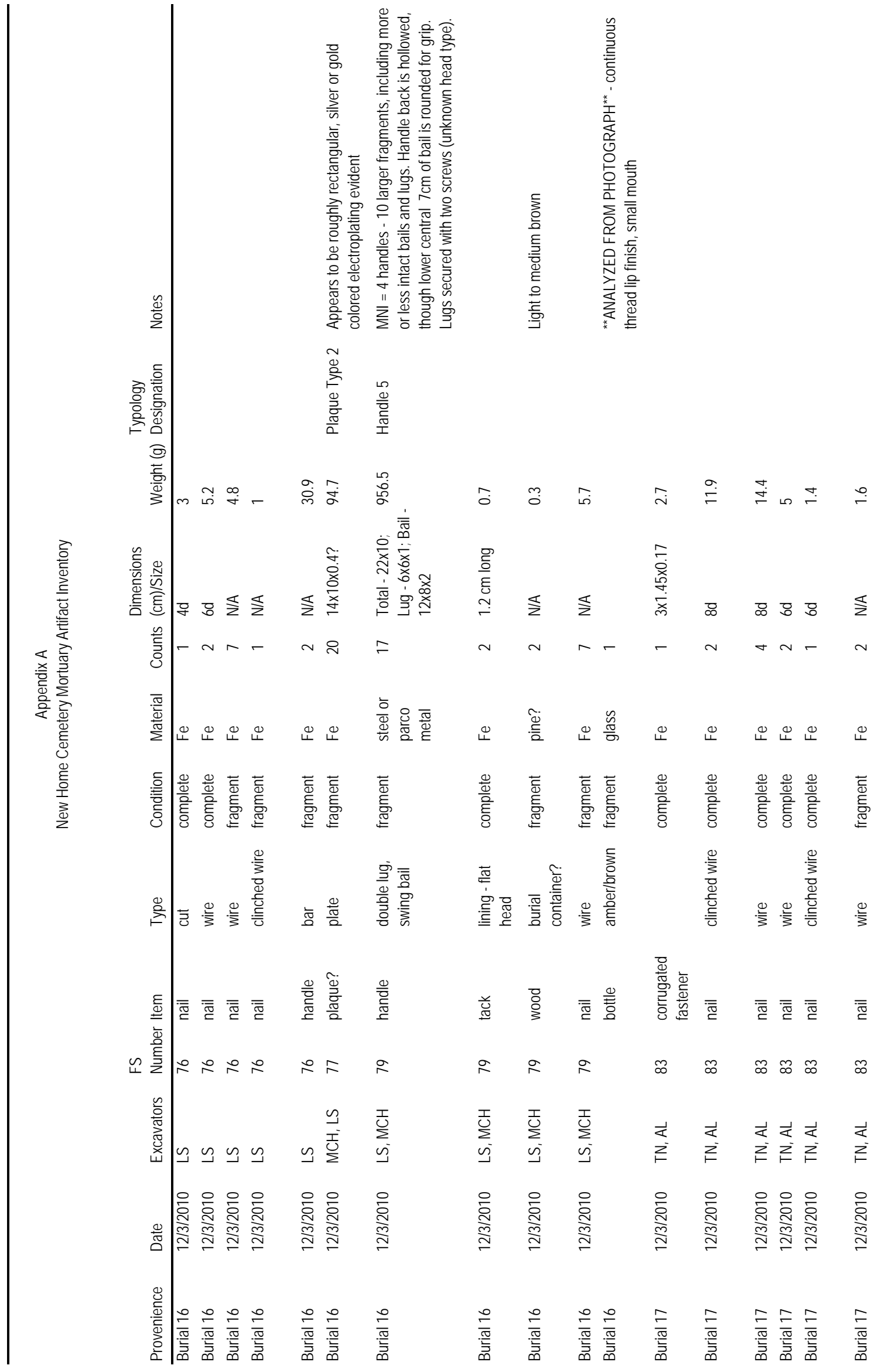




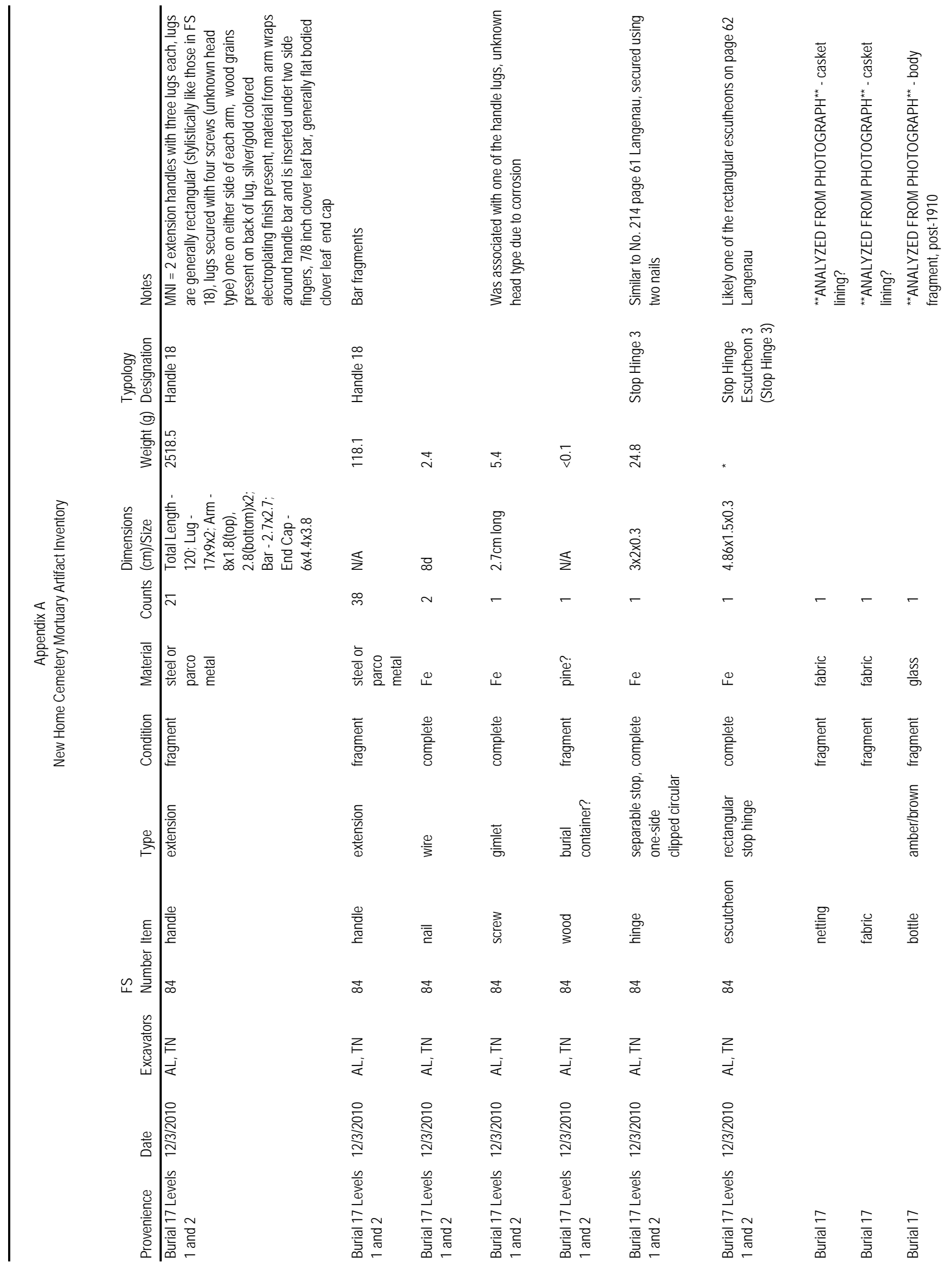




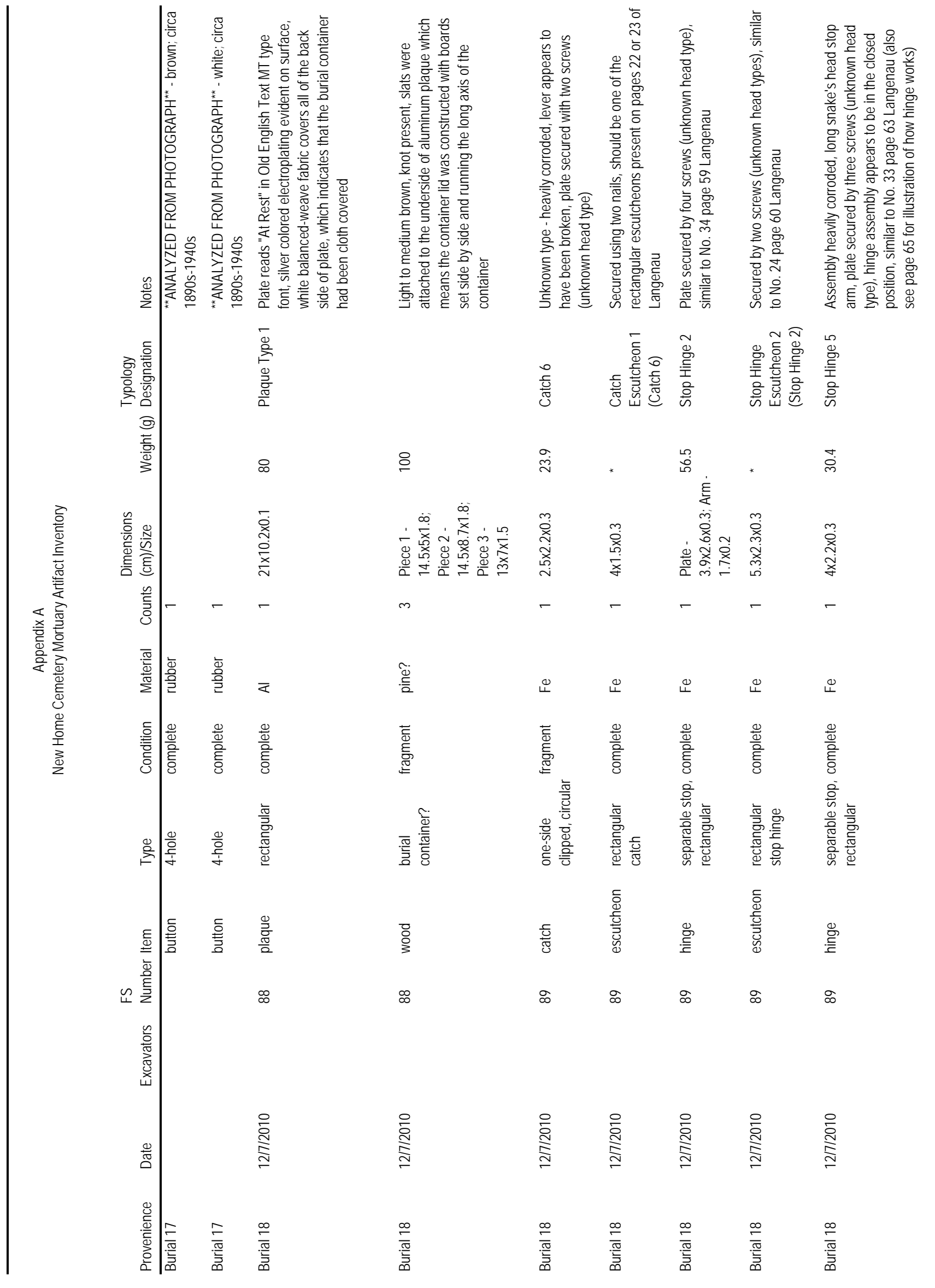




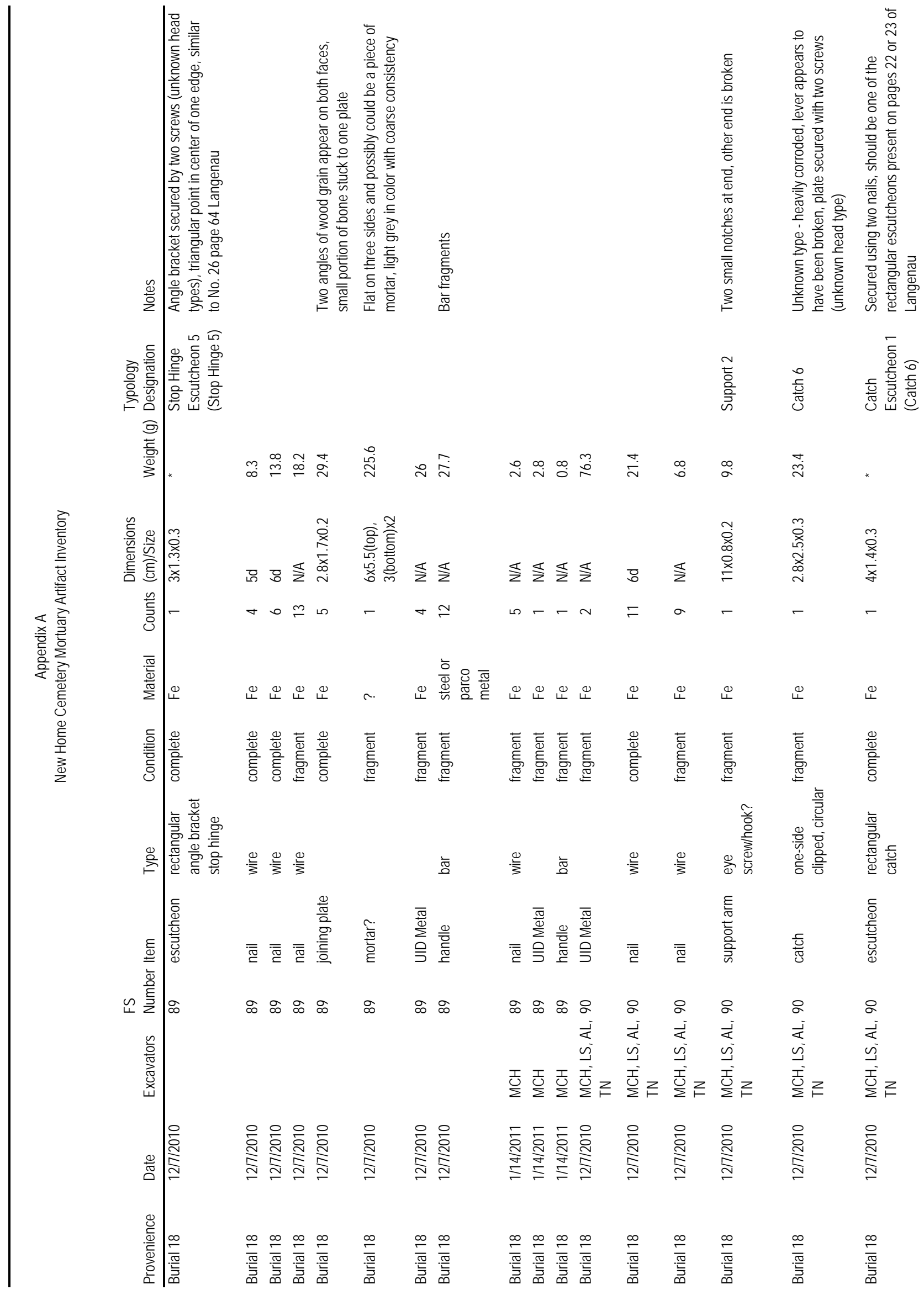




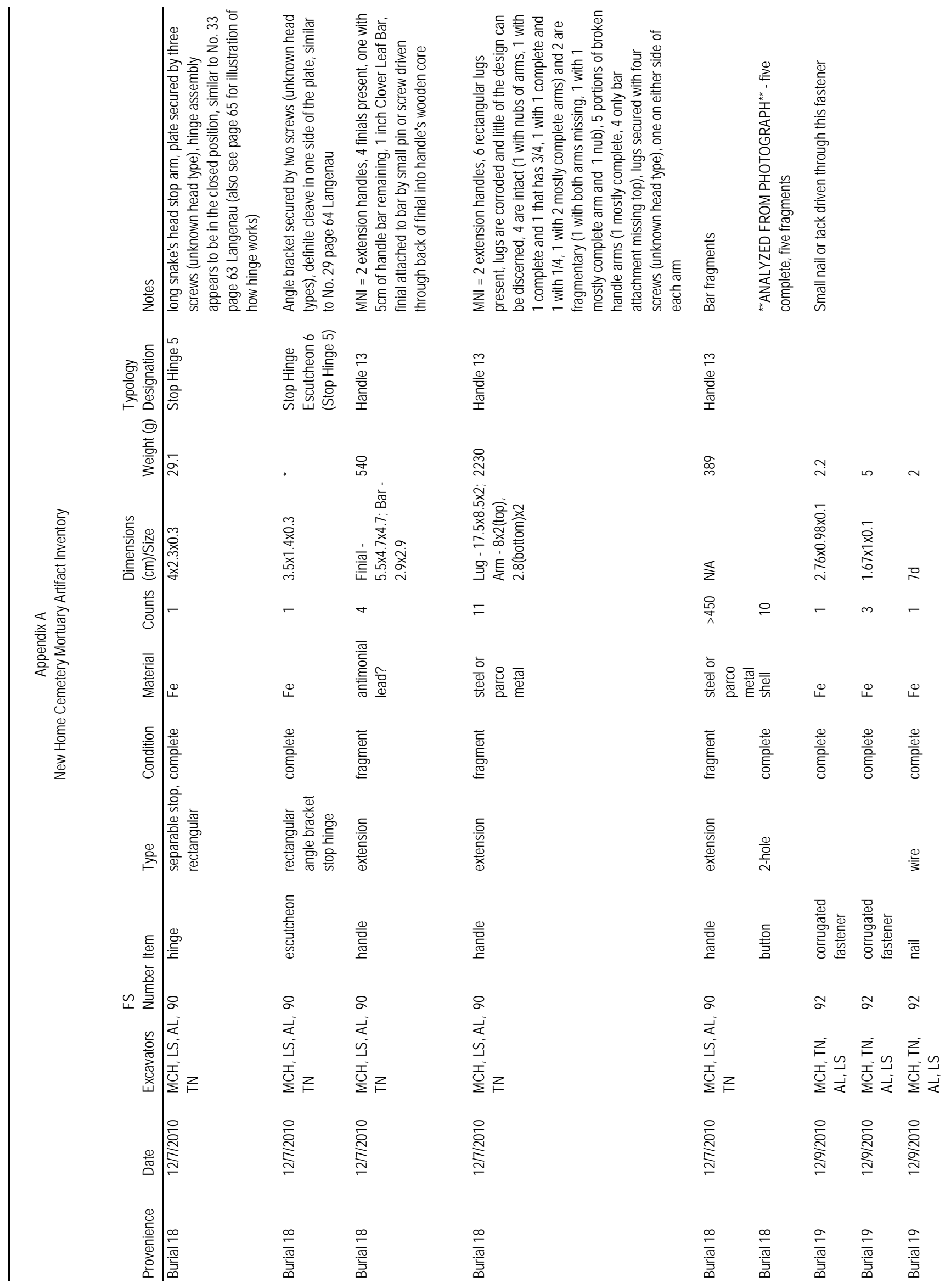




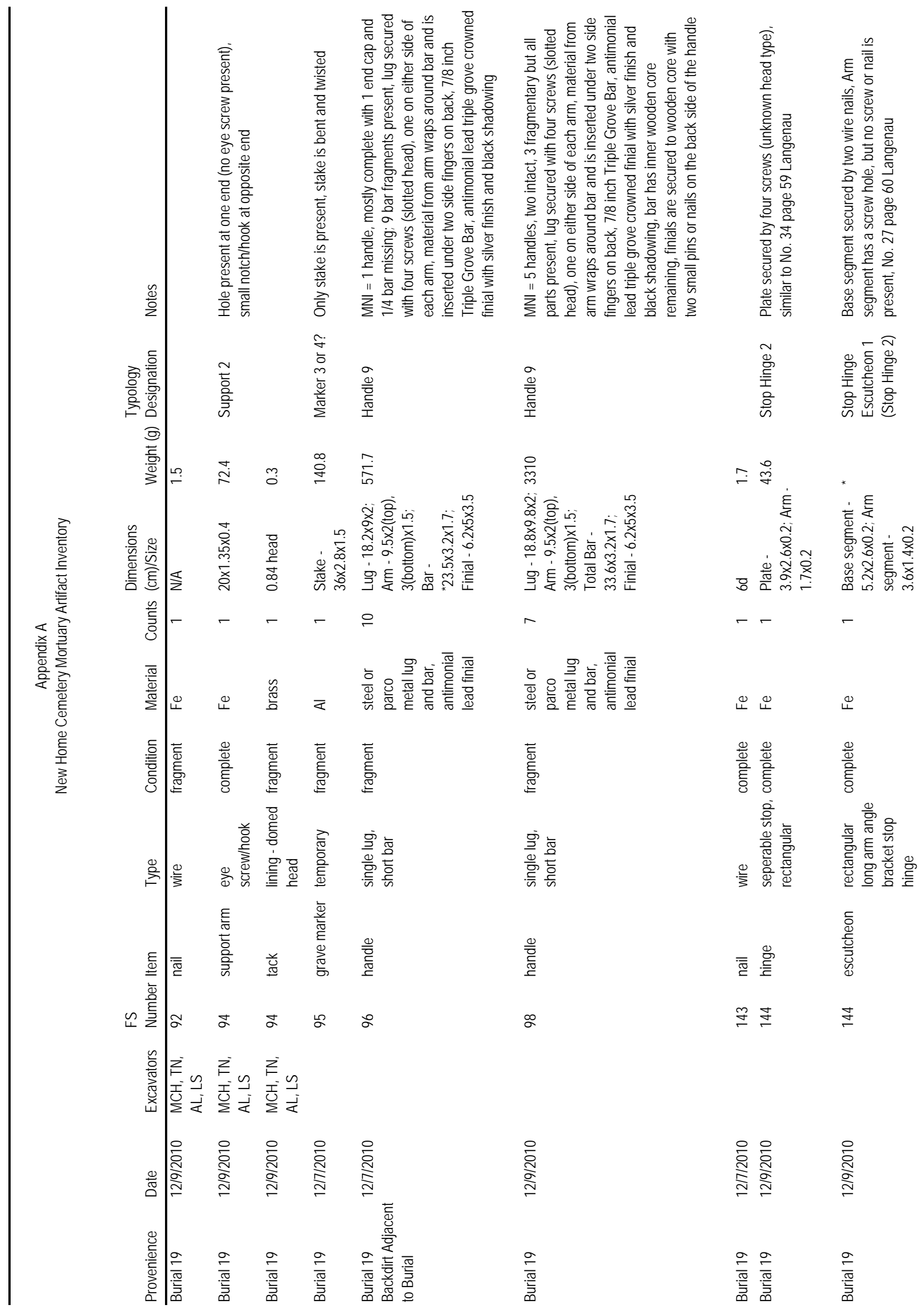




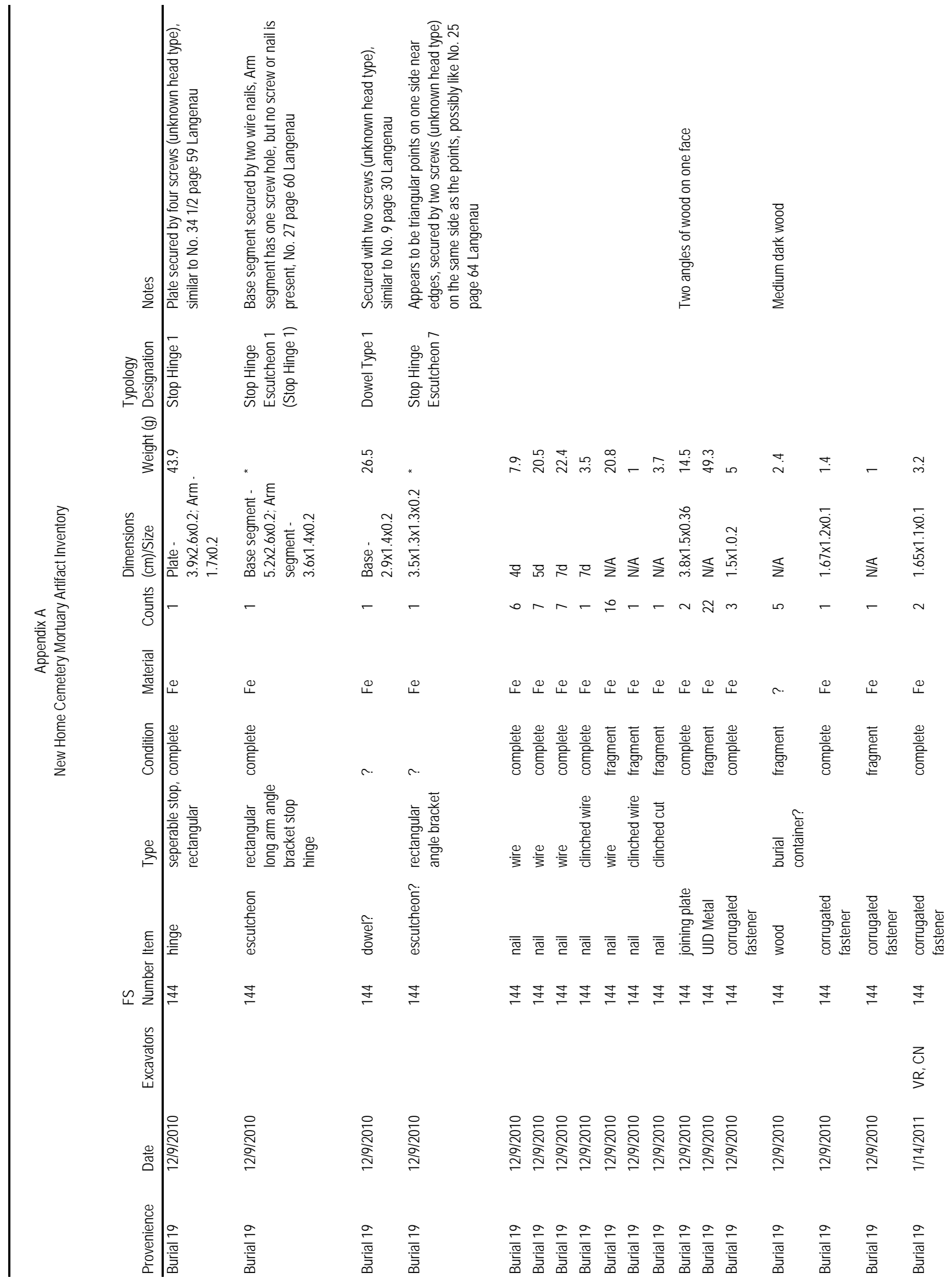




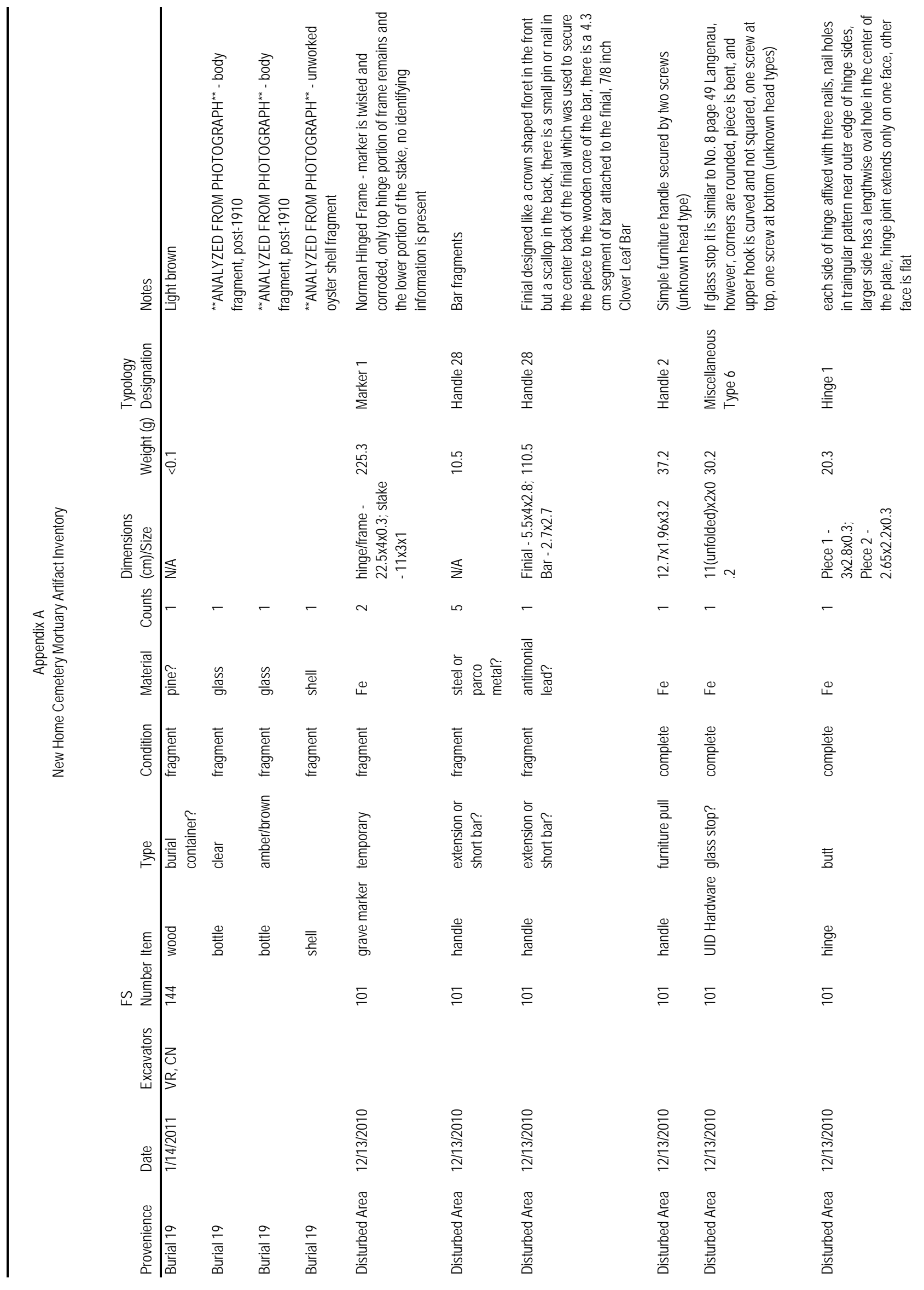




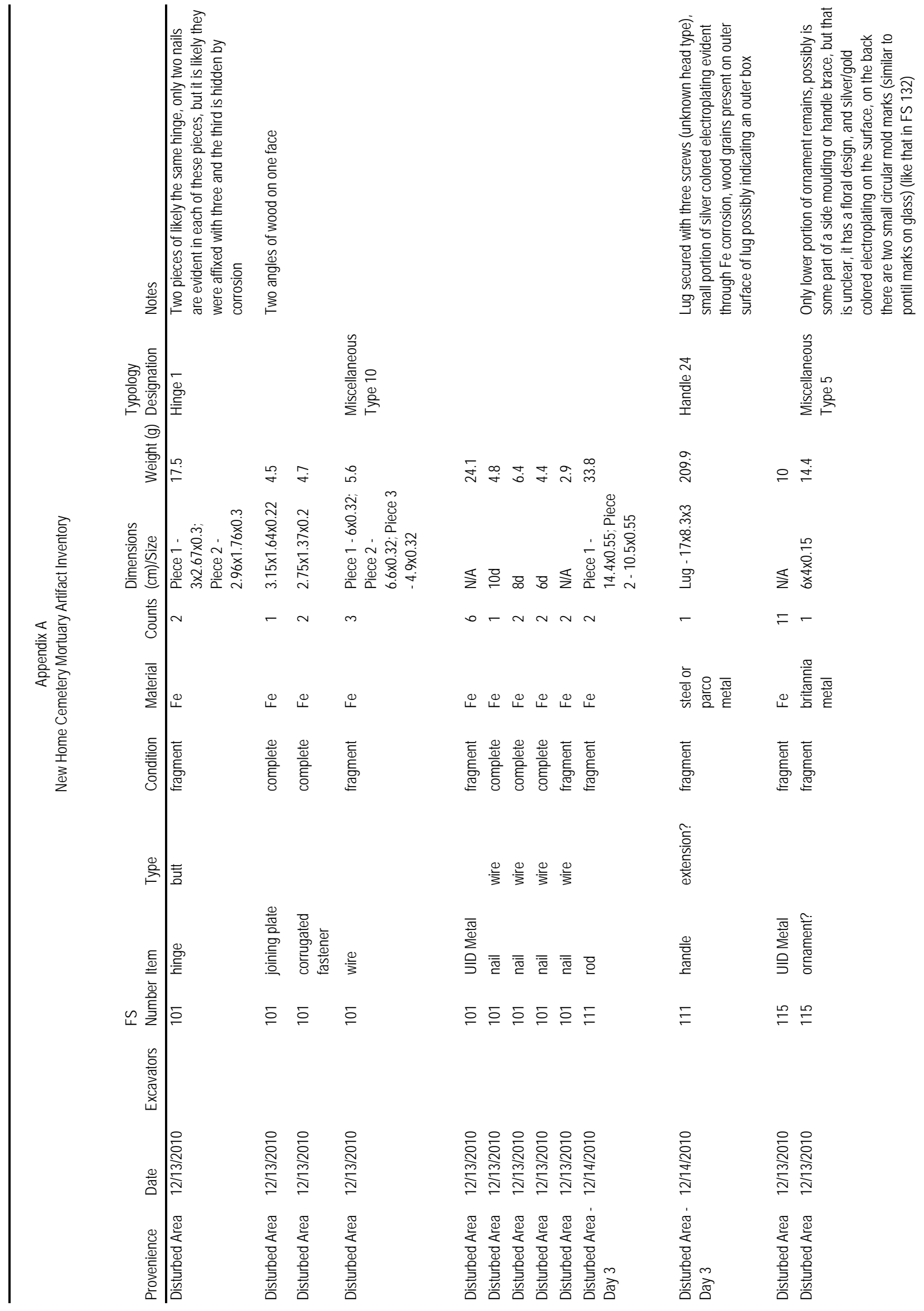




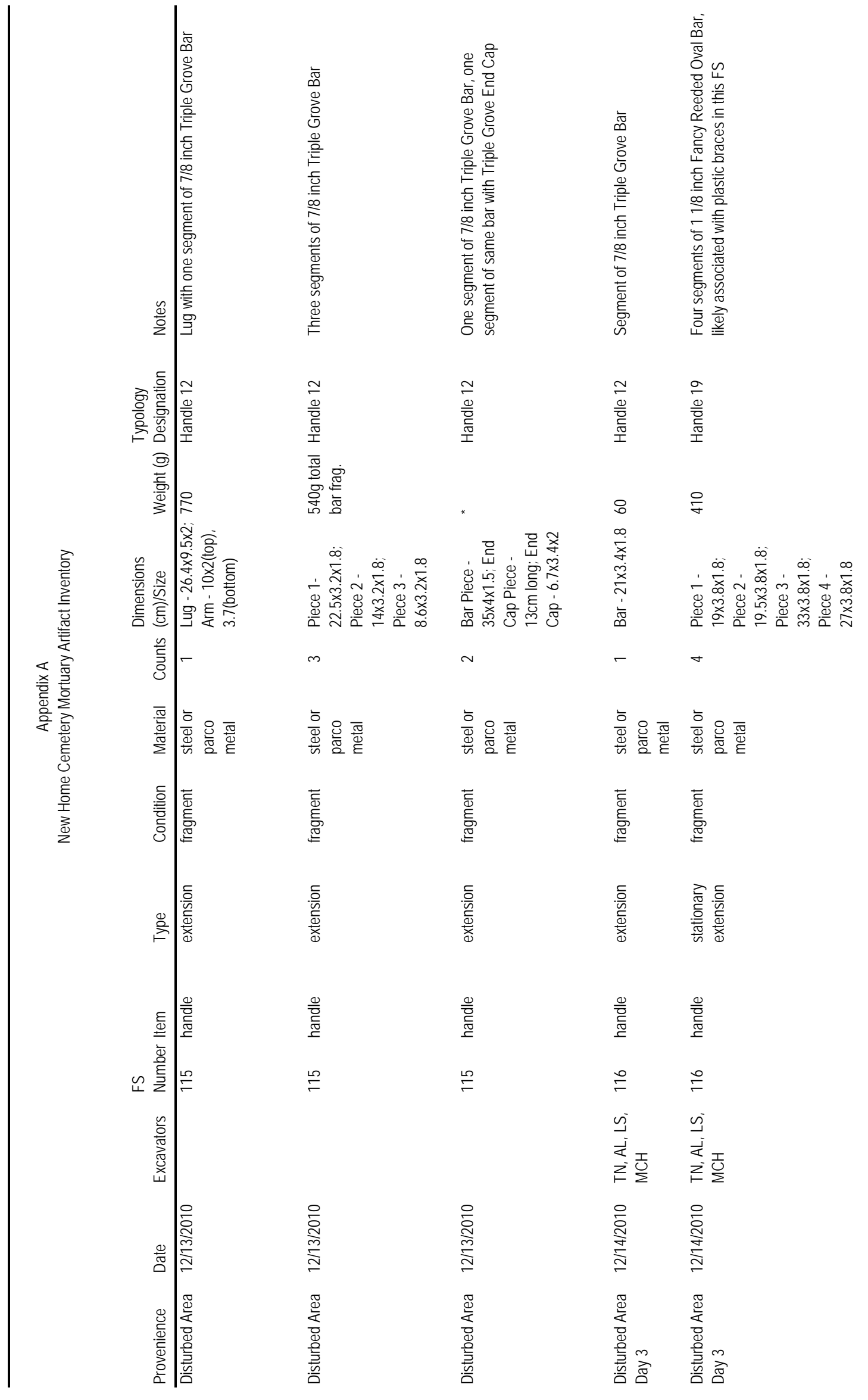



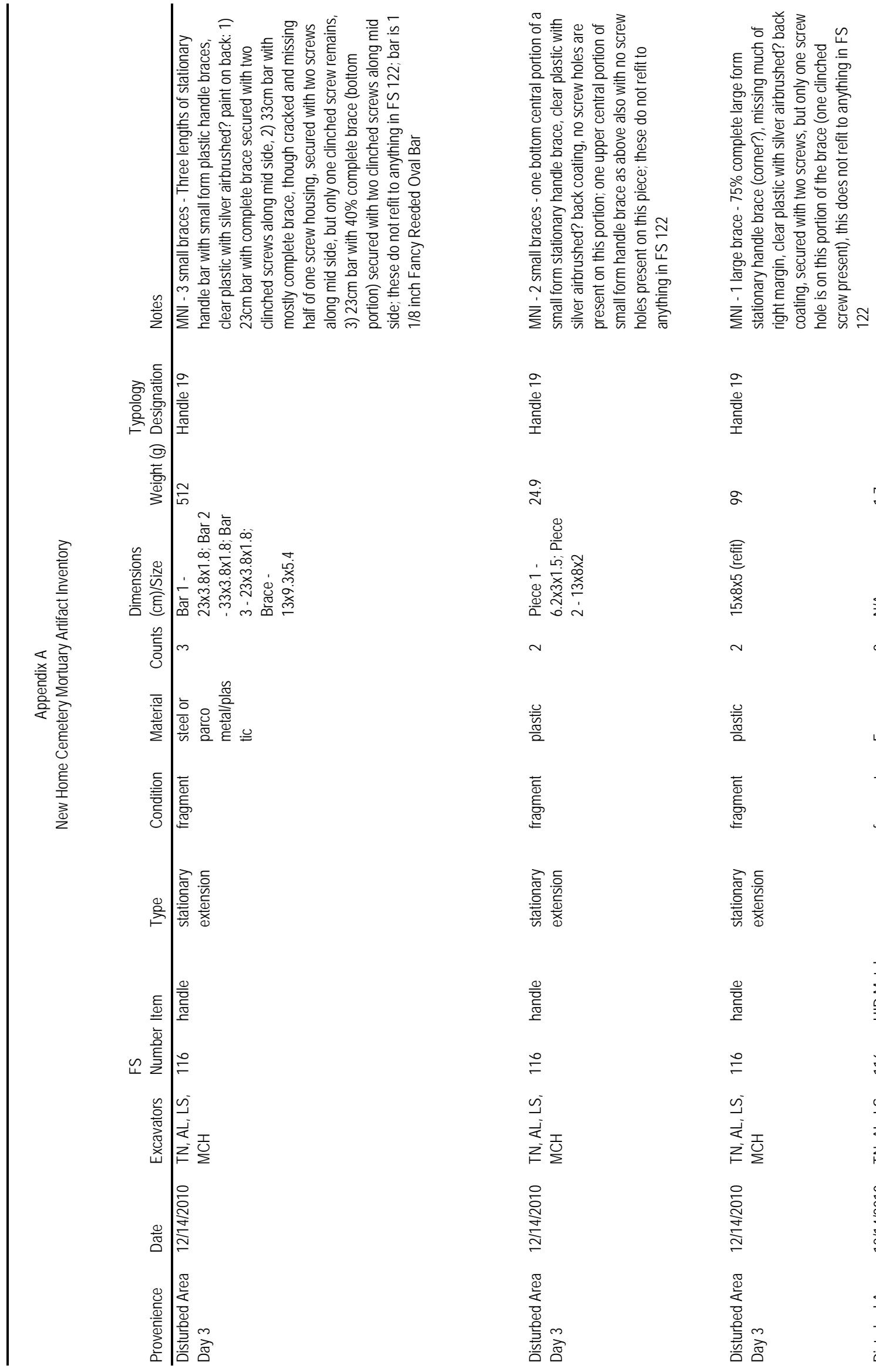

$\frac{9}{9}$
$\frac{0}{\overline{0}}$
$\frac{\bar{c}}{\bar{T}}$

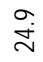

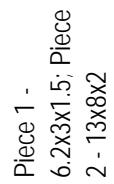

\&

సi

$\frac{0}{w}$
$\frac{\pi}{2}$

$\frac{0}{4}$
$\frac{\pi}{2}$

ષு.

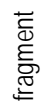

䒕

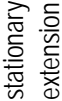

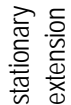

읗

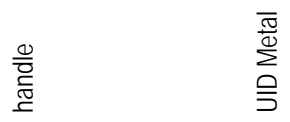

$\stackrel{ }{\exists}$

이

夏

崩

突

옳

웜

옹

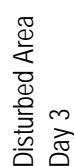

趂

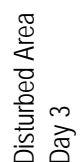




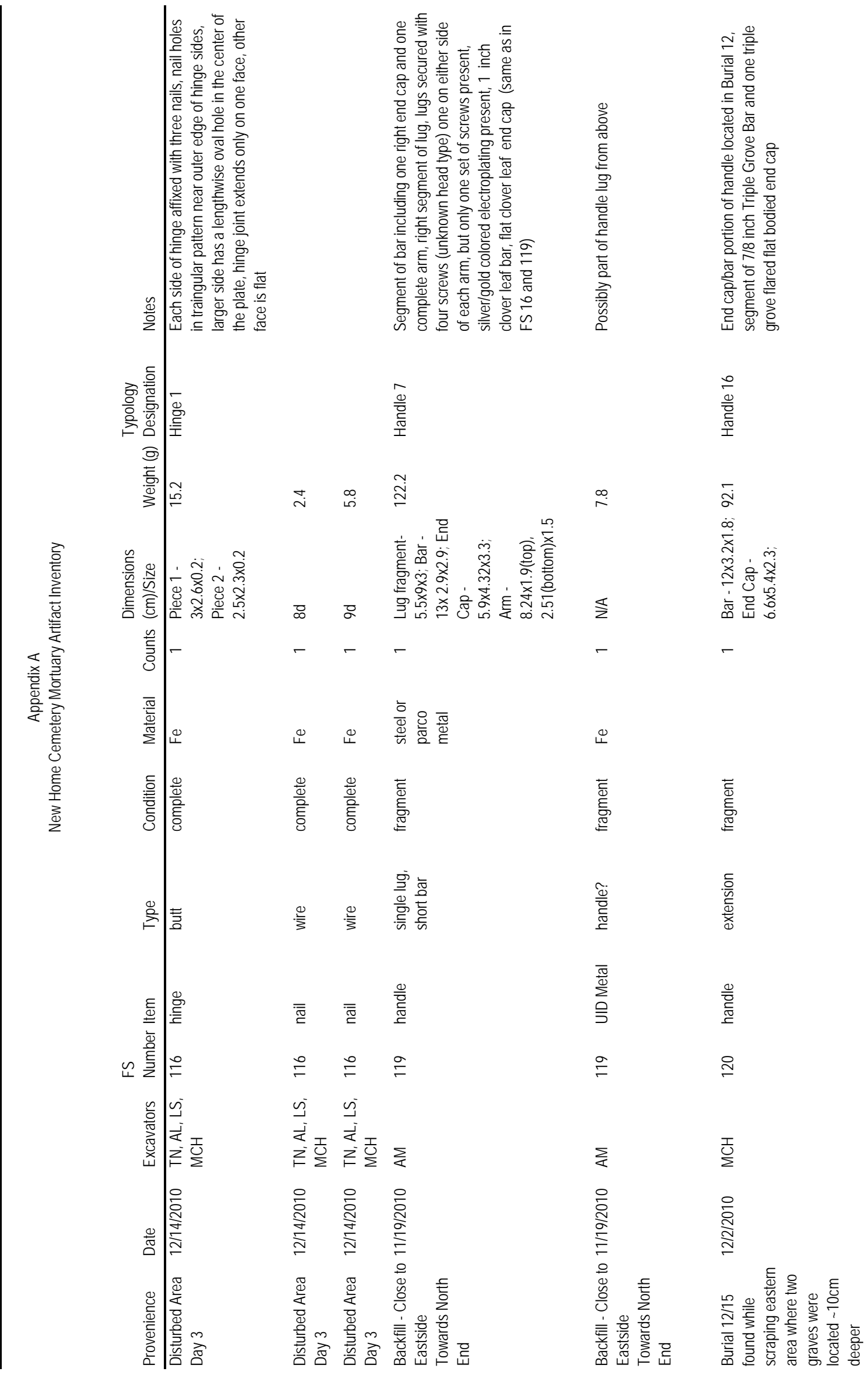




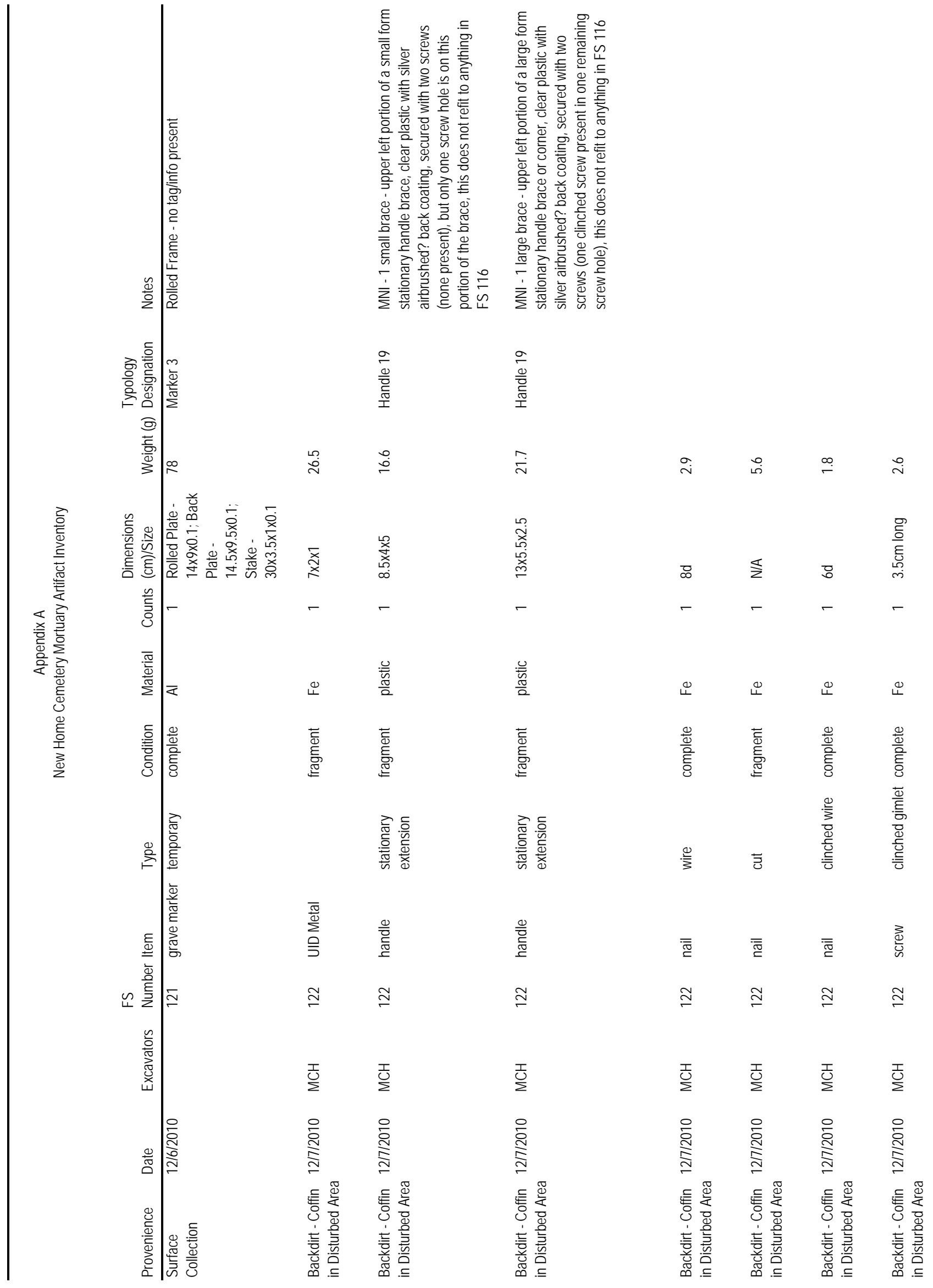




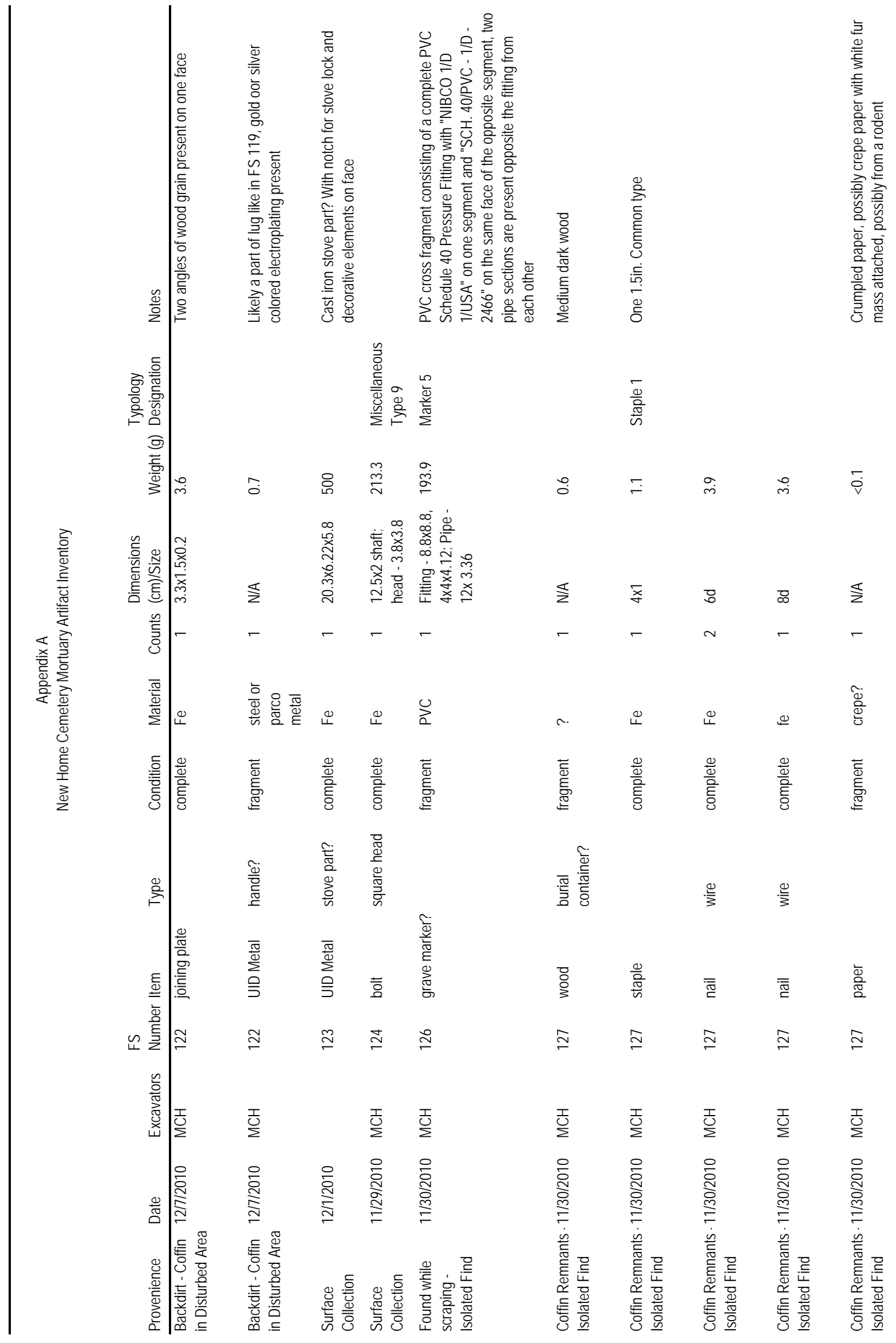




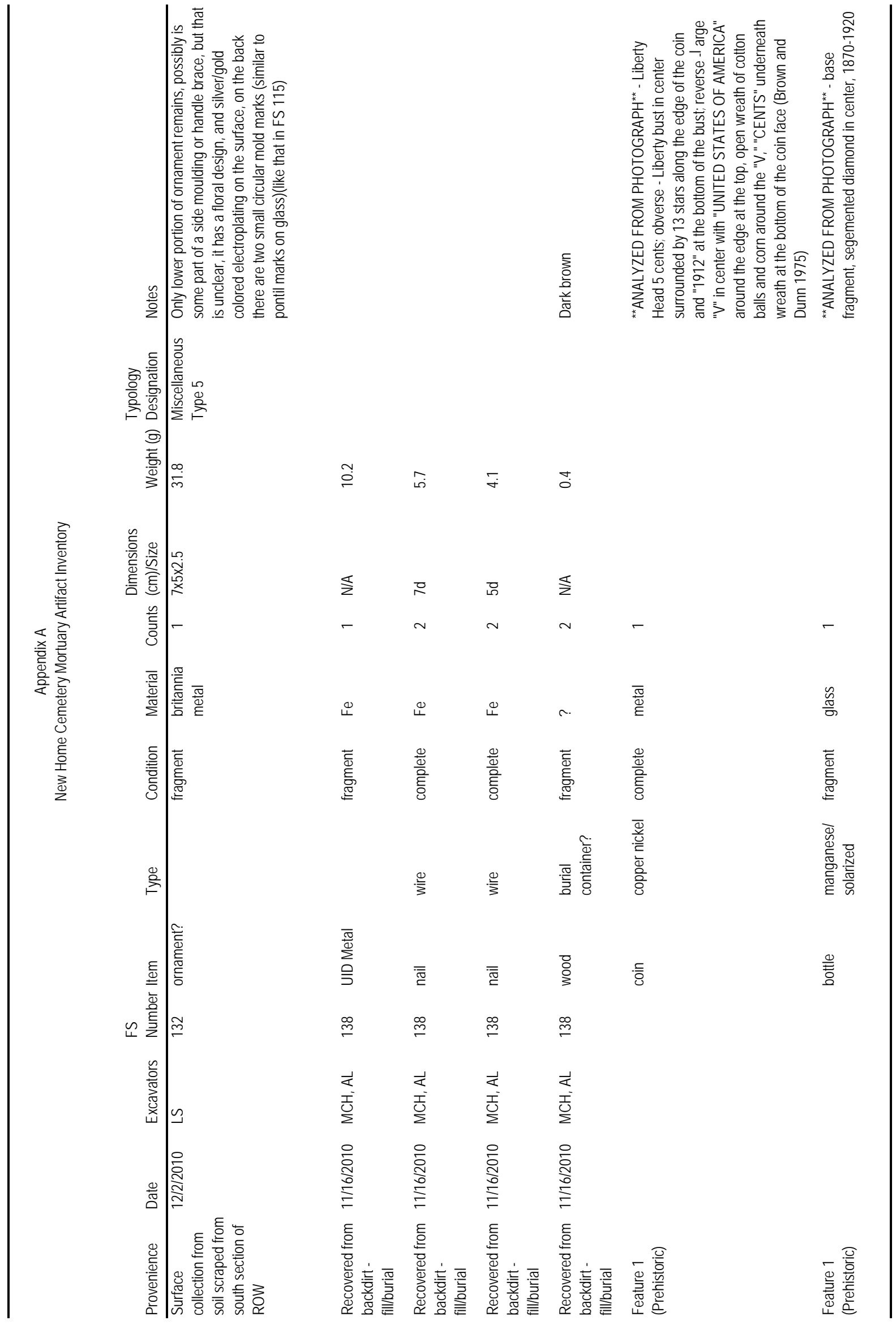


APPENDIX B

GEO-MARINE, INC., BURIAL FORM 

Project Name/Number:

Site Number:

Burial Number of

Excavator(s):

Date:

\section{Grave Shaft:}

Shape:

Orientation of long axis:

Length:

Width:

\section{Soil Type/Color:}

Grave fill:

Surrounding soil:

Depth to top of burial container:

Depth to bottom of burial container/grave:

\section{Burial Container:}

Outer Box:

(check one) Yes:

No: Other (specify):

Indeterminate:

Shape:

Material:

Length:

Width at headboard:

Width at shoulders:

Width at footboard:

Inner Box:

(check one) Yes:

No:

Indeterminate:

Other (specify)

Shape:

Material:

Length:

Width at headboard:

Width at shoulders:

Width at footboard: 
Burial Number of

Excavator(s):

Date:

\section{Viewing Window:}

(check one) Yes:___ No:

Shape:

Long axis length:

Short axis length:

Description of grave, burial container(s), associated artifacts, and unassociated artifacts: 
Burial Number of

Excavator(s):

Date:

Interment Type: (check all that apply)

Single Multiple Primary Secondary

Burial Position: (check one)

Extended Flexed Semi-flexed Indeterminate

Body Deposition: (check one)

Supine Prone Other (specify)

Indeterminate

Articulation: (check one)

Articulated Disturbed

Indeterminate

Preservation: (check one)

Excellent Good

Fair

Poor

Skull:

Top of skull facing:

Orientation:

Face to:

Arms: (check all that apply)

Hands: (check all that apply)

$$
\text { Left Right }
$$

Vertebral column:

Extended

Folded

Crossed
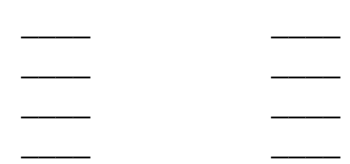

(

Over R. Hip

Over L. Hip

Over R. Shoulder

Over L. Shoulder

At Side

Other (specify)

Indeterminate 
Burial Number

Excavator(s):

Date:

Description of skeleton and burial position (include any measurements or demographic, pathological, or taphonomic observations): 
Burial Number

Excavator(s):

Date:

List number of artifacts present for each area.

\begin{tabular}{|c|c|c|c|c|c|}
\hline & \multicolumn{5}{|c|}{ AREA } \\
\hline MORTUARY ARTIFACTS & A & B & C & D & $\mathbf{E}$ \\
\hline Outer Box Handle & & & & & \\
\hline Iron Band & & & & & \\
\hline Outer Box Lid Fastener & & & & & \\
\hline Outer Box Hinge & & & & & \\
\hline Inner Box Hinge & & & & & \\
\hline Coffin/Casket Handle & & & & & \\
\hline Coffin/Casket Lid Fastener & & & & & \\
\hline Coffin Screw & & & & & \\
\hline Thumbscrew & & & & & \\
\hline Escutcheon & & & & & \\
\hline Caplifter & & & & & \\
\hline Plaque & & & & & \\
\hline Ornamental Tack & & & & & \\
\hline Cut Nail & & & & & \\
\hline Wire Nail & & & & & \\
\hline Lining Tack & & & & & \\
\hline Utilitarian Screw & & & & & \\
\hline Corrugated Fastener & & & & & \\
\hline Viewing Window Glass & & & & & \\
\hline Coffin/Casket Fabric (exterior) & & & & & \\
\hline Coffin/Casket Fabric (interior) & & & & & \\
\hline Unidentified Metal & & & & & \\
\hline Other_ & & & & & \\
\hline
\end{tabular}

A=Skull $\quad$ B=Upper Right $\quad$ C=Upper Left $\quad$ D=Lower Right $\quad$ E=Lower Left

(Superior iliac crest separates upper half from lower). 
Burial Number

Excavator(s):

Date:

List number of artifacts present for each area.

\begin{tabular}{|c|c|c|c|c|c|}
\hline & \multicolumn{5}{|c|}{ AREA } \\
\hline $\begin{array}{l}\text { PERSONAL ARTIFACT } \\
\text { (historic) }\end{array}$ & $\mathbf{A}$ & $\mathbf{B}$ & $\mathbf{C}$ & $\mathbf{D}$ & $\mathbf{E}$ \\
\hline \multicolumn{6}{|l|}{ Glass Button } \\
\hline \multicolumn{6}{|l|}{ Bone Button } \\
\hline \multicolumn{6}{|l|}{ Shell Button } \\
\hline \multicolumn{6}{|l|}{ Porcelain Button } \\
\hline \multicolumn{6}{|l|}{ Metal Button } \\
\hline \multicolumn{6}{|l|}{ Synthetic Button } \\
\hline \multicolumn{6}{|l|}{ Stud } \\
\hline \multicolumn{6}{|l|}{ Straight Pin } \\
\hline \multicolumn{6}{|l|}{ Safety Pin } \\
\hline \multicolumn{6}{|l|}{ Snap Fastener } \\
\hline \multicolumn{6}{|l|}{ Hook Fastener } \\
\hline \multicolumn{6}{|l|}{ Metal Cuff Link } \\
\hline \multicolumn{6}{|l|}{ Metal Pin Jewelry } \\
\hline \multicolumn{6}{|l|}{ Metal Finger Ring } \\
\hline \multicolumn{6}{|l|}{ Metal Earring } \\
\hline \multicolumn{6}{|l|}{ Glass Bead } \\
\hline \multicolumn{6}{|l|}{ Suspender Buckle } \\
\hline \multicolumn{6}{|l|}{ Shoe Leather Fragment } \\
\hline \multicolumn{6}{|l|}{ Eyelet } \\
\hline \multicolumn{6}{|l|}{ Fabric } \\
\hline \multicolumn{6}{|l|}{ Dental Appliance } \\
\hline \multicolumn{6}{|l|}{ Comb } \\
\hline \multicolumn{6}{|l|}{ Coin } \\
\hline \multicolumn{6}{|l|}{ Knife } \\
\hline Other & & & & & \\
\hline
\end{tabular}

A=Skull $\quad$ B=Upper Right $\quad$ C=Upper Left $\quad$ D=Lower Right $\quad$ E=Lower Left

(Superior iliac crest separates upper half from lower). 
APPENDIX C

STANDARDIZED DATA FORMS 



\section{INVENTORY RECORDING FORM FOR COMPLETE SKELETONS}

Site Name/Number 1

Feature/Burial Number

Burial/Skeleton Number

Present Location of Collection
Observer

Date

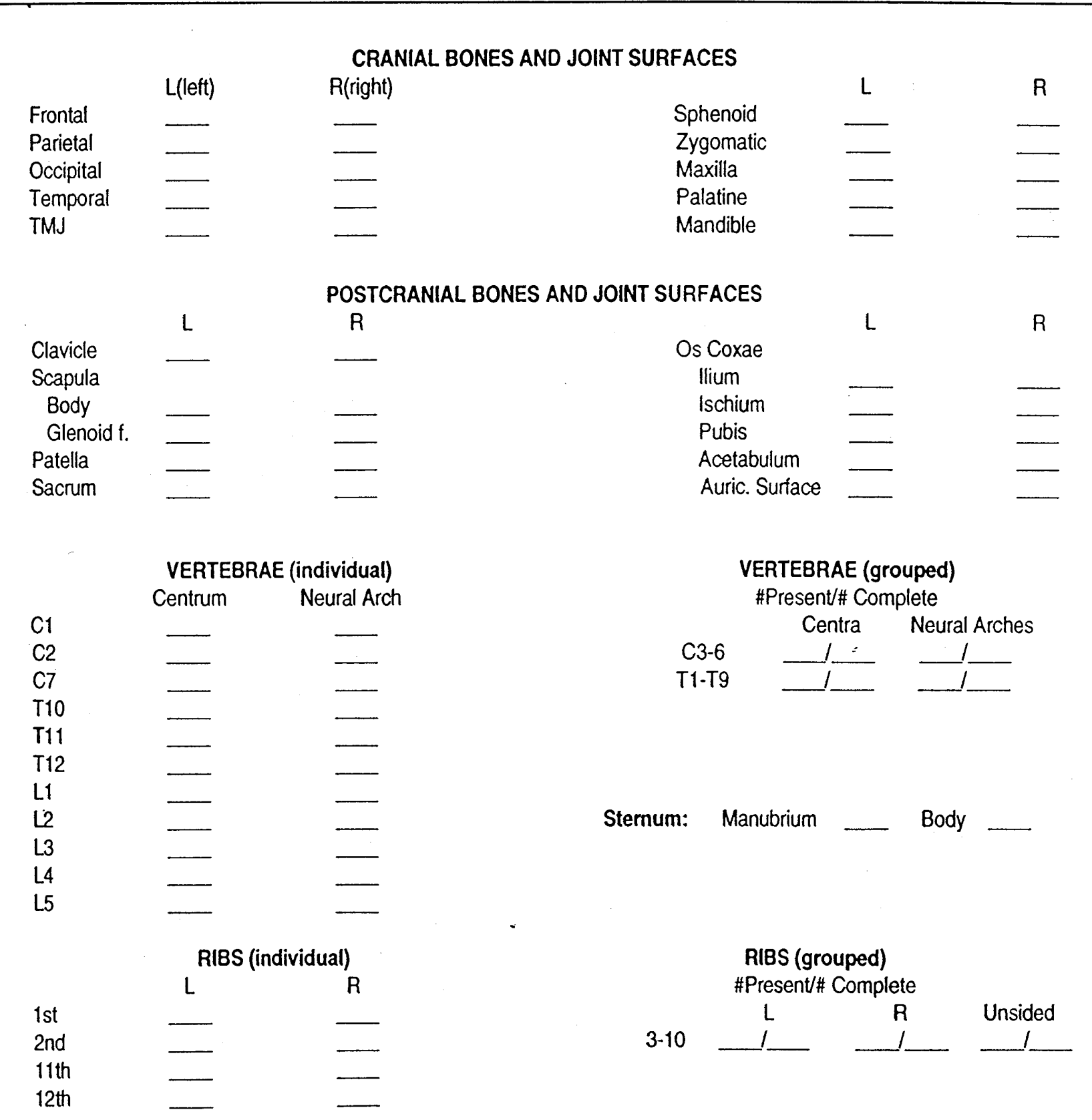




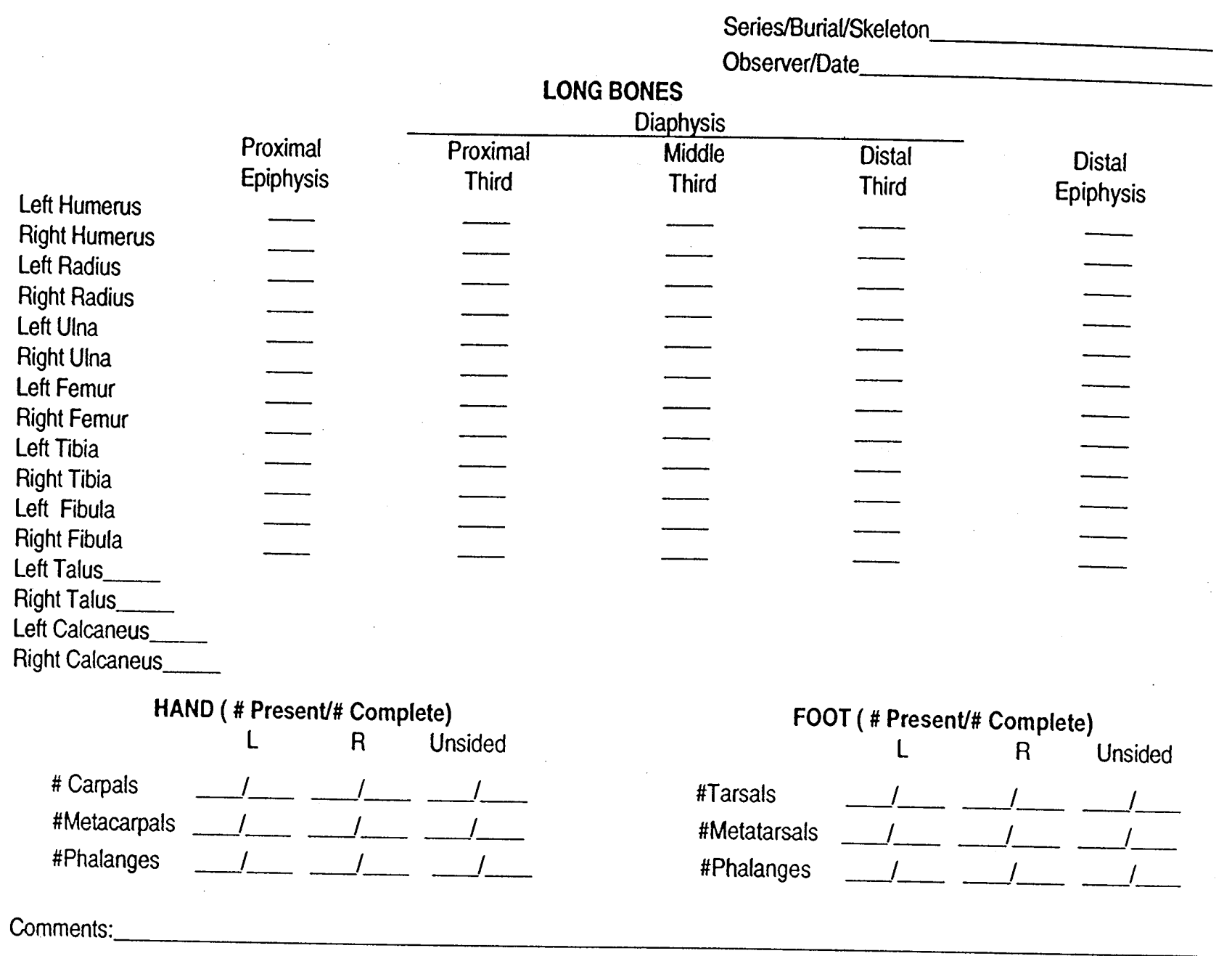




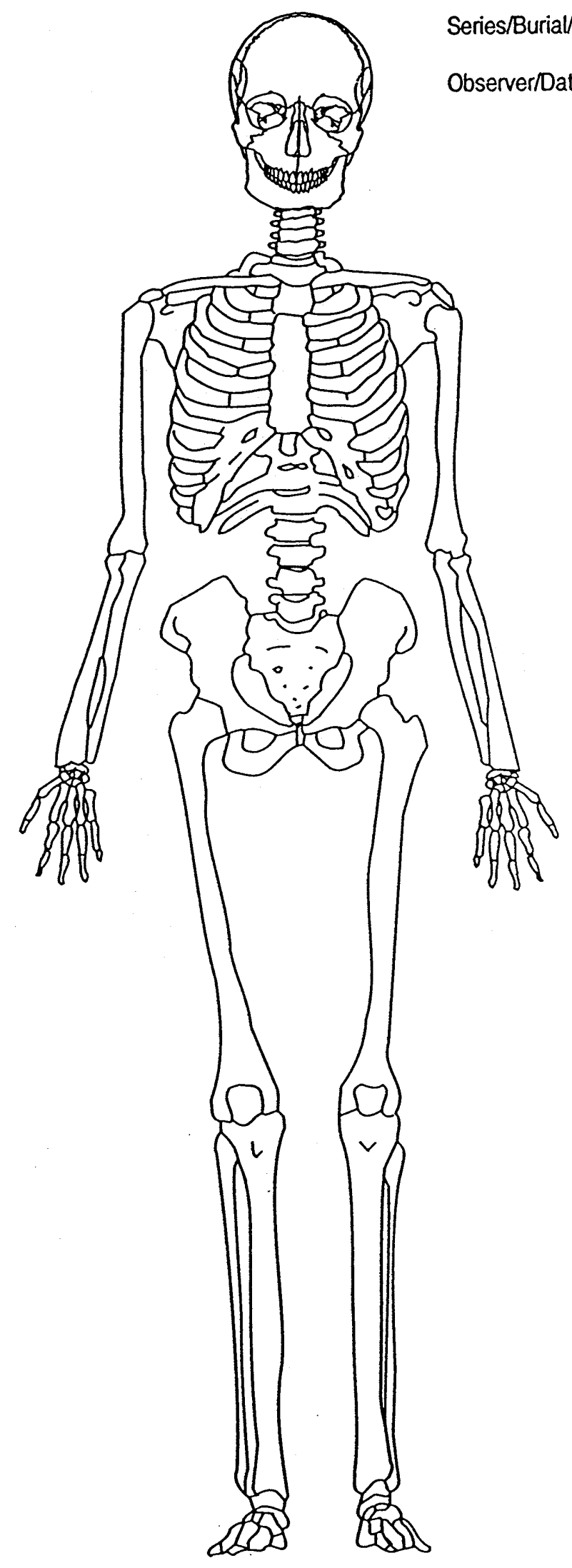




\section{ADULT SKELETON RECORDING FORM: POSTERIOR VIEW}

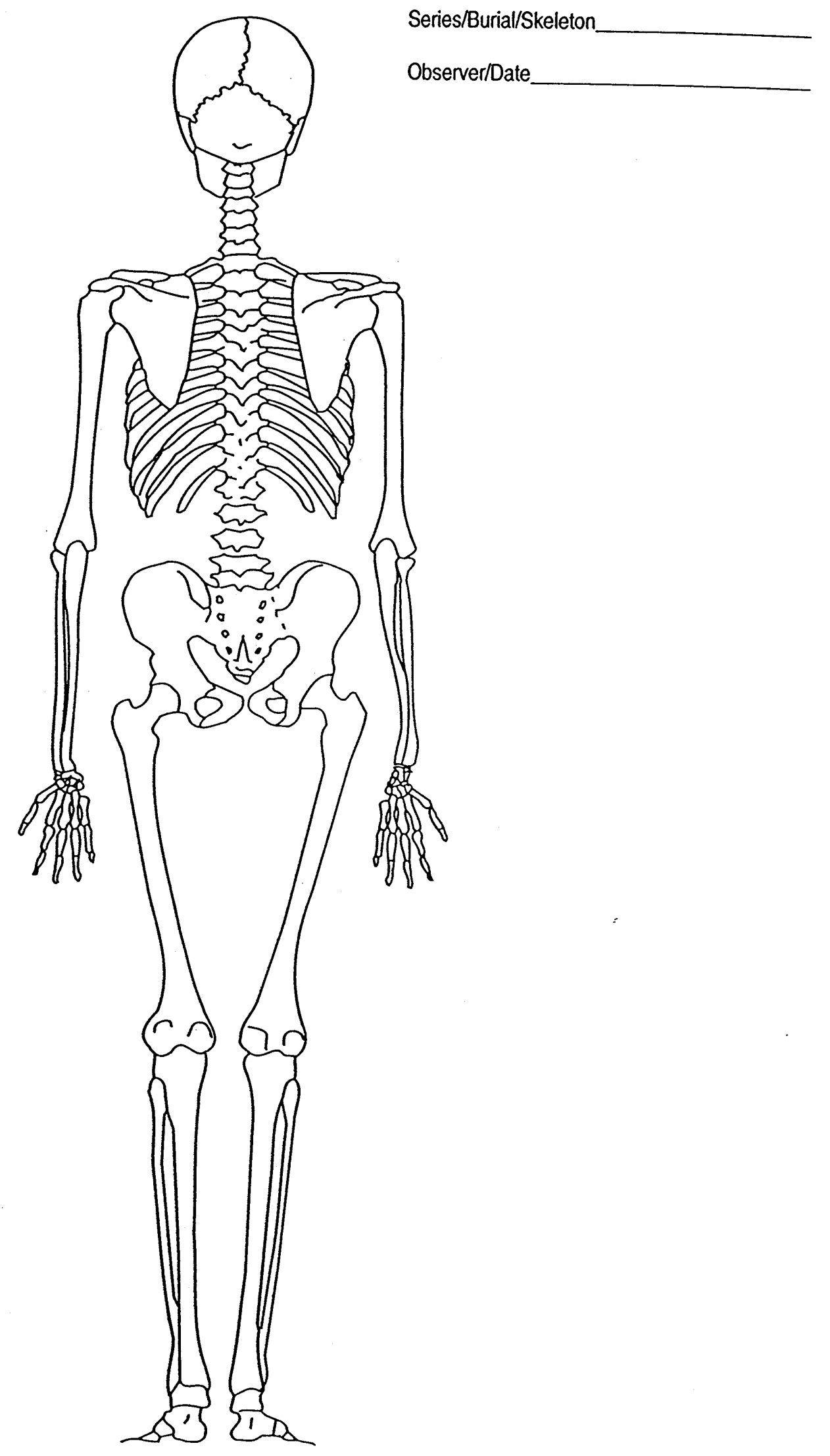




\section{JUVENILE SKELETON VISUAL RECORDING FORM}

\section{a. CHILD ANTERIOR VIEW}

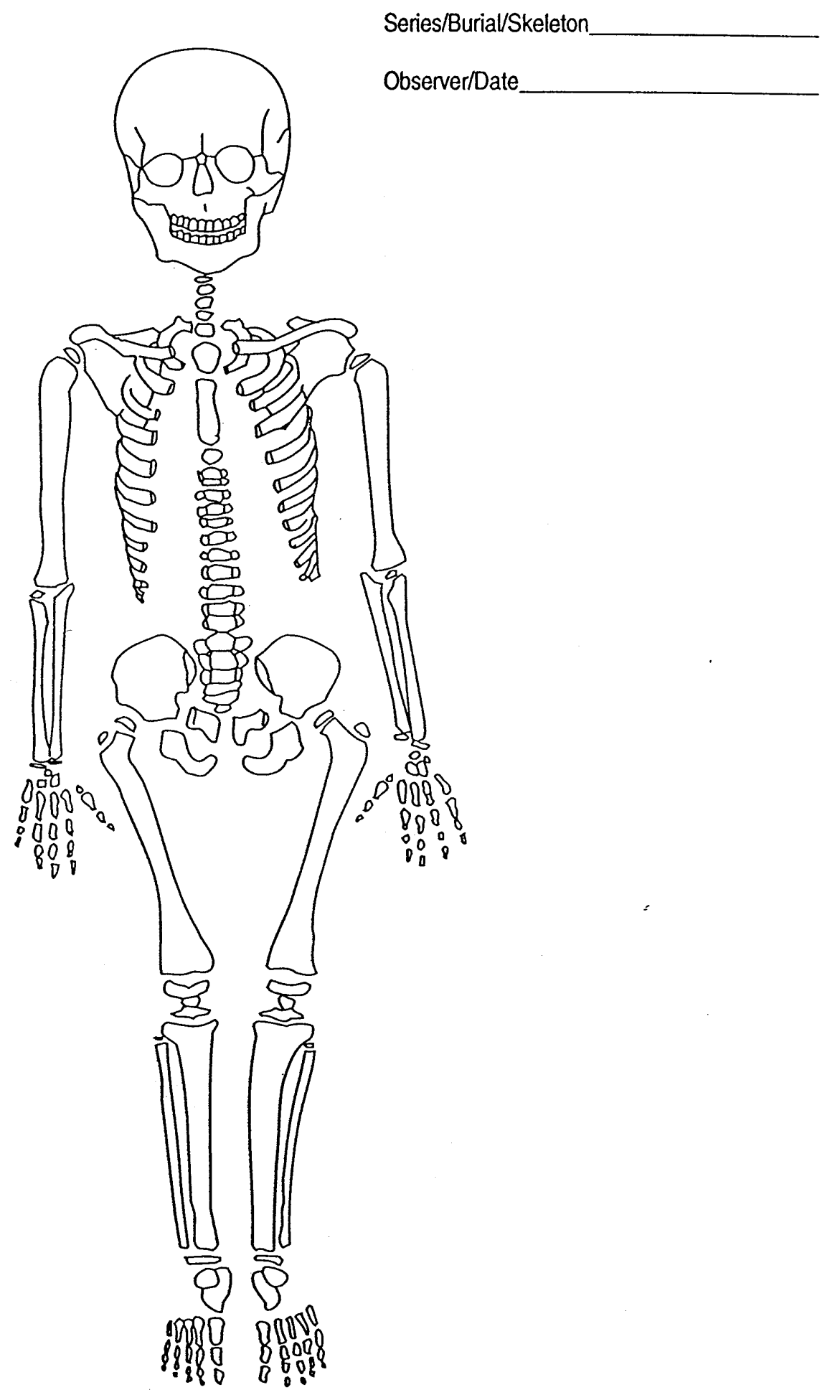




\section{JUVENILE SKELETON VISUAL RECORDING FORM b. FETUS (NEWBORN), ANTERIOR VIEW}

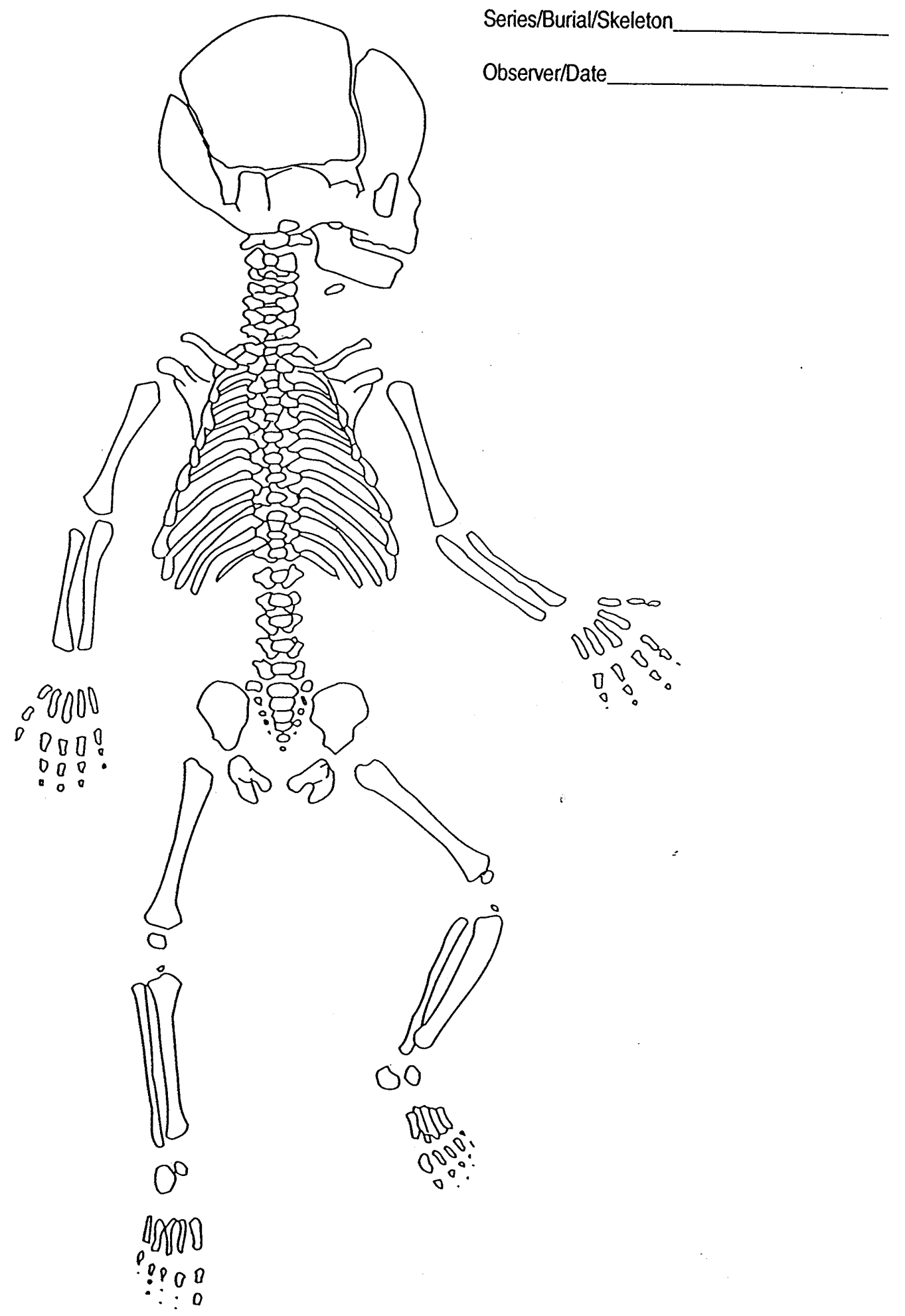




\section{ADULT SEXIAGE RECORDING FORM}

Site Name/Number
Feature/Burial Number
Burial/Skeleton Number
$\begin{aligned} & \text { Pelvis } \\ & \text { Ventral Arc (1-3) } \\ & \text { Subpubic Concavity (1-3) } \\ & \text { Ischiopubic Ramus Ridge (1-3) } \\ & \text { Greater Sciatic Notch (1-5) } \\ & \text { Preauricular Sulcus (0-4) }\end{aligned}$
$\begin{aligned} & \text { Estimated Sex, Pelvis (0-5) } \\ & \text { Comments: }\end{aligned}$


Series/Burial/Skeleton

Observer/Date

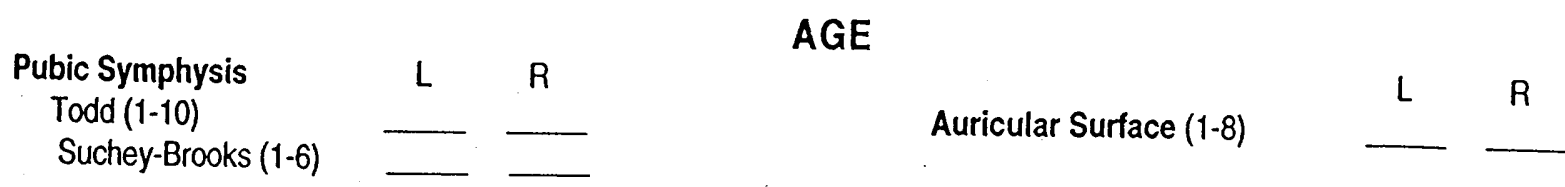

Suture Closure (blank $=$ unobservable; $0=$ open; $1=$ minimal $; 2=$ significant $3=$ complete)

$\begin{array}{cl}\text { External } & \text { 1. Midlambdoid } \\ \text { Cranial } & \text { 2. Lambda } \\ \text { Vault } & \text { 3. Obelion } \\ \text { 4. Anterior Sagittal } \\ \text { 5. Bregma } \\ \text { 6. Midcoronal } \\ \text { 7. Pterion } \\ \text { 8. Sphenofrontal } \\ \text { 9. Inferior Sphenotemporal } \\ \text { 10. Superior Sphenotemporal }\end{array}$

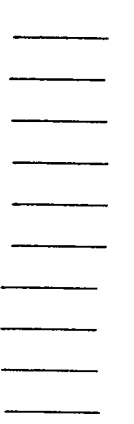
Palate 11. Incisive
12. Anterior Median Palatine
13. Posterior Median Palatine
14. Transverse Palatine
Internal 15. Sagittal
Cranial 16. Left Lambdoid
Vault 17. Left Coronal

Estimated Age: Young Adult (20-35 years)

Middle Adult ( $35-50$ years)

Comments:

Old Adult (50+ years) 


\section{IMMATURE REMAINS RECORDING FORM: BONE UNION AND EPIPHYSEAL CLOSURE}

Site Name/Number 1

Feature/Burial Number

Burial/Skeleton Number

Present Location of Collection

1

\section{EPIPHYSEAL FUSION}

Bone Epiphysis

Cenvical Vertebrae

superior

inferior

Thoracic Vertebrae

Lumbar Vertebrae

inferior

Scapula

coracoid acromion

Clavicle

Humerus

sternal

head

distal

medial epicondyle

Radius proximal distal

Uina proximal distal

Os Coxae iliac crest ischial tuberosity

Femur superior inferior superior $\begin{array}{lll} & \begin{array}{l}\text { greater trochanter } \\ \text { lesser trochanter } \\ \text { Tibia }\end{array} & - \\ & \text { distal } & - \\ \text { Fibula } & \text { distal } & - \\ & \text { proximal } & - \\ & \text { distal } & -\end{array}$

$\begin{array}{lll} & \begin{array}{l}\text { greater trochanter } \\ \text { lesser trochanter } \\ \text { Tibia }\end{array} & - \\ & \text { distal } & - \\ \text { Fibula } & \text { distal } & - \\ & \text { proximal } & - \\ & \text { distal } & -\end{array}$

\section{Stage of Union}
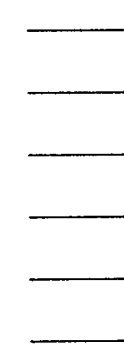

L R

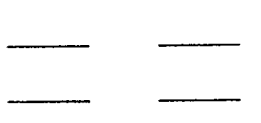

Observer

Date

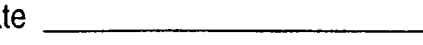


Series/Burial/Skeleton

Observer/Date

Estimate of chronological age based on postcranial maturation
Fetal
b-5
5-10
10-15
15-20
$20+$

Comments: 


\section{IMMATURE MEASUREMENTS RECORDING FORM}

Site Name/Number 1

Observer

Feature/Burial Number 1

Date

Burial/Skeleton Number

1

Present Location of Collection

CRANIAL MEASUREMENTS

$L$

M

R

1. Lesser Wing of the Sphenoid

(a) Length:

(b) Width:

2. Greater Wing of the Sphenoid
(a) Length
(b) Width:

3. Body of the Sphenoid

(a) Length:

(b) Width:

4. Petrous and Mastoid

Portions of the Temporal
(a) Length:
(b) Width:

5. Basilar Part of the Occipital

(a) Length:

(b) Width:

\section{Zygomatic}
(a) Length:
(b) Width:

\section{Maxilla}
(a) Length:
(b) Height:
(c) Width:
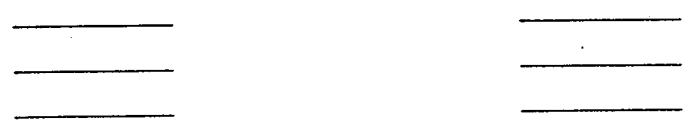

8. Mandible

(a) Length of the Body:

(b) Width of the Arc:

(c) Full Length of Half Mandible: 


\section{Series/Burial/Skeleton}

Observer/Date

\section{POSTCRANIAL MEASUREMENTS}

L

9. Clavicle

(a) Length:

(b) Diameter:

10. Scapula

(a) Length (height):

(b) Width:

(c) Length of the Spine:

11. llium
(a) Length:
(b) Width:

12. Ischium
(a) Length:
(b) Width:

13. Pubis

(a) Length:

14. Humerus
(a) Length:
(b) Width:
(c) Diameter:

R

L

R
15. Ulna
(a) Length:
(b) Diameter:

16. Radius
(a) Length:
(b) Diameter:

\section{Femur}
(a) Length:
(b) Width:
(c) Diameter:

18. Tibia
(a) Length:
(b) Diameter:

19. Fibula
(a) Length:
(b) Diameter:
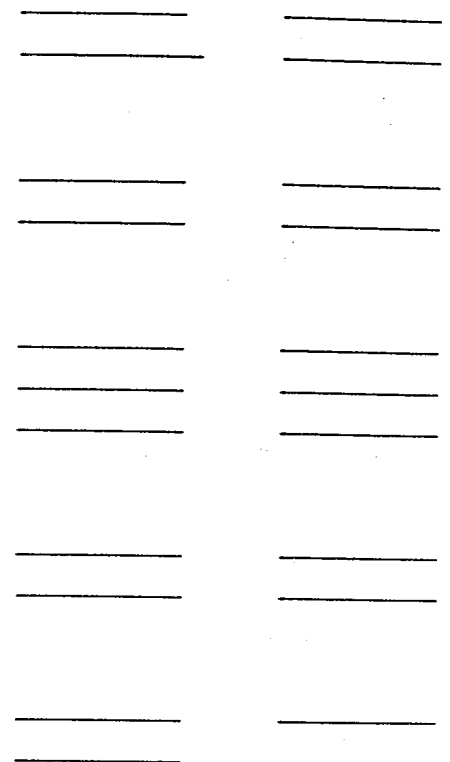

Comments: 


\section{DENTAL INVENTORY VISUAL RECORDING FORM: PERMANENT DENTITION}

Site Name/Number

Feature/Burial Number

Burial/Skeleton Number

Present Location of Collection
Observer

Date

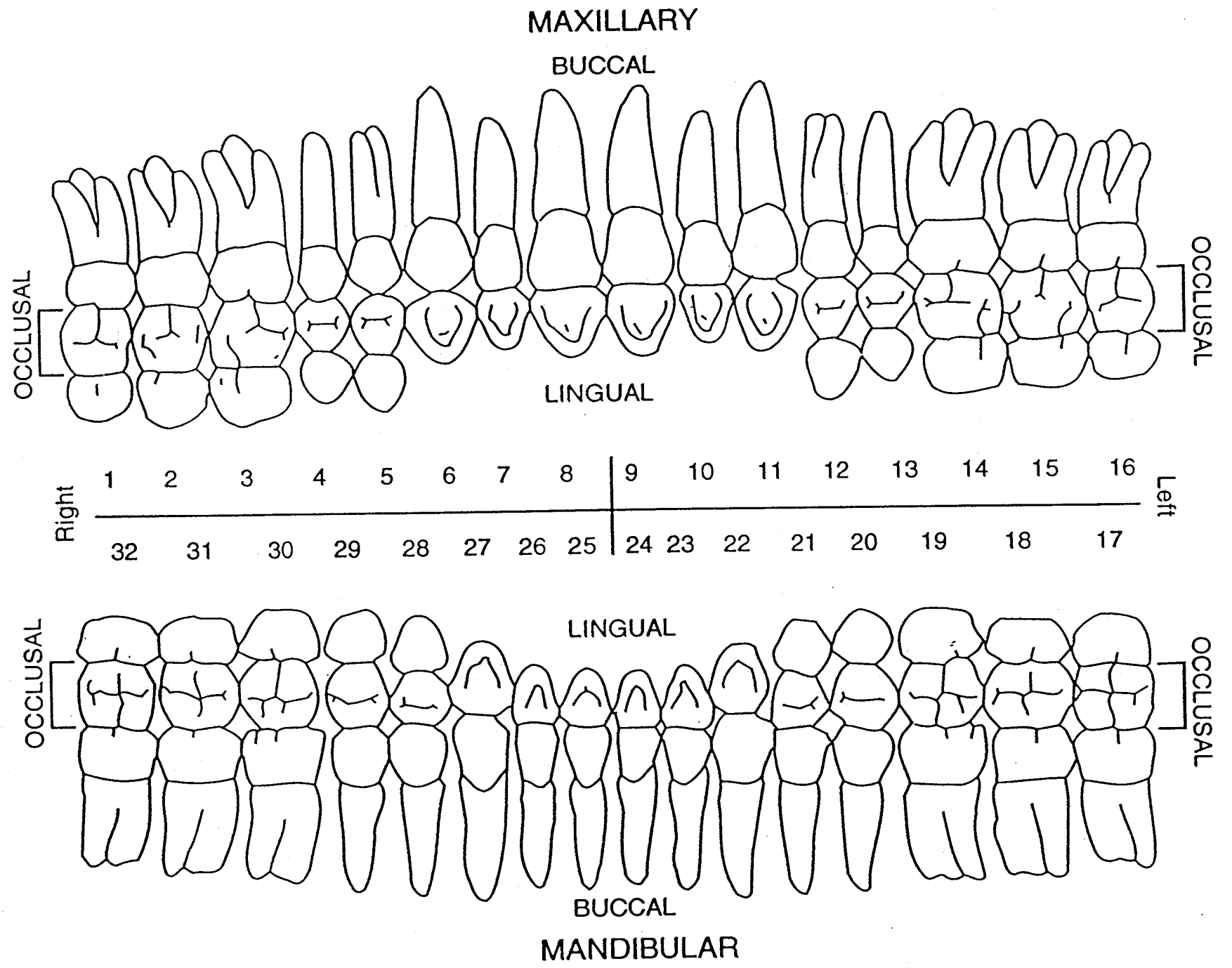




\section{VISUAL RECORDING FORM: DECIDUOUS DENTITION}

Site Name/Number 1

Observer

Feature/Burial Number

Date

Burial/Skeleton Number

Present Location of Collection

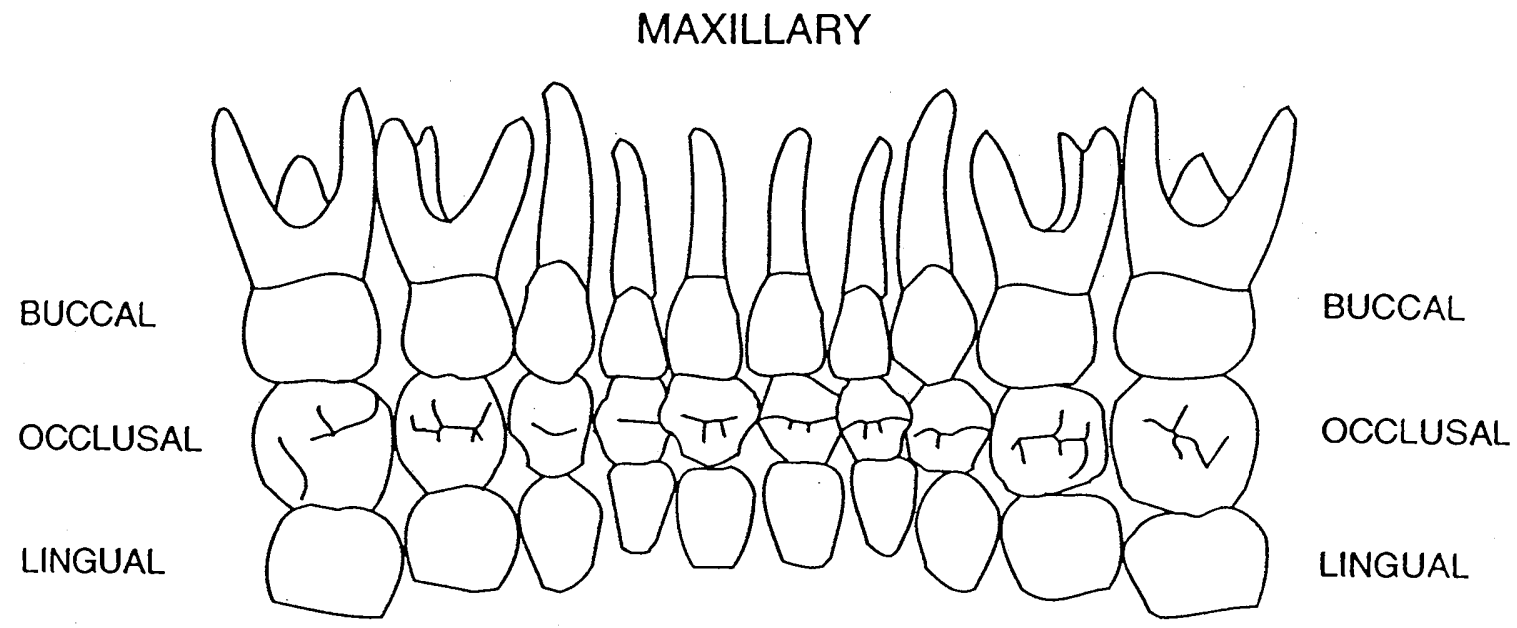

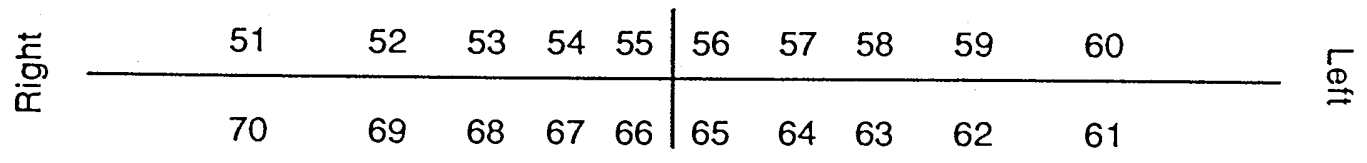

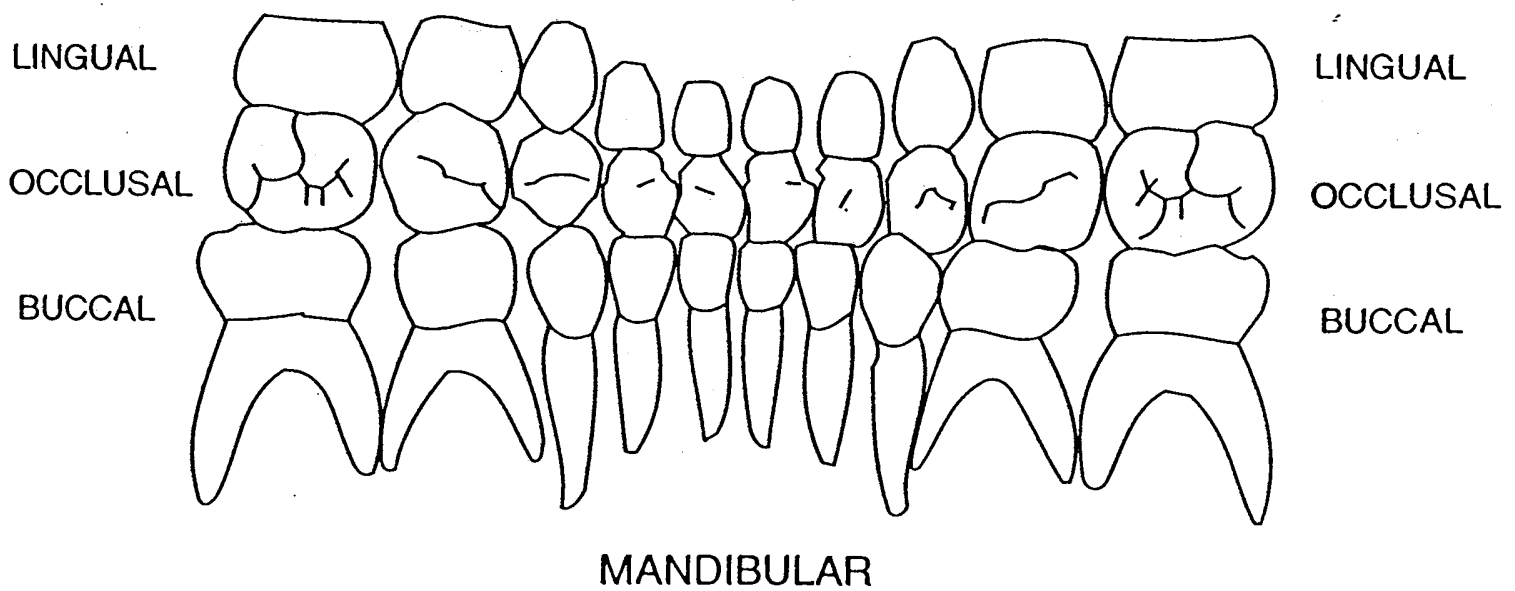




\section{SUPERNUMERARY TEETH AND ABSCESSES VISUAL RECORDING FORM: MAXILLARY DENTITION}

Site Name/Number

Feature/Burial Number

Burial/Skeleton Number

Present Location of Collection
Observer

Date
Right Buccal View

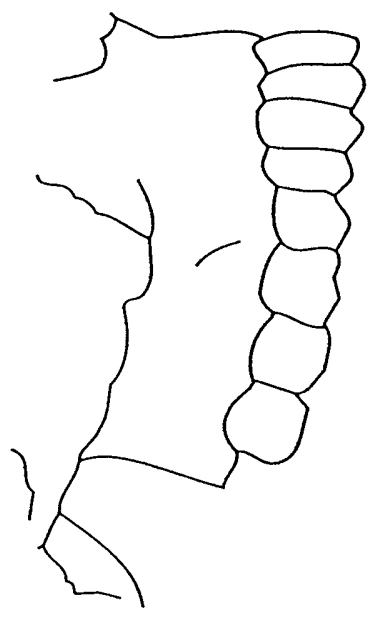

Left Buccal View

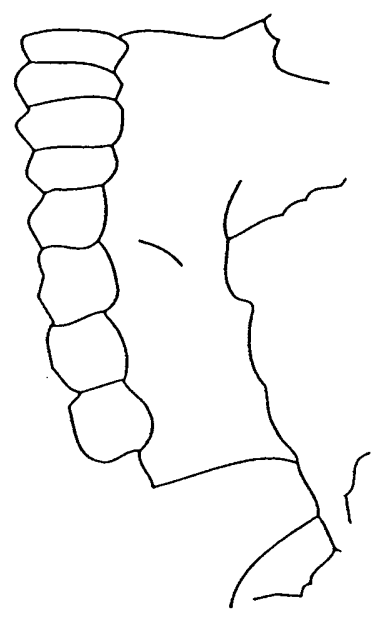

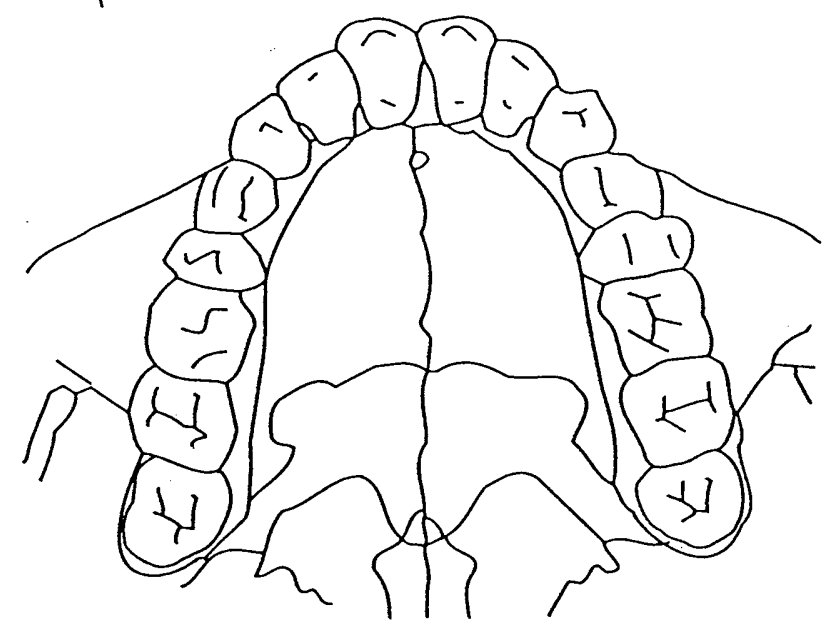

Palatal View 


\section{SUPERNUMERARY TEETH AND ABSCESSES VISUAL RECORDING FORM: MANDIBULAR DENTITION}

Site Name/Number 1

Observer

Feature/Burial Number 1

Date

Burial/Skeleton Number

Present Location of Collection

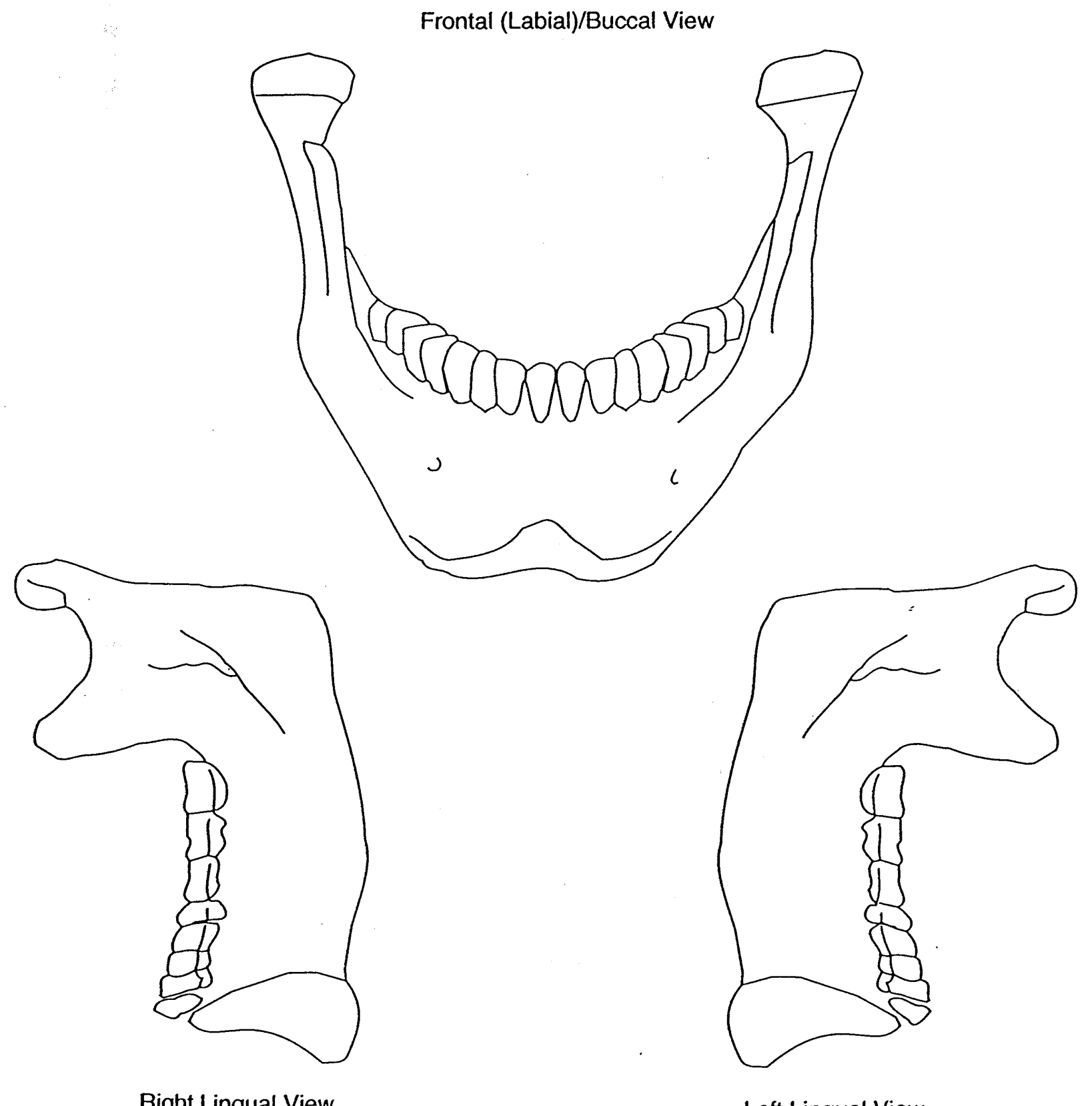

Right Lingual View

Left Lingual View 


\section{DENTAL INVENTORY RECORDING FORM DEVELOPMENT, WEAR, AND PATHOLOGY: PERMANENT TEETH}

Site Name/Number 1

Observer

Feature/Burial Number 1

Date

Burial/Skeleton Number

1

Present Location of Collection

Tooth presence and development: code 1-8. For teeth entered as "1" (present, but not in occlusion), record stage of crown/root formation under "Development." Occlusal surface wear: use left teeth, following Smith (1984) for anterior teeth (code 1-8) and Scott (1979) for molars (code 0-10). If marked asymmetry is present, record both sides. Record each molar quadrant separate in the spaces provided $(t)$ and the total for all four quadrants under "Total." Caries: code each carious lesion separately (1-7); Abscesses: code location (1-2). Calculus: code 0-3, 9. Note surface affected (buccal/abial or lingual).

Maxillary

Tooth Presence Development Wear /Total

Caries

Abscess Calculus/Affected

Right
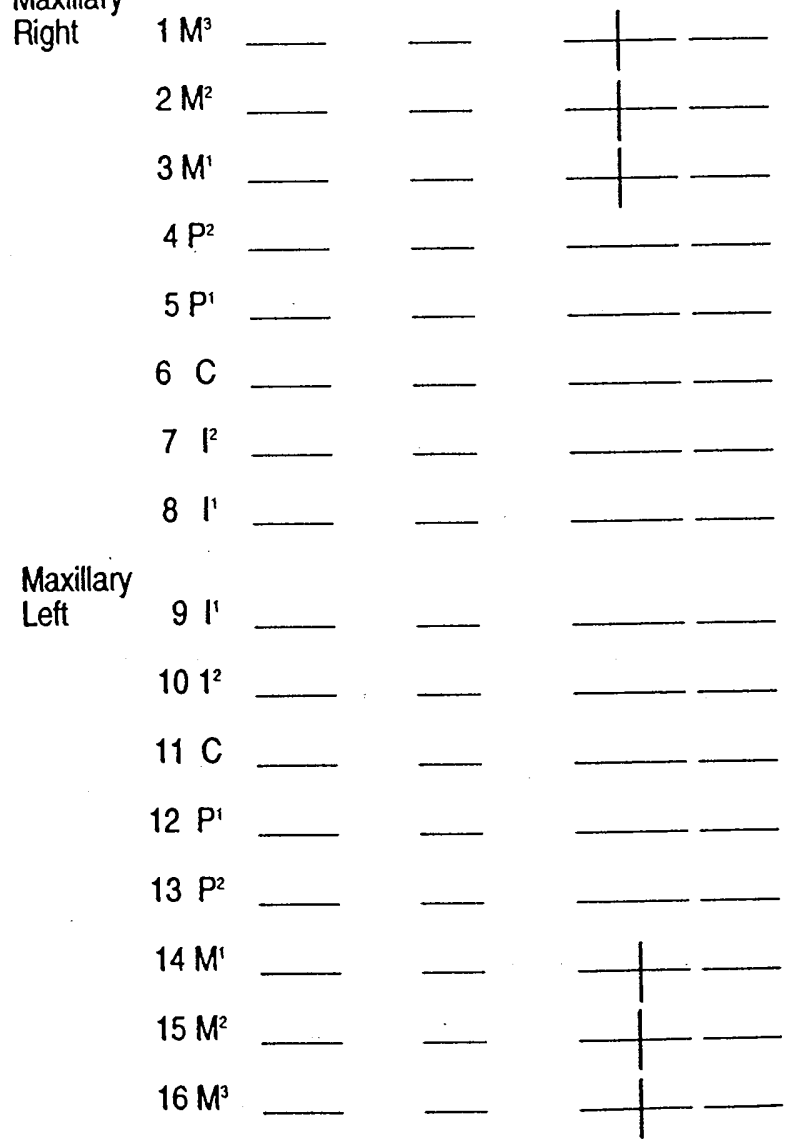
Mandibular

\section{Tooth Presence Development Wear/Total}

Left $\quad 17 \mathrm{M}_{3}$

$18 \mathrm{M}_{2}$

$19 M_{1}$

$20 \mathrm{P}_{2}$

$21 P_{1}$

$22 \mathrm{C}$

$23 \mathrm{H}_{2}$

$24 \mathrm{~h}$

Mandibular

Right 25 ।

$261_{2}$

$27 \mathrm{C}$

$28 P_{1}$

$29 \mathrm{P}_{2}$

$30 \mathrm{M}_{1}$

$31 \mathrm{M}_{2}$

$32 \mathrm{M}_{3}$

Estimated dental age (juveniles only)

Supernumerary Teeth: Position between teeth
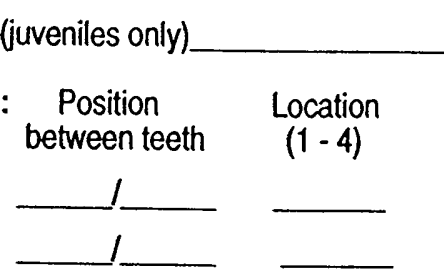
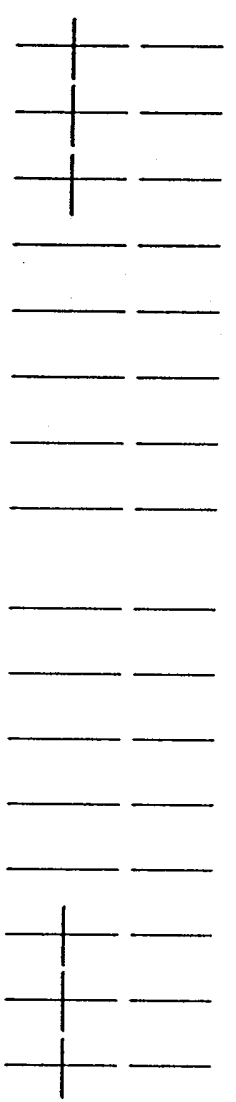

Caries
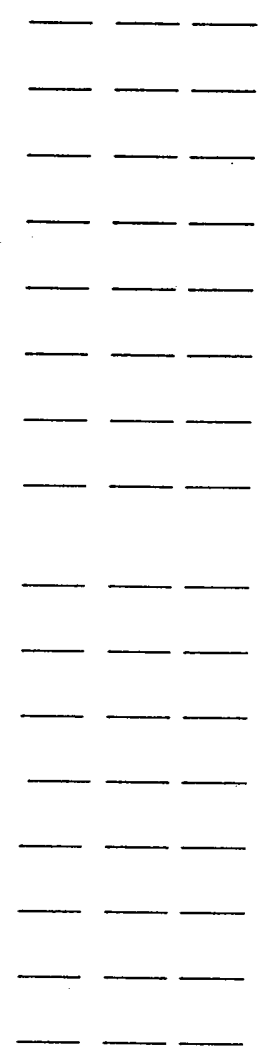

$$
\text { (y) }
$$

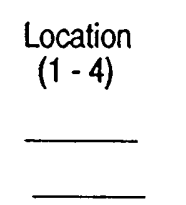

\section{Position \\ Location} between teeth

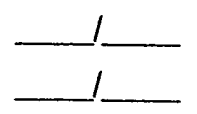
$(1-4)$
Abscess

Calculus/Affected
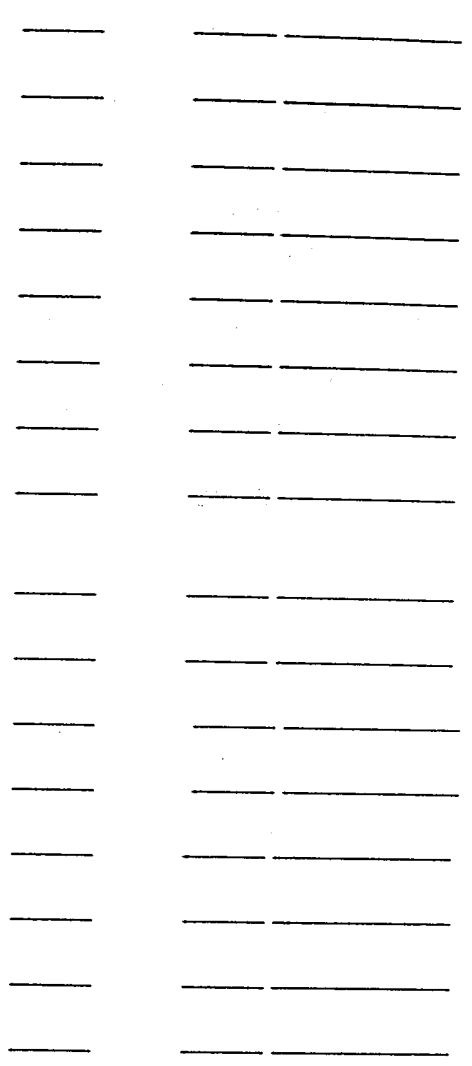

Position Location between teeth $(1-4)$

Comments: 


\section{DENTAL INVENTORY RECORDING FORM DEVELOPMENT AND PATHOLOGY: DECIDUOUS TEETH}

Site Name/Number

Feature/Burial Number

Burial/Skeleton Number

Present Location of Collection
Observer

Date

Tooth presence and development: code 1-8. For teeth entered as " 1 " (present, but not in occlusion), record stage of crown/root formation under "Development." Caries: code each carious lesion separately (17); Abscesses: code location (1-2). Calculus: code 0-3, 9. Note surface affected (buccal/labial or lingual).

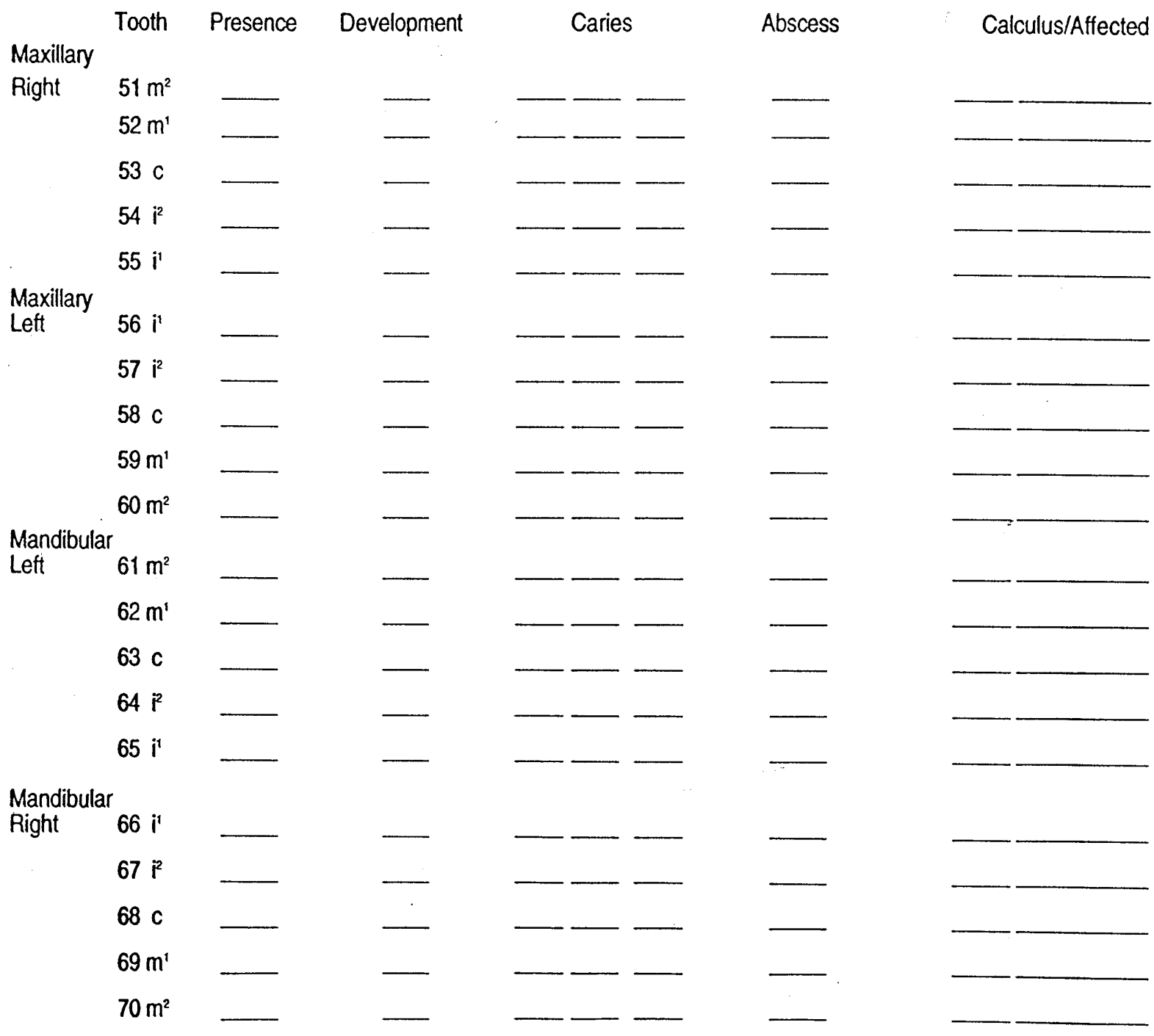


Series/Burial/Skeleton

Observer/Date

Estimated dental age (juveniles only)

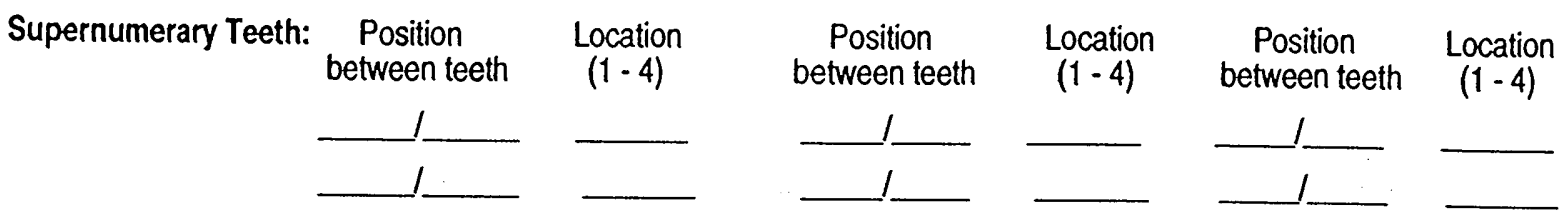

Comments: 


\section{ENAMEL DEFECTS (HYPOPLASIAS AND OPACITIES) RECORDING FORM: PERMANENT TEETH}

Site Name/Number

Feature/Burial Number

Burial/Skeleton Number

Present Location of Collection
Observer

Date

Type: code 0-7 or 9; Location: measure distance from the CEJ to most occlusal portion of defect;

Color: code 1-4 for hypocalfications (type 6 or 7 ) only.

Maxilla, Right

\begin{tabular}{|c|c|c|c|c|c|c|c|c|c|c|c|c|c|c|c|c|c|c|c|c|c|c|c|c|}
\hline Tooth & \multicolumn{3}{|c|}{$M^{3}$} & \multicolumn{3}{|c|}{$M^{2}$} & \multicolumn{3}{|c|}{$M^{1}$} & \multicolumn{3}{|c|}{$P M^{2}$} & \multicolumn{3}{|c|}{$P M^{1}$} & \multicolumn{3}{|c|}{ C } & \multicolumn{3}{|c|}{$\dot{1}^{2}$} & \multicolumn{3}{|c|}{$I^{1}$} \\
\hline Defect & 1 & 2 & 3 & 1 & 2 & 3 & 1 & 2 & 3 & 1 & 2 & 3 & 1 & 2 & 3 & 1 & 2 & 3 & 1 & 2 & 3 & 1 & 2 & 3 \\
\hline Iype & & & & & & & & & & & & & & & & & & & & & & & & \\
\hline Location & & & & & & & & & & & & & & & & & & & & & & & & \\
\hline Color & & & & & & & & & & & & & & & & & & & & & & & & \\
\hline
\end{tabular}

Maxilla, Left

\begin{tabular}{|c|c|c|c|c|c|c|c|c|c|c|c|c|c|c|c|c|c|c|c|c|c|c|c|c|}
\hline Tooth & \multicolumn{3}{|c|}{$l^{1}$} & \multicolumn{3}{|c|}{$1^{2}$} & \multicolumn{3}{|c|}{ C } & \multicolumn{3}{|c|}{$P M^{1}$} & \multicolumn{3}{|c|}{$P M^{2}$} & \multicolumn{3}{|c|}{$M^{\prime}$} & \multicolumn{3}{|c|}{$M^{2}$} & \multicolumn{3}{|c|}{$M^{3}$} \\
\hline Defect & 1 & 2 & 3 & 1 & 2 & 3 & 1 & 2 & 3 & 1 & 2 & 3 & 1 & 2 & 3 & 1 & 2 & 3 & 1 & 2 & 3 & 1 & 2 & 3 \\
\hline Type & & & & & & & & & & & & & & & & & & & & & & & & \\
\hline Location & & & & & & & & & & & & & & & & & & & & & & & & \\
\hline Color & & & & & & & & & & & & & & & & & & & & & & & & \\
\hline
\end{tabular}

Mandible, Left

\begin{tabular}{|c|c|c|c|c|c|c|c|c|c|c|c|c|c|c|c|c|c|c|c|c|c|c|c|c|}
\hline Tooth & \multicolumn{3}{|c|}{$M_{3}$} & \multicolumn{3}{|c|}{$M_{2}$} & \multicolumn{3}{|c|}{$M_{1}$} & \multicolumn{3}{|c|}{$\mathrm{PM}_{2}$} & \multicolumn{3}{|c|}{$\mathrm{PM}_{1}$} & \multicolumn{3}{|c|}{ C } & \multicolumn{3}{|c|}{$l_{2}$} & \multicolumn{3}{|c|}{ It } \\
\hline Defect & 1 & 2 & 3 & 1 & 2 & 3 & 1 & 2 & 3 & 1 & 2 & 3 & 1 & 2 & 3 & 1 & 2 & 3 & 1 & 2 & 3 & 1 & 2 & 3 \\
\hline Type & & & & & & & & & & & & & & & & & & & & & & & & \\
\hline Location & & & & & & & & & & & & & & & & & & & & & & & & \\
\hline Color & & & & & & & & & & & & & & & & & & & & & & & & \\
\hline
\end{tabular}

Mandible, Right

\begin{tabular}{|c|c|c|c|c|c|c|c|c|c|c|c|c|c|c|c|c|c|c|c|c|c|c|c|c|}
\hline Tooth & \multicolumn{3}{|c|}{ It } & \multicolumn{3}{|c|}{$I_{2}$} & \multicolumn{3}{|c|}{ C } & \multicolumn{3}{|c|}{$P M_{1}$} & \multicolumn{3}{|c|}{$\mathrm{PM}_{2}$} & \multicolumn{3}{|c|}{$M_{1}$} & \multicolumn{3}{|c|}{$M_{2}$} & \multicolumn{3}{|c|}{$M_{3}$} \\
\hline Defect & 1 & 2 & 3 & 1 & 2 & 3 & 1 & 2 & 3 & 1 & 2 & 3 & 1 & 2 & 3 & 1 & 2 & 3 & 1 & 2 & 3 & 1 & 2 & 3 \\
\hline Type & & & & & & & & & & & & & & & & & & & & & & & & \\
\hline Location & & & & & & & & & & & & & & & & & & & & & & & & \\
\hline Color & & & & & & & & & & & & & & & & & & & & & & & & \\
\hline
\end{tabular}




\section{ENAMEL DEFECTS (HYPOPLASIAS AND OPACITIES) RECORDING FORM: DECIDUOUS TEETH}

Site Name/Number

Feature/Burial Number

Observer

Burial/Skeleton Number

Present Location of Collection

Type: code $0-7$ or 9 .

Location: measure distance from the CEJ to the most occlusal portion of defect.

Color: code 1-4 for hypocalfications (type 6 or 7 ) only.

\begin{tabular}{|c|c|c|c|c|c|c|c|c|c|c|c|c|c|c|c|}
\hline & \multicolumn{15}{|c|}{ Maxilla, Right } \\
\hline Tooth & \multicolumn{3}{|c|}{$\mathrm{m}^{2}$} & \multicolumn{3}{|c|}{$\mathbf{m}^{1}$} & \multicolumn{3}{|c|}{ c } & \multicolumn{3}{|c|}{$i^{2}$} & \multicolumn{3}{|c|}{$\mathbf{i}^{1}$} \\
\hline Defect & 1 & 2 & 3 & 1 & 2 & 3 & 1 & 2 & 3 & 1 & 2 & 3 & 1 & 2 & 3 \\
\hline Type & & & & & & & & & & & & & & & \\
\hline Location & & & & & & & & & & & & & & & \\
\hline Color & & & & & & & & & & & & & & & \\
\hline
\end{tabular}

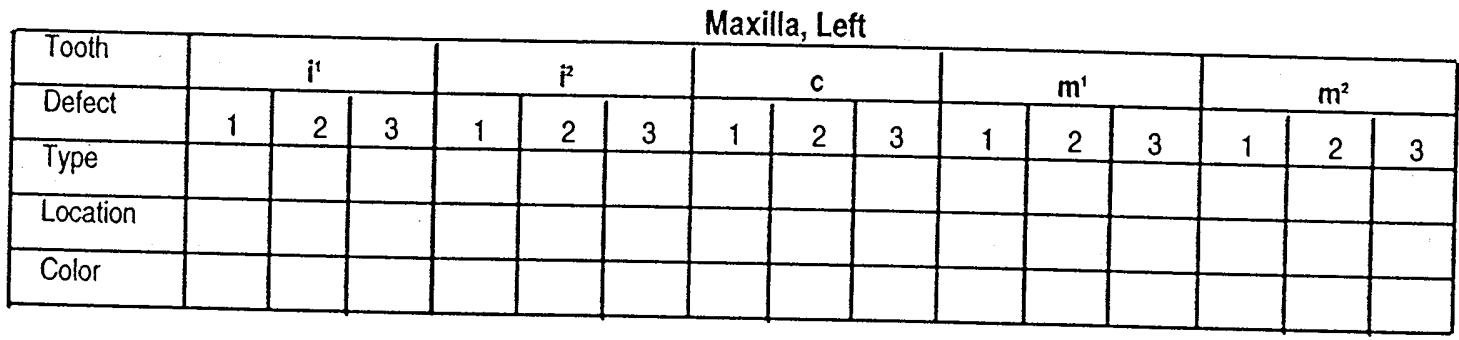

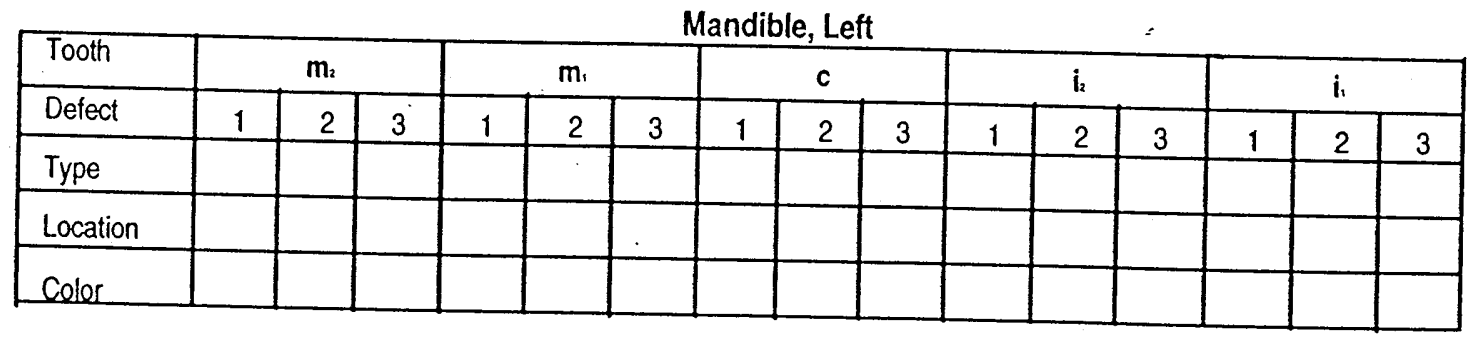

\begin{tabular}{|c|c|c|c|c|c|c|c|c|c|c|c|c|c|c|c|}
\hline \multicolumn{16}{|c|}{ Mandible, Right } \\
\hline Tooth & \multicolumn{3}{|c|}{$\mathbf{i}_{\mathbf{s}}$} & \multicolumn{3}{|c|}{$i_{2}$} & \multicolumn{3}{|c|}{ C } & \multicolumn{3}{|c|}{$\mathrm{m}_{\mathrm{r}}$} & \multicolumn{3}{|c|}{$m_{2}$} \\
\hline Defect & 1 & 2 & 3 & 1 & 2 & 3 & 1 & 2 & 3 & 1 & 2 & 3 & 1 & 2 & 3 \\
\hline Type & & & & & & & & & & & & & & & \\
\hline Location & & & & & & & & & & & & & & & \\
\hline Color & & & & & & & & & & & & & & & \\
\hline
\end{tabular}




\section{PREMORTEM DENTAL MODIFICATIONS RECORDING FORM}

Site Name/Number

Feature/Burial Number

Burial/Skeleton Number

Present Location of Collection

Type of Modification:

1. Aesthetic modification: filing

2. Aesthetic modification: drilling

3. Dental restorations and appliances

4. Dental wear associated with artifact use or production

5. Tooth ablation
Observer

Date

Comments: 
Series/Burial/Skeleton

Observer/Date

Attachment 19: CHAPTER 5 


\section{DENTAL MEASUREMENTS AND MORPHOLOGY RECORDING FORM}

Site Name/Number

Observer

Feature/Burial Number $+1$

Date

Burial/Skeleton Number

Present Location of Collection

Dental Measurements

Record left side of arcade only; substitute antimere when left not observable.

\begin{tabular}{|c|c|c|c|c|c|c|c|c|c|}
\hline \multirow{2}{*}{ Left } & \multicolumn{9}{|c|}{ Maxilla } \\
\hline & Tooth & $I^{1}$ & $p^{2}$ & $\mathrm{C}$ & $P M^{\prime}$ & $P M^{2}$ & $M^{\prime}$ & $M^{2}$ & $M^{3}$ \\
\hline \multirow{7}{*}{$R: g h t$} & Mesiodistal diameter & & & & & & & & \\
\hline & Buccolingual diameter & & & & & & & & \\
\hline & Crown height & & & & & & & & \\
\hline & Tooth & $M_{3}$ & $M_{2}$ & $M_{i}$ & $\mathrm{PM}_{2}$ & $P M_{1}$ & C & $I_{z}$ & l, \\
\hline & Mesiodistal diameter & & & & & & & & \\
\hline & Buccolingual diameter & & & & & & & & \\
\hline & Crown height & & & & & & & & \\
\hline
\end{tabular}

\begin{tabular}{l|l|l|l|l|l|l|l|l|l|}
\hline & Left & $M_{3}$ & $M_{2}$ & $M_{1}$ & $\mathrm{PM}_{2}$ & $\mathrm{PM}_{1}$ & $\mathrm{C}$ & $\mathrm{I}_{2}$ & $\mathrm{I}_{1}$ \\
\hline Tooth & & & & & & & & \\
\hline Mesiodistal diameter & & & & & & & & \\
\hline Buccolingual diameter & & & & & & & & \\
\hline Crown height
\end{tabular}

\begin{tabular}{|l|l|l|l|l|l|l|l|l|}
\hline Right & $\mathrm{I}^{\prime}$ & $\mathrm{I}^{2}$ & $\mathrm{C}$ & $\mathrm{PM}^{1}$ & $\mathrm{PM}^{2}$ & $\mathrm{M}^{1}$ & $\mathrm{M}^{2}$ & $\mathrm{M}^{3}$ \\
\hline Mesiodistal diameter & & & & & & & & \\
\hline Buccolingual diameter & & & & & & & & \\
\hline Crown height & & & & & & & & \\
\hline
\end{tabular}


Series/Burial/Skeleton

Observer/Date

Dental Morphology

\begin{tabular}{|c|c|c|c|c|c|c|c|c|c|c|c|c|}
\hline \multirow{2}{*}{\multicolumn{7}{|c|}{ Right }} & & & & & & \\
\hline & & & & & & & \multicolumn{6}{|c|}{ Left } \\
\hline Tooth & $M^{3}$ & $M^{2}$ & $M^{\prime}$ & $P M^{1}$ & $p^{2}$ & $I^{\prime}$ & 1 & $p^{2}$ & $\mathrm{PM}^{\prime}$ & $M^{1}$ & $M^{2}$ & $M^{3}$ \\
\hline \multicolumn{13}{|l|}{ Winging $(0-4)$} \\
\hline \multicolumn{13}{|l|}{ Shoveling (0-7) } \\
\hline \multicolumn{13}{|l|}{ Double-shoveling $(0-6)$} \\
\hline \multicolumn{13}{|l|}{ Peg-shaped Incisor (0-2) } \\
\hline \multicolumn{13}{|l|}{ Premolar Root Number (1-3) } \\
\hline \multicolumn{13}{|l|}{ Hypocone $(0-6)$} \\
\hline \multicolumn{13}{|l|}{ Metaconule (0-5) } \\
\hline \multicolumn{13}{|l|}{ Carabelli's Trait (0-7) } \\
\hline Enamel Extensions (0-3) & & & & & & & & & & & & \\
\hline
\end{tabular}

\begin{tabular}{|l|l|l|l|l|l|l|l|l|}
\hline \multicolumn{7}{|c|}{ Mandible } \\
\hline Tooth & $M_{3}$ & $M_{2}$ & $M_{1}$ & $P_{1}$ & $P_{1}$ & $M_{1}$ & $M_{2}$ & $M_{3}$ \\
\hline Premolar Root Number (0-5) & \multicolumn{3}{|c|}{ Left } \\
\hline Groove Pattern $(Y,+, X)$ & & & & & & & & \\
\hline Cusp Numbuer (4-6) & & & & & & & & \\
\hline Protostylid (0-7) & & & & & & & & \\
\hline Cusp 5 (0-5) & & & & & & & & \\
\hline Cusp 6 (0-5) & & & & & & & & \\
\hline Cusp 7 (0-4) & & & & & & & & \\
\hline Molar Root Number (1-3) & & & & & & & & \\
\hline
\end{tabular}




\section{CRANIAL AND POSTCRANIAL MEASUREMENT RECORDING FORM: ADULT REMAINS}

Site Name/Number

Feature/Burial Number

Burial/Skeleton Number

Present Location of Collection
Observer

Date

Record all measurements to the nearest millimeter; in the case of bilateral measurements, take measurement on the left side. If right side is substituted, place an $(R)$ next to the the measurement. If bones are fragmented, measurements should not be taken, but dimensions should be estimated for minor erosion or reconstruction; identify these with an asterick ${ }^{4 * \pi}$

\section{Cranial Measurements}

1. Maximum Cranial Length:

2. Maximum Cranial Breadth:

3. Bizygomatic Diameter:

4. Basion-Bregma Height:

5. Cranial Base Length:

6. Basion-Prosthion Length:

7. Maxillo-Alveolar Breadth:

8. Maxillo-Alveolar Length:

9. Biauricular Breadth:

10. Upper Facial Height:

11. Minimum Frontal Breadth:

12. Upper Facial Breadth:

13. Nasal Height:

14. Nasal Breadth:

15. Orbital Breadth:

16. Orbital Height:

17. Biorbital Breadth:
18. Interorbital Breadth:

19. Frontal Chord:

20. Parietal Chord:

21. Occipital Chord:

22. Foramen Magnum Length:

23. Foramen Magnum Breadth:

24. Mastoid Length:

25. Chin Height

26. Height of the Mandibular Body:

27. Breadth of the Mandibular Body:

28. Bigonial Width:

29. Bicondylar Breadth:

30. Minimum Ramus Breadth:

31. Maximum Ramus Breadth:

32. Maximum Ramus Height:

33. Mandibular Length:

34. Mandibular Angle: 
Observer/Date

Record all measurements to the nearest millimeter; in the case of bilateral measurements, take measurement on the left side. If right side is substituted, place an $(R)$ next to the the measurement. If bones are fragmented, measurements should not be taken, but dimensions should be estimated for minor erosion or reconstruction; identify these with an asterick"**

\section{Postcranial Measurements}

35. Clavicle: Maximum Length:

36. Clavicle: Ant.-Post. Diameter at Midshaft:

37. Clavicle: Sup.-Inf. Diameter at Midshaft:

38. Scapula: Height:

39. Scapula: Breadth:

40. Humerus: Maximum Length:

41. Humerus: Epicondylar Breadth:

42. Humerus: Vertical Diameter of Head:

43. Humerus: Maximum Diameter at Midshaft:

44. Humerus: Minimum Diameter at Midshaft:

45. Radius: Maximum Length:

46. Radius: Anterior-Posterior Diameter at Midshaft :

47. Radius: Medial-Lateral Diameter at Midshaft:

48. Ulna: Maximum Length:

49. Ulna: Anterior-Posterior Diameter:

50. Ulna: Medial-Lateral Diameter:

51. Ulna: Physiological Length:

52. Ulna: Minimum Circumference:

53. Sacrum: Anterior Length:

54. Sacrum: Anterior Superior Breadth:

55. Sacrum: Max. Transverse Diameter of Base:

56. Os Coxae: Height:
57. Os Coxae: lliac Breadth:

58. Os Coxae: Pubis Length:

59. Os Coxae: Ischium Length:

60. Femur: Maximum Length:

61. Femur: Bicondylar Length:

62. Femur: Epicondylar Breadth:

63. Femur: Maximum Diameter of the Femur Head:

64. Femur: Ant.-Post. Subtrochanteric Diameter:

65. Femur: Medial-Lateral Subtrochanteric Diameter:

66. Femur: Anterior-Posterior Midshaft Diameter:

67. Femur: Medial-Lateral Midshaft Diameter:

68. Femur: Midshaft Circumference:

69. Tibia: Length:

70. Tibia: Maximum Proximal Epiphyseal Breadth:

71. Tibia: Maximum Distal Epiphyseal Breadth:

72. Tibia: Max. Diameter at the Nutrient Foramen:

73. Tibia: Med.-Lat. Diameter at Nutrient Foramen:

74. Tibia: Circumference at the Nutrient Foramen:

75. Fibula: Maximum Length:

76. Fibula: Maximum Diameter at Midshaft:

77. Calcaneus: Maximum Length:

78. Calcaneus: Middle Breadth: 


\section{PRIMARY NONMETRIC TRAITS RECORDING FORM}

Site Name/Number 1

Observer

Feature/Burial Number 1

Date

Burial/Skeleton Number

Present Location of Collection

1. Metopic Suture:

$0=$ absent

$1=$ partial

$2=$ complete

$9=$ unobservable

\section{Supraorbital Structures}

a. Supraorbital notch:

$0=a b s e n t$

$1=$ present $<1 / 2$ occluded by spicules

$2=$ present $>1 / 2$ occluded by spicules

$3=$ present, degree of occlusion unknown

$4=$ multiple notches

$9=$ unobservable

b. Supraorbital foramen:

$0=$ absent

$1=$ present

$2=$ multiple foramina

$9=$ unobservable

3. Infraorbital Suture:

$0=$ absent

$1=$ partial

$2=$ complete

$9=$ unobservable

4. Multiple Infraorbital Foramina:

$0=$ absent

$1=$ internal division only

$2=$ two distinct foramina

$3=$ more than two distinct foramina

$9=$ unobservable

5. Zygomatico-facial Foramina:

$0=$ absent

$1=1$ large

$2=1$ large plus smaller $f$.

$3=2$ large

$4=2$ large plus smaller $f$.

$5=1$ small

$6=$ multiple small

$9=$ unobservable

\section{Parietal Foramen:}

$0=$ absent

$1=$ present, on parietal

$2=$ present, sutural

$9=$ unobservable

\section{Sutural Bones}

L $M \quad R$

$$
0=a b s e n t
$$

$1=$ present

$9=$ unobservable

a. epipteric bone:

b. coronal ossicle:

c. bregmatic bone:

d. sagittal ossicle:

e. apical bone:

f. lambdoid ossicle:

g. asterionic bone:

$h$ ossicle in occipitomastoid suture:

i. parietal notch bone:

8. Inca Bone

$0=a b s e n t$

$1=$ complete, single bone

$2=$ bipartite

$3=$ tripartite

$4=$ partial

$9=$ unobservable

9. Condylar Canal:

$0=$ not patent

$1=$ patent

$9=$ unobservable

10. Divided Hypoglossal Canal:

$0=$ absent

$1=$ partial, internal surface

$2=$ partial, within canal

$3=$ complete, internal surface

$4=$ complete, within canal

$9=$ unobservable

11. Flexure of Superior Sagittal Sulcus

$$
\begin{aligned}
& 1=\text { Right } \\
& 2=\text { Left } \\
& 3=\text { Bifurcate } \\
& 9=\text { Unobservable }
\end{aligned}
$$

12. Foramen Ovale Incomplete:

$$
\begin{aligned}
& 0=\text { absent } \\
& 1=\text { partial formation } \\
& 2=\text { no definition of foramen } \\
& 9=\text { unobservable }
\end{aligned}
$$

$\mathbf{R}$
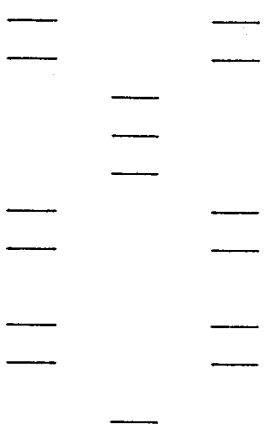

$$
\text { (1) }
$$


Series/Burial/Skeleton

Observer/Date

\section{L $\quad$ M R}

13. Foramen Spinosum Incomplete:
$0=$ absent
$1=$ partial formation
$2=$ no definition of foramen
$9=$ unobservable

14. Pterygo-spinous Bridge:

$0=$ absent

$1=$ trace (spicule only)

$2=$ partial bridge

$3=$ complete bridge

$9=$ unobservable

15. Pterygo-alar Bridge:

$0=$ absent

$1=$ trace (spicule only)

$2=$ partial bridge

$3=$ complete bridge

$9=$ unobservable

16. Tympanic Dihiscence:

$0=$ absent

$1=$ foramen only

2 = full defect present

$9=$ unobservable

17. Auditory Exostosis:

$0=a b s e n t$

$1=<1 / 3$ canal occluded

$2=1 / 3-2 / 3$ canal occluded

$3=>2 / 3$ canal occluded

$9=$ unobservable

18. Mastoid Foramen

a. Location:

$0=$ absent

$1=$ temporal

$2=$ sutural

$3=$ occipital

$4=$ both sutural and temporal

$5=$ both occipital and temporal

$9=$ unobservable

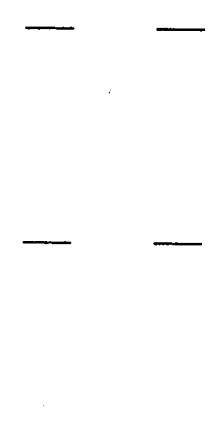

b. Number:

$0=$ absent

$1=1$

$2=2$

$3=$ more than 2

$9=$ unobservable

19. Mental Foramen:

$0=a b s e n t$

$1=1$

$2=2$

$3=>2$

$9=$ unobservable

20. Mandibular Torus:

$$
\begin{aligned}
& 0=\text { absent } \\
& 1=\text { trace (can palpate but not see) } \\
& 2=\text { moderate: elevation between } 2-5 \mathrm{~mm} . \\
& 3=\text { marked: elevation greater than } 5 \mathrm{~mm} . \\
& 9=\text { unobservable }
\end{aligned}
$$

21. Mylohyoid Bridge

a. Location:

$0=$ absent

$1=$ near mandibular foramen

$2=$ center of groove

$3=$ both bridges described in 1) and 2), with hiatus

$4=$ both bridges described in 1) and 2), no hiatus

$9=$ unobservable

b. Degree:

$0=a b s e n t$

$1=$ partial

$2=$ complete

$9=$ unobservable

22. Atlas Bridging

a. Lateral Bridging:

$0=a b s e n t$

$1=$ partial

$2=$ complete

$9=$ unobservable

b. Posterior Bridging:

$0=a b s e n t$

$1=$ partial

$2=$ complete

$9=$ unobservable

23. Accessory Transverse Foramina:

-in 7th Cervical Vertebra

$0=$ absent

$1=$ partial

$2=$ complete

$9=$ unobservable

24. Septal Aperture:

$0=$ absent

$1=$ small foramen (pinhole) only

$2=$ true perforation

$9=$ unobservable

L $\quad$ M R

\section{R}


PALEOPATHOLOGY RECORDING FORM I

SHAPE, SIZE, BONE LOSS, FORMATION, FRACTURES, AND POROTIC HYPEROSTOSIS

Site Name/Number

Observer

Feature/Burial Number

Date

Burial/Skeleton Number

Present Location of Collection

\subsection{SHAPE}

\begin{tabular}{|c|c|c|c|c|c|}
\hline Bone & Bone & Bone & Bone & Bone & Bone \\
\hline Side_ & Side & Side & Side_ & Side_ & Side \\
\hline Bone & Bone & Bone & Bone & Bone & Bone \\
\hline Side_ & Side & Side & Side & Side & Side \\
\hline Obs1_ & Obs1 & Obs1 & Obs1 & Obs1 & Obs1. \\
\hline Obs2 & Obs2 & Obs2 & Obs2 & Obs2 & Obs2 \\
\hline
\end{tabular}

\subsection{SIZE}

Bone
Side
Obs

Bone

Side

Obs

Bone
Side
Obs

Bone

Side

Bone

Side

Obs

Obs

Bone

Side

Obs

\subsection{BONE LOSS}

\begin{tabular}{|c|c|}
\hline Bone_ & Bone \\
\hline Side & Side \\
\hline Section_ & Section \\
\hline Aspect & Aspect \\
\hline Obs1_ & Obs1 \\
\hline Obs2 & Obs2 \\
\hline Obs3 & Obs3 \\
\hline Obs4_ & Obs4 \\
\hline Obs5 & Obs5 \\
\hline Obs6 & Obs6 \\
\hline Obs7 & Obs7 \\
\hline Obs8 & Obs8 \\
\hline
\end{tabular}

Bone__-
Side_-
Section
Aspect_-
Obs1_-
Obs2
Obs3
Obs4_-
Obs5
Obs6
Obs7
Obs8

\begin{tabular}{|c|}
\hline Bone \\
\hline Side _ \\
\hline Section_ \\
\hline Aspect_ \\
\hline Obs1 \\
\hline Obs2 \\
\hline Obs3 \\
\hline Obs4_ \\
\hline Obs5 \\
\hline Obs6_ \\
\hline Obs7_ \\
\hline Obs8 \\
\hline
\end{tabular}

Bone
Side_-
Section
Aspect
Obs1
Obs2
Obs3
Obs4
Obs5
Obs6
Obs7
Obs8

Bone

Side Section Aspect

Obs1

Obs2

- Obs3

Obs 4

Obs5

Obs6

Obs7

Obs8

\subsection{FORMATION}

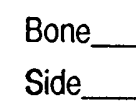

Section

Aspect

Obs1

Obs2

Obs3

Obs4

Obs5

Obs6

\section{Bone}

Side

Section

Aspect

Obs1

Obs2

Obs3

Obs 4

Obs5

Obs6
Bone

Side

Section

Aspect

Obs1

Obs2

Obs3

Obs4

Obs5

Obs6

Bone___
Side__
Section___
Aspect__
Obs1__
Obs2_
Obs3_
Obs4_
Obs5_
Obs6_

\begin{tabular}{|c|c|}
\hline Bone & Bone \\
\hline Side & Side_ \\
\hline Section & Section \\
\hline Aspect & Aspect_ \\
\hline Obs1_ & Obs1 \\
\hline Obs2 & Obs2 \\
\hline Obs3_ & Obs3 \\
\hline Obs4_ & Obs4 \\
\hline Obs5_ & Obs5 \\
\hline Obs6_ & Obs6 \\
\hline
\end{tabular}


Series/Burial/Skeleton

Observer/Date

\subsection{FRACTURES}

\begin{tabular}{|c|c|c|c|c|c|}
\hline Bone & Bone & Bone & Bone_ & Bone & Bone \\
\hline Side_ & Side_ & Side_ & Side_ & Side & Side \\
\hline Section & Section & Section & Section & Section & Section \\
\hline Obs1_ & Obs1_ & Obs1 & Obs1 & Obs1 & Obs1_ \\
\hline Obs2 & Obs2 & Obs2 & Obs2 & Obs2 & Obs2 \\
\hline Obs3 & Obs3_ & Obs3 & Obs3 & Obs3 & Obs3 \\
\hline Obs4 & Obs4 & Obs4 & Obs4 & Obs4 & Obs4 \\
\hline Obs5 & Obs5 & Obs5 & Obs5 & Obs5 & Obs5 \\
\hline Obs6 & Obs6 & Obs6 & Obs6 & Obs6 & Obs6_ \\
\hline Obs7 & Obs7 & Obs7_ & Obs7_ & Obs7 & Obs7 \\
\hline Obs8_ & Obs8_ & Obs8 & Obs8_ & Obs8 & Obs8_ \\
\hline Obs9 & Obs9 & Obs9_ & Obs9 & Obs9 & Obs9 \\
\hline Obs10 & Obs10 & Obs10 & Obs10_ & Obs10 & Obs10 \\
\hline Obs11 & Obs11_ & Obs11_ & Obs11_ & Obs11 & Obs11_ \\
\hline \multicolumn{6}{|c|}{ 6.0 POROTIC HYPEROSTOSIS } \\
\hline Bone & Bone & Bone & Bone & Bone & Bone \\
\hline Side & Side & Side_ & Side & Side_ & Side \\
\hline Obs1_ & Obs1 & Obs1 & Obs1 & Obs1_ & Obs1_ \\
\hline Obs2 & Obs2 & Obs2 & Obs2 & Obs2 & Obs2 \\
\hline Obs3 & Obs3 & Obs3 & Obs3 & Obs3 & Obs3 \\
\hline
\end{tabular}

Comments: 


\section{PALEOPATHOLOGY RECORDING FORM II VERTEBRAL PATHOLOGY, ARTHRITIS, AND MISCELLANEOUS}

Site Name/Number

Observer

Feature/Burial Number 1

Date

Burial/Skeleton Number

Present Location of Collection

\subsection{VERTEBRAL PATHOLOGY}

Bone___
Side__
Aspect___
Bone___
Side__
Aspect
Obs1__
Obs2
Obs3

\begin{tabular}{|c|c|}
\hline Bone_ & Bone \\
\hline Side_ & Side \\
\hline Aspect_ & Aspect_ \\
\hline Bone & Bone \\
\hline Side & Side \\
\hline Aspect & Aspect \\
\hline Obs1_ & Obs1_ \\
\hline Obs2 & Obs2 \\
\hline Obs3 & Obs3 \\
\hline
\end{tabular}

Bone_-
Side_-
Aspect
Bone_-
Side_-
Aspect__
Obs1
Obs2
Obs3

Bone
Side__-
Aspect_-
Bone_-
Side_-
Aspect__
Obs1
Obs2
Obs3

Bone_-
Side_-
Aspect_-
Bone_-
Side_-
Aspect
Obs1
Obs2
Obs3

\subsection{ARTHRITIS}

\begin{tabular}{|c|c|}
\hline Bone & Bone \\
\hline Side_. & Side_ \\
\hline Section/ & Section/ \\
\hline Aspect_ & Aspect \\
\hline Bone & Bone_ \\
\hline Side_ & Side_ \\
\hline Section/ & Section/ \\
\hline Aspect_ & Aspect_ \\
\hline Obs1_ & Obs1_ \\
\hline Obs2 & Obs2 \\
\hline Obs3 & Obs3 \\
\hline Obs4_ & Obs4 \\
\hline Obs5_ & Obs5 \\
\hline Obs6_ & Obs6_ \\
\hline Obs7 & Obs7_ \\
\hline Obs8_ & Obs8_ \\
\hline Obs9_ & Obs9 \\
\hline
\end{tabular}

\section{MISCELLANEOUS}

$\begin{array}{ll}\text { Bone___ } & \text { Bone__ } \\ \text { Side___ } & \text { Obs__ } \\ \text { Obs___ } & \text { Bone__ } \\ \text { Bone___ } & \text { Side } \\ \text { Side__ } & \text { Obs }\end{array}$

\begin{tabular}{|c|c|c|}
\hline Bone_ & Bone & Bone \\
\hline Side & Side & Side _. \\
\hline Section/ & Section/ & Section/ \\
\hline Aspect & Aspect & Aspect \\
\hline Bone & Bone & Bone \\
\hline Side & Side__ & Side \\
\hline Section/ & Section/ & Section/ \\
\hline Aspect & Aspect & Aspect \\
\hline Obs1 & Obs1 & Obs1 \\
\hline Obs2 & Obs2 & Obs2 \\
\hline Obs3 & Obs3 & Obs3 \\
\hline Obs4 & Obs4 & Obs4_ \\
\hline Obs5 & Obs5 & Obs5_ \\
\hline Obs6_ & Obs6 & Obs6 \\
\hline Obs7 & Obs7_ & Obs7 \\
\hline Obs8 & Obs8_ & Obs8 \\
\hline Obs9 & Obs9 & Obs9 \\
\hline
\end{tabular}

Bone

Side Section

Aspect

Bone

Side

Section

Aspect

Obs1

Obs2

Obs3

Obs4

Obs5

Obs6

Obs7

Obs8

Obs9

\begin{tabular}{|c|c|}
\hline Bone & Bone \\
\hline Side & Side \\
\hline Obs & Obs \\
\hline Bone & Bone \\
\hline Side & Side \\
\hline Obs & Obs_ \\
\hline
\end{tabular}

Bone
Side
Obs

Bone

Side

Obs

$\begin{array}{ll}\text { Bone___ } & \text { Bone__ } \\ \text { Side___ } & \text { Side } \\ \text { Obs__ } & \text { Obs }\end{array}$


Series/Burial/Skeleton

Observer/Date

\section{COMMENTS}




\section{APPENDIX D \\ LISTING OF COMPARISON CATALOGS}





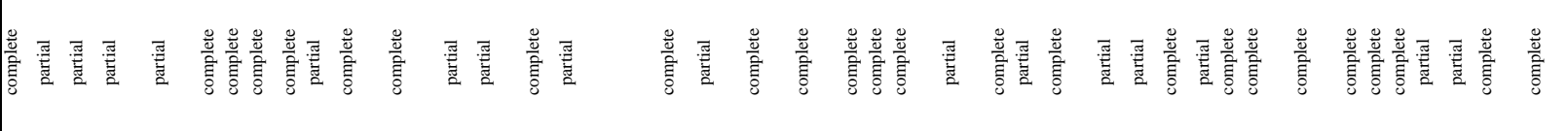

-1)

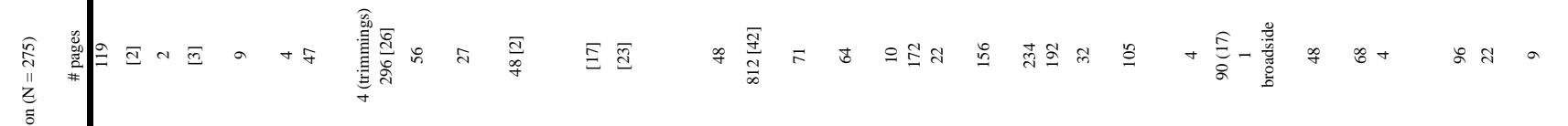

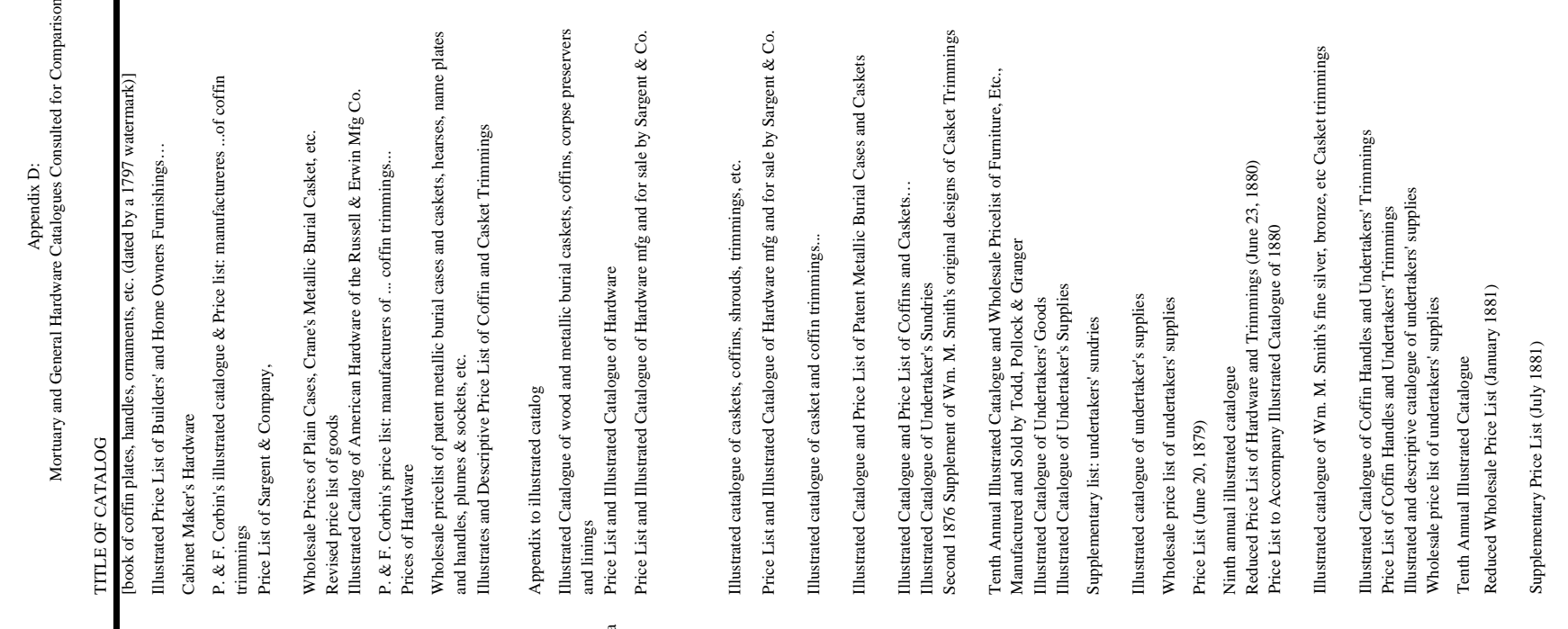

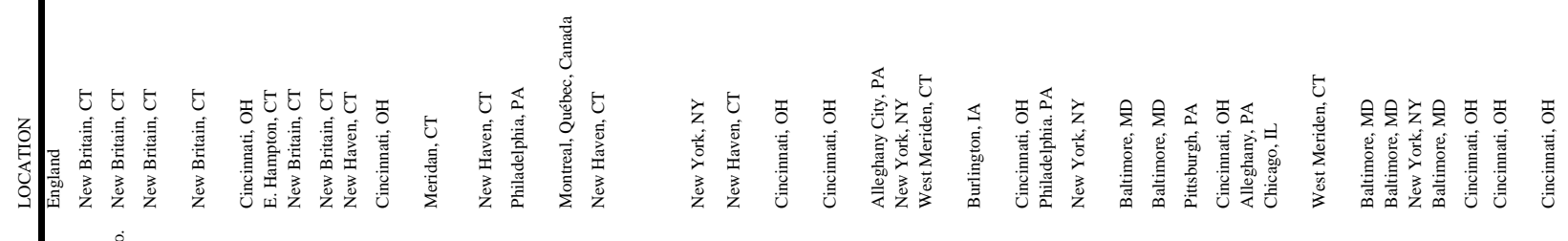

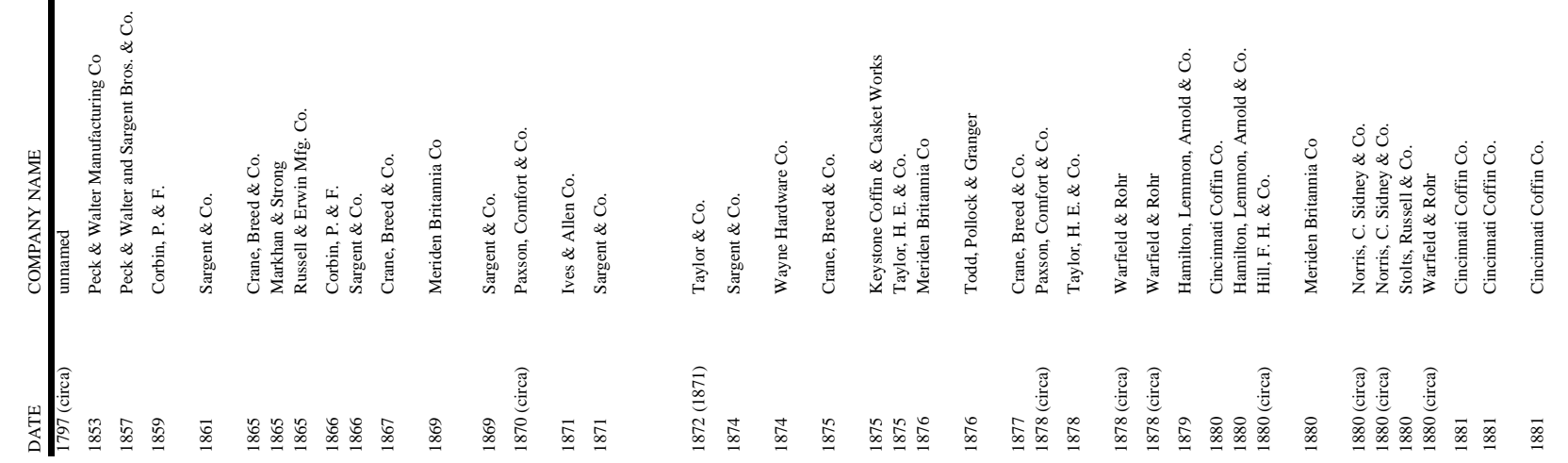




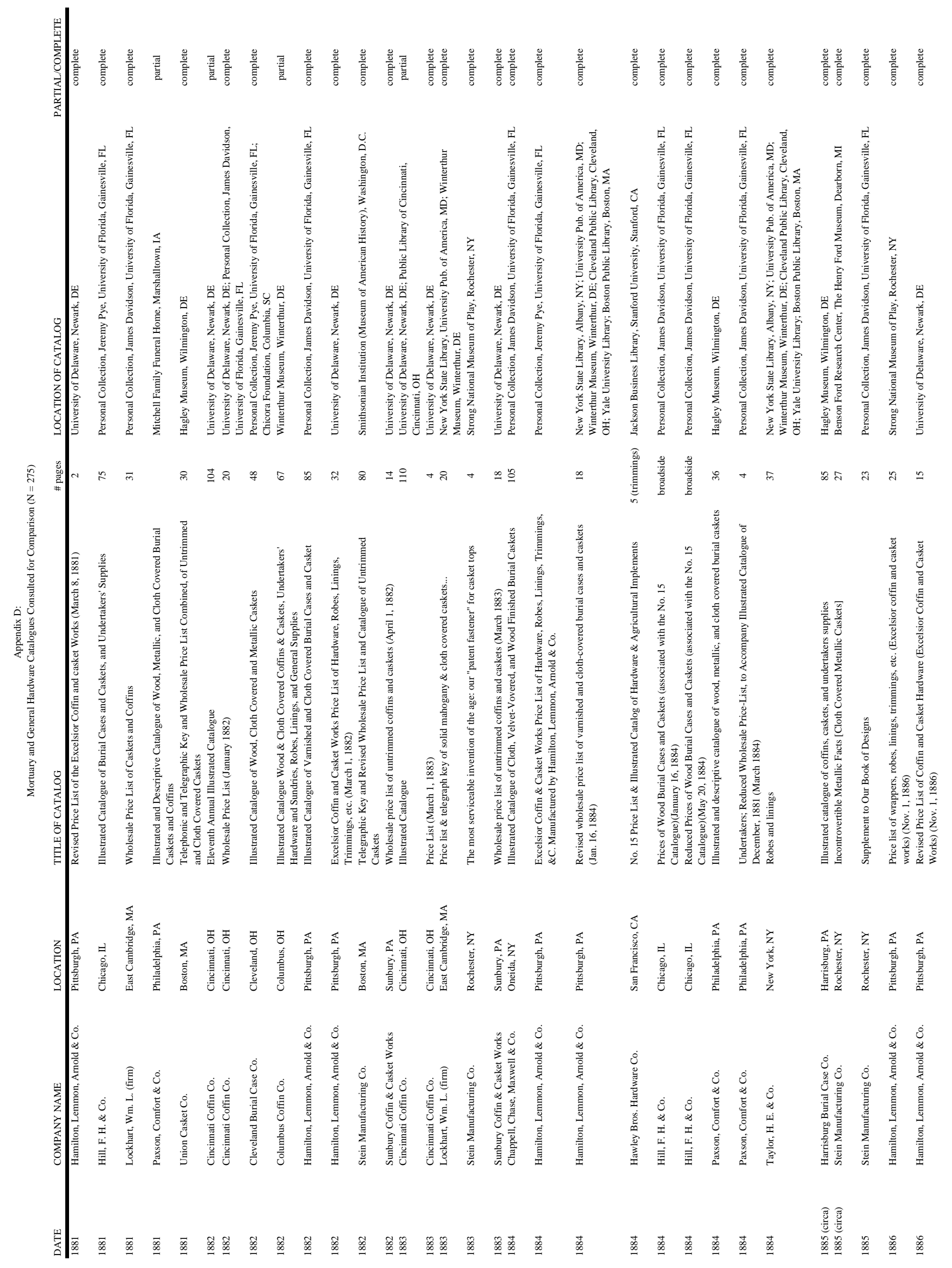




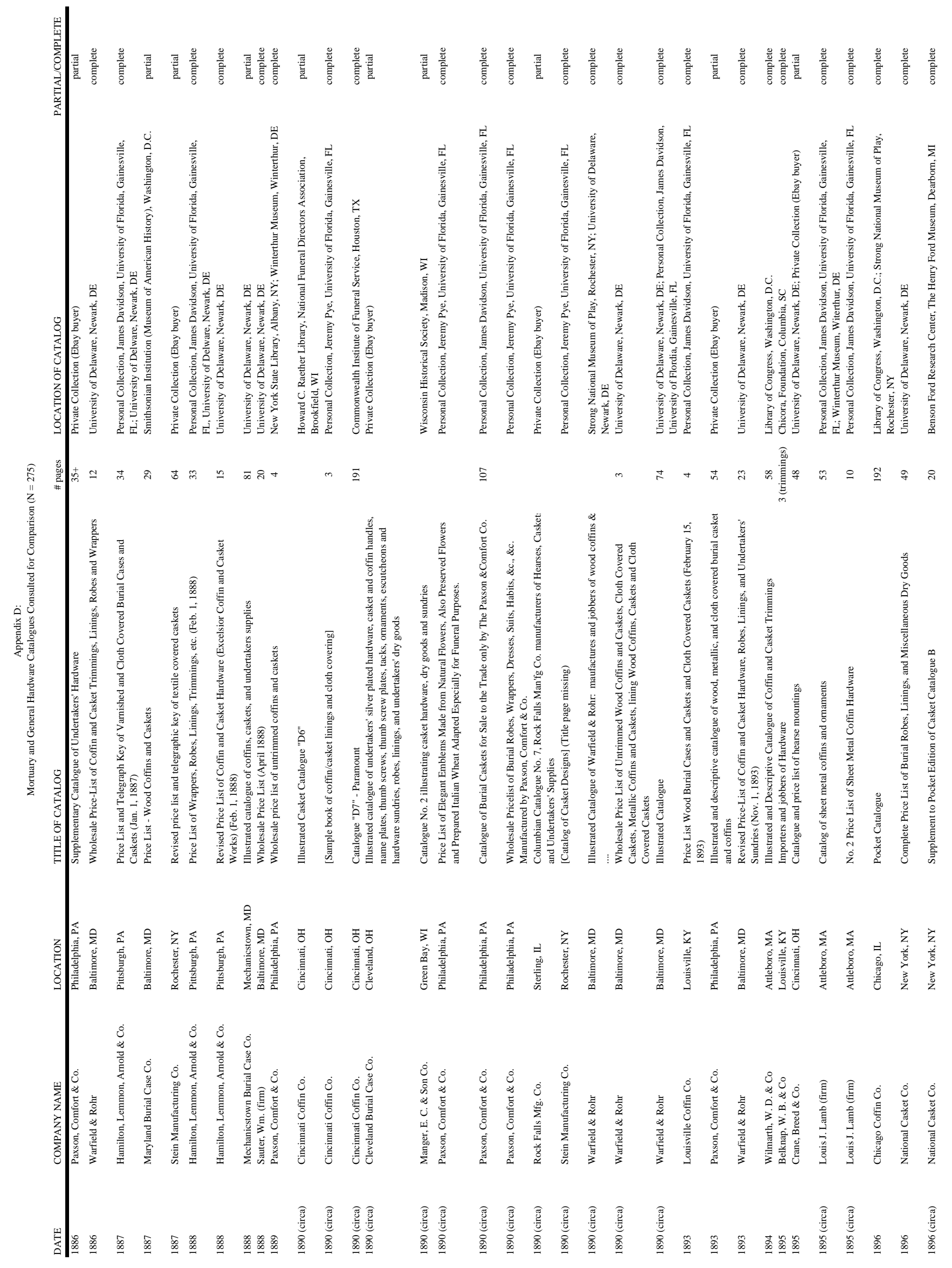




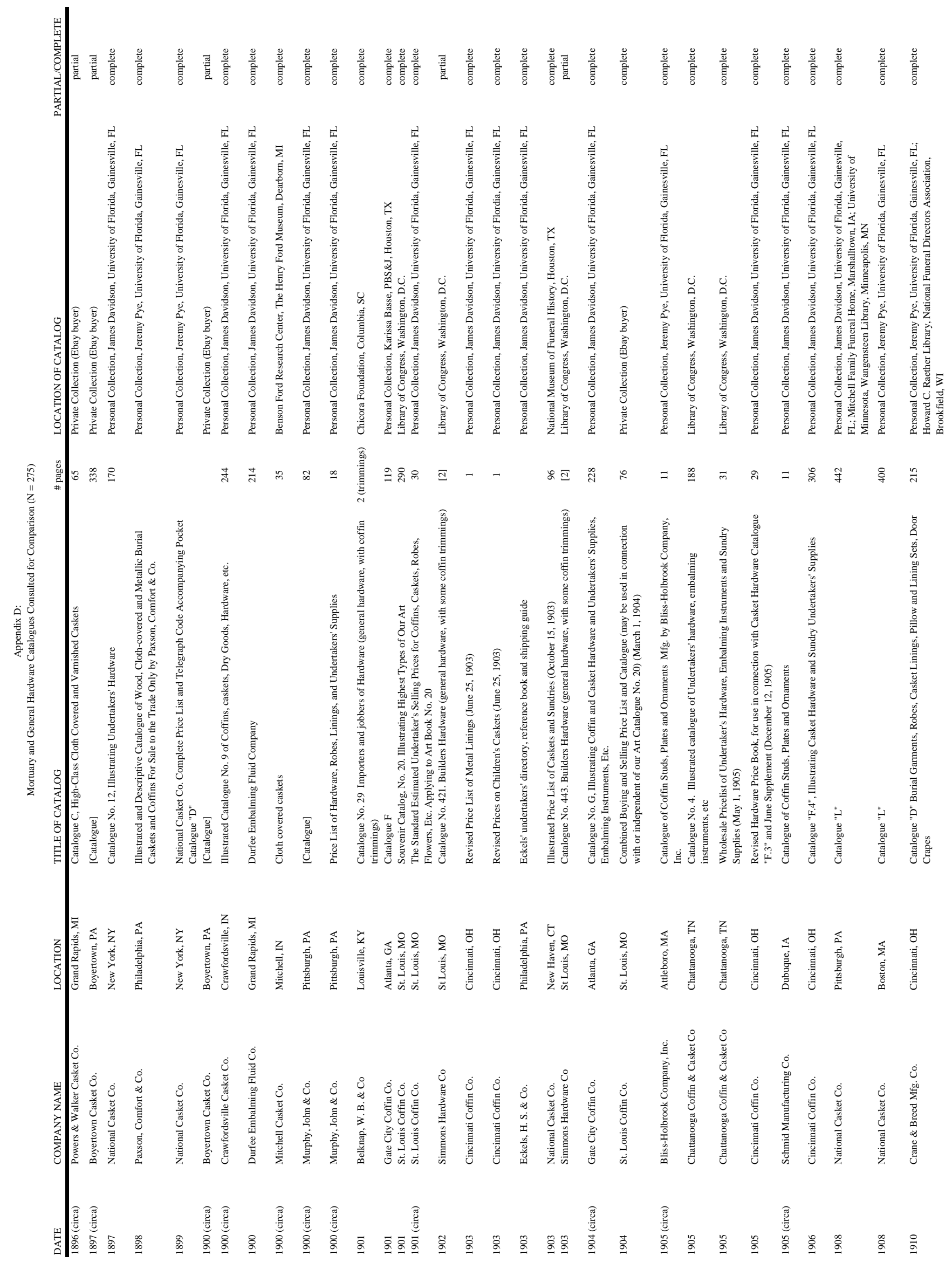




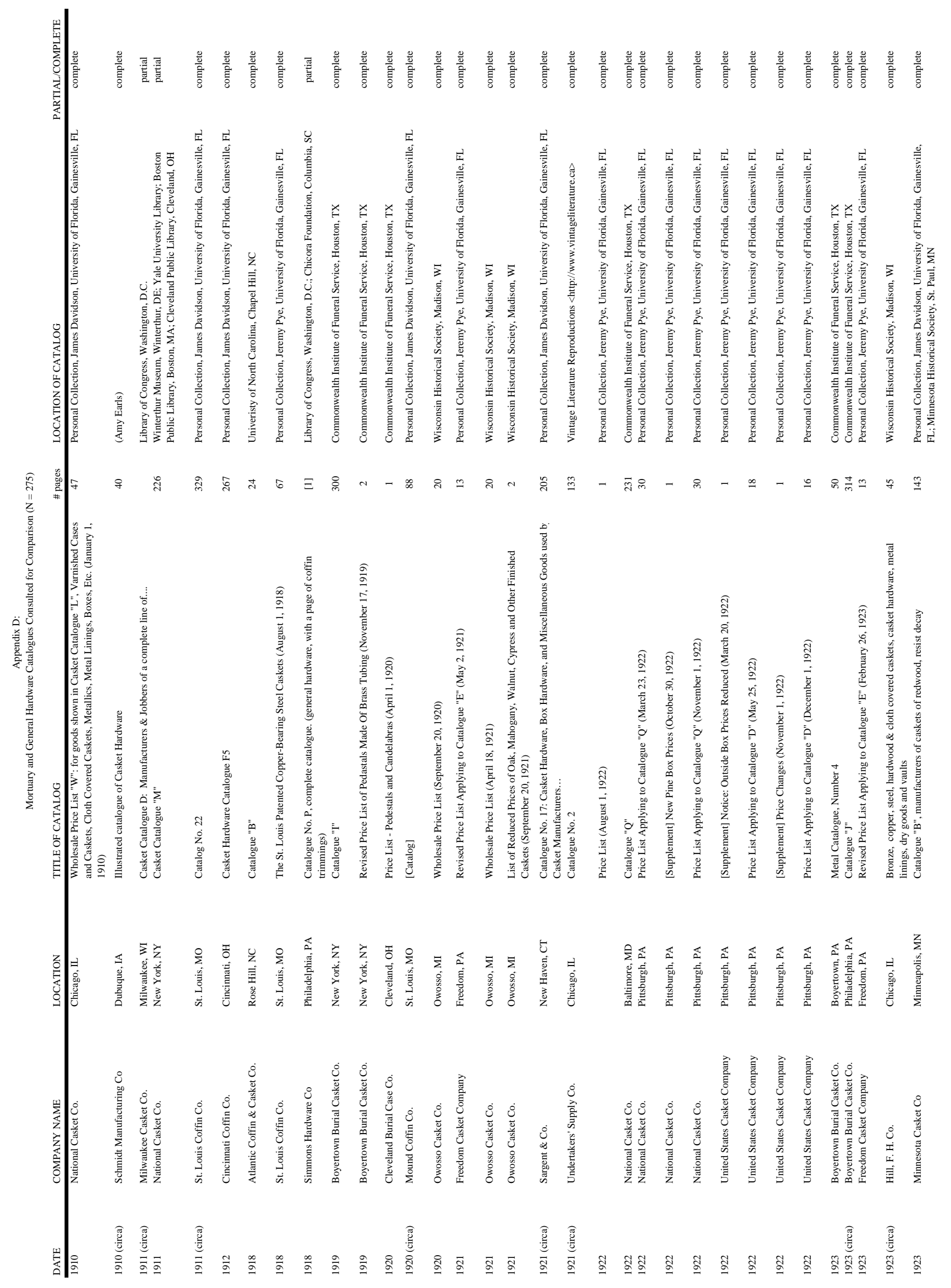




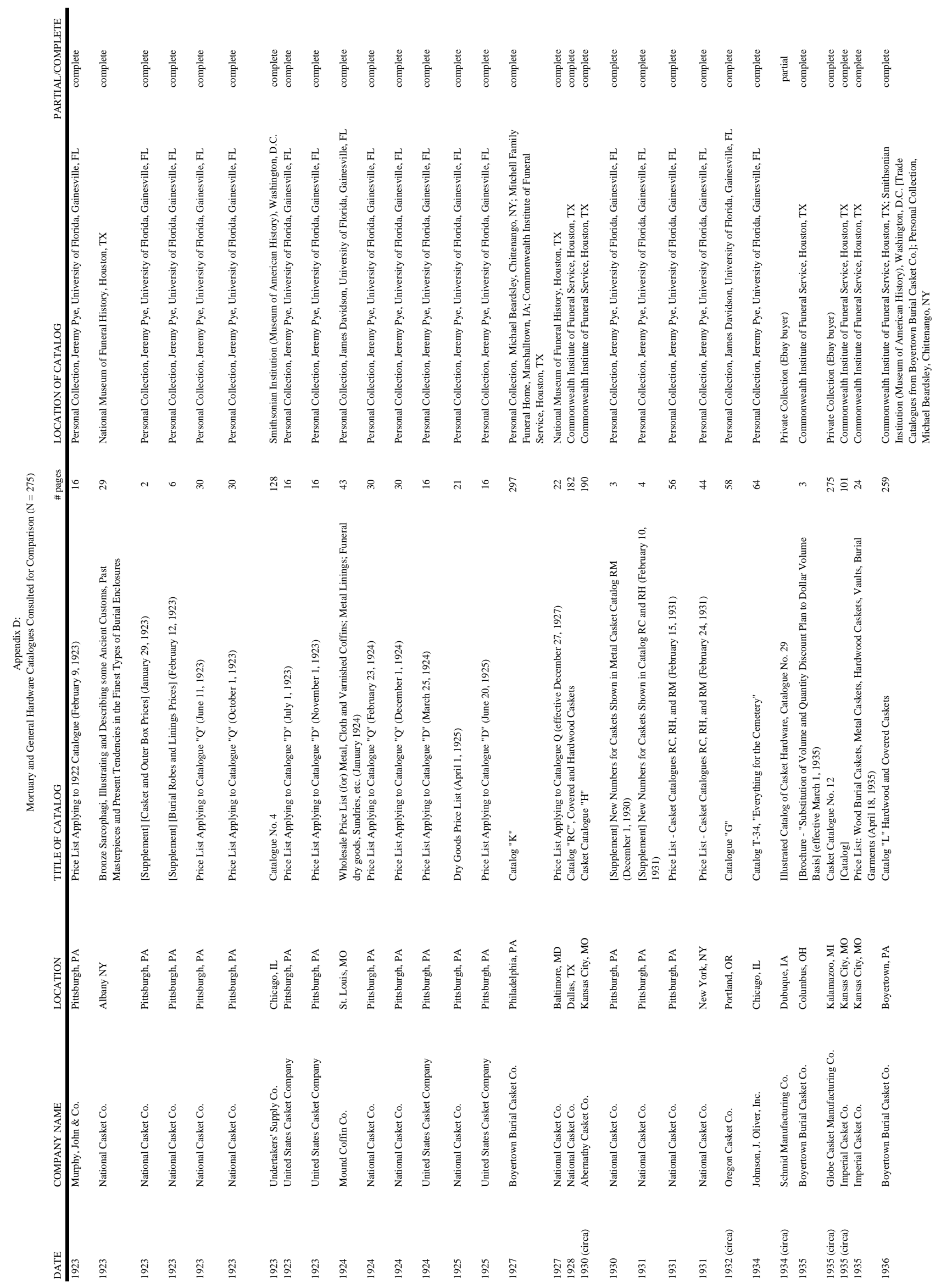




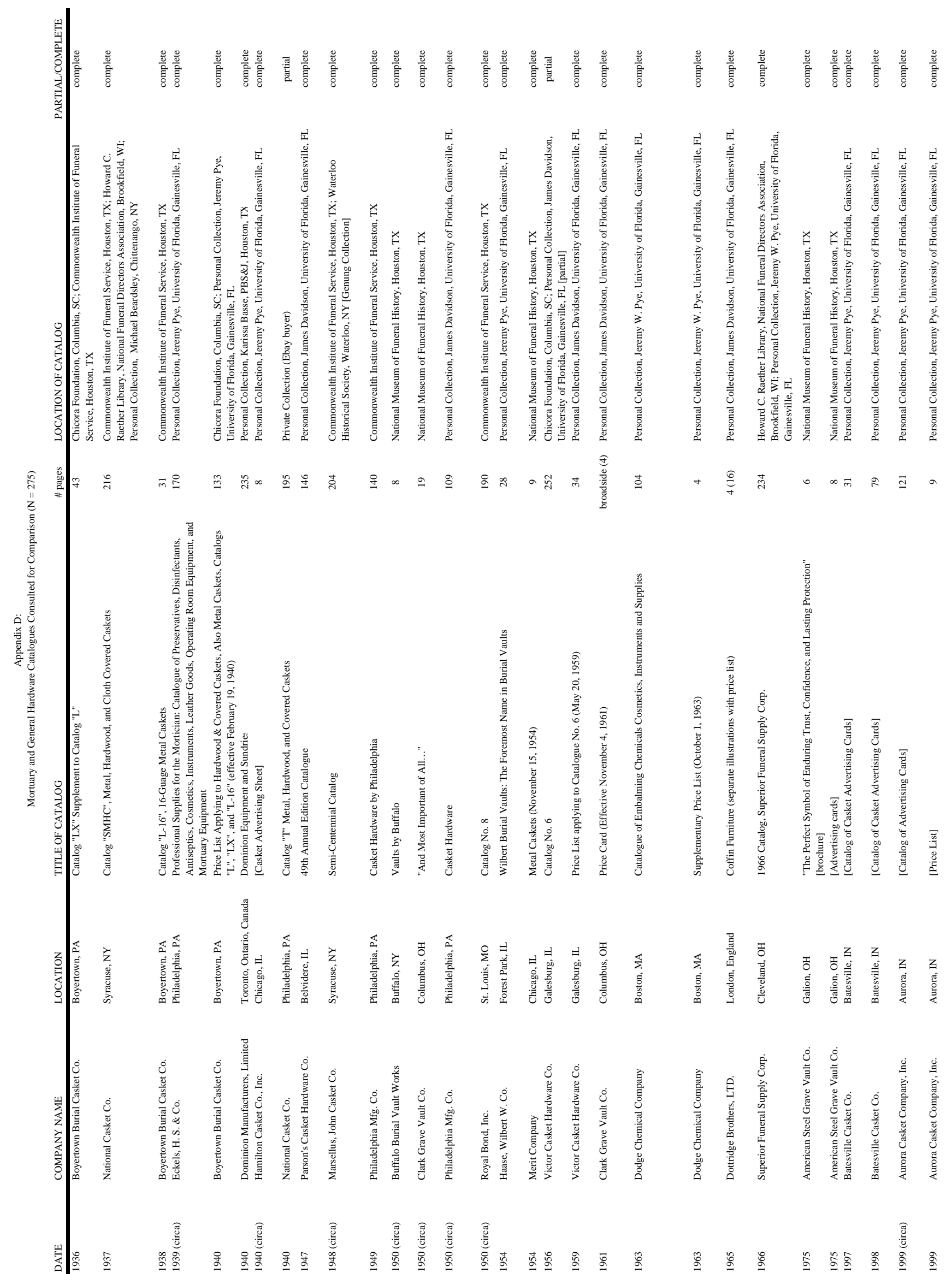




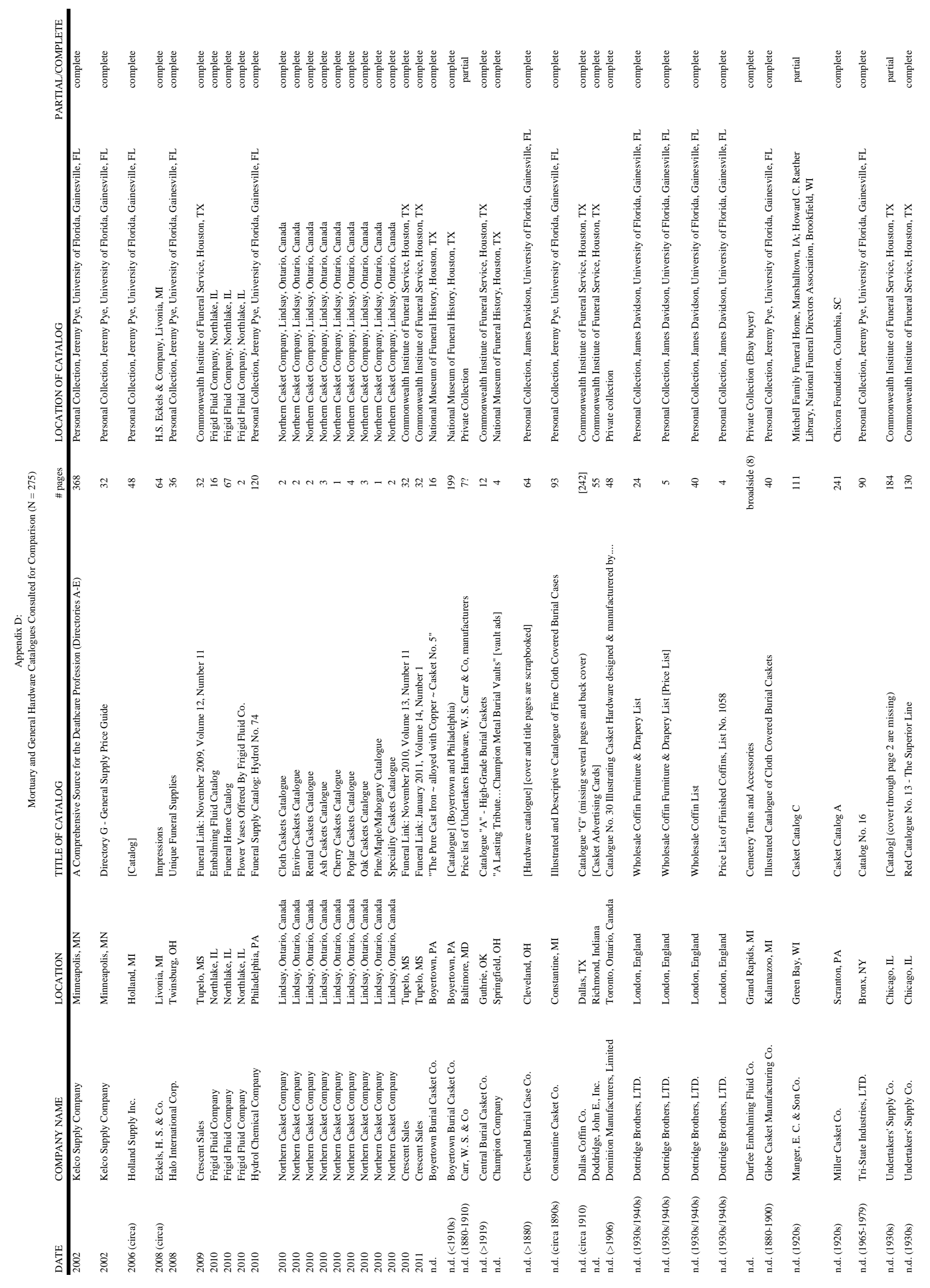




\section{APPENDIX E \\ LISTING OF COMPARISON CEMETERIES}





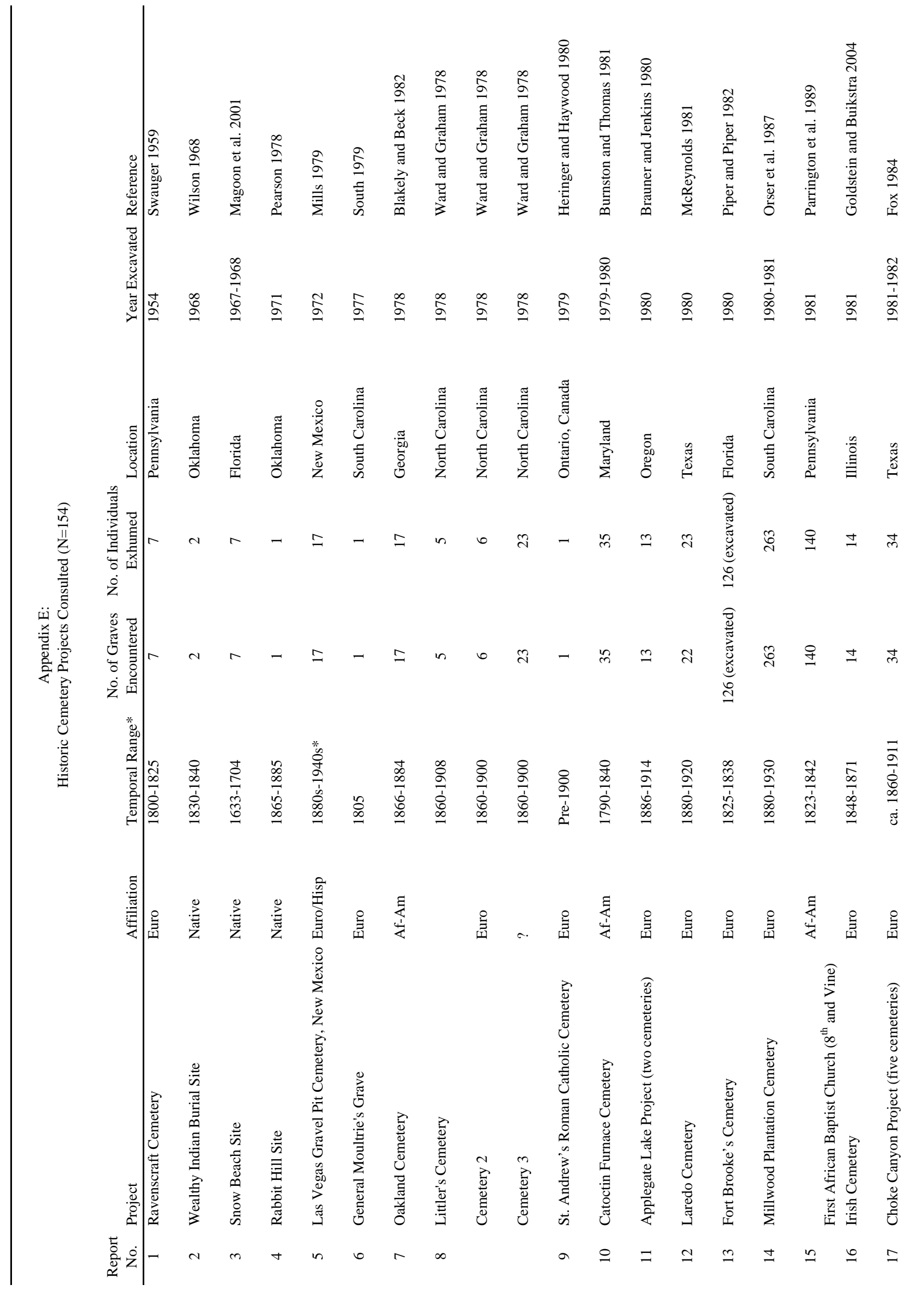




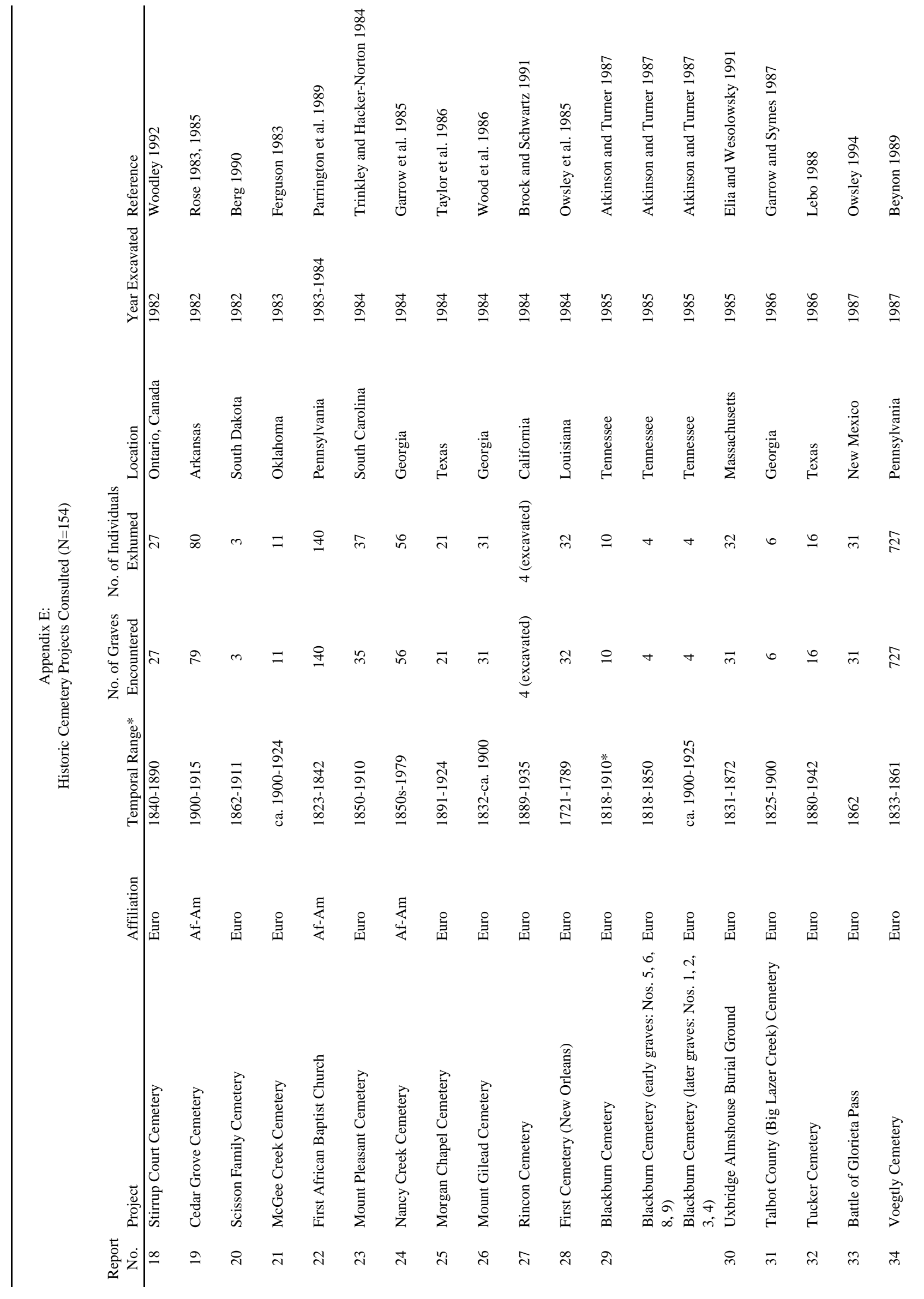




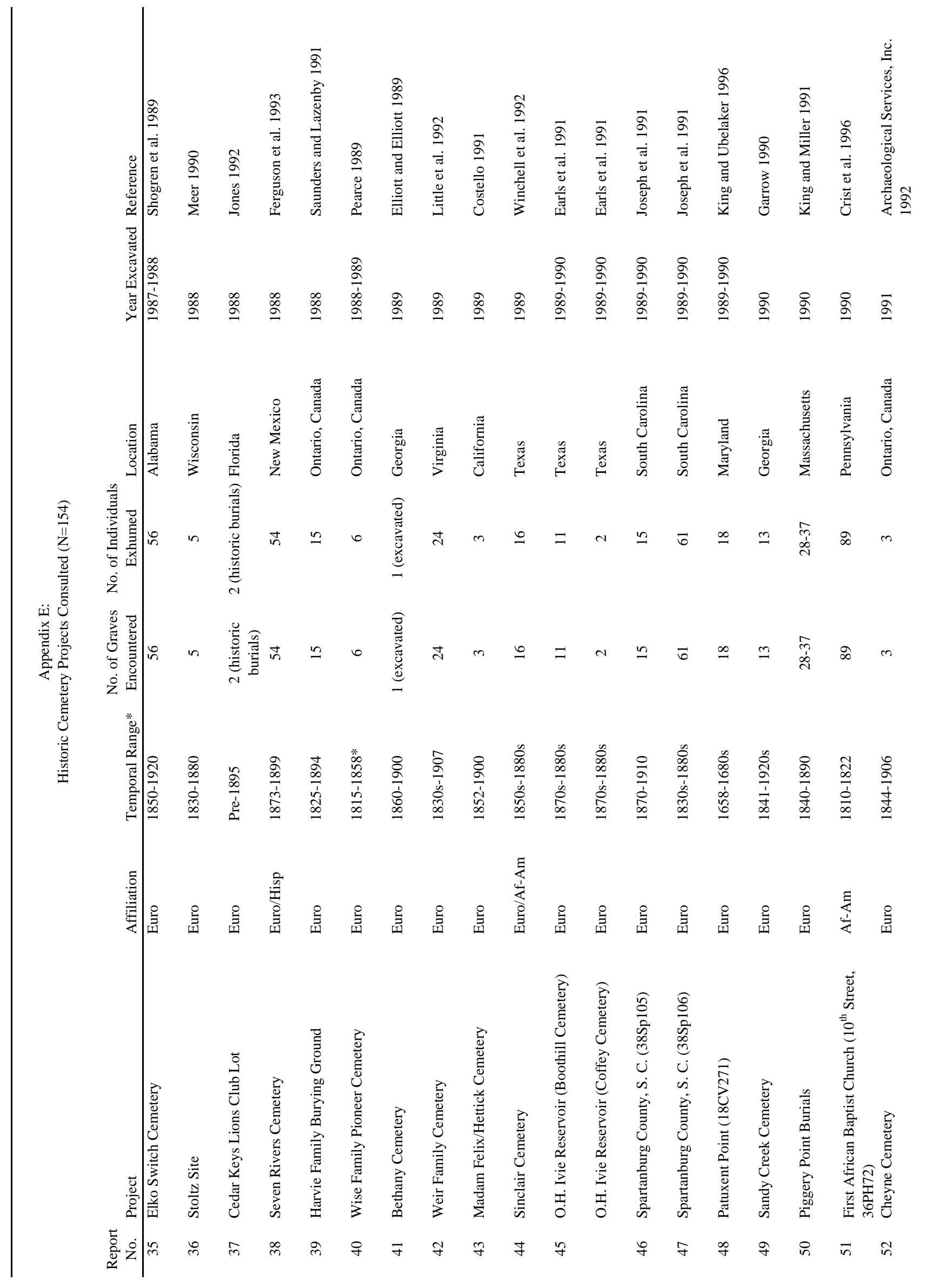




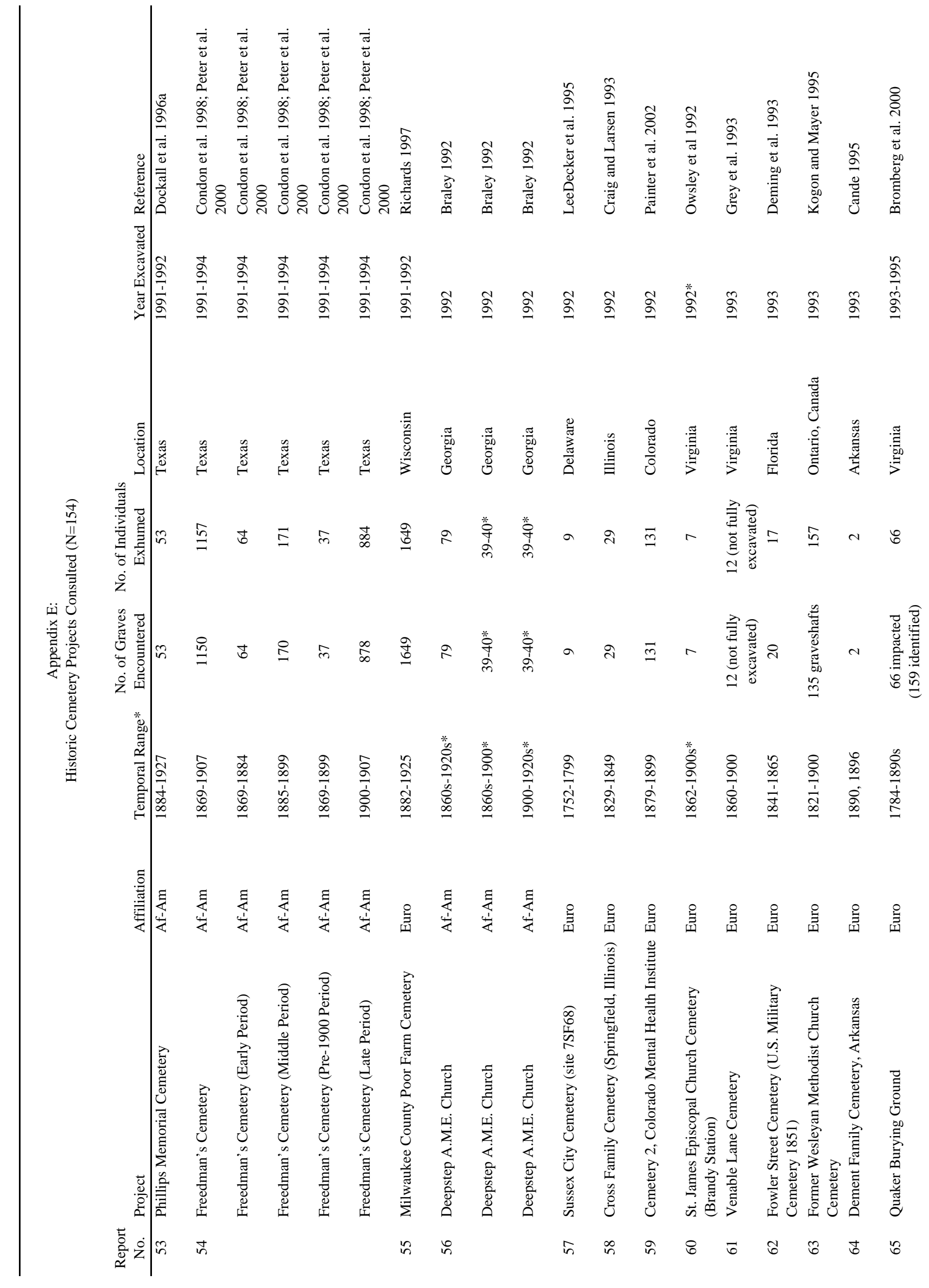




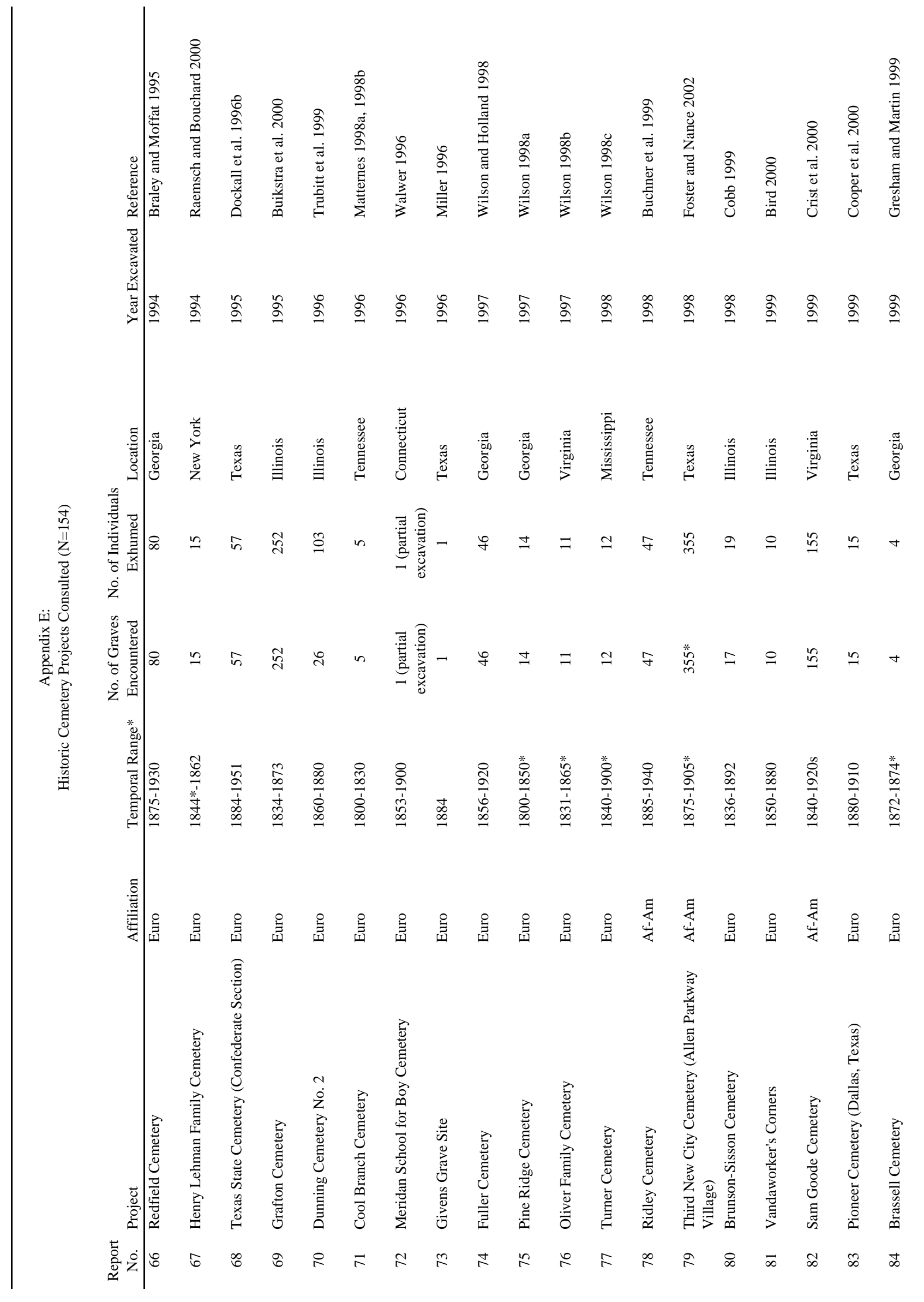




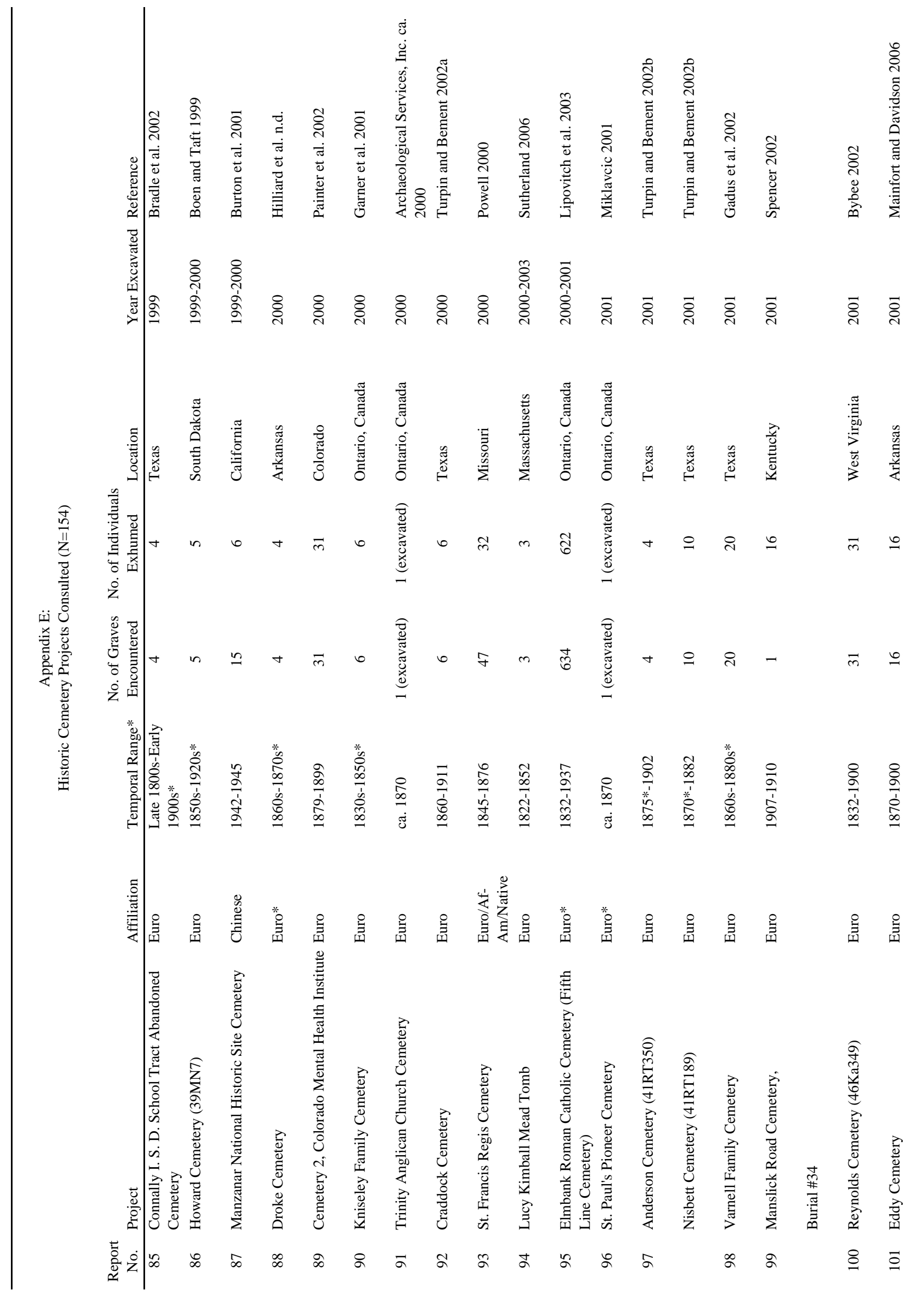




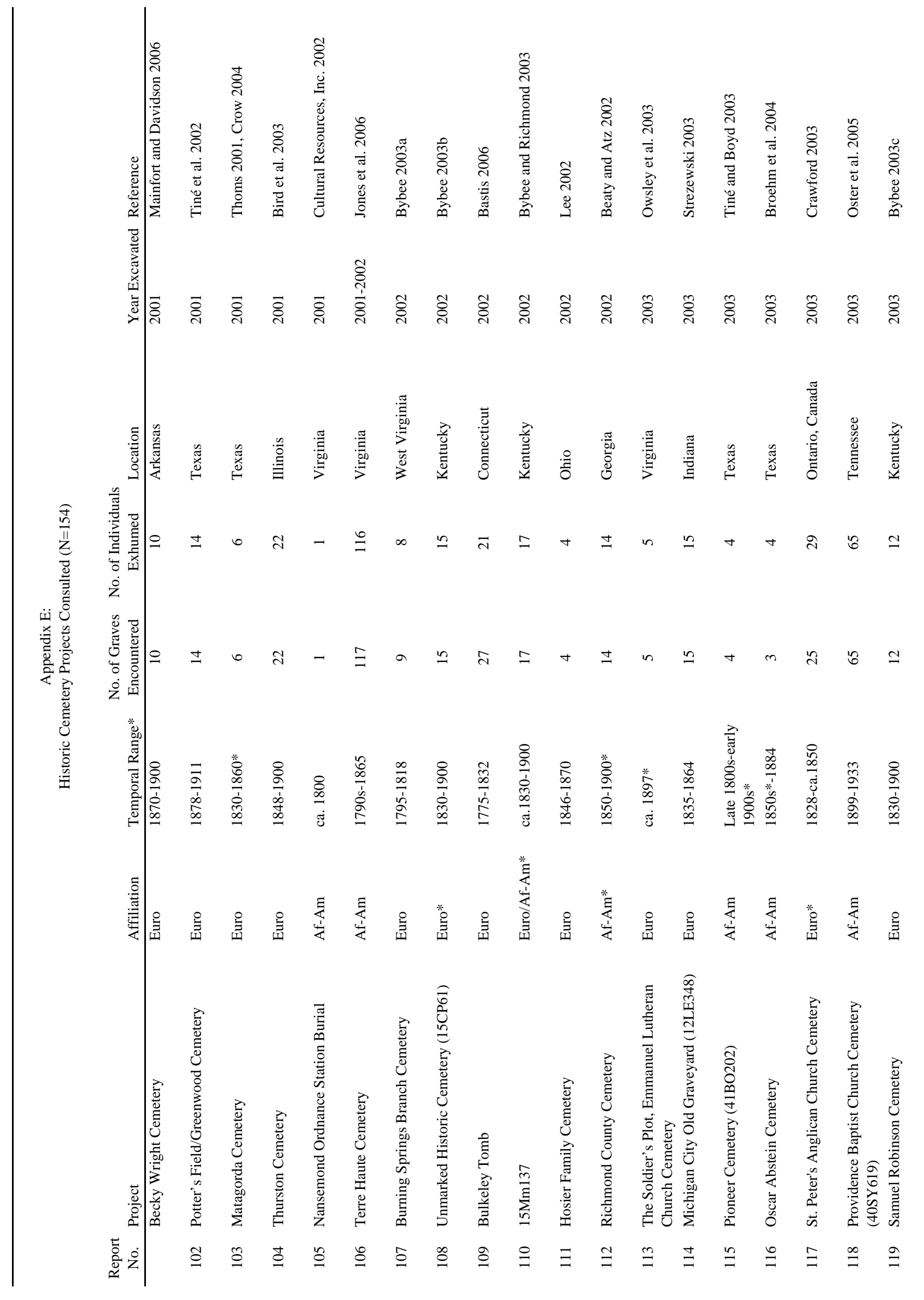




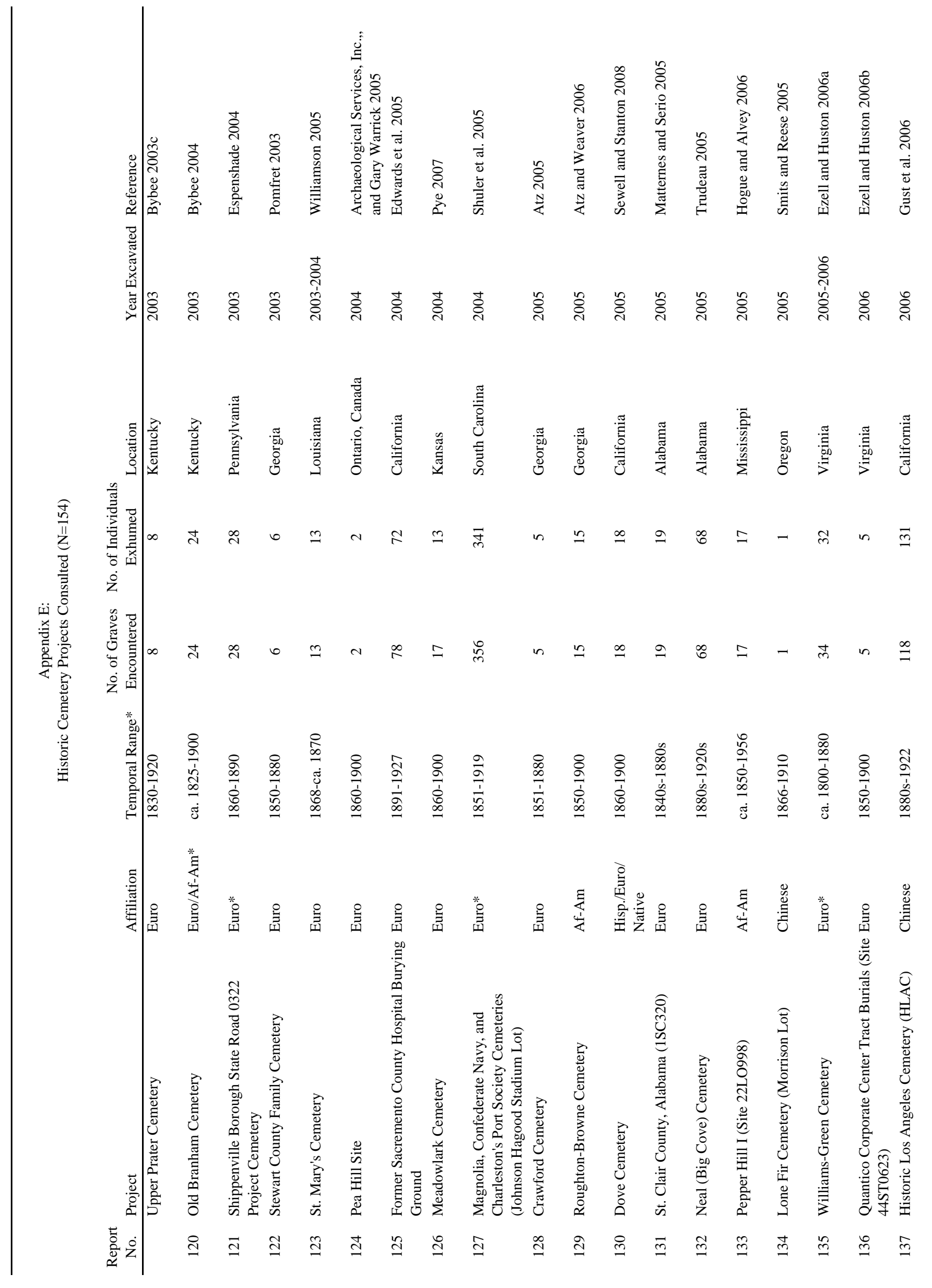




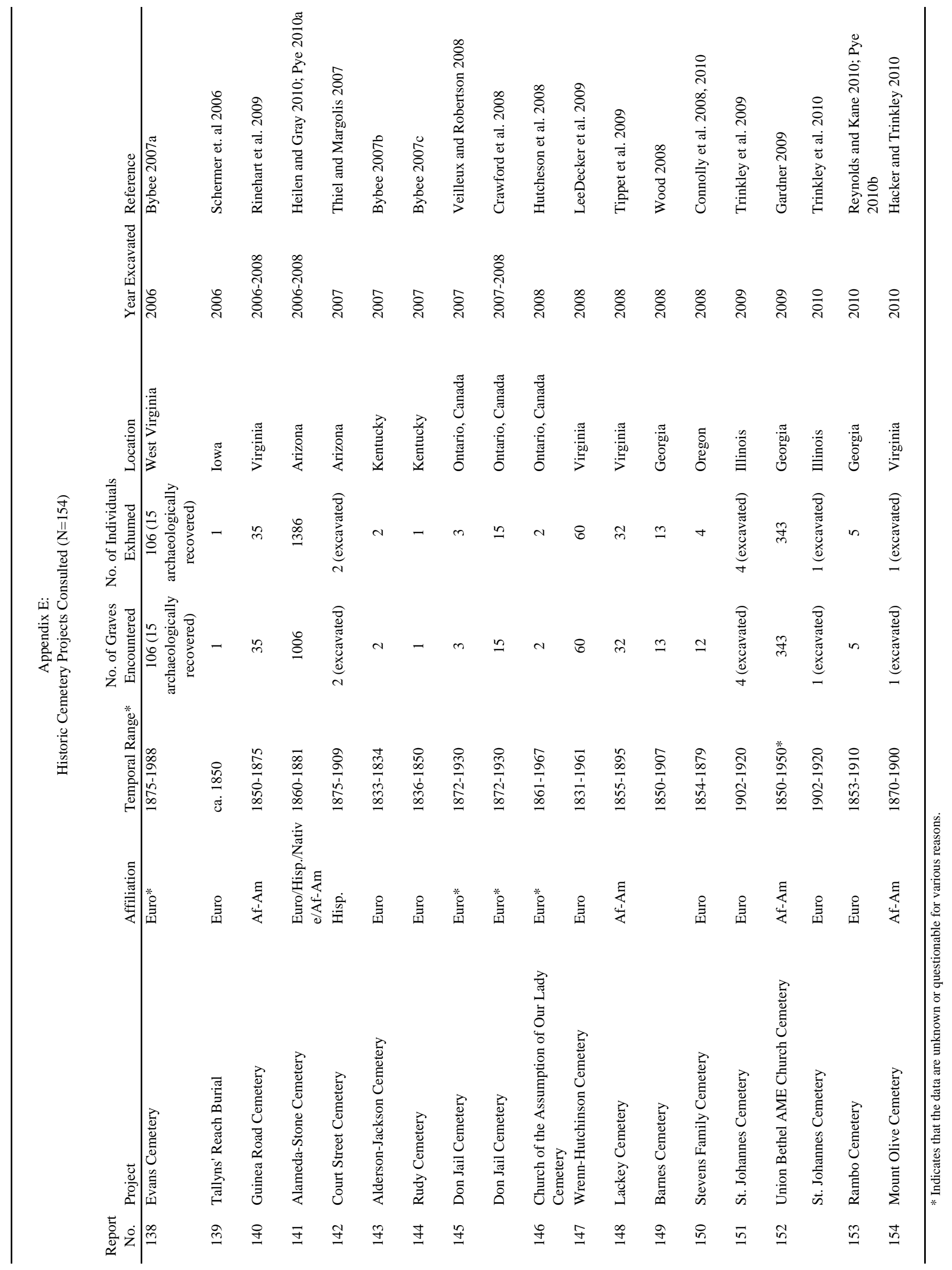





\section{APPENDIX F \\ EVIDENCE OF PREHISTORIC OCCUPATION AND \\ LITHIC ARTIFACT RAW DATA}





\section{EVIDENCE OF PREHISTORIC OCCUPATION}

The search for burials and their disinterment resulted in the discovery of evidence of prehistoric use of this landform. This evidence was in the form of an area of past thermal activity, designated Feature 1, and the presence of six lithic artifacts.

\section{FEATURE 1}

During the search and recovery for human remains, a large dark soil stain with the appearance of ash like deposits and charcoal was uncovered approximately 1.26 meters (4.1 feet) below surface. The majority of the stain matrix consisted of a dense, dry, dark brown (7.5YR 3/4) clay with charcoal stains (10YR 2/1), a burned/baked clay appearance of strong brown (7.5YR 4/6) clay, and a lighter color of reddish brown (5YR 4/4) clay inclusions. Artifacts collected near the surface of the stain, out of the wall of the trench cut by the backhoe, included a piece of glass, two small pieces of lithics, and a 1912 5-cent piece. The surface of the stain was irregular in shape (Figure F-1) and there appeared to be no recognizable pattern. The area with the highest concentration of charcoal and ash was bisected and excavated (Figure F-2). Only small amounts of charcoal and baked clay were collected and no additional artifacts including debitage or bone were observed. Only two levels of the feature were hand-excavated by trowel in 5-cm levels before the dark staining began to fade at approximately 1.36 meters ( 4.46 feet) below surface. The profile of the feature also did not reveal any recognizable pattern and appeared to be highly

disturbed. Excavation with the backhoe continued down to the proper depth for the detection of burials and no additional soil stains, artifacts, or burials were encountered.

\section{LITHIC ARTIFACTS}

In addition to this potential cultural feature, six pieces of lithics were recovered. Two lithic specimens were recovered in association with Burial 19 at approximately 0.50 meters (1.6 feet) below surface; two specimens were recovered from the exposure of Feature 1, and the remaining lithic specimens were isolated/surface finds collected as the backhoe was moving through the soil. All of these finds were derived from river cobbles. The two specimens associated with Feature 1 consist of a quartzite flake and a piece of shatter from a very glossy, honey-colored chert. The quartzite flake is 27.5 millimeters $(\mathrm{mm})$ long, $30.7 \mathrm{~mm}$ wide, and $11.6 \mathrm{~mm}$ thick. It exhibits a cortical platform. The maximum dimension of the shatter measures $23.3 \mathrm{~mm}$ and one surface exhibits a waxy patina. The specimens associated with Burial 19 consist of a tested quartzite cobble and a possible core fragment of a fossiliferous tan chert. The discoidal-shaped cobble exhibits cortex at several points around its perimeter and measures $41.4-x-37.3-x-20.2$ $\mathrm{mm}$. The core fragment $(27.2-\mathrm{x}-20.7-\mathrm{x}-19.0 \mathrm{~mm})$ is a portion of the platform of the core with cortex on two surfaces. The surface finds consist of a tested chert cobble and a quartzite flake. The tested cobble is a tannish brown chert with a darker brown cortex present. It is $49 \mathrm{~mm}$ long, $46.2 \mathrm{~mm}$ wide, and $28.3 \mathrm{~mm}$ thick. The quartzite flake (47.8-x-46.1-x-19.9 mm) exhibits cortex over 100 percent of its dorsal surface and a prepared platform. 


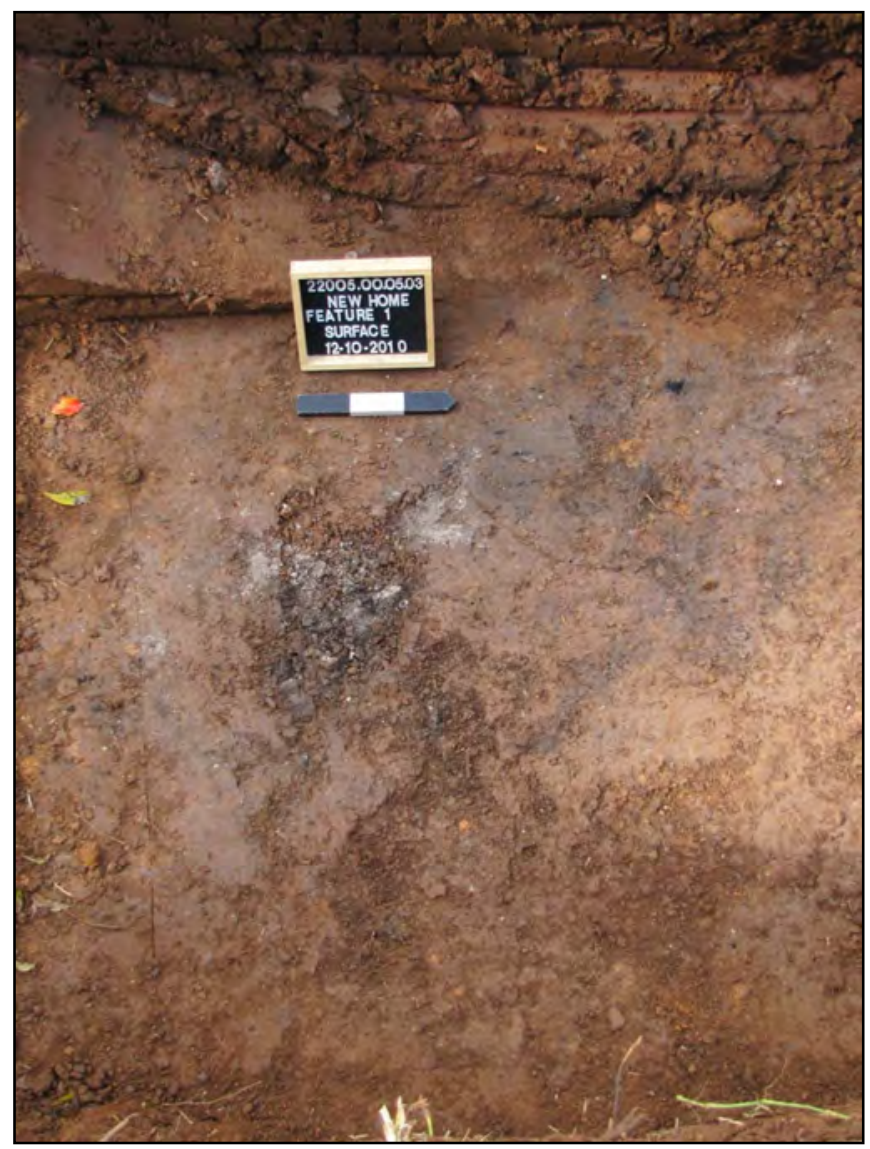

Figure F-1. Plan view of Feature 1.

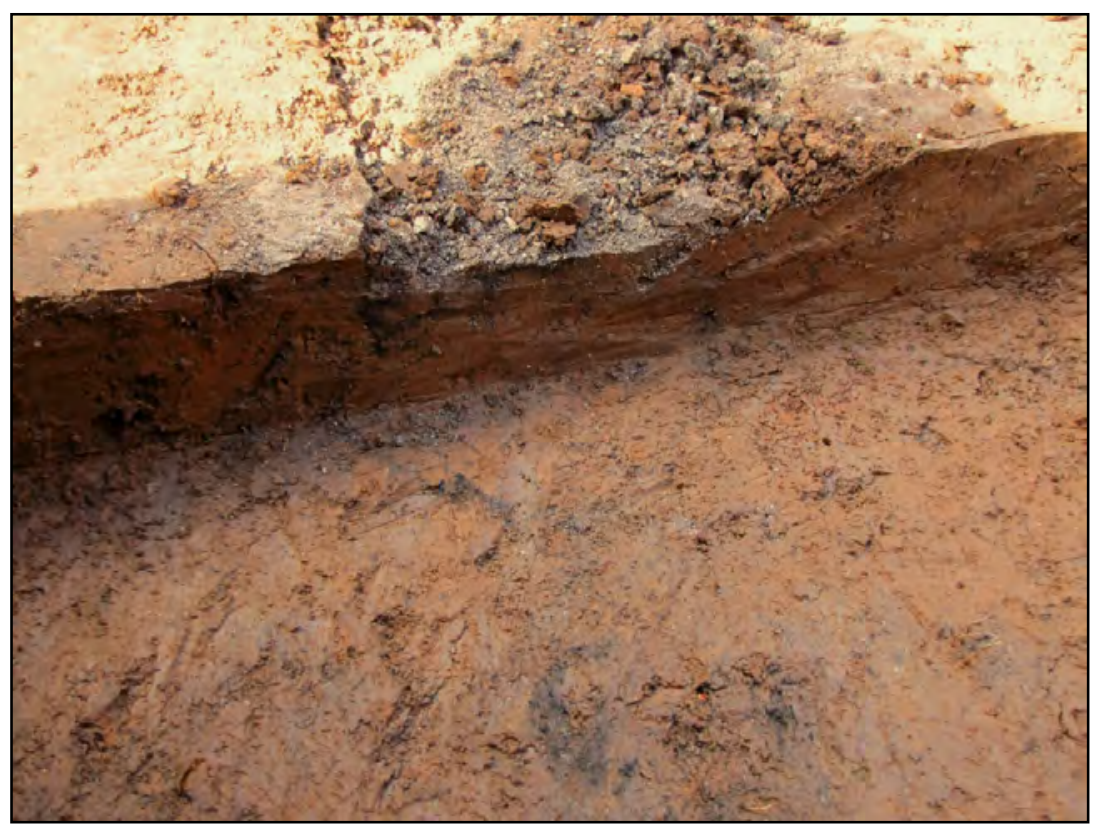

Figure F2. Profile view of bisected Feature 1. 
It is apparent that limited prehistoric period activities occurred on this landform; however, the disturbance to the site through interments, disinterments, prior archeological testing and excavation of the right-of-way have severely damaged any contextual integrity that the prehistoric assemblage might have had. Furthermore, the introduction of fill dirt to level the surface of the cemetery and prevent flooding may have inadvertently introduced some of the these prehistoric artifacts, particularly those found within the upper $61 \mathrm{~cm}(2 \mathrm{ft})$ of the deposits. 



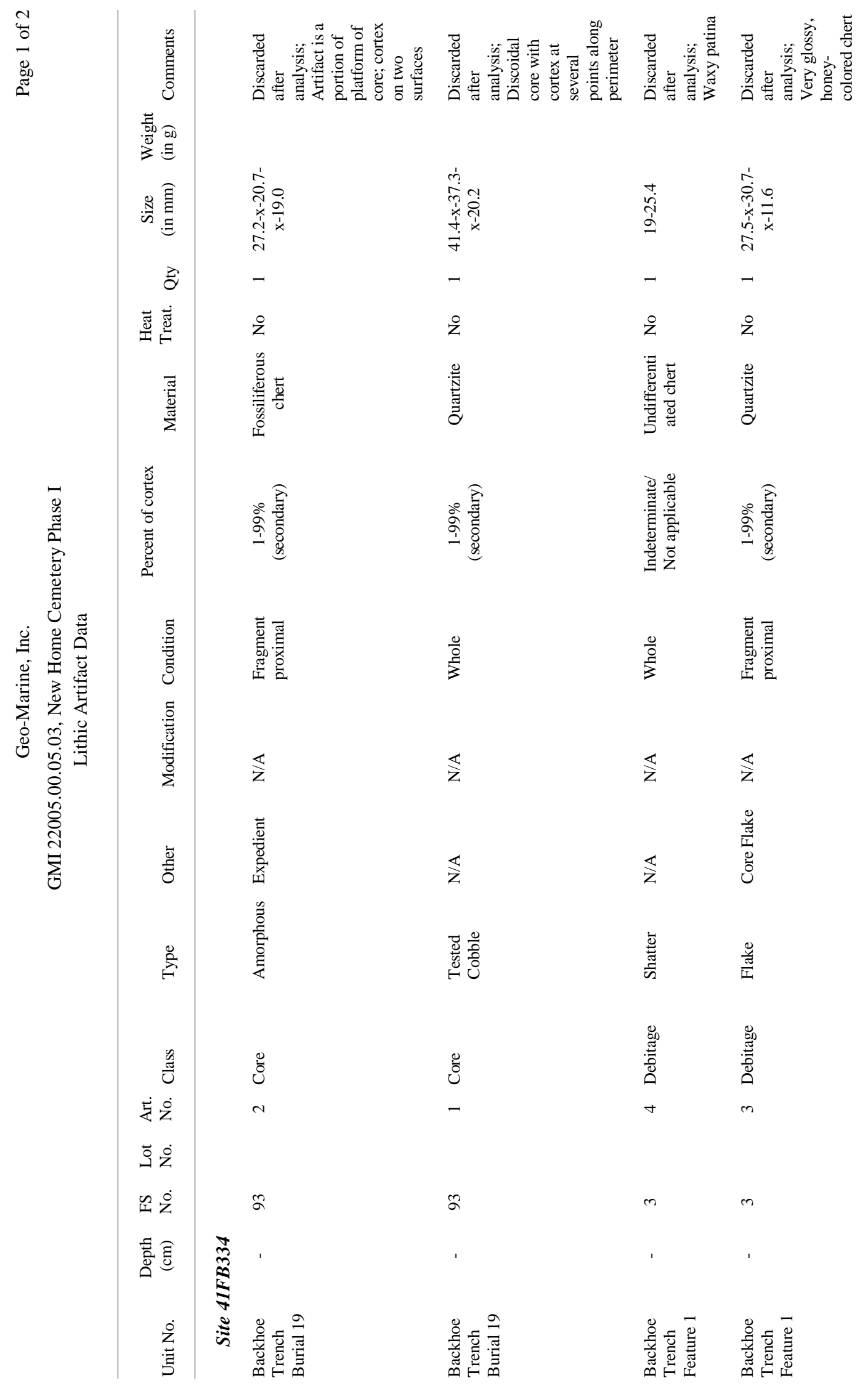




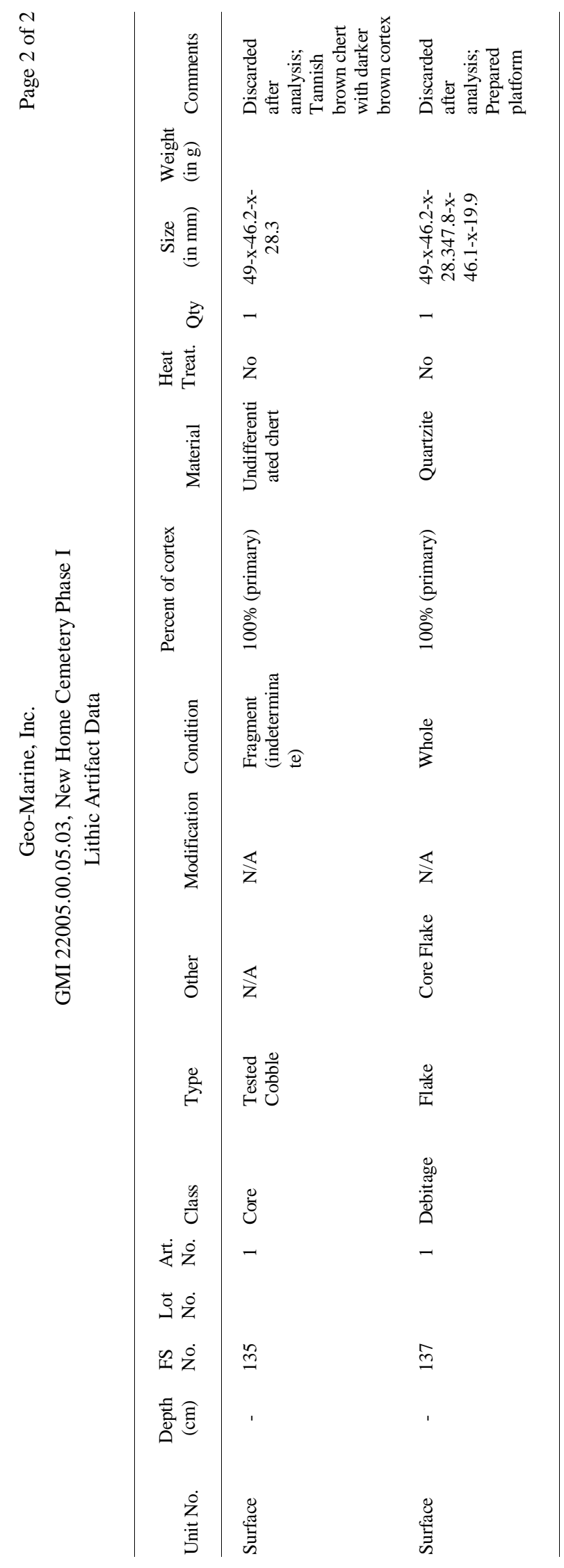

